\title{
SELF-ASSEMBLY OF CALIX[4]ARENE AMINE DERIVATIVES
}

\author{
A thesis submitted by \\ Laura O’Toole B.Sc. (Double Hons.) \\ to the

\begin{abstract}
National University of Ireland, Maynooth
\end{abstract} \\ For the degree of Doctor of Philosophy

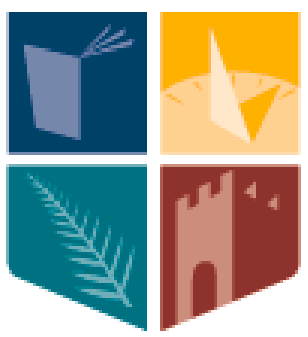 \\ NUI MAYNOOTH \\ Ollscoil na hÉireann Má Nuad \\ Volume 1 of 1
}

Based on the research carried out in the

Department of Chemistry, Faculty of Science, National University of Ireland, Maynooth

under the supervision and direction of

$$
\text { Dr. John McGinley }
$$

Head of Department: Dr. John Stephens

June 2013 


\section{Declaration}

I hereby certify that the material, which I now submit for assessment on the programmes of study leading to the award of a $\mathrm{PhD}$, is entirely my own work and has not been taken from the work of others save to the extent that such work has been cited and acknowledged within the text of my own work. No portion of work contained in this thesis has been submitted in support of an application for another degree or qualification to this or any other institution.

Signed:

Date:

Laura O’Toole

I hereby certify that the unreferenced work described in this thesis and being submitted for award of a $\mathrm{PhD}$, is entirely the work of Laura O'Toole. No portion of the work contained in this thesis has been submitted in support of an application for another degree or qualification to this or any other institution.

Signed:

Date:

Dr. John McGinley 


\section{Acknowledgements}

First of all I would like to thank my supervisor Dr. John McGinley for his continued help, support and friendship over the last four years. At many stages in the course of this research project I benefited from his advice, particularly so when exploring new ideas. His positive outlook and confidence in my research inspired me and gave me confidence. One could not ask for a better supervisor and for that I am truly grateful. I would like to thank Dr. Bernie Creaven for all her help and support over the past four years, the time spent with me on the NMR machine, as well as the tea-breaks, will never be forgotten.

I would sincerely like to thank some of the most important people in the department, Niamh, Donna, Carol and Noel, whose advice, support and friendship have been invaluable over the years. The department could not function without you, from the photocopying machine, to fixing computers, you are truly the heart of the department.

I would also like to thank the academic staff, in particular Dr. John Stephens and Dr. John Colleran for all there help and support over the years at NUIM. From queries in the lab and playing sports on the field, it was fantastic experience. I would like to extend a heartfelt thanks to all the technicians Ria, Ken, Barbara, Ollie, Ann, Walter, Orla and Maryanne whose help with demonstrating labs, training on analysing instruments as well as any safety questions were invaluable.

Thanks to all the postgrads, old and new, who welcomed me when I started, particularly Ciarán M, Declan, Denis, Walshy, Eoin and Martin. I would also like to thank Niall M. who started alongside with for all his help and support over the last four years. I would also like to thank my parents for their support over the past few years. 


\begin{abstract}
A new family of calix[4]arene amine derivatives, which have been functionalised on both the upper and lower rim have been developed as potential duel metal ion binding complexes, and all were fully characterised. The lower rim was first functionalised with various groups such as benzyls and pyridyls to give stability to the calix[4]arene unit, followed by functionalisation of the upper rim with an amine, such as 2-(aminomethyl)pyridine, and/or a bipyridine moiety. The derivatives were tested with a wide range of metal salts, $\mathrm{MX}_{2},\left(\mathrm{M}=\mathrm{Zn}^{2+}, \mathrm{Cu}^{2+}, \mathrm{Ni}^{2+}, \mathrm{Hg}^{2+}, \mathrm{Co}^{2+}\right.$ and $\mathrm{X}=$ acetate, chloride, perchlorate).

During the course of this project it was noted that the ${ }^{1} \mathrm{H}$ NMR spectra were much more complex than had been expected. To this end a series of nOe, serial dilution, high temperature and HMBC NMR spectroscopic techniques were employed. The results of these revealed that self-assembly was occurring in solution and it is believed that two kinds of self-assembled structures are present, namely the heterodimerisation form and the homodimerisation form. It is believed that due to the loss of the $t$-butyl groups early in the synthesis that this allows extra flexibility in the calix[4]arene unit, thereby allowing it to alternate between its different conformers. Metal ion complexation was performed to try and control the selfassembly but to no avail.
\end{abstract}

Fluorescence studies were performed on several compounds to further examine the metal-ligand interactions. It was found that with the addition of the metal ions intensity had an overall increase in intensity and the fluorescence bands were blueshifted with respect to the parent compound fluorescence bands.

Unfortunately suitable crystals were not obtained for X-ray analysis. SEM was performed on several of the compounds and their corresponding metal complexes and the images obtained give some insight into the morphology of the structures. 


\section{List of Abbreviations}

\begin{tabular}{|c|c|}
\hline $\mathrm{AcOH}$ & Acetic Acid \\
\hline $\mathrm{ACN}$ & Acetonitrile \\
\hline $\mathrm{AlCl}_{3}$ & Ammonium chloride \\
\hline $\mathrm{Ar}$ & Aryl \\
\hline $\mathrm{Br}$ & Broad Signal \\
\hline $\mathrm{CDCl}_{3}$ & Deuterated Chloroform \\
\hline $\mathrm{CH}_{2} \mathrm{Cl}_{2}$ & Dichloromethane \\
\hline $\mathrm{CHCl}_{3}$ & Chloroform \\
\hline $\mathrm{Cq}$ & Quaternary Carbon \\
\hline$\delta$ & Chemical Shift \\
\hline $\mathrm{d}$ & Doublet \\
\hline dd & Doublet of Doublets \\
\hline DCM & Dichloromethane \\
\hline DMF & Dimethylformamide \\
\hline DMSO & Dimethyl sulphoxide \\
\hline${ }^{\circ} \mathrm{C}$ & Degrees Celsius \\
\hline $\mathrm{EtOH}$ & Ethanol \\
\hline $\mathrm{HCl}$ & Hydrochloric acid \\
\hline $\mathrm{Hz}$ & Hertz \\
\hline $\mathrm{HNO}_{3}$ & Nitric acid \\
\hline $\mathrm{Hr}$. & Hour \\
\hline IR & Infrared Spectroscopy \\
\hline$J$ & Coupling constant \\
\hline $\mathrm{K}_{2} \mathrm{CO}_{3}$ & Potassium Carbonate \\
\hline $\mathrm{KBr}$ & Potassium Bromide \\
\hline
\end{tabular}


$\mathrm{KOH}$

L

$\mathrm{MgSO}_{4}$

m

M

$\mathrm{MeOH}$

$\mathrm{Me}_{4} \mathrm{Si}$

m.p.

$\mathrm{N}_{2}$

$\mathrm{NaBH}_{4}$

$\mathrm{NaOH}$

$\mathrm{NaSO}_{4}$

NMR

ppm

q

S

$\mathrm{Sn}(\mathrm{II}) \mathrm{Cl}_{2}$

$\mathrm{Sn}(\mathrm{IV}) \mathrm{Cl}_{4}$

$\mathrm{t}$

TLC

TEA
Potassium Hydroxide

Litre

Magnesium Sulphate

Multiplet

Molar

Methanol

Tetramethyl silane

Melting point

Nitrogen atmosphere

Sodium Borohydride

Sodium Hydroxide

Sodium Sulphate

Nuclear Magnetic Resonance

Parts per million

Quartet

Singlet

Tin(II) Chloride

Tin(IV) Chloride

Triplet

Thin layer chromatography

Triethylamine 


\section{Table of Contents}

Declaration

Acknowledgements ii

Abstract iii

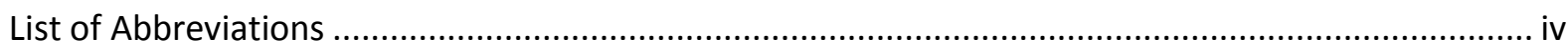

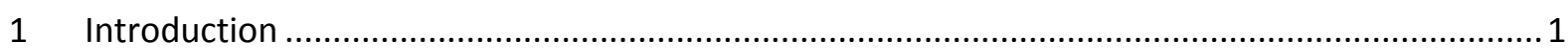

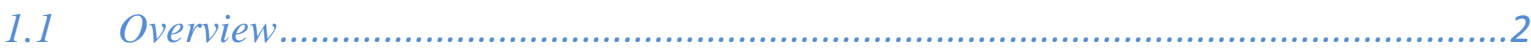

1.2 The chemistry of calix[4] arenes .................................................................

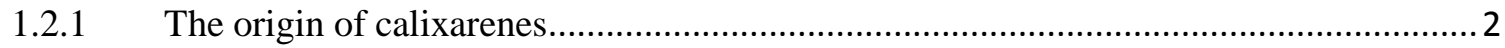

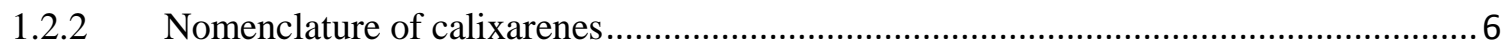

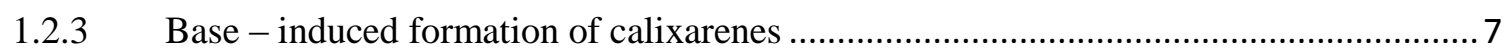

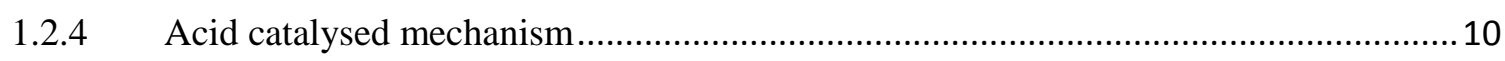

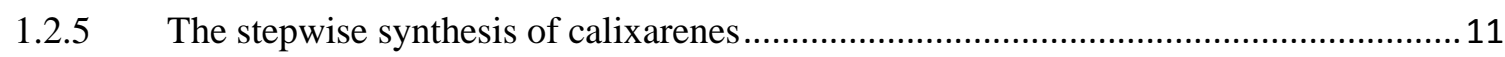

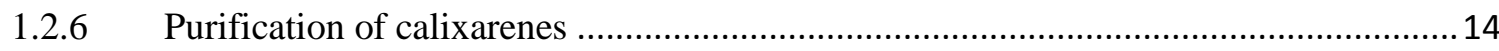

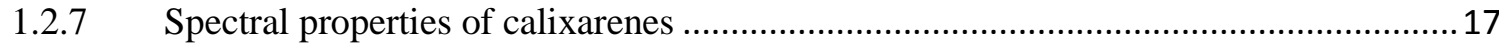

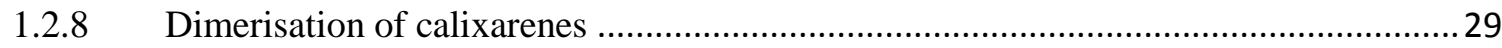

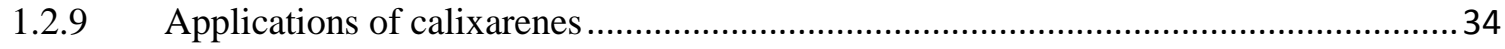

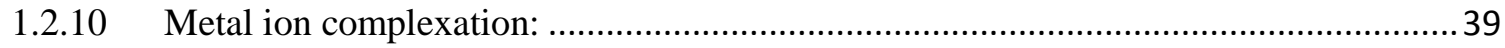

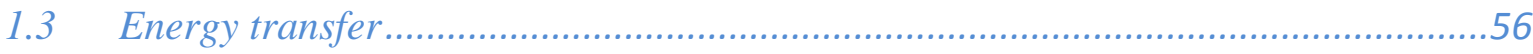

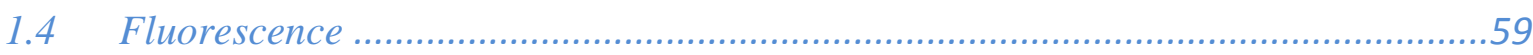

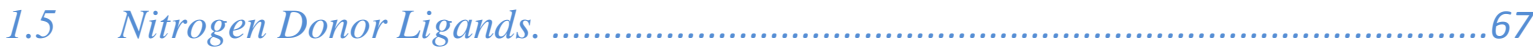

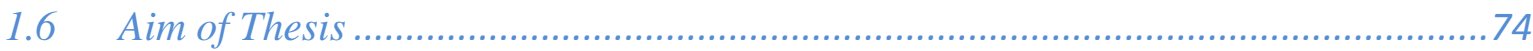

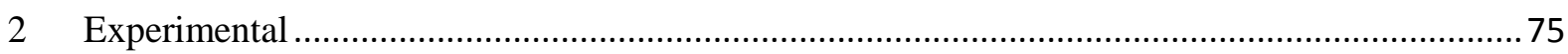

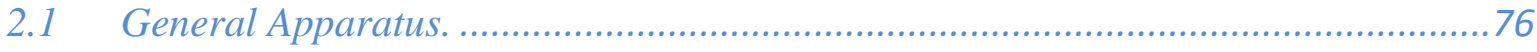

$2.25,11,17,23,29,35,41,47$-Octa-tert-butyl-49,50,51,52,53,54,55,56-octa-

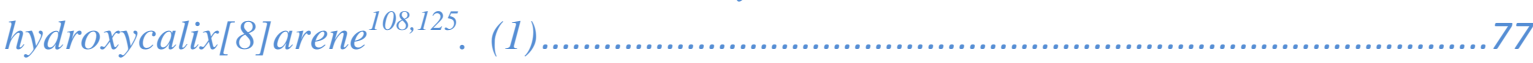

2.3 5,11,17,23-Tetra-tert-butyl-25,26,27,28-tetrahydroxycalix[4]arene $e^{108,125}$

(2) 78

2.4 5,11,17,23-Tetra-tert-butyl-25,27-diethoxycarbonylmethoxy-26,28dihydroxycalix[4]arene $e^{108,126}$.

2.5 5,17-Di-tert-butyl-11,23-dinitro-25,27-diethoxycarbonylmethoxy-26,28dihydroxycalix[4]arene ${ }^{124}$. (4)

2.6 5,17-Di-tert-butyl-11,23-diamino-25,27-dimethoxycarbonylmethoxy-26,28dihydroxycalix[4]arene ${ }^{124}$. (5) 
2.7 5,11,17,23-Tetra-tert-butyl-25,27-dibromoethyl-26,28-

dihydroxycalix[4] arene ${ }^{124}$. (6)

2.8 5,17-Di-tert-butyl-11,23-dinitro-25,27-dibromoethyl-26,28-

dihydroxycalix[4]arene ${ }^{124}$. (7) 83

2.9 5,11,17,23-Tetra-tert-butyl-25,27-dibenzyl-26,28-dihydroxycalix[4]arene ${ }^{124}$.

(8) 84

2.10 5,11,17,23-Tetra-tert-butyl-25,27-dibutoxy-26,28-

dihydroxycalix [4]arene $e^{124}$.

(9) . 85

$2.115,11,17,23$-Tetra-tert-butyl-25,27-dimethoxycalix[4]arene ${ }^{127}$. (10). 86

2.12 5,17-Di-tert-butyl-25,27-dimethoxycalix[4]arene ${ }^{127}$. (11). 87

2.13 25,26,27,28-Tetrahydroxycalix[4]arene. (12) 88

2.14 25,27-Dimethoxy-26,28-dihydroxycalix[4]arene. (13) .88

2.15 25,26,27-Trihydroxy-25-phenylmethoxycalix[4]arene ${ }^{128}$. (14). 89

2.16 25,26,27-tris(ethoxycarbonyl)methoxy-28-phenylmethoxy-calix[4]arene ${ }^{128}$. (15) 90

2.17 25,26,27-Tri[(ethoxycarbony)methoxy]-28-hydroxycalix[4]arene ${ }^{128}$. (16). .91

2.18 11,23-Dichloromethyl-25,26,27,28-tetrahydroxycalix[4]arene. (17) 92

2.19 11,23-Dibromomethyl-25,26,27,28-tetrahydroxycalix[4]arene. (18) .92

2.20 Bis(2-pyridylmethyl)amine (DPA $\left.{ }^{108}\right)$. (19). 93

2.21 4-Methyl-4-carbaldehyde-2,2-bipyridine ${ }^{129}$. (20) 94

2.22 4-Methyl-4-hydroxymethyl-2,2-bipyridine ${ }^{129} \cdot(21)$... .95

2.23 4-Methyl-4-bromomethyl-2,2-bipyridine ${ }^{129} \cdot(22)$ 96

2.24 11,23-Dibromomethyl-25,27-diethoxycarbonylmethoxy-26,28dihydroxycalix[4]arene. (23)..... .97

2.25 11,23-Dibromomethyl-25,27-dibutoxy-26,28-dihydroxycalix[4]arene. (24). 98

2.26 11,23-Dibromomethyl-25,27-dibutoxy-26,28-diethoxycarbonylmethoxycalix[4]arene. (25) .99

2.27 11,23-Di[bis(2-pyridylmethyl)amine]-25,27-dibutoxy-26,28-dihydroxycalix[4]arene. (26) 100

2.28 11,23-Di[N,N'-(2 'pyridylmethyl)aminomethyl]-25,27-diethoxycarbonylmethoxy-26,28-dibutoxycalix[4]arene. (27) 101

2.29 11,23-Di[N,N'-di(2'-pyridylmethyl)aminomethyl]-25,27-diethoxycarbonyl(methyl-2-pyridine)-26,28-dihydroxycalix[4]arene. (28) 102

2.30 11,23-Dibromomethyl-25,27-dimethylbenzyl-26,28-dihydroxycalix[4]arene. (29) 104 
2.31 11,23-Di[N-(2'-pyridylmethyl)aminomethyl]-25,27-dibenzyl-26,28-

dihydroxycalix[4]arene. (30)

2.32 11,23-Di[N,N'-di(2'-pyridylmethyl)aminomethyl]-25,27-dibenzyl-26,28-

dihydroxycalix[4]arene (31)

2.33 11,23-di[N,N'-(2'-pyridylmethyl)(4,4-dimethyl-2,2-

bipyridine)aminomethyl]-25,27-dibenzyl-26,28-dihydroxycalix[4]arene. (32) 109

2.34 11,23-dibromomethyl-25,27-di(aminomethyl-2-pyridine)-26,28-

dihydroxycalix[4]arene. (33).....

2.35 11,23-Di[N-(2'pyridylmethyl)aminomethyl]-25,27-di(methyl-2-pyridine)-

26,28-dihydroxycalix [4]arene. (34)

2.36 11,23-Di[N,N'-bis(2'-pyridylmethyl)aminomethyl]-25,27-di(methyl-2-

pyridine)-26,28-dihydroxycalix[4]arene. (35)

2.37 11,23-Di[N-(2'pyridylethyl)aminomethyl]-25,27-di(methyl-2-pyridine)-

26,28-dihydroxycalix [4]arene. (36)

2.38 11,23-Di[N-(2'-pyridylethyl)aminomethyl]-25,27-dibenzy-26,28-

dihydroxycalix[4]arene (37)

2.39 11,23-Di[N,N'-di(2'-pyridylethyl)(4-methyl-2,2-bipyridine-4'-

aminomethyl)]-25,27-dibenzyl-26,28-dihydroxycalix[4]arene (38)

$2.40 \quad 11,23-D i\left[N, N^{\prime}\right.$-di(2'-pyridylmethyl)(2'-pyridylethyl)aminomethyl]-25,27-

dibenzyl-26,28-dihydroxycalix [4]arene. (39)

2.41 11,23-Dibromomethyl-25,27-di(methyl-2-pyridine)-26,28-

diethoxycarbonylmethoxycalix[4]arene. (40)....

2.42 11,23-Di[N-(2-pyridiylethyl)aminomethyl]-25,27-dimethylpyridyl-26,28-

diethoxycarbonylmethoxycalix[4]arene. (41).... 136

2.43 11,23-Di[N,N'-di((2'-pyridylethyl)(4-dimethyl-2,2-bipyridine)-4'-

aminomethyl)]-25,27-dibenzyl-26,28-diethoxycarbonylmethoxy-calix[4]arene.

(42) 140

2.44 11,23-Di[N,N'-(2 'pyridylethyl)(4-methyl-2,2'-bipyridyl)-4'-aminomethyl]-

25,27-dibenzyl-26,28-diethoxycarbonyl(methyl-2-pyridine)calix[4]arene. (43) .143

2.45 11,23-Di[N,N'-(2'-pyridylethyl)(4-methyl-2,2-bipyridyl)aminomethyl]-

25,27-di(methyl-2-pyridine)-26,28-diethyoxycarbonylmethoxycalix[4]arene. (44) .144

2.46 11,12-Di[N,N'-(2 'pyridylethyl)(4-methyl-2,2'-bipyridyl)-4'-aminomethyl]-

25,27-di(methyl-2-pyrdine)-26,28-di(2'-pyridylmethyl)methoxy-calix[4]arene.

(45) 146

2.47 11,23-di[N,N'-di(2'-pyridylmethyl)aminomethyl]-25,27-di(methyl-2-

pyridine)-26,28-dihydroxy-calix[4]arene. (46).... 148 
2.48 11,23-Di[N,N'-di(2'-pyridylmethyl)aminomethyl]-25,27-di(methyl-2-

pyridine)-26,28-diethoxycarbonylmethoxycalix[4]arene. (47).....

2.49 11,23-dibromomethyl-25,27-dibenzyl-26,28-diethoxycarbonylmethoxy-

calix[4]arene. (48)

2.50 11,23-Di[N-(2'-pyridylethyl(aminomethyl]-25,27-dibenzyl-26,28-

diethoxycarbonylmethoxycalix[4]arene. (49)....

2.51 11,23-Di[N,N'-(2'-pyridylmethyl) (2'-pyridylethyl(aminomethyl]-25,27-

dibenzyl-26,28-diethoxycarbonylmethoxycalix[4] arene. (50)

2.52 11,23-Di[N,N'-(2'-pyridylmethyl)(2'-pyridylethyl)aminomethyl]-25,27-

di(methyl-2-pyridine)-26,28-diethoxycarbonylmethoxycalix[4]arene. (51).

2.53 11,23-Di[N-(2'-pyridylethyl)aminomethyl]-25,27-dibenzyl-26,28-di(2'-

pyridylmethylmethoxy)calix[4] arene (52).....

2.54 11,23-Di[N,N'-(2'-pyridylmethyl)(2'-pyridylehtyl)aminomethyl]-25,27-

dibenzyl-26,28-di(2'pyridylmethyl)methoxycalix[4] arene. (53).....

3 Discussion

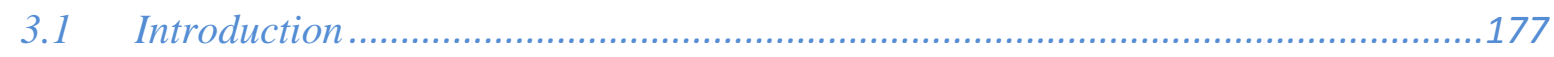

3.2 Synthesis of calixarenes as potential central scaffolds ......................................177

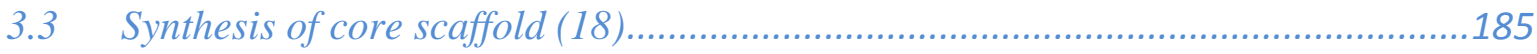

3.4 Functionalisation of the lower rim ........................................................ 188

3.4.1 DPA Synthesis and upper rim Functionalisation .................................................... 190

3.4.2 Lower rim functionalised pyridyl complexes .......................................................... 196

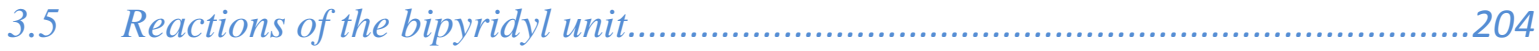

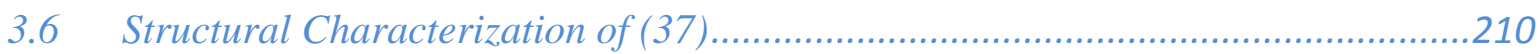

3.7 Self-assembly investigations of (37) and other benzyl compounds ......................211

3.7.1 Self-Assembly of lower rim pyridyl complexes ........................................................230

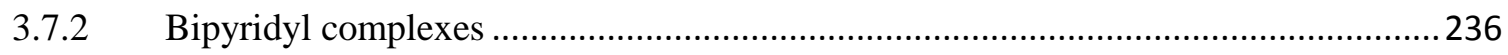

3.7.3 Lower rim benzyl complexes containing additional ester groups on the lower rim 240

3.8 Metal ion complexation reactions ...........................................................246

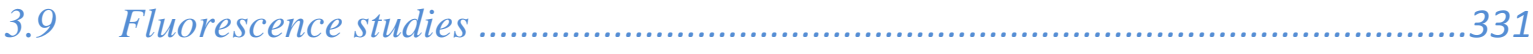

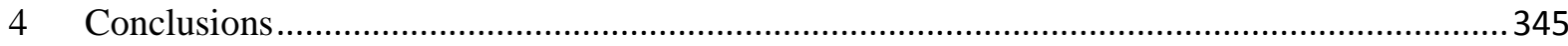

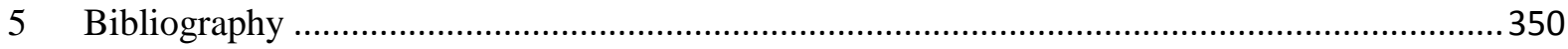


1 Introduction 


\subsection{Overview}

The work discussed in this Ph.D. thesis is based on using derivatives of calix[4]arene with functionalisation of both the upper and lower rims using $\mathrm{N}$-donor ligands including the bipy group. This introduction will cover the history of calixarenes, from their synthesis to their spectroscopic analysis.

\subsection{The chemistry of calix[4]arenes}

\subsubsection{The origin of calixarenes}

Work on calixarenes initially began over a century ago when Adolph von Baeyer displayed an interest in the reaction between formaldehyde and phenols ${ }^{1}$. His work in this area began in 1872 where he reported that when formaldehyde and phenols are reacted together a thickening of the reaction mixture is observed resulting in a 'cement-like' substance. Unfortunately he was unable to obtain a pure product and so he could not carry out any elemental analysis which may have allowed him to propose possible structures. Despite these odds he did manage to provide some insight by proposing a dimesitylmethane structure referring to a better known product obtained when mesitylene and formaldehyde are reacted (Figure 1$)^{2}$.

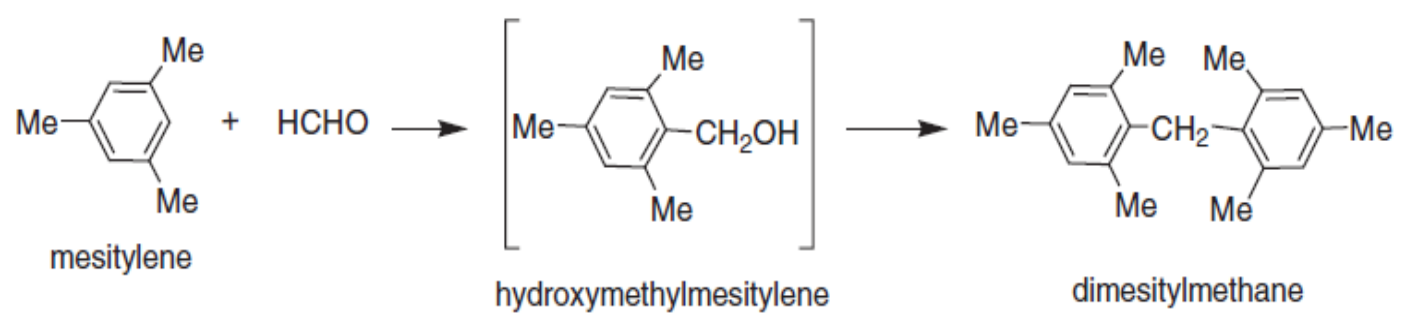

Figure 1 : The reaction between mesitylene and formaldehyde to form dimesitylmethane ${ }^{2}$.

Due to the lack of available analytical techniques, the first crystal structure of a calixarene wasn't determined until $1942^{3}$. Credit for the usefulness of Baeyer's 'cement-like' substance was to go to Leo Baekeland, who, after many years of research, applied for his first patent in 1907 on his synthetic process for the first synthetic plastic ${ }^{4}$. Alois Zinke made the first contribution in the determination of the 
parent compound structure. He began his work in 1936 with co-worker Erich Ziegler ${ }^{5}$. The results of their first compound were exciting if not confusing as it gave a high molecular weight of 1725 , which suggested that 8 to 9 p-tert-butylphenol units were connected in a cyclic array ${ }^{6}$. The next development was from B. T. Hayes and R. F. Hunter from Birmingham England ${ }^{7,8}$. The Hayes and Hunter synthesis, otherwise known as the ten step synthesis, showed a classic example of protection chemistry (Figure 2). They chose a bromine atom as their protecting group and introduced it at the initial step to one of the two ortho-positions of $p$-cresol to give 2bromo-4-methylphenol.

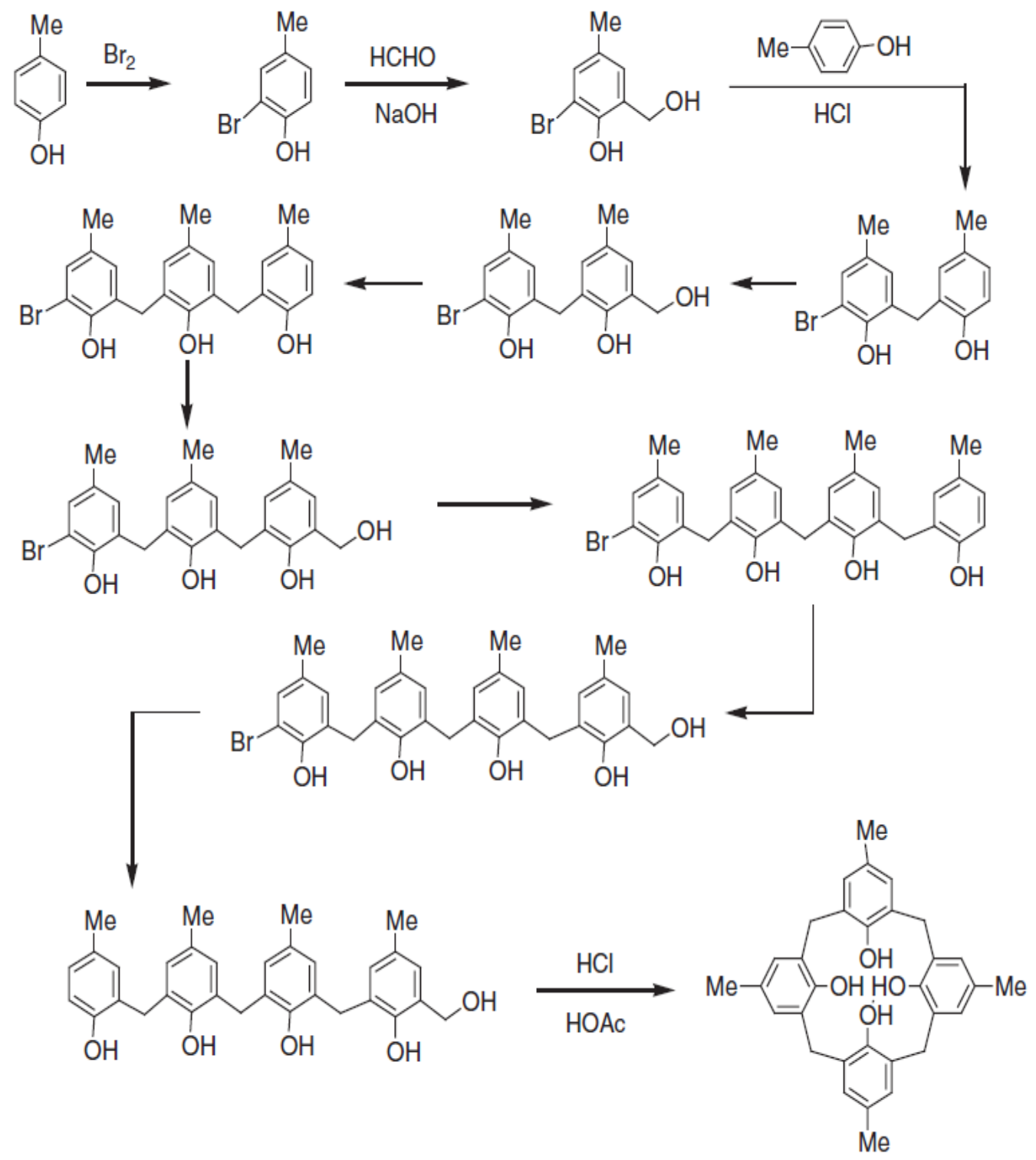

Figure 2: Hayes and Hunter ten step synthesis ${ }^{7}$. 
Base-induced hydroxymethylation gave 2-bromo-4-methyl-6-hydroxymethylphenol. Treatment with concentrated $\mathrm{HCl}$ and excess $p$-cresol resulted in the formation of 3bromo-2:2-dihydroxy-5:5'-dimethyldiphenyl methane. Repeating this base-induced hydroxylation and following with acid arylation produced the dimer, trimer and eventually the calix[4]arene. A final hydroxylation and removal of the protecting group afforded the mono-hydroxymethylated linear calix[4]arene. Acid-catalyzed treatment initiated cyclization to the cyclic calix[4]arene. They reported the material as a light brown solid with a melting point $>300{ }^{\circ} \mathrm{C}$.

The actual molecular weight of their compound was found at $525 \mathrm{~g}$ which was close to their calculated value of $480 \mathrm{~g}$. They put the difference in weight down to residual water in their compound. By the use of infrared techniques they observed an absorption band at $854 \mathrm{~cm}^{-1}$, which indicated the 1,2,4,6-tetrasubstitution pattern on the aromatic rings. They concluded that this synthesis showed that cyclic structures can be produced under suitable conditions during the hardening process of the phenol-formaldehyde resins thereby confirming the possibility that the compounds formed by Zinke were sterically plausible ${ }^{8}$. Overall this synthesis provided a significant insight into the world of phenol-formaldehyde chemistry.

John Cornforth was the next to make considerable input to calixarene chemistry. When he repeated Zinke's reaction, he found that he obtained two materials rather than a single product as Zinke had observed ${ }^{9,10}$. He repeated the reaction using $p$ tert-octylphenol as the starting material and found that the outcome was the same. From both sets of reactions he found that one of the materials had a higher melting point than the other in both cases, but that they all possessed the physical chemical properties of a cyclic oligomer. He code named the substances HOC, HBC, LOC and LBC in relation to high $(\mathrm{H})$ or $(\mathrm{L})$ melting points and whether the starting material was p-tert-octylphenol (O) or p-tert-butylphenol (B). As Zinke had given no information on whether the product may be a mixture, Cornforth began to investigate the matter. At first he thought that maybe one of the materials may be a linear oligomer. This was shown not to be so due to the presence of the same ratio of $\mathrm{CH}_{2}$ groups and phenolic residues in all compounds. Next he wanted to see if the compounds could be functionalized at other positions on the ring, so he reacted the 
compounds with $p$-nitrobenzene-diazonium chloride but to no avail. The possibility that there were different size rings in his compounds was ruled out by the help of Dorothy Crowfoot Hodgkin, a British X-ray crystallographer ${ }^{9,10}$. After considering his evidence, he came to the conclusion that the compounds were diastereoisomers which arose from hindered rotation. Four possible models were produced, (Figure 3) today know as conformers, each with their own independent existence with different chemical and physical properties.
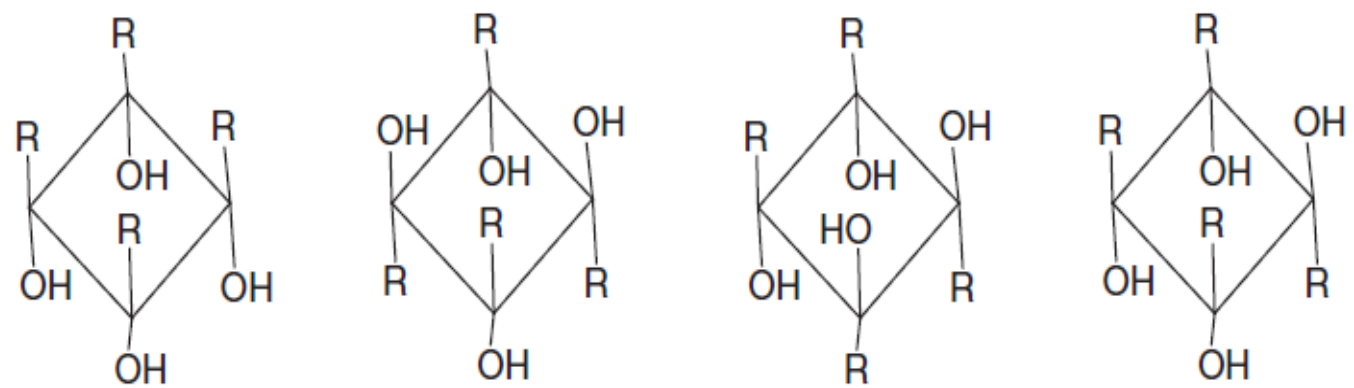

Figure 3: Cornforth's 4 models ${ }^{11}$.

The early 1970's was the era when bioorganic chemistry, specifically enzyme mimics, were emerging and the possibility existed to put Zinke's cyclic calix[4]areneic compounds to work as molecular baskets. In 1972 C. D. Gutsche began his work on building his enzyme mimic by functionalizing the calix[4]areneic compounds. He named his new cyclic calix[4]arenes 'Mehrkernmethylenephenolverbindungen'12. 


\subsubsection{Nomenclature of calixarenes}
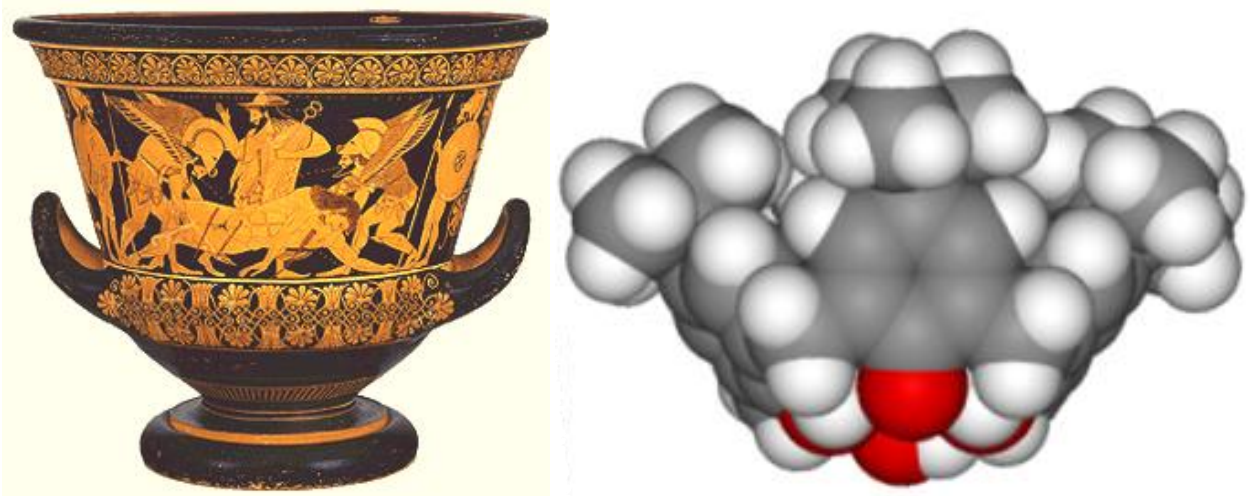

Figure 4: Similarities between the shape of the Greek vase and the cyclic calix[4]arene ${ }^{11}$.

Cornforth and others had previously pointed out that the cyclic calix[4]arene had a non-planer structure ${ }^{12,13}$ shown on the right hand side of Figure 4 in a space filling model, to which Gutsche noticed a resemblance to that of a Greek vase, the calix crater. From this he gave the name calixarene, coming from calix meaning vase or chalice and arene from the aryl groups (Figure 4). Initially this name was rejected by International Union of Pure and Applied Chemistry (IUPAC), but eventually it gained acceptance but only for use with the basic structure without any substituents. A more systematic nomenclature has risen for substituted calixarenes, for example, the cyclic calix[4]arene from p-tert-butylphenol and formaldehyde is known as 5,11,17,23-tetra-tert-butyl-calix[4]arene-25,26,27,28-tetrol. The numbering system for the carbon core of an un-substituted calix[4]arene unit is shown in Figure 5. 


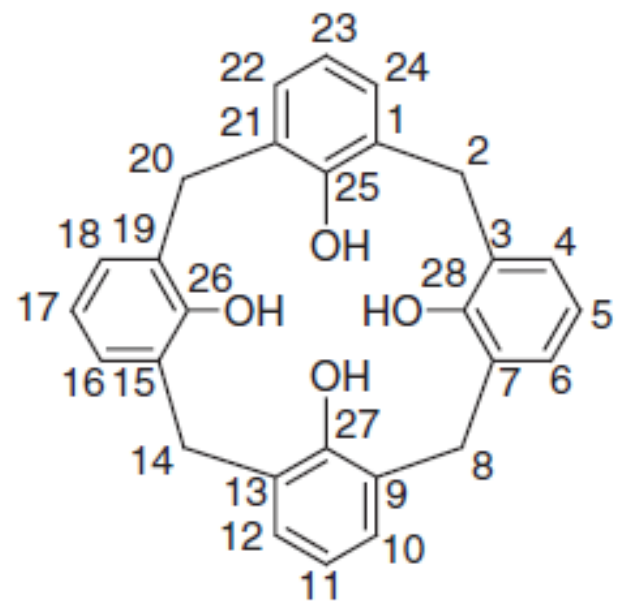

Figure 5: Calix[4]arene carbon numbering system ${ }^{11}$.

As vases are constructed to stand upright on the bases, it can be concluded that calixarenes should be drawn with their $\mathrm{OH}$ groups pointing downwards (endo) and their $p$-substituted groups pointing upwards (exo) to maintain this similarity. The endo face is also known as the lower / narrow and the exo face as the upper / wide rim. For the most part of this thesis the terms upper and lower will be used to designate the rims.

\subsubsection{Base - induced formation of calixarenes}

Initially, the synthesis of p-tert-butylcalix[4]arene was a painstaking task as obtaining consistent yields was rare despite the reaction conditions being identical. Eventually after long investigations with varying the concentration of base, a new more cost efficient procedure was found ${ }^{14,15}$. Even now, the reasons for the differences in the yields are still puzzling. The new procedure is a combination of the work of Zinke ${ }^{5}$ Cornforth $^{10}$ and Gutsche ${ }^{15,16}$ and involves using 0.045 equivalents of base $(\mathrm{NaOH})$ with respect to the phenol and also requires the use of a neutraliser to remove some of the base added. During neutron activation analysis ${ }^{17}$ it was seen that some of the Na remained, most likely in the phenoxide form. From numerous trials an optimum amount of base was found for producing the calix[4] arene as shown in Figure $6^{18}$. From the graph it can be seen that the maximum yield for calix[4]arene production falls between 0.03-0.04 equivalents of base. With less than this amount 
the yield decreases to zero and with more the cyclic hexamer is obtained. Different kinds of base were also investigated and the results indicated that in general $\mathrm{LiOH}$ is inferior for cyclooligomerization, $\mathrm{NaOH}$ can give higher yields of the calix[8]arene and $\mathrm{KOH}, \mathrm{RbOH}$, and $\mathrm{CsOH}$ give higher yields of the cyclic hexamer in the above reaction.

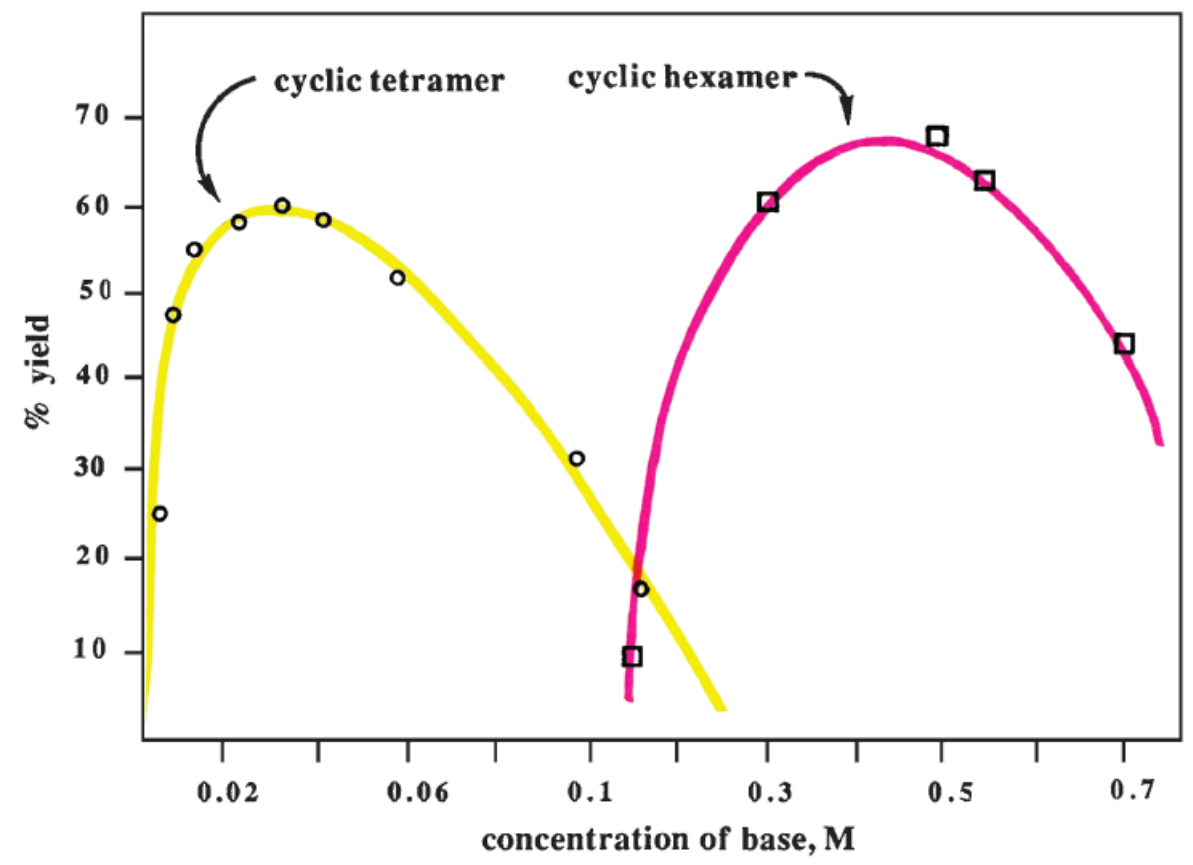

Figure 6: Effect of base on calix[4]arene formation ${ }^{11}$.

Since the beginning of the twentieth century, the pathway for the base induced mechanism has been widely studied. The reaction proceeds with the formation of the phenoxide ion which acts as a nucleophile to allow nucleophilic addition to occur at the carbonyl carbon (Figure 7). 


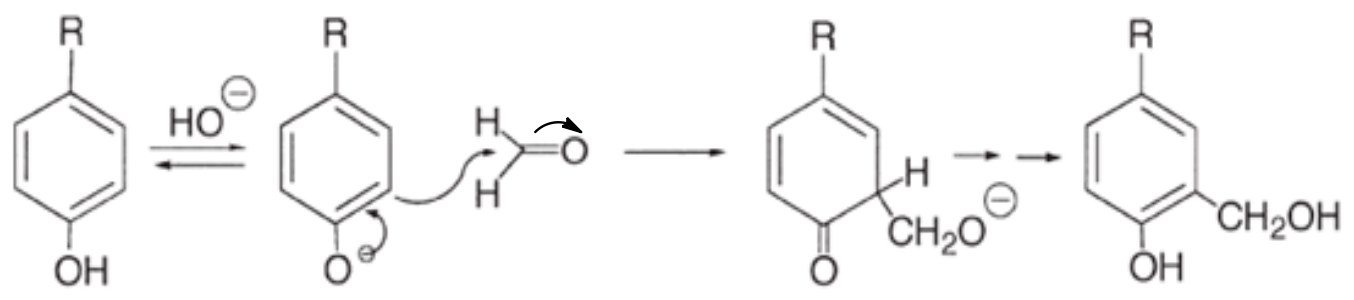

Figure 7: Reaction of phenol and formaldehyde under catalytic basic conditions ${ }^{19}$.

From here the reaction may continue to give diarylmethyl compounds, which is thought to proceed via $o$-quinonemethide intermediates, capable of undergoing a Michael-like process with the phenoxide (Figure 8).

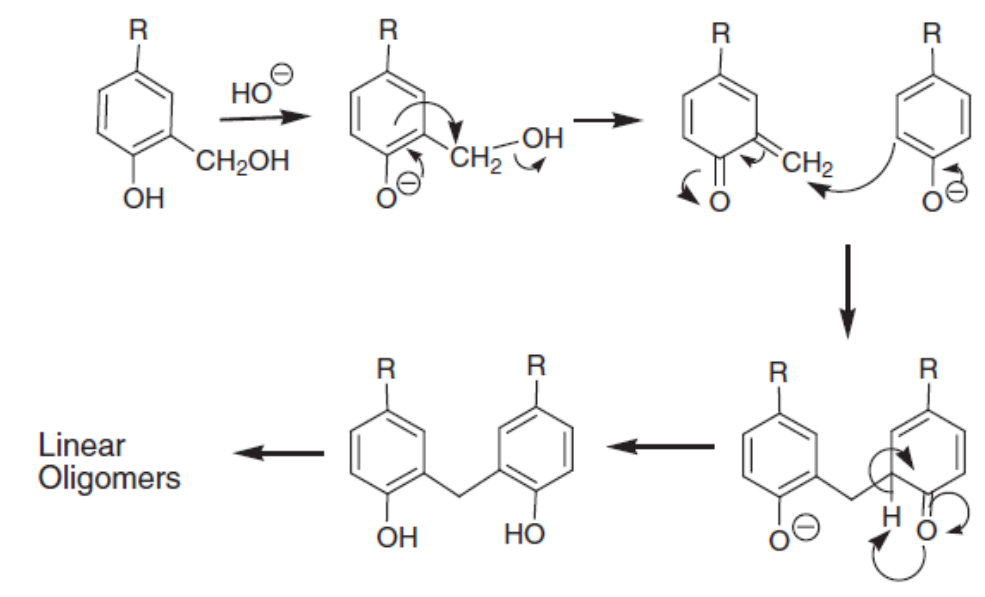

Figure 8: Base catalyzed synthesis of calixarenes ${ }^{20}$.

This process is involved when heating $p$-tert-butylphenol and aqueous formaldehyde at $50{ }^{\circ} \mathrm{C}$ for four days to yield the bis-hydroxymethyl dimer ${ }^{21}$. The $o$-quinonemethide intermediates were suggested as early as $1912^{22}$ and further supported by Hultzsch ${ }^{23}$ and Euler $^{24}$. While the usual synthesis of quinonemethide required high temperatures, it is known that some reactions, such as the oxy-Cope rearrangement, are able to occur more easily with the anions than with the neutral equivalents. From this it is plausible that quinonemethides may well exist under these conditions during oligomerization reactions ${ }^{25}$. 


\subsubsection{Acid catalysed mechanism}

The alternative calixarene synthesis involves the use of an acid catalyst which also has the ability to yield large cyclic products over linear oligomers in larger quantities compared to the base catalysed product. The product from the acid catalysed synthesis was first discovered in 1892 and was at first considered to contain a linear dimeric structure ${ }^{26}$. However, due to its non-crystalline and wayward character (decomposes at $120{ }^{\circ} \mathrm{C}$ without melting) it was suggested that it may have a polymeric structure instead. Högberg paid close attention to these resorcinol-derived calixarenes and devised a synthesis which was later adapted by Cram and co-workers for the large scale production of calix[4]resorcarenes ${ }^{21,27}$. A wide variety of aldehydes can be used in this synthesis from simple aliphatic aldehydes to functionalised ones containing halogens, hydroxyl groups, etc. ${ }^{21,27}$. However highly hindered aldehydes fail to react such as 2,4,6-trimethylbenzaldehyde ${ }^{27}$. The acidcatalyzed mechanism proceeds by the use of cation intermediates and electrophilic aromatic substation reactions (Figure 9).

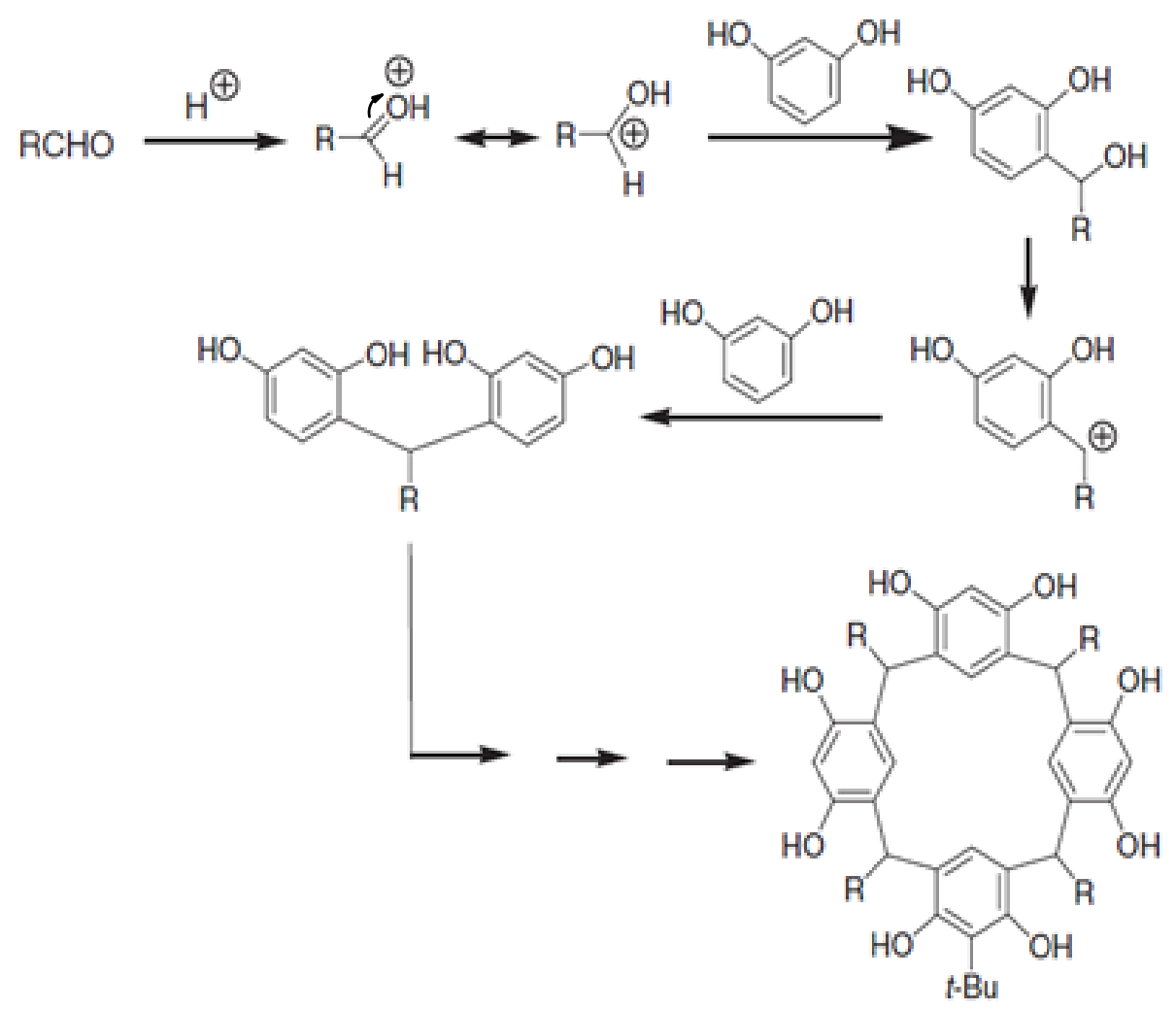

Figure 9: Acid catalyzed formation of calixarenes ${ }^{28}$. 
The calix[4]arenes, calix[6]arenes, and the calix[8]arenes are known as the major calixarenes whose synthesis can be easily carried out in the laboratory by the procedure above. Minor calixarenes also exist such as the calix[5]arenes and the calix[7]arenes which are obtained in lesser yields. The synthesis of other larger calixarenes is also known but will not be discussed here. An overview of the calixarene family is shown in (Figure 10). In this thesis the synthesis of two of the major calixarenes (calix[4]arenes and calix[8]arenes) is discussed.

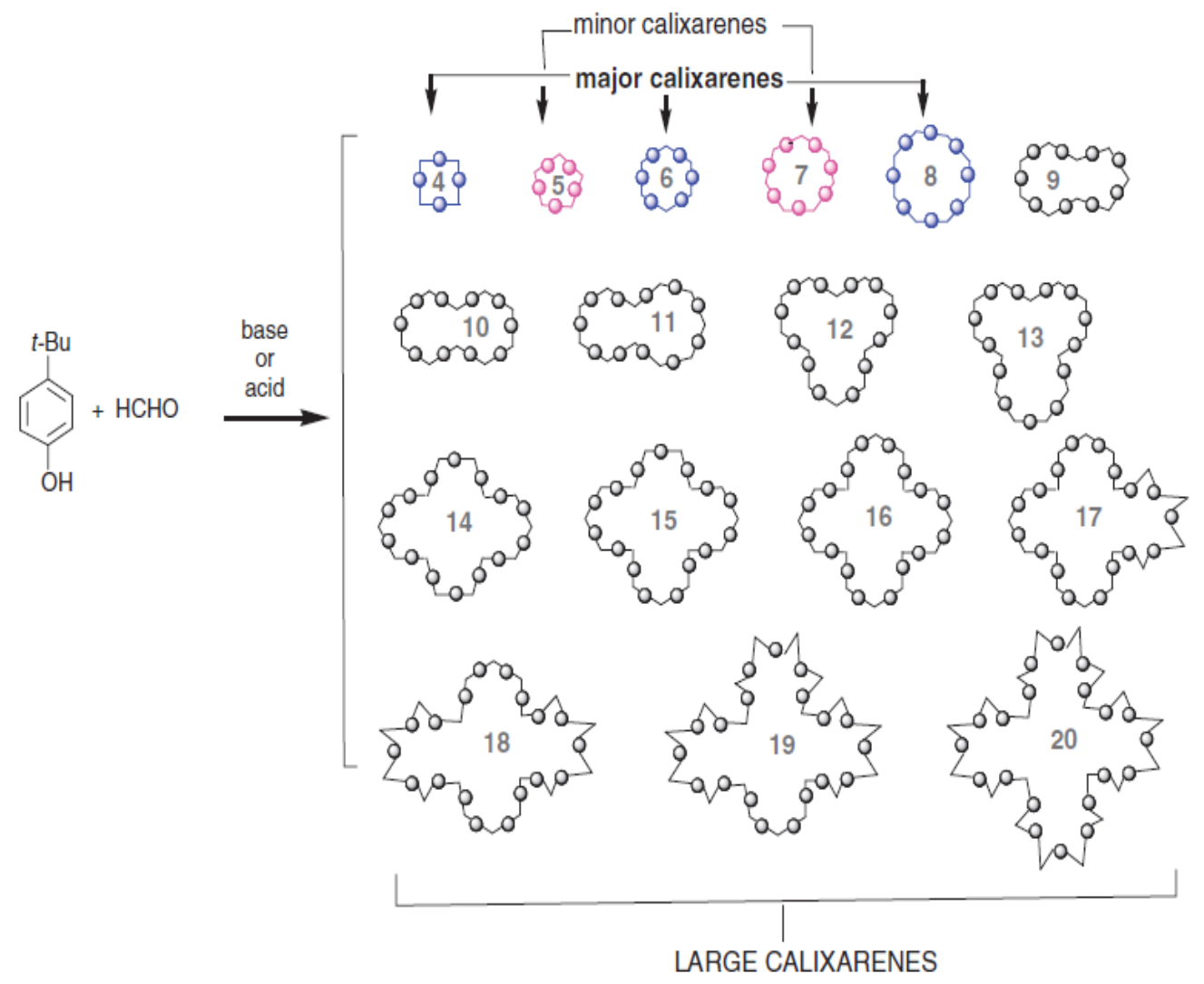

Figure 10: The extended family ${ }^{29}$.

\subsubsection{The stepwise synthesis of calixarenes}

Previously mentioned was the Hayes and Hunter method for the formation of calixarenes but this synthesis is not limited to just the synthesis of cyclic calix[4]arenes as demonstrated by Kämmerer in $1975^{30}$. He used this method for the synthesis of linear oligomers with the ability to cyclise to the corresponding 
cyclooligomers. He, along with co-workers synthesised over a dozen cyclooligomers consisting of various aromatic residues with various groups in the para positions ${ }^{31}$. The Hayes and Hunter synthesis, while a breakthrough at its time, is cumbersome with a maximum yield of $30 \%$ at the best of times. Several groups began to investigate new methods but success fell to Volker Böhmer, a student of Kämmerer. He worked with cyclic oligomers that had more functionality than those of his predecessor, and it is these compounds that lead to his ' $3+1$ ' approach where a linear trimer is condensed using a 2,5-bis-halomethyl phenol as shown in Figure $11^{32}$. Route $\mathrm{A}$ is the shorter pathway and uses a symmetrical linear trimer which is prepared from treating a hydroxymethylphenol in excess phenol whereas Route B is the longer pathway as it incorporates unsymmetrical linear trimers and it involves protection and deprotection steps. These two convergent fragmentation condensation procedures can give yields up to $30 \%$, but more often in the range $10-20 \%$. While the yield obtained with these two routes is less than what would be obtained with the Hayes and Hunter method, the two routes overall are much more cost and time efficient than the previous ten step synthesis. 


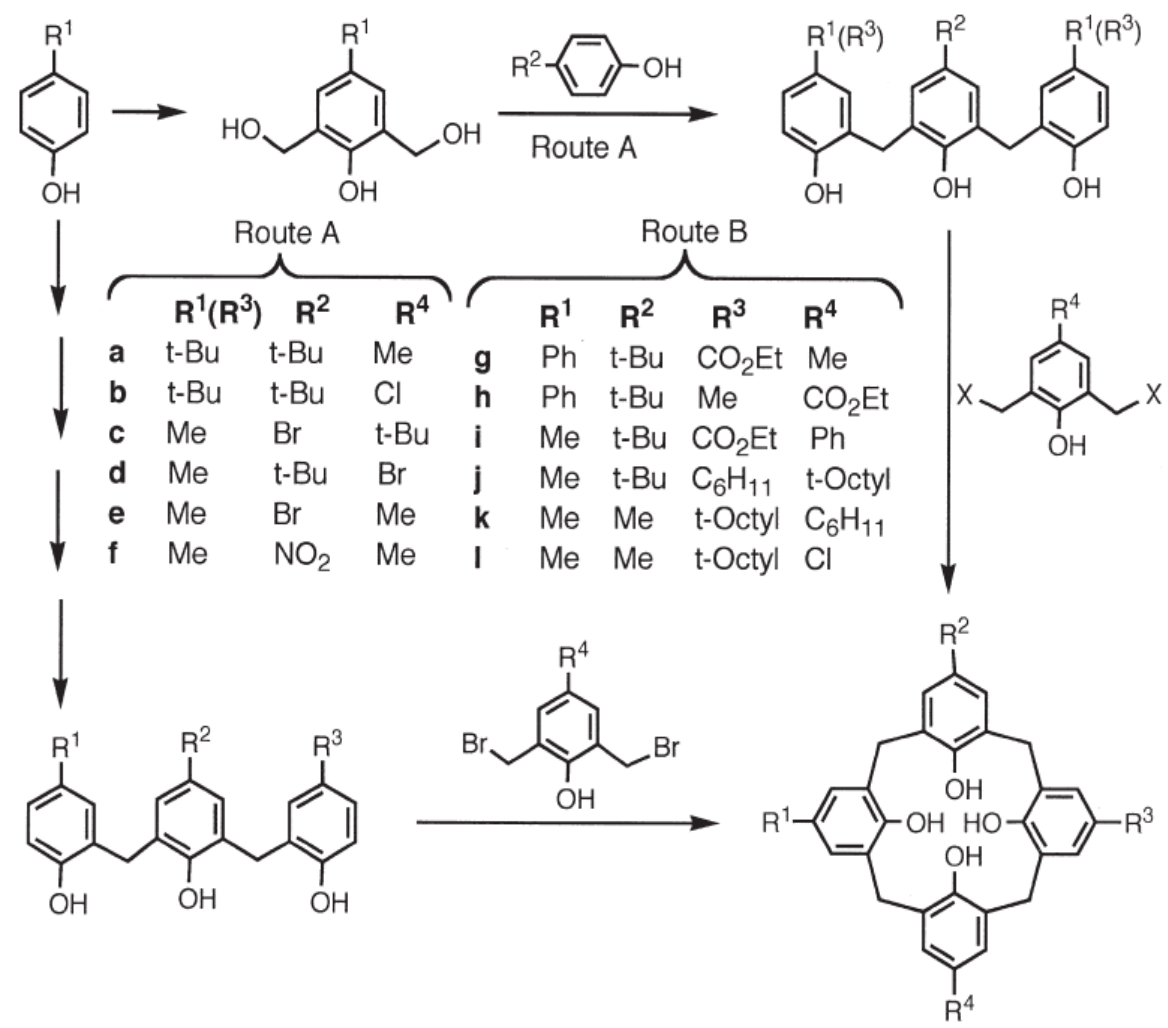

Figure 11: The ' $3+1$ ' approach $^{32}$.

The ' $2+2$ ' procedure was also studied by Böhmer and co-workers which involves the condensation of $p, p$-dichlorodiphenylmethane to produce $p$-chlorocalix[4]arene (Figure 12) ${ }^{33}$. 


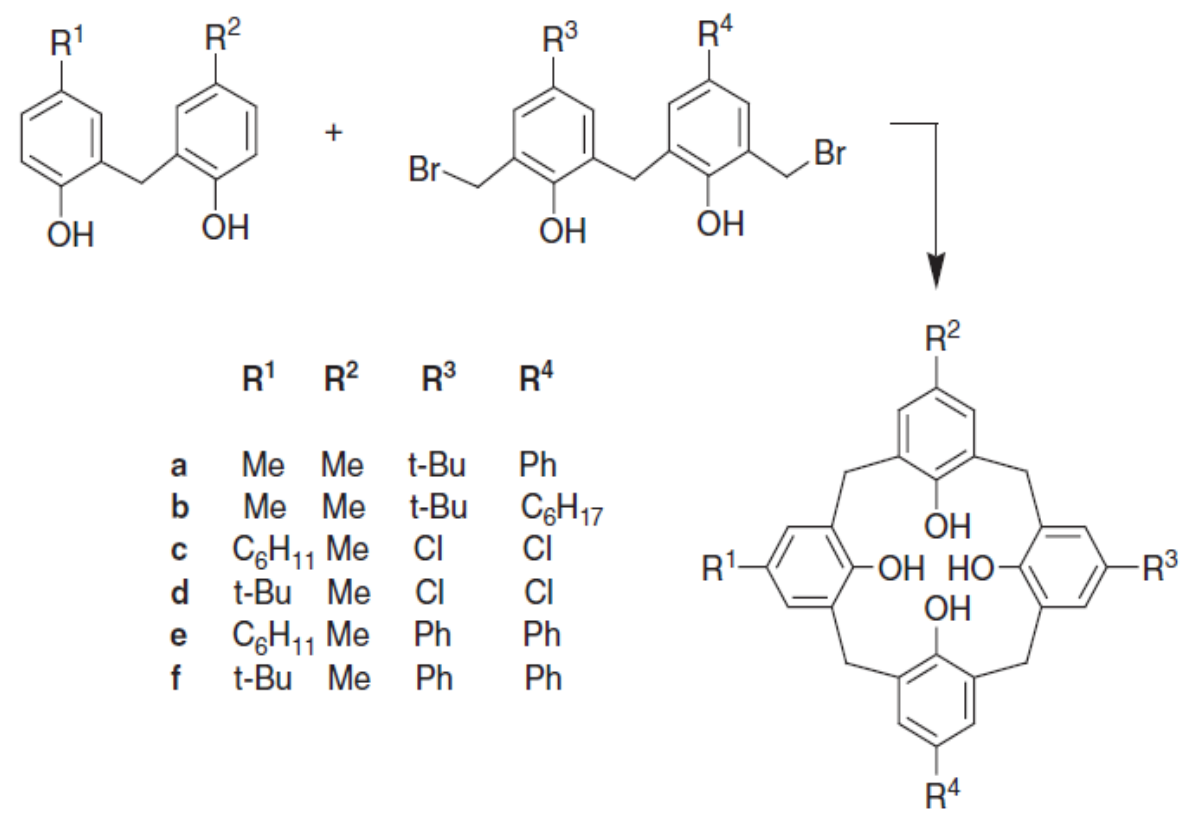

Figure 12: The ' $2+2$ ' approach $^{33}$.

The choice between the two procedures often depends on the ease of constructing the intermediate parts. The use of the fragment condensation for the synthesis of larger calixarenes has had some success. DeMendozza and co-workers have used the ' $3+$ $3^{\prime}$, procedure to form the calix[6] arene in a $9 \%$ yield $^{34}$. Various calix[5] $\operatorname{arenes}^{33}$ have also been reported and even a calix[8]arene ${ }^{35,36}$ by the fragmentation condensation method. A one step synthesis ' $1+1+1+1$ ' process has been developed for the synthesis of calix[4]arenes. It involves the use of $\mathrm{TiCl}_{4}$ with 2hydroxymethylphenols with a yield up to $30 \%{ }^{33}$.

\subsubsection{Purification of calixarenes}

For accurate characterization of a compound, the compound itself must be of high grade i.e. a pure compound. However, with the synthesis described above, the product often contains a mixture of compounds which need to be seperated before characterization can begin. Sometimes recrystallization suffices but in many other instances other techniques such as chromatographic procedures (HPLC) are required. Figure 13 shows the possible compounds from a $p$-tert-butylphenol/formaldehyde reaction mixture as observed by HPLC $^{37}$. 


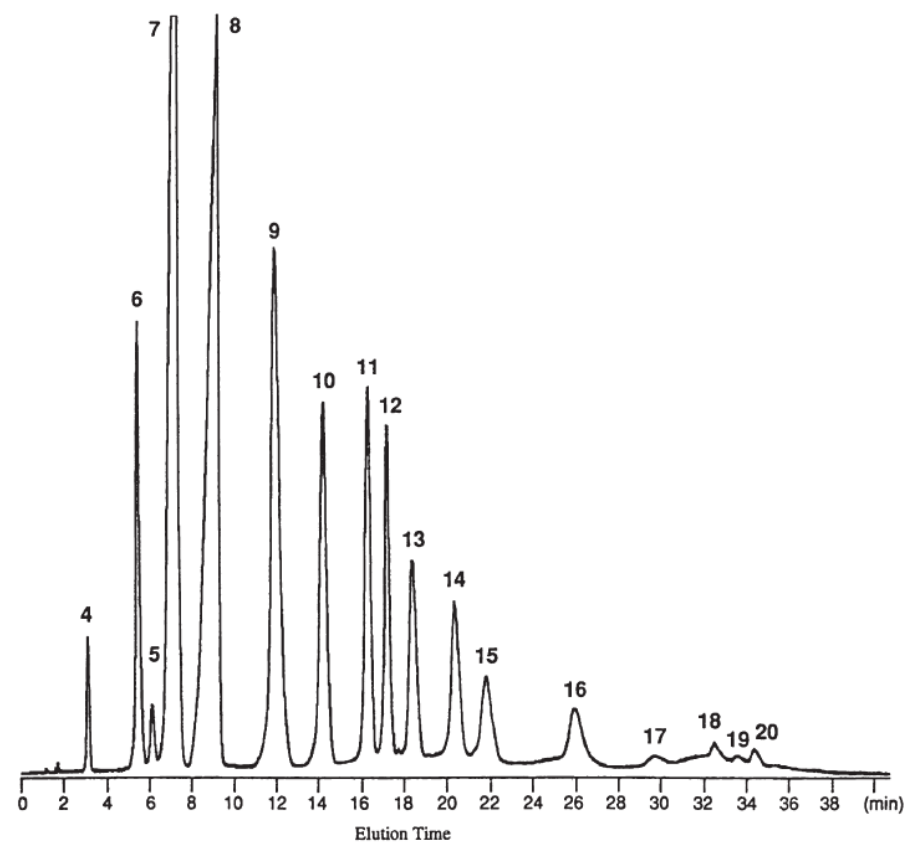

Figure 13: HPLC of p-tert-butylcalix[n]arene mixture ${ }^{37}$. The value of $\mathrm{n}$ is indicated by the numbers visible above the peaks.

X-ray crystallography is the ultimate way a chemist can 'see' into their molecules to determine their structure. An X-ray structure of a phenol-derived calixarene ${ }^{38}$ was obtained in 1979 by Andreetti, Ungaro and Pochini ${ }^{39}$ which also revealed the presence of a toluene molecule within the calix[4]arene. This gave rise to the idea that calixarenes could be used to form inclusion complexes. With the larger calixarenes, X-ray structures were difficult to obtain due to small crystal size but with advancements in the technology itself this problem was resolved and X-ray structures for the calix[6]arene and calix[7]arene were obtained ${ }^{40}$. The calix[8]arene also had problems with crystal growing, in that the crystals quickly lose their morphology and crumble to a powder. This was eventually overcome in $1985^{41}$ and four X-ray structures of common p-tert-butylcalixarenes are presented in Figure 14 below.

Melting point determination is one of the oldest techniques in a chemist's arsenal and it was the unusually high melting points of calixarenes that first attracted Zinke's attention. For calixarenes with free hydroxy groups the melting point is normally 
above $250{ }^{\circ} \mathrm{C}$. The calix[4]arene has a range between $342-344{ }^{\circ} \mathrm{C}$, while the calix[8] arene is higher at $418-420{ }^{\circ} \mathrm{C}$. Altering the calixarene framework has a big influence on the melting point range e.g. the addition of esters or ethers result in a melting point lower than the parent compound.

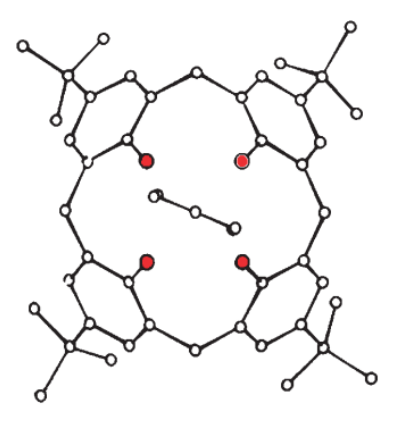

p-tert-Butylcalix $|4|$ arene

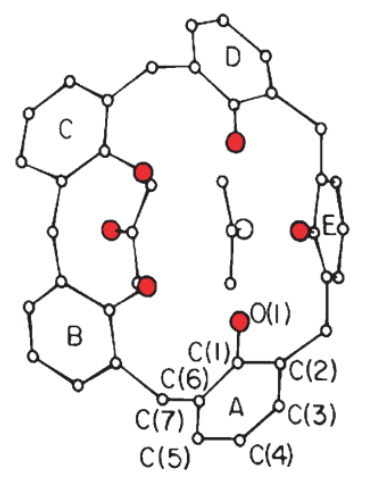

Calix|5|arene

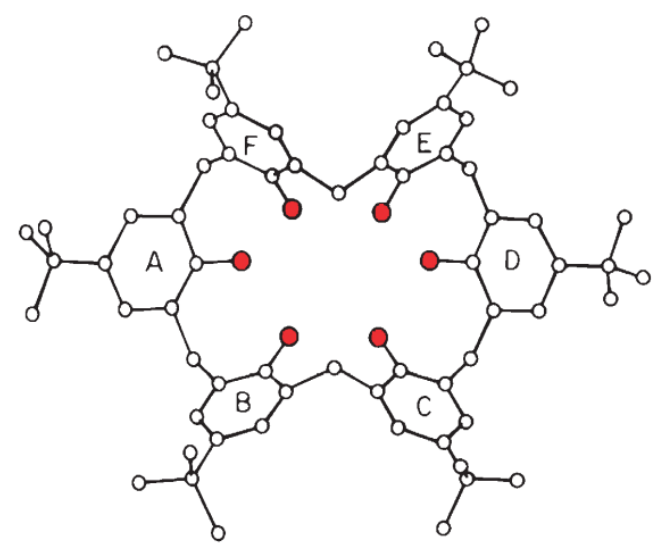

p-tert-Butylcalix|6|arene

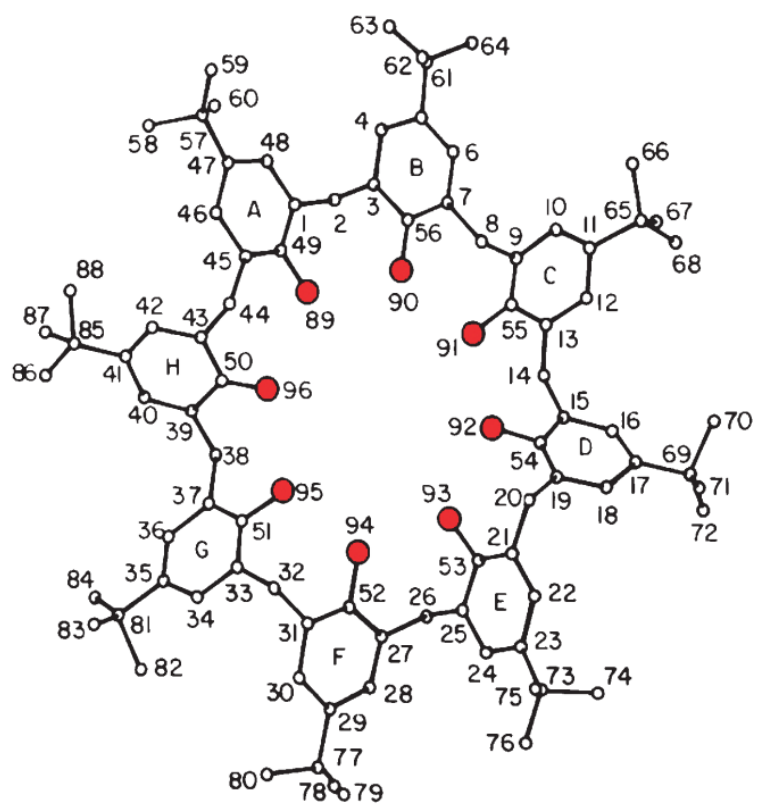

p-tert-Butylcalix $|8|$ arene

Figure 14: X-ray crystallographic structures of p-tert-butylcalixarenes. The calix[4]arene and calix[5]arene containing toluene or acetone respectively as an guest ${ }^{41}$. 
The parent calixarenes are insoluble in water and have very low solubility in organic solvents and it is this feature that makes them so hard to purify and characterize ${ }^{7}$. But fortunately most have sufficient solubility in chloroform, DCM and a handful of other solvents to allow some spectral measurements to be recorded. The addition of esters, ethers and other functional groups into the calixarenes does widen the solubility range and often quite significantly.

\subsubsection{Spectral properties of calixarenes}

In this subsection several characterisation techniques will be visited including IR and NMR spectroscopies and mass spectrometry. Infrared spectroscopy is a very useful tool in the analysis of a compound. Calixarenes have a very distinctive trait in that the $\mathrm{OH}$ of the calixarene occurs at different frequencies for different sized calixarene units. It is this characteristic quality that can allow rapid determination of calixarenes in an unknown mixture. In the calix[4]arene the hydroxyl band appears at $3179 \mathrm{~cm}^{-1}$, in the calix[8]arene it appears at $3191 \mathrm{~cm}^{-1}$ and the in calix[5] arene it occurs at 3300 $\mathrm{cm}^{-1}$. All other forms of unsubstituted calixarenes fall within this range. This is due to the strong intramolecular H-bonding interactions (Figure 15) at the lower rim. In the 'fingerprint region' calixarenes share a similar spectrum with some variations for particular forms e.g. signals at $693 \mathrm{~cm}^{-1}$ and $571 \mathrm{~cm}^{-1}$ for the pentamer and another set of lines for the calix[8]arene at $600-500 \mathrm{~cm}^{-1}$.

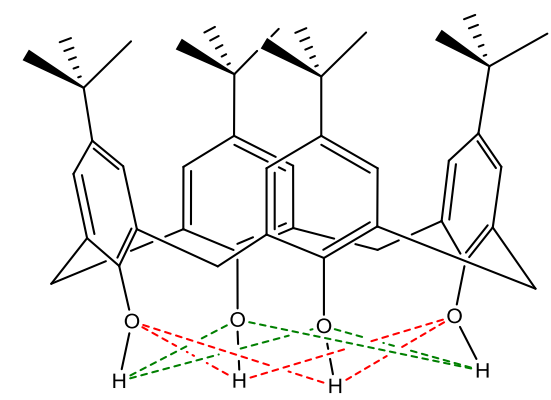

Figure 15: Intramolecular H-Bonding at the lower rim ${ }^{42}$.

Since the development of NMR spectroscopy, the analysis of calixarenes has become easier in that there is another analytical technique which can be used to confirm the 
molecules structure. While NMR spectroscopy provides invaluable insight into the possible structure it is important to note that NMR spectroscopy alone is not sufficient to precisely determine an accurate structure and further analysis such as Xray crystallography should be employed. Shown below (Figure 16) is a spectrum of the calix[4]arene containing four t-butyl groups on the upper rim. The $t$-butyl groups appear as a singlet at $1.2 \mathrm{ppm}$. The bridging methylene protons appear as two doublets around 3.2 and $4.2 \mathrm{ppm}$ respectively due to the two different environments the protons find themselves in. Four of the bridging protons are in an axial position which brings them into proximity with the lower rim hydroxyl group, particularly the oxygen which is able to deshield them slightly causing a doublet to appear around $4.2 \mathrm{ppm}$. The remaining four protons adopt an equatorial position away from the hydroxy groups and as a result are not subjected to the oxygen atoms deshielding properties causing a doublet to appear around $3.2 \mathrm{ppm}$. This was shown by Ungaro and co-workers who used NOESY experiments to assign the two sets of doublets, the higher doublet at $3.2 \mathrm{ppm}$ corresponding to the equatorial protons which lie closer to the aromatic rings, and the lower doublet at $4.2 \mathrm{ppm}$ to the axial protons which lie more towards the $\mathrm{OH}$ groups ${ }^{43}$. The aromatics also appear as a singlet around $7.10 \mathrm{ppm}$ and the hydroxyl protons are shifted further downfield to around $10.3 \mathrm{ppm}$ at room temperature.

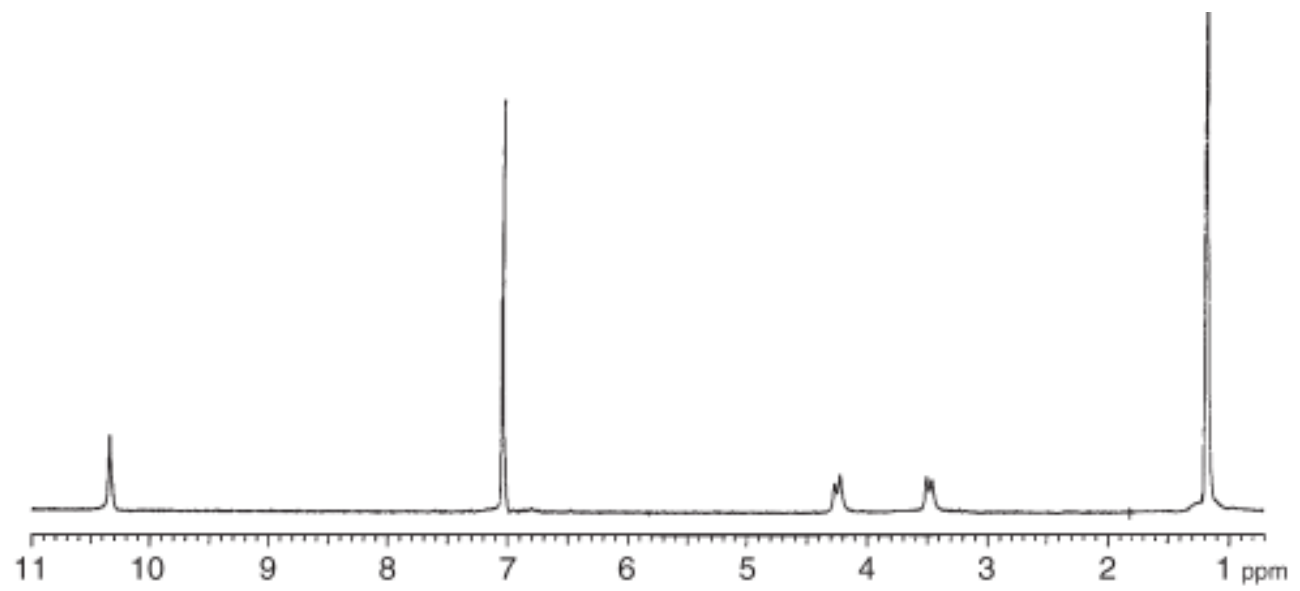

Figure 16: ${ }^{1} \mathrm{H}$ NMR of calix[4] $\operatorname{arene}^{31}$ 
Kämmerer was the first to demonstrate the flexibility of the molecule at raised temperatures, whereby the doublets change pattern to produce a sharp singlet at 60 ${ }^{\circ} \mathrm{C}^{31}$. The parent calix[4]arenes and calix[8]arenes can be determined from each other from the position of the $\mathrm{OH}$ groups in the ${ }^{1} \mathrm{H}$ NMR spectra. In the calix[8]arene spectrum the $\mathrm{OH}$ appears below $10 \mathrm{ppm}$ and in the calix[4]arene spectrum it appears above $10 \mathrm{ppm}^{44}$. Alternatively, low temperature NMR spectra can be obtained in a pyridine solution where the bridging methylene protons become the focus of attention. The calix[4]arene retains a pair of doublets at $0{ }^{\circ} \mathrm{C}$ but the calix[8]arene shows only a singlet even as low as $-90{ }^{\circ} \mathrm{C}$. This will be explained in further detail under conformation changes. The position of the resonance of the $\mathrm{OH}$ groups can be used as a measure of the strength of intramolecular $\mathrm{H}$-bonding represented by $\delta_{\mathrm{OH}} \mathrm{A}$ large value indicates strong hydrogen bonds. Intramolecular $\mathrm{H}$-bonding is quite powerful in the calix[4]arene but not as strong in the calix[8]arene ${ }^{11}$. Work on the ${ }^{13} \mathrm{C}$ NMR spectroscopy has been carried out by DeMendoza and co-workers who came up with a rule of thumb for designating the conformation of calixarenes - The DeMendoza Rule ${ }^{45,46}$. This rule states that in ${ }^{13} \mathrm{C}$ NMR spectroscopy, signals for the bridging methylene's appear around 33-31 ppm when the neighbouring aromatic ring in the syn-position, and when the bridging carbon methylene's produce signals around $37-40 \mathrm{ppm}$ the neighbour is in the anti- orientation (Figure 17).

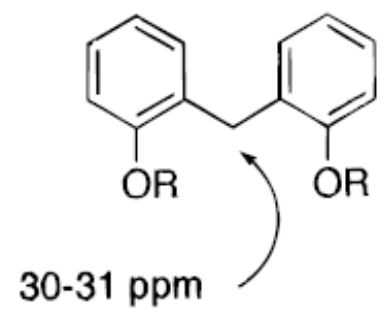<smiles>CCCCCc1ccccc1Cc1ccccc1O</smiles>

Figure 17: The DeMendoza Rule ${ }^{46}$

However it has been noted that in partially substituted calixarenes this rules breaks down such as that observed in syn- and anti-dibenzoylcalix[4]arenes (Figure 18) ${ }^{46}$. They performed several tests to account for this. NOESY NMR spectroscopy studies showed an interaction between the $\mathrm{H}_{\mathrm{i}}$ and $\mathrm{H}_{\mathrm{h}}$ methylene protons, as well as an 
interaction between $\mathrm{H}_{\mathrm{d}}$ and $\mathrm{H}_{\mathrm{f}}$ protons. This pattern indicated an all-syn cone conformation. Additional analysis was done using COSY NMR spectroscopy studies and it detected long distance coupling between the methylene protons and the aromatics which further supported the syn orientation (Figure 19). It was observed that the $\mathrm{H}_{\mathrm{h}}$ protons interaction solely with $\mathrm{H}_{\mathrm{f}}$, while $\mathrm{H}_{\mathrm{i}}$ interacted solely with $\mathrm{H}_{\mathrm{d}}$. Given the information from these spectra it was suggested that the orientation was that of a flattened cone conformation where the two free phenols are flattened and are pointed inside the cavity and hydrogen bonded to each other. The experimental value of around $32 \mathrm{ppm}$ for the bridging methylene carbons is a result of this flattened cone conformation which relieves some of the steric clash that would be observed in the cone conformation.

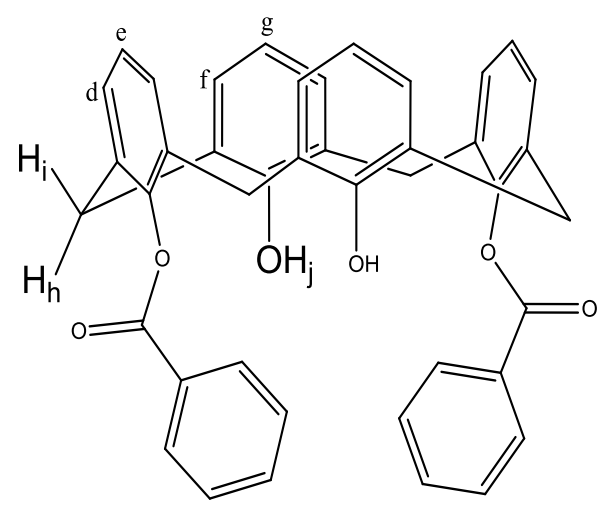

Syn

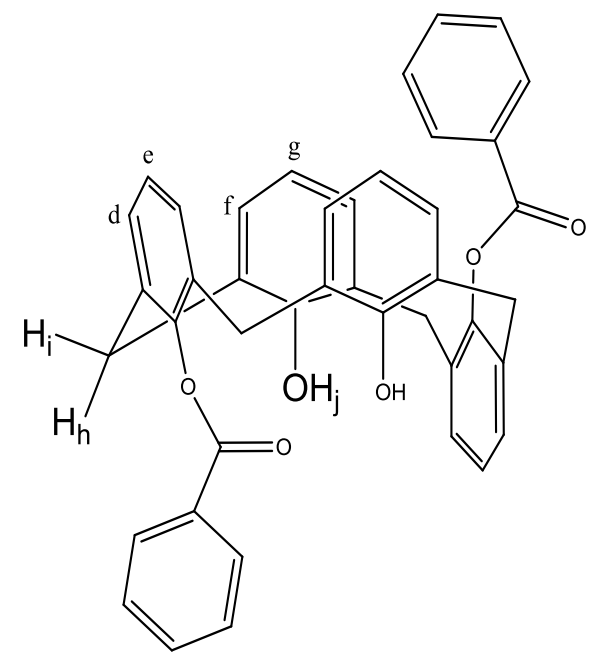

Anti

Figure 18: syn (I) and anti (II) conformations of 1,3-dibenzoylcalix[4]arenes ${ }^{46}$. 


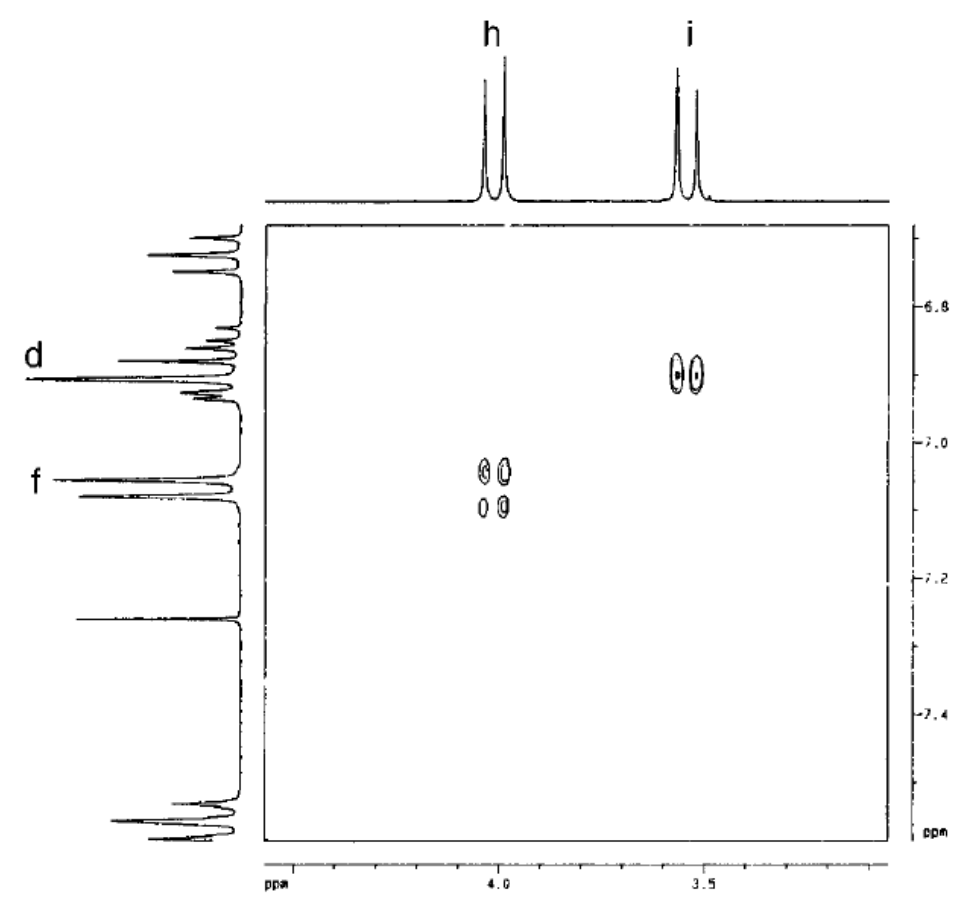

Figure 19: COSY indicating long distance coupling between methylene and aromatic protons $^{46}$

For the partial cone form the same tests were carried out and the NOESY NMR spectroscopy showed that interactions were seen between $\mathrm{H}_{h}$ and $\mathrm{H}_{\mathrm{i}}$ or $\mathrm{H}_{\mathrm{f}}$ at both room temperature and $-90{ }^{\circ} \mathrm{C}$. These two NOESY interactions suggest that a rapid equilibrium exists which involves rotation of the non-substituted (free) rings. It is known that this kind of rotation can give rise to two forms the partial cone and the 1,2-alternate (Figure 20). When variable temperature proton NMR spectroscopy was conducted on this compound a chemical shift of 0.08 ppm was observed for $\mathrm{H}_{\mathrm{h}}$. This suggested that there was only a small energy barrier between the two forms and that a change in temperature could easily influence the forms that are present. As $\mathrm{H}_{\mathrm{h}}$ is the only methylene proton that has a shift it stands to reason that at $-90{ }^{\circ} \mathrm{C}$ only this proton comes into range of the aromatic protons allowing NOESY coupling to occur $^{45,46}$. A possible explanation for the low barrier between the two forms may be due to disruption of weaker H-bonding interactions than would be seen for the unsubstituted calixarene which would have a much higher level of $\mathrm{H}$-bonding at the lower rim. 

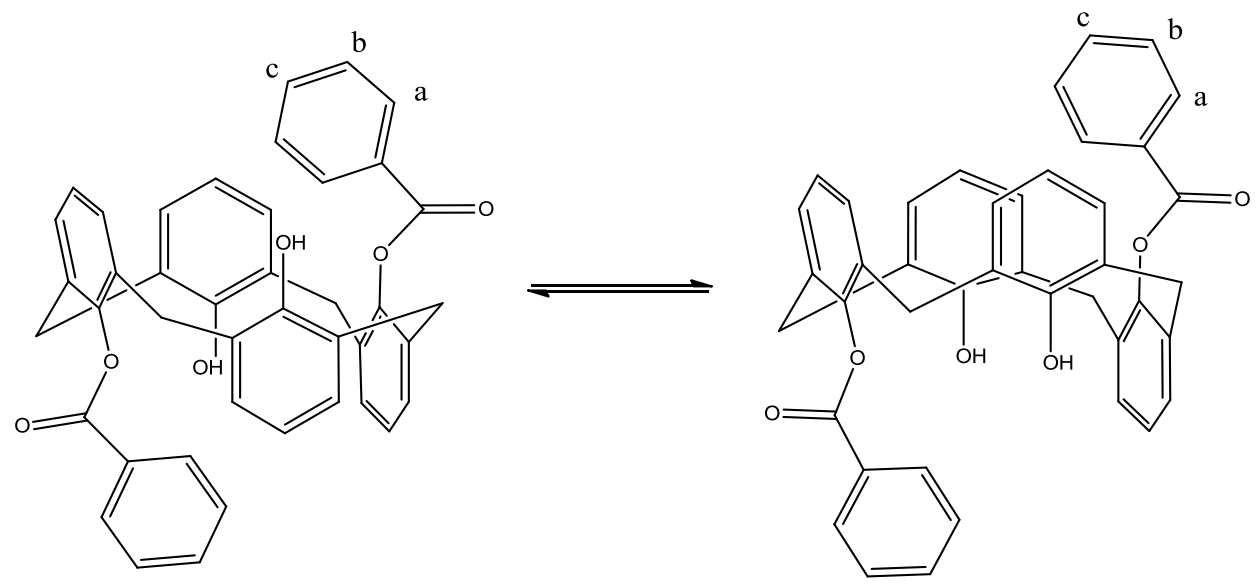

Figure 20: equilibrium between the 1,2-alt and partial cone conformations for (II) ${ }^{46}$.

The last test conducted was molecular modelling studies which suggested that one of the phenyl rings may be held inside the cavity. Shifts were seen in protons $\mathrm{H}_{\mathrm{a}}$ and $\mathrm{H}_{\mathrm{b}}$ to support this theory and inclusion could be stabilized by $\pi$-stacking via the walls of the calixarene and by $\mathrm{CH}_{2}$ bridges. From their results DeMendoza et al. propose that if signals are found between the standard of $31 \mathrm{ppm}$ and $37 \mathrm{ppm}$ for pure syn- and anti- forms respectively, then the results can be interpreted as either a distorted cone conformation or a rapid equilibrium between the syn and anti- forms ${ }^{46}$.

As previously mentioned, calixarenes are symmetrical molecules which can exist in a range of conformers. Those containing a higher number of aromatic rings are often more flexible and can adopt a wider range of forms. To start, we will consider the forms the 'free' calixarenes can adopt (those with free extra/intra annular $\mathrm{OH}$ groups) and then proceed to consider those 'fixed' (constrained via larger functional groups). Cornforth was the first to identify this 'shape-shifting' ability between four major forms or conformers of calix[4]arenes, see Figure 21. He assigned them in terms of the positions of their hydroxyl groups $(\mathrm{u}=\mathrm{up}, \mathrm{d}=\mathrm{down})$, and later these were renamed by Gutsche ${ }^{11,47}$ as cone, partial cone, 1,2-alternate and 1,3-alternate. 


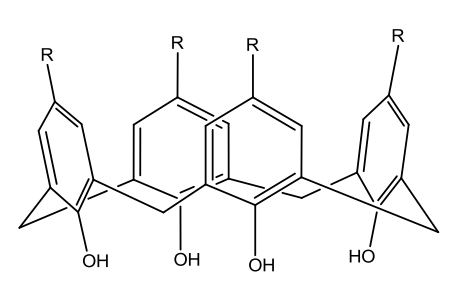

Cone d,d,d,d

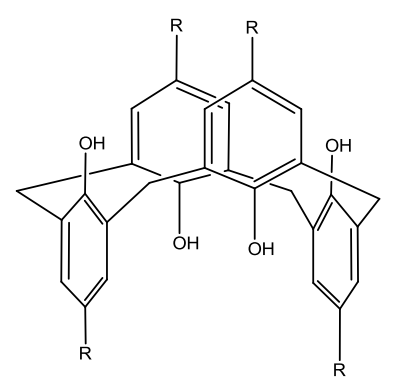

1,3-Alternate d,u,d,u
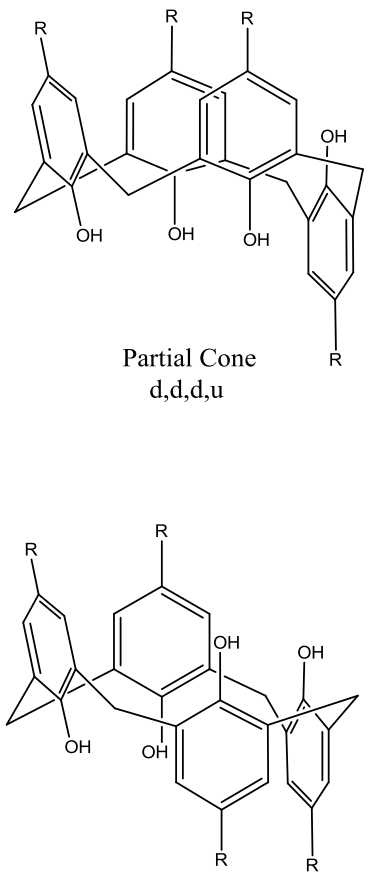

1,2-Alternate d,d,u,u

Figure 21: The 4 major conformers of calix[4]arenes ${ }^{11,47}$

The most conclusive way for determining the structure of a molecule is by X-Ray crystallography ${ }^{48,49}$. But when this method cannot be employed, one must rely on another simple but effective method such as ${ }^{1} \mathrm{H}$ NMR spectroscopy. The most common forms that the calix[4]arene can adopt are shown in Figure 21, and their characteristic peaks are shown in

Table 1. The most noticeable differences occur with the methylene bridging doublets i.e. between 3 - 4.5 ppm, and Figure 22 shows the difference in the methylene signals (circled) for the cone, partial cone and 1,3-alternate respectively. 


\begin{tabular}{|c|c|c|c|}
\hline Conformation & \multicolumn{3}{|c|}{ Resonance Signals $(\mathrm{s}=$ singlet, $\mathrm{d}=$ doublet $)$} \\
\hline & $\mathrm{ArH}$ & $\mathrm{ArCH}_{2} \mathrm{Ar}$ & $\mathrm{C}\left(\mathrm{CH}_{3}\right)_{3}$ \\
\hline Cone & $1 \times \mathrm{s}$ & $1 \mathrm{x}$ pair $\mathrm{d}(J=12 \mathrm{~Hz})$ & $1 \times \mathrm{s}$ \\
\hline Partial cone & $\begin{array}{l}2 \times \mathrm{s} \& 2 \times \mathrm{d} \text {, or } \\
4 \times \mathrm{s}\end{array}$ & $\begin{array}{l}2 \text { pair x d, }(J=12 \mathrm{~Hz}) \text { or } 1 \mathrm{x} \\
\text { pair d \& } 1 \text { x s }(J=12 \mathrm{~Hz})\end{array}$ & $\begin{array}{l}3 \times \mathrm{x} \\
(1: 2: 1)\end{array}$ \\
\hline 1,2-Alternate & $2 \times s$ & $1 \times \mathrm{s}, 2 \times \mathrm{d}(J=12 \mathrm{~Hz})$ & $1 \times \mathrm{s}$ \\
\hline 1,3-Alternate & $1 \times \mathrm{s}$ & $1 \times \mathrm{s}$ & $1 \times \mathrm{s}$ \\
\hline
\end{tabular}

Table 1: ${ }^{1} \mathrm{H}$ NMR for the four dominant calix[4]arene conformers

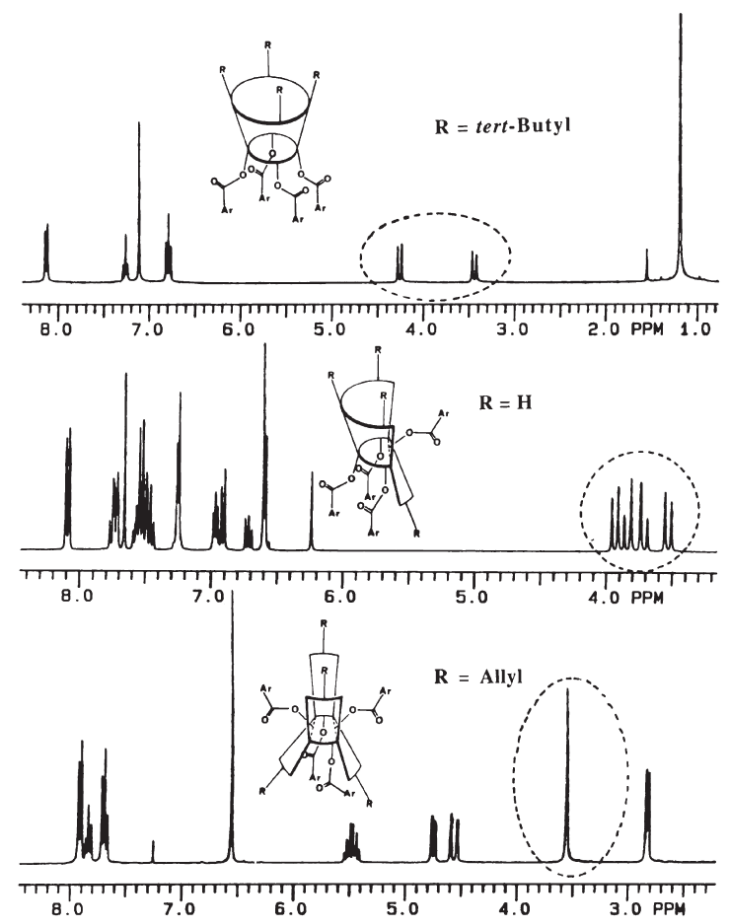

Figure 22: ${ }^{1} \mathrm{H}$ NMR of (a) the cone, (b) the partial cone and (c) the 1,3-alternate ${ }^{50}$.

Obviously as the number of aryl groups increase, so does the number of possible conformations, e.g. the calix[4]arene has four conformations while the calix[8]arene has a possibility of sixteen conformations. Additionally all the calixarenes have the ability to distort slightly from the standard cone shapes in that they protrude outwards and are so called distorted cone etc. This ability further increases the conformers available. 
Calixarene conformational change can be followed using proton NMR spectroscopy. The ${ }^{1} \mathrm{H}$ NMR spectrum of p-tert-butylcalix[4]arene, Figure 23, shows a resonance pattern of the bridging methylene groups, which at room temperature appear as a set of broad doublets but a higher temperature appears as a sharp singlet. The conclusion from this is that the cone conformation of the calix[4]arene is slowly interconverting at the lower temperature but doing so rapidly at higher temperature. The rate of coalescence is given as $\mathrm{k}_{\text {coalescence }}=\frac{\pi \Delta v}{\sqrt{2}}$, where $\Delta \mathrm{v}$ is the difference in chemical shift $(\mathrm{Hz})$ between the centres of the two peaks of the doublet ${ }^{51}$. From this the activation of rotation can be determined using $\Delta G=R T c\left[23+\operatorname{In} \frac{T c}{\Delta v}\right]$, where $\mathrm{R}$ is the gas constant and $\mathrm{T}_{\mathrm{c}}$ is the coalescence temperature in Kelvin ${ }^{51}$.

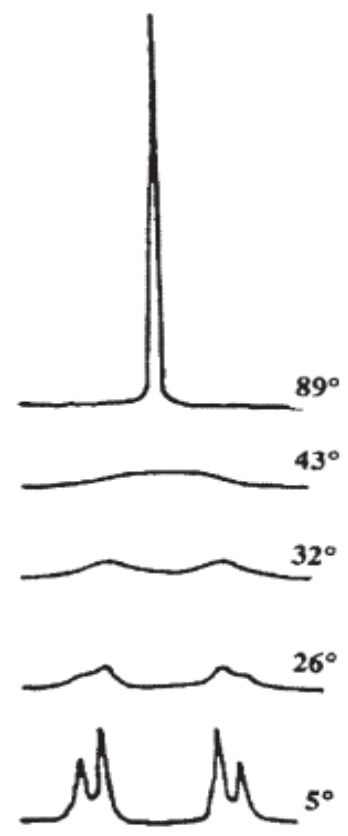

Figure 23: Temperature dependent ${ }^{1} \mathrm{H}$ NMR of the $p$-tert-butylcalix[4]arene in $\mathrm{CDCl}_{3}{ }^{11}$.

Changing the para-substituted group on the upper rim has very little effect on the conformational ability of the calixarene but reducing the number of hydroxyl groups does lower the barrier slightly. However solvent effects have an influence on the adopted form. For example, polar solvents (pyridine) can interfere with the intramolecular hydrogen bonding interactions on the lower rim thus lowering the conformation energy barrier. 
${ }^{13} \mathrm{C}$ NMR spectroscopy can also be used along with ${ }^{1} \mathrm{H}$ NMR spectroscopy to help assign the different conformations in solution ${ }^{52}$. In the cone form, two signals are found around $31 \mathrm{ppm}$ which corresponds to the two methylene doublets around 3.33 and $4.30 \mathrm{ppm}$ in the proton spectra, whereas in the partial cone four doublets are seen in the proton bridging region and signals between 50 and $60 \mathrm{ppm}$ are seen in the ${ }^{13} \mathrm{C}$ spectra. The 1,2-alternate form shows the two doublets about 3.30 and $4.30 \mathrm{ppm}$ along with a singlet about $4.02 \mathrm{ppm}$ in the ${ }^{1} \mathrm{H}$ NMR spectra and its corresponding carbon data shows signals around 30 and $38 \mathrm{ppm}$. The 1,3-alternate form often shows a broad singlet around $3.9-4.0 \mathrm{ppm}$ for the bridging protons and its carbon signal appears around $38 \mathrm{ppm}$ in the carbon spectra. Using this data can be helpful in determining what forms are present in the spectrum. However, deviations from these standards are regularly seen.

To prevent the interconversion of conformers, it is necessary to block the two pathways which allow movement, namely the endo and exo routes, (Figure 24). This can be done with large bulky groups which are unable to pass through the annulus of the ring or by a bridge across one or both rims. With the calix[4]arenes, the exo rim pathway is normally so hindered that only the endo rim allows conformational inversion, even if the upper rim contains only a $\mathrm{H}$ atom as its substituent. This is not true for larger calixarenes and will not be discussed here. Therefore for calix[4]arene inversion, the endo pathway through the annulus is adopted ${ }^{53}$. 


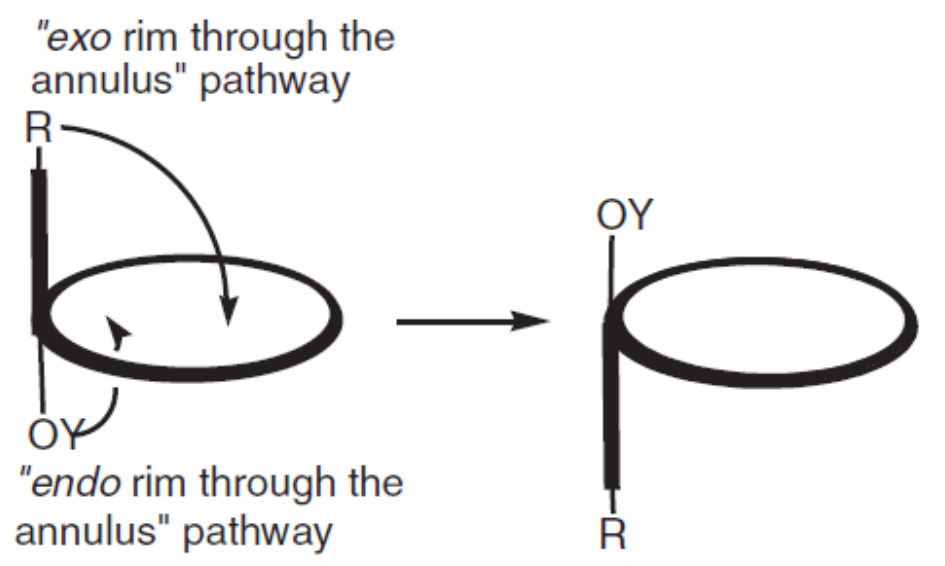

Figure 24: The exo and endo routes through the annulus ${ }^{53}$.

Since this is the adopted route several attempts to determine the minimum size of the OR group capable to pass through the annulus has been investigated ${ }^{54}$. Iwamoto et $a l$. investigated the minimum size requirements for passage through the annulus to occur $^{53}$. They found that both the tetramethyl and tetraethyl substituted calix[4]arenes were flexible enough to allow interconversion at raised temperature, but when the tetrapropyl ether was investigated it was found to be rigid even at higher temperatures. When larger groups were tested the same result as the tetrapropyl was observed. The conclusion from Iwamoto's work is that the tetraethers substituted with propyl groups or higher are unable to undergo interconversion between various conformations ${ }^{55}$.

The other possibility of preventing interconversion is by bridging the aryl groups ${ }^{54}$. Generally it is easier to bridge the lower rim over the upper rim and double bridging (double cavity) is also possible. By using bridging, compounds are created that are less conformationally mobile which helps to prevent complete inversion. However, partial inversion is still possible as shown in Figure 25, where the non-bridged aryl groups partake in flipping between their up and down forms. 


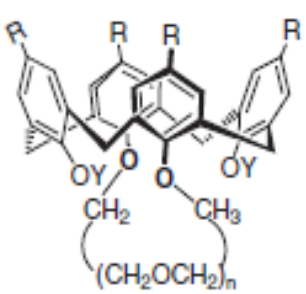

a

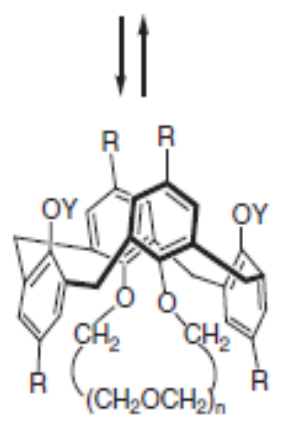

b

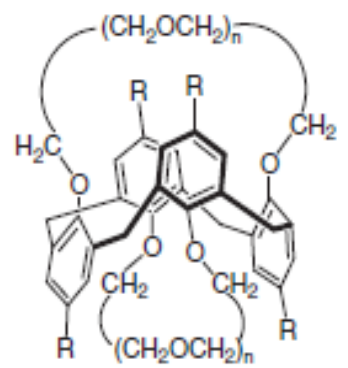

$c$

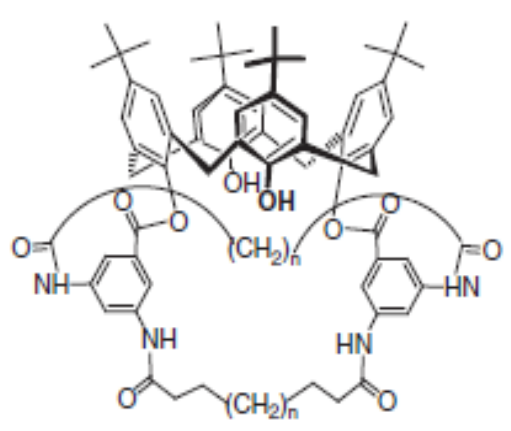

d

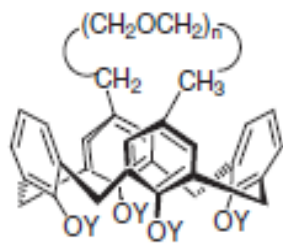

e

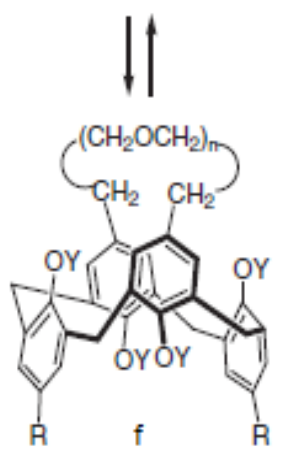

Figure 25: Partial inversion of endo bridged calix[4]arenes. (a) and (b), (e) and (f) are examples of partial conversion between cone and 1,3-Alt. (c) and (d) are examples of doubly bridged calix[4] $\operatorname{arenes}^{54}$.

If all the hydrogen's on the lower rim were replaced with large bulky groups, such as large alkyl groups, conformational immobilization may be achieved providing that the desired form is the only one present in the beginning. The ratios of conformers depend on a variety of factors including reaction conditions, the para-substituent and the steric constraints of the applied functional group. The type of base used can greatly influence the type of conformations obtained. For example, with small strong bases, such as $\mathrm{NaH}$, a mixture of cone, partial cone and 1,3-alternate forms are produced whereas larger bases, such as $\mathrm{K}_{2} \mathrm{CO}_{3}$ or $\mathrm{Cs}_{2} \mathrm{CO}_{3}$ produces mixtures consisting of only a small fraction of cone conformer and large amounts of partial cone and 1,3-alternate forms ${ }^{11}$. Other factors to consider are the para-substituent on the calixarene and the leaving group on the alkylating agent ${ }^{55}$.

Stepping away from NMR spectroscopy, we come to mass spectrometry. It is an extremely useful tool as it gives a detailed account of all the fragments present in a 
compound thereby allowing the analyst to determine the contents of their sample logically. Initially work was done on a calixresorcarene in 1964 by Erdtman and coworkers $^{56}$, and slowly moved up to the analysis of calixarenes in the 1970's. The unsubstituted calix[8]arene shows a strong signal at $\mathrm{m} / \mathrm{e}$ 648, which is the exact weight expected for the cyclic calix[4]arene. This was accepted for years as data for the cyclic calix[4]arene structure of the cyclooligomers. Often it was noted that several smaller peaks would occur above this and this caused confusion. Eventually using a trimethylsilyl derivative a signal for the cyclic calix[8]arene was observed at $\mathrm{m} / \mathrm{e} 1872$ with a smaller peak at $\mathrm{m} / \mathrm{e} 936$ representing the cyclic calix[4]arene. A possible explanation for the smaller peak could be due to the ion beam causing a conversion from the calix[8]arene to the calix[4]arene. Kämmerer and co-workers have provided mass spectrometry data for a range of calixarenes and their derivatives but it is worth noting that fragmentation is a common occurrence. Even the unsubstituted calix[4]arene is prone to fragmentation with the appearance of peaks for one, two and three phenolic units ${ }^{31}$. It is also worth mentioning here that limited mass spectrometry use was available during the course of this project and fragmentation was seen in the majority of samples.

\subsubsection{Dimerisation of calixarenes}

It has been well documented that molecules in solution can combine to form larger assemblies from relatively few starting materials ${ }^{57}$. This amazing area of 'selfassembly' attracts the interests of many scientists as large range of different architectures can be built from only a few starting building blocks ${ }^{58}$. Another attraction of this area is that the synthesis is usually straight forward, with encouraging yields ${ }^{58}$. There are several forces which can be employed during the construction of these architectures such as dipole-dipole interactions, hydrogen bonding, coordination to metal ions and many others. Hydrogen bonding is one of the main forces used, as can be shown by numerous examples in nature ${ }^{57}$.

The self-assembly of calix[4]arenes is well known and to date there are a number of papers referring to a wide range of differently functionalised calixarenes which are capable of undergoing various types of dimerisation ${ }^{59-62}$. Rudzevich et al. have 
previously reported the dimerisation of tetraureacalix[4]arene (III) in aprotic apolar solvents ${ }^{54}$. The cavity formed from the formation of these dimers has a large internal volume capable of housing solvent molecules of various sizes. In simple cases the dimerisation of calixarenes can be observed from NMR spectroscopy, as shown in Figure 26 where the signal for the amide $\alpha$ protons are shifted downfield due to the interaction with the carbonyl group of another calixarene ${ }^{54}$.

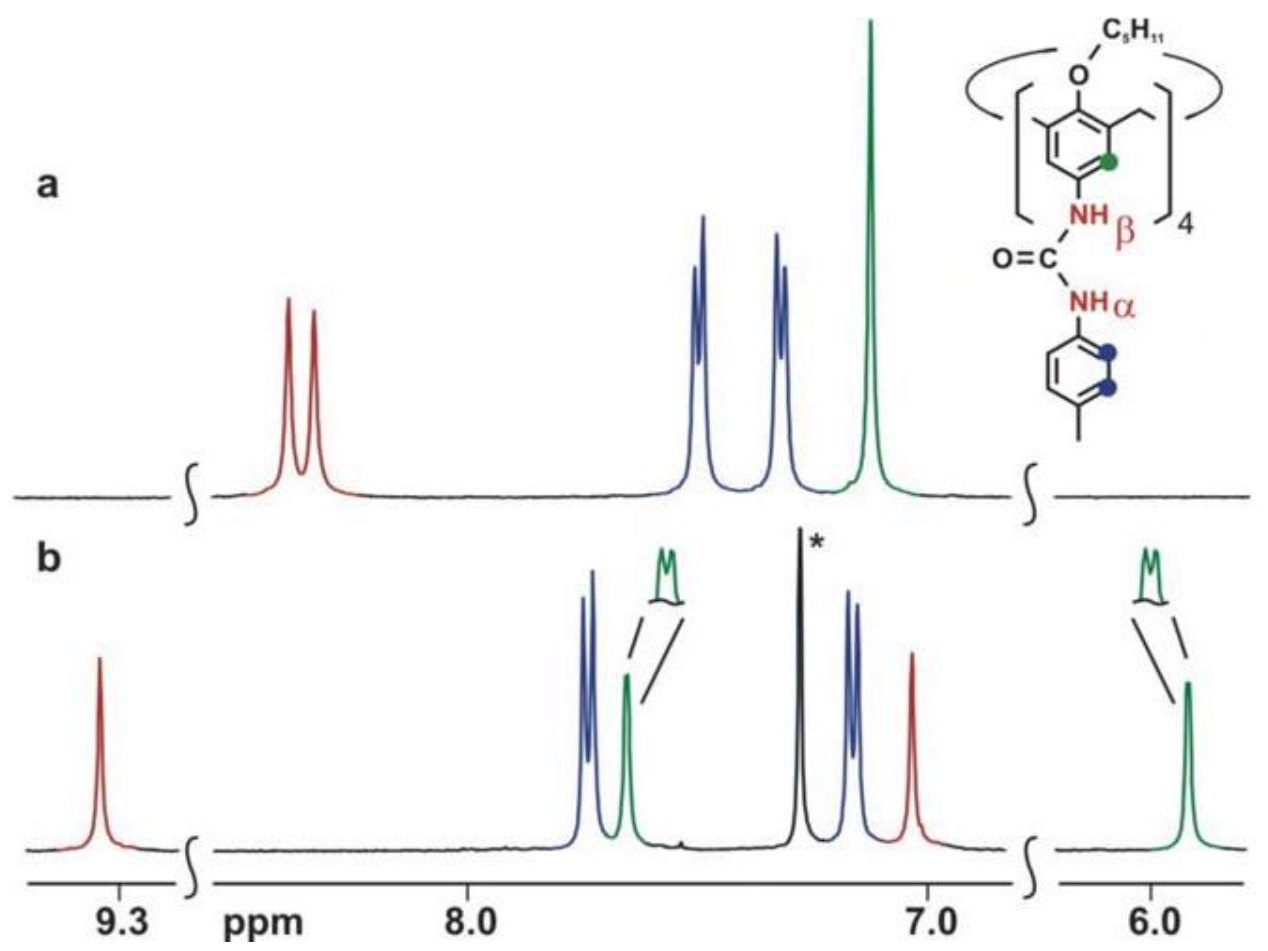

Figure 26: ${ }^{1} \mathrm{H}$ NMR spectra of the tetraureacalix[4] arene (III) (a) in $\mathrm{d}_{6}-\mathrm{DMSO}$ and (b) in $\mathrm{CDCl}_{3}$. The solvent is marked with an asterisk ${ }^{57}$.

There are a few ways that a dimer can assemble, such as head-to-head or tail-to-tail representing a homodimerisation fashion, or it can adopt a head-to-tail orientation giving the heterodimerisation form and it is not uncommon to find a mixture of the two forms in solution. One could continue on the train of thought that if the rims were substituted with large bulky groups, this may prevent dimerisation or allow some form of selectivity to occur. To this end Rudzevich et al. conducted a series of experiments involving bulky groups on the lower rim as well as the use of loops in an attempt to selectivity dimerise their compounds (IVa \& IVb) ${ }^{54}$. They tried to 
attach a number of these bulky residues to their tetraurea calixarenes in the hope that some selectivity may be obtained. However they observed that the formation of the dimer still occurred with their compounds (Figure 27).

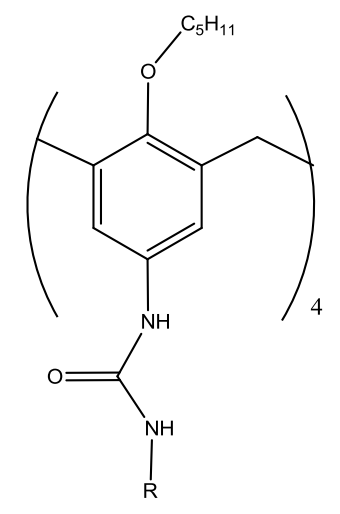

$$
\mathrm{R}=
$$

(a)

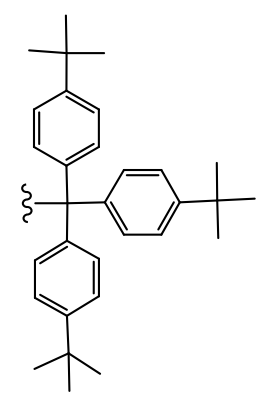

(b)

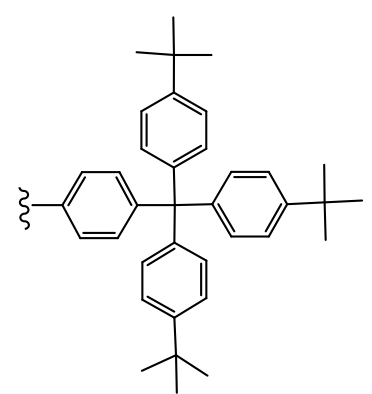

Figure 27: Tetraurea calix[4] arenes substituted with various bulky groups (IVa \& IVb) ${ }^{54}$.

As similar results were seen for other bulky residues, they concluded that the selective dimerisation of the tetraureacalix[4]arenes could not be controlled by use of bulky groups alone. To this end, they began work with 'loops' where two adjacent urea residues were covalently connected by an aliphatic chain. Various combinations were tried as shown in Figure 28 in the hope that these loops would bring selective dimerisation as the urea residue of the other calixarene must be able to penetrate the loop for dimerisation to occur. 

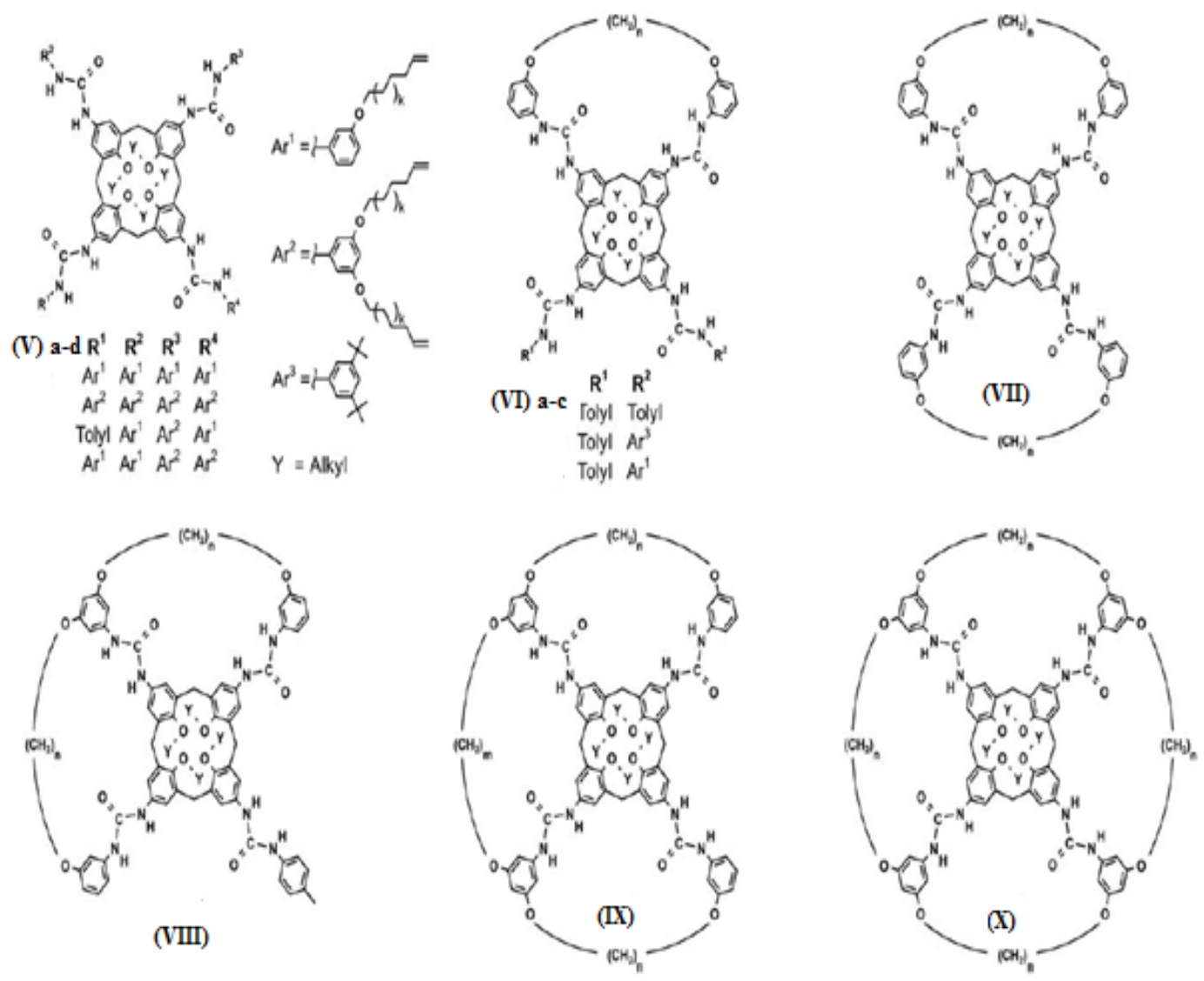

Figure 28: Possible tetraureas calixarenes where adjacent urea residues are connected by 1-4 loops $(\mathrm{V}-\mathrm{X})^{54}$.

They observed that compounds containing a single loop were still able to form homodimers but only one of two possible regioisomeric dimers of the AABB type was formed, Figure 29. This was more than likely due to the fact that overlapping loops is strongly disfavoured for steric reasons.

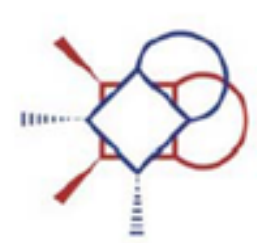

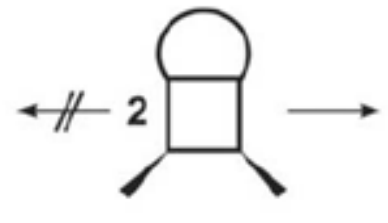

$\mathrm{VIa}$

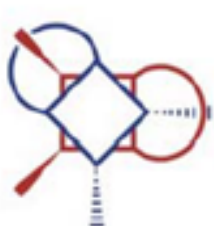

$\mathrm{VIa}-\mathrm{VIa}$

Figure 29: Regioisomeric dimerisation of mono-loop tetraurea calix[4] arene (VIa) ${ }^{54}$. 
Furthermore they found that bis-, tris- and tetra-loop substituted tetraureacalix[4]arenes did not form homodimers at all as dimerisation wasn't possible without loop overlap. The final step in their investigation was to combine loops and bulky residues to test for additional selectivity. The idea behind this was that if the loop was sufficiently small then large bulky groups should be unable to penetrate the loop, but should still allow some form of dimerisation, Figure 30 . In other words, the small loops would prevent the bulky groups from interacting at particular sites, so if dimerisation was to occur, it would selectively happen at the sites opposite from the loops. This was tested with various loops and bulky groups and from the NMR spectra they observed that homodimerisation had occurred where the bulky group was 'slim' enough to penetrate the loop.

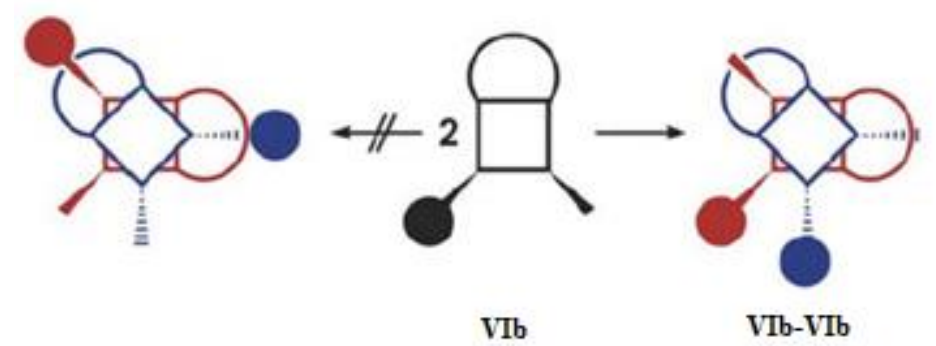

Figure 30: Homodimerisation of tetraurea calix[4]arene with small bis-substituted loops and bulky groups $(\mathrm{VIb})^{54}$.

Overall from their studies, they concluded that within a different mixture of tetraureacalix[4]arene only one or several dimeric pairs are formed (intermolecular selectivity) and even if only a single tetraureacalix[4]arene is present various regioisomeric dimers are possible (regioisomeric dimerisation) ${ }^{54}$.

Rudkevich et al. have also synthesised calixarene-based dimeric capsules capable of housing nitric oxide gas ${ }^{63}$. In most mammals nitric oxide is involved in the regulation of many pathways including the cardiovascular, respiratory and nervous systems ${ }^{64}$. Nitric oxide has also been proven to show beneficial effects against some diseases and as such have found roles as antianginal drugs and some anti- 
inflammatories ${ }^{65}$. It has also shown some antibacterial properties and as such the search of synthetic compounds which could chemically store and release nitric oxide is a current hot area for chemists ${ }^{66}$. Rathore et al. have developed calixarene-based units which are capable of storing nitric oxide in the form of entrapped $\mathrm{NO}^{+}$ion $^{67}$. Botta et al. have also mentioned the formation of stable complexes between the $\mathrm{NO}^{+}$ ion and a calixarene derivative, where the ion is tightly bound within the cavity of the calixarene through cation- $\pi$ interactions ${ }^{68}$.

\subsubsection{Applications of calixarenes}

Functionalised calix[4]arenes have a wide variety of promising applications and will be discussed here briefly. Calixarenes are an attractive family across the sciences particularly in the biomedical sciences ${ }^{69}$. Their amphiphilic properties make them potential candidates in the formation of new ion channels and ion transporters. The hydrophobic cavity offers an ideal binding locality for non-covalent groups and its hydrophilic character allows the unit as a whole to move into the bilayer and either form a channel or simply deposit its carrier. Since 1999, great strides have been made in biomedical chemistry with metal binding calixarenes. It is well known that intra- and extracellular concentration of different biologically necessary metal ions differ and that the ratio between these two is very important for good health e.g. cell signalling $^{70}$. The transport of inorganic cations into or out of the cell can occur by two ways, by ionophore mediated transport where it is bound by a chelator and is carried to the bilayer or by the formation of a trans-membrane channel which is generally selective for a particular ion ${ }^{69}$. Ion channels have a faster flow rate than individual ionophores. As a result there is more focus on the development of ion channels which create a hydrophilic passage inside the hydrophobic cell membrane. Clearly, disruption of these channels can lead to various diseases and, given the complexity of the task, any synthetic system needs to be able to allow charged metal ions to pass through the hydrophobic bilayer but also to be selective for a specific cation. In theory calixarenes can form channels in membranes in two ways. The first involves the cone conformation of calixarenes with the cavity at either end of the membrane and the second occurs with the 1,3-alternate which has the potential to form a channel with the cavity sitting in the middle of the membrane (Figure 31). Ideally the best channel mimics would sit into the membrane and provide binding 
sites for the metal ions to facilitate their transport into and out of the cell via the hydrophobic cavity.

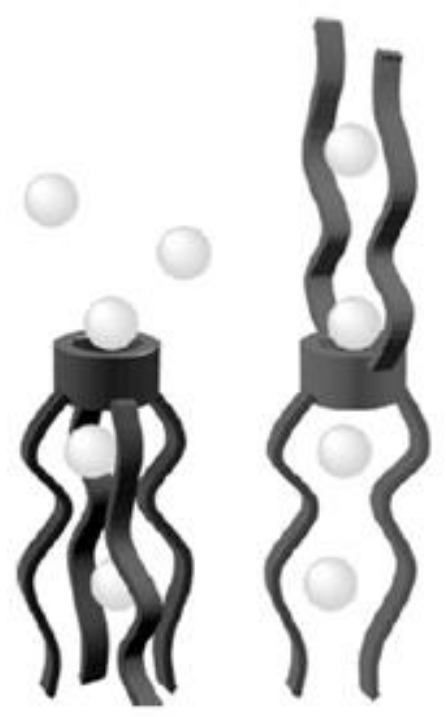

Figure 31: The two current possibilities of how calixarenes can form a channel in the membrane $^{69}$. The cone form the left and the 1,2-alternate on the right.

So far, research on calixarene ion channels has focused mainly on the $\mathrm{Na}^{+}$and $\mathrm{K}^{+}$ transport systems. Some calixarenes which are not large enough to span the membrane alone have being reported to self-assemble within the bilayer and form a channel $^{71}$. Jin et al. have shown that the cone conformation of the tetra-ester derivative (XI \& XII) of the calix[4] arene is highly selective for $\mathrm{Na}^{+}$ions (Figure 32) and that there is a possibility that the calixarene forms a dimer in the membrane to function either as a channel or as a shuttle ${ }^{72}$. The 1,3-alternate calixarenes are much more efficient than the cone calixarenes and this has led to the idea that they may function by a different transport procedure ${ }^{73}$. Maulucci et al. carried out work on the calix[4]arenes derivatives (XIII a-e) shown in Figure 33 and have reasoned that the 1,3-alternate forms are large enough to span the bilayer but that the cone forms need to dimerise ${ }^{73}$. Without this need to dimerise, it is possible that the 1,3 -alternate is able to form a more efficient channel. 


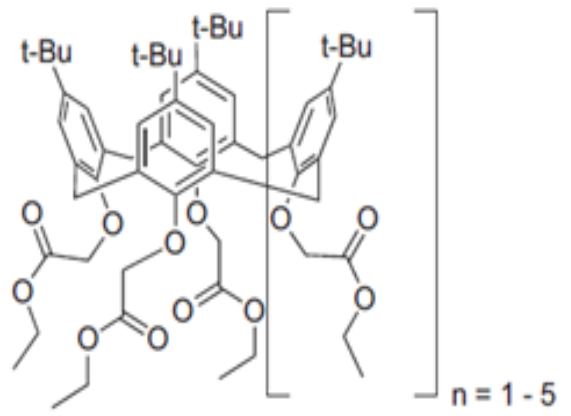

(XI)

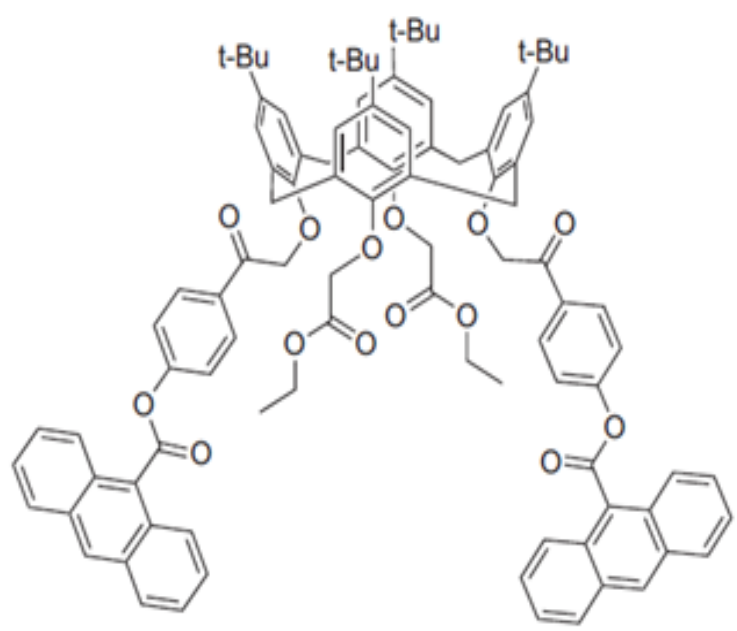

(XII)

Figure 32: $\mathrm{Na}^{+}$selective cone conformation calixarenes ${ }^{72}$.
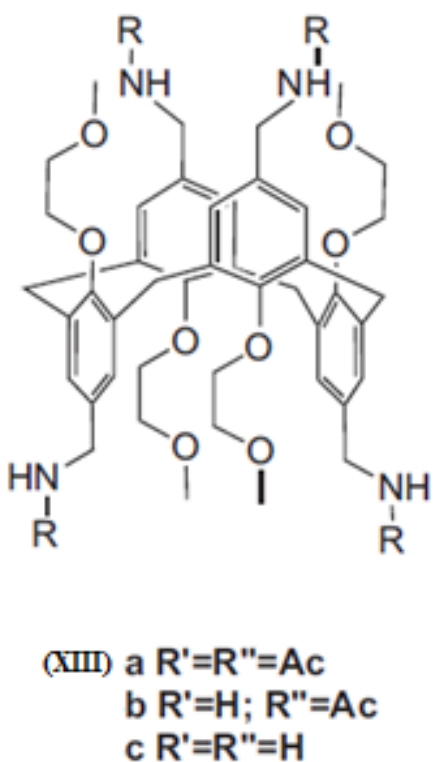

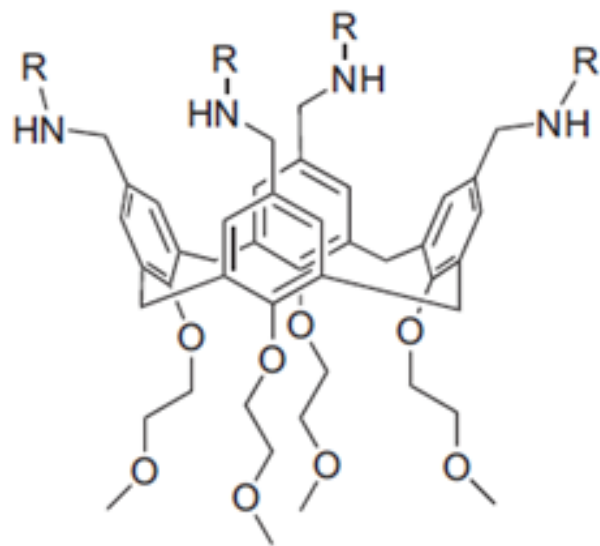

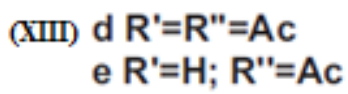

Figure 33: 1,3-alternate and cone conformations (XIII a-e $)^{73}$.

Very few reports have been published on the potassium channels and their transport pathways into and out of the cells. Arduini et al. ${ }^{74}$ constructed a promising mimic of a $\mathrm{K}^{+}$channel (XIV) using a tubular calixarene which can span the bilayer to form a channel, (Figure 34). 


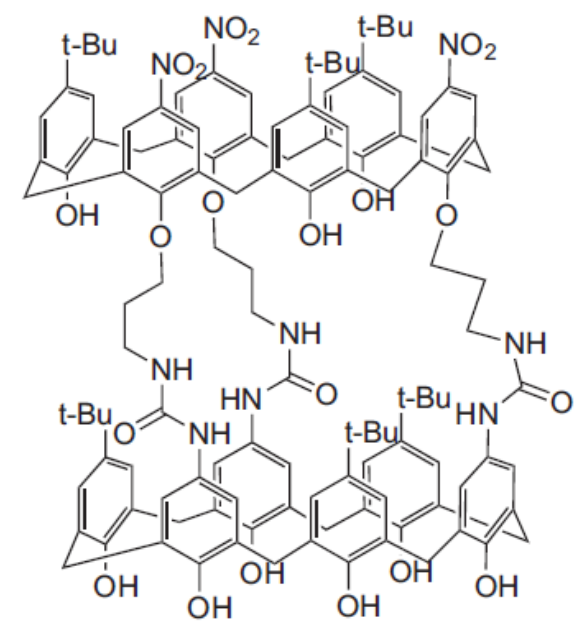

Figure 34: Arduini's synthetic $\mathrm{K}^{+}$channel (XIV) ${ }^{74}$.

In this set-up, the $\mathrm{K}^{+}$ion is bound by both the phenolic and carbonyl oxygens to allow it to pass through the channel into the cell. Hydrogen bonding is an important stabilizing factor, resulting in weak binding of the metal cation, i.e. hydrogen bonding is actually competing with the metal ion complexation reaction. This is suspected to play a role in the release of the ion allowing translocation to and from cells ${ }^{74}$.

Apart from roles as synthetic ion channels calix[4]arene derivatives have also been employed in the medical industry in Magnetic Resonance Imaging (MRI) and radiotherapy as imaging agents ${ }^{69}$. Lanthanides and actinides are often used in these techniques and calixarenes have been found to bind these effectively. Radiotherapy is most commonly used in cancer treatments but to prevent damage to healthy cells the radioactive metal ion must be complexed by strong chelator's. Gansey et al. have complexed several calix[4]arene derivatives (XV \& XVI) with several transition metal ions, including the $\operatorname{Re}(\mathrm{V})$ ion, (Figure 35), and tested the effects in vivo in mice ${ }^{75}$. However, they found that the binding to the target vector strongly depends on the peptides present on its surface along with the dosage injected. Gansey et al. concluded that complexes of this type would be unsuitable for human usage. However, further research into alternative calixarene complexes is currently being investigated. 

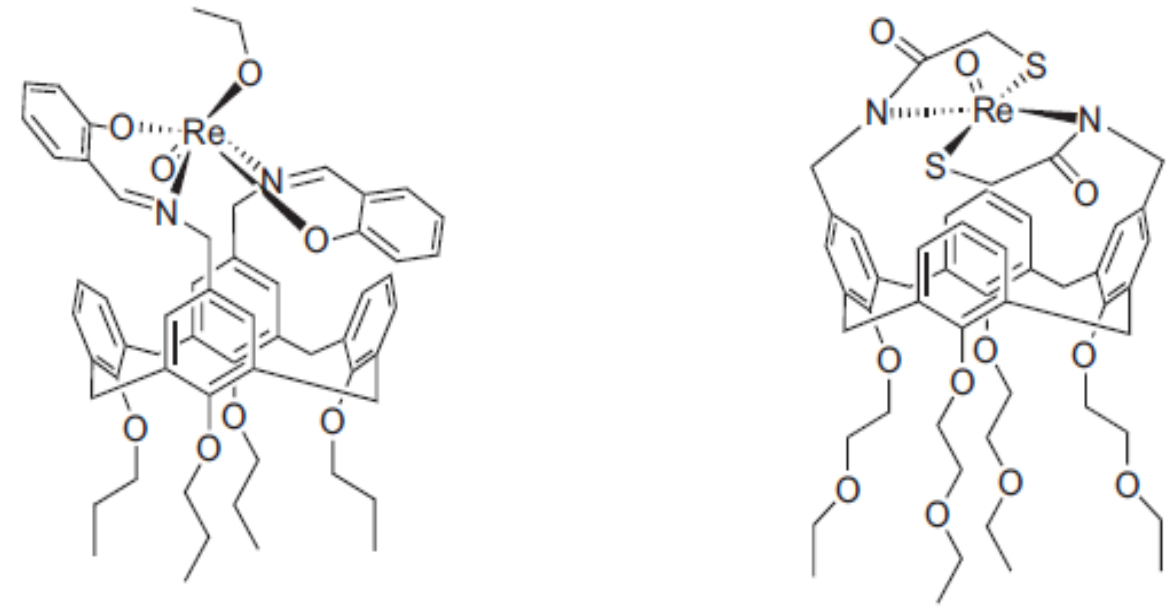

Figure 35: Calix[4]arene complexes used for Radiotherapy, XV (left) \& XVI (right) ${ }^{75}$

Most MRI scans rely on the proton signal of water molecules inside body compartments to produce usable images. This is done either by observing the proton density or the different relaxation times in different areas of the body. Most MRI contrast agents use $\mathrm{Gd}^{3+}$ ions which help to shorten the relaxation times and brighten the image of areas which contain the contrast agent. Because a higher dose of the MRI contrast agent is needed, the agents used must be stable to prevent adverse sideeffects to the patient. To this end, strong chelator's with the ability to bind $\mathrm{Gd}^{3+}$ very efficiently as well as high relaxivity are needed. Georgiev et al. prepared a calix[4]arene tetraamide (XVII) derivative which was used to chelate $\mathrm{Gd}^{3+}$, but unfortunately the stability and water solubility of the compound were found to be much too low so no further research was performed ${ }^{76}$. Bryant et al. synthesised a new chelator (XVIII) with four additional chelating groups compared to Georgiev ${ }^{77}$. While the presence of the four new chelating groups added extra stability to the molecule the complex itself was still not stable enough for human trials. 

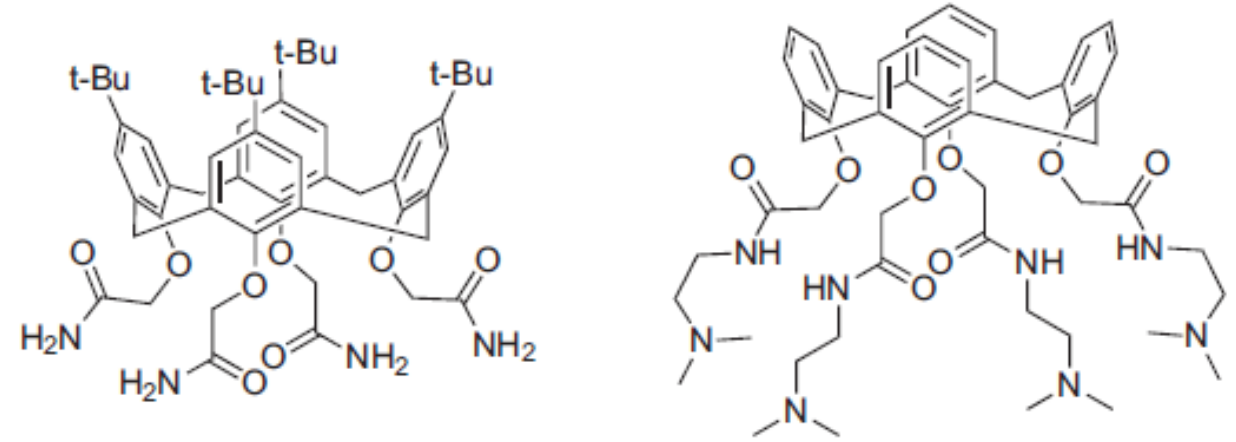

Figure 36: Georgiev's (XVII) ${ }^{76}$ and Bryant's (XVIII) ${ }^{77}$ calix[4]arene MRI contrast agents

Krishnan et al. formed a calix[8]arene derivative (XIX) which was capable of chelating eight $\mathrm{Gd}^{3+}$ ions. This compound was found to be very stable and was highly soluble in water making it the first calixarene based MRI contrast agent ${ }^{78}$. This compound is currently undergoing trials for human use.

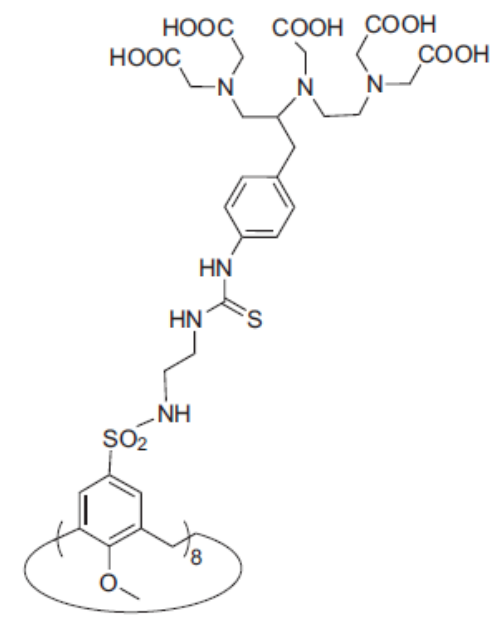

Figure 37: Krishnan's calix[8]arene MRI constant agent (XIX) ${ }^{78}$

\subsubsection{Metal ion complexation.}

It has long been known that calix[4]arenes which have been functionalised at the upper or lower rim or both possess the ability to bind metal ions such as zinc, copper, nickel, mercury and many others ${ }^{79}$. This complexation ability has given rise 
to various applications of the calix[4]arenes and has drawn a lot of interest to this area.

Zinc.

Zinc is a member of the transition metals located in group 12 of the periodic table ${ }^{58}$. The $\mathrm{Zn}^{2+}$ cation is diamagnetic and so is often used in NMR spectroscopy studies. The preferred oxidation state of zinc is +2 where it adopts a tetrahedral, square planar or octahedral form depending on the nature of the ligands. Zinc is a relatively hard Lewis acid and will form stable complexes with hard bases, such as oxygen donors, medium hard bases - nitrogen donors, and some soft bases such as the halogens ${ }^{58}$.

Zinc is an essential trace element needed for good health ${ }^{80}$. It is involved in all aspects of metabolism as well as other functions such as the regulation of gene expression. Zinc ions are key structural components of many peptides with highly specific functions. Zinc binding sites in proteins are made up of the sulphur atom of cysteine, the nitrogen of histidine or the oxygen of aspartate or a combination of the latter two $^{81}$. Minor changes in zinc concentration within the body can result in multiple biological and clinical effects. Cell death is normally carried out by apoptosis, the regulated biological pathway for the removal of dead or damaged cells and it is influenced mainly by zinc levels. The signalling mechanism to initiate cell death involves fourteen members of the caspase family and studies have shown that changes in zinc concentration can influence cell sensitivity to apoptosis. Zinc has been proven to block some of the caspases involved in regulating cell death, protecting the cell from apoptosis ${ }^{82}$.

Brown et al. experimented with the unsubstituted calix[4]arene (XX) and the structural orientation it adopts in terms of self-assembly and they also investigated the effect metal binding has on this orientation ${ }^{83}$. It is known that the unsubstituted calix[4]arene has low solubility and so its use as a model for self-assembly 
interactions was poor, but they had recrystallized the unsubstituted calix[4]arene from $n$-butylamine (nBA) and found that a self-assembled construct containing a hydrogen-bonded inclusion amine guest was obtained as well as the inclusion of some water molecules, (Figure 38).

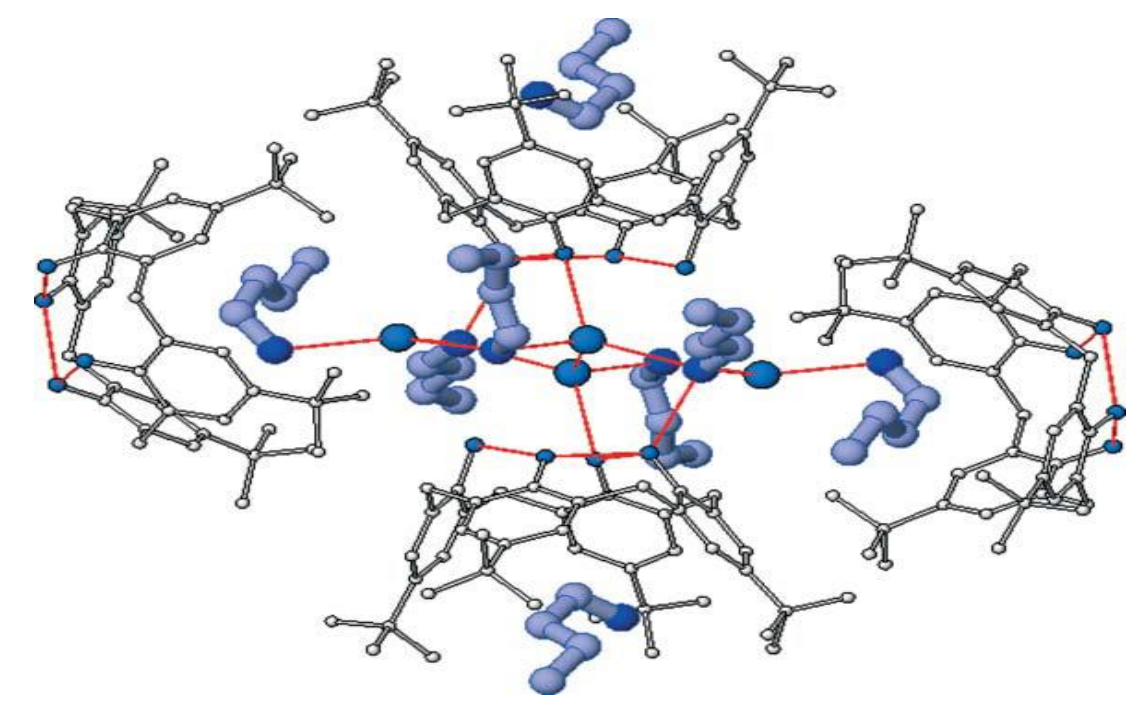

Figure 38: $\mathrm{X}$-ray structure of $(\mathrm{XX})$ with $\mathrm{nBA}$ in both the calixarene main cavity and the extended cavity. Water molecules are shown as large blue spheres, phenolic hydroxyls as small blue spheres, nBA large blue structures and hydrogen bonding in $\operatorname{red}^{83}$.

They also determined that the water molecule and two of the nBA molecules were forming hydrogen bonds with the phenolic hydroxyls of two adjacent calix[4]arene units of the self-assembled complex. They rationalised that both the hydrophobic cavity and the hydroxyls offered ideal sites for secondary coordination which encouraged them to introduce metal ions to the construct. They began their work with zinc and silver salts. The zinc chloride calix[4]arene complex was dissolved in $\mathrm{nBA}$ and water whose structure was determined by X-ray analysis (Figure 39). They found that the calix[4]arene molecules were arranged as layers of 'self-included dimers' with two different and distinct bundles occupying the space between the layers, and each bundle was found to contain a $\mathrm{Zn}^{2+}$ center. They suggest that the difference between the parent crystal structure (Figure 38) and the metal coordination structure (Figure 39), is due to the fact that coordination bonds are more 
thermodynamically favourable than hydrogen bonds formed with surrounding calixarenes.

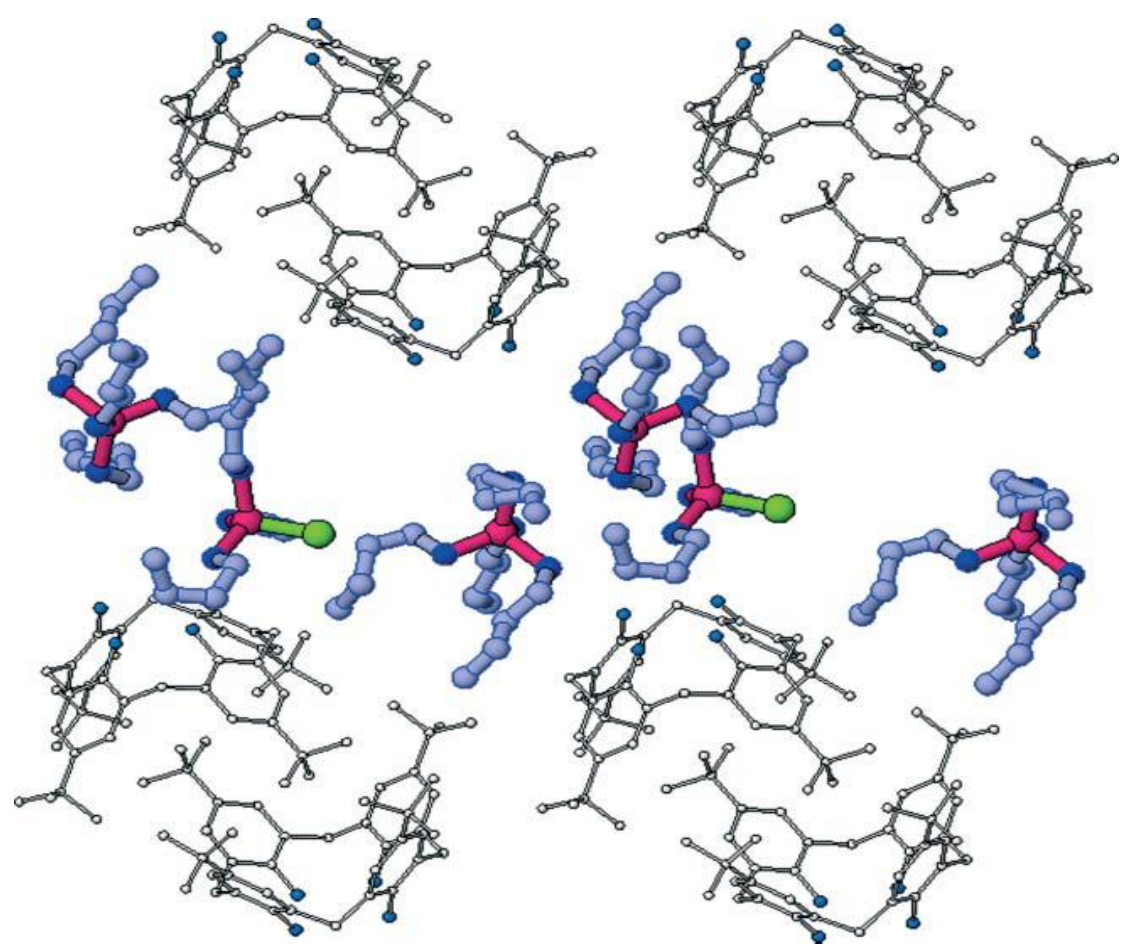

Figure 39: X-ray structure of $(\mathrm{XX})$ with $\mathrm{nBA}$ and phenolic hydroxyls in blue, $\mathrm{Zn}^{2+}$ in red and $\mathrm{Cl}^{-}$in green ${ }^{83}$.

McGinley et al. have published several papers involving zinc complexation, one of which describes calix[4]arene schiff base derivatives (XXI) as potential ligands for fluorescence studies ${ }^{84}$. They attempted to complex zinc to their ligand (Figure 40) on the lower rim using three different zinc salts (acetate, chloride, perchlorate). From analysis of their data, it was noted that only the acetate form of the zinc metal was binding to their ligands despite several metal ion acceptor sites available on the ligand. 


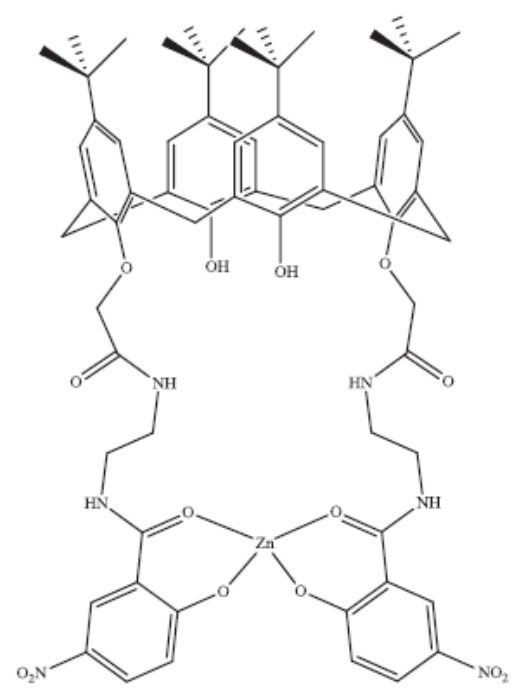

Figure 40: N-(2-aminoethyl)-2-hydroxy-5-nitrobenzamide functionalised calix[4]arene as potential $\mathrm{Zn}^{2+}$ metal ion binding construct $(\mathrm{XI})^{84}$.

After a series of ${ }^{1} \mathrm{H}$ NMR spectroscopy experiments an explanation was put forward to help to explain the unusual binding. The reasoning came from the examination of the relative basicity's of the ligand and the respective counter-ions. The lower rim of their compound was functionalised with para-nitrophenol which has an approximate pKa value of 10.8 in $\mathrm{DMSO}^{85}$. The acetate ion, the conjugate base of acetic acid, has a pKa of 12.3 in $\mathrm{DMSO}^{86}$. This shows that the acetate ion is more basic than the ligand and as a result would be able to deprotonate the phenol group to form the phenoxide. This negatively charged species now a greater binding site for the $\mathrm{Zn}^{2+}$ ion than the previous neutral ligand. When the chloride and perchlorate ions were examined for their pKa values (1.8 and 10 respectively) they were found to be more acidic than the ligand, and would not have a strong enough basic character to deprotonate the phenol groups. Being unable to deprotonate the phenol group's means that the preferred metal binding site is not formed and complexation will not occur.

\section{Copper.}

Copper is alongside zinc in the periodic table ${ }^{58}$. It is found in group 11. The $\mathrm{Cu}^{2+}$ cation is paramagnetic and can exist in a range of colours such as blues and greens (copper perchlorate and copper chloride). Metallic copper is a bright red colour. 
Copper can be found in its pure form in small amounts but most of the world's copper is produced from iron-containing ores via smelting and some of the chief impurities include zinc, tin, lead and arsenic. Copper is essential for good health in many life forms. In humans, it is incorporated into a variety of proteins and metalloenzymes which perform essential metabolic functions, as well as being involved in the formation of red blood cells, absorption of iron and various other roles around the body. In molluscs and crustaceans it replaces iron in haemoglobin in oxygen transport. A deficiency in copper can lead to many diseases such as Wilson's disease and anaemia. Copper is highly linked with Alzheimer's disease. Currently it is unknown whether copper is a causative or preventive agent of Alzheimer's. Some research suggests that copper may lead to the abnormal growth of protein clumps in the brain ${ }^{87}$, while other studies indicate that copper has beneficial roles in treating Alzheimer's ${ }^{88}$. Copper is most commonly employed in electrical wires and cables, roofing and cooking utensils ${ }^{58}$.

While copper is essential for good health of plants and animals, too much of it can lead to serious problems so the detection and determination of copper levels has received increased attention over the last few years ${ }^{79}$. Zhou et al. have designed piperidinoacetyl-containing calix[4]arenes (XXII) and have tested their ability to uptake metal ions in the hope of exploiting them to scavenge heavy metal ion pollutants in water supplies ${ }^{89}$. They formed their calix[4]arene copper complex by stirring 5,11,17,23-tetra-tert-butyl-25,26,27,28tetrakis(piperidinocarbonylmethoxy)calix[4]arene in acetonitrile and adding $\mathrm{Cu}\left(\mathrm{ClO}_{4}\right)_{2} \cdot 6 \mathrm{H}_{2} \mathrm{O}$. Crystals of the resulting complex was determined by X-ray crystallography (Figure 41). 


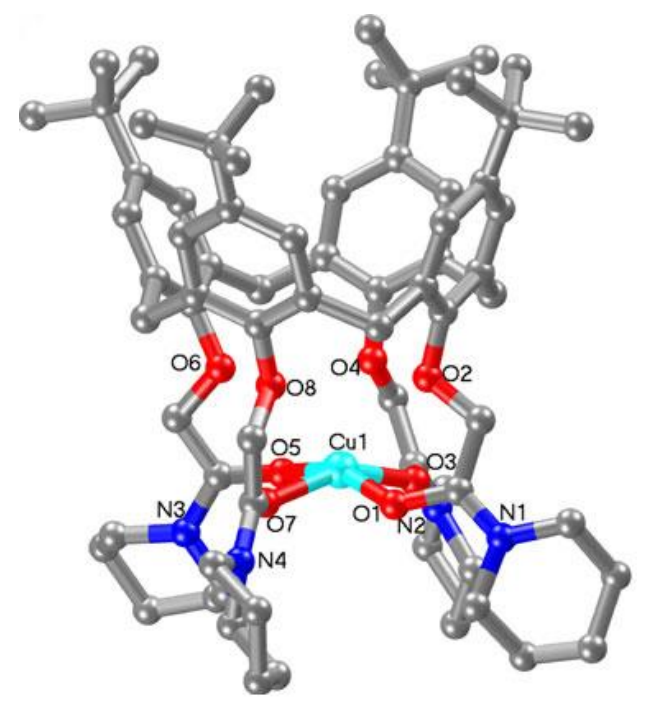

Figure 41: X-ray crystal structure of Zhou's complex $(\text { XXII })^{89}$.

They described the complexation geometry as a distorted tetragonal pyramid where the copper cation is coordinated by the four acyl oxygen atoms on the lower rim of the calixarene. The overall shape of the calix[4]arene resulting from the complexation, is that of a distorted cone conformation due to the metal ion coordinating at the lower rim and therefore 'locking' the conformation ${ }^{89}$. Creaven et al. have previously reported work on calix[4]arene derivatives which have been complexed with copper(II) ions $(\mathrm{XXIII})^{90}$. The calix[4]arene unit has being functionalised on the lower rim to form metal binding cages. Transition metals such as copper which are covalently linked through a ligand system are an area of interest as they can be used for studies into light-induced energy and electron transfer ${ }^{91}$. Supramolecular compounds complexed with transition metals can also be used as models for photochemical devices and the conversion of light energy into useable chemical energy is of current research. To this end Creaven et al. complexed their calix[4]arene derivatives with copper(II) salts $\mathrm{CuX}_{2}(\mathrm{X}=$ acetate, chloride or perchlorate) to test the binding ability and as a consequence the possibility of their compounds to be used in photochemical devices. One complex formed from the reaction with copper acetate, resulted in blue crystals, and was subjected to X-ray diffraction studies (Figure 42). It was observed that the $\mathrm{Cu}^{2+}$ ion was not complexed within the calix[4]azacrown ${ }^{90}$. 


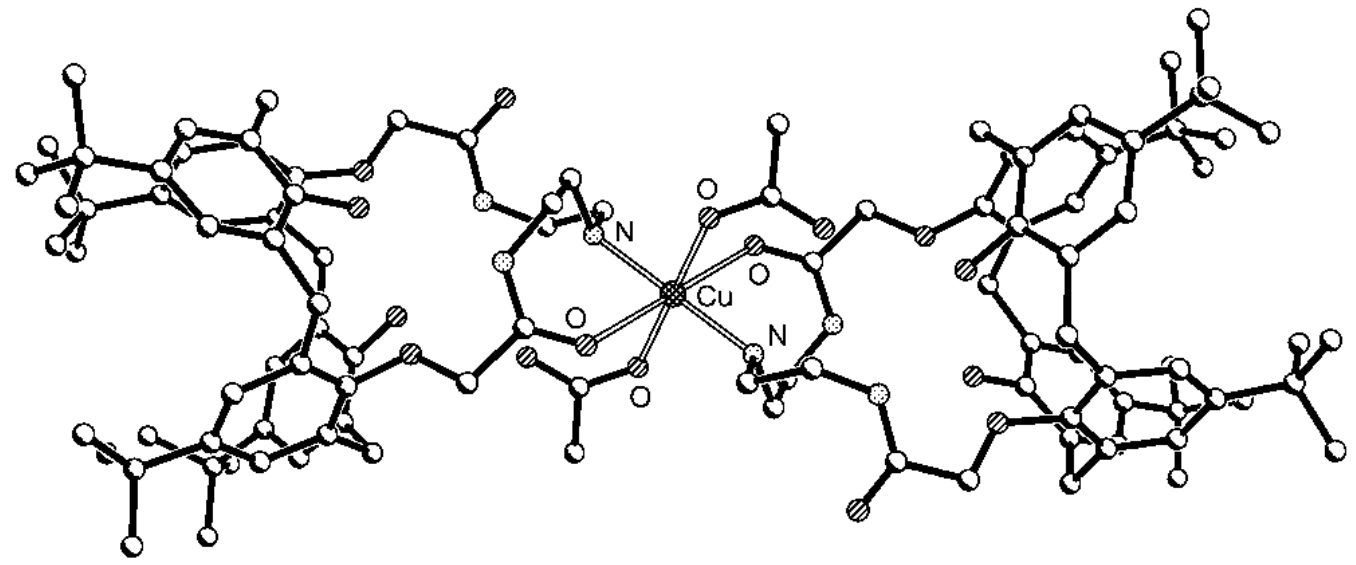

Figure 42: $\mathrm{X}$-ray image showing $\mathrm{Cu}^{2+}$ binding between two calix[4]azacrown units $(\mathrm{XXIII})^{90}$

Instead it was bound between two such units through binding between the lone pairs of electrons on the $\mathrm{NH}$ of the crown ring and the carbonyl groups of the amides on the lower rims of the two calix[4]azacrown units. From this they concluded that their compounds were acting as bidentate N,O ligands and the $\mathrm{Cu}^{2+}$ adopted a typical $[4+$ 2] distorted octahedral geometry. They also observed that the amine group retained its proton and was able to form $\mathrm{N}-\mathrm{H}^{\cdots} \mathrm{O}$ hydrogen bonds with the unbound oxygen of the acetate group. Similar results were obtained with the perchlorate form. Complexation with the copper chloride salts were a bit different in that two different products were obtained. The first was similar to the acetate and appeared to bind in the same way, whereas the second was only observed if the reaction was continued for prolonged periods and which cleaved the amide bond. Bond cleavage of this type has been previously reported ${ }^{92}$ and Creaven et al. concluded that the first was the more stable compound and requires a lot of energy in order to cleave the amide bond. A possible mechanism for this involves the copper(II) ion bring cleaved from an externally coordinated site (XXIV) as shown in Figure 42, to an internally coordinated site, Figure 43. It is suggested that this movement would allow the amide bond to cleave. 


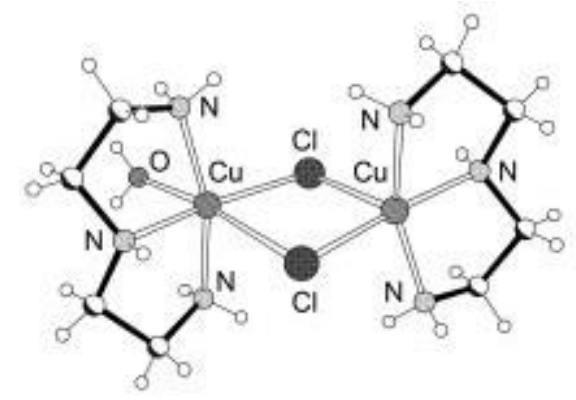

Figure 43: Cleaved product $(\mathrm{XXIV})^{\mathbf{9 0}}$

\section{Mercury.}

Mercury is found in group 12 along with zinc and it occurs naturally as $\mathrm{HgS}^{58}$. Mercury is the only metal which is a liquid at room temperature and boils in temperatures in excess of $350{ }^{\circ} \mathrm{C}$. Its preferred state is the +2 cation and tends to adopt a linear arrangement where possible. It can form a distorted octahedral complex with two of the arms shorter than the other four $(2+4$ coordination). Because of its high density mercury is commonly employed in barometers to measure atmospheric pressure. Mercury is non-essential in humans and as a result is quite toxic. Some early symptoms of mercury poisoning include bleeding gums; headaches etc. and more severe symptoms include loss of memory, damage to the central nervous system and death. Previously mercury was employed as a treatment for some skin diseases and to cure syphilis, but due to a better understanding of the toxic effects of this metal it is rarely used in medical treatments. Chelation therapy with D-penicillamine or another similar chelator is the most effective treatment in overcoming mercury poisoning and so the detection and development of new mercury chelator's is an area of increasing importance ${ }^{58}$.

Memon et al. are one group involved in developing new mercury chelators ${ }^{93}$. They synthesised biscalix[4]arenes containing nitrile derivatives with various bridges, (Figure 44) where in compounds such as $\mathrm{XXVa}(\mathrm{i}), \mathrm{x}$ - the linking agent, would be substituted with $\mathrm{CH}_{2} \mathrm{COCH}_{2}$ and the $\mathrm{R}$ groups as a proton, and in $\mathrm{XXVb}(\mathrm{i})$, the linking agent $\mathrm{x}$, is the same as $\mathrm{XXVa}(\mathrm{i})$, but the $\mathrm{R}$ groups are now functionalized with $\mathrm{CH}_{2} \mathrm{CN}$. 


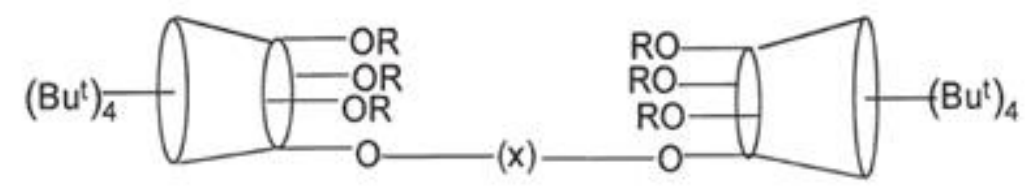

$(\mathrm{x})$

(a)

(b)

(i) $\mathrm{CH}_{2} \mathrm{COCH}_{2}$

$\mathrm{R}=\mathrm{H} \quad \mathrm{R}=\mathrm{CH}_{2} \mathrm{CN}$

(ii)

$\mathrm{CH}_{2} \mathrm{CH}_{2} \mathrm{OCH}_{2} \mathrm{CH}_{2}$

$\mathrm{R}=\mathrm{H}$

$\mathrm{R}=\mathrm{CH}_{2} \mathrm{CN}$

(iii) $\mathrm{CH}_{2} \mathrm{CONH}\left(\mathrm{CH}_{2}\right)_{2} \mathrm{NHCOCH}_{2}$

$\mathrm{R}=\mathrm{H}$

$\mathrm{R}=\mathrm{CH}_{2} \mathrm{CN}$

(iv) $\mathrm{CH}_{2} \mathrm{CONH}\left(\mathrm{CH}_{2}\right)_{8} \mathrm{NHCOCH}_{2}$

$\mathrm{R}=\mathrm{H}$

$\mathrm{R}=\mathrm{CH}_{2} \mathrm{CN}$

Figure 44: Nitrile derivatives of biscalix[4]arenes (XXV a(i)-a(iv) \& b(i)-b(iv) $)^{93}$.

They tested the extraction abilities of their compounds against various metal cations and found that compounds XXVa(i)-XXVa(iv) were poor extractors of $\mathrm{Hg}^{2+}$ but compounds XXVb(i)-XXVb(iv), with nitrile derivatives attached, proved to be more selective for $\mathrm{Hg}^{2+}$ (Table 2).

\begin{tabular}{|l|l|l|l|l|l|l|l|l|l|}
\hline Ligand & $\mathrm{Li}^{+}$ & $\mathrm{Na}^{+}$ & $\mathrm{K}^{+}$ & $\mathrm{Cs}^{+}$ & $\mathrm{Cu}^{2+}$ & $\mathrm{Co}^{2+}$ & $\mathrm{Cd}^{2+}$ & $\mathrm{Ni}^{2+}$ & $\mathrm{Hg}^{2+}$ \\
\hline XXVb(i) & 1.9 & 1.0 & 7.4 & 2.9 & 2.9 & 7.6 & 8.7 & 12.7 & 78.1 \\
\hline XXVb(ii) & 1.8 & 1.0 & 6.5 & 3.0 & 3.0 & 6.0 & 3.5 & 6.0 & 73.2 \\
\hline XXVb(iii) & 1.0 & 3.0 & 1.0 & 1.0 & 4.2 & 1.8 & 3.0 & 8.5 & 53.0 \\
\hline XXVb(iv) & 1.0 & 1.0 & 2.5 & 1.0 & 3.5 & 4.5 & 4.8 & 6.5 & 72.0 \\
\hline
\end{tabular}

Table 2: Summery of extraction abilities of biscalix[4]arene nitrile derivatives ${ }^{93}$.

They suggest this is due to the hard/soft acid-base principle where the $\mathrm{CN}$ group is a soft base and shows a stronger affinity towards soft acidic metal cations such as mercury. The development of new mercury chelator's to remove this pollutant from the environment has sparked the attention of many calixarene chemists as calixarenes can be functionalised to provide a wide range of potential mercury 
selective binding sites. This could make them strong competitors in chelation products.

Colleran et al. have performed extensive works on mercury binding to calix[4]arenes which have their lower rims functionalized with pyridine arms (XXVI) ${ }^{94}$ (Figure 45).

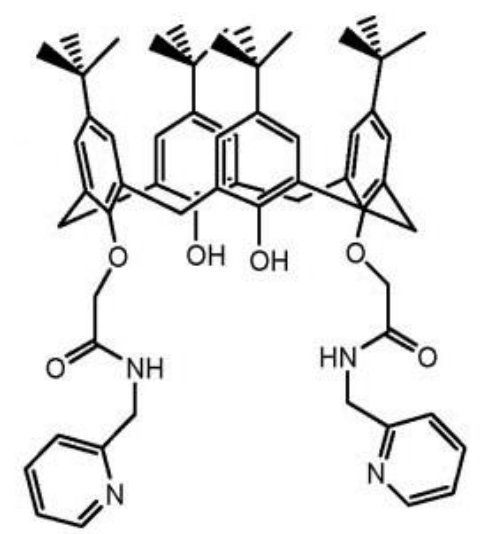

Figure 45: Colleran et al. lower rim functionalised metal binding derivative (XXVI) ${ }^{94}$

The complexation reactions were carried out at room temperature in methanol. When they examined their compound with ${ }^{1} \mathrm{H}$ NMR spectroscopy in DMSO and $\mathrm{CD}_{3} \mathrm{CN}$ they found that a clean spectrum containing a single set of signals was obtained. It was evident from the downfield shifts in the aromatic region, corresponding to the lower rim pyridyl's that mercury complexation had occurred and was preferably binding to the pyridyl's. Shifts were also seen for the lower rim hydroxyls and amide protons further supporting the metal binding choice. However when the ${ }^{1} \mathrm{H}$ NMR spectroscopy was repeated in $\mathrm{CDCl}_{3}$ a doubling of all the signals occurred. This suggests that either there are two different species present or that mercury complexation is producing a highly asymmetric species. To double-check the results, the solvent was removed from this sample and then the sample was re-dissolved in DMSO. The resulting ${ }^{1} \mathrm{H}$ NMR spectrum returned to what had previously been observed. Colleran et al. suggested that this may be due to a lack of solvation around the counter-anion. In this theory, the mercury cation binds to the lower rim of the ligand and the resulting counter-anion is free in solution where donor solvents such 
as DMSO or $\mathrm{CD}_{3} \mathrm{CN}$ can surround and solvate it. But if the solvent has weak donating ability such as $\mathrm{CDCl}_{3}$ then it can't solvate the anion, resulting in close ionparing and a high degree of asymmetry in the complex. When the methylene signals were examined in the proton spectra, it was noted that four sets of doublets were now present indicating that each proton of the methylene bridge was in a different environment further supporting the asymmetry. They also report a series of titration studies with the mercury(II) perchlorate in $\mathrm{CD}_{3} \mathrm{CN}$ and DMSO. From the NMR spectra it is evident that the pyridyl and amide protons show the largest shifts corresponding well with previously published values ${ }^{95}$. Colleran et al. repeated these complexation reactions under different conditions (refluxing in ethanol for two hours) they found that the calix[4]arene derivative had lost both its lower rim pendant arms and that cleavage had occurred between the amide carbonyl and nitrogen atoms to reform starting materials. They proposed a possible mechanism (Figure 46) whereby the ethanol molecule attacks the carbonyl carbon forming a new bond between the alcohol and the carbonyl carbon resulting in breaking the carbonoxygen double bond. Removal of a proton followed by reformation of the carbonoxygen double bond cleaves the carbon-nitrogen amide bond and produces the ester and the 2-aminomethylpyridine compound.

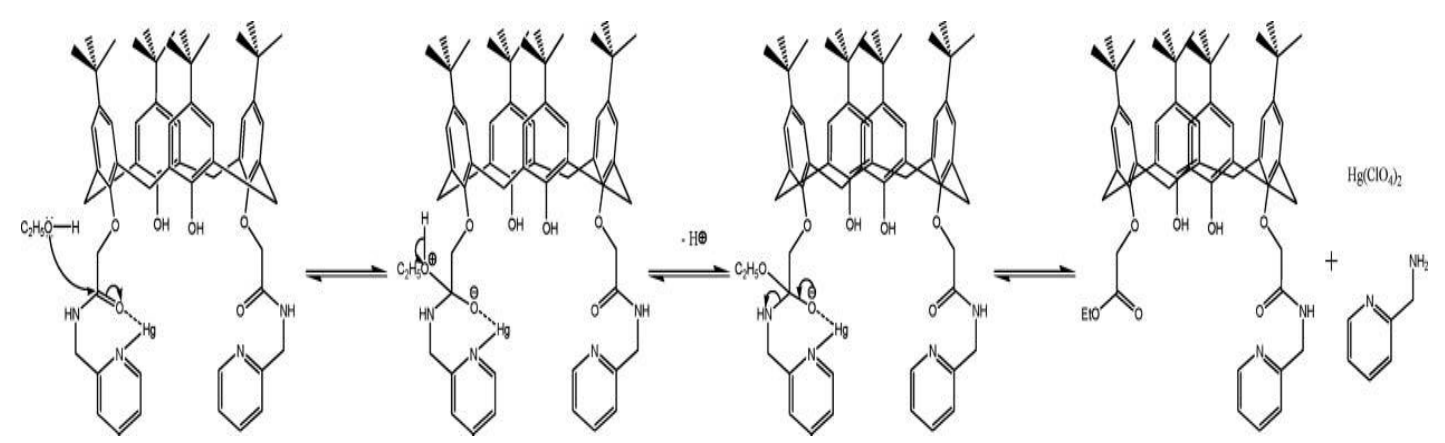

Figure 46: Proposed mechanism for the cleavage of the lower rim ${ }^{94}$

To test this pathway, the reaction was repeated by refluxing in methanol for two hours, as if it was feasible the methyl ester should be formed. From the ${ }^{1} \mathrm{H}$ NMR spectrum the methyl ester was indeed formed and as added support, if the reaction only proceeded for one hour then only one pendant arm was lost. 


\section{Nickel.}

Nickel is found in group 10 in the periodic table ${ }^{58}$. It prefers to exist as the $\mathrm{Ni}^{2+}$ cation and is normally found in its elemental form alloyed with iron. About $9 \%$ of pure nickel supplies comes from meteorites and 7\% from the earth's crust. The rest is obtained as impurities in other alloys. It is a silvery-white metal which melts at $1453{ }^{\circ} \mathrm{C}$ and boils at $2730{ }^{\circ} \mathrm{C}$, and it is a good thermal and electrical conductor. It's most common uses are in electroplating or welding household appliances with nickel to strengthen them and increase their resistance to corrosion or in industry to produce steel.

Nickel is essential for good health as it is involved in carbohydrate metabolism but too much of it is toxic to living beings. The most serious problems associated with nickel poisoning is when it's inhaled, as it can lead to an increased risk of respiratory infections including asthma and sinus problems ${ }^{58}$. As nickel is used in the steel industry, such as in stainless steel cookware, it is possible to inadvertently introduce harmful levels of nickel into the body ${ }^{96}$. Nickel has recently found new application in aerospace technologies. Aluminium alloys are often employed in the construction of these devices due to their lightness and strength ${ }^{97}$. Unfortunately severe operating conditions limit the use of aluminium alloys directly ${ }^{98}$. Obviously to launch a spacecraft requires huge sums of money, so to reduce the structural mass of machines and equipment light alloys are employed wherever possible. Nickel-plating offers unique properties such as; corrosion and wear resistance, increased hardness and durability, and a uniform deposit regardless of the shape of the object ${ }^{99}$. Because of these qualities nickel has become an attractive metal in finishing industries. Along with light alloys ultra-high black coatings are extremely important in terrestrial and space-borne optical equipment as these black coatings aid in improving thermal detectors and suppressing unwanted reflections or scattered light in optical systems (telescope housings). Saxena et al. have investigated different ways of combining the nickel plating with black paints as black paints with organic binders are of no use due to the high mass and their volatile material ${ }^{100}$. The volatile material has the ability to deposit on the optical mirrors of telescopes reducing its performance. Saxena et al. found that metal oxides or metal phosphate components were 
extremely stable in space environments and they could blacken the nickel effectively by etching it in acid solutions for small periods of time. The black coated specimens were uniform with no patches or discolouration at any point. The specimens were then tested for corrosion, thermal stability and humidity (pre-launch atmosphere). In all tests the specimens were found to be very stable and no discolouration of patching of the plating occurred. At all times the optical properties remained constant. They concluded from this that the black-nickel coated samples were of low weight as well as being capable of withstanding the environment in space and so could be used in metal finishing works for aerospace-craft ${ }^{100}$.

As seen with mercury, nickel poisoning is also a concern, and Sun et al. have developed a nickel chelator using Schiff bases as a potential treatment to nickel poisoning ${ }^{101}$. They derived their Schiff bases from S-alkyldithiocarbazate and attached them to the lower rim of the calix[4]arene (XXVII) to construct a metal binding platform. X-ray analysis was obtained on their compound and it can be seen from the structure (Figure 47) that the calix[4]arene complex has remained in the cone conformation and that the two arms of the Schiff base allow metal coordination to occur. 


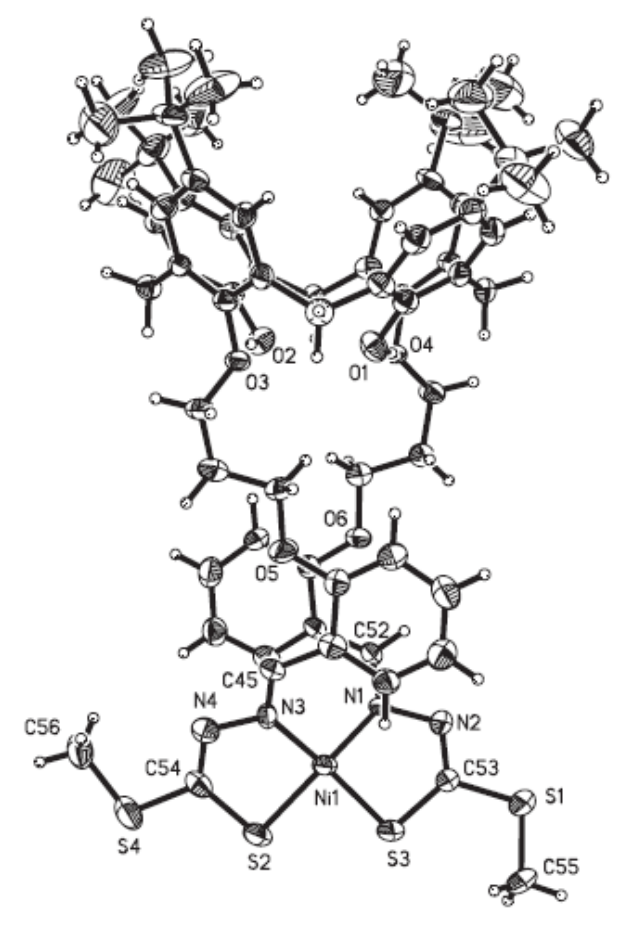

Figure 47: Crystal structure of $\mathrm{Ni}^{2+}$ Schiff base calix[4]arene derivative (XXVII) ${ }^{101}$.

It can be seen that the $\mathrm{Ni}^{2+}$ metal ion is coordinated through the nitrogen and sulphur atoms on the lower rim of the calix[4]arenes. Again this can probably be attributed to the hard/soft acid-base principle as the $\mathrm{Ni}^{2+}$ is a relativity soft Lewis acid and nitrogen and sulphur atoms are relativity soft Lewis bases. Work is currently underway to examine the feasibility of these compounds as potential nickel treatments for human use.

Chakrabarti et al. ${ }^{102}$ have researched into the structures of mono-substituted tetrathiacalix[4]arenes complexed with thiazoleazo groups on the upper rim (Figure 48). They hoped that this new compound could be used as molecular ion recognition agents to be incorporated into molecular filters. As the four methylene bridging are replaced with sulfide linkages, the compounds are expected to possess different chemical behaviour to the classical calix[4]arenes, primarily due to the varying oxidation states of the bridging sulphur atoms. 

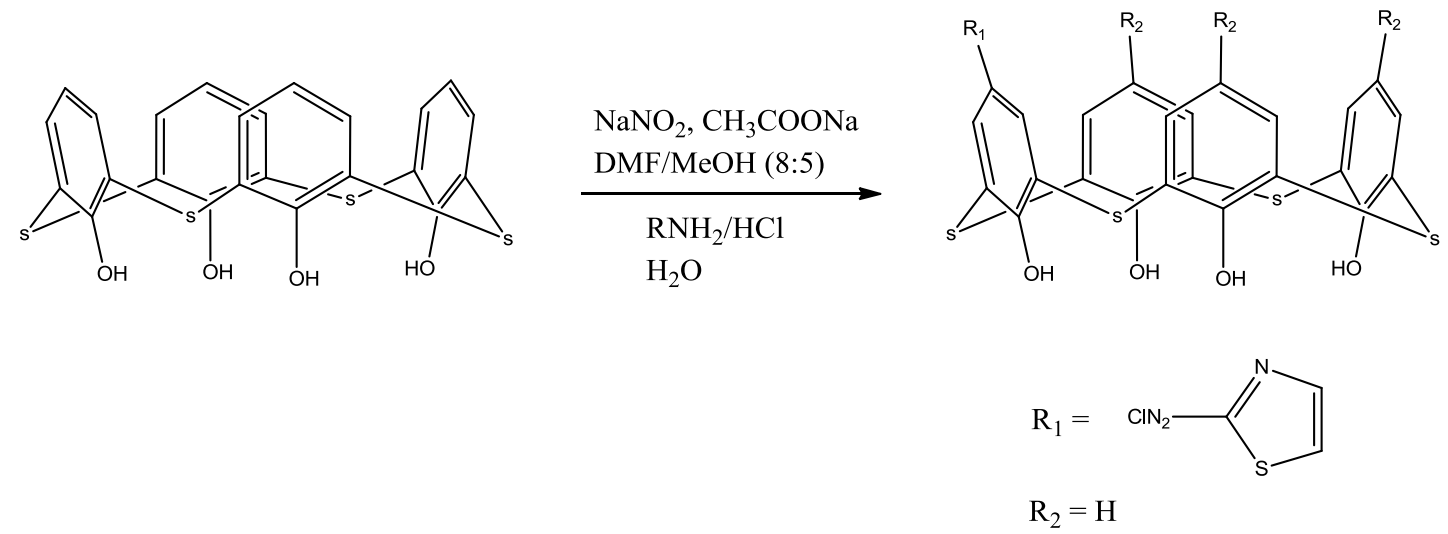

Figure 48: Chakrabarti's thiazoleazo coupled tetrathiacalix[4] $\operatorname{arene}^{102}$ (XXVIII) on the right-hand side where $\mathrm{R}_{1}=$ thiazoleazo and $\mathrm{R}_{2}-\mathrm{R}_{4}=\mathrm{H}$.

Chakrabarti's thiazoleazo coupled tetrathiacalix[4]arene (XXVIII) was synthesised by reacting the parent compound ${ }^{103}$ (left-hand side of Figure 48) in the presence of a weak base with a diazotised solution of 2-aminothiazole. The reaction was carried out at $5{ }^{\circ} \mathrm{C}$ in a mixed solvent system of dimethylformaldehye and methanol in a 8:5 ratio respectively. Unfortunately as the methylene bridges have been replaced by sulphur bridges, the conformation of the resulting complexes could not be determined by ${ }^{1} \mathrm{H}$ NMR spectroscopy. The conformation was confirmed by X-ray crystallography (Figure 49). The crystals were obtained from a chloroform solution and the X-ray revealed that a chloroform molecule was located outside the cavity.

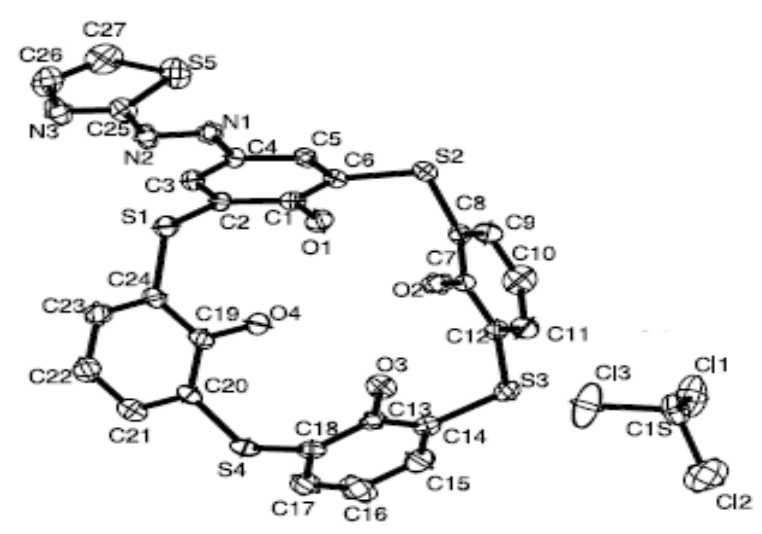

Figure 49: X-ray crystal structure of (XXVIII) ${ }^{102}$. 
The authors believe that a combination of hydrogen bonding interactions on the lower rim and sulphur-sulphur interactions aid the stability of the cone conformation. UV-Vis studies were performed to investigate metal ion affinity of the compounds with hard and soft metal cations (Figure 50). The UV-Vis was performed in a mixed solvent solution of chloroform and methanol at a 1:1 ratio. Compound (XXVIII) showed little to no shifts for the small alkali metal cations, but showed significant shifts with the larger alkali cations. This indicates that the size of the cavity and the size of the cation are of particular importance during metal ion complexation.

(a)

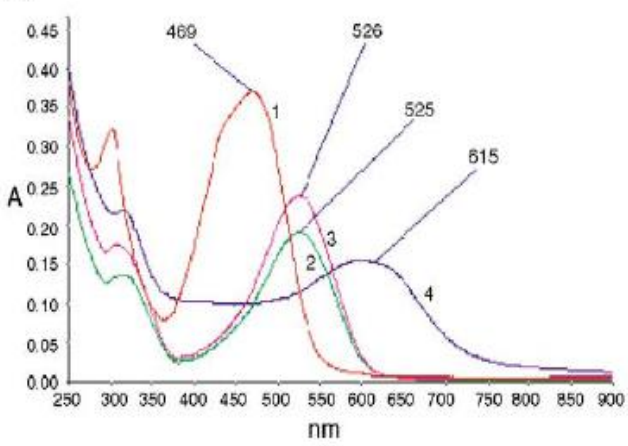

(b)

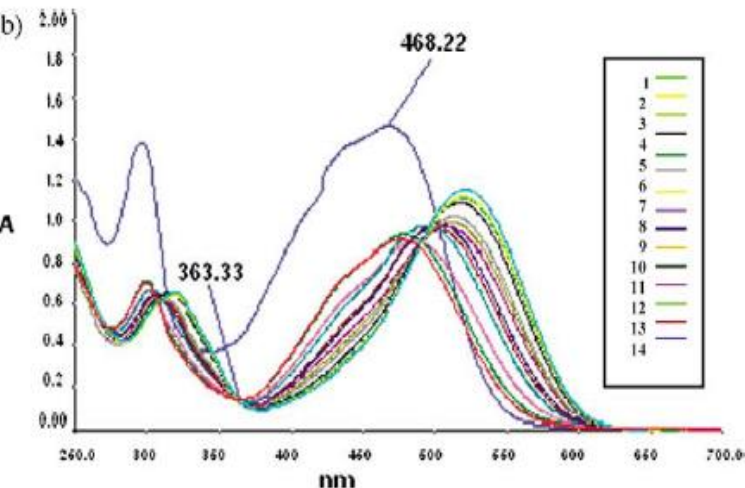

Figure 50: UV-Vis spectra of (XXVIII). (a) (1) $=10^{-4} \mathrm{M}$ solution of (XXVIII) and its shifts in $\lambda_{\max }$ upon addition of $10^{-4} \mathrm{M}$ solutions of (2) $\mathrm{Cs}_{2} \mathrm{CO}_{3}$, (3) $\mathrm{Rb}_{2} \mathrm{CO}_{3}$, (4) $\mathrm{K}_{2} \mathrm{PdCl}_{4}$. (b) changes in UV-Vis spectra as concentration of $\mathrm{Cs}_{2} \mathrm{CO}_{3}$ is increased from $(1)=1 \times 10^{-4}$ to (9) $=9 \times 10^{-4} \mathrm{M}$ and $(10)=1 \times 10^{-3}$ to $(14)=5 \times 10^{-3} \mathrm{M}^{102}$.

When UV-Vis was attempted with transition metal cations no interaction was seen with the exception of palladium. The spectra experienced a red-shift upon complexation to palladium and, which was accompanied by a visual colour change from yellow to blue-green. This indicates a change in the coordination sphere of palladium in the presence of (XXVIII). It was found that compound (XXVIII) in the uncomplexed form exhibited a broad band at $468 \mathrm{~nm}$ which decreased upon complexation to palladium with a corresponding new peak at $617 \mathrm{~nm}$ (red-shift). It is previously known that the colour change in palladium complexes from yellow to blue-green is a result of $d-d$ transitions ${ }^{104}$ and the authors conclude that this new peak at $617 \mathrm{~nm}$ most likely represents this transition. Mass spectrometry confirmed that binding was in a 1:2 ratio of compound (XXVIII) to metal cation for palladium and 1:1 for large alkali metal cations. The authors conclude that the coordination of 
palladium may be due to metal-oxygen or metal-sulphur interactions and are currently conducting investigations to prove this.

\subsection{Energy transfer}

Solar energy has received a new influx of interest over the last few years as alternative energy becomes all the more necessary. The conversion of solar energy into chemical energy is one of the hottest topics currently and transition metal ions can give information about electron transfer when they are covalently linked to a ligand system ${ }^{105}$. Generally, in order to observe energy transfer between two metal centres they must be linked by a bridging conjugated ligand. ${ }^{106}$ However McGinley et al. have reported that this isn't necessarily the case ${ }^{107}$. Previous work done by our research group has involved functionalising both the upper and lower rims with various functional groups to form a calixarene derivative with potentially two metal ion binding centres ${ }^{107,108}$. In particular, work was done with ruthenium and manganese ions, with the $\mathrm{Ru}$ (II) ion bound to the upper rim and the $\mathrm{Mn}$ (II) ion on the lower rim $^{107}$. The complex was tested using electron paramagnetic resonance (EPR) spectroscopy to determine if the metals were able to communicate with each other by electron transfer. They synthesised a series of calix[4]azacrowns (XXIX a $\&$ b) (Figure 51) and complexed them with ruthenium and manganese ions. 


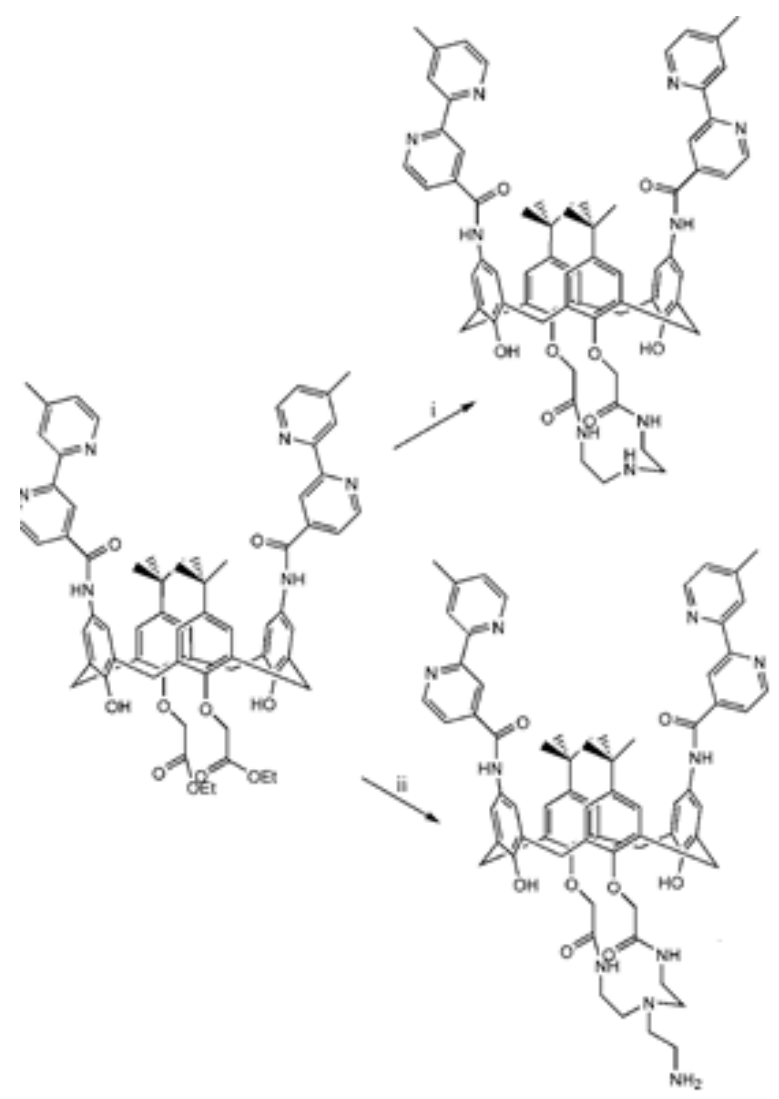

Figure 51: Calix[4]azacrown derivatives (XXIX a - top \& b - bottom $)^{107}$.

These metal complexes were then oxidised using ammonium cerium(IV) nitrate to obtain mixed valence $\mathrm{Ru}^{\mathrm{III}} / \mathrm{Mn}^{\mathrm{II}}$ complexes. The oxidation was surveyed under EPR. Initially only an increase in the $\mathrm{Ru}^{\mathrm{III}}$ species signal was seen, representing that oxidation had occurred at this site. The samples were then heated to $273 \mathrm{~K}$ and allowed to cool to allow electron transfer to occur and this revealed that there was a $30 \%$ decrease in both the $\mathrm{Ru}^{\mathrm{III}}$ and $\mathrm{Mn}^{\mathrm{II}}$ species demonstrating that some form of energy/electron transfer had occurred resulting in the oxidation of $\mathrm{Mn}^{\mathrm{II}}$ to $\mathrm{Mn}^{\mathrm{III}}$. This data suggests that the two metals are in communication with each other despite the fact that they are not directly linked through a conjugated system to one another ${ }^{107}$.

De Cola et al. ${ }^{109}$. have developed the first dye sensitization by a polymer-loaded mesoporous silica complex for potential use in LED'S, imaging probes etc. Their work began by developing a photostable system where an encapsulated donor compound can transfer excitation energy directly to an acceptor compound located 
on the surface of the silica polymer. The donor is the Super Yellow polymer (SY) encapsulated within the silica pores, and the acceptor is a cyanide dye (IRIS3) which is covalently attached to the outside of the silica pores (XXX), (Figure 52).

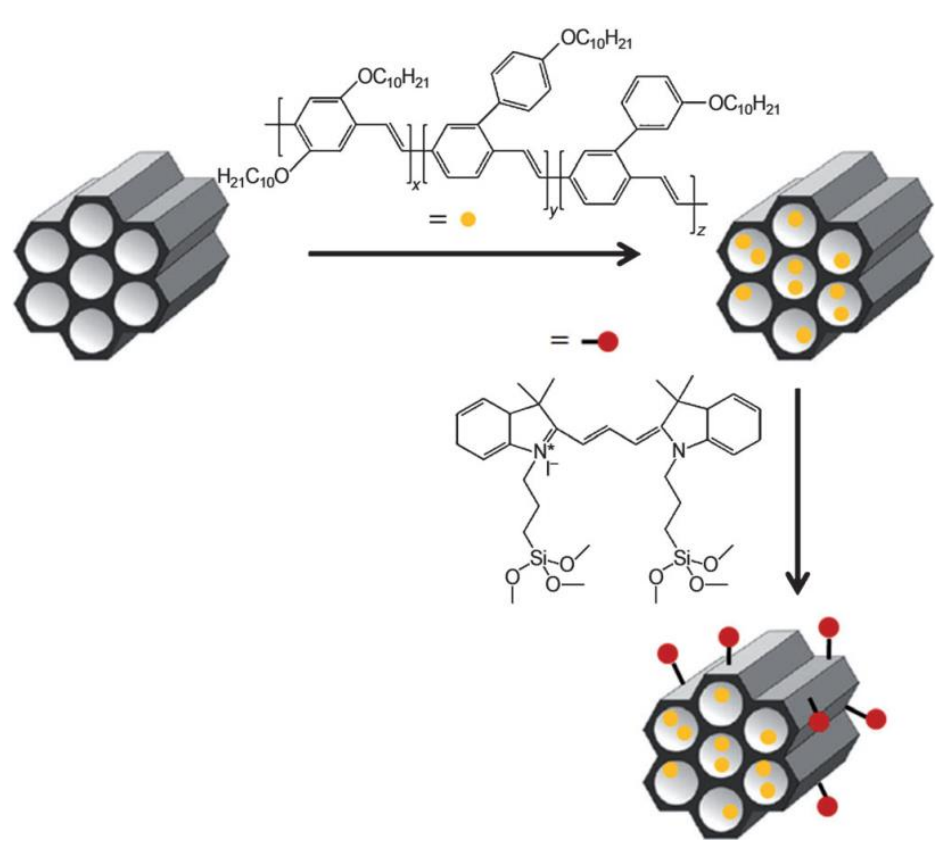

Figure 52: Encapsulation of SY donor and attachment of IRIS3 acceptor $(X X X)^{109}$.

For successful energy transfer between the donor and acceptor specific requirements have to be met. The first is that the donor unit must be efficient at absorbing light and have high photostability. Secondly, there must be good spectral overlap between the donor emission spectrum and the acceptor absorption spectrum. Thirdly, the transfer process must be fast enough to compete with other deactivation processes such as thermal relaxation. Their compound was found to meet these requirements, as both the donor and acceptor display good spectral overlap (Figure 53), and the donor's encapsulation also results in a higher degree of rigidity reducing thermal deactivation of its excited state. 


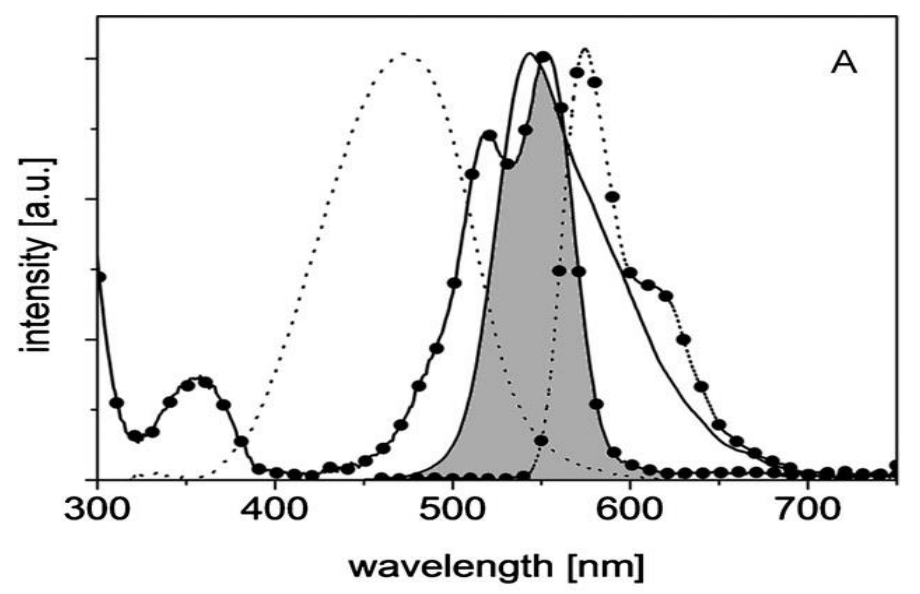

Figure 53: Spectral overlap of the donor-acceptor complex. The donor absorption and emission spectra are given by the dotted and solid line respectively. The acceptor complex absorption and emission bands are given by the solid line with circles and the dotted line with circles. The donor-acceptor overlap area is in grey ${ }^{109}$.

Bleaching tests were also conducted to investigate the photostability of the donoracceptor complex compared to the just the acceptor complex IRIS3-Silica polymer. It was found that after irradiation with light for three hours the IRIS3-Silia compound showed a decrease in activity of $40 \%$ but the donor-acceptor complex only displayed a $15 \%$ decrease. This shows that it is advantageous to encapsulate the donor as oxygen diffusion (bleaching agent) into the silica polymer is significantly slowed. They conclude that the donor and acceptor species are localized in space due to selective functionalisation of the silica polymer host, which reduced leakage of the polymer out of the silica as the pores are partially blocked by the acceptor. This also slows down the bleaching agents increasing the lifetime of the complex, increasing its photostability.

\subsection{Fluorescence}

Fluorescence spectroscopy is a widely used technique in the analysis of organic compounds in terms of their concentration and several calix[4]arenes derivatives have implemented as fluoroionophores. As a result from the interest in fluorescence a large number of papers have been published regarding the topic. As such only the basics will be discussed here. 
Fluorescence is part of the luminescence family where susceptible molecules emit light from electronically excited states which have been created by physical, mechanical or chemical means ${ }^{110}$ (Figure 54). The absorption of light is a physical effect and is the only one of the three mentioned that will be discussed here. The generation of luminescence through the excitation of a molecule by UV or visible light is termed photoluminescence, which can be sub-divided into fluorescence and phosphorescence. Fluorescence is defined as the property of some molecules to absorb light at a particular wavelength and subsequently to emit a light of a longer wavelength (low energy) for a brief interval of time.

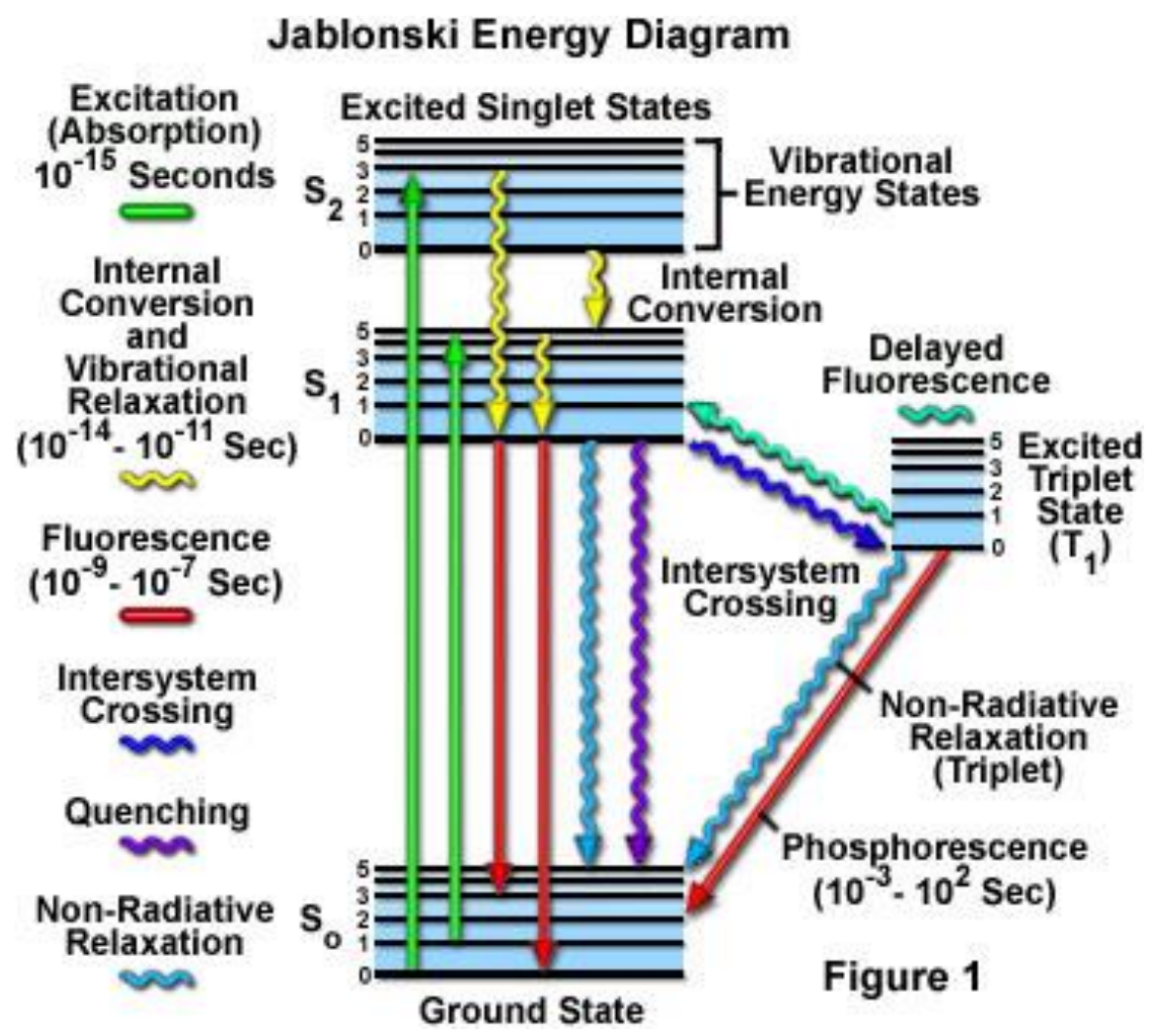

Figure 54: Jablonski Energy Diagram ${ }^{110}$.

The thick lines are electronic energy levels, thinner ones are vibrational energy states. Vertical upwards arrows indicate instantaneous nature of excitation process, wavy arrows occur on longer timescale. 
Fluorescence is a three step process and each step occurs at a different rate (Figure 54). The process starts with the absorption of a photon which is the quickest step at $10^{-15} \mathrm{~s}$ (femtoseconds). This excites the electron from the ground state all the way to one of the excited vibrational levels of $S_{2}$. The second step sees the relaxation of the excited vibrational electrons to the lowest energy level of the excited levels $S_{1}$. This occurs in a few picoseconds $\left(10^{-12} \mathrm{~s}\right)$. The final step is the emission of a longer wavelength photon from the excited electron which results in the visible fluorescence and the return of the molecule to its ground state form (nanoseconds $10^{-}$ $\left.{ }^{9} \mathrm{~S}\right)$.

Fluorescence is generally seen with highly conjugated polycyclic aromatic molecules that can exist in any one of several energy levels in the ground state, and the energy levels are associated with the specific arrangement of electronic orbitals. For any given molecule, several different electronic states exist $\left(S_{0}, S_{1}, S_{2}\right.$ etc. $)$ depending on the total electron energy and the relative electron spins. Each state is then further sub-divided into a number of vibrational and rotational energy levels associated with the atomic nuclei and the bonding orbitals. The ground state for most organic molecules is an electronic singlet in which all electrons are spin-paired i.e. opposite spins. At room temperature very few molecules have the energy to exist in any states higher than this and so excitation processes usually begin from this level.

The absorption of light is a rapid process in which discrete amounts of energy 'quanta' are absorbed resulting in the excitation of a molecule from the ground state to the excited state. The emission of a photon via fluorescence is also measured in terms of quanta and the energy in a quantum is expressed by Planck's Law:

$\mathbf{E}=\mathbf{h v}=\mathbf{h c} / \boldsymbol{\lambda}(\mathbf{E}=$ energy, $\mathbf{h}=$ Planck's constant, $\mathbf{v}$ and $\lambda$ are frequency and wavelength respectively and $\mathbf{c}=$ speed of light).

This law states that the radiation energy of an absorbed photon is directly proportional to the frequency and inversely proportional to the $\lambda$. This means that a 
shorter wavelength has a greater quantum of energy and is found to the left of a fluorescence spectrum. When a photon is absorbed due to an interaction of light with the electrons in a molecule, it is an all or nothing phenomenon and only occurs with light of a specific wavelength (absorption bands). If the absorbed photon contains more energy than required the excess energy is usually converted into rotational and vibrational energy, but if there is not enough energy present to promote a transition no absorption occurs. Broad absorption bands arise from closely spaced vibrational levels as well as thermal motion which allows a range of photon energies to be suitable for a transition.

The excited molecule exists in the lowest state $\left(S_{1}\right)$ normally for a few nanoseconds before relaxing into the ground state. If the relaxation occurs with the emission of a photon the process is called fluorescence. As the vibrational levels of the ground state are close together and as many photons of different energies are produced during emission, fluorescence is generally seen as an emission intensity over a range of wavelengths rather than a sharp line over on particular wavelength.

The possibility of a transition occurring from $S_{0} \rightarrow S_{1}$ depends on the similarity between the vibrational and rotational energy levels in both the ground and excited states when an electron is present. The Franck-Condon energy diagram (Figure 55) shows the vibrational energy distribution among the various levels in $S_{0}$ and $S_{1}$ for a given molecule. 


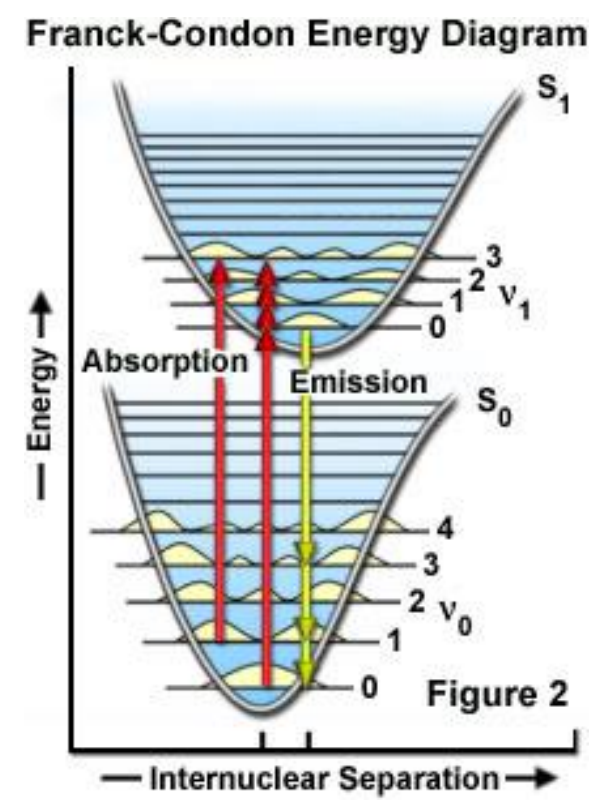

Figure 55: The Franck-Condon Energy Diagram

Excitation transitions are shown by the red lines and relaxation by the yellow. Excitation only lasts briefly (femtoseconds) and so the internuclear distance associated with the bonding orbital's doesn't change - Franck-Condon principle. The red line in the center represents the wavelength of maximum absorption and the most likely internuclear separation in the ground state to an allowed excited state. The most favoured transitions will occur when the vibrational and rotational electron population densities maximally overlap in both the ground and excited states. However transitions are not limited to this ideal condition. If enough energy is available then another transition may occur.

During an electronic transition an electron is promoted from a ground state orbital to a higher excited state by the absorption of energy, often in the form of a photon. With $\pi$ bonds, a $\pi$ electron can be promoted to an antibonding orbital $\pi^{*}$ and is referred to as $\pi \rightarrow \pi^{*}$ (Figure 56). Promotion of a $\sigma$ electron requires much more energy and as a result will not be described in this thesis. Some compounds will also contain heteroatoms such as oxygen or nitrogen, which have non-bonding electrons (lone pair of electrons). The orbital's in which these electrons are contained are 
called $\mathrm{n}$ orbital's and promotion of electron from this orbital's to the $\pi^{*}$ orbital's is denoted as $n \rightarrow \pi^{* 110}$.
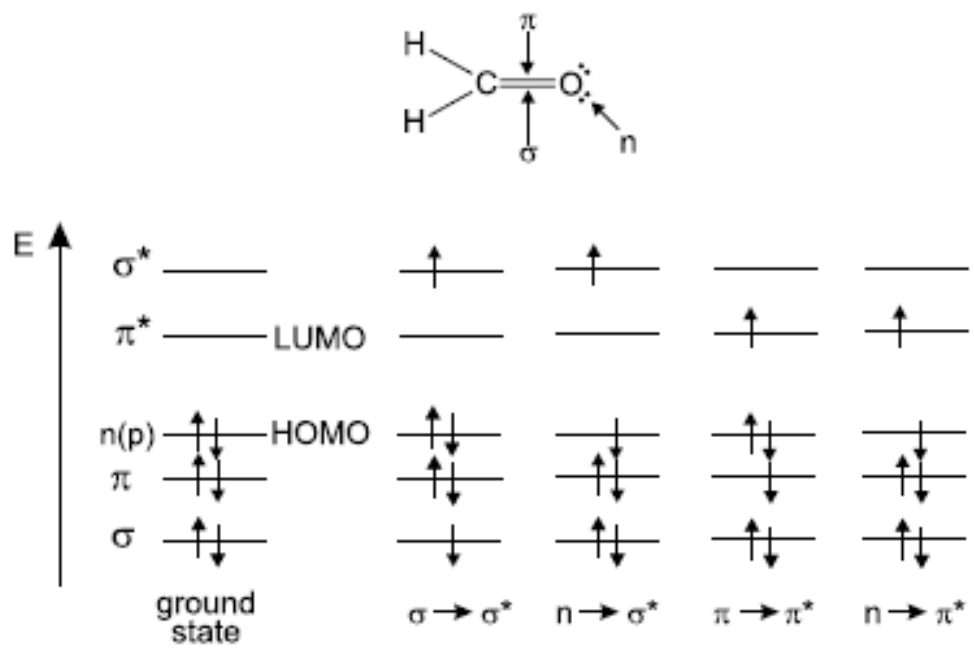

Figure 56: General trend of electronic transitions in spectroscopy

General trend: $\mathrm{n} \rightarrow \pi^{*}<\pi \rightarrow \pi^{*}<\mathrm{n} \rightarrow \sigma<\sigma \rightarrow \pi^{*}<\sigma \rightarrow \sigma^{*}$

Patra et al. have worked on developing calix[4]arene quinoline derived fluoroionophores (XXX a-c), where the quinoline acts as the fluorescent unit ${ }^{111}$. In this they hoped to form an excited state dimer (excimer) upon metal ion complexation. They developed three main chemosensors (Figure 57) where the amide functionality provided the metal ion binding center and the quinoline group as the fluorogenic unit. 


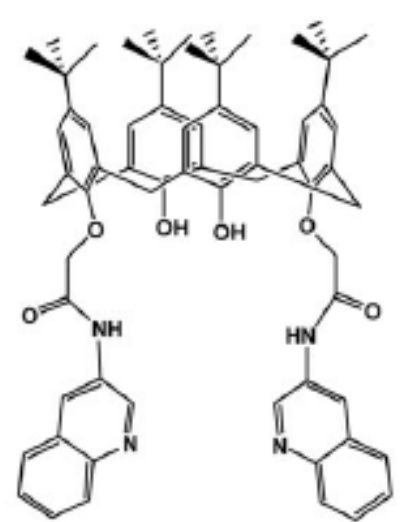

(a)

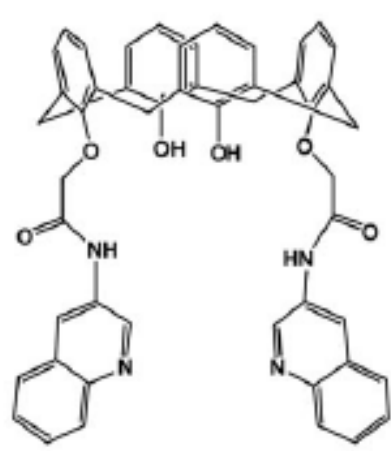

(b)

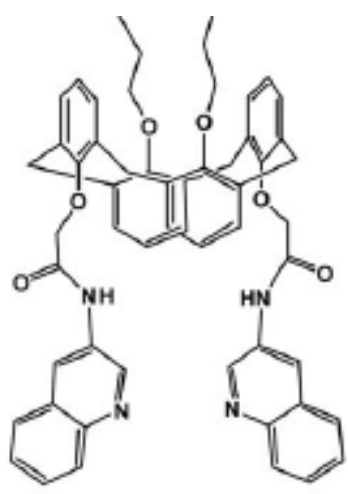

(c)

Figure 57: Calixarene based chemosensors $(\mathrm{XXX} \text { a-c })^{111}$.

The absorption spectra of all three were performed in acetonitrile and several common bands were observed. The band at $264 \mathrm{~nm}$ was assigned to the $n \rightarrow \pi$ transition, the bands at 318 and $333 \mathrm{~nm}$ where assigned to $\pi \rightarrow \pi^{*}$ transition, and the band at $280 \mathrm{~nm}$ is due to the $\pi \rightarrow \pi^{*}$ transition of the calixarene itself. The metal complexes of these were then examined by fluorescence spectroscopy in acetonitrile. In the parent compounds a band at $355 \mathrm{~nm}$ was observed representing the emission from the monomer but in the metal-complexed forms this band was quenched and a new band at $410 \mathrm{~nm}$ appeared which suggests that an excimer has formed due to metal ion complexation. Patra et al. concluded from this that it was possible to form the excimer with the aid of metal ion coordination, most encouragingly $\mathrm{Hg}^{2+}$, and that the complexation results in significant changes in the UV-Vis and fluorescence spectra due to the coordination of the metal ion with the nitrogen's of the quinoline $\operatorname{group}^{111}$. 
Lee et al. have investigated the use of lower rim functionalised calix[4]arene derivatives (XXXI) as potential chemosensors for cations and anions ${ }^{112}$. They functionalised all four positions of the lower rim. The first compound had two of its positions functionalized with a linking azacrown and the other two positions were functionalised with amide-pyrene units (Figure 58). The second had the amidepyrene units in two of the lower rim positions but it did not contain the linking azacrown. Instead two butoxy units were attached.

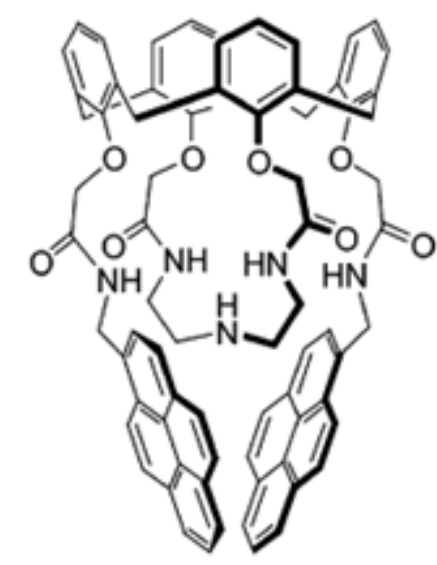

(a)

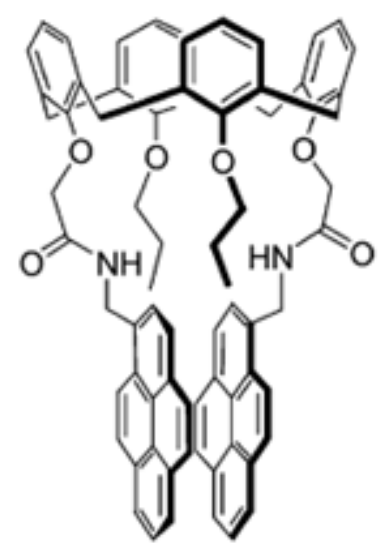

(b)

Figure 58: Lee et al. compounds for fluorescence studies with various ions (XXXI a \& b) ${ }^{112}$ 
Under fluorescence studies compound (XXXIb) showed an emission peak at $472 \mathrm{~nm}$ whereas compound (XXXIa) exhibited a peak at $448 \mathrm{~nm}$, a difference of $24 \mathrm{~nm}$. Lee et al. attributed this to the steric hindrance experienced by the first compound due to the presence of the triazacrown. The two compounds were tested with a range of cations with respect to fluorescence changes and their results are shown in (Table 3).

\begin{tabular}{|l|l|l|l|l|l|l|l|}
\hline Ligand & $\lambda_{\text {em }}(\mathrm{nm})$ & $\mathrm{Li}^{+}$ & $\mathrm{Na}^{+}$ & $\mathrm{K}^{+}$ & $\mathrm{Cs}^{+}$ & $\mathrm{Pb}^{2+}$ & $\mathrm{Co}^{2+}$ \\
\hline a & 396 & 76 & 7.0 & 1.0 & -3.0 & -17 & -168 \\
\hline & 448 & -74 & 26 & 23 & 23 & -223 & -242 \\
\hline b & 396 & -64 & -29 & 11 & -3.0 & -103 & -92 \\
\hline & 472 & 29 & 66 & 15 & 18 & -352 & -194 \\
\hline
\end{tabular}

Table 3: Fluorescence changes of the first and second compounds upon addition of various metal cations ${ }^{112}$.

$\mathrm{Pb}^{2+}$ and $\mathrm{Co}^{2+}$ are well known fluorescence quenching agents and as expected when both compounds were complexed with these two metal ions the fluorescence of both compounds was quenched. This is because there are two factors controlling fluorescence, the heavy metal ion effect and a reverse Photoinduced Electron Transfer (PET) from the pyrene units to the carbonyl groups where the electron density is decreased by metal ion complexation ${ }^{113}$. As well as testing the binding ability towards cations, their ability to bind anions was also investigated. It was found that compound (XXXIa) is highly selective for $\mathrm{F}^{-}$, which was followed by ${ }^{1} \mathrm{H}$ NMR spectroscopy to bind to the amide groups of the triazacrown ring on the lower rim. Compound (XXXIb) showed no binding to any of the anions tested.

\subsection{Nitrogen Donor Ligands.}

Nitrogen donor ligands can come in many forms from the simple pyridine units to the more complex bipyridine (bipy), bis(2-pyridylmethyl)amine (DPA), or porphyrin ligands. They are well known to bind metal cations including zinc, mercury, copper and many more. During this project both the bipy and DPA ligands were used and as such a brief discussion of both will be included here. 
DPA has found several uses in biological aspects resulting from the uptake of metal ions ${ }^{114}$ and its synthesis is relatively straight forward (Figure 59) ${ }^{115}$. Its synthesis involves the drop-wise addition of 2-pyridinecarboxaldehyde in ethanol to a solution of 2-(aminomethyl)-pyridine also in ethanol, to form the imine which is then reduced by sodium borohydride in ethanol to give the desired product.<smiles>O=Cc1ccccn1</smiles><smiles>NCc1ccccn1</smiles>

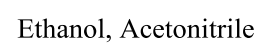<smiles>C(=N/Cc1ccccn1)\c1ccccn1</smiles><smiles>c1ccc(CNCc2ccccn2)nc1</smiles>

Figure 59: The synthesis of $\mathrm{DPA}^{116}$.

DPA is most commonly functionalised at the central nitrogen and Srećko et al. have investigated developing a new metal chaperone using a modified DPA ligand (XXXII) (Figure 60) ${ }^{114}$. They complexed their compounds with metal salts and some crystal structures (Figure 61) were obtained.

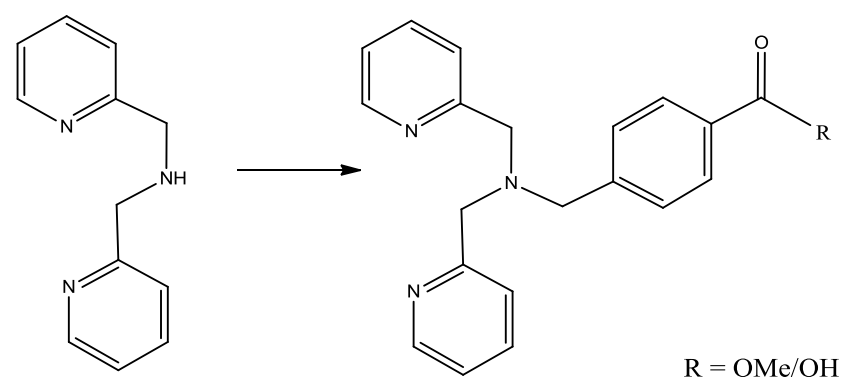

Figure 60: Modified DPA ligand (XXXII) ${ }^{114}$. 


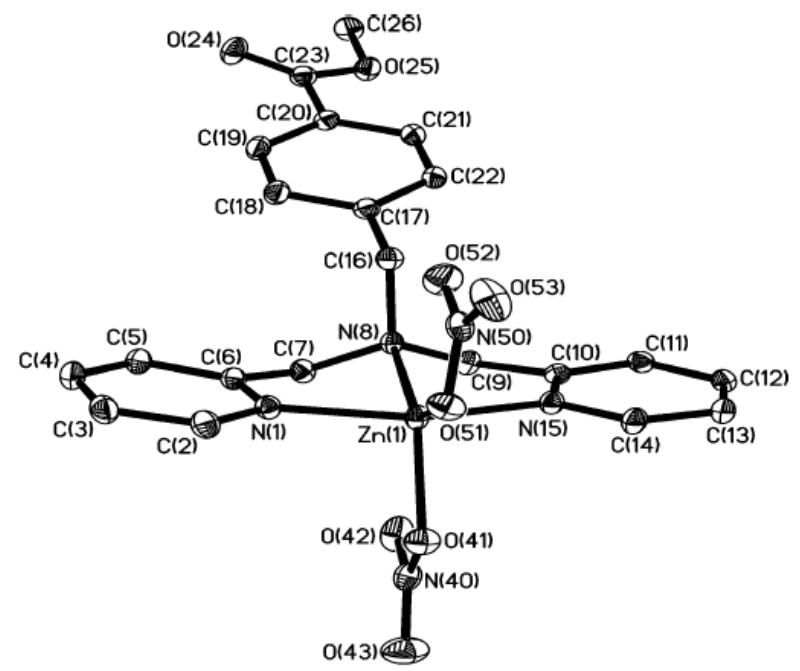

Figure 61: Crystal structure of (XXXII) complexed with zinc nitrate ${ }^{114}$.

They observed from the crystal structure that the DPA ligand acts as a tridentate ligand taking up a meridional position around the zinc ion. The structure also showed the two nitrate counter-ions coordinated to the zinc resulting in a distorted trigonal bipyramid geometry about the zinc ion. They concluded from their research that these DPA modified compounds could have the potential to act as artificial chaperones inside the body and work is on-going to test the feasibility of this ${ }^{114}$.

BeBout et al. have been investigating the geometries DPA adopts when complexed to $\mathrm{Hg}^{2+}$ ion $(\mathrm{XXXIII})^{117}$. DPA has been used to construct metal complexes to model the metal ion coordination's found in many proteins. Several publications have shown that DPA-metal complexes can adopt a range of coordination geometries including bidentate ${ }^{118}$ and tridentate ${ }^{119}$ facial (fac) and meridional (mer) orientations. From their crystal structures they concluded that the DPA ligand is acting as a tridentate ligand and that the $\mathrm{Hg}^{2+}$ ion was also coordinated to two perchlorate anions and one acetonitrile molecule (Figure 62), and that the DPA ligand was coordinating to the $\mathrm{Hg}^{2+}$ ion in a mer orientation ${ }^{117}$. 


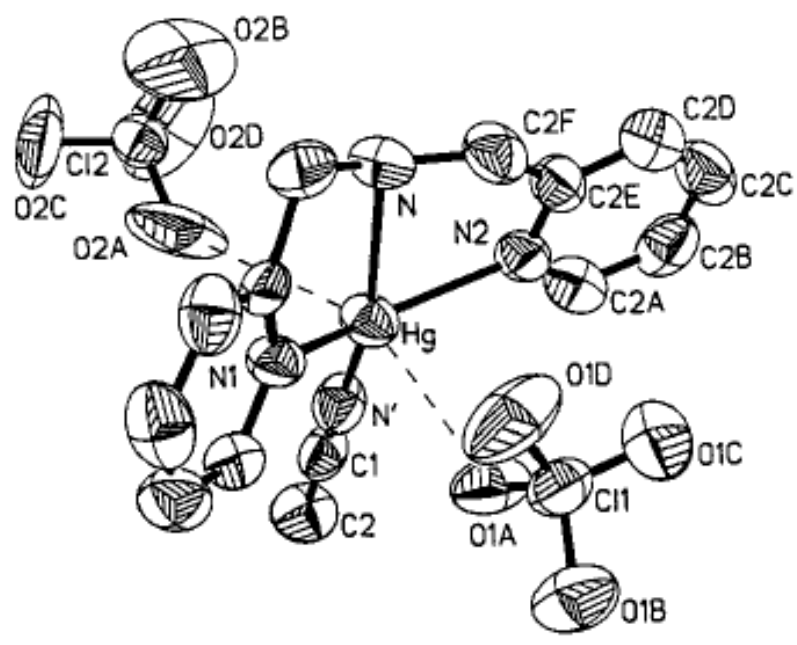

Figure 62: Crystal structure of $\mathrm{Hg}^{2+}$-DPA complex (XXXIII) ${ }^{117}$.

Jenkins et al. used platinium based DPA complexes to compare preferred orientations of the DPA ligand (XXXIV a-d) ${ }^{119}$. DPA has the potential to bind either in a bidentate or tridentate manner, (Figure 63). Jenkins et al. have used it in the study of oxidation-addition reactions of platinium(II).<smiles></smiles>

a<smiles>[M]C(C)N(Cc1ccccn1)Cc1ccccn1</smiles>

$\mathrm{b}$

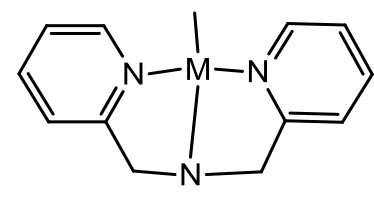

$\mathrm{c}$<smiles></smiles>

d

Bidentate

Tridentate

Figure 63: Bidentate and tridentate orientations of DPA ligand (XXXIV a-d) ${ }^{119}$.

They conducted a series of NMR experiments in deutrated chloroform which revealed that only one of the pyridine arms of the DPA ligand along with the central nitrogen were bound to the platinum(II). This was seen when they observed the ortho coupling for ${ }^{3} J_{(\mathrm{Pt}-\mathrm{H})}$ for only one of the pyridyl groups as well as observing two 
different resonance peaks for the two bridging methyl's, further suggesting that the $\mathrm{Pt}^{2+}$ ion was bound unsymmetrically. They suggested that the ligand is adopting a bidentate orientation (Figure 63) with one pyridyl and one amine group coordinated $^{119}$.

Constructs which have been designed to simultaneously complex metals often employ the bipyridine $\mathrm{N}$ - donor ligand as the second metal binding site ${ }^{120}$. McKenzie et al. have reported isolating cobalt complexed phenanthroline derivative $(\mathrm{XXXV})$, cis-[Co(phen $\left.)_{2} \mathrm{Cl}_{2}\right]^{121}$. In this compound, the cobalt ion is complexed through two phenanthroline units acting as bidentate chelator's and two chloride anions occupying two cis- positions from the octahedral complex. The cobalt complex was found to exhibit a distorted octahedral geometry and it crystallizes with 1.5 acetonitrile solvent molecules per complex Figure 64.
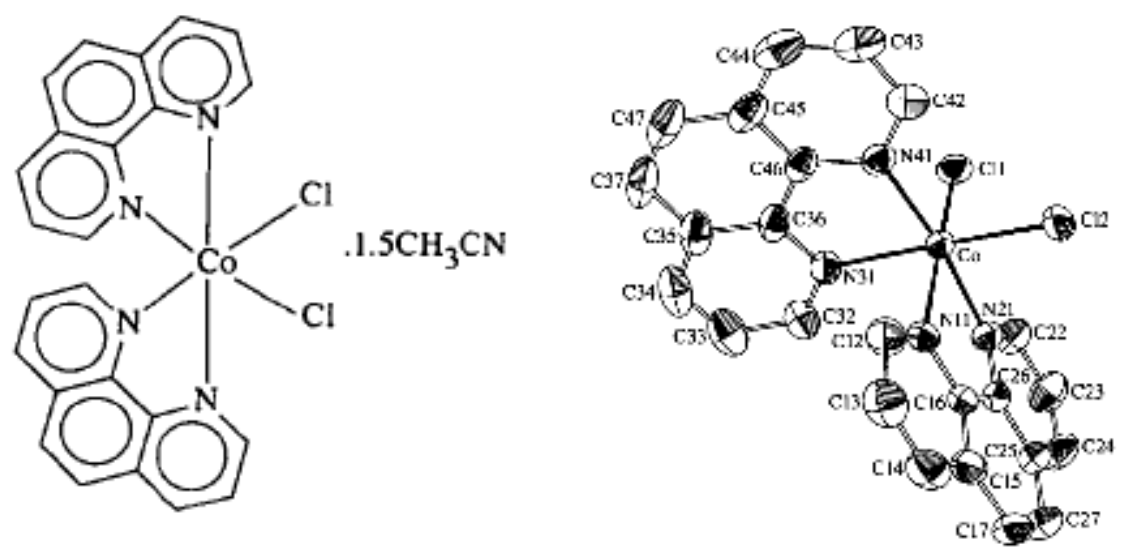

Figure 64: Structure and X-ray image of $c i s-\left[\mathrm{Co}(\text { phen })_{2} \mathrm{Cl}_{2}\right](\mathrm{XXXV})$. Solvent and $\mathrm{H}$ atoms omitted in X-ray image for clarity ${ }^{121}$.

Many 2,2'-bipyridine derivatives show biological activity as well as being good chelator's - due to the rings adoption of a co-planar orientation ${ }^{122}$. Spillane et al. have investigated the ability of bipyridine derivatives to act as potential anti-tumor agents $^{123}$. They wanted to design a 'tailored' agent which would bind to specific minor grooves in DNA resulting in a close interaction consisting of a number of base pairs which would ideally allow for sequence specificity. It was hoped that the 
compounds would give an alternative treatment to cisplatin as some tumors have become resistant to current cisplatin treatments. They developed several ruthenium based bipyridyl complexes (XXXVI), (Figure 65) and subjected them to several different human cancer cell models to test the antitumor activity. The results indicated that all their complexes were active against the tumor cells and also noticed that colon cancer cells were slightly more sensitive than the other cells tested. They concluded from this that the ruthenium bipyridyl complexes were having a noticeable effect on the cancerous cells but further research is required to determine exactly where the target of action is ${ }^{123}$.

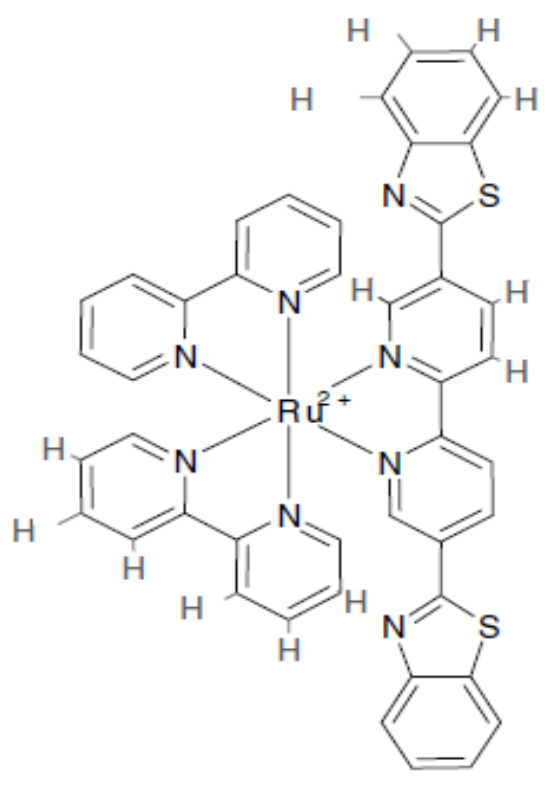

Figure 65: Ruthenium based bipyridine derivative (XXXVI) ${ }^{123}$.

Creaven et al. have published the functionalisation of the upper rim of calix[4]arenes with bipy groups ${ }^{124}$. Following the 1,3-functionalisation of the lower rim, the upper rim was functionalised first by nitration and then by reduction of the nitro groups to the amine. Both of these steps produced poor yields $(\max 17 \%)$. This amine compound was then reacted with 4-methyl-4'-carboxylicacid-2,2'-bipyridine to afford the upper rim functionalised calix[4]arene derivative (XXXVII) (Figure 66). 


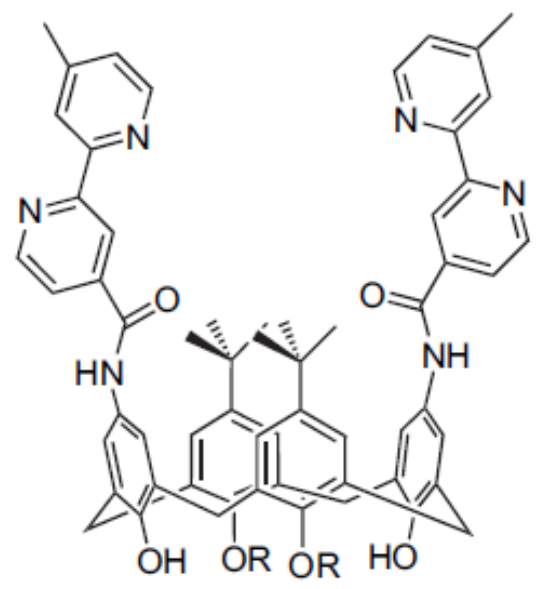

Figure 66: Upper rim functionalised bipy calix[4]arene derivative were $\mathrm{R}$ on the lower rim could be diester, butyl or butoxy groups (XXXVII) ${ }^{124}$.

The binding ability of this structure was then tested using $\left[\mathrm{Ru}(\text { bipy })_{2} \mathrm{Cl}_{2}\right]$. The complexation was carried out in ethanol and from the ${ }^{1} \mathrm{H}$ NMR spectrum (Figure 67), it can be clearly shown that complexation has occurred.
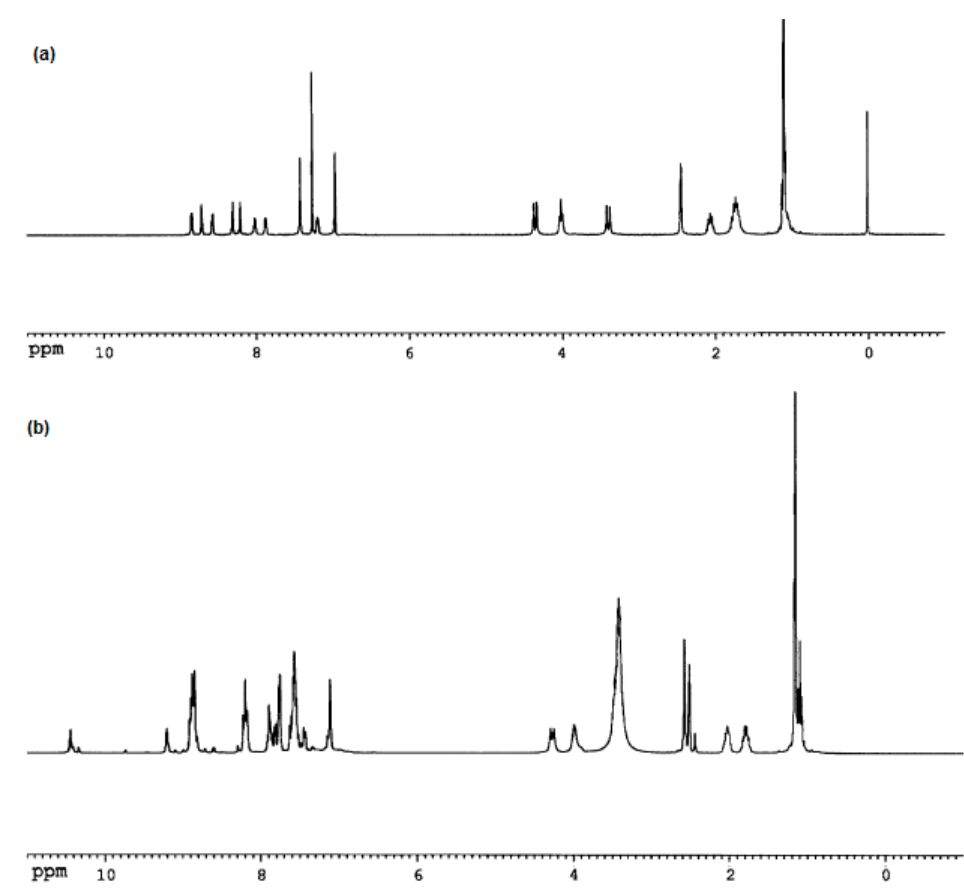

Figure 67: ${ }^{1} \mathrm{H}$ NMR of Creaven's 1,3-difunctionalised compound. (XXXVIIa) is uncomplexed, (XXXVIIb) complexed ${ }^{124}$.

The ${ }^{1} \mathrm{H}$ NMR spectrum shows the attachment of the $\left[\mathrm{Ru}(\mathrm{bipy})_{2}\right]$ to the upper rim of the calixarene. This produces the desired $\left[\mathrm{Ru}(\text { bipy })_{3}\right]$ moiety on the upper rim of the 
functionalised calix[4]arene, and was found to be produced in good yields (64\%). Creaven et al. showed from this that functionalisation of the upper rim of calix[4]arenes with the bipy unit works well and complexation to metal such as ruthenium was also possible in high yields.

\subsection{Aim of Thesis}

The work presented in this thesis involves the development of novel calix[4]arene metal ion binding compounds which have been modified at both the upper and lower rims. These compounds were designed with the goal of achieving two metal binding sites in each calix[4]arene scaffold, one on the upper rim and one on the lower rim. The initial design was developed in the hope that these compounds may one day be used as solar energy converters. The energy would be captured by one of these metal ions and would travel through the calix[4]arene system to the other metal ion where it could be collected and utilised.

Modification of these rims involves the use of various groups e.g. amides, amines, esters and several nitrogen donor containing ligands such as bipy and pyridyl units. It was envisioned that these groups would complex metal ions to allow electron transfer between the two rims. Functionalisation began with the lower rim, often with the ester linkage which could then be further modified at a later stage to create the metal ion binding site. Starting with the lower rim would also provide some rigidity to the calix[4]arene scaffold and help to decrease the likely-hood of interconversion between the four calix[4]arene conformers. Once the desired calix[4]arene scaffold had been constructed the metal ion binding ability would be investigated using a range of metal salts and the resulting complexes would be examined using various techniques.

However, during the course of this research an interesting observation was made, in that the NMR spectra of the compounds were becoming increasingly more complex than expected. As such the majority of the project has been spent investigating this unusual albeit interesting observation. 
2 Experimental 


\subsection{General Apparatus.}

All reagents were supplied by either Alfa Aesar or Sigma Aldrich and used without further purification. All solvents used were distilled and dried before use. Products were characterised by NMR spectroscopy using either a Bruker Avance III $500 \mathrm{MHz}$ NMR spectrometer (IT Tallaght with the assistance of Dr. Brian Murray), with a resolution of $0.005 \mathrm{~Hz}$ or a Bruker Avance $300 \mathrm{MHz}$ NMR spectrometer (NUIM) with a resolution of $0.18 \mathrm{~Hz}$. Both used $\mathrm{Me}_{4} \mathrm{Si}$ (TMS) as a reference and both had a probe temperature of $25{ }^{\circ} \mathrm{C}$ unless otherwise stated. Spectra were recorded using deuterated chloroform $\left(\mathrm{CDCl}_{3}\right)$ solutions, at a concentration of $10^{-3}$ millimolar unless otherwise indicated. Chemical shifts are given in ppm downfield from the internal reference $\mathrm{Me}_{4} \mathrm{Si}$ (TMS). While detailed analysis of the NMR spectra has been carried out, in the ${ }^{1} \mathrm{H}$ NMR spectra of the assembled compounds, assignment of the numbers of protons as well as proton association of the signals has not been accurately recorded here due to excessive overlap. However, were possible peaks have been assigned and discussed.

Infrared (IR) spectra $\left(\mathrm{cm}^{-1}\right)$ were recorded in the region $4000-400 \mathrm{~cm}^{-1}$ using a Perkin Elmer System 2000 FT-IR spectrophotometer (NUIM). Solid samples were ground with an excess of anhydrous potassium bromide (KBr) and compacted into a disc under a pressure of 10 tonnes. Liquid samples were run between $\mathrm{NaCl}$ plates.

UV-Vis spectra were recorded in a mixture of dichloromethane and methanol at a 1:2 ratio on a UNICAM UV 500 UV-visible spectrometer in the region $400-900 \mathrm{~nm}$, and analysed using Vision software V3.40 (NUIM). All samples presented in this thesis were performed in a solvent mixture consisting of $\mathrm{DCM}-\mathrm{MeOH}$ at a 1:2 ratio. Excitation and emission spectra were recorded on a JASCO FP-6300 spectrofluorometer in the same solvent mixture as the UV (NUIM).

Melting point analysis was carried out using a Stewart Scientific SMP-1 melting point apparatus and data are uncorrected. Mass spectrometry was conducted on an 
Agilent LC/TOF-MS model 6210 Time-Of-Flight LC/MS. The column employed was an Agilent Eclipse XBD-C18 consisting of a diameter of $4.6 \mathrm{~mm}$ and a column length of $150 \mathrm{~mm}$. The mobile phase consisted of a mixture of acetonitrile and formic acid with a gradient of $5 \%$ of acetonitrile rising to $100 \%$ at a flow rate of 0.5 $\mathrm{mL} / \mathrm{min}$ over a 15 minute period.

Microanalysis was carried out at the Chemistry Department (NUIM). Samples were analysed using an Exeter Analytical CE-440 elemental analyser in oxygen with a helium carrier gas at $975{ }^{\circ} \mathrm{C}$ in a combustion tube.

Metal ion complexation reactions were carried out with some of the compounds presented here and while it has been reported that the use of perchlorate salts have potentially explosive properties no encounters of this nature were found in this project. However, only small amounts were used and care was taken in their handling.

$2.25,11,17,23,29,35,41,47-O c t a-t e r t-b u t y l-49,50,51,52,53,54,55,56-$ octahydroxycalix[8]arene $\mathrm{e}^{108,125}$.

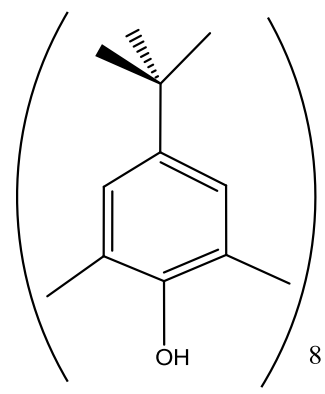

p-tert-Butylphenol (112 g, $746 \mathrm{mmol})$, paraformaldehyde (36 g, $1200 \mathrm{mmol}$ ) and 10 M potassium hydroxide $(1.7 \mathrm{~mL})$ in xylene $(600 \mathrm{~mL})$ was refluxed for $4 \mathrm{hr}$. in a 1 litre round-bottom flask, equipped with a Dean and Stark water collector. After 
cooling, the precipitate was filtered and washed in succession with $100 \mathrm{~mL}$ portions of toluene, ether, acetone and water. The compound was then dried in an oven for two weeks to give a white solid (1).

Yield $=20$ g, $17 \%$; m.p. $>300{ }^{\circ} \mathrm{C}\left(\right.$ lit $\left.411-412{ }^{\circ} \mathrm{C}\right){ }^{125} ; \mathrm{v}_{\max } / \mathrm{cm}^{-1}(\mathrm{KBr}): 3190(\mathrm{OH})$, $2955(\mathrm{CH}), 1487,1362,1204,874 ; \delta_{\mathrm{H}} / \mathrm{ppm}$ : Conc. of $10^{-3} \mathrm{~mm} ;\left(\mathrm{CDCl}_{3}\right): 9.62(\mathrm{~s}, 8 \mathrm{H}$, $\operatorname{Ar}-\underline{\mathrm{OH}}), 7.18$ (s, 16H, Ar- $\underline{\mathrm{H}}), 4.36\left(\mathrm{~d}, 8 \mathrm{H}, \underline{\mathrm{C}}_{2}, J=13.2 \mathrm{~Hz}\right), 3.48\left(\mathrm{~d}, 8 \mathrm{H}, \mathrm{C}_{2}, J=\right.$ $13.2 \mathrm{~Hz}), 1.25\left(\mathrm{~s}, 72 \mathrm{H}, \mathrm{C}\left(\mathrm{CH}_{3}\right)\right) ; \delta_{\mathrm{c}} / \mathrm{ppm}\left(\mathrm{CDCl}_{3}\right): 146.5,143.6,128.4,125.5,34.2$, $32.3,31.4$.

\section{$2.3 \quad 5,11,17,23-T e t r a-t e r t-b u t y l-25,26,27,28-$} tetrahydroxycalix[4]arene $\mathrm{ar}^{108,125}$.

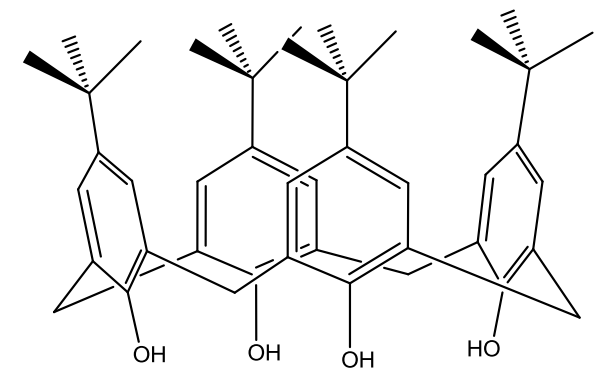

Compound 1 (22 g, $0.017 \mathrm{mmol})$ and $10 \mathrm{M}$ sodium hydroxide $(0.5 \mathrm{~mL})$ in diphenyl ether $(200 \mathrm{~mL})$ was refluxed for $1.5 \mathrm{hr}$. in a 1 litre round-bottomed flask equipped with a Dean and Stark water collector. The solution was cooled to between 80-100 ${ }^{\circ} \mathrm{C}$ when ethyl acetate $(200 \mathrm{ml})$ was added. The solution was then filtered while still hot. The sugar-like precipitate was washed with $100 \mathrm{~mL}$ portions of toluene, ether, acetone and water. The resulting powder was then dried in the oven for 3 days to give the white solid (2).

Yield $=11.5 \mathrm{~g}, 52 \% ; \mathrm{m} . \mathrm{p}>300{ }^{\circ} \mathrm{C}\left(\mathrm{lit} 342-344{ }^{\circ} \mathrm{C}\right){ }^{125} ; \mathrm{v}_{\max } / \mathrm{cm}^{-1}(\mathrm{KBr}): 3119(\mathrm{OH})$, $2950(\mathrm{CH}), 1482,1361,1199,1040,871 ; \delta_{\mathrm{H}} / \mathrm{ppm}$ : Conc. of $10^{-3} \mathrm{~mm} ;\left(\mathrm{CDCl}_{3}\right): 10.78$

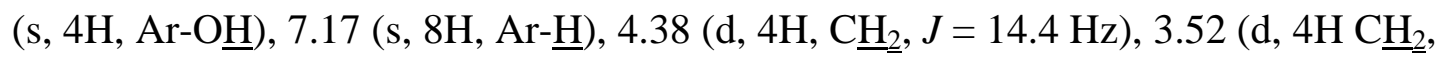
$J=14.4 \mathrm{~Hz}), 1.25\left(\mathrm{~s}, 36 \mathrm{H}, \mathrm{C}\left(\mathrm{C}_{3}\right)\right) ; \delta_{\mathrm{c}} / \mathrm{ppm}\left(\mathrm{CDCl}_{3}\right): 146.5,144.3,127.5,125.9$, $34.2,32.2,31.4$. 


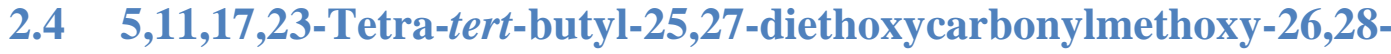

dihydroxycalix[4]arene ${ }^{108,126}$. (3)

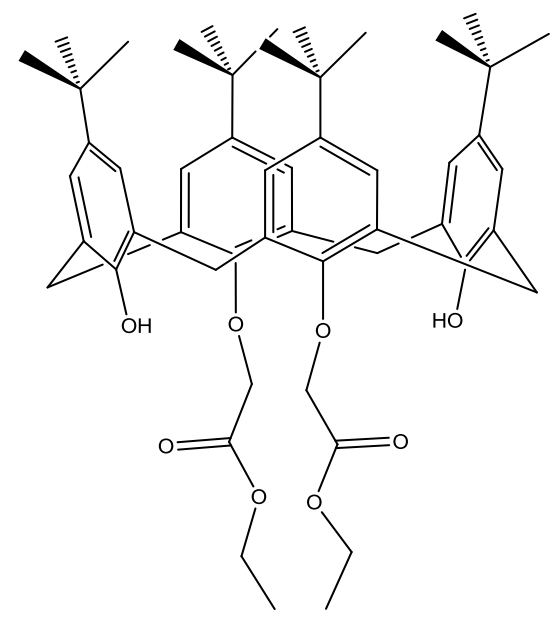

Compound (2) $(3.97 \mathrm{~g}, 6.2 \mathrm{mmol})$ and potassium carbonate $(2.11 \mathrm{~g}, 15.4 \mathrm{mmol})$ in acetonitrile $(120 \mathrm{~mL})$ were stirred under nitrogen in a $250 \mathrm{~mL}$ round bottom flask. Ethyl bromoacetate $(1.50 \mathrm{~mL}, 13.6 \mathrm{mmol})$ in acetonitrile $(10 \mathrm{~mL})$ was then added to the mixture. The solution was then refluxed for $18 \mathrm{hr}$. The mixture was cooled, filtered and the filtrate was then concentrated under reduced pressure to yield a white solid (3).

Yield $=6.70$ g, $90.9 \%$; m.p. $178-181{ }^{\circ} \mathrm{C}$ (lit. $182-184{ }^{\circ} \mathrm{C}$ ) ${ }^{126} ; \mathrm{v}_{\max } / \mathrm{cm}^{-1}(\mathrm{KBr}): 3434$ $(\mathrm{OH}), 2962(\mathrm{CH}), 1745(\mathrm{C}=\mathrm{O}), 1630,1586,1459,1183,760 ; \delta_{\mathrm{H}} / \mathrm{ppm}$ : Conc. of $10^{-3}$

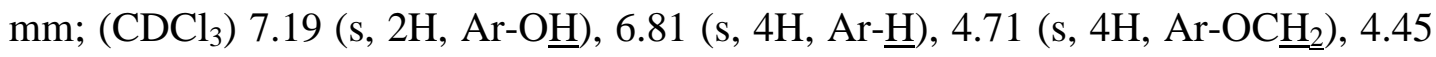
(d, 4H, $\left.\underline{\mathrm{H}}_{2}, J=13.1 \mathrm{~Hz}\right), 4.30$ (q, 4H, OC$\left.\underline{\mathrm{H}}_{2} \mathrm{CH}_{3}, J=7.6 \mathrm{~Hz}\right), 3.33$ (d, $4 \mathrm{H}, \mathrm{C}_{2}, J=$ $13.1 \mathrm{~Hz}), 1.33$ (t, $\left.6 \mathrm{H}, \mathrm{OCH}_{2} \mathrm{CH}_{3}, J=7.4 \mathrm{~Hz}\right), 1.25\left(\mathrm{~s}, 18 \mathrm{H}, \mathrm{C}\left(\mathrm{CH}_{3}\right)\right), 1.00(\mathrm{~s}, 18 \mathrm{H}$, $\left.\mathrm{C}\left(\mathrm{C}_{3}\right)\right) ; \delta_{\mathrm{c}} / \mathrm{ppm}\left(\mathrm{CDCl}_{3}\right): 169.5,151.0,149.6,143.1,126.5,126.0,125.1,72.4$, 61.3, 31.8, 31.6, 31.4, 14.2. 


\subsection{5,17-Di-tert-butyl-11,23-dinitro-25,27-diethoxycarbonylmethoxy- 26,28-dihydroxycalix[4] $\operatorname{arene}^{124}$. (4)}

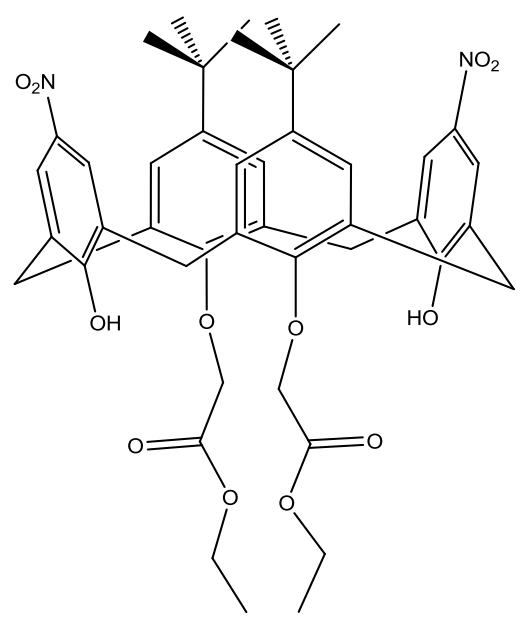

To a solution of compound (3) $(7.18 \mathrm{~g}, 9 \mathrm{mmol})$ in glacial acetic acid $(75 \mathrm{~mL})$ and dichloromethane $(75 \mathrm{~mL}), 70 \%$ nitric acid $(10 \mathrm{~mL})$ was added. This mixture was stirred at room temperature under nitrogen for $1.5 \mathrm{hr}$. A colour change from yellow to red was observed. The reaction was quenched with $150 \mathrm{~mL}$ of ice-cold water and left stirring overnight. The organic layer was then separated. The aqueous layer was extracted with DCM (2 x $50 \mathrm{~mL})$. The organic extracts were combined and washed with water $(2 \times 50 \mathrm{~mL})$. The organic layer was then dried over magnesium sulphate, filtered and the volatiles were removed under reduced pressure to give a yellow solid (4).

Yield $=1.3$ g, $18 \%$; m.p. $193-195{ }^{\circ} \mathrm{C}$ (lit 198-200 $\left.{ }^{\circ} \mathrm{C}\right){ }^{124} ; \mathrm{v}_{\max } / \mathrm{cm}^{-1}(\mathrm{KBr}): 3356$ (OH), $2966(\mathrm{CH}), 1769(\mathrm{C}=\mathrm{O}), 1516(\mathrm{NO}), 1340,1182,760 ; \delta_{\mathrm{H}} / \mathrm{ppm}$ : Conc. of $10^{-3}$ mm; $\left(\mathrm{CDCl}_{3}\right): 8.78$ (s, 2H, Ar-Oㅂ) $), 8.12$ (s, 4H, Ar- $\left.\underline{\mathrm{H}}\right), 7.03$ (s, 4H, Ar- $\left.\underline{\mathrm{H}}\right), 4.81$ (s, $\left.4 \mathrm{H}, \operatorname{Ar}-\mathrm{OC}_{2}\right), 4.51$ (d, 4H, $\left.\underline{\mathrm{C}}_{2}, J=13.2 \mathrm{~Hz}\right), 4.33$ (q, 4H, $\mathrm{OC}_{2} \underline{\mathrm{CH}}_{3}, J=7.2 \mathrm{~Hz}$ ), $3.47\left(\mathrm{~d}, 4 \mathrm{H}, \underline{\mathrm{C}}_{2}, J=13.2 \mathrm{~Hz}\right), 1.36\left(\mathrm{t}, 6 \mathrm{H}, \mathrm{OCH}_{2} \underline{\mathrm{CH}}_{3}, J=7.53 \mathrm{~Hz}\right), 1.10$ (s, $18 \mathrm{H}$, $\left.\mathrm{C}\left(\mathrm{C}_{3}\right)\right) ; \delta_{\mathrm{c}} / \mathrm{ppm}\left(\mathrm{CDCl}_{3}\right): 169.4,157.6,153.2,145.1,142.3,133.3,129.3,127.5$, 124.6, 69.0, 60.9, 34.1, 31.3, 30.8, 14.0. 
2.6 5,17-Di-tert-butyl-11,23-diamino-25,27-dimethoxycarbonylmethoxy26,28-dihydroxycalix[4] $\operatorname{arene}^{124}$. (5)

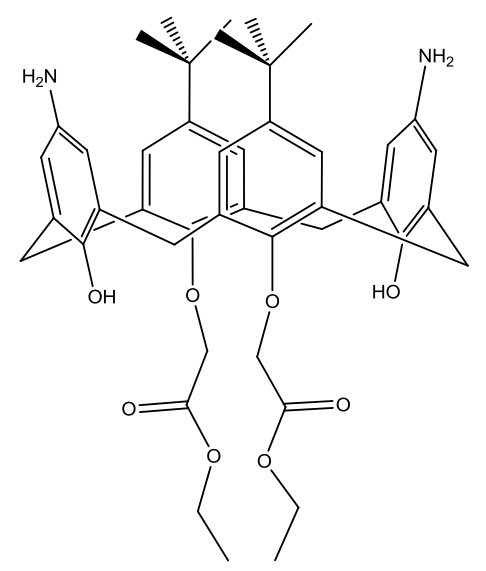

A mixture of compound (4) $(0.5 \mathrm{~g}, 0.68 \mathrm{mmol})$ and $\mathrm{Sn}(\mathrm{II}) \mathrm{Cl}_{2}(1.28 \mathrm{~g}, 6.81 \mathrm{mmol})$ in ethanol $(25 \mathrm{~mL})$ was refluxed under nitrogen for $24 \mathrm{hr}$. The solution was cooled to room temperature and was then poured onto crushed ice $(25 \mathrm{~mL})$ where the $\mathrm{pH}$ was then adjusted to 9-10 using a $5 \mathrm{M} \mathrm{KOH}$ solution. The solution was then extracted with DCM and the combined organic layers were washed with brine $(2 \times 50 \mathrm{~mL})$ and dried over $\mathrm{Na}_{2} \mathrm{SO}_{4}$. The volatiles were removed under reduced pressure to give a yellow-orange solid. Analysis of this solid showed it to be the starting calix[4]arene 4. 


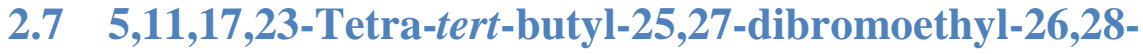 dihydroxycalix[4]arene ${ }^{124}$. (6)}

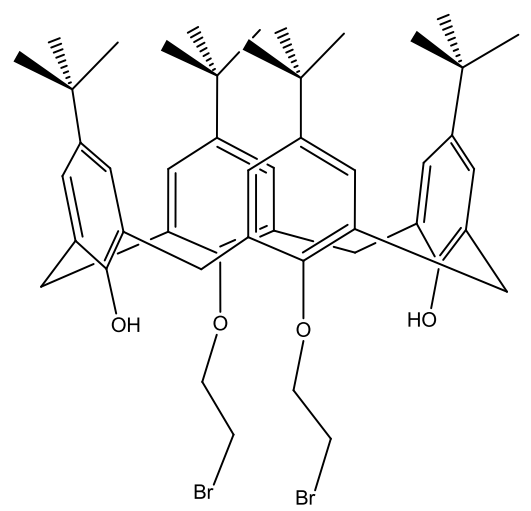

A mixture of calix[4]arene (2) $(5 \mathrm{~g}, 7.7 \mathrm{mmol})$, potassium carbonate $(2.74 \mathrm{~g}, 15.4$ $\mathrm{mmol})$, and dibromoethane $(2.89 \mathrm{~g}, 15.4 \mathrm{mmol})$ in acetonitrile $(150 \mathrm{~mL})$ was refluxed under nitrogen for two days. The solution was concentrated under reduced pressure to $10 \mathrm{~mL}$, and then added to ice-cold water $(250 \mathrm{~mL})$ with vigorous stirring. The solution was filtered to leave a cream-white, sticky solid (6) which was dried in the oven.

Yield $=5.2$ g, $80 \%$; m.p. $=208-210{ }^{\circ} \mathrm{C}$; (lit. 207-209 $\left.{ }^{\circ} \mathrm{C}\right){ }^{124} ; \mathrm{v}_{\max } / \mathrm{cm}^{-1}(\mathrm{KBr}): 3450$ $(\mathrm{OH}), 2963(\mathrm{CH}), 1485,1459,1195,759 ; \delta_{\mathrm{H}} / \mathrm{ppm}$ : Conc. of $10^{-3} \mathrm{~mm} ;\left(\mathrm{CDCl}_{3}\right): 7.19$ (s, 2H, Ar-OH) $7.01(\mathrm{~s}, 4 \mathrm{H}, \mathrm{Ar}-\underline{\mathrm{H}}), 6.81(\mathrm{~s}, 4 \mathrm{H}, \mathrm{Ar}-\underline{\mathrm{H}}), 4.45\left(\mathrm{~d}, 4 \mathrm{H}, \mathrm{CH}_{2}, J=13.2\right.$ $\mathrm{Hz}), 3.85\left(\mathrm{t}, \mathrm{OC}_{2} \mathrm{CH}_{2} \mathrm{Br}, J=5.1 \mathrm{~Hz}\right), 3.33\left(\mathrm{~d}, 4 \mathrm{H}, \underline{\mathrm{C}}_{2}, J=13.2 \mathrm{~Hz}\right), 1.87(\mathrm{t}, 4 \mathrm{H}$, $\left.\mathrm{OCH}_{2} \underline{\mathrm{C}}_{2} \mathrm{Br}, J=8.0 \mathrm{~Hz}\right), 1.25\left(\mathrm{~s}, 18 \mathrm{H}, \mathrm{C}\left(\mathrm{C}_{3}\right)\right), 1.00\left(\mathrm{~s}, 18 \mathrm{H}, \mathrm{C}\left(\mathrm{C}_{3}\right)\right) ; \delta_{\mathrm{c}} / \mathrm{ppm}$ $\left(\mathrm{CDCl}_{3}\right): 152.3,148.7,143.5,125.6,125.1,75.4,31.7,31.7,30.9,29.3$. 


\subsection{5,17-Di-tert-butyl-11,23-dinitro-25,27-dibromoethyl-26,28-}

dihydroxycalix[4]arene ${ }^{124}$. (7)

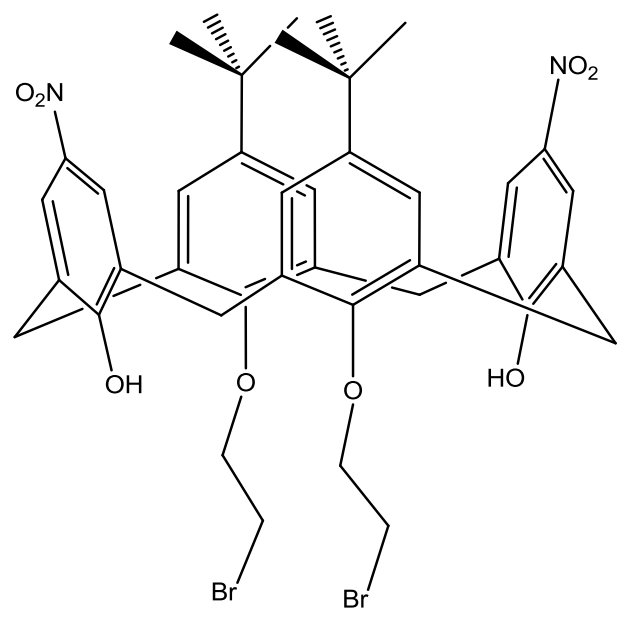

A solution of compound $(6)(1.42 \mathrm{~g}, 1.6 \mathrm{mmol})$ in DCM $(15 \mathrm{~mL})$ was stirred at $0{ }^{\circ} \mathrm{C}$. Then $70 \%$ nitric acid $(1.5 \mathrm{~mL})$ was added. The solution was then allowed to warm up to room temperature and stirred for $2.5 \mathrm{hr}$. Water $(10 \mathrm{~mL})$ was then added and the organic layer was extracted with DCM $(2 \times 10 \mathrm{~mL})$. The organic layer was dried over $\mathrm{Na}_{2} \mathrm{SO}_{4}$, filtered and the volatiles removed under reduced pressure. Addition of methanol (20 mL) gave a light copper coloured solid (7).

Yield $=1.1$ g, $16 \%$; m.p. $=220-222{ }^{\circ} \mathrm{C} ;\left(\right.$ lit. $\left.221-223{ }^{\circ} \mathrm{C}\right){ }^{124} ; \mathrm{v}_{\max } / \mathrm{cm}^{-1}(\mathrm{KBr}): 3449$ (OH), $2964(\mathrm{CH}), 1559$ (NO), 1484, 1456, 761; $\delta_{\mathrm{H}} / \mathrm{ppm}$ : Conc. of $10^{-3} \mathrm{~mm} ;\left(\mathrm{CDCl}_{3}\right)$;

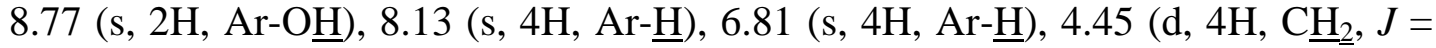
$13.1 \mathrm{~Hz}), 3.85\left(\mathrm{q}, \mathrm{O}-\mathrm{C}_{2} \mathrm{CH}_{2} \mathrm{Br}, J=3.8 \mathrm{~Hz}\right), 3.33\left(\mathrm{~d}, 4 \mathrm{H}, \mathrm{C}_{2}, J=13.2 \mathrm{~Hz}\right), 1.87$ (t, $\left.4 \mathrm{H}, \mathrm{OCH}_{2} \underline{\mathrm{C}}_{2} \mathrm{Br}, J=6.8 \mathrm{~Hz}\right), 1.00\left(\mathrm{~s}, 18 \mathrm{H}, \mathrm{C}\left(\mathrm{C}_{\underline{H}}\right)\right) ; \delta_{\mathrm{c}} / \mathrm{ppm} ; 156.5,152.3,144.0$, $141.2,133.0,129.8,125.6,123.9,68.1,34.1,31.5,31.0,28.2$. 


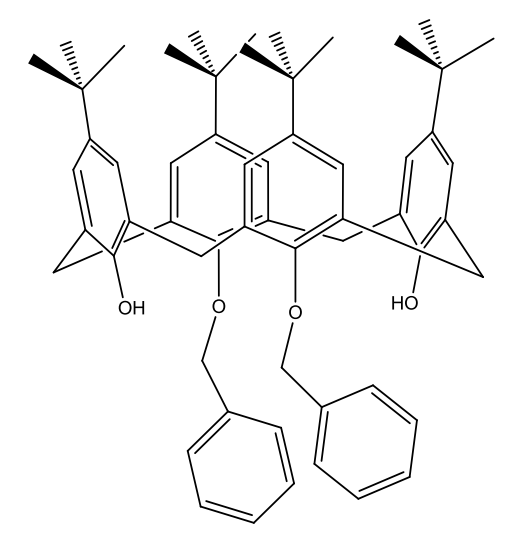

To a solution of compound (2) (2.0 g, $3.1 \mathrm{mmol})$, potassium carbonate $(1.07 \mathrm{~g}, 7.7$ $\mathrm{mmol})$ in $\mathrm{CH}_{3} \mathrm{CN}(60 \mathrm{~mL})$ stirring under nitrogen, benzyl bromide $(0.86 \mathrm{~g}, 6.8$ mmol) in $\mathrm{CH}_{3} \mathrm{CN}(100 \mathrm{~mL})$ was added. The mixture was refluxed under nitrogen for $26 \mathrm{hr}$. It was then cooled to room temperature and filtered to remove any inorganic salts. The filtrate was concentrated under reduced pressure yielding a white solid $(\mathbf{8})$.

Yield $=1.9$ g, $100 \%$; m.p. $=210-213{ }^{\circ} \mathrm{C},\left(\right.$ lit. $\left.210-212{ }^{\circ} \mathrm{C}\right){ }^{124} ; \mathrm{v}_{\max } / \mathrm{cm}^{-1}(\mathrm{KBr}): 3343$ $(\mathrm{OH}), 2959(\mathrm{CH}), 1591,1464,1189,1088,765 ; \delta_{\mathrm{H}} / \mathrm{ppm}$ : Conc. of $10^{-3} \mathrm{~mm}$; $\left(\mathrm{CDCl}_{3}\right): 7.64$ (s, 2H, Ar-O프), 7.64 (d, 4H, Bn- $\left.\underline{\mathrm{H}}, J=5.8 \mathrm{~Hz}\right), 7.36$ (t, 6H, Bn- $\underline{\mathrm{H}}, J=$ $3.0 \mathrm{~Hz}$ ), 7.03 (s, 4H, Ar- $\underline{\mathrm{H}}), 7.01$ (s, 4H, Ar-H), 5.04 (s, 4H, O-C $\left.\underline{\mathrm{H}}_{2}\right), 4.28$ (d, 4H, $\left.\mathrm{C}_{2}, J=13.3 \mathrm{~Hz}\right), 3.24\left(\mathrm{~d}, 4 \mathrm{H}, \underline{\mathrm{CH}}_{2}, J=13.3 \mathrm{~Hz}\right), 1.25\left(\mathrm{~s}, 18 \mathrm{H}, \mathrm{C}\left(\mathrm{C}_{\underline{H}}\right)\right), 1.00$ (s, $\left.18 \mathrm{H}, \mathrm{C}\left(\underline{\mathrm{CH}}_{3}\right)\right) ; \delta_{\mathrm{c}} / \mathrm{ppm} 150.7,149.8,143.2,142.6,141.3,137.2,132.6,128.6,127.6$, $127.3,125.5,124.9,76.5,34.7,31.7,31.4$. 


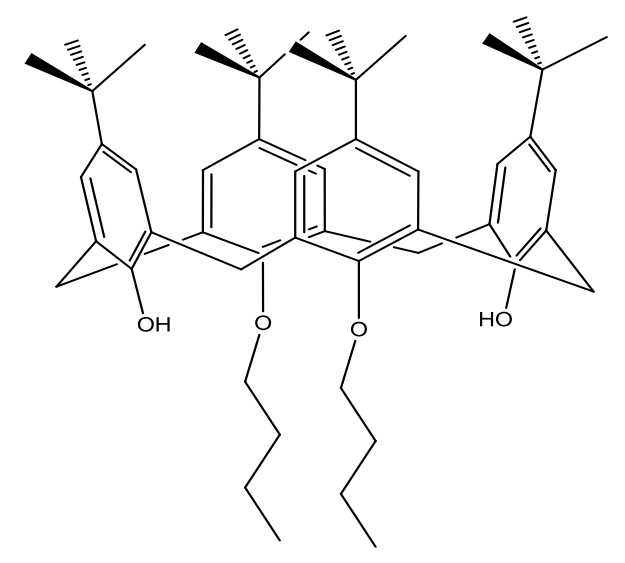

To a solution of compound (2) $(5 \mathrm{~g}, 7.5 \mathrm{mmol})$ and potassium carbonate $(2.34 \mathrm{~g}$, $16.9 \mathrm{mmol})$ in distilled $\mathrm{CH}_{3} \mathrm{CN}(250 \mathrm{~mL})$, under nitrogen, 1-bromobutane $(1.82 \mathrm{~mL}$, $16.9 \mathrm{mmol}$ ) was added. The solution was then refluxed under nitrogen for $24 \mathrm{hr}$. After cooling, the solution was filtered and the filtrate was concentrated under reduced pressure to give a white solid (9).

Yield $=4.5$ g, $86 \%$; m.p. $=218-220{ }^{\circ} \mathrm{C} ;\left(\text { lit. } 219-221{ }^{\circ} \mathrm{C}\right)^{124} ; \mathrm{v}_{\max } / \mathrm{cm}^{-1}(\mathrm{KBr}): 3332$, 2965, 2926, 1189, 1088, 976, 746; $\delta_{\mathrm{H}} / \mathrm{ppm}$ : Conc. of $10^{-3} \mathrm{~mm}$; $\left(\mathrm{CDCl}_{3}\right): 7.95$ (s, $2 \mathrm{H}$, Ar-O프), 7.05 (s, 4H, Ar- $\underline{H}), 6.86$ (s, 4H, Ar- $\underline{\mathrm{H}}), 4.31$ (d, 4H, $\left.\underline{\mathrm{C}}_{2}, J=13.1 \mathrm{~Hz}\right), 4.00$ (t, $\left.2 \mathrm{H}, \mathrm{O}-\mathrm{C}_{2} \mathrm{CH}_{2}, J=5.6 \mathrm{~Hz}\right), 3.28\left(\mathrm{~d}, 4 \mathrm{H}, \mathrm{C}_{2}, J=13.1 \mathrm{~Hz}\right), 2.04(\mathrm{q}, 4 \mathrm{H}, \mathrm{O}-$ $\mathrm{CH}_{2} \mathrm{CH}_{2} \mathrm{CH}_{2} \mathrm{CH}_{3}, J=7.1 \mathrm{~Hz}$ ), 1.75 (q, 4H, O- $\left.\mathrm{CH}_{2} \mathrm{CH}_{2} \mathrm{CH}_{2} \mathrm{CH}_{3}, J=7.8 \mathrm{~Hz}\right), 1.25$ (s, $\left.18 \mathrm{H}, \mathrm{C}\left(\mathrm{CH}_{3}\right)\right), 1.08$ (t, $\left.6 \mathrm{H}, \mathrm{O}-\mathrm{CH}_{2} \mathrm{CH}_{2} \mathrm{CH}_{2} \mathrm{C}_{3}, J=7.4 \mathrm{~Hz}\right), 1.00$ (s, $18 \mathrm{H}, \mathrm{C}\left(\mathrm{C}_{3}\right)$ ); $\delta_{\mathrm{c}} / \mathrm{ppm}\left(\mathrm{CDCl}_{3}\right): 150.6,149.8,146.4,143.5,142.7,140.1,127.1,124.7,124.2,69.1$, $33.1,32.9,31.3,31.0,18.4,13.2$. 


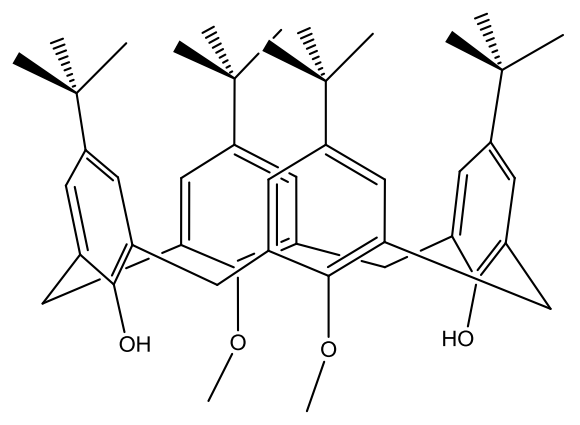

To a mixture of compound (2) $(2.0 \mathrm{~g}, 3.1 \mathrm{mmol})$, potassium carbonate $(1.07 \mathrm{~g}, 7.7$ mmol) in $\mathrm{CH}_{3} \mathrm{CN}(60 \mathrm{~mL})$, under nitrogen, was added methyl iodide $(0.38 \mathrm{~mL}, 6.2$ mmol). The solution was refluxed under nitrogen for $26 \mathrm{hr}$. Afterwards it was allowed to cool to room temperature where it was filtered to remove any inorganic salts and the volatiles were removed under reduced pressure. Addition of fresh $\mathrm{CH}_{3} \mathrm{CN}(10 \mathrm{~mL})$ yielded a white solid (10).

Yield $=1.94$ g, $95 \%$; m.p. $=215-218^{\circ} \mathrm{C} ;\left(\text { lit. } 217-219^{\circ} \mathrm{C}\right)^{127} ; \mathrm{v}_{\max } / \mathrm{cm}^{-1}(\mathrm{KBr}): 3329$, 3214, 2958, 2923, 1195, 1089, 753; $\delta_{\mathrm{H}} / \mathrm{ppm}$ : Conc. of $10^{-3} \mathrm{~mm} ;\left(\mathrm{CDCl}_{3}\right): 7.28(\mathrm{~s}$, $2 \mathrm{H}, \mathrm{Ar}-\mathrm{O} \underline{\mathrm{H}}$ ), 7.16 (s, 4H, Ar- $\underline{\mathrm{H}}$ ), 6.78 (s, 4H, Ar- $\left.\underline{\mathrm{H}}_{2}\right), 4.30$ (d, 4H, $\underline{\mathrm{C}}_{2}, J=12.8 \mathrm{~Hz}$ ), 3.95 (s, 6H, O- $\left.\mathrm{CH}_{3}\right), 3.30\left(\mathrm{~d}, 4 \mathrm{H}, \mathrm{C}_{2}, J=13.1 \mathrm{~Hz}\right), 1.29$ (s, 18H, C( $\left.\left.\underline{\mathrm{C}}_{3}\right)\right), 0.94$ (s, $\left.18 \mathrm{H}, \mathrm{C}\left(\mathrm{C}_{3}\right)\right) ; \delta_{\mathrm{c}} / \mathrm{ppm} 151.1,150.4,146.9,146.0,132.3,127.9,125.5,125.0,63.5$, $33.1,33.4,31.7$. 


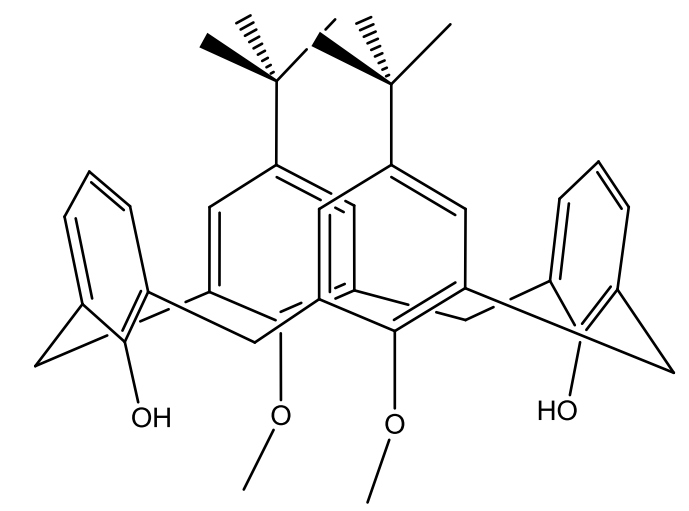

A mixture of aluminium chloride $(1.07 \mathrm{~g}, 7.7 \mathrm{mmol})$ in $\mathrm{CH}_{2} \mathrm{Cl}_{2}(10 \mathrm{ml})$ was stirred under nitrogen for $15 \mathrm{~min}$. To this a solution of compound (2) $(1.0 \mathrm{~g}, 1.54 \mathrm{mmol})$ in dry toluene $(25 \mathrm{~mL})$ was added. The reaction was stirred vigorously at room temperature for $3 \mathrm{hr}$. After this, the reaction was then quenched with $1 \mathrm{M} \mathrm{HCl}$ (30 $\mathrm{mL})$ and extracted with ethyl acetate $(30 \mathrm{~mL})$. The organic layer was washed twice with brine and the volatiles removed under reduced pressure to give a yellow solid (11).

Yield $=0.4$ g, $46 \%$; m.p. $=210-212{ }^{\circ} \mathrm{C} ;\left(\right.$ lit. $\left.208-210{ }^{\circ} \mathrm{C}\right){ }^{127} ; \mathrm{v}_{\max } / \mathrm{cm}^{-1}(\mathrm{KBr}): 3329$, 3048, 2958, 1485, 1362, 1205, 1010, 784; $\delta_{\mathrm{H}} / \mathrm{ppm}$ : Conc. of $10^{-3} \mathrm{~mm} ;\left(\mathrm{CDCl}_{3}\right)$ : 10.27 (s, 2H, Ar-OH), 7.15 (s, 4H, Ar-H), 7.12 (d, 4H, Ar-H, J = $6.7 \mathrm{~Hz}), 6.97$ (t, $4 \mathrm{H}, \mathrm{Ar}-\mathrm{H}, J=6.6 \mathrm{~Hz}), 4.31\left(\mathrm{~d}, 4 \mathrm{H}, \underline{\mathrm{C}}_{2}, J=13.2 \mathrm{~Hz}\right), 4.01$ (s, 6H, O-C $\left.\underline{\mathrm{H}}_{3}\right), 3.75$ (d, $\left.4 \mathrm{H}, \underline{\mathrm{CH}}_{2}, J=13.2 \mathrm{~Hz}\right), 1.27\left(\mathrm{~s}, 18 \mathrm{H}, \mathrm{CCH}_{3}\right) ; \delta_{\mathrm{C}} / \mathrm{ppm}\left(\mathrm{CDCl}_{3}\right): 153.0,152.6,146.6$, 133.3, 128.5, 127.6, 125.2, 123.5, 62.8, 33.8, 31.4, 30.9. 


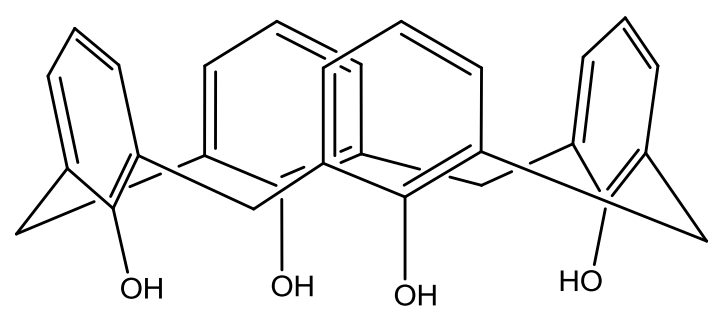

Aluminium chloride (2.0 g, $15.4 \mathrm{mmol})$ in DCM (20 mL) was stirred under nitrogen at room temperature for $15 \mathrm{~min}$. To this a solution of compound (2) (1.0 g, 1.5 $\mathrm{mmol})$ in dry toluene $(25 \mathrm{~mL})$ was added. The mixture was stirred vigorously for 3 hr. at room temperature and then quenched with $1 \mathrm{M} \mathrm{HCl}(3 \times 10 \mathrm{~mL})$. Extraction with ethyl acetate $(3 \times 10 \mathrm{~mL})$ then followed. The organic layer was separated, washed with brine and the volatiles removed under reduced pressure to yield a white solid (12).

Yield $=0.523$ g, $52 \%$; m.p. $=310-315^{\circ} \mathrm{C} ;\left(\text { lit. } 311-313{ }^{\circ} \mathrm{C}\right)^{127} ; \mathrm{v}_{\max } / \mathrm{cm}^{-1}(\mathrm{KBr})$ : 3416, 3329, 2927, 1465, 1244, 753; $\delta_{\mathrm{H}} / \mathrm{ppm}$ : Conc. of $10^{-3} \mathrm{~mm} ;\left(\mathrm{CDCl}_{3}\right): 10.17(\mathrm{~s}$, $4 \mathrm{H}, \operatorname{Ar}-\mathrm{O} \underline{\mathrm{H}}), 7.05(\mathrm{~d}, 8 \mathrm{H}, \mathrm{Ar}-\underline{\mathrm{H}}, J=12.1 \mathrm{~Hz}), 6.69$ (t, 4H, Ar- $\underline{\mathrm{H}}, J=8.7 \mathrm{~Hz}), 4.21(\mathrm{~d}$, $\left.4 \mathrm{H}, \underline{\mathrm{C}}_{2}, J=13.8 \mathrm{~Hz}\right), 3.44\left(\mathrm{~d}, \underline{\mathrm{C}}_{2}, J=13.6 \mathrm{~Hz}\right) ; \delta_{\mathrm{C}} / \mathrm{ppm}\left(\mathrm{CDCl}_{3}\right): 148.7,128.9$, 128.2, 122.5, 31.7. ESI-HRMS: calcd for $\mathrm{C}_{28} \mathrm{H}_{24} \mathrm{O}_{4}[\mathrm{M}+1]^{+} 447.2100$, found 447.2000; Anal. Calc. for $\mathrm{C}_{28} \mathrm{H}_{24} \mathrm{O}_{4}$ : C, 79.22; H, $5.70 \%$, found; C, 79.32; H, 5.71 $\%$.

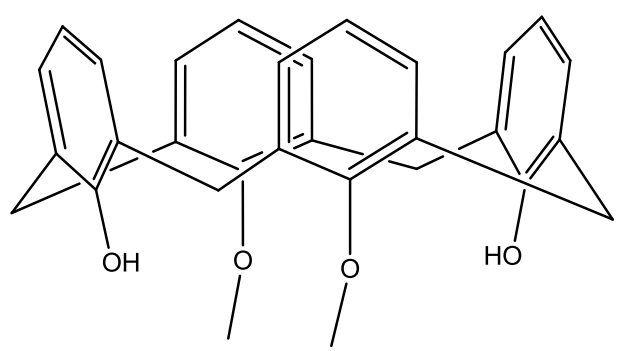


A solution of aluminium chloride $(1.0 \mathrm{~g}, 11.7 \mathrm{mmol})$ and DCM $(6 \mathrm{~mL})$ was allowed to stir under nitrogen for $15 \mathrm{~min}$ at room temperature. Then compound (10) $(1.0 \mathrm{~g}$, $1.47 \mathrm{mmol}$ ), in dry toluene was then added to the solution. The mixture was allowed to stir vigorously under nitrogen at room temperature for $3 \mathrm{hr}$. The reaction was then quenched with $1 \mathrm{M} \mathrm{HCL}(3 \times 10 \mathrm{~mL})$, extracted with ethyl acetate $(3 \times 10 \mathrm{~mL})$ and the organics were washed twice with brine $(2 \times 15 \mathrm{~mL})$, and the volatiles were removed under reduced pressure to give a yellow solid (13). On analysis of this compound showed it to be the starting calix[4]arene (10).

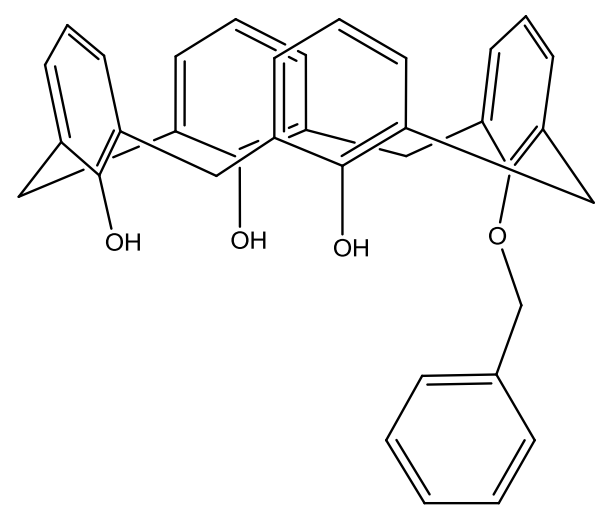

Compound (12) (0.1 g, $0.23 \mathrm{mmol})$, potassium carbonate $(0.02 \mathrm{~g}, 0.11 \mathrm{mmol})$ were stirred in $\mathrm{CH}_{3} \mathrm{CN}(10 \mathrm{~mL})$ for $30 \mathrm{~min}$. Benzyl bromide $(0.02 \mathrm{~mL}, 0.23 \mathrm{mmol})$ was then added and the mixture was refluxed overnight. The solution was allowed to cool and the solvent was removed under reduced pressure. The resulting residue was stirred with water $(10 \mathrm{~mL})$ and DCM $(10 \mathrm{~mL})$ for $10 \mathrm{~min}$. The aqueous phase was extracted with DCM (2 x 20mL), the organics were combined and the solvent removed under reduced pressure. The residue was stirred with diethyl ether $(20 \mathrm{~mL})$ for $1 \mathrm{hr}$. and the resulting white solid (14) was filtered under suction and air dried.

Yield $=0.52 \mathrm{~g}, 52 \% ; \mathrm{v}_{\max } / \mathrm{cm}^{-1}(\mathrm{KBr}): 3342,3089,2981,1459,1022,760 ; \delta_{\mathrm{H}} / \mathrm{ppm}:$ Conc. of $10^{-3} \mathrm{~mm} ;\left(\mathrm{CDCl}_{3}\right): 9.23(\mathrm{~s}, 3 \mathrm{H}, \mathrm{OH}), 7.56(\mathrm{~m}, 5 \mathrm{H}, \mathrm{Ar}-\underline{\mathrm{H}}), 7.07$ (d, 4H, Ar$\underline{\mathrm{H}}, J=7.8 \mathrm{~Hz}), 6.94(\mathrm{~d}, 4 \mathrm{H}, \mathrm{Ar}-\underline{\mathrm{H}}, J=6.9 \mathrm{~Hz}), 6.69$ (t, 4H, Ar- $\underline{\mathrm{H}}, J=8.4 \mathrm{~Hz}), 5.00$ 
(s, 2H, O-C $\left.\underline{\mathrm{H}}_{2}-\mathrm{Bn}\right), 4.31$ (d, 4H, $\left.\underline{\mathrm{C}}_{2}, J=13.4 \mathrm{~Hz}\right), 3.47$ (d, 4H, $\left.\underline{\mathrm{CH}}_{2}, J=13.4 \mathrm{~Hz}\right)$, $3.37\left(\mathrm{~d}, 4 \mathrm{H}, \underline{\mathrm{C}}_{2}, J=13.4 \mathrm{~Hz}\right)$.

\subsection{5,26,27-tris(ethoxycarbonyl)methoxy-28-phenylmethoxy-} calix[4] $\operatorname{arene}^{128} \cdot(15)$

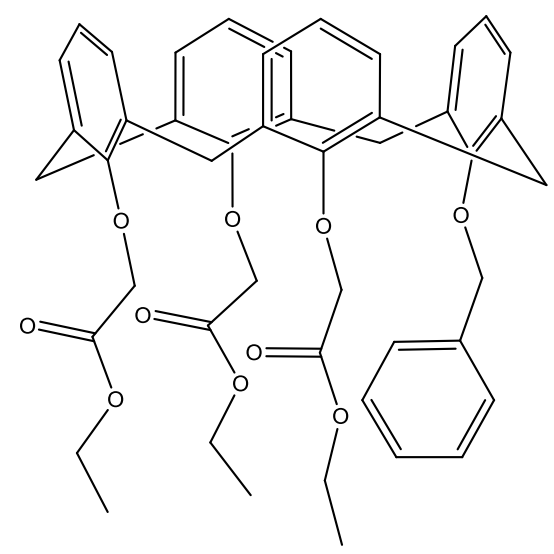

Compound (14) (0.2 g, $0.27 \mathrm{mmol})$ and $\mathrm{K}_{2} \mathrm{CO}_{3}(0.07 \mathrm{~g}, 0.54 \mathrm{mmol})$ were stirred for $30 \mathrm{~min}$ in $\mathrm{CH}_{3} \mathrm{CN}(20 \mathrm{~mL})$. Ethyl bromoacetate $(0.18 \mathrm{~mL}, 1.62 \mathrm{mmol})$ was added and the reaction was refluxed overnight. Ethyl bromoacetate $(0.03 \mathrm{~mL}, 0.27 \mathrm{mmol})$ was added and refluxing continued for another $24 \mathrm{hr}$. The reaction was allowed to cool and the solvent was removed under reduced pressure. The resulting residue was stirred with water $(10 \mathrm{~mL})$ and DCM $(10 \mathrm{~mL})$ for $20 \mathrm{~min}$. The aqueous layer was extracted with DCM (2 x $10 \mathrm{~mL})$, the organics were combined and the solvent removed under reduced pressure. The residue was stirred in ether $(20 \mathrm{~mL})$ for $1 \mathrm{hr}$., filtered and washed with ether. Again the solvent was removed under reduced pressure and the resulting solid was stirred in ethanol for $2 \mathrm{hr}$., filtered and dried to give a white solid (15).

Yield $=0.5 \mathrm{~g}, 19.7 \%$; $\mathrm{v}_{\max } / \mathrm{cm}^{-1}(\mathrm{KBr}): 3429,2927,1758,1459,1183,1094,761$; $\delta_{\mathrm{H}} / \mathrm{ppm}$ : Conc. of $10^{-3} \mathrm{~mm} ;\left(\mathrm{CDCl}_{3}\right) ; 7.88(\mathrm{~d}, 2 \mathrm{H}, \mathrm{Bn}-\underline{\mathrm{H}}, J=8.7 \mathrm{~Hz}), 7.65(\mathrm{t}, 3 \mathrm{H}, \mathrm{Bn}-$ $\underline{\mathrm{H}}, J=3.9 \mathrm{~Hz}), 7.02$ (d, 6H, Ar- $\underline{\mathrm{H}}, J=3.5 \mathrm{~Hz}), 7.00$ (d, 2H, Ar- $\underline{\mathrm{H}}, J=1.7 \mathrm{~Hz}), 6.74$ $\left(\mathrm{t}, 1 \mathrm{H}, \mathrm{Ar}-\underline{\mathrm{H}}, J=6.5 \mathrm{~Hz}\right.$ ), 5.06 (s, 2H, O-C $\left.\underline{\mathrm{H}}_{2}-\mathrm{Bn}\right), 4.33$ (q, 6H, O- $\underline{\mathrm{H}}_{2}{ }_{2} \mathrm{CH}_{3}, J=10.4$ $\mathrm{Hz}$ ), 4.21 (d, 4H, $\left.\underline{\mathrm{H}}_{2}, J=13.6 \mathrm{~Hz}\right), 3.45$ (s, 6H, O-C $\left.\underline{\mathrm{H}}_{2}-\mathrm{CO}\right), 3.33$ (d, 4H, $\underline{\mathrm{C}}_{2}, J=$ 
$13.3 \mathrm{~Hz}), 1.32\left(\mathrm{t}, 9 \mathrm{H}, \mathrm{OCH}_{2} \mathrm{CH}_{3}, J=7.5 \mathrm{~Hz}\right) ; \delta_{\mathrm{C}} / \mathrm{ppm}\left(\mathrm{CDCl}_{3}\right) ; 160.5,154.6,154.3$, $145.1,136.0,134.3,128.7,127.8,127.4,126.2,79.2$, 78.3, 61.1, 34.3, 31.6, 31.4, 21.9.

$2.1725,26,27-T r i[(e t h o x y c a r b o n y) m e t h o x y]-28-h y d r o x y c a l i x[4] \operatorname{arene}^{128}$.

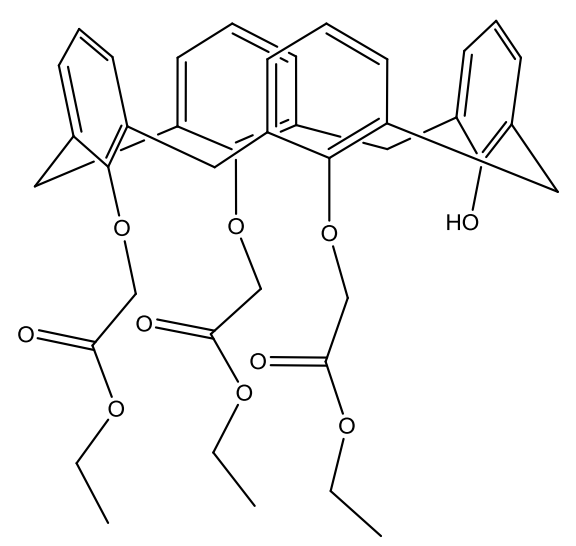

To compound (15) (0.5 g, $0.6 \mathrm{mmol})$ stirring in EtOH $(30 \mathrm{~mL})$ was added an excess of $10 \% \mathrm{Pd}$ on carbon $(0.06 \mathrm{~g}, 0.65 \mathrm{mmol})$ and an excess of anhydrous ammonium formate $(0.04 \mathrm{~g}, 0.65 \mathrm{mmol})$ and the mixture was refluxed for $3 \mathrm{hr}$. The solution was allowed to cool to room temperature and DCM $(30 \mathrm{~mL})$ was added which was filtered through a celite pad. The celite was washed with DCM $(2 \times 25 \mathrm{~mL})$ and the washings were combined with the filtrate. The solvent was removed under reduced pressure and the residue was re-dissolved in the minimum amount of DCM and EtOH. The resulting solid was filtered under suction and dried. Analysis of this compound showed that the desired product was not formed. 


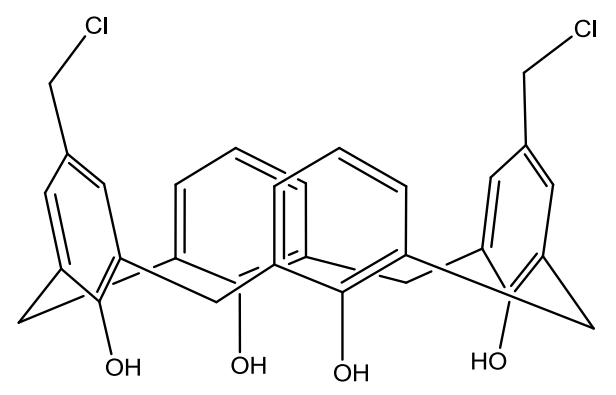

Compound (12) $(0.5 \mathrm{~g}, 1.17 \mathrm{mmol})$ and chloromethyl-n-octyl ether $(0.69 \mathrm{~mL})$ in DCM $(20 \mathrm{~mL})$ under nitrogen at $-40{ }^{\circ} \mathrm{C}$ was added $\mathrm{SnCl}_{4}(1.0 \mathrm{~mL})$. The mixture was allowed to warm up to room temperature and was stirred until all the starting material had dissolved. The reaction was followed by TLC analysis using hexane: DCM (1:1) as the solvent mixture. After $4 \mathrm{hr}$., no product was observed.

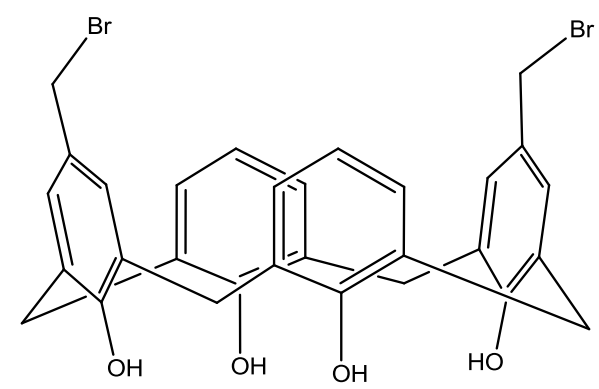

Zinc powder $(0.18 \mathrm{~g}, 2.85 \mathrm{mmol})$ was added to glacial acetic acid $(100 \mathrm{~mL})$ followed by $\mathrm{HBr}$ solution ( $33 \%$ wt in acetic acid, $12 \mathrm{~mL}$ ) and stirred for 30 min under nitrogen gas. Compound (12) $(3.0 \mathrm{~g}, 7.07 \mathrm{mmol})$ paraformaldehyde $(0.106 \mathrm{~g}, 3.5$ mmol) and $\mathrm{HBr}(60 \mathrm{~mL})$ were added and refluxed at $90{ }^{\circ} \mathrm{C}$ for $72 \mathrm{hr}$. The solvent was removed under reduced pressure and the residue was washed with water (30 $\mathrm{mL})$ and ether $(30 \mathrm{~mL})$ and the solvent removed under reduced pressure again to afford a brown solid (18). 
Yield $=2.98$ g, $99 \%$, m.p. $=255-230{ }^{\circ} \mathrm{C} ; \mathrm{v}_{\max } / \mathrm{cm}^{-1}(\mathrm{KBr}): 3175,1608,1594,1446$, 1192, 753; $\delta_{\mathrm{H}} / \mathrm{ppm}$ : Conc. of $10^{-3} \mathrm{~mm} ;\left(\mathrm{CDCl}_{3}\right) ; 10.19$ (s, 4H, Ar-O프), $7.24(\mathrm{~s}, 1 \mathrm{H}$, $\operatorname{Ar}-\underline{\mathrm{H}}), 7.05(\mathrm{~d}, 1 \mathrm{H}, \operatorname{Ar}-\underline{\mathrm{H}}, J=12.1 \mathrm{~Hz}), 6.72(\mathrm{t}, 1 \mathrm{H}, \operatorname{Ar}-\underline{\mathrm{H}}, J=8.6 \mathrm{~Hz}), 4.73(\mathrm{~s}, 4 \mathrm{H}$, $\left.\underline{\mathrm{C}}_{2}-\mathrm{Br}\right), 4.24\left(\mathrm{~d}, 4 \mathrm{H}, \underline{\mathrm{CH}}_{2}, J=14.0 \mathrm{~Hz}\right), 3.51\left(\mathrm{~d}, 4 \mathrm{H}, \underline{\mathrm{CH}}_{2}, J=14.0 \mathrm{~Hz}\right) ; \delta_{\mathrm{c}} / \mathrm{ppm}$ $\left(\mathrm{CDCl}_{3}\right) ; 148.7,131.6$ 128.9, 128.2, 122.2, 76.5, 34.1, 31.7. ESI-HRMS: calcd for $\mathrm{C}_{30} \mathrm{H}_{26} \mathrm{Br}_{2} \mathrm{O}_{4}[\mathrm{M}+1]^{+}$608.0010, found 609.0200; Anal. Calc. for $\mathrm{C}_{30} \mathrm{H}_{26} \mathrm{Br}_{2} \mathrm{O}_{4}: \mathrm{C}$, 59.04; H, $4.29 \%$, found; C, 58.78; H, $4.19 \%$.

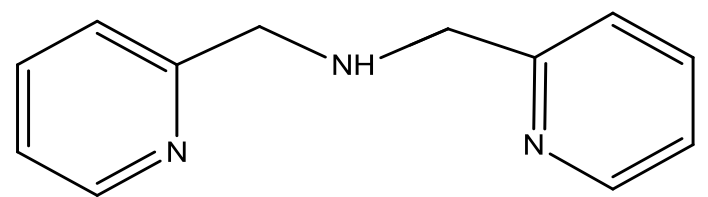

To a suspension of magnesium sulphate $(82 \mathrm{~g}, 683 \mathrm{mmol})$ in ethanol $(160 \mathrm{~mL})$ was added 2-pyridinecarboxaldehyde (15 g, $138 \mathrm{mmol})$ and 2-aminomethylpyridine (14.8 $\mathrm{g}, 138 \mathrm{mmol}$ ). The resulting mixture was then allowed to stir for $3 \mathrm{hr}$. at room temperature. During this time the mixture became warm and a colour change to yellow was observed. After stirring, the inorganic salts were removed by filtration and the filtrate was chilled to $-5{ }^{\circ} \mathrm{C}$. To this glacial acetic acid was added $(8 \mathrm{~mL}, 138$ mmol) and another colour change to a light yellow was observed. To this solution was then slowly added, a suspension of sodium borohydride $(10.7 \mathrm{~g}, 276 \mathrm{mmol})$ in ethanol $(200 \mathrm{~mL})$ over a $1 \mathrm{hr}$. period with the temperature maintained at $-5{ }^{\circ} \mathrm{C}$. Vigorous bubbling occurred during the addition followed by the precipitation of a white solid. A final colour change to bright red was seen when the addition was complete. The resulting suspension was allowed to stir at room temperature for a further $18 \mathrm{hr}$. upon which the reaction was quenched with $12 \mathrm{M} \mathrm{HCl}(160 \mathrm{~mL}, 1.9$ mmol) and then heated to $60{ }^{\circ} \mathrm{C}$ for $2 \mathrm{hr}$. until the evolution of gas had ceased. The white precipitate was removed by filtration and the filtrate was concentrated under reduced pressure. The residue was taken up in water $(70 \mathrm{~mL})$ and the resulting yellow solution was basified by the addition of sodium hydroxide pellets (64 g, 1600 
mmol) with adequate cooling. A red oil seperated almost immediately and was extracted with diethyl ether portions ( $3 \times 400 \mathrm{~mL}$ ) which were then collected and dried over anhydrous sodium sulphate. Removal of the inorganic salts followed by evaporation of the solvent gave a crude red oil which upon distillation, gave the pure product as a yellow oil (19).

Yield $=20.69 \mathrm{~g}, 69 \% ; \mathrm{v}_{\max } / \mathrm{cm}^{-1}(\mathrm{KBr}): 3450,2990,1595(\mathrm{NCH}), 1569,1437,1424$; $\delta_{\mathrm{H}} / \mathrm{ppm}$ : Conc. of $10^{-3} \mathrm{~mm} ;\left(\mathrm{CDCl}_{3}\right) ; 8.54(\mathrm{~d}, 2 \mathrm{H}, \mathrm{Py}-\underline{\mathrm{H}}, J=4.6 \mathrm{~Hz}), 7.63$ (t, 2H, Py$\underline{\mathrm{H}}, J=8.3 \mathrm{~Hz}), 7.35$ (d, 2H, Py- $\underline{\mathrm{H}}, J=6.4 \mathrm{~Hz}), 7.15$ (t, 2H, Py- $\underline{\mathrm{H}}, J=5.5 \mathrm{~Hz}), 3.96$ $\left(\mathrm{s}, 4 \mathrm{H}, \underline{\mathrm{CH}}_{2}\right), 2.58(\mathrm{~s}, 1 \mathrm{H}, \mathrm{N} \underline{\mathrm{H}}) ; \delta \mathrm{c}\left(\mathrm{CDCl}_{3}\right): 162.7,149.3,136.2,122.8,121.5,54.1$.

\subsection{4-Methyl-4-carbaldehyde-2,2-bipyridine ${ }^{129}$ (20)}

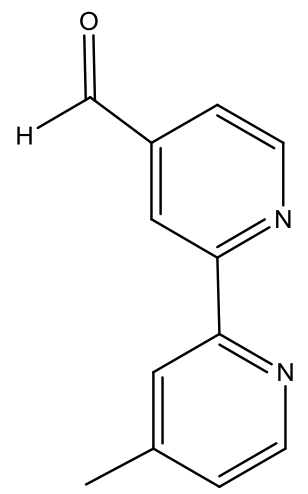

1,4-Dioxane $(100 \mathrm{~mL})$ was added to a $250 \mathrm{~mL}$ round bottom flask and 4,4-dimethyl2,2-dipyridine ( $3.0 \mathrm{~g}, 16.4 \mathrm{mmol})$ was then added. The contents were stirred for 5 min to form a suspension. To this, selenium dioxide $(2.26 \mathrm{~g}, 20.4 \mathrm{mmol})$ was added and the reactants were refluxed under nitrogen for two days. The solution was allowed to cool to room temperature and the solution was filtered to remove any unreacted selenium. The solvent was removed under reduced pressure to leave a yellow solid (20).

Yield $=3.15 \mathrm{~g}, 96.8 \% ; \mathrm{v}_{\max } / \mathrm{cm}^{-1}(\mathrm{KBr}): 2826,1705,1594,1556,1459,1208,821$;

$\delta_{\mathrm{H}} / \mathrm{ppm}$ : Conc. of $10^{-3} \mathrm{~mm} ;\left(\mathrm{CDCl}_{3}\right) ; 10.19$ (s, $\left.1 \mathrm{H}, \mathrm{C} \underline{\mathrm{HO}}\right), 8.90$ (d, $1 \mathrm{H}, \mathrm{Ar}-\underline{\mathrm{H}}, J=5.0$ $\mathrm{Hz}), 8.82$ (s, 1H, Ar- $\underline{\mathrm{H}}), 8.84$ (d, 1H, Ar- $\underline{\mathrm{H}}, J=5.0 \mathrm{~Hz}), 8.58$ (d, 1H, Ar- $\underline{\mathrm{H}}, J=4.3$ 
$\mathrm{Hz}), 8.28$ (s, 1H, Ar- $\underline{\mathrm{H}}), 7.73$ (d, 1H, Ar- $\underline{\mathrm{H}}, J=4.9 \mathrm{~Hz}), 7.21$ (d, 1H, Ar- $\underline{\mathrm{H}}, J=4.0$ $\mathrm{Hz}), 2.47$ (s, 3H, $\left.\underline{\mathrm{C}}_{3}\right) ; \delta \mathrm{c}\left(\mathrm{CDCl}_{3}\right): 191.7,158.2$, 154.6, 150.4, 149.3, 148.4, 142.6, 125.3, 122.4, 121.7, 120.6, 21.3; Anal. Calc. for $\mathrm{C}_{12} \mathrm{H}_{10} \mathrm{~N}_{2} \mathrm{O}: \mathrm{C}, 72.71 ; \mathrm{H}, 5.08 ; \mathrm{N}$, $14.13 \%$, found; C, 72.48; H, 5.05; N, $14.02 \%$.

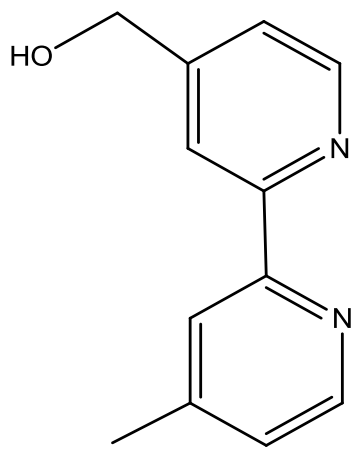

Compound (20) (1.0 g, $5.04 \mathrm{mmol})$ was suspended in $\mathrm{MeOH}(25 \mathrm{~mL})$. Sodium borohydride $(0.20 \mathrm{~g}, 6.0 \mathrm{mmol})$ in a sodium hydroxide solution $(0.25 \mathrm{M}, 12.5 \mathrm{~mL})$ was added drop-wise to the suspension which was cooled on ice. The solution was then stirred for $1 \mathrm{hr}$. at room temperature, after which the $\mathrm{MeOH}$ was then removed under reduced pressure to give a pale yellow solid (21).

Yield $=0.98 \mathrm{~g}, 99 \% ; \mathrm{v}_{\max } / \mathrm{cm}^{-1}(\mathrm{KBr}): 3431,1595,1551,1458,1125,824 ; \delta_{\mathrm{H}} / \mathrm{ppm}:$ Conc. of $10^{-3} \mathrm{~mm}$; $\left(\mathrm{CDCl}_{3}\right) ; 8.65$ (d, $\left.1 \mathrm{H}, \mathrm{Ar}-\underline{\mathrm{H}}, J=5.0 \mathrm{~Hz}\right), 8.54$ (d, 1H, Ar- $\underline{\mathrm{H}}, J=$ $4.3 \mathrm{~Hz}), 8.35$ (s, 1H, Ar- $\underline{\mathrm{H}}$ ), 8.22 (s, 1H, Ar- $\underline{\mathrm{H}}), 7.35$ (d, 1H, Ar- $\underline{\mathrm{H}}, J=4.9 \mathrm{~Hz}), 7.14$ $(\mathrm{d}, 1 \mathrm{H}, \operatorname{Ar}-\underline{\mathrm{H}}, J=4.0 \mathrm{~Hz}), 4.83\left(\mathrm{~s}, 2 \mathrm{H}, \mathrm{OC}_{\underline{2}}\right), 2.44\left(\mathrm{~s}, 2 \mathrm{H}, \underline{\mathrm{CH}}_{3}\right) ; \delta_{\mathrm{C}} / \mathrm{ppm}\left(\mathrm{CDCl}_{3}\right)$ : 156.0, 151.0, 149.3, 148.9, 148.2, 148.1, 124.8, 122.1, 121.0, 118.5, 63.7, 21.1; Anal. Calc. for $\mathrm{C}_{12} \mathrm{H}_{12} \mathrm{~N}_{2} \mathrm{O}: \mathrm{C}, 71.98 ; \mathrm{H}, 6.04 ; \mathrm{N}, 13.19 \%$, found; C, 71.52; H, 5.90; $\mathrm{N}, 13.01 \%$. 


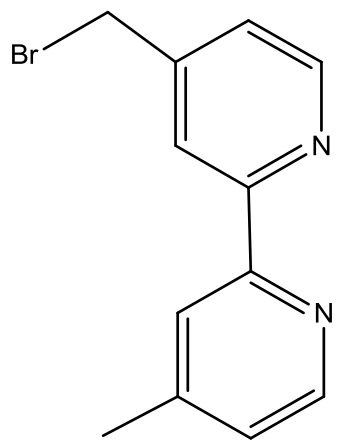

Compound (21) $(0.5 \mathrm{~g}, 2.5 \mathrm{mmol})$ was dissolved in $\mathrm{HBr}(20 \mathrm{~mL})$ and to this $\mathrm{H}_{2} \mathrm{SO}_{4}$ $(2.0 \mathrm{~mL})$ was added. The reaction was refluxed for $6 \mathrm{hr}$. and then the solution was allowed to cool to room temperature. DCM $(40 \mathrm{~mL})$ and distilled water $(40 \mathrm{~mL})$ were added and the aqueous layer was adjusted to a $\mathrm{pH}$ of 8 using a saturated sodium carbonate solution. This layer was then extracted with $5 \mathrm{~mL}$ portions of DCM until the organic layer had changed from pink to colourless. The organics were combined and dried over magnesium sulphate and the solvent removed under reduced pressure to afford a pink oil (22).

Yield $=0.60 \mathrm{~g}, 91 \%, \mathrm{v}_{\max } / \mathrm{cm}^{-1}(\mathrm{KBr}): 1660,1631,1563,1411,1102,608 ; \delta_{\mathrm{H}} / \mathrm{ppm}:$ Conc. of $10^{-3} \mathrm{~mm}$; $\left(\mathrm{CDCl}_{3}\right) ; 8.64$ (d, $\left.1 \mathrm{H}, \mathrm{Ar}-\underline{\mathrm{H}}, J=4.6 \mathrm{~Hz}\right), 8.59$ (d, $1 \mathrm{H}, \mathrm{Ar}-\underline{\mathrm{H}}, J=$ $5.1 \mathrm{~Hz}), 8.41$ (s, $1 \mathrm{H}, \mathrm{Ar}-\underline{\mathrm{H}}), 8.23$ (s, 1H, Ar- $\underline{\mathrm{H}}), 7.33(\mathrm{~d}, 1 \mathrm{H}, \mathrm{Ar}-\underline{\mathrm{H}}, J=5.8 \mathrm{~Hz}), 7.14$ (d, $1 \mathrm{H}, \mathrm{Ar}-\underline{\mathrm{H}}, J=4.5 \mathrm{~Hz}), 4.49$ (s, $\left.2 \mathrm{H}, \mathrm{C}_{2}-\mathrm{Br}\right), 2.43$ (s, 3H, $\left.\underline{\mathrm{C}}_{3}\right) ; \delta_{\mathrm{C}} / \mathrm{ppm}\left(\mathrm{CDCl}_{3}\right)$ : 156.0, 149.6, 149.0, 148.9, 148.3, 148.2, 147.2, 125.0, 123.5, 122.0, 121.0, 30.7, 21.1. ESI-HRMS: calcd for $\mathrm{C}_{12} \mathrm{H}_{11} \mathrm{BrN}_{2}[\mathrm{M}+1]^{+} 262.0100$, found 262.0101; Anal. Calc. for $\mathrm{C}_{12} \mathrm{H}_{11} \mathrm{BrN}_{2}$ : C, 54.77; H, 4.21; N, $10.65 \%$, found; C, 54.31; H, 3.95; N, $10.22 \%$. 


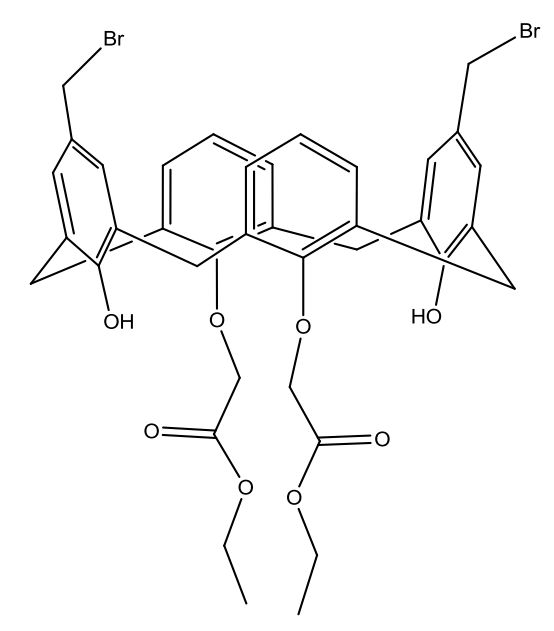

Compound (18) (1.0 g, $1.38 \mathrm{mmol})$, potassium carbonate (0.38 g, $2.77 \mathrm{mmol})$ and ethyl bromoacetate $(0.55 \mathrm{~g}, 2.77 \mathrm{mmol})$ were refluxed in $\mathrm{CH}_{3} \mathrm{CN}(80 \mathrm{~mL})$ under nitrogen for $18 \mathrm{hr}$. The solution was allowed to cool to room temperature, inorganic salts were removed by filtration and the solvent was removed under reduced pressure to give a red-brown waxy solid (23).

Yield $=1.0 \mathrm{~g}, 91 \%, \mathrm{v}_{\max } / \mathrm{cm}^{-1}(\mathrm{KBr}): 3218,2984,1705,1457,1183,1092,758$; $\delta_{\mathrm{H}} / \mathrm{ppm}$ : Conc. of $10^{-3} \mathrm{~mm} ;\left(\mathrm{CDCl}_{3}\right) ; 7.12(\mathrm{~d}, 4 \mathrm{H}, \mathrm{Ar}-\underline{\mathrm{H}}, J=6.7 \mathrm{~Hz}), 7.00(\mathrm{~s}, 2 \mathrm{H}$, $\mathrm{O} \underline{\mathrm{H}}), 6.91(\mathrm{~d}, 4 \mathrm{H}, \mathrm{Ar}-\underline{\mathrm{H}}, J=6.9 \mathrm{~Hz}), 6.76(\mathrm{~s}, 4 \mathrm{H}, \mathrm{Ar}-\underline{\mathrm{H}}), 6.59$ (t, 2H, Ar- $\underline{\mathrm{H}}, J=6.6$ $\mathrm{Hz}), 4.77$ (s, 4H, $\left.\underline{\mathrm{C}}_{2} \mathrm{Br}\right), 4.52$ (d, 4H, $\underline{\mathrm{H}}_{2}, J=13.2 \mathrm{~Hz}$ ), 4.34 (br m, 4H, OC$\underline{H}_{2}$ ), 4.23 (br m, 4H, OC$\left.\underline{H}_{2} \mathrm{CH}_{3}\right), 3.45$ (d, 4H, $\left.\underline{\mathrm{C}}_{2}, J=13.2 \mathrm{~Hz}\right), 1.33$ (t, $6 \mathrm{H}, \mathrm{OCH}_{2} \underline{\mathrm{C}}_{3}, J$ $=7.5 \mathrm{~Hz}) ; \delta_{\mathrm{C}} / \mathrm{ppm}\left(\mathrm{CDCl}_{3}\right) ; 169.2,154.5,153.2,134.6,132.7,128.5,128.1,124.3$, 123.8, 71.4, 61.0, 34.2, 33.1, 14.3; Anal. Calc. for $\mathrm{C}_{38} \mathrm{H}_{38} \mathrm{Br}_{2} \mathrm{O}_{8}: \mathrm{C}, 58.33$; H, $4.89 \%$, found; C, 58.03; H, $4.90 \%$. 


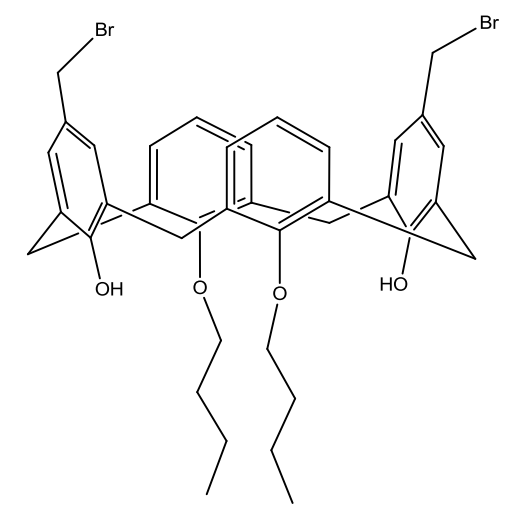

Compound (18) (2.0 g, $3.28 \mathrm{mmol})$, potassium carbonate $(0.9 \mathrm{~g}, 6.56 \mathrm{mmol})$ and 1bromobutane $(0.70 \mathrm{ml}, 6.56 \mathrm{mmol})$ in $\mathrm{CH}_{3} \mathrm{CN}(80 \mathrm{~mL})$ were refluxed under nitrogen gas for $24 \mathrm{hr}$. The solution was allowed to cool to room temperature, inorganic salts removed by filtration and the solvent was removed under reduced pressure to yield a brown solid (24).

Yield $=2.15 \mathrm{~g}, 91 \%: \mathrm{v}_{\max } / \mathrm{cm}^{-1}(\mathrm{KBr}): 3212,2916,1461,1185,1091,763 ; \delta_{\mathrm{H}} / \mathrm{ppm}:$ Conc. of 10 $0^{-3} \mathrm{~mm}$; $\left(\mathrm{CDCl}_{3}\right)$ : 7.82 (s, 2H, OH), 7.08 (s, $\left.4 \mathrm{H}, \mathrm{Ar}-\underline{\mathrm{H}}\right), 7.03$ (d, 4H, Ar- $\underline{\mathrm{H}}$, $J=4.9 \mathrm{~Hz}$ ), 6.65 (br t, $2 \mathrm{H}, \mathrm{Ar}-\underline{\mathrm{H}}), 4.35$ (d, 4H, $\left.\underline{\mathrm{H}}_{2}, J=12.9 \mathrm{~Hz}\right), 4.25$ (br t, $4 \mathrm{H}$, $\mathrm{OC}_{2}$ ), 4.12 (br q, $4 \mathrm{H}, \mathrm{OCH}_{2} \underline{\mathrm{CH}}_{2}$ ), 3.48 (d, $4 \mathrm{H} \mathrm{C}_{2}, J=12.9 \mathrm{~Hz}$ ), 1.69 (br q, $4 \mathrm{H}$, $\left.\mathrm{OCH}_{2} \mathrm{CH}_{2} \underline{\mathrm{C}}_{2}\right), 1.09$ (t, $6 \mathrm{H}, \mathrm{OCH}_{2} \mathrm{CH}_{2} \mathrm{CH}_{2} \underline{\mathrm{C}}_{3}, J=9.8 \mathrm{~Hz}$ ); $\delta_{\mathrm{C}} / \mathrm{ppm}\left(\mathrm{CDCl}_{3}\right)$ : 155.4, 153.2, 133.1, 131.5, 128.4, 127.2, 125.0, 123.1, 68.7, 34.2, 31.8, 31.3, 18.9, 13.7; Anal. Calc. for $\mathrm{C}_{38} \mathrm{H}_{42} \mathrm{Br}_{2} \mathrm{O}_{4}: \mathrm{C}, 63.17 ; \mathrm{H}, 5.86 \%$, found; C, 63.16; H, $5.80 \%$. 


\subsection{1,23-Dibromomethyl-25,27-dibutoxy-26,28-}

diethoxycarbonylmethoxy-calix[4]arene. (25)

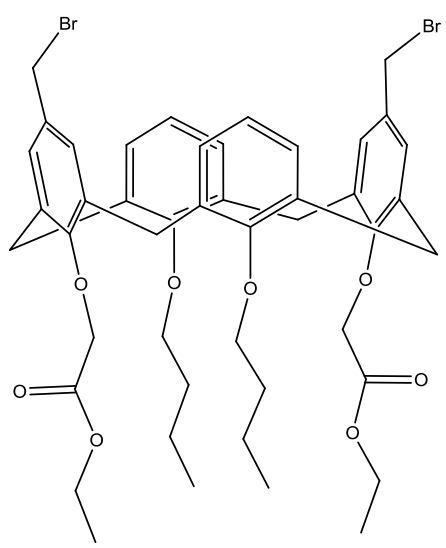

Compound (24) $(2.0 \mathrm{~g}, 2.84 \mathrm{mmol})$ potassium carbonate $(0.78 \mathrm{~g}, 5.66 \mathrm{mmol})$ and ethyl bromoacetate $(0.62 \mathrm{~mL}, 5.66 \mathrm{mmol})$ in $\mathrm{CH}_{3} \mathrm{CN}(80 \mathrm{~mL})$ were refluxed overnight under nitrogen gas. The solution was allowed to cool to room temperature, the inorganic salts removed by filtration and the solvent was removed under reduced pressure to afford a light brown solid (25)

Yield $=2.3 \mathrm{~g}, 90 \% ; \mathrm{v}_{\max } / \mathrm{cm}^{-1}(\mathrm{KBr}): 1746,1464,1299,1048,966,754 ; \delta_{\mathrm{H}} / \mathrm{ppm}:$ Conc. of $10^{-3} \mathrm{~mm}$; $\left(\mathrm{CDCl}_{3}\right): 7.10$ (d, 4H, Ar- $\left.\underline{\mathrm{H}}, J=7.9 \mathrm{~Hz}\right), 6.97$ (s, $\left.4 \mathrm{H}, \mathrm{Ar}-\underline{\mathrm{H}}\right), 6.55$ (t, $2 \mathrm{H}, \mathrm{Ar}-\underline{\mathrm{H}}, J=6.4 \mathrm{~Hz}$ ), 4.77 (br s, $\left.4 \mathrm{H}, \mathrm{C}_{2}-\mathrm{Br}\right), 4.53$ (s, $\left.4 \mathrm{H}, \mathrm{OC}_{\underline{2}}\right), 4.37$ (d, $4 \mathrm{H}$, $\left.\mathrm{C}_{2}, J=10.0 \mathrm{~Hz}\right), 4.28\left(\mathrm{t}, 4 \mathrm{H}, \mathrm{OCH}_{2} \mathrm{C}_{2}, J=7.7 \mathrm{~Hz}\right), 4.19$ (t, $4 \mathrm{H}, \mathrm{COC}_{2} \underline{\mathrm{H}}_{2} \mathrm{CH}_{3}, J=$ $5.4 \mathrm{~Hz}$ ), 4.09 (br q, 4H, $\mathrm{OCH}_{2} \mathrm{C}_{2}$ ), 3.44 (d, 4H, $\left.\underline{\mathrm{C}}_{2}, J=12.0 \mathrm{~Hz}\right), 2.11$ (m, 4H, $\mathrm{OCH}_{2} \mathrm{CH}_{2} \mathrm{CH}_{2}, J=6.9 \mathrm{~Hz}$ ), 1.83 (br t, $6 \mathrm{H}, \mathrm{OCH}_{2} \mathrm{C}_{3}$ ), 1.15 (br m, $6 \mathrm{H}$, $\left.\mathrm{OCH}_{2} \mathrm{CH}_{2} \mathrm{CH}_{2} \mathrm{CH}_{3}\right) ; \delta_{\mathrm{C}} / \mathrm{ppm}\left(\mathrm{CDCl}_{3}\right): 170.2,154.2,152.3,133.4,131.0,128.7$, 124.9, 123.1, 72.6, 68.7, 60.2, 33.3, 32.4, 31.3, 18.9, 14.5; Anal. Calc. for $\mathrm{C}_{46} \mathrm{H}_{54} \mathrm{Br}_{2} \mathrm{O}_{8}: \mathrm{C}, 61.75 ; \mathrm{H}, 6.08 \%$, found; $\mathrm{C}, 61.42 ; \mathrm{H}, 5.96 \%$. 


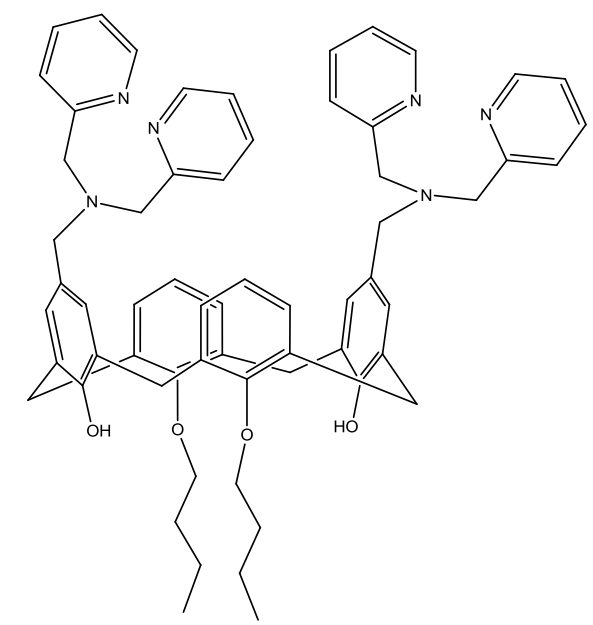

Compound (24) $(1.0 \mathrm{~g}, 1.28 \mathrm{mmol})$, triethylamine $(0.26 \mathrm{~g}, 2.57 \mathrm{mmol})$, and DPA $(0.54 \mathrm{~g}, 2.56 \mathrm{mmol})$ were refluxed in DCM $(80 \mathrm{~mL})$ under nitrogen gas overnight. The solution was allowed to cool to room temperature to give a suspension. The inorganic salts were removed by filtration and the volatiles removed under reduced pressure to afford a red waxy solid (26).

Yield $=1.2 \mathrm{~g}, 97 \% ; \mathrm{v}_{\max } / \mathrm{cm}^{-1}(\mathrm{KBr}): 3401,1591,1570,1436,1247,1047,755$; $\delta_{\mathrm{H}} / \mathrm{ppm}$ : Conc. of $10^{-3} \mathrm{~mm} ;\left(\mathrm{CDCl}_{3}\right) ; 8.57(\mathrm{~d}, 4 \mathrm{H}, \mathrm{Py}-\underline{\mathrm{H}}, J=5.0 \mathrm{~Hz}), 7.70(\mathrm{t}, 2 \mathrm{H}, \mathrm{Py}-$ $\underline{\mathrm{H}}, J=7.9 \mathrm{~Hz}$ ), 7.63 (t, 4H, Py- $\underline{\mathrm{H}}, J=7.0 \mathrm{~Hz}), 7.46$ (d. $4 \mathrm{H}, \mathrm{Py}-\underline{\mathrm{H}}, J=7.9 \mathrm{~Hz}), 7.16$ (s, 4H, Ar- $\underline{-}$ ), 7.00 (d, 4H, Ar- $\underline{H}, J=7.9 \mathrm{~Hz}), 6.62(\mathrm{t}, 2 \mathrm{H}, \mathrm{Ar}-\underline{\mathrm{H}}, J=8.3 \mathrm{~Hz}), 4.67$ (s, $4 \mathrm{H}, \mathrm{N}-\underline{\mathrm{C}}_{2}$ ), 4.25 (br d, $4 \mathrm{H}, \mathrm{C}_{2}$ ), 4.15 (t, $4 \mathrm{H}, \mathrm{O}-\mathrm{C}_{2}, J=6.6 \mathrm{~Hz}$ ), 3.96 (s, $8 \mathrm{H}$, $\mathrm{N}-\underline{\mathrm{C}}_{2}$ ), 3.43 (br d, $4 \mathrm{H}, \mathrm{C}_{2}$ ), 1.71 (m, 4H, OCH $\left.{ }_{2} \underline{\mathrm{CH}}_{2} J=7.5 \mathrm{~Hz}\right), 1.08$ (m, $4 \mathrm{H}$, $\left.\mathrm{OCH}_{2} \mathrm{CH}_{2} \mathrm{C}_{2} \underline{\underline{H}} J=7.5 \mathrm{~Hz}\right), 0.90$ (t, $\left.6 \mathrm{H}, \mathrm{CH}_{2} \mathrm{C}_{3}, J=7.9 \mathrm{~Hz}\right) ; \delta_{\mathrm{C}} / \mathrm{ppm}\left(\mathrm{CDCl}_{3}\right)$ : 156.2, 155.6, 153.4, 149.2, 138.7, 134.8, 133.2, 129.7, 128.3, 125.4, 124.9, 123.2, 120.4, 69.5, 63.1, 32.1, 31.3, 18.7, 14.3; Anal. Calc. for $\mathrm{C}_{62} \mathrm{H}_{66} \mathrm{~N}_{6} \mathrm{O}_{4}$ : C, 77.63; H, 6.94; N, $8.76 \%$, found; C, 77.60; H, 6.87; N, $8.61 \%$. 


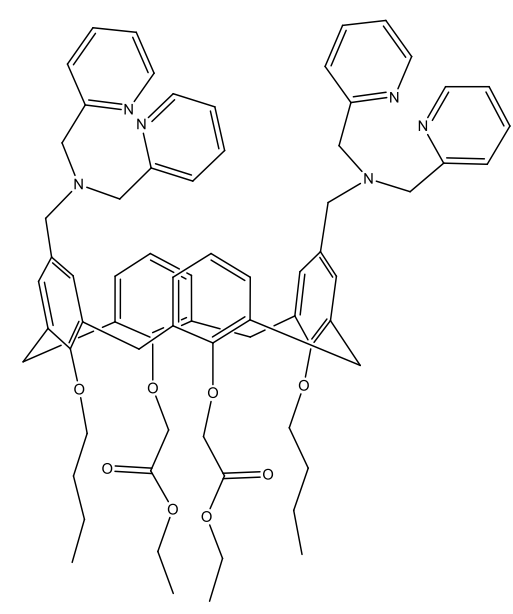

Compound (25) (2.0 g, $2.24 \mathrm{mmol})$, potassium carbonate $(0.62 \mathrm{~g}, 4.49 \mathrm{mmol})$ and dipyridyl-amine (DPA) $(0.89 \mathrm{~g}, 4.49 \mathrm{mmol})$ in $\mathrm{CH}_{3} \mathrm{CN}(200 \mathrm{~mL})$ were refluxed overnight under nitrogen gas. The solution was allowed to cool to room temperature, the inorganic salts were removed by filtration and the solvent removed under reduced pressure to give a dark red waxy solid (27).

Yield $=1.25 \mathrm{~g}, 0.49 \% ; \mathrm{v}_{\max } / \mathrm{cm}^{-1}(\mathrm{KBr}): 3426,2927,1753,1591,1436,1190,753$; $\delta_{\mathrm{H}} / \mathrm{ppm}$ : Conc. of $10^{-3} \mathrm{~mm}$; $\left(\mathrm{CDCl}_{3}\right): 8.56(\mathrm{br} \mathrm{d}, 4 \mathrm{H}, \mathrm{Py}-\underline{\mathrm{H}}, J=5.3 \mathrm{~Hz}), 7.62(\mathrm{t}, 4 \mathrm{H}$, Py- $\underline{\mathrm{H}}, J=9.3 \mathrm{~Hz}$ ), 7.35 (d, 4H, Py- $\underline{\mathrm{H}}, J=9.3 \mathrm{~Hz}$ ), 7.15 (br t, 4H, Py- $\underline{\mathrm{H}}, J=11.9 \mathrm{~Hz}$ ), 7.05 (d, 4H, Ar- $\underline{\mathrm{H}}, J=7.9 \mathrm{~Hz}), 6.90$ (d, 2H, Ar- $\underline{\mathrm{H}}, J=6.6 \mathrm{~Hz}), 6.73(\mathrm{t}, 2 \mathrm{H}, \mathrm{Ar}-\underline{\mathrm{H}}, J=$ $4.5 \mathrm{~Hz}), 4.74$ (s, 4H, OC$\left.\underline{H}_{2} \mathrm{CO}\right), 4.21\left(\mathrm{~d}, 4 \mathrm{H}, \mathrm{C}_{2}, J=13.0 \mathrm{~Hz}\right), 4.15(\mathrm{q}, 4 \mathrm{H}$, $\mathrm{OC}_{2} \mathrm{CH}_{3}, J=7.0 \mathrm{~Hz}$ ), 4.02 (t, $4 \mathrm{H}, \mathrm{OCH}_{2} \mathrm{CH}_{2} \mathrm{CH}_{2} \mathrm{CH}_{3} J=7.6 \mathrm{~Hz}$ ), 3.97 (s, $12 \mathrm{H}, \mathrm{N}-$ $\left.\mathrm{C}_{2}\right), 3.90\left(\mathrm{~d}, 4 \mathrm{H}, \underline{\mathrm{CH}}_{2}, J=7.0 \mathrm{~Hz}\right), 1.78\left(\mathrm{~m}, 4 \mathrm{H}, \mathrm{OCH}_{2} \underline{\mathrm{C}}_{2} \mathrm{CH}_{2} \mathrm{CH}_{3}, J=7.6 \mathrm{~Hz}\right)$, $1.32\left(\mathrm{~m}, 4 \mathrm{H}, \mathrm{OCH}_{2} \mathrm{CH}_{2} \mathrm{C}_{2} \mathrm{CH}_{3}, J=6.5 \mathrm{~Hz}\right), 1.29$ (t, $6 \mathrm{H}, \mathrm{OCH}_{2} \mathrm{C}_{3}, J=7.6 \mathrm{~Hz}$ ), 1.15 (t, $\left.6 \mathrm{H}, \mathrm{OCH}_{2} \mathrm{CH}_{2} \mathrm{CH}_{2} \mathrm{CH}_{3}, J=8.2 \mathrm{~Hz}\right) ; \delta_{\mathrm{C}} / \mathrm{ppm}\left(\mathrm{CDCl}_{3}\right): 172.5,161.8,157.2$, 156.3, 148.8, 137.6, 134.2, 133.5, 128.8, 128.4, 126.4, 123.1, 122.9, 122.9, 70.9, 64.1, 60.4, 60.14, 59.8, 32.4, 30.7, 21.3, 14.1, 13.9; Anal. Calc. for $\mathrm{C}_{70} \mathrm{H}_{78} \mathrm{~N}_{6} \mathrm{O}_{8}: \mathrm{C}$, $74.31 ; \mathrm{H}, 6.95 ; \mathrm{N}, 7.43 \%$, found; C, 74.30; H, 6.91; N, $7.39 \%$; 


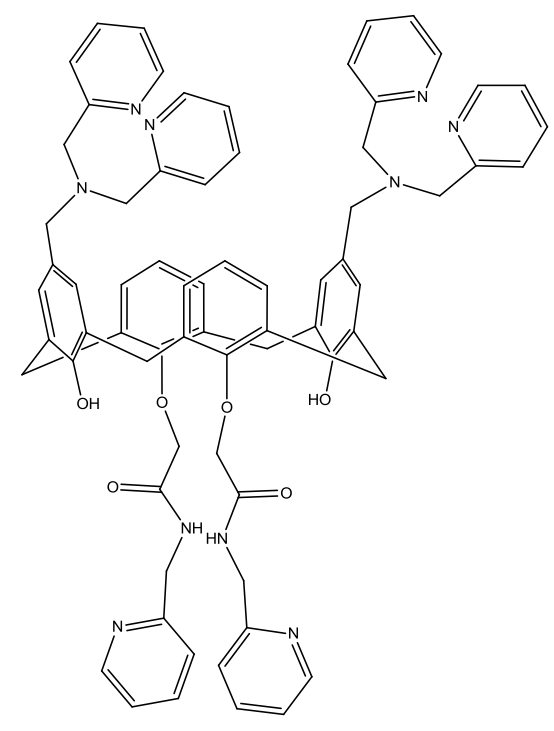

Compound (27) (0.70 g, $0.78 \mathrm{mmol})$ and 2-(aminomethyl)pyridine (0.16 mL, 1.57 $\mathrm{mmol})$ in $\mathrm{MeOH}(70 \mathrm{~mL})$ and dry toluene $(100 \mathrm{~mL})$ were refluxed for 10 days under nitrogen gas. The solution was allowed to cool, the inorganic salts were removed by filtration and the solvent was removed under reduced pressure to give a dark red waxy solid (28).

Yield $=0.41 \mathrm{~g}, 44 \% ; \mathrm{v}_{\max } / \mathrm{cm}^{-1}(\mathrm{KBr}): 3290,2923,1605,1571,1438,1261,1018$, 761: $\delta_{\mathrm{H}} / \mathrm{ppm}$ : Conc. of $10^{-3} \mathrm{~mm} ;\left(\mathrm{CDCl}_{3}\right): 8.55$ (br d, $4 \mathrm{H}, \mathrm{Py}-\underline{\mathrm{H}}, J=7.5 \mathrm{~Hz}$ ), 7.63 (t, 4H, Py- $\underline{\mathrm{H}}, J=7.5 \mathrm{~Hz}$ ), 7.25 (d, 4H, Py- $\underline{\mathrm{H}}, J=10.7 \mathrm{~Hz}$ ), 7.15 (br t, H, Py- $\underline{\mathrm{H}}, J=6.3$ $\mathrm{Hz}), 7.07$ (d, 4H, Ar- $\underline{\mathrm{H}}, J=8.2 \mathrm{~Hz}), 6.90(\mathrm{~d}, 2 \mathrm{H}, \mathrm{Ar}-\underline{\mathrm{H}}, J=6.9 \mathrm{~Hz}), 6.65$ (t, $2 \mathrm{H}, \mathrm{Ar}-$ $\underline{\mathrm{H}}, J=7.6 \mathrm{~Hz}), 4.74\left(\mathrm{~s}, 4 \mathrm{H}, \mathrm{OC} \underline{H}_{2} \mathrm{CO}\right), 4.21\left(\mathrm{~m}, 4 \mathrm{H}, \mathrm{OC}_{2} \mathrm{CH}_{3}, J=16.2 \mathrm{~Hz}\right), 4.15$ $\left(\mathrm{d}, 4 \mathrm{H}, \quad \underline{\mathrm{C}}_{2}, J=13.7 \mathrm{~Hz}\right), 3.98(\mathrm{~s}, 16 \mathrm{H}, \mathrm{N}-\mathrm{CH} 2-\mathrm{Py}), 3.44$ (t, $4 \mathrm{H}$, $\left.\mathrm{OC}_{2} 2 \mathrm{CH}_{2} \mathrm{CH}_{2} \mathrm{CH}_{3}, J=10.4 \mathrm{~Hz}\right), 3.25\left(\mathrm{~d}, 4 \mathrm{H}, \mathrm{C}_{2}, J=13.3 \mathrm{~Hz}\right), 1.83(\mathrm{~m}, 4 \mathrm{H}$, $\left.\mathrm{OCH}_{2} \mathrm{CH}_{2} \mathrm{CH}_{2} \mathrm{CH}_{3}, J=11.2 \mathrm{~Hz}\right), 1.35\left(\mathrm{~m}, 4 \mathrm{H}, \mathrm{OCH}_{2} \mathrm{CH}_{2} \mathrm{C}_{2} \mathrm{CH}_{3}, J=14.4 \mathrm{~Hz}\right)$, 1.09 (t, $\left.6 \mathrm{H}, \mathrm{OCH}_{2} \mathrm{CH}_{3}, J=6.4 \mathrm{~Hz}\right) ; \delta_{\mathrm{C}} / \mathrm{ppm}\left(\mathrm{CDCl}_{3}\right): 169.5,158.4,155.4,154.7$, 153.4, 148.8, 148.6, 137.6, 136.8, 134.3, 129.1, 128.1, 127.6, 127.4, 126.4, 123.7, 123.6, 121.8, 120.8, 73.5, 70.1, 69.7, 44.0, 32.4; Anal. Calc. for $\mathrm{C}_{70} \mathrm{H}_{66} \mathrm{~N}_{10} \mathrm{O}_{6}$ : C, 73.53; H, 5.82; N, $12.25 \%$, found; C, 73.39; H, 5.80; N, $12.15 \%$. 


\section{Metal complexes of (28)}

All metal ion complexation reactions were performed at room temperature in a solvent mixture of DCM-MeOH at a 1:1 ratio. All reactions used $0.159 \mathrm{mmol}$ of (28) and corresponding metal salt. The solution was stirred for $15 \mathrm{~min}$ and then the solvent was slowly evaporated off. A water-DCM wash was performed to remove any uncomplexed metal and the solvent was removed under reduced pressure.

Compound (28i): Zinc acetate; Yield $=0.25 \mathrm{~g}, 48 \% ; \mathrm{v}_{\max } / \mathrm{cm}^{-1}(\mathrm{KBr}): 3321,2945$, 1698, 1621, 1590, 1466, 1091, 764; $\delta_{\mathrm{H}} / \mathrm{ppm}$ : Conc. of $10^{-3} \mathrm{~mm}$; $\left(\mathrm{CDCl}_{3}\right): 9.43(\mathrm{~d}, J=$ $4.7 \mathrm{~Hz}), 8.75(\mathrm{~d}, J=3.7 \mathrm{~Hz}), 8.51(\mathrm{~d}, J=5.3 \mathrm{~Hz}), 7.89(\mathrm{t}, J=5.3 \mathrm{~Hz}), 7.72(\mathrm{t}, J=$ $8.3 \mathrm{~Hz}), 7.43(\mathrm{~d}, J=7.5 \mathrm{~Hz}), 7.38$ (d, $J=7.5 \mathrm{~Hz}), 7.18$ (s), 7.09 (s), 7.06 (d, $J=3.7$ Hz), $7.03(\mathrm{~d}, J=3.7 \mathrm{~Hz}), 6.96(\mathrm{~d}, J=7.4 \mathrm{~Hz}), 6.89(\mathrm{~d}, J=7.0 \mathrm{~Hz}), 6.70(\mathrm{br} \mathrm{t}), 4.88$ (d, $J=5.4 \mathrm{~Hz}), 4.68(\mathrm{~s}), 4.52(\mathrm{~s}), 4.32(\mathrm{~s}), 4.26(\mathrm{~d}, J=13.5 \mathrm{~Hz}), 4.13(\mathrm{~d}, J=13.5$ $\mathrm{Hz}$ ), 3.54 (s), 3.48 (d, J=5.0 Hz), 3.43 (s), 2.53 (s); Anal. Calc. for $\mathrm{C}_{74} \mathrm{H}_{72} \mathrm{~N}_{10} \mathrm{O}_{10} \mathrm{Zn}$ : C, 66.99; H, 5.47; N, $10.56 \%$, found; C, 70.01; H, 5.49; N, $10.50 \%$.

Compound (28ii): Zinc chloride; Yield $=0.10 \mathrm{~g}, 50 \% ; \mathrm{v}_{\max } / \mathrm{cm}^{-1}(\mathrm{KBr}): 3324$, 2954, 1688, 1621, 1591, 1444, 1089, 763; $\delta_{\mathrm{H}} / \mathrm{ppm}$ : Conc. of $10^{-3} \mathrm{~mm} ;\left(\mathrm{CDCl}_{3}\right) ; 9.47$ (br d), 9.09 (br d), 9.01 (d, $J=6.1 \mathrm{~Hz}), 8.91$ (br d), 8.69 (d, $J=5.3 \mathrm{~Hz}), 8.58$ (br t), 8.51 (br d), 8.28 (d, $J=5.3 \mathrm{~Hz}), 8.18$ (t, $J=8.4 \mathrm{~Hz}), 7.82$ (br t), $7.75(\mathrm{t}, J=7.7 \mathrm{~Hz})$, 7.69 (t, $J=8.0 \mathrm{~Hz}$ ), 7.60 (br t), 7.43 (d, $J=7.3 \mathrm{~Hz}$ ), 7.31 (br d), 7.22 (br d), 7.17 (s), $7.08(\mathrm{~s}), 7.05(\mathrm{~d}, J=5.1 \mathrm{~Hz}), 7.01(\mathrm{~s}), 6.98(\mathrm{~d}, J=5.1 \mathrm{~Hz}), 6.88(\mathrm{~d}, J=7.72 \mathrm{~Hz})$, $6.80(\mathrm{~d}, J=8.0 \mathrm{~Hz}), 6.75(\mathrm{t}, J=7.3 \mathrm{~Hz}), 6.69(\mathrm{t}, J=7.3 \mathrm{~Hz}), 6.53(\mathrm{t}, J=8.0 \mathrm{~Hz})$, $4.88(\mathrm{~d}, J=5.8 \mathrm{~Hz}), 4.80(\mathrm{~s}), 4.69(\mathrm{~d}, J=5.1 \mathrm{~Hz}), 4.65(\mathrm{~s}), 4.52(\mathrm{~s}), 4.27$ (d, $J=13.6$ $\mathrm{Hz}), 4.20$ (s), 4.13 (d, $J=13.6 \mathrm{~Hz}), 3.99$ (d, $J=13.2 \mathrm{~Hz}), 3.44$ (d, $J=13.6 \mathrm{~Hz}), 3.39$ $(\mathrm{d}, J=13.2 \mathrm{~Hz})$; Anal. Calc. for $\mathrm{C}_{70} \mathrm{H}_{66} \mathrm{Cl}_{2} \mathrm{~N}_{10} \mathrm{O}_{6} \mathrm{Zn}$ : C, 65.70; H, 5.20; N, 10.95\%, found; C, 65.55; H, 5.10; N, $10.64 \%$.

Compound (28iii): Zinc perchlorate; Yield $=0.11 \mathrm{~g}, 49 \% ; \mathrm{v}_{\max } / \mathrm{cm}^{-1}(\mathrm{KBr}): 3304$, 2951, 1672, 1610, 1590, 1462, 1099 (perchlorate peak); $\delta_{\mathrm{H}} / \mathrm{ppm}$ : Conc. of $10^{-3} \mathrm{~mm}$; (d6-DMSO); 9.26 (br d), 8.95 (br d), 8.90 (br d), 8.79 (br d), 8.56 (d, $J=4.9 \mathrm{~Hz}$ ), $8.44(\mathrm{~d}, J=4.9 \mathrm{~Hz}), 8.33$ (s), 8.16 (br t), 8.11 (br t), 8.05 (br t), 7.89 (br t), 7.81 (t, $J$ $=7.4 \mathrm{~Hz}), 7.71(\mathrm{t}, J=7.4 \mathrm{~Hz}), 7.50(\mathrm{~d}, J=7.4 \mathrm{~Hz}), 7.37(\mathrm{~d}, J=8.2 \mathrm{~Hz}), 7.30(\mathrm{t}, J=$ $6.6 \mathrm{~Hz}), 7.17(\mathrm{~s}), 7.12(\mathrm{~d}, J=7.4 \mathrm{~Hz}), 7.07$ (s), $7.02(\mathrm{~d}, J=8.2 \mathrm{~Hz}), 6.91(\mathrm{~d}, J=8.2$ 
$\mathrm{Hz}), 6.81(\mathrm{t}, J=7.4 \mathrm{~Hz}), 6.57(\mathrm{t}, J=7.4 \mathrm{~Hz}), 6.40(\mathrm{t}, J=7.4 \mathrm{~Hz}), 4.69(\mathrm{~s}), 4.64(\mathrm{~d}, J$ $=6.6 \mathrm{~Hz}), 4.38(\mathrm{~d}, J=12.4 \mathrm{~Hz}), 4.19(\mathrm{~s}), 4.10(\mathrm{~d}, J=13.2 \mathrm{~Hz}), 3.99$ (br s); Anal. Calc. for $\mathrm{C}_{70} \mathrm{H}_{66} \mathrm{Cl}_{2} \mathrm{~N}_{10} \mathrm{O}_{14} \mathrm{Zn}: \mathrm{C}, 59.73 ; \mathrm{H}, 4.73 ; \mathrm{N}, 9.95 \%$, found; C, 59.61; H, $4.70 ; \mathrm{N}, 9.89 \%$.

\subsection{1,23-Dibromomethyl-25,27-dimethylbenzyl-26,28-} dihydroxycalix[4]arene. $(29)^{142}$

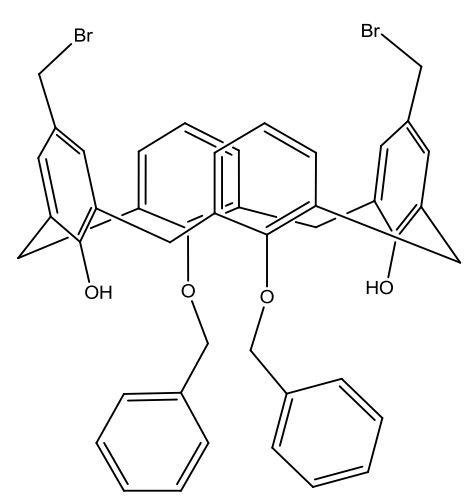

Compound (18) (1.0 g, $1.64 \mathrm{mmol})$, potassium carbonate $(0.45 \mathrm{~g}, 3.28 \mathrm{mmol})$ and benzyl bromide $(0.39 \mathrm{~mL}, 3.28 \mathrm{mmol})$ were refluxed in $\mathrm{CH}_{3} \mathrm{CN}(100 \mathrm{~mL})$ under nitrogen overnight. The solution was allowed to cool to room temperature, the inorganic salts were removed by filtration and the volatiles removed under reduced pressure to afford a red-wine waxy solid (30).

Yield $=0.65 \mathrm{~g}, 89 \% ; \mathrm{v}_{\max } / \mathrm{cm}^{-1}(\mathrm{KBr}): 3361,1608,1595,1448,1088,753 ; \delta_{\mathrm{H}} / \mathrm{ppm}:$ Conc. of $10^{-3} \mathrm{~mm} ;\left(\mathrm{CDCl}_{3}\right): 7.94(\mathrm{~s}, 2 \mathrm{H}, \mathrm{O} \underline{\mathrm{H}}), 7.72(\mathrm{t}, 4 \mathrm{H}, \mathrm{Bn}-\underline{\mathrm{H}}, J=2.8 \mathrm{~Hz}), 7.69$

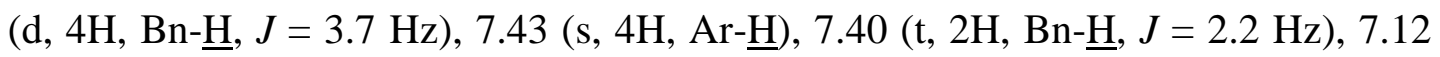
$(\mathrm{d}, 4 \mathrm{H}, \mathrm{Ar}-\underline{\mathrm{H}}, J=6.9 \mathrm{~Hz}), 6.96$ (d, 4H, Ar- $\underline{\mathrm{H}}, J=7.7 \mathrm{~Hz}), 6.77$ (t, 2H, Ar- $\underline{\mathrm{H}}, J=10.0$ $\mathrm{Hz}), 5.12$ (s, 4H, $\left.\underline{\mathrm{C}}_{2}-\mathrm{Bn}\right), 4.54$ (s, 4H, $\left.\underline{\mathrm{H}}_{2}-\mathrm{Br}\right), 4.38$ (d, 4H, $\underline{\mathrm{C}}_{2}, J=13.1 \mathrm{~Hz}$ ), $3.42\left(\mathrm{~d}, 4 \mathrm{H}, \underline{\mathrm{C}}_{2}, J=13.1 \mathrm{~Hz}\right) ; \delta \mathrm{c} / \mathrm{ppm}\left(\mathrm{CDCl}_{3}\right) ; 153.3,151.9,136.7,133.2,129.0$, 128.7, 128.4, 127.9, 127.4, 125.4, 118.3, 78.3, 76.9, 31.4. ESI-HRMS: calcd. for $\mathrm{C}_{44} \mathrm{H}_{38} \mathrm{Br}_{2} \mathrm{O}_{4}[\mathrm{M}+1]^{+}$788.1100, found 787.3558; Anal. Calc. for $\mathrm{C}_{44} \mathrm{H}_{38} \mathrm{Br}_{2} \mathrm{O}_{4}: \mathrm{C}$, $66.85 ; \mathrm{H}, 4.84 \%$, found; C, 66.65; H, $4.32 \%$. 


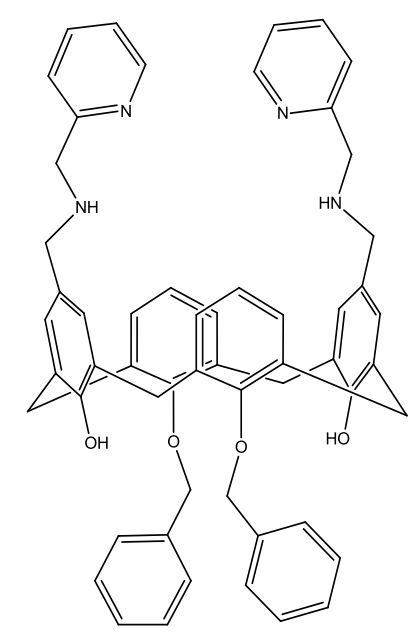

Compound (29) (0.7 g, $0.88 \mathrm{mmol})$, triethylamine $(0.18 \mathrm{~g}, 1.77 \mathrm{mmol})$, and 2aminomethyl pyridine $(0.28 \mathrm{~mL}, 1.77 \mathrm{mmol})$ were refluxed in $\mathrm{CH}_{3} \mathrm{CN}(60 \mathrm{~mL})$ overnight under nitrogen gas. The solution was allowed to cool to room temperature, the inorganic salts removed by filtration and the volatiles removed under reduced pressure to give a red-wine waxy solid (30).

Yield $=0.72 \mathrm{~g}, 96 \% ; \mathrm{v}_{\max } / \mathrm{cm}^{-1}(\mathrm{KBr}): 3414,3388,1590,1089,961,764 ; \delta_{\mathrm{H}} / \mathrm{ppm}:$ Conc. of $10^{-3} \mathrm{~mm} ;\left(\mathrm{CDCl}_{3}\right): 8.59$ (d, $\left.2 \mathrm{H}, \mathrm{Py}-\underline{\mathrm{H}}, J=3.8 \mathrm{~Hz}\right), 7.79$ (d, $2 \mathrm{H}, \mathrm{Bn}-\underline{\mathrm{H}}, J=$ $1.9 \mathrm{~Hz}$ ), 7.73 (t, 2H, Py- $\underline{\mathrm{H}}, J=2.1 \mathrm{~Hz}$ ), 7.42 (t, 2H, Py- $\underline{\mathrm{H}}, J=1.9 \mathrm{~Hz}$ ), 7.39 (s, $2 \mathrm{H}$, Ar-O프), 7.37 (d, $2 \mathrm{H}, \mathrm{Py}-\underline{\mathrm{H}}, J=2.1 \mathrm{~Hz}), 7.34$ (t, 4H, Bn- $\underline{\mathrm{H}}, J=2.2 \mathrm{~Hz}), 7.14$ (t, $2 \mathrm{H}$, Bn- $\underline{\mathrm{H}}, J=1.8 \mathrm{~Hz}), 7.10$ (s, 4H, Ar- $\underline{\mathrm{H}}), 6.89$ (d, 4H, Ar- $\underline{\mathrm{H}}, J=6.9 \mathrm{~Hz}), 6.57$ (t, $2 \mathrm{H}$, Ar- $\underline{\mathrm{H}}, J=8.1 \mathrm{~Hz}), 5.13$ (s, 4H, $\left.\underline{\mathrm{C}}_{2}-\mathrm{Bn}\right), 4.79$ (s, 8H, $\left.\underline{\mathrm{C}}_{2}-\mathrm{NH}\right), 4.41$ (d, 4H, $\underline{\mathrm{C}}_{2}, J$ $=13.2 \mathrm{~Hz}), 3.91$ (s, $\left.4 \mathrm{H}, \mathrm{C}_{\underline{2}}-\mathrm{py}\right), 3.23\left(\mathrm{~d}, 4 \mathrm{H}, \mathrm{C}_{2}, J=13.2 \mathrm{~Hz}\right), 2.43(\mathrm{br} \mathrm{s}, 2 \mathrm{H}$, $\mathrm{N} \underline{\mathrm{H}}) ; \delta_{\mathrm{C}} / \mathrm{ppm}\left(\mathrm{CDCl}_{3}\right): 154.2,152.3,149.2,135.6,134.0,133.2,129.9,128.8,128.6$, 128.5, 128.4, 128.3, 128.9, 128.0, 123.7, 121.3, 75.2, 71.7, 54.5, 31.5; Anal. Calc. for $\mathrm{C}_{56} \mathrm{H}_{52} \mathrm{~N}_{4} \mathrm{O}_{4}: \mathrm{C}, 79.59 ; \mathrm{H}, 6.20 ; \mathrm{N}, 6.63 \%$, found; $\mathrm{C}, 79.64 ; \mathrm{H}, 5.97 ; \mathrm{N}, 6.82 \%$; $\lambda_{\max }(\mathrm{DCM} / \mathrm{MeOH}) 260 \mathrm{~nm} @ 0.006 \mathrm{mM}, \varepsilon=30 \mathrm{M}^{-1} \mathrm{~cm}^{-1}$. 


\section{Metal complexes of (30)}

All metal ion complexation reactions were performed at room temperature in a solvent mixture of $\mathrm{DCM}-\mathrm{MeOH}$ at a 1:1 ratio. The solution was stirred for $15 \mathrm{~min}$ and then the solvent was slowly evaporated off. A water-DCM wash was performed to remove any uncomplexed metal and the solvent was removed under reduced pressure.

Compound (30i): Zinc acetate; Yield $=0.08 \mathrm{~g}, 66 \% ; \mathrm{v}_{\max } / \mathrm{cm}^{-1}(\mathrm{KBr}): 3426,3257$, 1607, 1462, 1183, 1092, 761; $\delta_{\mathrm{H}} / \mathrm{ppm}$ : Conc. of $10^{-3} \mathrm{~mm} ;\left(\mathrm{CDCl}_{3}\right) ; 9.16(\mathrm{~d}, J=5.5$ $\mathrm{Hz}), 9.07(\mathrm{~d}, J=5.5 \mathrm{~Hz}$ ), $8.92(\mathrm{dd}, J=5.5 \mathrm{~Hz}), 8.57(\mathrm{~d}, J=5.5 \mathrm{~Hz}), 8.46(\mathrm{br} \mathrm{d})$, $7.88(\mathrm{dd}, J=1.5,6.6 \mathrm{~Hz}), 7.77(\mathrm{~d}, J=1.5 \mathrm{~Hz}), 7.72$ (s), 7.69 (br d), 7.65 (d, $J=2.4$ Hz), $7.62(\mathrm{~d}, J=2.7 \mathrm{~Hz}), 7.47(\mathrm{~d}, J=3.9 \mathrm{~Hz}), 7.37(\mathrm{~d}, J=1.6 \mathrm{~Hz}), 7.36(\mathrm{~d}, J=2.2$ Hz), 7.34 (s), 7.32 (br t), 7.30 (br t), 7.28 (s), 7.21 (d, $J=7.7 \mathrm{~Hz}), 7.06$ (d, $J=7.3$ $\mathrm{Hz}), 6.84(\mathrm{~d}, J=7.2 \mathrm{~Hz}), 6.67(\mathrm{t}, J=7.2 \mathrm{~Hz}), 6.57(\mathrm{t}, J=7.2 \mathrm{~Hz}), 5.05(\mathrm{~s}), 4.82(\mathrm{br}$ s), $4.64(\mathrm{~s}), 4.31(\mathrm{~d}, J=12.7 \mathrm{~Hz}), 4.20(\mathrm{br} \mathrm{s}), 3.95(\mathrm{~d}, J=12.7 \mathrm{~Hz}), 3.35(\mathrm{~d}, J=11.6$ $\mathrm{Hz}$ ), $3.32\left(\mathrm{~d}, J=14.5 \mathrm{~Hz}\right.$ ); Anal. Calc. for $\mathrm{C}_{60} \mathrm{H}_{58} \mathrm{~N}_{4} \mathrm{O}_{8} \mathrm{Zn}: \mathrm{C}, 70.07 ; \mathrm{H}, 5.68 ; \mathrm{N}, 5.45$ $\%$, found; C, 69.98; H, 5.25; N, $5.50 \% ; \lambda_{\max }(\mathrm{DCM} / \mathrm{MeOH}) 417 \mathrm{~nm} @ 6.5 \mathrm{mM}, \varepsilon=$ $48 \mathrm{M}^{-1} \mathrm{~cm}^{-1}$.

Compound (30ii): Zinc chloride; Yield $=0.09 \mathrm{~g}, 75 \%$; $\mathrm{v}_{\max } / \mathrm{cm}^{-1}(\mathrm{KBr}): 3447$, 3386, 1607, 1453, 1183, 1093, 763; $\delta_{\mathrm{H}} / \mathrm{ppm}$ : Conc. of $10^{-3} \mathrm{~mm} ;\left(\mathrm{CDCl}_{3}\right) ; 9.30(\mathrm{~d}, J$ $=6.2 \mathrm{~Hz}), 9.15(\mathrm{~d}, J=6.2 \mathrm{~Hz}), 9.03(\mathrm{br} \mathrm{d}), 8.87(\mathrm{~d}, J=3.5 \mathrm{~Hz}), 8.69(\mathrm{~d}, J=4.4$ $\mathrm{Hz}), 8.63$ (d, $J=4.4 \mathrm{~Hz}), 8.50$ (d, $J=5.3 \mathrm{~Hz}), 8.36$ (br d), 7.89 (s), 7.64 (t, $J=4.1$, $3.2 \mathrm{~Hz}), 7.53(\mathrm{~d}, J=8.2 \mathrm{~Hz}), 7.43(\mathrm{~s}), 7.36(\mathrm{~d}, J=4.9 \mathrm{~Hz}), 7.28(\mathrm{~s}), 7.18(\mathrm{t}, J=8.2$, $7.4 \mathrm{~Hz}), 7.06(\mathrm{~d}, J=6.5 \mathrm{~Hz}), 6.84(\mathrm{~d}, J=7.4 \mathrm{~Hz}), 6.68(\mathrm{t}, J=7.4 \mathrm{~Hz}), 6.65$ (t, $J=$ $7.4 \mathrm{~Hz}), 5.05$ (s), 4.72 (d, $J=4.1 \mathrm{~Hz}), 4.31(\mathrm{~d}, J=13.3 \mathrm{~Hz}), 4.14(\mathrm{~s}), 4.08$ (br d), 3.45 (s), 3.34 (d, $J=13.2 \mathrm{~Hz}$ ); Anal. Calc. for $\mathrm{C}_{56} \mathrm{H}_{52} \mathrm{Cl}_{2} \mathrm{~N}_{4} \mathrm{O}_{4} \mathrm{Zn}: \mathrm{C}, 68.54$; H, 5.34; $\mathrm{N}, 5.71 \%$, found; C, 68.40; H, 5.94; N, $4.59 \% ; \lambda_{\max }(\mathrm{DCM} / \mathrm{MeOH}) 457 \mathrm{~nm} @ 6.5$ $\mathrm{mM}, \varepsilon=46 \mathrm{M}^{-1} \mathrm{~cm}^{-1}$.

Compound (30iii): Zinc perchlorate; Yield $=0.085 \mathrm{~g}, 70 \% ; \mathrm{v}_{\max } / \mathrm{cm}^{-1}(\mathrm{KBr}): 3444$, 3391, 1607, 1423, 1182 (perchlorate anion peak), 762; $\delta_{\mathrm{H}} / \mathrm{ppm}$ : Conc. of $10^{-3} \mathrm{~mm}$; $\left(d_{6}\right.$-DMSO): $8.71(\mathrm{~d}, J=5.5 \mathrm{~Hz}), 8.63(\mathrm{~d}, J=6.8 \mathrm{~Hz}), 8.57(\mathrm{~d}, J=6.8 \mathrm{~Hz}), 8.25(\mathrm{br}$ d), 8.19 (s), 7.94 (br t), 7.86 (td, $J=1.8 \mathrm{~Hz}), 7.65$ (d, $J=6.4 \mathrm{~Hz}), 7.48$ (s), 7.45 (s), 
$7.43(\mathrm{~d}, J=1.9 \mathrm{~Hz}), 7.41(\mathrm{br} \mathrm{d}), 7.35(\mathrm{~s}), 7.21(\mathrm{~d}, J=1.1 \mathrm{~Hz}), 7.20(\mathrm{~s}), 7.15(\mathrm{~s}), 7.11$ $(\mathrm{d}, J=7.0 \mathrm{~Hz}), 7.04(\mathrm{~d}, J=4.5 \mathrm{~Hz}), 7.02(\mathrm{t}, J=2.1 \mathrm{~Hz}), 6.92(\mathrm{~s}), 6.90(\mathrm{~d}, J=2.1$ $\mathrm{Hz}), 6.77(\mathrm{dd}, J=7.4,1.2 \mathrm{~Hz}), 6.61(\mathrm{dt}, J=7.4 \mathrm{~Hz}), 6.50(\mathrm{t}, J=7.4 \mathrm{~Hz}), 5.15(\mathrm{~d}, J$ $=12.8 \mathrm{~Hz}), 5.03(\mathrm{~s}), 4.68(\mathrm{~s}), 4.62(\mathrm{~d}, J=12.8 \mathrm{~Hz}), 4.45(\mathrm{~d}, J=7.4 \mathrm{~Hz}), 4.19$ (br m), $3.80(\mathrm{~d}, J=10.6 \mathrm{~Hz}), 3.72(\mathrm{~s}), 3.16(\mathrm{~d}, J=12.8 \mathrm{~Hz})$; Anal. Calc. for $\mathrm{C}_{56} \mathrm{H}_{52} \mathrm{Cl}_{2} \mathrm{~N}_{4} \mathrm{O}_{12} \mathrm{Zn}: \mathrm{C}, 60.63 ; \mathrm{H}, 4.72 ; \mathrm{N}, 5.05 \%$, found; $\mathrm{C}, 58.28 ; \mathrm{H}, 4.32 ; \mathrm{N}, 5.59$ $\% ; \lambda_{\max }(\mathrm{DCM} / \mathrm{MeOH}) 402 \mathrm{~nm} @ 6.5 \mathrm{mM}, \varepsilon=230 \mathrm{M}^{-1} \mathrm{~cm}^{-1}$.

\subsection{1,23-Di[ $N, N^{\prime}$-di(2'-pyridylmethyl)aminomethyl]-25,27-dibenzyl- 26,28-dihydroxycalix[4]arene (31)}

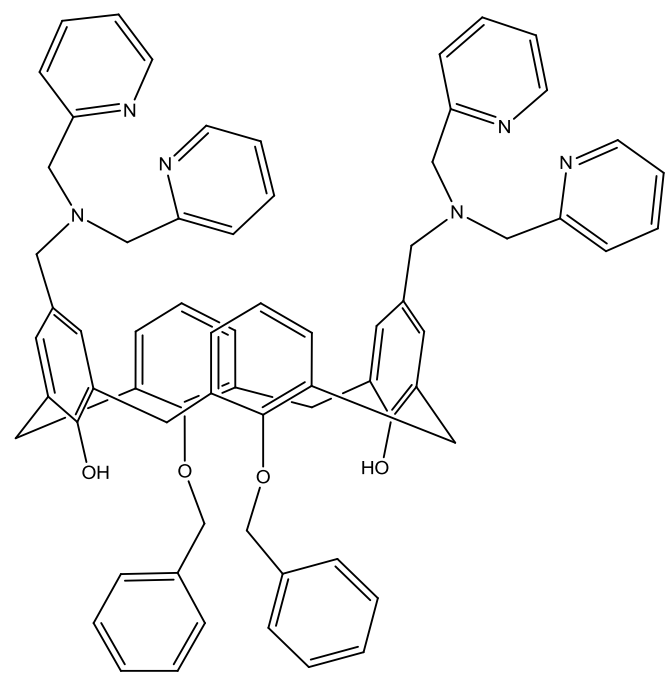

Compound (30) (0.9 g, $1.06 \mathrm{mmol})$, triethylamine (0.21 g, $2.12 \mathrm{mmol})$ and 2-picolyl chloride hydrochloride $(0.35 \mathrm{~g}, 2.12 \mathrm{mmol})$ were refluxed in DCM $(60 \mathrm{~mL})$ under nitrogen gas overnight. The solution was allowed to cool to room temperature, the resulting suspension was filtered to remove the inorganic salts and the volatiles removed under reduced pressure to afford a red-wine waxy solid (31).

Yield $=1.04 \mathrm{~g}, 96 \% ; \mathrm{v}_{\max } / \mathrm{cm}^{-1}(\mathrm{KBr}): 3388,1590,1570,1436,1191,1089,765$; $\delta_{\mathrm{H}} / \mathrm{ppm}$ : Conc. of $10^{-3} \mathrm{~mm} ;\left(\mathrm{CDCl}_{3}\right): 8.56(\mathrm{~d}, 4 \mathrm{H}, \mathrm{Py}-\underline{\mathrm{H}}, J=5.4 \mathrm{~Hz}), 7.85(\mathrm{~s}, 2 \mathrm{H}$, 
$\mathrm{O} \underline{\mathrm{H}}), 7.65$ (t, 4H, Py- $\underline{\mathrm{H}}, J=7.7 \mathrm{~Hz}), 7.63$ (t, 4H, Py- $\underline{\mathrm{H}}, J=5.0 \mathrm{~Hz}), 7.58$ (d, 4H, Py$\underline{\mathrm{H}}, J=8.1 \mathrm{~Hz}), 7.43(\mathrm{~d}, 4 \mathrm{H}, \mathrm{Bn}-\underline{\mathrm{H}}, J=1.6 \mathrm{~Hz}), 7.37$ (t, 4H, Bn- $\underline{\mathrm{H}}, J=1.3 \mathrm{~Hz}), 7.19$ (t, 2H, Bn- $\underline{\mathrm{H}}, J=2.5 \mathrm{~Hz}), 7.10$ (s, 2H, Ar- $\underline{\mathrm{H}}), 7.05$ (d, 4H, Ar- $\underline{\mathrm{H}}, J=6.5 \mathrm{~Hz}), 6.86$ $(\mathrm{d}, 4 \mathrm{H}, \operatorname{Ar}-\underline{\mathrm{H}}, J=7.7 \mathrm{~Hz}), 6.80(\mathrm{~d}, 4 \mathrm{H}, \mathrm{Ar}-\mathrm{H}, J=6.2 \mathrm{~Hz}), 6.49$ (t, $4 \mathrm{H}, \operatorname{Ar}-\underline{\mathrm{H}}, J=5.8$ $\mathrm{Hz}$ ), 6.27 (t, 2H, Ar- $\underline{\mathrm{H}}, J=7.3 \mathrm{~Hz}), 5.05$ (s, 4H, $\underline{\mathrm{C}}_{2}-\mathrm{Bn}$ ), 4.74 (s, 4H, $\left.\underline{\mathrm{C}}_{2}-\mathrm{py}\right), 4.39$ (s, 4H, $\left.\underline{\mathrm{H}}_{2}\right), 4.30$ (d, 4H, $\left.\underline{\mathrm{C}}_{2}, J=13.7 \mathrm{~Hz}\right), 3.85$ (s, 8H, $\left.\mathrm{NHC}_{2}\right), 3.77(\mathrm{~s}, 4 \mathrm{H}$, $\left.\mathrm{C}_{2}\right), 3.65$ (s, 4H, $\left.\underline{\mathrm{C}}_{2}\right), 3.60$ (d, 4H, $\left.\underline{\mathrm{C}}_{2}, J=8.3 \mathrm{~Hz}\right), 3.53$ (d, 4H, $\underline{\mathrm{C}}_{2}, J=13.9$ $\mathrm{Hz}), 3.34\left(\mathrm{~d}, 4 \mathrm{H}, \underline{\mathrm{C}}_{2}, J=11.2 \mathrm{~Hz}\right), 3.16\left(\mathrm{~d}, 4 \mathrm{H}, \mathrm{C}_{2}, J=13.84 \mathrm{~Hz}\right), 3.06(\mathrm{~d}, 4 \mathrm{H}$, $\left.\mathrm{C}_{2} \underline{2}, J=13.2 \mathrm{~Hz}\right) ; \delta \mathrm{c} / \mathrm{ppm}\left(\mathrm{CDCl}_{3}\right) ; 149.2,137.7,137.2,136.7,135.6,134.8,133.2$, 133.1, 131.0, 128.8, 128.6, 128.5, 128.3, 128.2, 128.0, 124.0, 123.6, 78.5, 76.9, 52.8, 31.9; Anal. Calc. for $\mathrm{C}_{68} \mathrm{H}_{62} \mathrm{~N}_{6} \mathrm{O}_{4}$ : C, 79.51; H, 6.08; N, 8.18\%, found; C, 79.42; H, $6.01 ; \mathrm{N}, 7.90 \% ; \lambda_{\max }(\mathrm{DCM} / \mathrm{MeOH}) 260 \mathrm{~nm} @ 0.006 \mathrm{mM}, \varepsilon=428 \mathrm{M}^{-1} \mathrm{~cm}^{-1}$.

\section{Metal complexes of (31) at 1:1 ratio}

All metal ion complexation reactions were performed at room temperature in a solvent mixture of DCM-MeOH at a 1:1 ratio. All reactions used $0.10 \mathrm{mmol}$ of (31) and corresponding metal salt. The solution was stirred for $15 \mathrm{~min}$ and then the solvent was slowly evaporated off. A water-DCM wash was performed to remove any uncomplexed metal and the solvent was removed under reduced pressure.

Compound (31i): Zinc acetate; Yield $=0.07 \mathrm{~g}, 70 \% ; \mathrm{v}_{\max } / \mathrm{cm}^{-1}(\mathrm{KBr}): 3448,1606$, 1590, 1437, 1181, 1092, 763; $\delta_{\mathrm{H}} / \mathrm{ppm}$ : Conc. of $10^{-3} \mathrm{~mm} ;\left(\mathrm{CDCl}_{3}\right) ; 8.69$ (br d), 8.63 (br d), 8.60 (d, $J=5.2 \mathrm{~Hz}), 7.88$ (s), $7.82(\mathrm{dt}, J=6.9,7.5 \mathrm{~Hz}), 7.73(\mathrm{td}, J=1.6,7.5$ $\mathrm{Hz}), 7.64(\mathrm{~d}, J=7.5 \mathrm{~Hz}), 7.49$ (d, $J=7.5 \mathrm{~Hz}), 7.38$ (d, $J=1.9 \mathrm{~Hz}), 7.23$ (br d), 7.17 $(\mathrm{d}, J=7.7 \mathrm{~Hz}), 7.07(\mathrm{~d}, J=7.7 \mathrm{~Hz}), 6.88(\mathrm{~d}, J=8.2 \mathrm{~Hz}), 6.68(\mathrm{t}, J=7.7 \mathrm{~Hz}), 5.06$ (s), 4.69 (s), 4.33 (d, $J=14.1 \mathrm{~Hz}), 4.08$ (br d), 3.35 (d, $J=14.1 \mathrm{~Hz})$; Anal. Calc. for $\mathrm{C}_{72} \mathrm{H}_{68} \mathrm{~N}_{6} \mathrm{O}_{8} \mathrm{Zn}: \mathrm{C}, 74.43 ; \mathrm{H}, 5.66 ; \mathrm{N}, 6.94 \%$, found; C, 74.21; H, 5.22; N, $6.67 \%$; $\lambda_{\max }(\mathrm{DCM} / \mathrm{MeOH}) 388 \mathrm{~nm} @ 0.6 \mathrm{mM}, \varepsilon=273 \mathrm{M}^{-1} \mathrm{~cm}^{-1}$.

Compound (31ii): Zinc chloride; Yield $=0.08 \mathrm{~g}, 80 \% ; \mathrm{v}_{\max } / \mathrm{cm}^{-1}(\mathrm{KBr}): 3426$, 1606, 1591, 1436, 1180, 1090, 762; $\delta_{\mathrm{H}} / \mathrm{ppm}$ : Conc. of $10^{-3} \mathrm{~mm} ;\left(\mathrm{CDCl}_{3}\right) ; 8.94(\mathrm{~d}, J=$ $5.2 \mathrm{~Hz}), 8.61(\mathrm{~d}, J=5.2 \mathrm{~Hz}), 7.82(\mathrm{t}, J=8.2 \mathrm{~Hz}), 7.73(\mathrm{t}, J=8.2 \mathrm{~Hz}), 7.64(\mathrm{~d}, J=$ $8.2 \mathrm{~Hz}), 7.52(\mathrm{~d}, J=9.0 \mathrm{~Hz}), 7.39$ (br s), $7.31(\mathrm{~d}, J=7.3 \mathrm{~Hz}), 7.08(\mathrm{~d}, J=6.5 \mathrm{~Hz})$, $6.83(\mathrm{~d}, J=8.2 \mathrm{~Hz}), 6.70(\mathrm{t}, J=7.3 \mathrm{~Hz}), 6.59$ (t, $J=7.3 \mathrm{~Hz}), 5.59$ (s), 5.05 (s), 4.99 
(s), 4.94 (s), 4.32 (d, $J=12.6 \mathrm{~Hz}), 4.11$ (br d), 3.35 (d, $J=12.6 \mathrm{~Hz})$; Anal. Calc. for $\mathrm{C}_{68} \mathrm{H}_{68} \mathrm{Cl}_{2} \mathrm{~N}_{6} \mathrm{O}_{4} \mathrm{Zn}$ : C, 70.19; H, 5.37; N, 7.22 \%, found; C, 70.07; H, 5.58; N, 7.03 $\% ; \lambda_{\max }(\mathrm{DCM} / \mathrm{MeOH}) 376 \mathrm{~nm} @ 0.6 \mathrm{mM}, \varepsilon=234 \mathrm{M}^{-1} \mathrm{~cm}^{-1}$.

Compound (31iii): Zinc perchlorate; Yield $=0.075 \mathrm{~g}, 63 \% ; \mathrm{v}_{\max } / \mathrm{cm}^{-1}(\mathrm{KBr}): 3455$, $1608,1591,1434,1181$ (perchlorate anion peak), 1092, 763; $\delta_{\mathrm{H}} / \mathrm{ppm}$ : Conc. of $10^{-3}$ mm; $\left(d_{6}\right.$-DMSO); 8.62 (br d), 8.40 (br d), 8.06 (s), 7.62 (d, $\left.J=8.8 \mathrm{~Hz}\right), 7.39$ (t, $J=$ $6.8 \mathrm{~Hz}), 7.31(\mathrm{~d}, J=7.9 \mathrm{~Hz}), 7.13(\mathrm{~d}, J=7.2 \mathrm{~Hz}), 6.93(\mathrm{br} \mathrm{t}), 6.76(\mathrm{~d}, J=7.2 \mathrm{~Hz})$, $6.44(\mathrm{t}, J=7.2 \mathrm{~Hz}), 5.06(\mathrm{~s}), 4.75(\mathrm{~s}), 4.33$ (d, $J=13.4 \mathrm{~Hz}), 3.37$ (d, $J=12.1 \mathrm{~Hz})$; Anal. Calc. for $\mathrm{C}_{68} \mathrm{H}_{62} \mathrm{Cl}_{2} \mathrm{~N}_{6} \mathrm{O}_{12} \mathrm{Zn}$ : C, 63.24; H, 4.84; N, 6.51\%, found; C, 63.15; H, 4.78; N, $6.45 \% ; \lambda_{\max }(\mathrm{DCM} / \mathrm{MeOH}) 320 \mathrm{~nm} @ 0.6 \mathrm{mM}, \varepsilon=207 \mathrm{M}^{-1} \mathrm{~cm}^{-1}$.

\subsection{1,23-di[ $N, N^{\prime}$-(2'-pyridylmethyl)(4,4-dimethyl-2,2-}

bipyridine)aminomethyl]-25,27-dibenzyl-26,28-

dihydroxycalix[4]arene. (32)

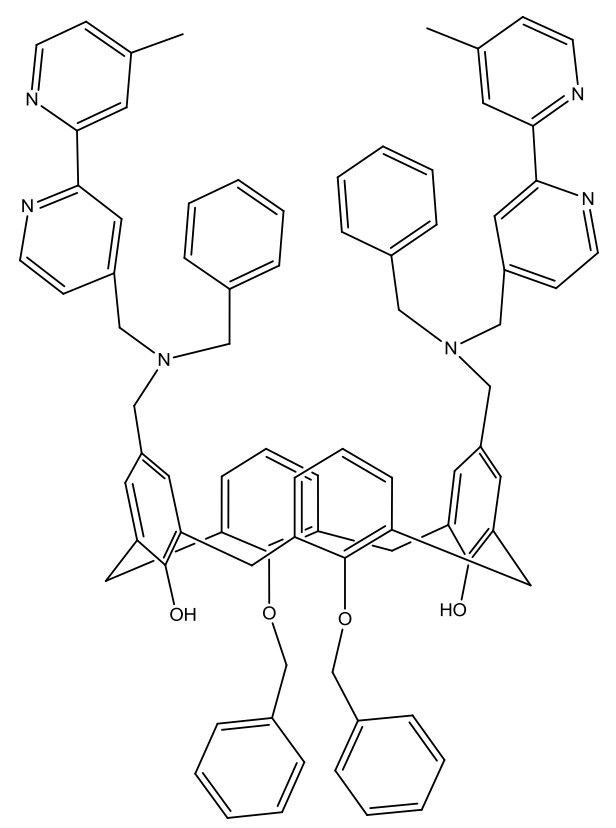

Compound (30) (0.026 g, $0.03 \mathrm{mmol})$, triethylamine $(0.025 \mathrm{~g}, 0.18 \mathrm{mmol})$ and compound (22) (0.049 g, $0.18 \mathrm{mmol})$ were refluxed in $\mathrm{CH}_{3} \mathrm{CN}$ (40 mL) under nitrogen gas for $18 \mathrm{hr}$. The solution was allowed to cool to room temperature, the 
inorganic salts were removed by filtration and the solvents removed under reduced pressure to give a brown-red waxy solid (32).

Yield $=0.025 \mathrm{~g}, 66 \% ; \mathrm{v}_{\max } / \mathrm{cm}^{-1}(\mathrm{KBr}): 3436,1630,1594,1458,1384,1016,825 ;$ $\delta_{\mathrm{H}} / \mathrm{ppm}$ : Conc. of $10^{-3} \mathrm{~mm} ;\left(\mathrm{CDCl}_{3}\right) ; 8.68(\mathrm{~d}, 4 \mathrm{H}$, bipy- $\underline{\mathrm{H}}, J=5.7 \mathrm{~Hz}), 8.63(\mathrm{br} \mathrm{d}$, 2H, Py- $\underline{\mathrm{H}}), 8.54$ (d, 4H, bipy-H, $J=4.6 \mathrm{~Hz}$ ), 8.41 (s, 4H, bipy- $\underline{\mathrm{H}}$ ), 8.35 (s, 4H, bipy$\underline{\mathrm{H}}$ ), 8.22 (br d, 4H, bipy-H), 7.82 (s, 2H, Ar-O吕), 7.65 (br t, 4H, Py- $\underline{\mathrm{H}}$ ), 7.63 (br t, 4H, Py- $\underline{\mathrm{H}}$ ), 7.48 (br d, 4H, Bn- $\underline{\mathrm{H}}$ ), 7.38 (d, 4H, bipy- $\underline{\mathrm{H}}, J=2.0 \mathrm{~Hz}$ ), 7.36 (d, 4H, Py$\underline{\mathrm{H}}, J=1.8 \mathrm{~Hz}$ ), 7.31 (br t, 2H, Bn- $\underline{\mathrm{H}}$ ), 7.17 (br t, 4H, Bn-H), 7.05 (d, 4H, Ar- $\underline{\mathrm{H}}, J=$ $7.2 \mathrm{~Hz}), 6.90(\mathrm{~d}, 4 \mathrm{H}, \mathrm{Ar}-\underline{\mathrm{H}}, J=7.5 \mathrm{~Hz}), 6.77$ (t, $2 \mathrm{H}, \operatorname{Ar}-\underline{\mathrm{H}}, J=7.8 \mathrm{~Hz}), 6.65$ (t, 2H, $\operatorname{Ar}-\underline{\mathrm{H}}, J=7.7 \mathrm{~Hz}$ ), 5.06 (s, 4H, O-C $\left.\underline{\mathrm{H}}_{2}-\mathrm{bn}\right), 4.83$ (s, 4H, Ar-C $\left.\underline{\mathrm{H}}_{2}\right), 4.63$ (s, 4H, N$\left.\mathrm{C}_{2}\right), 4.31\left(\mathrm{~d}, 4 \mathrm{H}, \underline{\mathrm{CH}}_{2}, J=13.4 \mathrm{~Hz}\right), 3.95$ (s, 4H, N-C $\left.\underline{\mathrm{H}}_{2}\right), 3.34\left(\mathrm{~d}, 4 \mathrm{H}, \underline{\mathrm{C}}_{2}, J=\right.$ $12.9 \mathrm{~Hz}), 2.23$ (s, 3H, $\left.\underline{\mathrm{H}}_{3}\right) ; \delta_{\mathrm{C}} / \mathrm{ppm}\left(\mathrm{CDCl}_{3}\right) ; 149.6,149.3,149.0,148.9,136.7$, $136.6,132.4,129.5,129.1,128.7,128.5,128.0,127.1,125.4,125.0,124.7,123.5$, 123.1, 122.9, 122.3, 122.0, 121.3, 121.1, 120.4, 78.4, 63.2, 60.8, 57.5, 31.4, 21.3; Anal. Calc. for $\mathrm{C}_{82} \mathrm{H}_{74} \mathrm{~N}_{6} \mathrm{O}_{4}$ : C, 81.56; H, 6.18; N, $6.95 \%$, found; C, 81.24; H, 6.03; $\mathrm{N}, 6.87 \%$.

\subsection{1,23-dibromomethyl-25,27-di(aminomethyl-2-pyridine)-26,28-} dihydroxycalix[4]arene. (33)

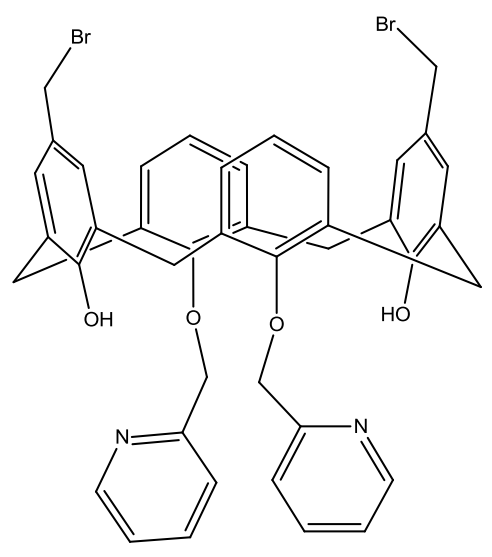

2-Picolylchloride hydrochloride $(0.26 \mathrm{~g}, 1.6 \mathrm{mmol})$ was dissolved in $\mathrm{CH}_{3} \mathrm{CN}$ (10 $\mathrm{mL})$ and to this sodium carbonate $(0.17 \mathrm{~g}, 1.6 \mathrm{mmol})$ was added to remove the 
hydrochloride acid. Compound (18) $(0.5 \mathrm{~g}, 0.82 \mathrm{mmol}) \mathrm{NaH}(0.04 \mathrm{~g}, 1.6 \mathrm{mmol})$ and the treated 2-picolylchloride hydrochloride were then refluxed in $\mathrm{CH}_{3} \mathrm{CN}(60 \mathrm{~mL})$ under nitrogen gas overnight. The solution was allowed to cool to room temperature, the inorganic salts were removed by filtration and volatiles removed under reduced pressure to give a red waxy solid (33).

Yield $=1.24 \mathrm{~g}, 95 \%, ; \mathrm{v}_{\max } / \mathrm{cm}^{-1}(\mathrm{KBr}): 3404,2919,1591,1466,1088,757 ; \delta_{\mathrm{H}} / \mathrm{ppm}:$ Conc. of $10^{-3} \mathrm{~mm} ;\left(\mathrm{CDCl}_{3}\right) ; 8.51(\mathrm{~d}, 2 \mathrm{H}, \mathrm{Py}-\underline{\mathrm{H}}, J=7.5 \mathrm{~Hz}), 7.59$ (t, 2H, Py- $\underline{\mathrm{H}}, J=$ $4.7 \mathrm{~Hz}$ ), 7.51 (s, 2H, Ar-OH), 7.39 (t, 2H, Py- $\underline{\mathrm{H}}, J=5.3 \mathrm{~Hz}), 7.15$ (d, 2H, Py- $\underline{\mathrm{H}}, J=$ $6.7 \mathrm{~Hz}), 7.09$ (s, 4H, Ar- $\underline{\mathrm{H}}), 6.89$ (d, 4H, Ar- $\underline{\mathrm{H}}, J=6.1 \mathrm{~Hz}), 6.63$ (t, 2H, Ar- $\underline{\mathrm{H}}, J=$ $8.6 \mathrm{~Hz}$ ), 5.16 (s, 4H, $\underline{\mathrm{H}}_{2}-\mathrm{Py}$ ), 4.64 (s, 4H, $\underline{\mathrm{C}}_{2}-\mathrm{Br}$ ), 4.37 (br d, 4H, $\underline{\mathrm{C}}_{2}, J=13.2$ $\mathrm{Hz}$ ), 3.42 (br d, $\left.4 \mathrm{H}, \underline{\mathrm{C}}_{2}, J=13.3 \mathrm{~Hz}\right) ; \delta_{\mathrm{C}} / \mathrm{ppm}\left(\mathrm{CDCl}_{3}\right)$; 156.2, 155.6, 152.6, 148.7 , 136.5, 133.6, 132.3, 128.7, 128.5, 128.1, 125.0, 124.9, 120.9, 78.2, 46.3, 31.6; Anal. Calc. for $\mathrm{C}_{42} \mathrm{H}_{36} \mathrm{Br}_{2} \mathrm{~N}_{2} \mathrm{O}_{4}: \mathrm{C}, 63.65 ; \mathrm{H}, 3.53 ; \mathrm{N}, 4.58 \%$, found; $\mathrm{C}, 63.54 ; \mathrm{H}, 3.48 ; \mathrm{N}$, $4.49 \%$.

\subsection{1,23-Di[N-(2'pyridylmethyl)aminomethyl]-25,27-di(methyl-2-} pyridine)-26,28-dihydroxycalix[4]arene. (34)

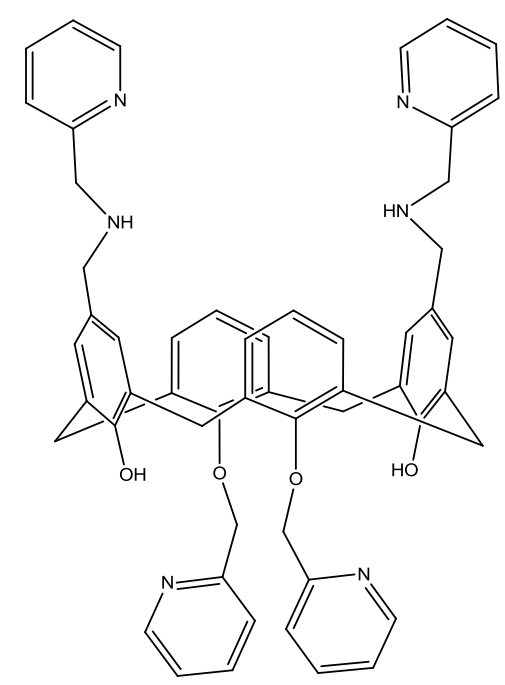

Compound (33) $(0.44 \mathrm{~g}, 0.55 \mathrm{mmol})$, triethylamine $(0.11 \mathrm{~g}, 1.11 \mathrm{mmol})$ and 2aminmomethyl-pyridine $(0.11 \mathrm{~mL}, 1.11 \mathrm{mmol})$ were refluxed overnight in $\mathrm{CH}_{3} \mathrm{CN}$ 
(70 mL) under nitrogen gas. The solution was allowed to cool to room temperature, the inorganic salts were removed by filtration and the volatiles were removed under reduced pressure. The residue was washed with DCM and water, the organics seperated and dried over anhydrous magnesium sulphate and solvent removed under reduced pressure to afford a red-brown waxy solid (34).

Yield $=0.46 \mathrm{~g}, 99 \%, \mathrm{v}_{\max } / \mathrm{cm}^{-1}(\mathrm{KBr}): 3418,2923,1591,1571,1435,1149,1088$, 755; $\delta_{\mathrm{H}} / \mathrm{ppm}$ : Conc. of $10^{-3} \mathrm{~mm} ;\left(\mathrm{CDCl}_{3}\right): 8.59$ (d, 2H, Py- $\left.\underline{\mathrm{H}}, J=4.8 \mathrm{~Hz}\right), 8.49$ (d, $2 \mathrm{H}, \mathrm{Py} \underline{-\mathrm{H}}, J=4.2 \mathrm{~Hz}), 7.63$ (t, 2H, Py- $\underline{\mathrm{H}}, J=5.4 \mathrm{~Hz}$ ), 7.58 (d, 2H, Py- $\underline{\mathrm{H}}, J=6.9$ $\mathrm{Hz}), 7.56$ (t, 2H, Py- $\underline{\mathrm{H}}, J=6.5 \mathrm{~Hz}), 7.52$ (t, 2H, Py- $\underline{\mathrm{H}}, J=6.9 \mathrm{~Hz}), 7.47$ (d, 2H, Py$\underline{\mathrm{H}}, J=7.7 \mathrm{~Hz}$ ), 7.41 (br t, 2H, Py- $\underline{\mathrm{H}}$ ), 7.09 (d, 4H, Ar- $\underline{\mathrm{H}}, J=7.6 \mathrm{~Hz}), 7.05$ (s, 4H, Ar$\underline{\mathrm{H}}), 7.03$ (s, 4H, Ar- $\underline{\mathrm{H}}), 6.98$ (d, 4H, Ar- $\underline{\mathrm{H}}, J=7.6 \mathrm{~Hz}), 6.94$ (d, 4H, Ar- $\underline{\mathrm{H}}, J=7.9$ $\mathrm{Hz}), 6.81$ (d, 4H, Ar- $\underline{\mathrm{H}}, J=7.3 \mathrm{~Hz}), 6.71$ (t, 2H, Ar- $\underline{\mathrm{H}}, J=7.6 \mathrm{~Hz}), 6.54$ (t, 2H, Ar$\underline{\mathrm{H}}, J=7.6 \mathrm{~Hz}), 5.24\left(\mathrm{~s}, 4 \mathrm{H}, \mathrm{OC}_{2} \underline{\mathrm{Py}}\right), 5.16\left(\mathrm{~s}, 4 \mathrm{H}, \mathrm{C}_{2} \underline{\mathrm{NH}}^{\mathrm{NH}}\right), 4.40\left(\mathrm{~d}, 2 \mathrm{H}, \mathrm{C}_{2}, J=\right.$ $14.5 \mathrm{~Hz}), 4.35\left(\mathrm{~d}, 4 \mathrm{H}, \underline{\mathrm{C}}_{2}, J=14.2 \mathrm{~Hz}\right), 4.25\left(\mathrm{~d}, 2 \mathrm{H}, \underline{\mathrm{C}}_{2}, J=14.5 \mathrm{~Hz}\right), 3.95$ (s, $\left.4 \mathrm{H}, \mathrm{NHC}_{2} \underline{2} \mathrm{Py}\right), 3.38\left(\mathrm{~d}, 4 \mathrm{H}, \underline{\mathrm{C}}_{2}, J=13.2 \mathrm{~Hz}\right) ; \delta_{\mathrm{c}} / \mathrm{ppm}\left(\mathrm{CDCl}_{3}\right) ; 158.3,156.0$, $154.7,152.3,150.7,149.9,136.4,135.5,133.4,131.1,128.4,127.8,127.1,126.9$, 125.1, 124.6, 120.1, 120.4, 77.7, 59.2, 53.6, 30.9; Anal. Calc. for $\mathrm{C}_{54} \mathrm{H}_{50} \mathrm{~N}_{6} \mathrm{O}_{4}$ : C, 76.57; H, 5.99; N, 9.92\%, found; C, 76.41; H 5.74; N, $9.56 \% ; \lambda_{\max }(\mathrm{DCM} / \mathrm{MeOH})$ $262 \mathrm{~nm} @ 0.005 \mathrm{mM}, \varepsilon=28 \mathrm{M}^{-1} \mathrm{~cm}^{-1}$.

\section{Metal complexes of (34)}

All metal ion complexation reactions were performed at room temperature in a solvent mixture of DCM-MeOH at a 1:1 ratio. All reactions used $0.09 \mathrm{mmol}$ of (34) and corresponding metal salt. The solution was stirred for $15 \mathrm{~min}$ and then the solvent was slowly evaporated off. A water-DCM wash was performed to remove any uncomplexed metal and the solvent was removed under reduced pressure.

Compound (34i): Zinc acetate; Yield $=0.08 \mathrm{~g}, 88 \% ; \mathrm{v}_{\max } / \mathrm{cm}^{-1}(\mathrm{KBr}): 3420,2786$, 1591, 1434, 939, 929, 675; $\delta_{\mathrm{H}} / \mathrm{ppm}$ : Conc. of $10^{-3} \mathrm{~mm}$; $\left(\mathrm{CDCl}_{3}\right) ; 8.65(\mathrm{~d}, J=5.5 \mathrm{~Hz})$, 8.54 (br d), 7.85 (d, $J=7.0 \mathrm{~Hz}), 7.83$ (s), 7.65 (d, $J=7.8 \mathrm{~Hz}), 7.46$ (s), 7.42 (d, $J=$ $6.3 \mathrm{~Hz}), 7.40$ (s), 7.37 (d, $J=7.8 \mathrm{~Hz}), 7.32$ (d, $J=4.7 \mathrm{~Hz}), 7.22$ (s), 7.19 (d, $J=6.3$ $\mathrm{Hz}), 7.15$ (t, $J=7.0 \mathrm{~Hz}), 7.01$ (s), 7.05 (d, $J=6.3 \mathrm{~Hz}), 7.03$ (s), 6.97 (t, $J=4.7 \mathrm{~Hz}$ ), $6.98(\mathrm{~d}, J=7.8 \mathrm{~Hz}), 6.80(\mathrm{~d}, J=7.0 \mathrm{~Hz}), 6.76(\mathrm{~d}, J=7.8 \mathrm{~Hz}), 6.73(\mathrm{~d}, J=3.1 \mathrm{~Hz})$, $6.68(\mathrm{~d}, J=7.0 \mathrm{~Hz}), 6.65(\mathrm{t}, J=7.0 \mathrm{~Hz}), 6.59(\mathrm{~d}, J=4.7 \mathrm{~Hz}), 6.49(\mathrm{t}, J=6.3 \mathrm{~Hz})$, 
$6.43(\mathrm{t}, J=4.7 \mathrm{~Hz}), 5.10(\mathrm{~d}, J=11.0 \mathrm{~Hz}), 5.07(\mathrm{~s}), 4.88(\mathrm{~s}), 4.80(\mathrm{~d}, J=11.8 \mathrm{~Hz})$, 4.75 (s), 4.73 (d, $J=10.2 \mathrm{~Hz}), 4.65$ (d, $J=2.6 \mathrm{~Hz}), 4.45$ (s), 4.31 (d, $J=14.1 \mathrm{~Hz})$, 4.11 (s), 4.01 (d, $J=14.9 \mathrm{~Hz}), 3.80(\mathrm{t}, J=7.0 \mathrm{~Hz}), 3.77$ (s), 3.72 (s), 3.70 (s), 3.64 (d, $J=7.9 \mathrm{~Hz}), 3.60$ (s), 3.52 (d, $J=7.9 \mathrm{~Hz}), 3.33$ (d, $J=13.5 \mathrm{~Hz}), 3.17$ (br d); Anal. Calc. for $\mathrm{C}_{58} \mathrm{H}_{56} \mathrm{~N}_{6} \mathrm{O}_{8} \mathrm{Zn}: \mathrm{C}, 67.60 ; \mathrm{H}, 5.48 ; \mathrm{N}, 8.16 \%$, found; C, 67.57; H, 5.46; N, $8.14 \% ; \lambda_{\max }(\mathrm{DCM} / \mathrm{MeOH}) 434 \mathrm{~nm} @ 5.0 \mathrm{mM}, \varepsilon=27 \mathrm{M}^{-1} \mathrm{~cm}^{-1}$.

Compound (34ii): Zinc chloride; Yield $=0.09 \mathrm{~g}, 81 \% ; \mathrm{v}_{\max } / \mathrm{cm}^{-1}(\mathrm{KBr}): 3446$, 2800, 1629, 1466, 724; $\delta_{\mathrm{H}} / \mathrm{ppm}$ : Conc. of $10^{-3} \mathrm{~mm} ;\left(\mathrm{CDCl}_{3}\right) ; 8.76(\mathrm{~d}, J=5.4 \mathrm{~Hz})$, 8.70 (br d), 8.66 (br d), 8.21 (s), 8.05 (td, $J=1.8 \mathrm{~Hz}$ ), 7.96 (s), 7.93 (d, $J=7.5 \mathrm{~Hz}$ ), 7.89 (s), 7.73 (td, $J=1.8 \mathrm{~Hz}), 7.61(\mathrm{~d}, J=6.6 \mathrm{~Hz}), 7.53$ (s), 7.51 (s), 7.47 (d, $J=4.7$ Hz), 7.45 (s), 7.30 (br t), 7.20 (br d), 7.17 (br d), 7.11 (s), 7.09 (s), 7.06 (s), 7.04 (s), $6.93(\mathrm{~d}, J=6.8 \mathrm{~Hz}), 6.85(\mathrm{~d}, J=8.0 \mathrm{~Hz}), 6.63(\mathrm{br} \mathrm{t}), 6.41(\mathrm{t}, J=7.6 \mathrm{~Hz}), 5.14(\mathrm{~s})$, 4.44 (d, $J=12.9 \mathrm{~Hz}$ ), 4.29 (s), 4.24 (s), 4.18 (s); Anal. Calc. for $\mathrm{C}_{54} \mathrm{H}_{50} \mathrm{Cl}_{2} \mathrm{~N}_{6} \mathrm{O}_{4} \mathrm{Zn}$ : $\mathrm{C}, 65.96 ; \mathrm{H}, 5.13 ; \mathrm{N}, 8.55 \%$, found; $\mathrm{C}, 65.61 ; \mathrm{H}, 5.09 ; \mathrm{N}, 8.54 \%$; $\lambda_{\max }(\mathrm{DCM} / \mathrm{MeOH}) 406 \mathrm{~nm} @ 5.0 \mathrm{mM}, \varepsilon=28 \mathrm{M}^{-1} \mathrm{~cm}^{-1}$.

Compound (34iii): Zinc perchlorate; Yield $=0.10 \mathrm{~g}, 83 \% ; \mathrm{v}_{\max } / \mathrm{cm}^{-1}(\mathrm{KBr}): 3437$, 2813, 1626, 1466, 1099 (perchlorate anion peak), 754, 622; $\delta_{\mathrm{H}} / \mathrm{ppm}$ : Conc. of $10^{-3}$ mm; $\left(d_{6}\right.$-DMSO); 8.75 (d, $\left.J=7.9 \mathrm{~Hz}\right), 8.66$ (d, $\left.J=5.6 \mathrm{~Hz}\right), 8.51$ (br d), 8.28 (br t), $8.20(\mathrm{~s}), 8.03($ br t), $7.92(\mathrm{~d}, J=6.8 \mathrm{~Hz}), 7.85$ (t, $J=9.0 \mathrm{~Hz}), 7.71(\mathrm{t}, J=7.9 \mathrm{~Hz})$, 7.54 (d, $J=6.8 \mathrm{~Hz}$ ), 7.51 (br d), 7.47 (s), 7.31 (br t), 7.19 (br d), 7.16 (br d), 7.14 (s), 7.07 (s), $7.04(\mathrm{~s}), 6.97$ (s), $6.83(\mathrm{~d}, J=7.5 \mathrm{~Hz}), 6.62(\mathrm{t}, J=7.5 \mathrm{~Hz}), 6.58(\mathrm{t}, J=6.8$ Hz), 5.11 (s), 4.41 (d, $J=12.3 \mathrm{~Hz}), 4.39$ (s), 4.22 (br t), 4.15 (s); Anal. Calc. for $\mathrm{C}_{54} \mathrm{H}_{50} \mathrm{Cl}_{2} \mathrm{~N}_{6} \mathrm{O}_{12} \mathrm{Zn}$ : C, 58.36; H, 4.53; N, 7.56 \%, found; C, 58.41; H, 4.21; N, 7.50 $\% ; \lambda_{\max }(\mathrm{DCM} / \mathrm{MeOH}) 432 \mathrm{~nm} @ 5.0 \mathrm{mM}, \varepsilon=36 \mathrm{M}^{-1} \mathrm{~cm}^{-1}$.

\section{Metal complexes of (34) at 1:2 ratio}

Same procedure was used as in the case of the 1:1 ratio, with the exception that the ratio of ligand to metal was $0.09 \mathrm{mmol}$ to $0.18 \mathrm{mmol}$.

Compound (34iv): Zinc acetate; Yield $=0.09 \mathrm{~g}, 81 \%$; $\mathrm{v}_{\max } / \mathrm{cm}^{-1}(\mathrm{KBr}): 3442$, 2818, 1608, 1436, 755, 403; $\delta_{\mathrm{H}} / \mathrm{ppm}$ : Conc. of $10^{-3} \mathrm{~mm} ;\left(\mathrm{CDCl}_{3}\right) ; 8.70(\mathrm{~d}, J=5.5$ $\mathrm{Hz}), 8.63(\mathrm{~d}, J=5.5 \mathrm{~Hz}), 8.56(\mathrm{br} \mathrm{t}), 8.25(\mathrm{~d}, J=6.6 \mathrm{~Hz}), 8.20(\mathrm{~d}, J=8.8 \mathrm{~Hz}), 8.09$ (s), 7.88 (s), 7.79 (s), 7.65 (br t), 7.56 (t, $J=7.7 \mathrm{~Hz}$ ), 7.36 (br t), 7.30 (s), 7.19 (br t), 
$7.09(\mathrm{~d}, J=7.2 \mathrm{~Hz}), 7.03(\mathrm{~d}, J=7.2 \mathrm{~Hz}), 6.99$ (d, $J=6.34 \mathrm{~Hz}), 6.90(\mathrm{~d}, J=7.2 \mathrm{~Hz})$, 6.74 (br t), 6.66 (t, $J=7.2 \mathrm{~Hz}), 5.20$ (s), 4.50 (d, $J=13.6 \mathrm{~Hz}), 4.38$ (s), 4.34 (s), 4.25 (d, $J=13.6 \mathrm{~Hz}$ ), 3.46 (s), 3.43 (s), 3.41 (s); Anal. Calc. for $\mathrm{C}_{62} \mathrm{H}_{62} \mathrm{~N}_{6} \mathrm{O}_{12} \mathrm{Zn}_{2}:$ C, 61.34; H, 5.15; N, $6.92 \%$, found; C, 61.04; H, 5.01; N, $6.45 \%$; $\lambda_{\max }(\mathrm{DCM} / \mathrm{MeOH})$ $434 \mathrm{~nm} @ 5.0 \mathrm{mM}, \varepsilon=33 \mathrm{M}^{-1} \mathrm{~cm}^{-1}$.

Compound (34v): Zinc chloride; Yield $=0.09 \mathrm{~g}, 70 \% ; \mathrm{v}_{\max } / \mathrm{cm}^{-1}(\mathrm{KBr}): 3429$, 1607, 1465, 936, 755, 517; $\delta_{\mathrm{H}} / \mathrm{ppm}$ : Conc. of $10^{-3} \mathrm{~mm} ;\left(\mathrm{CDCl}_{3}\right) ; 8.78(\mathrm{br} \mathrm{d}), 8.71(\mathrm{~d}$, $J=4.7 \mathrm{~Hz}), 8.67(\mathrm{~d}, J=4.7 \mathrm{~Hz}), 8.55(\mathrm{br} \mathrm{d}), 8.23(\mathrm{~s}), 8.08(\mathrm{t}, J=7.1 \mathrm{~Hz}), 7.95$ (t, $J=$ $8.3 \mathrm{~Hz}), 7.74(\mathrm{t}, J=8.3 \mathrm{~Hz}), 7.64$ (br t), 7.53 (d, $J=9.5 \mathrm{~Hz}), 7.48$ (t, $J=3.2 \mathrm{~Hz})$, 7.32 (br t), 7.20 (d, $J=7.1 \mathrm{~Hz}), 7.12$ (s), 7.09 (d, $J=7.6 \mathrm{~Hz}), 7.06$ (s), 7.69 (d, $J=$ $7.1 \mathrm{~Hz}), 6.86$ (t, $J=7.6 \mathrm{~Hz}), 6.64(\mathrm{br} \mathrm{t}), 6.45$ (t, $J=7.1 \mathrm{~Hz}), 5.15$ (s), 4.44 (d, $J=$ $11.7 \mathrm{~Hz}$ ), 4.30 (d, $J=10.5 \mathrm{~Hz}$ ), 4.23 (s), 4.18 (s); Anal. Calc. for $\mathrm{C}_{54} \mathrm{H}_{50} \mathrm{Cl}_{4} \mathrm{~N}_{6} \mathrm{O}_{4} \mathrm{Zn}_{2}$ : C, 57.93; H, 4.30; N, 7.51\%, found; C, 57.87; H, 4.21; N, $7.49 \%$; $\lambda_{\max }(\mathrm{DCM} / \mathrm{MeOH}) 402 \mathrm{~nm} @ 5.0 \mathrm{mM}, \varepsilon=22 \mathrm{M}^{-1} \mathrm{~cm}^{-1}$.

Compound (34vi): Zinc perchlorate; Yield $=0.11 \mathrm{~g}, 73 \% ; \mathrm{v}_{\max } / \mathrm{cm}^{-1}(\mathrm{KBr}): 3513$, 2801, 1614, 1466, 1086 (perchlorate anion peak), 759, 626; $\delta_{\mathrm{H}} / \mathrm{ppm}$ : Conc. of $10^{-3}$ mm; ( $d_{6}$-DMSO); 8.72 (d, $\left.J=4.7 \mathrm{~Hz}\right), 8.62$ (br d), 8.51 (br d), 8.24 (s), 8.16 (s), 8.00 (br t), 7.90 (t, $J=7.1 \mathrm{~Hz}), 7.81(\mathrm{~d}, J=8.2 \mathrm{~Hz}), 7.68(\mathrm{t}, J=7.1 \mathrm{~Hz}), 7.49$ (d, $J=9.4$ Hz), 7.43 (br t), 7.14 (d, $J=7.3 \mathrm{~Hz}$ ), 7.04 (s), 7.02 (s), 6.94 (s), 6.86 (d, $J=8.3 \mathrm{~Hz}$ ), $6.79(\mathrm{~d}, J=6.7 \mathrm{~Hz}), 6.60(\mathrm{t}, J=7.5 \mathrm{~Hz}), 5.08(\mathrm{~s}), 4.38(\mathrm{~d}, J=13.1 \mathrm{~Hz}), 4.27(\mathrm{~s}), 4.17$ (d, $J=13.1 \mathrm{~Hz}$ ), 4.16 (s); Anal. Calc. for $\mathrm{C}_{54} \mathrm{H}_{50} \mathrm{Cl}_{4} \mathrm{~N}_{6} \mathrm{O}_{20} \mathrm{Zn}_{2}: \mathrm{C}, 47.15 ; \mathrm{H}, 3.66 ; \mathrm{N}$, $6.11 \%$, found; C, 47.11; H, 3.62; N, $6.09 \%$; $\lambda_{\max }(\mathrm{DCM} / \mathrm{MeOH}) 432 \mathrm{~nm} @ 0=5.0$ $\mathrm{mM}, \varepsilon=31 \mathrm{M}^{-1} \mathrm{~cm}^{-1}$. 


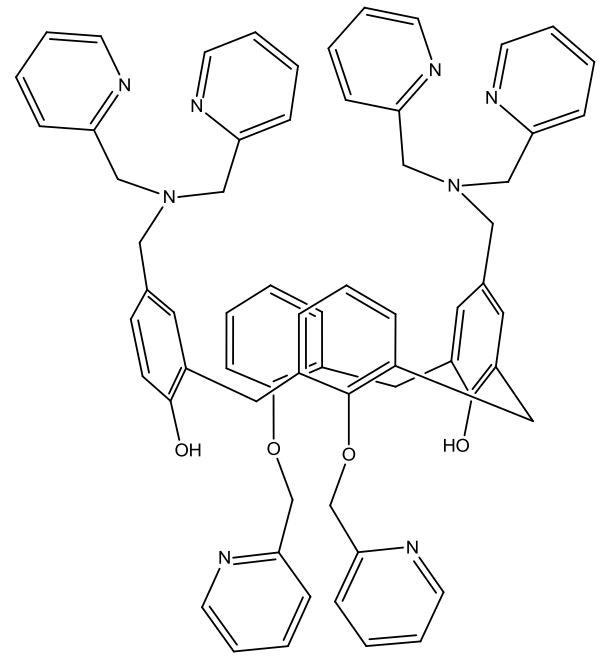

2-Picolylchloride hydrochloride $(0.21 \mathrm{~g}, 1.27 \mathrm{mmol})$ and sodium carbonate $(0.14 \mathrm{~g}$, $1.27 \mathrm{mmol}$ ) were suspended in $\mathrm{CH}_{3} \mathrm{CN}(20 \mathrm{~mL})$, stirred for $1 \mathrm{hr}$. and then filtered to remove the hydrochloric acid salts. Compound (33) (0.54 g, $0.64 \mathrm{mmol}), \mathrm{NaH}(0.03$ $\mathrm{g}, 1.27 \mathrm{mmol})$ were then added to the filtrate along with $\mathrm{CH}_{3} \mathrm{CN}$ :DCM $(40: 10 \mathrm{~mL})$. The solution was then refluxed overnight under nitrogen gas. The solution was allowed to cool to room temperature, the inorganic salts were removed by filtration and volatiles removed under reduced pressure. The residue was then washed with DCM and water to remove and excess 2-picolylchloride hydrochloride. The solvent was then removed under reduced pressure to give a red waxy solid (35).

Yield $=0.52 \mathrm{~g}, 76 \% ; \mathrm{v}_{\max } / \mathrm{cm}^{-1}(\mathrm{KBr}): 3432,1630,1435,1384,1090,747 . \delta_{\mathrm{H}} / \mathrm{ppm}:$ Conc. of $10^{-3} \mathrm{~mm}$; $\left(\mathrm{CDCl}_{3}\right) ; 8.60$ (d, 2H, Py- $\left.\underline{\mathrm{H}}, J=4.5 \mathrm{~Hz}\right), 8.49$ (d, 4H, Py- $\underline{\mathrm{H}}, J=$

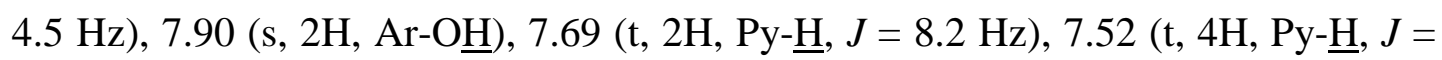
$7.4 \mathrm{~Hz}$ ), 7.23 (d, 2H, Py- $\underline{\mathrm{H}}, J=5.7 \mathrm{~Hz}), 7.17$ (d, 4H, Py- $\underline{\mathrm{H}}, J=5.3 \mathrm{~Hz}), 7.09$ (s, 4H, Ar- $\underline{\mathrm{H}}), 7.03$ (t, $2 \mathrm{H}, \mathrm{Py}-\underline{\mathrm{H}}, J=5.7 \mathrm{~Hz}), 7.00(\mathrm{t}, 4 \mathrm{H}, \mathrm{Py}-\underline{\mathrm{H}}, J=6.7 \mathrm{~Hz}), 6.74$ (d, $4 \mathrm{H}$, Ar- $\underline{\mathrm{H}}, J=7.6 \mathrm{~Hz}), 6.50(\mathrm{t}, 2 \mathrm{H}, \mathrm{Ar}-\underline{\mathrm{H}}, J=7.4 \mathrm{~Hz}), 5.21\left(\mathrm{~s}, 4 \mathrm{H}, \mathrm{OC}_{\underline{2}}-\mathrm{Py}\right), 4.62$ (s, $\left.12 \mathrm{H}, \underline{\mathrm{C}}_{2}-\mathrm{Py}\right), 4.36\left(\mathrm{~d}, 4 \mathrm{H}, \underline{\mathrm{C}}_{2}, J=13.7 \mathrm{~Hz}\right), 3.39\left(\mathrm{~d}, 4 \mathrm{H}, \underline{\mathrm{C}}_{2}, J=12.0 \mathrm{~Hz}\right)$; $\delta_{\mathrm{C}} / \mathrm{ppm}\left(\mathrm{CDCl}_{3}\right) ; 154.4,153.8,151.4,150.4,149.1,148.2,136.6,136.5,132.7$, 131.3, 127.6, 127.8, 127.0, 126.8, 126.5, 126.0, 120.3, 120.0, 76.5, 48.7, 44.9, 29.9; 
Anal. Calc. for $\mathrm{C}_{66} \mathrm{H}_{60} \mathrm{~N}_{8} \mathrm{O}_{4}$ : C, 77.02; H, 5.95; N, $11.02 \%$, found; C, 76.85; H, 5.65; $\mathrm{N}, 10.78 \% ; \lambda_{\max }(\mathrm{DCM} / \mathrm{MeOH}) 260 \mathrm{~nm} @ 0.004 \mathrm{mM}, \varepsilon=309 \mathrm{M}^{-1} \mathrm{~cm}^{-1}$.

\section{Metal complexes of (35) at 1:1 ratio}

All metal ion complexation reactions were performed at room temperature in a solvent mixture of DCM-MeOH at a 1:1 ratio. All reactions used $0.12 \mathrm{mmol}$ of (35) and corresponding metal salt. The solution was stirred for $15 \mathrm{~min}$ and then the solvent was slowly evaporated off. A water-DCM wash was performed to remove any uncomplexed metal and the solvent was removed under reduced pressure.

Compound (35i): Zinc acetate: Yield $=0.085 \mathrm{~g}, 70 \% ; \mathrm{v}_{\max } / \mathrm{cm}^{-1}(\mathrm{KBr}): 3429,2777$, 1621, 1384, 926, 760, 609; $\delta_{\mathrm{H}} / \mathrm{ppm}$ : Conc. of $10^{-3} \mathrm{~mm} ;\left(\mathrm{CDCl}_{3}\right) ; 8.72(\mathrm{~d}, J=5.3$ $\mathrm{Hz}), 8.63(\mathrm{~d}, J=5.2 \mathrm{~Hz}), 8.59(\mathrm{~d}, J=5.2 \mathrm{~Hz}), 7.91(\mathrm{~d}, J=6.6 \mathrm{~Hz}), 7.78(\mathrm{~s}), 7.70(\mathrm{~d}$, $J=3.0 \mathrm{~Hz}), 7.75(\mathrm{t}, J=2.2 \mathrm{~Hz}), 7.55(\mathrm{t}, J=2.1 \mathrm{~Hz}), 7.53(\mathrm{t}, J=2.1 \mathrm{~Hz}), 7.52(\mathrm{t}, J$ = $4.2 \mathrm{~Hz}), 7.47(\mathrm{~s}), 7.40(\mathrm{t}, J=1.4 \mathrm{~Hz}), 7.31(\mathrm{t}, J=1.1 \mathrm{~Hz}), 7.29($ br d), $7.23(\mathrm{~s})$, 7.22 (br t), 7.11 (s), 7.09 (d, $J=7.5 \mathrm{~Hz}), 7.06$ (s), 7.04 (d, $J=3.1 \mathrm{~Hz}), 7.00$ (d, $J=$ $1.2 \mathrm{~Hz}), 6.92(\mathrm{~d}, J=7.04 \mathrm{~Hz}), 6.79(\mathrm{t}, J=8.2 \mathrm{~Hz}), 6.72(\mathrm{t}, J=7.8 \mathrm{~Hz}), 6.68(\mathrm{t}, J=$ $7.8 \mathrm{~Hz}), 5.19$ (s), 4.69 (s), 4.51 (d, $J=15.3 \mathrm{~Hz}), 4.35$ (d, $J=13.3 \mathrm{~Hz}), 4.27$ (s), 4.23 $(\mathrm{d}, J=4.2 \mathrm{~Hz}), 4.21(\mathrm{~d}, J=2.9 \mathrm{~Hz}), 4.20(\mathrm{~s}), 3.47(\mathrm{~s}), 3.45(\mathrm{~d}, J=3.3 \mathrm{~Hz}), 3.43$ (d, $J=4.7 \mathrm{~Hz}$ ), 3.41 (s), 2.17 (s), 1.57 (s); Anal. Calc. for $\mathrm{C}_{69} \mathrm{H}_{66} \mathrm{~N}_{8} \mathrm{O}_{4} \mathrm{Zn}: \mathrm{C}, 69.02 ; \mathrm{H}$, 5.54; N, $9.33 \%$, found; C, 69.00; H, 5.31; N, $9.13 \%$; $\lambda_{\max }(\mathrm{DCM} / \mathrm{MeOH}) 483 \mathrm{~nm} @$ $0.4 \mathrm{mM}, \varepsilon=439 \mathrm{M}^{-1} \mathrm{~cm}^{-1}$.

Compound (35ii): Zinc chloride; Yield $=0.08 \mathrm{~g}, 72 \% ; \mathrm{v}_{\max } / \mathrm{cm}^{-1}(\mathrm{KBr}): 3434$, 2772, 1619, 1466, 1088, 1019, 554; $\delta_{\mathrm{H}} / \mathrm{ppm}$ : Conc. of $10^{-3} \mathrm{~mm}$; $\left(d_{6}\right.$-DMSO); $8.91(\mathrm{~d}$, $J=5.1 \mathrm{~Hz}), 8.80(\mathrm{~d}, J=5.7 \mathrm{~Hz}), 8.74(\mathrm{~d}, J=5.7 \mathrm{~Hz}), 8.66(\mathrm{~d}, J=6.3 \mathrm{~Hz}), 8.57(\mathrm{~d}$, $J=5.1 \mathrm{~Hz}), 8.18(\mathrm{~s}), 8.12(\mathrm{t}, J=7.4 \mathrm{~Hz}), 8.07$ (t, $J=7.4 \mathrm{~Hz}), 7.93$ (br d), 7.85 (d, $J$ $=7.4 \mathrm{~Hz}), 7.72(\mathrm{~d}, J=2.8 \mathrm{~Hz}), 7.71(\mathrm{~d}, J=1.7 \mathrm{~Hz}), 7.70(\mathrm{~s}), 7.69(\mathrm{~d}, J=3.1 \mathrm{~Hz})$, 7.63 (br d), 7.62 (t, $J=2.0 \mathrm{~Hz}), 7.59$ (t, $J=1.2 \mathrm{~Hz}), 7.57(\mathrm{~d}, J=1.0 \mathrm{~Hz}), 7.53$ (t, $J=$ $4.1 \mathrm{~Hz}), 7.46$ (d, $J=0.8 \mathrm{~Hz}), 7.45(\mathrm{t}, J=1.7 \mathrm{~Hz}), 7.44(\mathrm{~d}, J=1.0 \mathrm{~Hz}), 7.19$ (s), 7.17 (s), 7.16 (s), 7.09 (s), 7.07 (s), 7.06 (d, $J=6.4 \mathrm{~Hz}), 6.99$ (s), 6.83 (t, $J=7.8 \mathrm{~Hz}$ ), $6.61(\mathrm{t}, J=8.3 \mathrm{~Hz}), 5.11(\mathrm{~s}), 4.78(\mathrm{~s}), 4.41$ (d, $J=11.7 \mathrm{~Hz}), 4.30$ (s), 4.26 (d, $J=$ $13.3 \mathrm{~Hz}), 4.18(\mathrm{~s}), 4.14(\mathrm{~d}, J=6.6 \mathrm{~Hz})$; Anal. Calc. for $\mathrm{C}_{65} \mathrm{H}_{60} \mathrm{Cl}_{2} \mathrm{~N}_{8} \mathrm{O}_{4} \mathrm{Zn}: \mathrm{C}, 67.68$; H, 5.24; N, $9.71 \%$, found; C, 67.84; H, 4.97; N, $9.46 \%$; $\lambda_{\max }(\mathrm{DCM} / \mathrm{MeOH}) 467 \mathrm{~nm}$ @ $0.4 \mathrm{mM}, \varepsilon=198 \mathrm{M}^{-1} \mathrm{~cm}^{-1}$. 
Compound (35iii): Zinc perchlorate; Yield $=0.10 \mathrm{~g}, 66 \% ; \mathrm{v}_{\max } / \mathrm{cm}^{-1}(\mathrm{KBr}): 3444$, 1624,1089 (perchlorate anion peak), 924, 749, 628; $\delta_{\mathrm{H}} / \mathrm{ppm}$ : Conc. of $10^{-3} \mathrm{~mm} ;\left(d_{6^{-}}\right.$ DMSO); 8.95 (d, $J=6.0 \mathrm{~Hz}$ ), 8.70 (br d), 8.52 (br d), 8.35 (br t), 8.19 (d, $J=1.6$ $\mathrm{Hz}), 8.16(\mathrm{~d}, J=1.4 \mathrm{~Hz}), 8.14(\mathrm{~d}, J=1.6 \mathrm{~Hz}), 8.08(\mathrm{t}, J=7.4 \mathrm{~Hz}), 7.94($ br t $), 7.72$ $(\mathrm{t}, J=6.0 \mathrm{~Hz}), 7.67(\mathrm{~d}, J=7.7 \mathrm{~Hz}), 7.63(\mathrm{~s}), 7.59$ (d, $J=7.1 \mathrm{~Hz}), 7.52$ (d, $J=7.1$ Hz), 7.47 (br d), 7.38 (d, $J=8.2 \mathrm{~Hz}), 7.34$ (s), 7.22 (d, $J=7.1 \mathrm{~Hz}), 7.15$ (d, $J=7.7$ Hz), 7.08 (br d), 7.00 (s), 6.93 (br d), 6.86 (br d), 6.73 (br t), 6.69 (t, $J=7.7 \mathrm{~Hz}$ ), 5.01 (br s), 4.81 (d, $J=4.2 \mathrm{~Hz}), 4.77$ (d, $J=9.2 \mathrm{~Hz}), 4.71(\mathrm{br} \mathrm{s}), 4.75$ (d, $J=10.2$ $\mathrm{Hz}), 4.48$ (br d), 4.35 (s), 4.27 (d, J=6.4 Hz), 4.05 (s), 4.01 (d, J= 5.7 Hz), 3.94 (br d), $3.88(\mathrm{~d}, J=10.7 \mathrm{~Hz}), 3.10(\mathrm{~d}, J=12.8 \mathrm{~Hz})$; Anal. Calc. for $\mathrm{C}_{65} \mathrm{H}_{60} \mathrm{Cl}_{2} \mathrm{~N}_{8} \mathrm{O}_{12} \mathrm{Zn}$ : $\mathrm{C}, 60.92 ; \mathrm{H}, 4.72 ; \mathrm{N}, 8.74 \%$, found; $\mathrm{C}, 60.55 ; \mathrm{H}, 4.52 ; \mathrm{N}, 8.56 \%$; $\lambda_{\max }(\mathrm{DCM} / \mathrm{MeOH}) 478 \mathrm{~nm} @ 0.4 \mathrm{mM}, \varepsilon=2122 \mathrm{M}^{-1} \mathrm{~cm}^{-1}$.

\section{Metal complexes of (35) at $1: 2$ ratio}

Same procedure was used as in the case of the 1:1 with the exception that the ratio of ligand to metal was $0.12 \mathrm{mmol}$ to $0.24 \mathrm{mmol}$.

Compound (35iv): Zinc acetate; Yield $=0.10 \mathrm{~g}, 71 \% ; \mathrm{v}_{\max } / \mathrm{cm}^{-1}(\mathrm{KBr}): 3432$, 2925, 2780, 1607, 1442, 1023, 757; $\delta_{\mathrm{H}} / \mathrm{ppm}$ : Conc. of $10^{-3} \mathrm{~mm} ;\left(\mathrm{CDCl}_{3}\right) ; 8.78(\mathrm{br} \mathrm{d})$, $8.73(\mathrm{~d}, J=4.9 \mathrm{~Hz}), 8.62(\mathrm{~d}, J=5.8 \mathrm{~Hz}), 8.58(\mathrm{~d}, J=6.8 \mathrm{~Hz}), 8.55(\mathrm{~d}, J=5.8 \mathrm{~Hz})$, $8.26(\mathrm{~d}, J=9.8 \mathrm{~Hz}), 7.92(\mathrm{~d}, J=3.2 \mathrm{~Hz}), 7.91(\mathrm{~d}, J=2.1 \mathrm{~Hz}), 7.89(\mathrm{br} \mathrm{t}), 7.88$ (d, $J$ $=2.91 \mathrm{~Hz}), 7.78(\mathrm{~s}), 7.76($ br d), $7.75($ br t), $7.72($ br t), 7.71 (d, $J=1.6 \mathrm{~Hz}), 7.69$ (d, $J=1.9 \mathrm{~Hz}), 7.62(\mathrm{t}, J=5.0 \mathrm{~Hz}), 7.55(\mathrm{br} \mathrm{t}), 7.47(\mathrm{~s}), 7.44(\mathrm{t}, J=5.8 \mathrm{~Hz}), 7.40$ (br t), 7.33 (br t), 7.32 (br d), $7.31(\mathrm{~d}, J=1.5 \mathrm{~Hz}), 7.29$ (d, $J=1.9 \mathrm{~Hz}), 7.22($ br d), 7.18 (d, $J=7.8 \mathrm{~Hz}), 7.11(\mathrm{~s}), 7.09$ (d, $J=2.3 \mathrm{~Hz}), 7.08(\mathrm{~s}), 7.07$ (d, $J=2.6 \mathrm{~Hz}), 7.00(\mathrm{~s})$, $6.91(\mathrm{t}, J=8.5 \mathrm{~Hz}), 6.78(\mathrm{t}, J=7.6 \mathrm{~Hz}), 6.72(\mathrm{t}, J=7.9 \mathrm{~Hz}), 5.19(\mathrm{~s}), 4.51(\mathrm{~d}, J=$ $11.3 \mathrm{~Hz}), 4.35(\mathrm{~d}, J=14.6 \mathrm{~Hz}), 4.25(\mathrm{~d}, J=11.3 \mathrm{~Hz}), 3.4(\mathrm{~s}), 3.45(\mathrm{~d}, J=4.3 \mathrm{~Hz})$, $3.43\left(\mathrm{~d}, J=5.8 \mathrm{~Hz}\right.$ ), 3.41 (s); Anal. Calc. for $\mathrm{C}_{73} \mathrm{H}_{72} \mathrm{~N}_{8} \mathrm{O}_{12} \mathrm{Zn}_{2}: \mathrm{C}, 63.34 ; \mathrm{H}, 5.24 ; \mathrm{N}$, $8.10 \%$, found; C, 63.25; H, 5.03; N, $7.97 \%$; $\lambda_{\max }(\mathrm{DCM} / \mathrm{MeOH}) 483 \mathrm{~nm} @ 0.4 \mathrm{mM}$, $\varepsilon=286 \mathrm{M}^{-1} \mathrm{~cm}^{-1}$.

Compound (35v): Zinc chloride; Yield $=0.09 \mathrm{~g}, 69 \% ; \mathrm{v}_{\max } / \mathrm{cm}^{-1}(\mathrm{KBr}): 3437$, 2785, 1609, 1464, 755; $\delta_{\mathrm{H}} / \mathrm{ppm}$ : Conc. of $10^{-3} \mathrm{~mm}$; $\left(d_{6}\right.$-DMSO); $8.92(\mathrm{~d}, J=6.7 \mathrm{~Hz})$, $8.81(\mathrm{~d}, J=6.7 \mathrm{~Hz}), 8.74(\mathrm{~d}, J=5.3 \mathrm{~Hz}), 8.66(\mathrm{~d}, J=4.6 \mathrm{~Hz}), 8.57(\mathrm{~d}, J=3.3 \mathrm{~Hz})$, 8.54 (s), 8.19 (s), 8.15 (s), 8.05 (t, $J=8.6 \mathrm{~Hz}), 7.93(\mathrm{~d}, J=9.7 \mathrm{~Hz}), 7.85$ (t, $J=7.5$ 
Hz), 7.73 (s), 7.72 (d, $J=1.5 \mathrm{~Hz}), 7.71$ (s), 7.70 (d, $J=1.5 \mathrm{~Hz}), 7.69$ (br d), 7.68 (d, $J=3.5 \mathrm{~Hz}), 7.62(\mathrm{t}, J=4.3 \mathrm{~Hz}), 7.57(\mathrm{~d}, J=7.9 \mathrm{~Hz}), 7.45(\mathrm{t}, J=6.7 \mathrm{~Hz}), 7.17(\mathrm{t}, J$ = $7.0 \mathrm{~Hz}), 7.09$ (s), $7.06(\mathrm{~d}, J=7.0 \mathrm{~Hz}), 7.01$ (br d), 6.99 (s), 6.82 (t, $J=5.4 \mathrm{~Hz})$, $6.61(\mathrm{t}, J=7.7 \mathrm{~Hz}), 5.11(\mathrm{~s}), 4.78$ (s), 4.40 (br d), 4.28 (s), 4.25 (d, $J=13.6 \mathrm{~Hz}$ ), $4.18(\mathrm{~s}), 4.15(\mathrm{~d}, J=1.4 \mathrm{~Hz}), 4.14(\mathrm{~d}, J=5.5 \mathrm{~Hz})$; Anal. Calc. for $\mathrm{C}_{65} \mathrm{H}_{60} \mathrm{Cl}_{4} \mathrm{~N}_{8} \mathrm{O}_{4} \mathrm{Zn}_{2}: \mathrm{C}, 60.53 ; \mathrm{H}, 4.69 ; \mathrm{N}, 8.69 \%$, found; $\mathrm{C}, 60.43 ; \mathrm{H}, 4.37 ; \mathrm{N}, 8.51$ $\% ; \lambda_{\max }(\mathrm{DCM} / \mathrm{MeOH}) 426 \mathrm{~nm} @ 0.4 \mathrm{mM}, \varepsilon=2002 \mathrm{M}^{-1} \mathrm{~cm}^{-1}$.

Compound (36iii): Zinc perchlorate; Yield $=0.11 \mathrm{~g}, 57 \% ; \mathrm{v}_{\max } / \mathrm{cm}^{-1}(\mathrm{KBr}): 3504$, 2776, 1619, 1468, 1082 (perchlorate anion peak), 925, 760, 625; $\delta_{\mathrm{H}} / \mathrm{ppm}$ : Conc. of $10^{-3} \mathrm{~mm} ;\left(d_{6}\right.$-DMSO); $8.91(\mathrm{~d}, J=5.0 \mathrm{~Hz}), 8.75(\mathrm{~d}, J=5.0 \mathrm{~Hz}), 8.66(\mathrm{~d}, J=4.0$ $\mathrm{Hz}), 8.54$ (s), 8.18 (s), 8.11 (s), 8.06 (t, $J=1.4 \mathrm{~Hz}), 7.92$ (br d), 7.85 (d, $J=9.2 \mathrm{~Hz}$ ), $7.72(\mathrm{t}, J=1.6 \mathrm{~Hz}), 7.71(\mathrm{~s}), 7.71(\mathrm{~d}, J=1.6 \mathrm{~Hz}), 7.70(\mathrm{~d}, J=2.4 \mathrm{~Hz}), 7.68(\mathrm{~d}, J=$ $2.9 \mathrm{~Hz}), 7.61(\mathrm{~d}, J=5.3 \mathrm{~Hz}), 7.60(\mathrm{~d}, J=2.2 \mathrm{~Hz}), 7.57(\mathrm{~d}, J=7.9 \mathrm{~Hz}), 7.54(\mathrm{t}, J=$ $1.8 \mathrm{~Hz}), 7.47(\mathrm{~d}, J=10.0 \mathrm{~Hz}), 7.45(\mathrm{t}, J=1.5 \mathrm{~Hz}), 7.44(\mathrm{br} \mathrm{d}), 7.17$ (s), 7.16 (d, $J=$ $7.1 \mathrm{~Hz}), 7.09$ (d, $J=3.4 \mathrm{~Hz}), 7.08$ (d, $J=3.1 \mathrm{~Hz}), 7.06$ (d, $J=6.8 \mathrm{~Hz}) 7.04$ (s), 7.01 $(\mathrm{d}, J=1.8 \mathrm{~Hz}), 6.99(\mathrm{~s}), 6.87(\mathrm{t}, J=8.6 \mathrm{~Hz}), 6.83(\mathrm{t}, J=7.7 \mathrm{~Hz}), 6.61(\mathrm{t}, J=8.1$ $\mathrm{Hz}), 5.11(\mathrm{~s}), 4.78(\mathrm{~s}), 4.41(\mathrm{~d}, J=13.1 \mathrm{~Hz}), 4.26(\mathrm{~d}, J=13.4 \mathrm{~Hz}), 4.18(\mathrm{~s}), 4.14(\mathrm{~d}$, $J=5.6 \mathrm{~Hz}$ ); Anal. Calc. for $\mathrm{C}_{65} \mathrm{H}_{60} \mathrm{Cl}_{4} \mathrm{~N}_{8} \mathrm{O}_{20} \mathrm{Zn}_{2}: \mathrm{C}, 50.50 ; \mathrm{H}, 3.91 ; \mathrm{N}, 7.25 \%$, found; C, 50.48; H, 3.75; N, 7.01\%; $\lambda_{\max }(\mathrm{DCM} / \mathrm{MeOH}) 469 \mathrm{~nm} @ 0.4 \mathrm{mM}, \varepsilon=$ $1185 \mathrm{M}^{-1} \mathrm{~cm}^{-1}$. 


\subsection{1,23-Di[N-(2'pyridylethyl)aminomethyl]-25,27-di(methyl-2-}

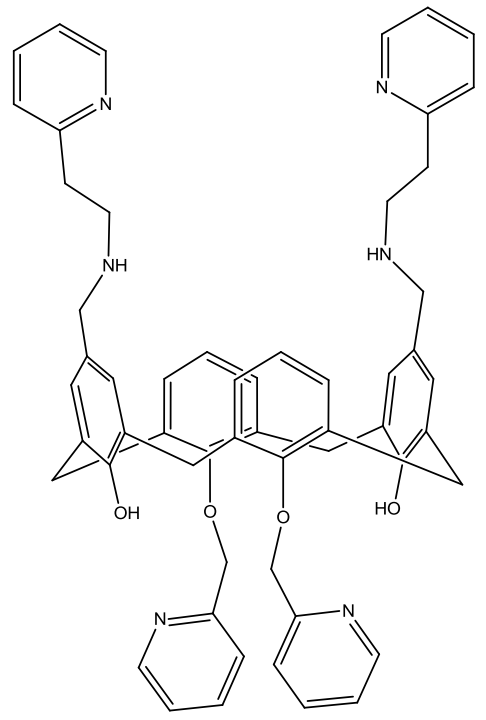

Compound (34) (1.24 g, $1.56 \mathrm{mmol})$, triethylamine $(0.32 \mathrm{~g}, 3.12 \mathrm{mmol})$ and 2aminoethyl- pyridine $(0.37 \mathrm{~mL}, 3.12 \mathrm{mmol})$ were refluxed in $\mathrm{CH}_{3} \mathrm{CN}: \mathrm{CH}_{3} \mathrm{Cl}(60: 20$ $\mathrm{mL}$ ) under nitrogen gas overnight. The solution was then allowed to cool to room temperature. The inorganic salts were removed by filtration and the volatiles removed under reduced pressure. The residue was then washed with DCM and water to remove any excess triethylamine and the solvents were removed under reduced pressure to afford a brown-red waxy solid (36).

Yield $=1.20 \mathrm{~g}, 63 \% ; \mathrm{v}_{\max } / \mathrm{cm}^{-1}(\mathrm{KBr}): 3404,2916,1591,1435,1087,752 ; \delta_{\mathrm{H}} / \mathrm{ppm}:$ Conc. of $10^{-3} \mathrm{~mm}$; $\left(\mathrm{CDCl}_{3}\right) ; 8.62$ (d, $\left.2 \mathrm{H}, \mathrm{Py}-\underline{\mathrm{H}}, J=4.6 \mathrm{~Hz}\right), 8.51$ (d, $2 \mathrm{H}, \mathrm{Py}-\underline{\mathrm{H}}, J=$ $5.3 \mathrm{~Hz}), 7.75(\mathrm{t}, 2 \mathrm{H}, \mathrm{Py}-\underline{\mathrm{H}}, J=8.3 \mathrm{~Hz}), 7.63(\mathrm{~d}, 2 \mathrm{H}, \mathrm{Py}-\underline{\mathrm{H}}, J=7.6 \mathrm{~Hz}), 7.59(\mathrm{t}, 2 \mathrm{H}$, Py- $\underline{\mathrm{H}}, J=6.4 \mathrm{~Hz}), 7.41$ (t, 2H, Py- $\underline{\mathrm{H}}, J=6.4 \mathrm{~Hz}), 7.08(\mathrm{~d}, 4 \mathrm{H}, \mathrm{Ar}-\underline{\mathrm{H}}, J=7.3 \mathrm{~Hz})$, 7.06 (t, 2H, Py- $\underline{\mathrm{H}}, J=7.3 \mathrm{~Hz}$ ), 7.04 (d, 2H, Py- $\underline{\mathrm{H}}, J=7.3 \mathrm{~Hz}$ ), 6.99 (d, 4H, Ar- $\underline{\mathrm{H}}, J=$ $6.8 \mathrm{~Hz}$ ), 6.89 (s, $2 \mathrm{H}, \mathrm{Ar}-\underline{\mathrm{H}}), 6.62$ (t, $2 \mathrm{H}, \mathrm{Ar}-\underline{\mathrm{H}}, J=7.7 \mathrm{~Hz}), 5.14$ (s, 4H, O-C $\left.\underline{\mathrm{H}}_{2}-\mathrm{Py}\right)$, 4.36 (br d, $4 \mathrm{H}, \underline{\mathrm{CH}}_{2}, J=13.3 \mathrm{~Hz}$ ), 3.94 (s, $\underline{\mathrm{C}}_{2}-\mathrm{NHR}$ ), 3.33 (br d, $4 \mathrm{H}, \mathrm{C}_{2}, J=13.9$ $\mathrm{Hz}$ ), 3.04 (t, $\left.4 \mathrm{H}, \mathrm{N}-\mathrm{CH}_{2} \underline{\mathrm{CH}}_{2}-\mathrm{Py}, J=6.6 \mathrm{~Hz}\right), 2.94$ (t, $\mathrm{NC}_{2} \mathrm{CH}_{2}-\mathrm{Py}, J=6.8 \mathrm{~Hz}$ ); $\delta_{\mathrm{c}}$ ppm $\left(\mathrm{CDCl}_{3}\right) ; 160.4,159.6,156.1,153.5,149.4,149.5,136.6,136.5,133.7$, 129.9, 128.5, 128.1, 127.8, 125.5, 123.9, 123.5, 123.3, 122.9, 78.7, 55.0, 53.6, 38.8, 31.3; Anal. Calc. for $\mathrm{C}_{56} \mathrm{H}_{54} \mathrm{~N}_{6} \mathrm{O}_{4}$ : C, 76.86; $\mathrm{H}, 6.22 ; \mathrm{N}, 9.60 \%$, found; C, 76.52; $\mathrm{H}$, 6.14; N, $9.12 \% ; \lambda_{\max }(\mathrm{DCM} / \mathrm{MeOH}) 261 \mathrm{~nm} @ 0.001 \mathrm{mM}, \varepsilon=92 \mathrm{M}^{-1} \mathrm{~cm}^{-1}$. 


\section{Metal complexes of (36) at 1:1 ratio}

All metal ion complexation reactions were performed at room temperature in a solvent mixture of $\mathrm{DCM}-\mathrm{MeOH}$ at a 1:1 ratio. All reactions used $0.01 \mathrm{mmol}$ of (36) and corresponding metal salt. The solution was stirred for $15 \mathrm{~min}$ and then the solvent was slowly evaporated off. A water-DCM wash was performed to remove any uncomplexed metal and the solvent was removed under reduced pressure.

Compound (36i): Zinc acetate; Yield $=0.01 \mathrm{~g}, 78 \% ; \mathrm{v}_{\max } / \mathrm{cm}^{-1}(\mathrm{KBr}): 3429,2922$, 1591, 1466, 1385, 1088, 756; $\delta_{\mathrm{H}} / \mathrm{ppm}$ : Conc. of $10^{-3} \mathrm{~mm} ;\left(\mathrm{CDCl}_{3}\right) ; 8.75$ (br d), 8.72 (br d), 8.63 (d, $J=5.4 \mathrm{~Hz}$ ), 8.58 (br d), 8.53 (br d), 8.23 (d, $J=8.7 \mathrm{~Hz}), 8.16$ (br t), 7.89 (br t), 7.75 (s), 7.69 (t, $J=7.2 \mathrm{~Hz}), 7.60($ br d), 7.54 (d, $J=7.6 \mathrm{~Hz}), 7.37$ (br t), $7.31(\mathrm{~d}, J=6.5 \mathrm{~Hz}), 7.21(\mathrm{~s}), 7.17(\mathrm{~d}, J=7.9 \mathrm{~Hz}), 7.07(\mathrm{t}, J=8.8 \mathrm{~Hz}), 7.01(\mathrm{~s}), 6.97$ $(\mathrm{d}, J=6.6 \mathrm{~Hz}), 6.90(\mathrm{~d}, J=7.5 \mathrm{~Hz}), 6.78(\mathrm{t}, J=7.5 \mathrm{~Hz}), 6.65(\mathrm{br} \mathrm{t}), 5.19$ (s), 4.51 (d, $J=12.4 \mathrm{~Hz}), 4.35(\mathrm{~d}, J=12.4 \mathrm{~Hz}), 4.25(\mathrm{~d}, J=12.5 \mathrm{~Hz}), 3.73(\mathrm{~s}), 3.44(\mathrm{br} \mathrm{d}), 3.39$ (br d), $3.24(\mathrm{br}$ d), $3.11(\mathrm{t}, J=6.9 \mathrm{~Hz}), 3.02(\mathrm{t}, J=6.2 \mathrm{~Hz})$; Anal. Calc. for $\mathrm{C}_{60} \mathrm{H}_{60} \mathrm{~N}_{6} \mathrm{O}_{8} \mathrm{Zn}: \mathrm{C}, 68.08 ; \mathrm{H}, 5.71 ; \mathrm{N}, 7.94 \%$, found; C, 68.03; H, 5.54; N, 7.81\%; $\lambda_{\max }(\mathrm{DCM} / \mathrm{MeOH}) 459 \mathrm{~nm} @ 1.0 \mathrm{mM}, \varepsilon=659 \mathrm{M}^{-1} \mathrm{~cm}^{-1}$.

Compound (36ii): Zinc chloride; Yield $=0.008 \mathrm{~g}, 72 \% ; \mathrm{v}_{\max } / \mathrm{cm}^{-1}(\mathrm{KBr}): 3430$, 1607, 1570, 1440, 1155, 1088, 757; $\delta_{\mathrm{H}} / \mathrm{ppm}$ : Conc. of $10^{-3} \mathrm{~mm}$; $\left(d_{6}\right.$-DMSO); 9.00 (d, $J=6.7 \mathrm{~Hz}$ ), 8.95 (d, $J=4.5 \mathrm{~Hz}), 8.84$ (br d), 8.70 (br d), 8.68 (br t), 8.63 (d, $J=4.5$ $\mathrm{Hz}$ ), 8.57 (br d), 8.48 (br d), 8.24 (s), 8.13 (br t), 8.06 (br d), 8.04 (t, $J=1.9 \mathrm{~Hz}$ ), $7.97(\mathrm{t}, J=8.4 \mathrm{~Hz}), 7.90(\mathrm{~s}), 7.87(\mathrm{~d}, J=1.4 \mathrm{~Hz}), 7.84(\mathrm{~d}, J=1.8 \mathrm{~Hz}), 7.82(\mathrm{~d}, J=$ $1.6 \mathrm{~Hz}), 7.80$ (d, $J=2.2 \mathrm{~Hz}), 7.61(\mathrm{t}, J=8.7 \mathrm{~Hz}), 7.54(\mathrm{br} \mathrm{t}), 7.34$ (s), 7.37 (br d), 7.33(s), $7.30(\mathrm{~s}), 7.21(\mathrm{td}, J=6.7,2.8 \mathrm{~Hz}), 7.14(\mathrm{~s}), 7.09$ (d, $J=7.9 \mathrm{~Hz}), 6.99(\mathrm{~d}, J=$ $7.3 \mathrm{~Hz}), 6.92(\mathrm{~d}, J=7.3 \mathrm{~Hz}), 6.85(\mathrm{t}, J=5.6 \mathrm{~Hz}), 6.66(\mathrm{~d}, J=7.1 \mathrm{~Hz}), 6.61(\mathrm{~d}, J=$ $7.6 \mathrm{~Hz}), 6.58(\mathrm{~s}), 6.46$ (t, $J=7.6 \mathrm{~Hz}), 6.37$ (t, $J=7.6 \mathrm{~Hz}), 5.15$ (s), 4.78 (br s), 4.45 (d, $J=12.2 \mathrm{~Hz}), 4.34(\mathrm{~s}), 4.27(\mathrm{~d}, J=14.5 \mathrm{~Hz}), 4.19(\mathrm{~s}), 4.12(\mathrm{~d}, J=6.1 \mathrm{~Hz}), 4.03$ (d, $J=8.6 \mathrm{~Hz}), 3.93(\mathrm{~s}), 3.88(\mathrm{br} \mathrm{t}), 3.72(\mathrm{~d}, J=7.68), 3.68(\mathrm{~s}), 3.55(\mathrm{~s}), 3.50(\mathrm{~s})$, 3.46 (d, $J=6.14), 3.33(\mathrm{~s}), 3.27(\mathrm{~d}, J=7.2 \mathrm{~Hz}), 3.22(\mathrm{t}, J=7.0 \mathrm{~Hz}), 3.11(\mathrm{t}, J=7.2$ $\mathrm{Hz}$ ), 2.96 (t, $J=7.2 \mathrm{~Hz}$ ); Anal. Calc. for $\mathrm{C}_{56} \mathrm{H}_{54} \mathrm{Cl}_{2} \mathrm{~N}_{6} \mathrm{O}_{4} \mathrm{Zn}: \mathrm{C}, 66.50 ; \mathrm{H}, 5.38 ; \mathrm{N}$, $8.31 \%$, found; C, 66.45; H, 5.24; N, $8.28 \% ; \lambda_{\max }(\mathrm{DCM} / \mathrm{MeOH}) 452 \mathrm{~nm} @ 1.0 \mathrm{mM}$, $\varepsilon=97 \mathrm{M}^{-1} \mathrm{~cm}^{-1}$. 
Compound (36iii): Zinc perchlorate; Yield $=0.009 \mathrm{~g}, 49 \% ; \mathrm{v}_{\max } / \mathrm{cm}^{-1}(\mathrm{KBr}): 3429$, 2928, 1647, 1610, 1571, 1446, 1094 (perchlorate anion peak), 760; $\delta_{\mathrm{H}} / \mathrm{ppm}$ : Conc. of $10^{-3} \mathrm{~mm}$; $\left(d_{6}\right.$-DMSO); $8.73(\mathrm{~d}, J=5.9 \mathrm{~Hz}), 8.66(\mathrm{~d}, J=5.9 \mathrm{~Hz}), 8.51(\mathrm{br} \mathrm{d}), 8.30(\mathrm{~s})$, 8.16 (s), 8.04 (br d), 7.99 (d, $J=6.6 \mathrm{~Hz}), 7.91(\mathrm{~d}, J=8.1 \mathrm{~Hz}), 7.84$ (d, $J=6.6 \mathrm{~Hz})$, $7.77(\mathrm{~d}, J=8.1 \mathrm{~Hz}), 7.71(\mathrm{br} \mathrm{d}), 7.57(\mathrm{~d}, J=8.9 \mathrm{~Hz}), 7.52(\mathrm{t}, J=6.8 \mathrm{~Hz}), 7.45(\mathrm{t}, J=$ $6.8 \mathrm{~Hz}), 7.33(\mathrm{~d}, J=8.2 \mathrm{~Hz}), 7.28(\mathrm{br} \mathrm{t}), 7.17$ (d, $J=4.7 \mathrm{~Hz}), 7.15(\mathrm{~d}, J=4.7 \mathrm{~Hz})$, $7.09(\mathrm{~d}, J=1.7 \mathrm{~Hz}), 7.06(\mathrm{~d}, J=2.5 \mathrm{~Hz}), 7.03(\mathrm{~d}, J=2.5 \mathrm{~Hz}), 6.97(\mathrm{~d}, J=6.9 \mathrm{~Hz})$, $6.88(\mathrm{~d}, J=8.2 \mathrm{~Hz}), 6.81(\mathrm{t}, J=6.4 \mathrm{~Hz}), 6.60(\mathrm{t}, J=8.2 \mathrm{~Hz}), 6.47(\mathrm{t}, J=8.2 \mathrm{~Hz})$, $5.26(\mathrm{~s}), 5.10(\mathrm{~s}), 4.40(\mathrm{~d}, J=11.8 \mathrm{~Hz}), 4.26(\mathrm{~d}, J=13.1 \mathrm{~Hz}), 4.16(\mathrm{~d}, J=13.8 \mathrm{~Hz})$, $3.51(\mathrm{~s}), 3.45$ (d, $J=5.3 \mathrm{~Hz}), 3.05(\mathrm{t}, J=8.0 \mathrm{~Hz}), 2.88(\mathrm{t}, J=7.1 \mathrm{~Hz}), 2.73(\mathrm{t}, J=1.9$ $\mathrm{Hz}$ ); Anal. Calc. for $\mathrm{C}_{56} \mathrm{H}_{54} \mathrm{Cl}_{2} \mathrm{~N}_{6} \mathrm{O}_{12} \mathrm{Zn}: \mathrm{C}, 59.03 ; \mathrm{H}, 4.78 ; \mathrm{N}, 7.38 \%$, found; $\mathrm{C}$, 58.64; H, 4.75; N, $7.32 \%$; $\lambda_{\max }(\mathrm{DCM} / \mathrm{MeOH}) 465 \mathrm{~nm} @ 1.0 \mathrm{mM}, \varepsilon=578 \mathrm{M}^{-1} \mathrm{~cm}^{-1}$.

\section{Metal complexes of (36) at 1:2 ratio}

Same procedure was used as in the case of the 1:1 with the exception that the ratio of ligand to metal was $0.01 \mathrm{mmol}$ to $0.02 \mathrm{mmol}$.

Compound (36iv): Zinc acetate; Yield $=0.009 \mathrm{~g}, 75 \% ; \mathrm{v}_{\max } / \mathrm{cm}^{-1}(\mathrm{KBr}): 3426$, 2926, 1592, 1441, 1386, 1021, 755; $\delta_{\mathrm{H}} / \mathrm{ppm}$ : Conc. of $10^{-3} \mathrm{~mm} ;\left(\mathrm{CDCl}_{3}\right) ; 8.77(\mathrm{~d}, J=$ $5.3 \mathrm{~Hz}), 8.72(\mathrm{~d}, J=5.3 \mathrm{~Hz}), 8.63(\mathrm{~d}, J=4.8 \mathrm{~Hz}), 8.53(\mathrm{~d}, J=4.8 \mathrm{~Hz}), 8.23(\mathrm{~d}, J=$ $8.2 \mathrm{~Hz}), 8.16(\mathrm{~s}), 7.87(\mathrm{~d}, J=8.4 \mathrm{~Hz}), 7.82$ (t, $J=7.4 \mathrm{~Hz}), 7.77$ (s), 7.69 (td, $J=1.9$, 1.6, 1.7 Hz), 7.61 (d, $J=7.7 \mathrm{~Hz}), 7.56$ (d, $J=7.7 \mathrm{~Hz}), 7.42$ (s), 7.30 (br d), 7.22 (d, $J$ = $6.5 \mathrm{~Hz}), 7.09(\mathrm{~s}), 7.03(\mathrm{~d}, J=7.7 \mathrm{~Hz}), 6.98(\mathrm{~d}, J=7.0 \mathrm{~Hz}) 6.90(\mathrm{~d}, J=8.0 \mathrm{~Hz})$, $6.62(\mathrm{t}, J=8.5 \mathrm{~Hz}), 5.19(\mathrm{~s}), 4.51(\mathrm{~d}, J=13.9 \mathrm{~Hz}), 4.36(\mathrm{~d}, J=12.6 \mathrm{~Hz}), 4.25(\mathrm{~d}, J=$ $13.9 \mathrm{~Hz}), 3.72(\mathrm{t}, J=6.6 \mathrm{~Hz}), 3.47(\mathrm{~d}, J=5.9 \mathrm{~Hz}), 3.44(\mathrm{~d}, J=4.3 \mathrm{~Hz}), 3.14(\mathrm{~d}, J=$ 3.96), $3.29(\mathrm{t}, J=9.2 \mathrm{~Hz}), 3.14(\mathrm{t}, J=8.5 \mathrm{~Hz}), 3.01(\mathrm{t}, J=5.9 \mathrm{~Hz})$; Anal. Calc. for $\mathrm{C}_{64} \mathrm{H}_{66} \mathrm{~N}_{6} \mathrm{O}_{12} \mathrm{Zn}_{2}: \mathrm{C}, 61.89 ; \mathrm{H}, 5.36 ; \mathrm{N}, 6.77 \%$, found; C, 61.76; H, 5.22; N, $6.68 \%$; $\lambda_{\max }(\mathrm{DCM} / \mathrm{MeOH}) 459 \mathrm{~nm} @ 1.0 \mathrm{mM}, \varepsilon=659 \mathrm{M}^{-1} \mathrm{~cm}^{-1}$.

Compound (36v): Zinc Chloride; Yield $=0.01 \mathrm{~g}, 76 \% ; \mathrm{v}_{\max } / \mathrm{cm}^{-1}(\mathrm{KBr}): 3437$, 2922, 1609, 1592, 1466, 1194, 755; $\delta_{\mathrm{H}} / \mathrm{ppm}$ : Conc. of $10^{-3} \mathrm{~mm} ;\left(d_{6}\right.$-DMSO); 8.96 (d, $J=5.5 \mathrm{~Hz}), 8.66(\mathrm{~d}, J=5.5 \mathrm{~Hz}), 8.63(\mathrm{~d}, J=4.7 \mathrm{~Hz}), 8.58(\mathrm{~d}, J=4.7 \mathrm{~Hz}), 8.52(\mathrm{~d}, J$ = $6.3 \mathrm{~Hz}), 8.39$ (d, $J=5.5 \mathrm{~Hz}), 8.32$ (s), 8.19 (s), 8.02 (d, J=7.8 Hz), 7.98 (s), 7.92 (d, $J=8.2 \mathrm{~Hz}), 7.88$ (br d), 7.79 (d, $J=7.4 \mathrm{~Hz}), 7.76$ (d, $J=8.2 \mathrm{~Hz}), 7.71$ (t, $J=2.8$ $\mathrm{Hz}), 7.59(\mathrm{t}, J=6.0 \mathrm{~Hz}), 7.52(\mathrm{~d}, J=8.4 \mathrm{~Hz}), 7.45$ (t, $J=7.2 \mathrm{~Hz}), 7.40$ (br t), 7.35 
$(\mathrm{d}, J=8.4 \mathrm{~Hz}), 7.31(\mathrm{~s}), 7.28(\mathrm{~d}, J=9.6 \mathrm{~Hz}), 7.23(\mathrm{t}, J=7.2 \mathrm{~Hz}), 7.16(\mathrm{~d}, J=9.6$ $\mathrm{Hz}), 7.06(\mathrm{~d}, J=6.0 \mathrm{~Hz}), 7.01(\mathrm{~d}, J=7.2 \mathrm{~Hz}), 6.88(\mathrm{~d}, J=9.3 \mathrm{~Hz}), 6.86$ (s), 6.82 (d, $J=7.7 \mathrm{~Hz}), 6.77(\mathrm{~d}, J=8.2 \mathrm{~Hz}), 6.61(\mathrm{t}, J=6.6 \mathrm{~Hz}), 6.57(\mathrm{t}, J=7.1 \mathrm{~Hz}), 6.39(\mathrm{t}, J=$ $6.0 \mathrm{~Hz}), 6.36(\mathrm{t}, J=8.2 \mathrm{~Hz}), 5.11(\mathrm{~s}), 4.91(\mathrm{~s}), 4.46(\mathrm{~d}, J=12.9 \mathrm{~Hz}), 4.26(\mathrm{~d}, J=11.6$ $\mathrm{Hz}), 4.10$ (s), 4.08 (s), 3.75 (t, $J=7.0 \mathrm{~Hz}), 3.68$ (t, $J=8.0 \mathrm{~Hz}), 3.60$ (d, $J=7.0 \mathrm{~Hz})$, $3.46(\mathrm{~d}, J=12.6 \mathrm{~Hz}), 3.21(\mathrm{t}, J=8.0 \mathrm{~Hz}), 3.15(\mathrm{~s}), 3.12(\mathrm{~s}), 3.05(\mathrm{t}, J=9.6 \mathrm{~Hz})$, $2.99(\mathrm{t}, J=7.3 \mathrm{~Hz}), 2.88(\mathrm{t}, J=6.6 \mathrm{~Hz})$; Anal. Calc. for $\mathrm{C}_{56} \mathrm{H}_{54} \mathrm{Cl}_{2} \mathrm{~N}_{6} \mathrm{O}_{4} \mathrm{Zn}_{2}: \mathrm{C}$, $58.61 ; \mathrm{H}, 4.74 ; \mathrm{N}, 7.32 \%$, found; C, 58.57; H, 4.86; N, $7.31 \% ; \lambda_{\max }(\mathrm{DCM} / \mathrm{MeOH})$ $465 \mathrm{~nm} @ 1.0 \mathrm{mM}, \varepsilon=64 \mathrm{M}^{-1} \mathrm{~cm}^{-1}$.

Compound (36vi): Zinc perchlorate; Yield $=0.011 \mathrm{~g}, 73 \% ; \mathrm{v}_{\max } / \mathrm{cm}^{-1}(\mathrm{KBr}): 3428$, 2928, 1610, 1466, 1092 (perchlorate anion peak), 900, 757; $\delta_{\mathrm{H}} / \mathrm{ppm}$ : Conc. of $10^{-3}$ mm; $\left(d_{6}\right.$-DMSO); $8.75(\mathrm{~d}, J=5.1 \mathrm{~Hz}), 8.66(\mathrm{~d}, J=5.1 \mathrm{~Hz}), 8.54(\mathrm{~s}), 8.18(\mathrm{~s}), 8.11$ (s), $8.03(\mathrm{t}, J=2.9 \mathrm{~Hz}), 7.92(\mathrm{~d}, J=8.1 \mathrm{~Hz}), 7.72(\mathrm{t}, J=1.1 \mathrm{~Hz}), 7.71(\mathrm{~s}), 7.71(\mathrm{~d}, J$ $=1.6 \mathrm{~Hz}), 7.69$ (d, $J=2.6 \mathrm{~Hz}), 7.68(\mathrm{~d}, J=3.4 \mathrm{~Hz}), 7.62(\mathrm{~d}, J=8.3 \mathrm{~Hz}), 7.56(\mathrm{~s})$, 7.55 (t, $J=1.8 \mathrm{~Hz}$ ), 7.53 (br t), 7.46 (br d), 7.45 (br d), 7.44 (br d), 7.19 (s), 7.16 (d, $J=7.3 \mathrm{~Hz}), 7.09$ (s), $7.06(\mathrm{~d}, J=7.3 \mathrm{~Hz}), 6.83(\mathrm{t}, J=7.9 \mathrm{~Hz}), 6.61(\mathrm{t}, J=7.9 \mathrm{~Hz})$, 5.26 (s), 5.11 (s), 4.78 (s), 4.40 (br d), 4.30 (s), 4.26 (d, J = 12.2 Hz), 4.18 (s), 4.14 $(\mathrm{d}, J=6.6 \mathrm{~Hz})$; Anal. Calc. for $\mathrm{C}_{56} \mathrm{H}_{54} \mathrm{Cl}_{4} \mathrm{~N}_{6} \mathrm{O}_{20} \mathrm{Zn}_{2}: \mathrm{C}, 47.92 ; \mathrm{H}, 3.88 ; \mathrm{N}, 5.99 \%$, found; C, 47.68; H, 3.89; N, $5.64 \%$; $\lambda_{\max }(\mathrm{DCM} / \mathrm{MeOH}) 460 \mathrm{~nm} @ 1.0 \mathrm{mM}, \varepsilon=64$ $\mathrm{M}^{-1} \mathrm{~cm}^{-1}$. 


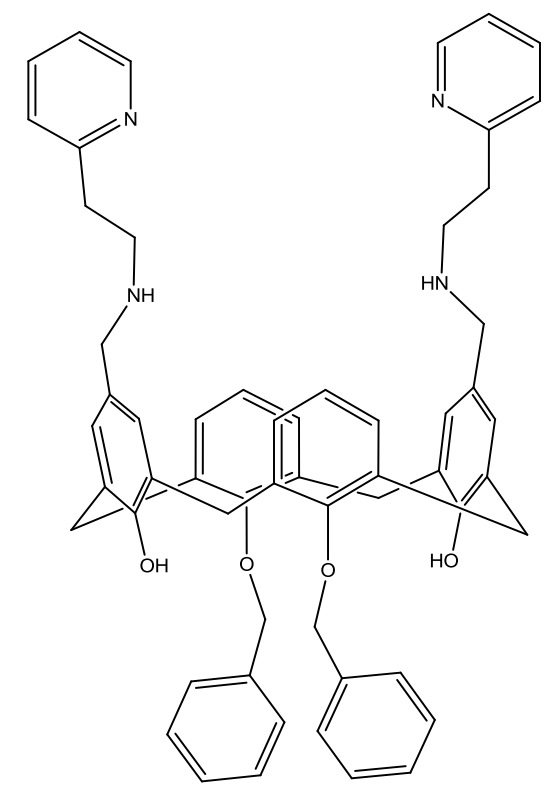

Compound (30) $(0.5 \mathrm{~g}, 0.71 \mathrm{mmol})$, triethylamine $(0.14 \mathrm{~g}, 1.42 \mathrm{mmol})$ and 2aminoethyl pyridine $(0.17 \mathrm{~g}, 1.42 \mathrm{mmol})$ in DCM $(60 \mathrm{~mL})$ were refluxed for two days under nitrogen gas. The solution was cooled to room temperature, the inorganic salts were removed by filtration and solvent was removed under reduced pressure to give a dark yellow waxy solid (37).

Yield $=1.18 \mathrm{~g}, 98 \% ; \mathrm{v}_{\max } / \mathrm{cm}^{-1}(\mathrm{KBr}): 3394,2926,1591,1464,1088,976,765 ;$ $\delta_{\mathrm{H}} / \mathrm{ppm}$ : Conc. of $10^{-3} \mathrm{~mm} ;\left(\mathrm{CDCl}_{3}\right) ; 8.53(\mathrm{~d}, 2 \mathrm{H}, \mathrm{Py}-\underline{\mathrm{H}}, J=3.6 \mathrm{~Hz}), 7.70(\mathrm{~d}, 2 \mathrm{H}$, Bn- $\underline{\mathrm{H}}, J=6.6 \mathrm{~Hz}$ ), 7.57 (t, 2H, Py- $\underline{\mathrm{H}}, J=7.1 \mathrm{~Hz}), 7.49$ (t, 2H, Py- $\underline{\mathrm{H}}, J=8.5 \mathrm{~Hz}$ ), 7.46 (d, 4H, Py- $\underline{\mathrm{H}}, J=4.6 \mathrm{~Hz}$ ), 7.43 (t, 4H, Bn- $\underline{\mathrm{H}}, J=6.7 \mathrm{~Hz}$ ), 7.37 (t, 2H, Bn- $\underline{\mathrm{H}}, J=$

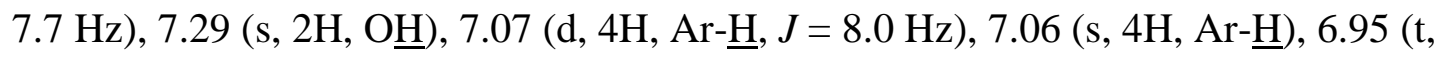
$2 \mathrm{H}, \mathrm{Ar}-\underline{\mathrm{H}}, J=7.6 \mathrm{~Hz}$ ), 5.14 (s, $\left.4 \mathrm{H}, \mathrm{C}_{2}-\mathrm{Bn}\right), 4.79$ (s, $\left.4 \mathrm{H}, \mathrm{C}_{2}-\mathrm{NH}\right), 4.23$ (d, $4 \mathrm{H}$, $\mathrm{C}_{2}, J=13.3 \mathrm{~Hz}$ ), 3.34 (d, 4H, $\left.\underline{\mathrm{C}}_{2}, J=13.3 \mathrm{~Hz}\right), 3.07$ (t, $4 \mathrm{H}, \mathrm{NH}_{-} \underline{\mathrm{C}}_{2}, J=6.4 \mathrm{~Hz}$ ), 2.99 (t, 4H, NH-CH $\left.{ }_{2}-\mathrm{CH}_{2}, J=8.4 \mathrm{~Hz}\right), 1.78($ br s, $2 \mathrm{H}, \mathrm{N} \underline{\mathrm{H}}) ; \delta_{\mathrm{C}} / \mathrm{ppm}\left(\mathrm{CDCl}_{3}\right) ; 158.6$, 154.1, 152.3, 149.2, 135.6, 135.4, 133.4, 129.9, 128.5, 128.3, 127.6, 127.5, 127.4, 125.0, 123.7, 74.2, 53.4, 48.8, 36.0, 31.0. ESI-HRMS: calcd. for $\mathrm{C}_{58} \mathrm{H}_{56} \mathrm{~N}_{4} \mathrm{O}_{4}[\mathrm{M}+$ 1] $]^{+}$872.4296, found 872.4262; Anal. Calc. for $\mathrm{C}_{58} \mathrm{H}_{56} \mathrm{~N}_{4} \mathrm{O}_{4}$ : C, 79.79; H, 6.46; N, 
$6.42 \%$, found; C, 79.56; H, 6.08; N, $6.21 \% ; \lambda_{\max }(\mathrm{DCM} / \mathrm{MeOH}) 260 \mathrm{~nm} @ 0.005$ $\mathrm{mM}, \varepsilon=181 \mathrm{M}^{-1} \mathrm{~cm}^{-1}$.

\section{Metal complexes of (37) at 1:1 ratio}

All metal ion complexation reactions were performed at room temperature in a solvent mixture of $\mathrm{DCM}-\mathrm{MeOH}$ at a 1:1 ratio. All reactions used $0.12 \mathrm{mmol}$ of (37) and corresponding metal salt. The solution was stirred for $15 \mathrm{~min}$ and then the solvent was slowly evaporated off. A water-DCM wash was performed to remove any uncomplexed metal and the solvent was removed under reduced pressure.

Compound (37i): Zinc acetate; Yield $=0.10 \mathrm{~g}, 78 \% ; \mathrm{v}_{\max } / \mathrm{cm}^{-1}(\mathrm{KBr}): 3419,2918$, 1591, 1570, 1435, 1198, 757; $\delta_{\mathrm{H}} / \mathrm{ppm}$ : Conc. of $10^{-3} \mathrm{~mm} ;\left(\mathrm{CDCl}_{3}\right) ; 8.73$ (br d), 8.63 $(\mathrm{d}, J=5.71 \mathrm{~Hz}), 8.57(\mathrm{~d}, J=5.1 \mathrm{~Hz}), 7.84(\mathrm{~s}), 7.83(\mathrm{~d}, J=2.1 \mathrm{~Hz}), 7.81(\mathrm{t}, J=2.5)$, $7.65(\mathrm{dd}, J=1.6,2.1 \mathrm{~Hz}), 7.42(\mathrm{~d}, J=8.0 \mathrm{~Hz}), 7.40(\mathrm{~d}, J=6.6 \mathrm{~Hz}), 7.37$ (s), 7.32 (d, $J=0.9 \mathrm{~Hz}), 7.28(\mathrm{~s}), 7.19(\mathrm{~d}, J=7.3 \mathrm{~Hz}), 7.15(\mathrm{t}, J=6.9 \mathrm{~Hz}), 7.07$ (s), 7.05 (d, $J=$ $7.7 \mathrm{~Hz}), 7.03$ (s), 6.98 (d, J=3.8 Hz), 6.88 (d, $J=7.2 \mathrm{~Hz}), 6.80$ (d, J=7.5 Hz), 6.78 $(\mathrm{d}, J=1.8 \mathrm{~Hz}), 6.75(\mathrm{~d}, J=1.5 \mathrm{~Hz}), 6.73(\mathrm{~s}), 6.72(\mathrm{~s}), 6.69$ (dd, 1.5, $7.0 \mathrm{~Hz}), 6.65$ (t, $J=7.3 \mathrm{~Hz}), 6.60(\mathrm{~d}, J=1.5 \mathrm{~Hz}), 6.59(\mathrm{~d}, J=1.8 \mathrm{~Hz}), 6.49(\mathrm{t}, J=7.3 \mathrm{~Hz}), 6.43(\mathrm{t}, J$ $=3.4 \mathrm{~Hz}), 5.09(\mathrm{~d}, J=12.5 \mathrm{~Hz}), 5.06(\mathrm{~s}), 4.88(\mathrm{~s}), 4.80(\mathrm{~d}, J=12.5 \mathrm{~Hz}), 4.75(\mathrm{~s})$, $4.65(\mathrm{~d}, J=2.9 \mathrm{~Hz}), 4.46(\mathrm{~s}), 4.31(\mathrm{~d}, J=13.5 \mathrm{~Hz}), 4.00(\mathrm{~d}, J=13.9 \mathrm{~Hz}), 3.77(\mathrm{~s})$, 3.73 (s), 3.59 (s), 3.34 (d, $J=13.9 \mathrm{~Hz}$ ), 3.28 (t, $J=5.3 \mathrm{~Hz}$ ), 3.18 (s), 3.15 (br t), 3.08 (br t); Anal. Calc. for $\mathrm{C}_{62} \mathrm{H}_{62} \mathrm{~N}_{4} \mathrm{O}_{8} \mathrm{Zn}$ : C, 70.48; H, 5.91; N, $5.30 \%$, found; C, 70.71; $\mathrm{H}, 5.69 ; \mathrm{N}, 5.24 \%$; $\lambda_{\max }(\mathrm{DCM} / \mathrm{MeOH}) 404 \mathrm{~nm} @ 0.5 \mathrm{mM}, \varepsilon=277 \mathrm{M}^{-1} \mathrm{~cm}^{-1}$.

Compound (37ii): Zinc chloride; Yield $=0.10 \mathrm{~g}, 73 \% ; \mathrm{v}_{\max } / \mathrm{cm}^{-1}(\mathrm{KBr}): 3429$, 2919, 1651, 1592, 1570, 1465, 1090, 757; $\delta_{\mathrm{H}} / \mathrm{ppm}$ : Conc. of $10^{-3} \mathrm{~mm}$; ( $d_{6}$-DMSO); $8.53(\mathrm{~d}, J=4 \mathrm{~Hz}), 8.50(\mathrm{~d}, J=4.3 \mathrm{~Hz}), 8.46$ (d, $J=4.7 \mathrm{~Hz}), 8.32$ (s), 8.16 (s), 7.95 (br d), $7.76(\mathrm{td}, J=1.6,7.8 \mathrm{~Hz}), 7.66(\mathrm{~d}, J=6.6 \mathrm{~Hz}), 7.41$ (d, $J=5.5 \mathrm{~Hz}), 7.32$ (br d) $7.22(\mathrm{~s}), 7.21(\mathrm{~d}, J=8.9 \mathrm{~Hz}), 7.14(\mathrm{~d}, J=7.8 \mathrm{~Hz}), 7.12(\mathrm{~d}, J=6.6 \mathrm{~Hz}), 7.05(\mathrm{~s}), 7.04$ $(\mathrm{d}, J=2.6 \mathrm{~Hz}), 7.00(\mathrm{~s}), 6.92(\mathrm{~d}, J=6.6 \mathrm{~Hz}), 6.80(\mathrm{~d}, J=6.6 \mathrm{~Hz}), 6.78(\mathrm{~d}, J=7.7$ $\mathrm{Hz}), 6.65(\mathrm{~d}, J=10.0 \mathrm{~Hz}), 6.57(\mathrm{t}, J=6.6 \mathrm{~Hz}), 6.51(\mathrm{t}, J=7.7 \mathrm{~Hz}), 6.31(\mathrm{t}, J=7.7$ $\mathrm{Hz}), 5.15$ (d, $J=10.5 \mathrm{~Hz}), 5.05$ (s), 4.79 (s), 4.70 (s), 4.63 (d, $J=10.5 \mathrm{~Hz}), 4.15$ (d, $J$ $=12.8 \mathrm{~Hz}), 3.86(\mathrm{~d}, J=13.6 \mathrm{~Hz}), 3.78(\mathrm{~d}, J=12.9 \mathrm{~Hz}), 3.69(\mathrm{~d}, J=14.7 \mathrm{~Hz}), 3.17$ (d, $J=13.6 \mathrm{~Hz}), 3.05$ (br t), 2.96 (br d), 2.88 (t, $J=6.5 \mathrm{~Hz}$ ); Anal. Calc. for 
$\mathrm{C}_{58} \mathrm{H}_{56} \mathrm{Cl}_{2} \mathrm{~N}_{4} \mathrm{O}_{4} \mathrm{Zn}: \mathrm{C}, 69.02 ; \mathrm{H}, 5.59 ; \mathrm{N}, 5.55 \%$, found; C, 68.84; H, 5.26; N, 5.30 $\% ; \lambda_{\max }(\mathrm{DCM} / \mathrm{MeOH}) 410 \mathrm{~nm} @ 0.5 \mathrm{mM}, \varepsilon=126 \mathrm{M}^{-1} \mathrm{~cm}^{-1}$.

Compound (37iii): Zinc perchlorate; Yield $=0.09 \mathrm{~g}, 74 \% ; \mathrm{v}_{\max } / \mathrm{cm}^{-1}(\mathrm{KBr}): 3432$, 1629, 1591, 1457, 1090 (perchlorate anion peak), 626; $\delta_{\mathrm{H}} / \mathrm{ppm}$ : Conc. of $10^{-3} \mathrm{~mm}$; $\left(d_{6}\right.$-DMSO); 8.53 (d, $\left.J=7.5 \mathrm{~Hz}\right), 8.17$ (s), 7.78 (br t), 7.67 (d, $\left.J=8.5 \mathrm{~Hz}\right), 7.41$ (d, $J$ $=6.5 \mathrm{~Hz}), 7.37$ (s), 7.36 (s), $7.34(\mathrm{~s}), 7.23$ (d, $J=7.4 \mathrm{~Hz}), 7.15$ (d, $J=8.5 \mathrm{~Hz}), 7.13$ $(\mathrm{d}, J=8.5 \mathrm{~Hz}), 7.05(\mathrm{dd}, J=7.0,6.0 \mathrm{~Hz}), 7.01(\mathrm{~s}), 6.93$ (d, $J=6.5 \mathrm{~Hz}), 6.79$ (d, $J=$ $7.9 \mathrm{~Hz}), 6.64(\mathrm{~d}, J=6.0 \mathrm{~Hz}), 6.61(\mathrm{~s}), 6.51(\mathrm{t}, J=7.4 \mathrm{~Hz}), 5.16(\mathrm{~d}, J=12.1 \mathrm{~Hz}), 5.05$ (s), 4.79 (s), $4.71(\mathrm{~s}), 4.64(\mathrm{~d}, J=13.2 \mathrm{~Hz}), 4.17$ (d, $J=13.2 \mathrm{~Hz}), 3.87$ (d, $J=13.9$ $\mathrm{Hz}), 3.78(\mathrm{~d}, J=12.7 \mathrm{~Hz}), 3.69(\mathrm{~d}, J=13.9 \mathrm{~Hz}), 3.19(\mathrm{~d}, J=13.9 \mathrm{~Hz}), 3.04(\mathrm{t}, J=$ $6.9 \mathrm{~Hz}$ ); Anal. Calc. for $\mathrm{C}_{58} \mathrm{H}_{56} \mathrm{Cl}_{2} \mathrm{~N}_{4} \mathrm{O}_{12} \mathrm{Zn}: \mathrm{C}, 61.25 ; \mathrm{H}, 4.96 ; \mathrm{N}, 4.93 \%$, found; $\mathrm{C}$, 61.08; H, 4.80; N, $4.82 \% ; \lambda_{\max }(\mathrm{DCM} / \mathrm{MeOH}) 410 \mathrm{~nm} @ 0.5 \mathrm{mM}, \varepsilon=115 \mathrm{M}^{-1} \mathrm{~cm}^{-1}$.

Compound (37iv): Mercury perchlorate; Yield $=0.07 \mathrm{~g}, 47 \% ; \mathrm{v}_{\max } / \mathrm{cm}^{-1}(\mathrm{KBr})$ : 3420, 1631, 1592, 1444, 1089 (perchlorate anion peak), 635; $\delta_{\mathrm{H}} / \mathrm{ppm}$ : Conc. of $10^{-3}$ $\mathrm{mm}$; $\left(d_{6}\right.$-DMSO); $8.98(\mathrm{br} \mathrm{d}), 8.80(\mathrm{~d}, J=5.3 \mathrm{~Hz}), 8.62(\mathrm{~d}, J=6.2 \mathrm{~Hz}), 7.88(\mathrm{t}, J=$ $7.8 \mathrm{~Hz}$ ), 7.73 (d, $J=7.8 \mathrm{~Hz}), 7.47$ (s), 7.46 (s), 7.27 (t, $J=8.5 \mathrm{~Hz}), 7.21$ (br t), 7.19 $(\mathrm{d}, J=6.1 \mathrm{~Hz}), 7.14$ (d, $J=7.8 \mathrm{~Hz}), 7.11(\mathrm{t}, J=6.1 \mathrm{~Hz}), 7.07$ (s), 7.04 (d, $J=7.5$ Hz), 6.90 (br d), 6.89 (d, $J=7.9 \mathrm{~Hz}), 6.84$ (d, $J=7.1 \mathrm{~Hz}), 6.81(\mathrm{~d}, J=7.19 \mathrm{~Hz}), 6.73$ (s), $6.70(\mathrm{t}, J=7.7 \mathrm{~Hz}), 6.66(\mathrm{~d}, J=7.1 \mathrm{~Hz}), 6.56(\mathrm{t}, J=7.1 \mathrm{~Hz}), 6.39(\mathrm{t}, J=7.1 \mathrm{~Hz})$, $6.27(\mathrm{~d}, J=8.3 \mathrm{~Hz}), 5.23(\mathrm{t}, J=11.1 \mathrm{~Hz}), 5.12(\mathrm{~s}), 4.86(\mathrm{~s}), 4.77(\mathrm{~d}, J=11.1 \mathrm{~Hz})$, $4.70(\mathrm{~d}, J=11.1 \mathrm{~Hz}), 4.24(\mathrm{~d}, J=13.4 \mathrm{~Hz}), 3.93(\mathrm{~d}, J=5.3 \mathrm{~Hz}), 3.84(\mathrm{~m}, J=6.2$ $\mathrm{Hz}), 3.76(\mathrm{t}, J=12.4 \mathrm{~Hz}), 3.58(\mathrm{br} d), 3.28(\mathrm{~d}, J=12.4 \mathrm{~Hz}), 3.21(\mathrm{t}, J=6.2 \mathrm{~Hz}), 3.13$ (t, $J=7.1 \mathrm{~Hz})$.

Compound (37v): Mercury Chloride; Yield = $0.08 \mathrm{~g}, 58 \% ; \mathrm{v}_{\max } / \mathrm{cm}^{-1}(\mathrm{KBr}): 3431$, 1628, 1590, 1449, 1198, 620; $\delta_{\mathrm{H}} / \mathrm{ppm}$ : Conc. of $10^{-3} \mathrm{~mm}$; $\left(d_{6}\right.$-DMSO); 8.53 (br d), $7.83(\mathrm{~s}), 7.64$ (d, $J=7.5 \mathrm{~Hz}), 7.43$ (t, $J=7.8 \mathrm{~Hz}), 7.36$ (br d), 7.34 (s), 7.19 (d, $J=$ $6.6 \mathrm{~Hz}), 7.16(\mathrm{~d}, J=7.4 \mathrm{~Hz}), 7.05(\mathrm{~d}, J=8.4 \mathrm{~Hz}), 6.97(\mathrm{t}, J=3.5 \mathrm{~Hz}), 6.89(\mathrm{~d}, J=$ $7.1 \mathrm{~Hz}), 6.80(\mathrm{~d}, J=7.1 \mathrm{~Hz}), 6.75(\mathrm{~d}, J=7.1 \mathrm{~Hz}), 6.68(\mathrm{t}, J=7.0 \mathrm{~Hz}), 6.59$ (s), 6.49 $(\mathrm{t}, J=7.7 \mathrm{~Hz}), 5.09(\mathrm{~d}, J=11.7 \mathrm{~Hz}), 5.06(\mathrm{~s}), 4.88(\mathrm{~d}, J=9.1 \mathrm{~Hz}), 4.80(\mathrm{~d}, J=13.0$ $\mathrm{Hz}), 4.75(\mathrm{~s}), 4.65(\mathrm{~d}, J=2.1 \mathrm{~Hz}), 4.30(\mathrm{~d}, J=14.3 \mathrm{~Hz}), 4.00(\mathrm{~d}, J=11.7 \mathrm{~Hz}), 3.77$ $(\mathrm{d}, J=13.0 \mathrm{~Hz}), 3.73$ (d, $J=14.6 \mathrm{~Hz}), 3.60$ (s), 3.34 (d, $J=14.6 \mathrm{~Hz}), 3.16$ (d, $J=$ $13.0 \mathrm{~Hz}$ ), 2.97 (br t), 2.85 (br t), 2.54 (br s). 


\section{Metal complexes of (37) at 1:2 ratio}

Same procedure was used as in the case of the 1:1 with the exception that the ratio of ligand to metal was $0.12 \mathrm{mmol}$ to $0.24 \mathrm{mmol}$.

Compound (37vi): Zinc acetate; Yield $=0.22 \mathrm{~g}, 67 \% ; \mathrm{v}_{\max } / \mathrm{cm}^{-1}(\mathrm{KBr}): 3419$, 3027, 1606, 1447, 1195, 1021, 760; $\delta_{\mathrm{H}} / \mathrm{ppm}$ : Conc. of $10^{-3} \mathrm{~mm} ;\left(\mathrm{CDCl}_{3}\right) ; 8.75(\mathrm{br} \mathrm{d})$, $8.64(\mathrm{~d}, J=5.0 \mathrm{~Hz}), 8.54($ br d), $7.83(\mathrm{~s}), 7.64(\mathrm{~d}, J=6.5 \mathrm{~Hz}), 7.42(\mathrm{~d}, J=7.2 \mathrm{~Hz})$, $7.37(\mathrm{~s}), 7.32(\mathrm{~d}, J=0.8 \mathrm{~Hz}), 7.19(\mathrm{~d}, J=7.5 \mathrm{~Hz}), 7.15(\mathrm{t}, J=7.5 \mathrm{~Hz}), 7.07(\mathrm{~s}), 7.05$ (d, $J=7.5 \mathrm{~Hz}), 7.03(\mathrm{~s}), 6.97(\mathrm{t}, J=3.7 \mathrm{~Hz}), 6.88(\mathrm{~d}, J=8.3 \mathrm{~Hz}), 6.80$ (d, $J=8.3$ Hz), 6.77 (s), 6.75 (br t), 6.73 (s), 6.72 (d, $J=1.3 \mathrm{~Hz}), 6.65$ (t, $J=8.3 \mathrm{~Hz}), 6.49$ (t, $J$ = 7.7 Hz), $5.11(\mathrm{~d}, J=12.5 \mathrm{~Hz}), 5.06(\mathrm{~s}), 4.89$ (s), 4.79 (d, $J=12.5 \mathrm{~Hz}), 4.75$ (s), 4.64 (s), 4.31 (d, $J=13.6 \mathrm{~Hz}), 4.00$ (d, $J=13.6 \mathrm{~Hz}), 3.77$ (d, $J=13.9 \mathrm{~Hz}), 3.72$ (d, $J$ $=14.9 \mathrm{~Hz}), 3.59(\mathrm{~s}), 3.32(\mathrm{~d}, J=12.9 \mathrm{~Hz}), 3.28($ br d), 3.15 (br t), 3.05 (br t); Anal. Calc. for $\mathrm{C}_{66} \mathrm{H}_{68} \mathrm{~N}_{4} \mathrm{O}_{12} \mathrm{Zn}_{2}$ : C, 63.93; H, 5.53; N, $4.52 \%$, found; $\mathrm{C}, 63.56 ; \mathrm{H}, 5.26$; $\mathrm{N}, 4.34 \% ; \lambda_{\max }(\mathrm{DCM} / \mathrm{MeOH}) 526 \mathrm{~nm} @ 0.5 \mathrm{mM}, \varepsilon=196 \mathrm{M}^{-1} \mathrm{~cm}^{-1}$.

Compound (37vii): Zinc chloride; Yield $=0.26 \mathrm{~g}, 77 \% ; \mathrm{v}_{\max } / \mathrm{cm}^{-1}(\mathrm{KBr}): 3420$, 2919, 1642, 1590, 1448, 1194, 759; $\delta_{\mathrm{H}} / \mathrm{ppm}$ : Conc. of $10^{-3} \mathrm{~mm} ;\left(d_{6}\right.$-DMSO); 8.54 (d, $J=4.9 \mathrm{~Hz}), 8.51(\mathrm{~d}, J=4.9 \mathrm{~Hz}), 8.32(\mathrm{~s}), 8.18(\mathrm{~s}), 7.67(\mathrm{~d}, J=6.8 \mathrm{~Hz}), 7.41(\mathrm{~d}, J=$ $9.8 \mathrm{~Hz}), 7.23(\mathrm{~d}, J=7.3 \mathrm{~Hz}), 7.15(\mathrm{~d}, J=7.3 \mathrm{~Hz}), 7.13(\mathrm{~d}, J=7.3 \mathrm{~Hz}), 7.05$ (s), 7.01 (s), $6.93(\mathrm{~d}, J=7.3 \mathrm{~Hz}), 6.82(\mathrm{~d}, J=7.9 \mathrm{~Hz}), 6.79(\mathrm{~d}, J=7.4 \mathrm{~Hz}), 6.66(\mathrm{t}, J=9.5$ Hz), $6.62(\mathrm{~d}, J=6.8 \mathrm{~Hz}), 6.52(\mathrm{t}, J=7.4 \mathrm{~Hz}), 6.33(\mathrm{t}, J=6.8 \mathrm{~Hz}), 5.16(\mathrm{~d}, J=12.6$ Hz), 5.06 (s), 4.71 (s), 4.65 (d, $J=13.7 \mathrm{~Hz}), 4.18(\mathrm{~d}, J=13.7 \mathrm{~Hz}), 3.86(\mathrm{~d}, J=13.8$ Hz), $3.79(\mathrm{~d}, J=11.6 \mathrm{~Hz}), 3.68(\mathrm{~d}, J=13.9 \mathrm{~Hz}), 3.18(\mathrm{~d}, J=13.9 \mathrm{~Hz}), 3.07(\mathrm{br} \mathrm{t})$, $2.88(\mathrm{t}, J=6.9 \mathrm{~Hz})$; Anal. Calc. for $\mathrm{C}_{58} \mathrm{H}_{56} \mathrm{Cl}_{4} \mathrm{~N}_{4} \mathrm{O}_{4} \mathrm{Zn}_{2}: \mathrm{C}, 60.81 ; \mathrm{H}, 4.93 ; \mathrm{N}, 4.89$ $\%$, found; C, 60.39; H, 4.62; N, $4.54 \%$; $\lambda_{\max }(\mathrm{DCM} / \mathrm{MeOH}) 441 \mathrm{~nm} @ 0.5 \mathrm{mM}, \varepsilon=$ $129 \mathrm{M}^{-1} \mathrm{~cm}^{-1}$.

Compound (37viii): Zinc perchlorate; Yield $=0.20 \mathrm{~g}, 72 \% ; \mathrm{v}_{\max } / \mathrm{cm}^{-1}(\mathrm{KBr}): 3432$, 3028, 1625, 1448, 1091 (perchlorate anion peak), 760, 627; $\delta_{\mathrm{H}} / \mathrm{ppm}$ : Conc. of $10^{-3}$ mm; $\left(d_{6}\right.$-DMSO); 8.92 (br d), 8.53 (d, $\left.J=6.6 \mathrm{~Hz}\right) 8.17$ (s), 7.78 (br d), 7.67 (d, $J=$ $8.0 \mathrm{~Hz}), 7.41(\mathrm{~d}, J=7.5 \mathrm{~Hz}), 7.21(\mathrm{~d}, J=8.2 \mathrm{~Hz}), 7.15(\mathrm{~d}, J=7.5 \mathrm{~Hz}), 7.12(\mathrm{~d}, J=$ $7.5 \mathrm{~Hz}), 7.06$ (s), 7.05 (d, $J=5.2 \mathrm{~Hz}), 7.01$ (s), $6.93(\mathrm{~d}, J=6.0 \mathrm{~Hz}), 6.81$ (d, $J=7.5$ $\mathrm{Hz}), 6.78(\mathrm{~d}, J=7.5 \mathrm{~Hz}), 6.51(\mathrm{t}, J=6.8 \mathrm{~Hz}), 5.16(\mathrm{~d}, J=12.6 \mathrm{~Hz}), 5.06(\mathrm{~s}), 4.71(\mathrm{~s})$, $4.65(\mathrm{~d}, J=13.8 \mathrm{~Hz}), 4.17(\mathrm{~d}, J=12.6 \mathrm{~Hz}), 3.86(\mathrm{~d}, J=14.5 \mathrm{~Hz}), 3.78(\mathrm{~d}, J=12.4$ 
Hz), $3.69(\mathrm{~d}, J=15.6 \mathrm{~Hz}), 3.19(\mathrm{~d}, J=13.5 \mathrm{~Hz}), 3.05(\mathrm{t}, J=7.2 \mathrm{~Hz}), 2.88(\mathrm{t}, J=7.2$ $\mathrm{Hz}$ ); Anal. Calc. for $\mathrm{C}_{58} \mathrm{H}_{56} \mathrm{Cl}_{2} \mathrm{~N}_{4} \mathrm{O}_{20} \mathrm{Zn}_{2}$ : C, 49.70; H, 4.03; N, $4.00 \%$, found; $\mathrm{C}$, 49.37; H, 3.81; N, $3.78 \%$; $\lambda_{\max }(\mathrm{DCM} / \mathrm{MeOH}) 524 \mathrm{~nm} @ 0.5 \mathrm{mM}, \varepsilon=144 \mathrm{M}^{-1} \mathrm{~cm}^{-1}$.

\section{Metal complexes of (37) at 1:3 ratio}

Same procedure was used as in the case of the 1:1 with the exception that the ratio of ligand to metal was $0.12 \mathrm{mmol}$ to $0.36 \mathrm{mmol}$.

Compound (37ix): zinc acetate; Yield $=0.40 \mathrm{~g}, 75 \% ; \mathrm{v}_{\max } / \mathrm{cm}^{-1}(\mathrm{KBr}): 3422,3017$, 1605, 1448, 1193, 1022, 760; $\delta_{\mathrm{H}} / \mathrm{ppm}$ : Conc. of $10^{-3} \mathrm{~mm} ;\left(\mathrm{CDCl}_{3}\right) ; 8.64$ (br d), 8.52 (br d), 7.82 (s), 7.65 (d, J= $7.2 \mathrm{~Hz}$ ), 7.47 (s), 7.43 (d, J= $7.3 \mathrm{~Hz}), 7.37$ (s), 7.36 (s), $7.32(\mathrm{t}, J=4.5 \mathrm{~Hz}), 7.19(\mathrm{t}, J=7.8 \mathrm{~Hz}), 7.15(\mathrm{~d}, J=7.4 \mathrm{~Hz}), 7.07(\mathrm{~s}), 7.05(\mathrm{~d}, J=$ $7.1 \mathrm{~Hz}), 7.03(\mathrm{~s}), 6.89$ (d, $J=7.1 \mathrm{~Hz}), 6.80(\mathrm{~d}, J=6.47$ ), $6.73(\mathrm{~d}, J=7.5 \mathrm{~Hz}), 6.73$ $(\mathrm{d}, J=2.5 \mathrm{~Hz}), 6.69(\mathrm{t}, J=1.9 \mathrm{~Hz}), 6.65(\mathrm{t}, J=6.6 \mathrm{~Hz}), 6.59(\mathrm{br} \mathrm{d}), 6.49(\mathrm{t}, J=7.9$ $\mathrm{Hz}), 5.10(\mathrm{~d}, J=10.7 \mathrm{~Hz}), 5.06(\mathrm{~s}), 4.88(\mathrm{~s}), 4.80(\mathrm{~d}, J=12.7 \mathrm{~Hz}), 4.75$ (s), 4.72 (d, $J$ $=9.4 \mathrm{~Hz}), 4.31(\mathrm{~d}, J=11.9 \mathrm{~Hz}), 4.00(\mathrm{~d}, J=14.3 \mathrm{~Hz}), 3.80(\mathrm{t}, J=6.3 \mathrm{~Hz}), 3.77(\mathrm{~s})$, 3.73 (s), 3.69 (s), 3.64 (br t), 3.59 (s), 3.52 (d, $J=7.1 \mathrm{~Hz}), 3.34$ (d, $J=13.4 \mathrm{~Hz}), 3.18$ (t, $J=6.3 \mathrm{~Hz}), 3.15$ (br t), 3.08 (br t).

\section{Metal complexes of (37) at 1:4 ratio}

Same procedure was used as in the case of the 1:1 with the exception that the ratio of ligand to metal was $0.12 \mathrm{mmol}$ to $0.48 \mathrm{mmol}$.

Compound (37x): zinc acetate; Yield $=0.61 \mathrm{~g}, 79 \% ; \mathrm{v}_{\max } / \mathrm{cm}^{-1}(\mathrm{KBr}): 3423,3018$, 1605, 1448, 1192, 1023, 760; $\delta_{\mathrm{H}} / \mathrm{ppm}$ : Conc. of $10^{-3} \mathrm{~mm} ;\left(\mathrm{CDCl}_{3}\right) ; 8.64$ (d, $J=4.8$ $\mathrm{Hz}$ ), 8.53 (br d), 7.85 (d, $J=8.4 \mathrm{~Hz}$ ), 7.82 (s), 7.65 (d, $J=7.2 \mathrm{~Hz}), 7.47$ (s), 7.43 (d, $J=8.4 \mathrm{~Hz}), 7.36$ (br s), 7.32 (s), 7.20 (d, $J=7.3 \mathrm{~Hz}), 7.15$ (t, $J=7.9 \mathrm{~Hz}), 7.07$ (s), $7.03(\mathrm{~s}), 6.89(\mathrm{~d}, J=6.9 \mathrm{~Hz}), 6.80(\mathrm{~d}, J=6.9 \mathrm{~Hz}), 6.76(\mathrm{~d}, J=8.2 \mathrm{~Hz}), 6.73(\mathrm{~d}, J=$ $3.4 \mathrm{~Hz}), 6.69(\mathrm{~d}, J=5.5 \mathrm{~Hz}), 6.65(\mathrm{t}, J=6.2 \mathrm{~Hz}), 6.59(\mathrm{~s}), 6.49(\mathrm{t}, J=5.9 \mathrm{~Hz}), 5.10$ $(\mathrm{d}, J=11.9 \mathrm{~Hz}), 5.06(\mathrm{~s}), 4.88(\mathrm{~s}), 4.79$ (d, $J=12.9 \mathrm{~Hz}), 4.75(\mathrm{~s}), 4.72(\mathrm{~d}, J=9.7$ $\mathrm{Hz}), 4.65(\mathrm{~d}, J=2.08 \mathrm{~Hz}), 4.31(\mathrm{~d}, J=12.9 \mathrm{~Hz}), 4.11(\mathrm{~d}, J=13.2 \mathrm{~Hz}), 4.00(\mathrm{~d}, J=$ $13.2 \mathrm{~Hz}), 3.80(\mathrm{t}, J=6.6 \mathrm{~Hz}), 3.76(\mathrm{~s}), 3.73(\mathrm{~s}), 3.70(\mathrm{~d}, J=9.2 \mathrm{~Hz}), 3.63(\mathrm{br} \mathrm{s}), 3.58$ (s), $3.52(\mathrm{~d}, J=8.1 \mathrm{~Hz}), 3.33(\mathrm{~d}, J=12.8 \mathrm{~Hz}), 3.17(\mathrm{t}, J=3.8 \mathrm{~Hz}), 3.14(\mathrm{br} \mathrm{t}), 3.02$ $(\mathrm{t}, J=6.4 \mathrm{~Hz})$. 


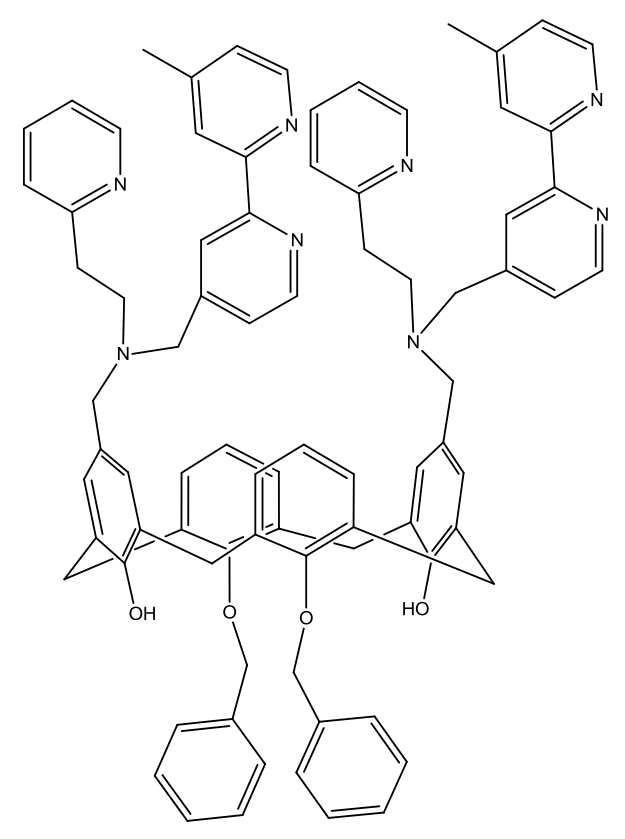

Compound (22) (0.06 g, $0.23 \mathrm{mmol})$, compound (37) (0.03 g, $0.04 \mathrm{mmol})$, and triethylamine $(0.02 \mathrm{~g}, 0.23 \mathrm{mmol})$ were refluxed under nitrogen gas in $\mathrm{CH}_{3} \mathrm{CN}(80$ $\mathrm{mL}$ ) for $48 \mathrm{hr}$. The solution was allowed to cool to room temperature. The inorganic salts were removed by filtration and the volatiles removed under reduced pressure to afford a dark yellow waxy solid (38).

Yield $=0.04 \mathrm{~g}, 14 \% ; \mathrm{v}_{\max } / \mathrm{cm}^{-1}(\mathrm{KBr}): 3401,1629,1593,1457,1371,991,825$; $\delta_{\mathrm{H}} / \mathrm{ppm}$ : Conc. of $10^{-3} \mathrm{~mm} ;\left(\mathrm{CDCl}_{3}\right) ; 8.64(\mathrm{~d}, 2 \mathrm{H}, \mathrm{Py}-\underline{\mathrm{H}}, J=4.3 \mathrm{~Hz}), 8.53(\mathrm{~d}, 4 \mathrm{H}$,

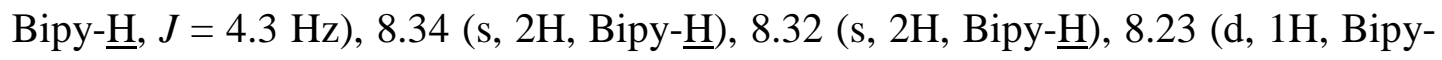

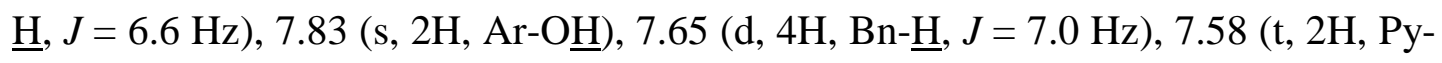
$\underline{\mathrm{H}}, J=8.5 \mathrm{~Hz}), 7.48(\mathrm{t}, 4 \mathrm{H}, \mathrm{Bn}-\underline{\mathrm{H}}, J=7.8 \mathrm{~Hz}), 7.32(\mathrm{~d}, 2 \mathrm{H}, \mathrm{Py}-\underline{\mathrm{H}}, J=4.4 \mathrm{~Hz}), 7.30$ (d, 2H, Bipy- $\underline{\mathrm{H}}, J=4.0 \mathrm{~Hz}$ ), $7.13(\mathrm{t}, 2 \mathrm{H}, \mathrm{Bn}-\underline{\mathrm{H}}, J=4.8 \mathrm{~Hz}$ ), 7.08 (s, 4H, Ar- $\underline{\mathrm{H}}$ ), 7.05

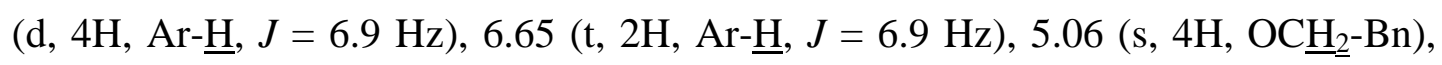
4.81 (s, $4 \mathrm{H}, \mathrm{C}_{2} \mathrm{NR}_{2}$ ), 4.31 (d, 4H, $\underline{\mathrm{C}}_{2}, J=13.0 \mathrm{~Hz}$ ), 3.46 (s, 4H, $\left.\underline{\mathrm{C}}_{2}-\mathrm{Bipy}\right), 3.34$ (d, $4 \mathrm{H}, \mathrm{CH}_{2}, J=13.0 \mathrm{~Hz}$ ), 3.06 (t, $4 \mathrm{H}, \mathrm{RNCH}_{2} \mathrm{C}_{2}-\mathrm{Py}, J=7.96 \mathrm{~Hz}$ ), 2.94 (t, $4 \mathrm{H}$, $\left.\mathrm{RNC}_{2} \mathrm{CH}_{2}-\mathrm{Py}, J=7.6 \mathrm{~Hz}\right), 2.44$ (s, 6H, Bipy-C $\left.\underline{\mathrm{H}}_{3}\right) ; \delta_{\mathrm{c}} / \mathrm{ppm}\left(\mathrm{CDCl}_{3}\right) ; 155.9,153.3$, $151.8,151.0,149.3,148.9,148.5,148.2,136.1,136.5,136.3,129.1,128.8,128.4$, 
128.3, 128.1, 127.6, 127.4, 127.1, 125.4, 124.8, 123.6, 122.8, 121.1, 78.4, 73.1, 63.6, 58.7, 35.7, 31.4, 21.2; Anal. Calc. for $\mathrm{C}_{82} \mathrm{H}_{76} \mathrm{~N}_{8} \mathrm{O}_{4}$ : C, 79.58; H, 6.19; N, $9.05 \%$, found; C, 79.18; $\mathrm{H}, 6.04 ; \mathrm{N}, 8.78 \%$.

\subsection{1,23-Di[ $N, N^{\prime}$-di(2'-pyridylmethyl)(2'-pyridylethyl)aminomethyl]- 25,27-dibenzyl-26,28-dihydroxycalix[4]arene. (39)}

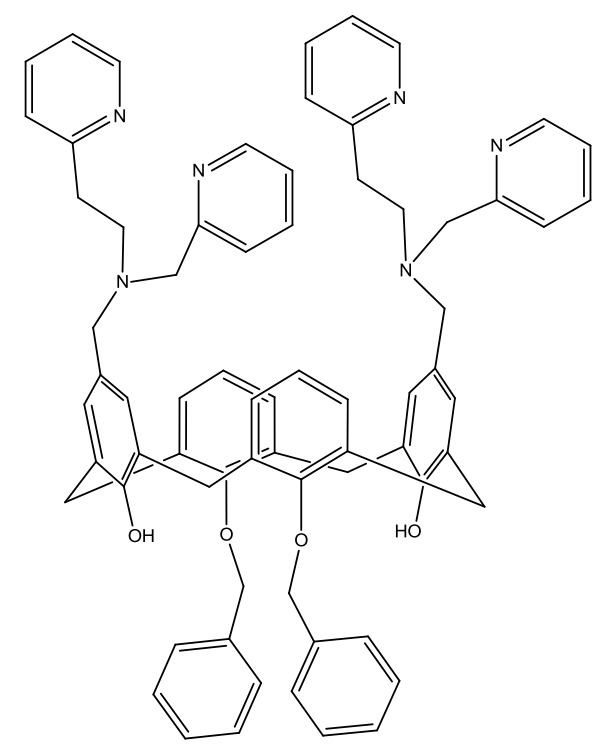

Compound (29) (0.2 g, $0.23 \mathrm{mmol})$ was stirred in $\mathrm{CH}_{3} \mathrm{CN}(50 \mathrm{~mL})$ to dissolve for 10 min. 2-Picolylchloride hydrochloride $(0.07 \mathrm{~g}, 0.46 \mathrm{mmol})$ and sodium carbonate (0.05 g, $0.46 \mathrm{mmol}$ ) were dissolved in $\mathrm{CH}_{3} \mathrm{CN}(20 \mathrm{~mL})$ and allowed to sit for $5 \mathrm{~min}$. The suspension was then filtered to remove the $\mathrm{HCl}$ salt and the filtrate was added to solution of compound (29). This mixture was allowed to stir under nitrogen gas for a further $5 \mathrm{~min}$. Sodium hydride $(0.01 \mathrm{~g}, 0.46 \mathrm{mmol})$ was then added to the mixture which was refluxed overnight under nitrogen gas. The solution was allowed to cool to room temperature, the inorganic salts were removed by filtration and the volatiles removed under reduced pressure to give a yellow waxy solid (39).

Yield $=0.24 \mathrm{~g}, 92 \% ; \mathrm{v}_{\max } / \mathrm{cm}^{-1}(\mathrm{KBr}): 3429,2921,1591,1456,1195,1088,994$, 759; $\delta_{\mathrm{H}} / \mathrm{ppm}$ : Conc. of $10^{-3} \mathrm{~mm} ;\left(\mathrm{CDCl}_{3}\right) ; 8.50(\mathrm{~d}, 2 \mathrm{H}, \mathrm{Py}-\underline{\mathrm{H}}, J=4.6 \mathrm{~Hz}), 8.47$ (d, $2 \mathrm{H}, \mathrm{Py}-\underline{\mathrm{H}}, J=4.9 \mathrm{~Hz}), 7.86(\mathrm{~s}, 2 \mathrm{H}, \mathrm{Ar}-\mathrm{O} \underline{\mathrm{H}}), 7.65$ (d, 4H, Bn- $\underline{\mathrm{H}}, J=6.8 \mathrm{~Hz}), 7.57$ (t, 
6H, Bn- $\underline{\mathrm{H}}, J=7.7 \mathrm{~Hz}), 7.50$ (t, 2H, Py- $\underline{\mathrm{H}}, J=4.7 \mathrm{~Hz}), 7.43$ (d, 2H, Py- $\underline{\mathrm{H}}, J=7.9$ Hz), 7.38 (d, 2H, Py-H, J = 4.6 Hz), 7.31 (t, 2H, Py-H, J =6.4 Hz), 7.25 (t, 2H, Py$\mathrm{H}, J=7.6 \mathrm{~Hz}), 7.20$ (d, 2H, Py- $\underline{\mathrm{H}}, J=6.7 \mathrm{~Hz}), 7.13$ (s, 4H, Ar- $\underline{\mathrm{H}}), 7.08$ (d, 4H, Ar$\underline{\mathrm{H}}, J=7.6 \mathrm{~Hz}), 6.76(\mathrm{t}, 2 \mathrm{H}, \mathrm{Ar}-\underline{\mathrm{H}}, J=8.1 \mathrm{~Hz}), 5.06\left(\mathrm{~s}, 4 \mathrm{H}, \mathrm{OC}_{2}{ }_{2} \mathrm{Bn}\right), 4.75(\mathrm{~s}, 4 \mathrm{H}$, $\mathrm{C}_{2} \mathrm{NR}_{2}$ ), 4.31 (d, 4H, $\left.\underline{\mathrm{H}}_{2}, J=12.9 \mathrm{~Hz}\right), 3.91$ (s, 4H, $\left.\underline{\mathrm{H}}_{2} \mathrm{Py}\right), 3.34$ (d, 4H, $\underline{\mathrm{H}}_{2}, J=$ $12.9 \mathrm{~Hz}$ ), 3.15 (t, 4H, $\left.\mathrm{CH}_{2} \underline{\mathrm{C}}_{2} \mathrm{Py}, J=14.4 \mathrm{~Hz}\right), 3.02$ (t, 4H, $\underline{\mathrm{C}}_{2} \mathrm{CH}_{2} \mathrm{Py}, J=14.4$ $\mathrm{Hz}) ; \delta_{\mathrm{c}} / \mathrm{ppm}\left(\mathrm{CDCl}_{3}\right) ; 160.3,159.3,154.1,151.9,148.9,148.5,137.7,136.5,136.3$, $133.9,129.0,128.5,128.3,128.0,127.6,127.2,126.4,125.4,123.1,122.3,121.2$, 71.6, 60.2, 58.5, 54.4, 39.1, 31.9; Anal. Calc. for $\mathrm{C}_{70} \mathrm{H}_{66} \mathrm{~N}_{6} \mathrm{O}_{4}: \mathrm{C}, 79.67 ; \mathrm{H}, 6.30 ; \mathrm{N}$, $7.96 \%$, found; C, 79.31; H, 6.13; N, $7.86 \%$; $\lambda_{\max }(\mathrm{DCM} / \mathrm{MeOH}) 260 \mathrm{~nm} @ 0.004$ $\mathrm{mM}, \varepsilon=623 \mathrm{M}^{-1} \mathrm{~cm}^{-1}$.

\section{Metal complexes of (39) at 1:1 ratio}

All metal ion complexation reactions were performed at room temperature in a solvent mixture of $\mathrm{DCM}-\mathrm{MeOH}$ in a 1:1 ratio. All reactions used $0.09 \mathrm{mmol}$ of (39) and corresponding metal salt. The solution was stirred for $15 \mathrm{~min}$ and then the solvent was slowly evaporated off. A water-DCM wash was performed to remove any uncomplexed metal and the solvent was removed under reduced pressure.

Compound (39i): Zinc acetate; Yield $=0.09 \mathrm{~g}, 76 \% ; \mathrm{v}_{\max } / \mathrm{cm}^{-1}(\mathrm{KBr}): 3412,2922$, 1607, 1447, 1195, 1089, 1018, 754; $\delta_{\mathrm{H}} / \mathrm{ppm}$ : Conc. of $10^{-3} \mathrm{~mm} ;\left(\mathrm{CDCl}_{3}\right) ; 9.00(\mathrm{~d}, J=$ $6.1 \mathrm{~Hz}), 8.65(\mathrm{~d}, J=6.1 \mathrm{~Hz}), 8.54(\mathrm{~d}, J=6.1 \mathrm{~Hz}), 8.15(\mathrm{~s}), 7.80(\mathrm{br} \mathrm{d}), 7.74(\mathrm{br} \mathrm{t})$, 7.71 (br t), 7.66 (d, $J=1.8 \mathrm{~Hz}), 7.64$ (d, $J=2.9 \mathrm{~Hz}$ ), 7.60 (s), 7.46 (br d), 7.44 (br d), 7.41 (br d), 7.30 (d, $J=2.4 \mathrm{~Hz}$ ), 7.27 (s), 7.22 (d, $J=1.8 \mathrm{~Hz}), 7.19$ (br t), 7.18 (br t), 7.17 (br d), 7.15 (br d), 7.12 (d, $J=2.7 \mathrm{~Hz}), 7.10$ (s), 6.99 (s), 6.87 (d, $J=7.0 \mathrm{~Hz}$ ), 6.81 (br t), 6.77 (br t), $6.73(\mathrm{~d}, J=7.0 \mathrm{~Hz}), 6.67(\mathrm{~d}, J=7.0 \mathrm{~Hz}), 6.64(\mathrm{~d}, J=3.9 \mathrm{~Hz})$, $6.42(\mathrm{t}, J=6.2 \mathrm{~Hz}), 5.12(\mathrm{~s}), 5.08(\mathrm{~s}), 5.02(\mathrm{~s}), 4.82(\mathrm{~s}), 4.77$ (d, $J=2.7 \mathrm{~Hz}), 4.74(\mathrm{~s})$, $4.72(\mathrm{~d}, J=4.1 \mathrm{~Hz}), 4.51(\mathrm{~s}), 4.31(\mathrm{~d}, J=13.7 \mathrm{~Hz}), 4.11(\mathrm{~d}, J=14.1 \mathrm{~Hz}), 4.01(\mathrm{~d}, J$ $=8.3 \mathrm{~Hz}), 3.97(\mathrm{~d}, J=8.3 \mathrm{~Hz}), 3.77(\mathrm{~s}), 3.74(\mathrm{~s}), 3.66(\mathrm{~d}, J=6.9 \mathrm{~Hz}), 3.61(\mathrm{br} \mathrm{d})$, 3.56 (s), 3.34 (d, $J=12.5 \mathrm{~Hz}), 3.18$ (br d), 3.15 (t, $J=4.5 \mathrm{~Hz}), 3.03$ (t, $J=5.0 \mathrm{~Hz}$ ), $2.90\left(\mathrm{t}, J=8.0 \mathrm{~Hz}\right.$ ); Anal. Calc. for $\mathrm{C}_{74} \mathrm{H}_{72} \mathrm{~N}_{6} \mathrm{O}_{8} \mathrm{Zn}: \mathrm{C}, 71.75 ; \mathrm{H}, 5.86 ; \mathrm{N}, 6.78 \%$, found; C, 71.54; H, 5.79; N, 6.72\%; $\lambda_{\max }(\mathrm{DCM} / \mathrm{MeOH}) 404 \mathrm{~nm} @ 0.4 \mathrm{mM}, \varepsilon=322$ $\mathrm{M}^{-1} \mathrm{~cm}^{-1}$. 
Compound (39iii): Zinc chloride; Yield $=0.09 \mathrm{~g}, 75 \% ; \mathrm{v}_{\max } / \mathrm{cm}^{-1}(\mathrm{KBr}): 3429$, 2920, 1607, 1448, 1194, 1089, 760; $\delta_{\mathrm{H}} / \mathrm{ppm}$ : Conc. of $10^{-3} \mathrm{~mm} ;\left(d_{6}\right.$-DMSO); 8.95 (d, $J=6.0 \mathrm{~Hz}$ ), 8.51 (br d), 8.16 (s), 8.04 (t, $J=6.8 \mathrm{~Hz}), 7.97$ (s), 7.93 (s), 7.87 (br t), 7.84 (br d), 7.82 (br t), 7.79 (br d), 7.77 (br d), 7.74 (br d), 7.70 (br d), 7.60 (s), 7.56 (d, $J=7.9 \mathrm{~Hz}), 7.53$ (br t), 7.50 (d, $J=2.4 \mathrm{~Hz}), 7.44$ (s), 7.41 (s), 7.39 (s), 7.35 (d, $J$ $=3.9 \mathrm{~Hz}), 7.31(\mathrm{~s}), 7.29(\mathrm{br} \mathrm{d}), 7.14(\mathrm{~d}, J=7.9 \mathrm{~Hz}), 7.10$ (s), 7.04 (d, J = $7.6 \mathrm{~Hz})$, 7.00 (s), 6.89 (d, J=8.3 Hz), 6.83 (s), 6.80 (s), 6.77 (d, J=2.9 Hz), 6.74 (s), 6.60 (t, $J=7.9 \mathrm{~Hz}$ ), 5.16 (s), 5.12 (s), 4.88 (s), 4.77 (s), 4.69 (s), 4.66 (s), 4.61 (s), 4.57 (s), $4.16(\mathrm{~d}, J=12.1 \mathrm{~Hz}), 4.06$ (s), 3.83 (br d), 3.78 (s), 3.76 (d, $J=6.7 \mathrm{~Hz}), 3.69$ (d, $J=$ $5.2 \mathrm{~Hz}), 3.65$ (s), 3.45 (s), $3.18(\mathrm{t}, J=7.6 \mathrm{~Hz}), 3.13(\mathrm{~s}), 3.05(\mathrm{t}, J=7.1 \mathrm{~Hz}), 2.88$ (t, $J$ $=6.1 \mathrm{~Hz}$ ); Anal. Calc. for $\mathrm{C}_{70} \mathrm{H}_{66} \mathrm{Cl}_{2} \mathrm{~N}_{6} \mathrm{O}_{4} \mathrm{Zn}: \mathrm{C}, 70.56 ; \mathrm{H}, 5.58 ; \mathrm{N}, 7.05 \%$, found; $\mathrm{C}$, 70.12; H, 5.21; N, $6.98 \%$; $\lambda_{\max }(\mathrm{DCM} / \mathrm{MeOH}) 403 \mathrm{~nm} @ 0.4 \mathrm{mM}, \varepsilon=381 \mathrm{M}^{-1} \mathrm{~cm}^{-1}$.

Compound (39ii): Zinc perchlorate; Yield $=0.08 \mathrm{~g}, 70 \% ; \mathrm{v}_{\max } / \mathrm{cm}^{-1}(\mathrm{KBr}): 3436$, 1609, 1448, 1091 (perchlorate anion peak), 760, 626; $\delta_{\mathrm{H}} / \mathrm{ppm}$ : Conc. of $10^{-3} \mathrm{~mm}$; $\left(d_{6}\right.$-DMSO); 8.95 (br d), 8.51 (br d), $8.15(\mathrm{~s}), 8.05$ (t, $\left.J=7.7 \mathrm{~Hz}\right), 7.77(\mathrm{t}, J=7.7$ $\mathrm{Hz}), 7.66$ (d, $J=7.2 \mathrm{~Hz}), 7.57$ (d, $J=7.6 \mathrm{~Hz}), 7.50$ (br t), 7.41 (s), 7.39 (s), 7.37 (s), $7.32(\mathrm{~d}, J=1.2 \mathrm{~Hz}), 7.23$ (br d), 7.14 (d, $J=7.2 \mathrm{~Hz}), 7.04$ (d, $J=7.3 \mathrm{~Hz}), 6.89$ (br t), $6.81(\mathrm{~d}, J=7.3 \mathrm{~Hz}), 6.77(\mathrm{~d}, J=3.8 \mathrm{~Hz}), 6.59(\mathrm{t}, J=8.0 \mathrm{~Hz}), 6.50(\mathrm{t}, J=8.0 \mathrm{~Hz})$, 5.12 (s), 5.05 (s), 4.89 (s), 4.77 (s), 4.79 (s), 4.57 (d, $J=12.7 \mathrm{~Hz}), 4.41$ (s), 4.30 (br d), $4.16(\mathrm{~d}, J=12.1 \mathrm{~Hz}), 3.82(\mathrm{~d}, J=7.2 \mathrm{~Hz}), 3.76(\mathrm{t}, J=6.2 \mathrm{~Hz}), 3.71(\mathrm{~d}, J=15.2$ $\mathrm{Hz}), 3.21(\mathrm{~d}, J=6.9 \mathrm{~Hz}), 3.14(\mathrm{br} \mathrm{t}), 3.04(\mathrm{t}, J=7.6 \mathrm{~Hz}), 2.89(\mathrm{t}, J=8.2 \mathrm{~Hz}), 2.73(\mathrm{t}$, $J=1.9 \mathrm{~Hz}$ ); Anal. Calc. for $\mathrm{C}_{70} \mathrm{H}_{66} \mathrm{Cl}_{2} \mathrm{~N}_{6} \mathrm{O}_{12} \mathrm{Zn}: \mathrm{C}, 63.71 ; \mathrm{H}, 5.04 ; \mathrm{N}, 6.37 \%$, found; $\mathrm{C}, 63.54 ; \mathrm{H}, 4.95 ; \mathrm{N}, 6.31 \% ; \lambda_{\max }(\mathrm{DCM} / \mathrm{MeOH}) 402 \mathrm{~nm} @ 0.4 \mathrm{mM}, \varepsilon=322 \mathrm{M}^{-1}$ $\mathrm{cm}^{-1}$.

\section{Metal complexes of (39) at 1:2 ratio}

Same procedure was used as in the case of the 1:1 with the exception that the ratio of ligand to metal was $0.09 \mathrm{mmol}$ to $0.18 \mathrm{mmol}$.

Compound (39iv): Zinc acetate; Yield $=0.20 \mathrm{~g}, 74 \% ; \mathrm{v}_{\max } / \mathrm{cm}^{-1}(\mathrm{KBr}): 3439$, 2754, 1607, 1436, 1188, 1095, 762, 672; $\delta_{\mathrm{H}} / \mathrm{ppm}$ : Conc. of $10^{-3} \mathrm{~mm} ;\left(\mathrm{CDCl}_{3}\right) ; 8.60$ $(\mathrm{d}, J=5.6 \mathrm{~Hz}), 8.56(\mathrm{~d}, J=5.6 \mathrm{~Hz}), 7.86(\mathrm{tt}, J=2.0,8.9 \mathrm{~Hz}), 7.79(\mathrm{~d}, J=6.3 \mathrm{~Hz})$, $7.71(\mathrm{~d}, J=7.8 \mathrm{~Hz}), 7.55(\mathrm{~d}, J=7.8 \mathrm{~Hz}), 7.06(\mathrm{~d}, J=7.8 \mathrm{~Hz}), 6.99(\mathrm{~d}, J=7.0 \mathrm{~Hz})$, $6.86(\mathrm{~d}, J=3.1 \mathrm{~Hz}), 6.81(\mathrm{~d}, J=4.7 \mathrm{~Hz}), 6.70(\mathrm{~d}, J=7.8 \mathrm{~Hz}), 6.48(\mathrm{~d}, J=7.8 \mathrm{~Hz})$, 
$6.42(\mathrm{t}, J=5.4 \mathrm{~Hz}), 6.32(\mathrm{~d}, J=10.2 \mathrm{~Hz}), 6.27(\mathrm{t}, J=7.8 \mathrm{~Hz}), 4.92(\mathrm{~s}), 4.74(\mathrm{~s}), 4.69$ (d, $J=10.5 \mathrm{~Hz}), 4.61$ (s), 4.39 (s), 4.20 (d, $J=7.3 \mathrm{~Hz}), 4.14$ (d, $J=6.7 \mathrm{~Hz}), 3.99$ (s), $3.90(\mathrm{~d}, J=7.3 \mathrm{~Hz}), 3.70(\mathrm{~d}, J=7.3 \mathrm{~Hz})$; Anal. Calc. for $\mathrm{C}_{78} \mathrm{H}_{78} \mathrm{~N}_{6} \mathrm{O}_{12} \mathrm{Zn}_{2}: \mathrm{C}, 65.87$; $\mathrm{H}, 5.53 ; \mathrm{N}, 5.91 \%$, found; C, 65.72; H, 5.10; N, $5.84 \%$; $\lambda_{\max }(\mathrm{DCM} / \mathrm{MeOH}) 405 \mathrm{~nm}$ @ $0.4 \mathrm{mM}, \varepsilon=494 \mathrm{M}^{-1} \mathrm{~cm}^{-1}$.

Compound (39v): Zinc chloride; Yield $=0.21 \mathrm{~g}, 83 \% ; \mathrm{v}_{\max } / \mathrm{cm}^{-1}(\mathrm{KBr}): 3430$, 1630, 1458, 1185, 918, 765, 540; $\delta_{\mathrm{H}} / \mathrm{ppm}$ : Conc. of $10^{-3} \mathrm{~mm}$; $\left(d_{6}\right.$-DMSO); 8.94 (d, $J$ $=4.1 \mathrm{~Hz}), 8.61(\mathrm{~d}, J=4.7 \mathrm{~Hz}), 7.93(\mathrm{~s}), 7.82(\mathrm{t}, J=8.7 \mathrm{~Hz}), 7.73(\mathrm{t}, J=7.8 \mathrm{~Hz})$, $7.64(\mathrm{~d}, J=7.8 \mathrm{~Hz}), 7.52$ (d, $J=7.8 \mathrm{~Hz}), 7.38$ (br d), 7.31 (d, $J=7.4 \mathrm{~Hz}), 7.08$ (d, $J$ $=6.9 \mathrm{~Hz}), 6.83(\mathrm{~d}, J=7.4 \mathrm{~Hz}), 6.70(\mathrm{t}, J=7.4 \mathrm{~Hz}), 6.59$ (t, $J=6.4 \mathrm{~Hz}), 5.59$ (s), 5.05 (s), 4.99 (s), 4.94 (s), 4.32 (d, $J=13.2 \mathrm{~Hz}), 4.11$ (br d), 3.35 (d, $J=13.2 \mathrm{~Hz}$ ), Anal. Calc. for $\mathrm{C}_{70} \mathrm{H}_{66} \mathrm{Cl}_{2} \mathrm{~N}_{6} \mathrm{O}_{4} \mathrm{Zn}_{2}: \mathrm{C}, 63.31 ; \mathrm{H}, 5.01 ; \mathrm{N}, 6.33 \%$, found; C, 63.11; H, 4.99; N, $6.18 \%$; $\lambda_{\max }(\mathrm{DCM} / \mathrm{MeOH}) 402 \mathrm{~nm} @ 0.4 \mathrm{mM}, \varepsilon=338 \mathrm{M}^{-1} \mathrm{~cm}^{-1}$.

Compound (39vi): Zinc perchlorate; Yield $=0.22 \mathrm{~g}, 73 \% ; \mathrm{v}_{\max } / \mathrm{cm}^{-1}(\mathrm{KBr}): 3569$, 1626, 1459, 1384, 1121 (perchlorate anion peak), 626; $\delta_{\mathrm{H}} / \mathrm{ppm}$ : Conc. of $10^{-3} \mathrm{~mm}$; $\left(d_{6}\right.$-DMSO); $8.62(\mathrm{~d}, J=6.2 \mathrm{~Hz}), 8.40(\mathrm{br} \mathrm{d}), 8.06(\mathrm{~s}), 7.62(\mathrm{~d}, J=7.5 \mathrm{~Hz}), 7.39$ (t, $J$ $=5.6 \mathrm{~Hz}), 7.31(\mathrm{~d}, J=8.0 \mathrm{~Hz}), 7.13(\mathrm{~d}, J=7.2 \mathrm{~Hz}), 6.93(\mathrm{t}, J=7.2 \mathrm{~Hz}), 6.76(\mathrm{~d}, J=$ $7.2 \mathrm{~Hz}), 6.44$ (t, $J=8.0 \mathrm{~Hz}$ ), 5.06 (s), 4.75 (s), 4.33 (br d), 3.37 (br d); Anal. Calc. for $\mathrm{C}_{70} \mathrm{H}_{66} \mathrm{Cl}_{4} \mathrm{~N}_{6} \mathrm{O}_{20} \mathrm{Zn}_{2}: \mathrm{C}, 53.08 ; \mathrm{H}, 4.20 ; \mathrm{N}, 5.31 \%$, found; C, 52.88; H, 4.15; N, $5.23 \% ; \lambda_{\max }(\mathrm{DCM} / \mathrm{MeOH}) 402 \mathrm{~nm} @ 0.4 \mathrm{mM}, \varepsilon=381 \mathrm{M}^{-1} \mathrm{~cm}^{-1}$. 


\subsection{1,23-Dibromomethyl-25,27-di(methyl-2-pyridine)-26,28-}

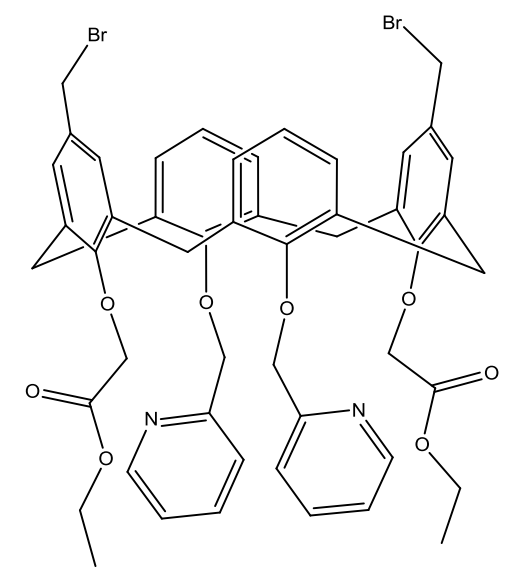

Compound (34) $(0.4 \mathrm{~g}, 0.5 \mathrm{mmol})$, ethyl bromoacetate $(0.17 \mathrm{~g}, 1.0 \mathrm{mmol})$ and sodium hydride ( $0.3 \mathrm{~g}, 1.3 \mathrm{mmol}$ (excess)) were refluxed in $\mathrm{CH}_{3} \mathrm{CN}$ :DCM (50 $\mathrm{mL}: 20 \mathrm{~mL}$ ) overnight under nitrogen gas. The solution was allowed to cool to room temperature, the inorganic salts were removed by filtration and the volatiles removed under reduced pressure to afford a red waxy solid (40).

Yield $=0.36 \mathrm{~g}, 74 \% ; \mathrm{v}_{\max } / \mathrm{cm}^{-1}(\mathrm{KBr}): 2924,1747,1590,1464,1192,1092,761$; $\delta_{\mathrm{H}} / \mathrm{ppm}$ : Conc. of $10^{-3} \mathrm{~mm} ;\left(\mathrm{CDCl}_{3}\right) ; 8.66(\mathrm{~d}, 2 \mathrm{H}, \mathrm{Py}-\underline{\mathrm{H}}, J=5.0 \mathrm{~Hz}), 7.72$ (t, $2 \mathrm{H}, \mathrm{Py}-$ $\underline{\mathrm{H}}, J=8.8 \mathrm{~Hz}), 7.40$ (d, 2H, Py- $\underline{\mathrm{H}}, J=8.8 \mathrm{~Hz}), 7.16(\mathrm{t}, 2 \mathrm{H}, \mathrm{Py}-\underline{\mathrm{H}}, J=5.0 \mathrm{~Hz}), 7.05$ (s, 4H, Ar- $\underline{\mathrm{H}}$ ), 7.00 (d, 4H, Ar- $\underline{\mathrm{H}}, J=7.1 \mathrm{~Hz}), 6.67$ (t, 2H, Ar- $\underline{\mathrm{H}}, J=6.5 \mathrm{~Hz}), 5.12$ (s, 4H, O-C $\underline{\mathrm{H}}_{2}-\mathrm{Py}$ ), 4.75 (s, 4H, O-C $\underline{\mathrm{H}}_{2}-\mathrm{CO}$ ), 4.68 (s, 4H, $\underline{\mathrm{CH}}_{2}-\mathrm{Br}$ ), 4.33 (q, 4H, O$\mathrm{C}_{2} \mathrm{CH}_{3}, J=4.5 \mathrm{~Hz}$ ), 4.22 (br d, $4 \mathrm{H}, \mathrm{C}_{2}, J=12.8 \mathrm{~Hz}$ ), 3.22 (br d, 4H, $\underline{\mathrm{C}}_{2}, J=$ $12.8 \mathrm{~Hz}), 1.37$ (t, $\left.6 \mathrm{H}, \mathrm{O}-\mathrm{CH}_{2} \mathrm{CH}_{3}, J=7.2 \mathrm{~Hz}\right) ; \delta_{\mathrm{C}} / \mathrm{ppm}\left(\mathrm{CDCl}_{3}\right) ; 170.6,155.3,150.7$, 150.4, 149.2, 136.3, 134.4, 134.1, 131.2, 128.8, 128.2, 126.1, 122.2, 121.1, 77.3, 72.0, 61.4, 31.8, 31.4, 14.2; Anal. Calc. for $\mathrm{C}_{50} \mathrm{H}_{48} \mathrm{Br}_{2} \mathrm{~N}_{2} \mathrm{O}_{8} \mathrm{CH}_{3} \mathrm{CN}$ : C, 62.25; $\mathrm{H}$, $5.01 ; \mathrm{N}, 2.90 \%$, found; C, 62.18; H, 4.79; N, $2.44 \%$.

\section{Metal complexes of (40) at 1:1 ratio}

All metal ion complexation reactions were performed at room temperature in a solvent mixture of DCM-MeOH in a 1:4 ratio. All reactions used $0.10 \mathrm{mmol}$ of (40) and corresponding metal salt. The solution was stirred for $15 \mathrm{~min}$ and then the 
solvent was slowly evaporated off. A water-DCM wash was performed to remove any uncomplexed metal and the solvent was removed under reduced pressure.

Compound (40i): Zinc acetate; Yield $=0.10 \mathrm{~g}, 84 \% ; \mathrm{v}_{\max } / \mathrm{cm}^{-1}(\mathrm{KBr}): 2925,1750$, 1629, 1591, 1463, 1189, 1093, 756; $\delta_{\mathrm{H}} / \mathrm{ppm}$ : Conc. of $10^{-3} \mathrm{~mm} ;\left(\mathrm{CDCl}_{3}\right) ; 9.78(\mathrm{br} \mathrm{d})$, 9.47 (br d), 9.15 (br d), 8.74 (s), 8.58 (br d), 8.22 (br t), 7.88 (br d), 7.83 (s), 7.75 (br t), 7.59 (s), 7.09 (s), 7.07 (s), 7.03 (br t), 6.99 (d, J= $7.9 \mathrm{~Hz}), 6.92$ (d, J = 7.4 Hz), $6.86(\mathrm{t}, J=9.4 \mathrm{~Hz}), 6.68(\mathrm{~d}, J=3.3 \mathrm{~Hz}), 6.65(\mathrm{~d}, J=4.2 \mathrm{~Hz}), 6.63(\mathrm{~d}, J=3.3 \mathrm{~Hz})$, 6.51 (br t), 5.08 (s), 4.88 (s), 4.71 (s), 4.66 (br d), 4.58 (br d), 4.50 (s), 4.45 (d, $J=$ $4.8 \mathrm{~Hz}), 4.41$ (d, $J=9.6 \mathrm{~Hz}), 4.33(\mathrm{~d}, J=5.5 \mathrm{~Hz}), 4.29$ (s), 4.26 (dd, $J=3.4,6.9 \mathrm{~Hz})$, $4.20(\mathrm{~d}, J=8.3 \mathrm{~Hz}$ ), 4.13 (br t), 3.96 (s), 3.47 (s), 3.36 (br d), 3.27 (br t), 1.31 (br t); Anal. Calc. for $\mathrm{C}_{54} \mathrm{H}_{54} \mathrm{Br}_{2} \mathrm{~N}_{2} \mathrm{O}_{12} \mathrm{Zn}$ : C, 56.49; H, 4.74; N, $2.44 \%$, found; C, 56.33; H, $4.44 ; \mathrm{N}, 2.29 \%$.

Compound (40ii): Zinc chloride; Yield $=0.12 \mathrm{~g}, 79 \% ; \mathrm{v}_{\max } / \mathrm{cm}^{-1}(\mathrm{KBr}): 2926$, 1746, 1628, 1464, 1189, 1093, 755; $\delta_{\mathrm{H}} / \mathrm{ppm}$ : Conc. of $10^{-3} \mathrm{~mm}$; $\left(d_{6}\right.$-DMSO); 8.94 (br d), 8.76 (t, $J=6.9 \mathrm{~Hz}), 8.59$ (br d), 8.49 (br t), 8.40 (d, $J=8.41$ ), 8.38 (d, $J=7.7$ $\mathrm{Hz}), 8.26(\mathrm{t}, J=7.7 \mathrm{~Hz}), 8.13(\mathrm{~s}), 7.96(\mathrm{t}, J=5.6 \mathrm{~Hz}), 7.84(\mathrm{t}, J=7.7 \mathrm{~Hz}), 7.71(\mathrm{t}, J$ = 6.3 Hz), 7.61 (s), 7.45 (br t), 7.21 (br t), 7.09 (br d), 7.02 (s), 6.98 (d, J=5.1 Hz), 6.93 (s), 6.91 (s), 6.88 (d, J=6.7 Hz), 6.85 (s), 6.82 (d, J=6.0 Hz), 6.76 (s), 6.70 (br t), 6.65 (s), 6.60 (br t), 5.50 (s), 5.08 (s), 4.81 (s), 4.72 (s), 4.59 (d, J = 9.7 Hz), 4.49 (br d), 4.35 (d, $J=14.6 \mathrm{~Hz}$ ), 4.19 (s), 4.16 (d, $J=6.7 \mathrm{~Hz}$ ), 4.09 (br t), 4.04 (d, $J=5.2$ Hz), 4.01 (s), 3.97 (d, $J=4.2 \mathrm{~Hz}$ ), 3.84 (br t), 3.66 (br t), 3.25 (br d), 1.32 (br t); Anal. Calc. for $\mathrm{C}_{50} \mathrm{H}_{48} \mathrm{Br}_{2} \mathrm{Cl}_{2} \mathrm{~N}_{2} \mathrm{O}_{8} \mathrm{Zn}$ : C, 54.54; H, 4.39; N, 2.54\%, found; C, 54.23; $\mathrm{H}, 4.14 ; \mathrm{N}, 2.27 \%$.

Compound (40iii): Zinc perchlorate; Yield $=0.13 \mathrm{~g}, 78 \% ; \mathrm{v}_{\max } / \mathrm{cm}^{-1}(\mathrm{KBr}): 2925$, 1747, 1628, 1465, 1089 (perchlorate anion peak), 754, 624; $\delta_{\mathrm{H}} / \mathrm{ppm}$ : Conc. of $10^{-3}$ mm; ( $d_{6}$-DMSO); 9.09 (br d), 8.92 (d, $\left.J=6.8 \mathrm{~Hz}\right), 8.75$ (br d), 8.66 (s), 8.59 (d, $J=$ $5.9 \mathrm{~Hz}), 8.55(\mathrm{~d}, J=8.8 \mathrm{~Hz}), 8.43(\mathrm{t}, J=8.8 \mathrm{~Hz}), 8.14(\mathrm{~s}), 8.10(\mathrm{~d}, J=7.8 \mathrm{~Hz}) 8.05$ $(\mathrm{d}, J=6.0 \mathrm{~Hz}), 8.00(\mathrm{~d}, J=7.0 \mathrm{~Hz}), 7.90(\mathrm{~d}, J=6.7 \mathrm{~Hz}), 7.81$ (s), 7.64 (br d), 7.41 (d, $J=7.1 \mathrm{~Hz}), 7.19$ (s), $7.16(\mathrm{~d}, J=5.7 \mathrm{~Hz}), 7.11(\mathrm{~s}), 7.08$ (d, $J=8.5 \mathrm{~Hz}), 7.03$ (s), $6.87(\mathrm{t}, J=7.5 \mathrm{~Hz}), 6.82(\mathrm{~s}), 6.77(\mathrm{~d}, J=7.8 \mathrm{~Hz}), 6.94(\mathrm{~s}), 6.16(\mathrm{t}, J=6.4 \mathrm{~Hz}), 5.67$ (s), 5.00 (s), 4.89 (s), 4.76 (s), 4.67 (s), 4.52 (d, J=12.8 Hz), 4.36 (br d), 4.33 (d, $J=$ $5.8 \mathrm{~Hz}), 4.26$ (br t), 4.20 (t, $J=6.4 \mathrm{~Hz}), 4.14(\mathrm{~d}, J=5.3 \mathrm{~Hz}), 4.02($ br t), 3.86 (s), 
3.17 (s), 2.76 (s), 1.32 (br t); Anal. Calc. for $\mathrm{C}_{50} \mathrm{H}_{48} \mathrm{Br}_{2} \mathrm{Cl}_{2} \mathrm{~N}_{2} \mathrm{O}_{16} \mathrm{Zn}: \mathrm{C}, 48.86$; $\mathrm{H}$, $3.94 ; \mathrm{N}, 2.28 \%$, found; C, 48.63; H, 3.65; N, $2.19 \%$.

\section{Metal complexes of (40) at 1:2 ratio}

Same procedure was used as in the case of the 1:1 with the exception that the ratio of ligand to metal was $0.10 \mathrm{mmol}$ to $0.20 \mathrm{mmol}$.

Compound (40iv): Zinc acetate; Yield $=0.20 \mathrm{~g}, 75 \% ; \mathrm{v}_{\max } / \mathrm{cm}^{-1}(\mathrm{KBr}): 1751$, 1590, 1469, 1111, 1090, 757, 626; $\delta_{\mathrm{H}} / \mathrm{ppm}$ : Conc. of $10^{-3} \mathrm{~mm} ;\left(\mathrm{CDCl}_{3}\right) ; 9.78(\mathrm{br} \mathrm{d})$, 9.45 (br d), 9.13 (br d), 8.74 (s), 7.83 (s), 7.74 (br t), 7.59 (s), 7.10 (d, J=2.8 Hz), 7.07 (br d), 7.03 (d, $J=2.4 \mathrm{~Hz}), 7.01$ (d, $J=2.4 \mathrm{~Hz}), 6.99$ (d, $J=3.3 \mathrm{~Hz}), 6.93$ (br d), $6.90(\mathrm{~d}, J=3.5 \mathrm{~Hz}), 6.88(\mathrm{t}, J=3.0 \mathrm{~Hz}), 6.68(\mathrm{~d}, J=2.1 \mathrm{~Hz}), 6.68(\mathrm{t}, J=2.8 \mathrm{~Hz})$, 6.65 (s), 6.62 (br d), 6.52 (br t), 6.49 (br t), 5.09 (br t), 5.04 (br t), 4.88 (s), 4.71 (d, J $=3.1 \mathrm{~Hz}), 4.65$ (br t), 4.59 (br d), 4.49 (d, $J=2.6 \mathrm{~Hz}), 4.44$ (br t), 4.42 (s), 4.38 (t, $J$ $=3.7 \mathrm{~Hz}), 4.32(\mathrm{br} \mathrm{t}), 4.28(\mathrm{~d}, J=3.4 \mathrm{~Hz}), 4.21(\mathrm{~d}, J=2.8 \mathrm{~Hz}), 4.21(\mathrm{~d}, J=3.4 \mathrm{~Hz})$, 4.15 (br t), 3.95 (d, $J=3.7 \mathrm{~Hz}), 3.48$ (s), 3.43 (s), 3.36 (br t), 3.30 (br t), 1.29 (br t); Anal. Calc. for $\mathrm{C}_{58} \mathrm{H}_{60} \mathrm{Br}_{2} \mathrm{~N}_{2} \mathrm{O}_{16} \mathrm{Zn}_{2}$ : C, 52.31; H, 4.54; N, $2.10 \%$, found; C, 52.03; $\mathrm{H}, 4.41 ; \mathrm{N}, 2.00 \%$.

Compound (40v): Zinc chloride; Yield $=0.21 \mathrm{~g}, 84 \% ; \mathrm{v}_{\max } / \mathrm{cm}^{-1}(\mathrm{KBr}): 1747$, 1621, 1465, 1384, 1191, 1094, 756; $\delta_{\mathrm{H}} / \mathrm{ppm}$ : Conc. of $10^{-3} \mathrm{~mm}$; $\left(d_{6}\right.$-DMSO); 9.10 (br d), $8.92(\mathrm{~d}, J=6.8 \mathrm{~Hz}), 8.58(\mathrm{~d}, J=8.8 \mathrm{~Hz}), 8.43(\mathrm{t}, J=6.8 \mathrm{~Hz}), 8.17$ (br t), 8.13 (s), $8.01(\mathrm{~d}, J=9.8 \mathrm{~Hz}), 7.91(\mathrm{~d}, J=7.8 \mathrm{~Hz}), 7.23(\mathrm{~d}, J=8.7 \mathrm{~Hz}), 7.13(\mathrm{~d}, J=9.6 \mathrm{~Hz})$, 7.10 (s), 7.05 (d, $J=9.6 \mathrm{~Hz}), 7.00$ (s), 6.89 (d, $J=7.0 \mathrm{~Hz}), 6.83$ (s), 6.68 (d, $J=5.2$ $\mathrm{Hz}), 6.61(\mathrm{~d}, J=3.5 \mathrm{~Hz}), 6.60(\mathrm{~d}, J=6.1 \mathrm{~Hz}), 6.38$ (t, $J=7.0 \mathrm{~Hz}), 4.86(\mathrm{~s}), 4.76(\mathrm{~s})$, 4.63 (br t), 4.52 (d, $J=16.2 \mathrm{~Hz}), 4.37$ (s), 4.32 (t, $J=5.6 \mathrm{~Hz}), 4.27$ (br t), 4.21 (s), $4.15(\mathrm{~d}, J=8.9 \mathrm{~Hz}), 3.80(\mathrm{~s}), 3.56(\mathrm{br} \mathrm{d}), 1.31(\mathrm{br} \mathrm{t})$; Anal. Calc. for $\mathrm{C}_{50} \mathrm{H}_{48} \mathrm{Br}_{2} \mathrm{Cl}_{4} \mathrm{~N}_{2} \mathrm{O}_{8} \mathrm{Zn}_{2}$ : C, 48.54; H, 3.91; N, $2.26 \%$, found; C, 48.09; H, 3.78; N, $2.08 \%$.

Compound (40vi): Zinc perchlorate; Yield $=0.20 \mathrm{~g}, 87 \% ; \mathrm{v}_{\max } / \mathrm{cm}^{-1}(\mathrm{KBr}): 2926$, 1747, 1625, 1590, 1439, 1189 (perchlorate anion peak), 759; $\delta_{\mathrm{H}} / \mathrm{ppm}$ : Conc. of $10^{-3}$ mm; $\left(d_{6}\right.$-DMSO); 9.07 (d, $\left.J=8.4 \mathrm{~Hz}\right), 8.90(\mathrm{~d}, J=8.4 \mathrm{~Hz}), 8.74(\mathrm{br} \mathrm{t}), 8.66$ (s), 8.58 $(\mathrm{d}, J=6.3 \mathrm{~Hz}), 8.53$ (d, $J=5.2 \mathrm{~Hz}), 8.41(\mathrm{t}, J=8.4 \mathrm{~Hz}), 8.27(\mathrm{~s}), 8.12(\mathrm{t}, J=8.4$ Hz), 7.99 (d, $J=8.4 \mathrm{~Hz}), 7.90$ (t, $J=7.3 \mathrm{~Hz}), 7.81$ (s), 7.62 (br t), 7.38 (d, $J=7.7$ 
Hz), 7.19 (s), 7.15 (d, $J=5.9 \mathrm{~Hz}), 7.10$ (s), 7.06 (d, $J=7.8 \mathrm{~Hz}), 7.02$ (s), 6.99 (s), $6.93(\mathrm{~d}, J=6.4 \mathrm{~Hz}), 6.86(\mathrm{t}, J=6.4 \mathrm{~Hz}), 6.82(\mathrm{~s}), 6.79$ (br d), 6.75 (br d), 6.63 (br d), $6.61(\mathrm{~d}, J=2.3 \mathrm{~Hz}), 6.58(\mathrm{~d}, J=2.3 \mathrm{~Hz}), 4.88$ (s), 4.77 (br d), 4.67 (br d), 4.52 (d, $J$ $=15.7 \mathrm{~Hz}), 4.35(\mathrm{~s}), 4.32(\mathrm{~d}, J=7.4 \mathrm{~Hz}), 4.25(\mathrm{br} \mathrm{t}), 4.19$ (t, $J=5.4 \mathrm{~Hz}), 4.14(\mathrm{~d}, J=$ $5.9 \mathrm{~Hz}$ ), 4.01 (br t), 3.84 (br t), 3.17 (s), 2.74 (d, $J=4.5 \mathrm{~Hz}$ ), 1.33 (br t); Anal. Calc. for $\mathrm{C}_{50} \mathrm{H}_{48} \mathrm{Br}_{2} \mathrm{Cl}_{4} \mathrm{~N}_{2} \mathrm{O}_{24} \mathrm{Zn}_{2}: \mathrm{C}, 40.22 ; \mathrm{H}, 3.24 ; \mathrm{N}, 1.88 \%$, found; $\mathrm{C}, 40.08 ; \mathrm{H}, 3.09$; $\mathrm{N}, 1.73 \%$.

\subsection{1,23-Di[ $N$-(2-pyridiylethyl)aminomethyl]-25,27-dimethylpyridyl-} 26,28-diethoxycarbonylmethoxycalix[4]arene. (41)

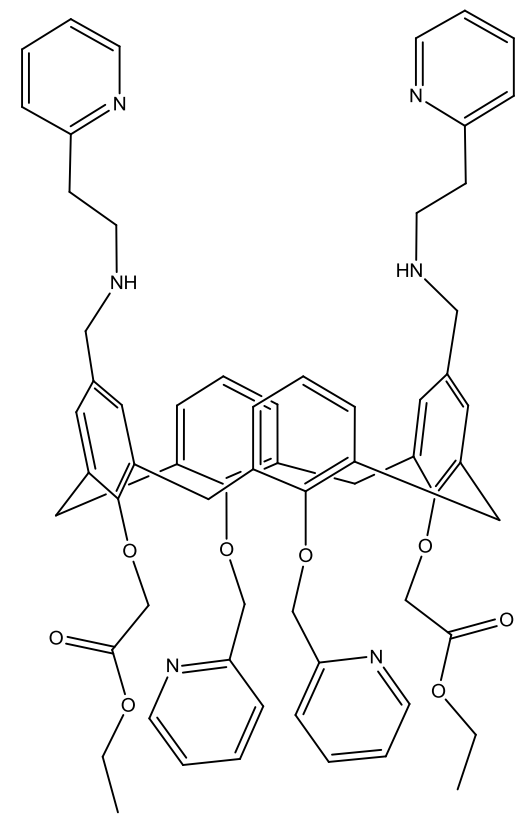

Compound (40) $(0.4 \mathrm{~g}, 0.41 \mathrm{mmol})$ triethylamine $(0.08 \mathrm{~g}, 0.83 \mathrm{mmol})$ and 2 aminoethylpyridine $(0.10 \mathrm{~g}, 0.83 \mathrm{mmol})$ were refluxed in $\mathrm{CH}_{3} \mathrm{CN}: \mathrm{DCM}$ (50 mL:20 $\mathrm{mL}$ ) overnight under nitrogen gas. The solution was allowed to cool to room temperature and the volatiles removed under reduced pressure. The residue was then washed with water $(30 \mathrm{~mL})$ and DCM $(30 \mathrm{~mL})$ to remove any excess triethylamine and the solvent removed under reduced pressure to afford a yellow waxy solid (41). 
Yield $=0.40 \mathrm{~g}, 84 \% ; \mathrm{v}_{\max } / \mathrm{cm}^{-1}(\mathrm{KBr}): 3419,2924,1751,1666,1591,1435,1191$, 1091, 757; $\delta_{\mathrm{H}} / \mathrm{ppm}$ : Conc. of $10^{-3} \mathrm{~mm} ;\left(\mathrm{CDCl}_{3}\right) ; 8.58(\mathrm{~d}, 4 \mathrm{H}, \mathrm{Py}-\underline{\mathrm{H}}, J=5.2 \mathrm{~Hz}), 7.75$ (t, 4H, Py- $\underline{\mathrm{H}}, J=3.3 \mathrm{~Hz}), 7.56$ (t, 4H, Py- $\underline{\mathrm{H}}, J=8.7 \mathrm{~Hz}), 7.20$ (d, 4H, Py- $\underline{\mathrm{H}}, J=7.6$ $\mathrm{Hz}), 6.99$ (s, 4H, Ar- $\underline{\mathrm{H}}$ ), 6.89 (d, 4H, Ar- $\underline{\mathrm{H}}, J=9.1 \mathrm{~Hz}), 6.77$ (t, 2H, Ar- $\underline{\mathrm{H}}, J=1.8$ $\mathrm{Hz}$ ), 5.19 (s, 4H, O-C $\underline{\mathrm{H}}_{2}-\mathrm{Py}$ ), 4.64 (s, 4H, $\underline{\mathrm{C}}_{2}-\mathrm{NR}_{2}$ ), 4.37 (t, $4 \mathrm{H}, \mathrm{O}-\underline{\mathrm{C}}_{2} \mathrm{CH}_{3}, J=7.2$ $\mathrm{Hz}), 4.32\left(\mathrm{~d}, 4 \mathrm{H}, \underline{\mathrm{C}}_{2}, J=13.8 \mathrm{~Hz}\right), 3.42\left(\mathrm{~s}, 4 \mathrm{H}, \mathrm{O}-\underline{\mathrm{C}}_{2}-\mathrm{CO}\right), 3.37\left(\mathrm{~d}, 4 \mathrm{H}, \mathrm{C}_{2}, J=\right.$ $13.8 \mathrm{~Hz}), 3.17$ (t, $\left.4 \mathrm{H}, \mathrm{N}-\mathrm{CH}_{2} \underline{\mathrm{C}}_{2}, J=8.6 \mathrm{~Hz}\right), 3.01$ (t, $4 \mathrm{H}, \mathrm{N}-\underline{\mathrm{C}}_{2} \underline{\mathrm{CH}}_{2}, J=7.6 \mathrm{~Hz}$ ), $1.15\left(\mathrm{t}, 6 \mathrm{H}, \mathrm{O}-\mathrm{CH}_{2} \underline{\mathrm{C}}_{3}, J=7.4 \mathrm{~Hz}\right) ; \delta_{\mathrm{C}} / \mathrm{ppm}\left(\mathrm{CDCl}_{3}\right) ; 170.9,166.9,156.9,155.8$, $154.5,147.8,147.6,136.3$, 135.7, 134.4, 132.8, 127.9, 127.6, 125.9, 124.5, 124.5, 122.5, 121.0, 120.6, 76.8, 70.7, 59.2, 58.8, 49.4, 37.4, 31.3, 12.6; Anal. Calc. for $\mathrm{C}_{64} \mathrm{H}_{66} \mathrm{~N}_{6} \mathrm{O}_{8}: \mathrm{C}, 73.40 ; \mathrm{H}, 6.35 ; \mathrm{N}, 8.02 \%$, found; C, 73.09; H, 6.21; N, $7.96 \%$.

\section{Metal complexes of (41) at 1:1 ratio}

All metal ion complexation reactions were performed at room temperature in a solvent mixture of DCM-MeOH in a 1:1 ratio. All reactions used $0.09 \mathrm{mmol}$ of (41) and corresponding metal salt. The solution was stirred for $15 \mathrm{~min}$ and then the solvent was slowly evaporated off. A water-DCM wash was performed to remove any uncomplexed metal and the solvent was removed under reduced pressure.

Compound (41i): Zinc acetate; Yield $=0.09 \mathrm{~g}, 77 \% ; \mathrm{v}_{\max } / \mathrm{cm}^{-1}(\mathrm{KBr}): 3422,2923$, 1755, 1591, 1435, 1192, 1092, 759; $\delta_{\mathrm{H}} / \mathrm{ppm}$ : Conc. of $10^{-3} \mathrm{~mm} ;\left(\mathrm{CDCl}_{3}\right) ; 8.72(\mathrm{br} \mathrm{d})$, 8.62 (br d), 8.61 (br d), 8.57 (br d), 8.09 (d, $J=8.6 \mathrm{~Hz}$ ), 7.92 (s), 7.83 (dd, $J=2.1$, $7.5 \mathrm{~Hz}), 7.76(\mathrm{t}, J=8.9 \mathrm{~Hz}), 7.66(\mathrm{t}, J=1.7 \mathrm{~Hz}), 7.63(\mathrm{~s}), 7.41(\mathrm{t}, J=8.0 \mathrm{~Hz}), 7.31$ (d, $J=5.1 \mathrm{~Hz}), 7.23$ (br d), 7.12 (t, $J=6.8 \mathrm{~Hz}), 7.07$ (s), 7.05 (d, $J=2.2 \mathrm{~Hz}), 7.02$ (d, $J=2.2 \mathrm{~Hz}$ ), 6.99 (br t), 6.97 (br t), 6.94 (t, $J=1.8 \mathrm{~Hz}), 6.91$ (br d), 6.89 (s), 6.86 (br t), $6.83(\mathrm{~d}, J=4.4 \mathrm{~Hz}), 6.78(\mathrm{t}, J=7.8 \mathrm{~Hz}), 6.67(\mathrm{~d}, J=7.5 \mathrm{~Hz}), 6.63(\mathrm{~d}, J=3.4 \mathrm{~Hz})$, $5.21(\mathrm{~s}), 5.01$ (s), 4.75 (s), 4.72 (s), 4.64 (s), 4.48 (d, J=13.8 Hz), 4.40 (s), 4.38 (s), $4.34(\mathrm{~d}, J=3.2 \mathrm{~Hz}), 4.31(\mathrm{~d}, J=3.8 \mathrm{~Hz}), 4.27(\mathrm{~s}), 4.14(\mathrm{t}, J=5.5 \mathrm{~Hz}), 4.10(\mathrm{~s}), 3.48$ (s), $3.42(\mathrm{~d}, J=5.6 \mathrm{~Hz}), 3.38(\mathrm{~d}, J=5.6 \mathrm{~Hz}), 3.28(\mathrm{br} \mathrm{t}), 3.23(\mathrm{~s}), 3.17(\mathrm{~s}), 3.15(\mathrm{t}, J=$ $3.0 \mathrm{~Hz}$ ), 3.13 (s), 1.11 (br t); Anal. Calc. for $\mathrm{C}_{68} \mathrm{H}_{72} \mathrm{~N}_{6} \mathrm{O}_{12} \mathrm{Zn}: \mathrm{C}, 66.36 ; \mathrm{H}, 5.90 ; \mathrm{N}$, $6.83 \%$, found; C, 66.14; H, 5.86; N, $6.56 \%$.

Compound (41ii): Zinc chloride; Yield $=0.85 \mathrm{~g}, 75 \% ; \mathrm{v}_{\max } / \mathrm{cm}^{-1}(\mathrm{KBr}): 3407$, 2929, 1743, 1608, 1464, 1192, 1090, 762; $\delta_{\mathrm{H}} / \mathrm{ppm}$ : Conc. of $10^{-3} \mathrm{~mm}$; ( $d_{6}$-DMSO); $8.61(\mathrm{~d}, J=4.9 \mathrm{~Hz}), 8.56(\mathrm{~d}, J=4.0 \mathrm{~Hz}), 8.51(\mathrm{~d}, J=5.9 \mathrm{~Hz}), 8.31(\mathrm{~d}, J=6.5 \mathrm{~Hz})$, 
$8.13(\mathrm{~s}), 8.02(\mathrm{~d}, J=7.3 \mathrm{~Hz}), 7.81(\mathrm{~s}), 7.70(\mathrm{~d}, J=9.0 \mathrm{~Hz}), 7.55(\mathrm{~d}, J=6.5 \mathrm{~Hz}), 7.44$ (t, $J=7.3 \mathrm{~Hz}), 7.07$ (s), 7.05 (s), 7.03 (d, $J=4.5 \mathrm{~Hz}), 7.00$ (d, $J=4.5 \mathrm{~Hz}), 6.98$ (s), $6.81(\mathrm{~d}, J=6.7 \mathrm{~Hz}), 6.77(\mathrm{~s}), 6.69(\mathrm{t}, J=6.7 \mathrm{~Hz}), 6.60(\mathrm{~d}, J=3.1 \mathrm{~Hz}), 6.57(\mathrm{~d}, J=3.1$ $\mathrm{Hz}), 6.49$ (d, $J=7.6 \mathrm{~Hz}), 6.33(\mathrm{t}, J=7.6 \mathrm{~Hz}), 5.13(\mathrm{~s}), 4.98$ (s), $4.92(\mathrm{~s}), 4.75$ (d, $J=$ $9.0 \mathrm{~Hz}), 4.69$ (s), 4.61 (s), 4.36 (d, $J=5.2 \mathrm{~Hz}), 4.31(\mathrm{t}, J=5.2 \mathrm{~Hz}), 4.26$ (d, J= 7.5 $\mathrm{Hz}), 4.22$ (br t), 4.16 (d, $J=7.5 \mathrm{~Hz}), 4.12$ (d, $J=7.9 \mathrm{~Hz}), 4.04$ (s), 4.01 (d, $J=3.9$ $\mathrm{Hz}$ ), 3.89 (d, $J=7.5 \mathrm{~Hz}$ ), 1.14 (br t); Anal. Calc. for $\mathrm{C}_{64} \mathrm{H}_{66} \mathrm{Cl}_{2} \mathrm{~N}_{6} \mathrm{O}_{8} \mathrm{Zn}: \mathrm{C}, 64.95 ; \mathrm{H}$, $5.62 ; \mathrm{N}, 7.10 \%$, found; C, 64.52; H, 5.41; N, $6.96 \%$.

Compound (41iii): Zinc perchlorate; Yield $=0.10 \mathrm{~g}, 80 \% ; \mathrm{v}_{\max } / \mathrm{cm}^{-1}(\mathrm{KBr}): 3413$, 2929, 1747, 1611, 1465, 1093 (perchlorate anion peak), 761, 622; $\delta_{\mathrm{H}} / \mathrm{ppm}$ : Conc. of $10^{-3} \mathrm{~mm}$; $\left(d_{6}\right.$-DMSO); 8.61 (br d), 8.55 (br d), 8.30 (br d), 8.13 (s), 7.98 (t, $J=7.9$ Hz), 7.94 (s), 7.80 (s), 7.75 (br t), 7.55 (br t), 7.44 (br t), 7.36 (d, J= 7.6 Hz), 7.14 (d, $J=7.7 \mathrm{~Hz}), 7.10(\mathrm{~s}), 7.07$ (s), 7.04 (d, $J=4.0 \mathrm{~Hz}), 7.01$ (d, $J=3.0 \mathrm{~Hz}), 7.00$ (d, $J=$ $1.5 \mathrm{~Hz}), 6.97$ (s), 6.82 (br t), 6.79 (s), 6.77 (s), 6.60 (br t), 6.49 (d, J=8.4 Hz), 6.33 (t, $J=8.0 \mathrm{~Hz}), 6.03(\mathrm{~s}), 5.13(\mathrm{~s}), 4.98(\mathrm{~s}), 4.91(\mathrm{~s}), 4.83$ (d, $J=12.0 \mathrm{~Hz}), 4.75$ (d, $J=$ $8.8 \mathrm{~Hz}$ ), 4.67 (br d), 4.61 (s), 4.35 (d, $J=6.6 \mathrm{~Hz}), 4.33$ (s), 4.30 (d, $J=3.3 \mathrm{~Hz}), 4.27$ $(\mathrm{d}, 7.7 \mathrm{~Hz}), 4.22(\mathrm{t}, J=3.0 \mathrm{~Hz}), 4.18(\mathrm{~s}), 4.01(\mathrm{~d}, J=5.0 \mathrm{~Hz}), 3.90(\mathrm{~d}, J=6.9 \mathrm{~Hz})$, $3.19(\mathrm{~d}, J=13.5 \mathrm{~Hz}), 3.02$ (br t), 2.89 (s), 2.73 (s), 1.16 (br t); Anal. Calc. for $\mathrm{C}_{64} \mathrm{H}_{66} \mathrm{Cl}_{2} \mathrm{~N}_{6} \mathrm{O}_{16} \mathrm{Zn}: \mathrm{C}, 58.61 ; \mathrm{H}, 5.07 ; \mathrm{N}, 6.41 \%$, found; C, 58.54; H, 4.91; N, 6.17 $\%$.

\section{Metal complexes of (41) at $1: 2$ ratio}

Same procedure was used as in the case of the 1:1 with the exception that the ratio of ligand to metal was $0.09 \mathrm{mmol}$ to $0.18 \mathrm{mmol}$.

Compound (41iv): Zinc acetate; Yield $=0.22 \mathrm{~g}, 81 \% ; \mathrm{v}_{\max } / \mathrm{cm}^{-1}(\mathrm{KBr}): 3429$, $1751,1610,1436,1188,1088,762 ; \delta_{\mathrm{H}} / \mathrm{ppm}$ : Conc. of $10^{-3} \mathrm{~mm} ;\left(\mathrm{CDCl}_{3}\right) ; 8.73(\mathrm{t}, J=$ $6.1 \mathrm{~Hz}), 8.62(\mathrm{~d}, J=6.1 \mathrm{~Hz}), 8.53(\mathrm{br} \mathrm{d}), 7.77(\mathrm{td}, J=1.7,7.5 \mathrm{~Hz}), 7.61(\mathrm{t}, J=6.8$ $\mathrm{Hz}), 7.36$ (t, $J=6.9 \mathrm{~Hz}), 7.23(\mathrm{~d}, J=8.2 \mathrm{~Hz}), 7.17$ (d, $J=6.9 \mathrm{~Hz}), 7.10$ (s), 7.08 (s), $7.05(\mathrm{~d}, J=2.7 \mathrm{~Hz}), 7.01(\mathrm{~d}, J=4.8 \mathrm{~Hz}), 6.91(\mathrm{~s}), 6.87(\mathrm{~d}, J=6.21), 6.71(\mathrm{t}, J=6.2$ Hz), 5.19 (s), 5.07 (s), 4.99 (s), 4.88 (s), 4.76 (s), 4.64 (d, J= 4.2 Hz), 4.52 (d, J= $12.7 \mathrm{~Hz}$ ), 4.40 (s), 4.36 (d, J=4.2 Hz), 4.32 (br t), 4.28 (s), 4.23 (br t), 4.15 (s), 4.11 (s), 4.05 (s), 3.98 (d, J=6.0 Hz), 3.89 (d, $J=6.0 \mathrm{~Hz}), 3.72$ (t, $J=6.7 \mathrm{~Hz}), 3.46(\mathrm{~d}, J$ 
$=12.9 \mathrm{~Hz}), 3.16(\mathrm{br} \mathrm{t}), 3.06(\mathrm{t}, J=5.5 \mathrm{~Hz}), 1.15(\mathrm{br} \mathrm{t})$; Anal. Calc. for $\mathrm{C}_{72} \mathrm{H}_{78} \mathrm{~N}_{6} \mathrm{O}_{16} \mathrm{Zn}_{2}:$ C, 61.15; H, 5.56; N, $5.94 \%$, found; C, 61.03; H, 5.31; N, $6.24 \%$.

Compound (41v): Zinc chloride; Yield $=0.21 \mathrm{~g}, 83 \% ; \mathrm{v}_{\max } / \mathrm{cm}^{-1}(\mathrm{KBr}): 3429$, 1747, 1595, 1438, 1191, 1092, 762; $\delta_{\mathrm{H}} / \mathrm{ppm}$ : Conc. of $10^{-3} \mathrm{~mm}$; $\left(d_{6}\right.$-DMSO); 8.57 (br d), 7.79 (br d), 7.61 (br t), 7.06 (br d), 6.90 (br t), 6.69 (br t), 5.20 (s), 4.78 (br d), 4.64 (s), 4.57 (br d), 3.45 (br d), 3.16 (br t), 1.12 (br t); Anal. Calc. for $\mathrm{C}_{64} \mathrm{H}_{66} \mathrm{Cl}_{4} \mathrm{~N}_{6} \mathrm{O}_{8} \mathrm{Zn}_{2}: \mathrm{C}, 58.24 ; \mathrm{H}, 5.04 ; \mathrm{N}, 6.37 \%$, found; $\mathrm{C}, 58.03 ; \mathrm{H}, 4.87 ; \mathrm{N}, 6.15$ $\%$.

Compound (41vi): Zinc perchlorate; Yield $=0.30 \mathrm{~g}, 83 \% ; \mathrm{v}_{\max } / \mathrm{cm}^{-1}(\mathrm{KBr}): 3423$, 2938, 1744, 1610, 1445, 1096 (perchlorate anion peak), 763, 622; $\delta_{\mathrm{H}} / \mathrm{ppm}$ : Conc. of $10^{-3} \mathrm{~mm}$; $\left(d_{6}\right.$-DMSO); $8.66(\mathrm{~d}, J=5.0 \mathrm{~Hz}), 8.60(\mathrm{~d}, J=4.0 \mathrm{~Hz}), 8.55(\mathrm{br} \mathrm{d}), 8.29$ (d, $J=7.0 \mathrm{~Hz}), 8.13(\mathrm{~s}), 7.98(\mathrm{t}, J=10.0 \mathrm{~Hz}), 7.80(\mathrm{t}, J=7.0 \mathrm{~Hz}), 7.70(\mathrm{t}, J=9.0 \mathrm{~Hz})$, 7.55 (br t), 7.44 (t, $J=9.0 \mathrm{~Hz}), 7.35(\mathrm{t}, J=9.0 \mathrm{~Hz}), 7.13(\mathrm{t}, J=8.9 \mathrm{~Hz}), 7.06(\mathrm{~d}, J=$ $8.2 \mathrm{~Hz}), 7.02$ (d, $J=4.4 \mathrm{~Hz}), 6.99(\mathrm{~d}, J=7.4 \mathrm{~Hz}), 6.80$ (t, $J=8.0 \mathrm{~Hz}), 6.70(\mathrm{br}$ t), $6.59(\mathrm{t}, J=7.2 \mathrm{~Hz}), 6.48(\mathrm{~d}, J=7.6 \mathrm{~Hz}), 6.33(\mathrm{t}, J=8.0 \mathrm{~Hz}), 5.12$ (s), 4.99 (s), 4.91 (s), 4.75 (d, $J=7.5 \mathrm{~Hz}), 4.67$ (br d), 4.61 (d, $J=12.2 \mathrm{~Hz}), 4.36$ (d, $J=2.1 \mathrm{~Hz}), 4.33$ (s), $4.31(\mathrm{t}, J=2.1 \mathrm{~Hz}), 4.28(\mathrm{~d}, J=2.8 \mathrm{~Hz}), 4.26(\mathrm{t}, J=2.5 \mathrm{~Hz}), 4.22(\mathrm{t}, J=3.2 \mathrm{~Hz})$, 4.18 (s), 4.16 (br t), 4.10 (br t), 4.01 (d, $J=12.2 \mathrm{~Hz}), 3.89$ (q, $J=7.5 \mathrm{~Hz}), 3.17$ (d, $J=$ $14.5 \mathrm{~Hz}), 3.03$ (br t), 2.88 (s), 2.73 (s), 1.13 (br t); Anal. Calc. for $\mathrm{C}_{64} \mathrm{H}_{66} \mathrm{Cl}_{4} \mathrm{~N}_{6} \mathrm{O}_{24} \mathrm{Zn}_{2}: \mathrm{C}, 48.78 ; \mathrm{H}, 4.22 ; \mathrm{N}, 5.33 \%$, found; $\mathrm{C}, 48.55 ; \mathrm{H}, 4.11 ; \mathrm{N}, 5.19$ $\%$. 


\subsection{1,23-Di[ $N, N^{\prime}$-di((2'-pyridylethyl)(4-dimethyl-2,2-bipyridine)-4'-}

aminomethyl)]-25,27-dibenzyl-26,28-diethoxycarbonylmethoxycalix[4]arene. (42)

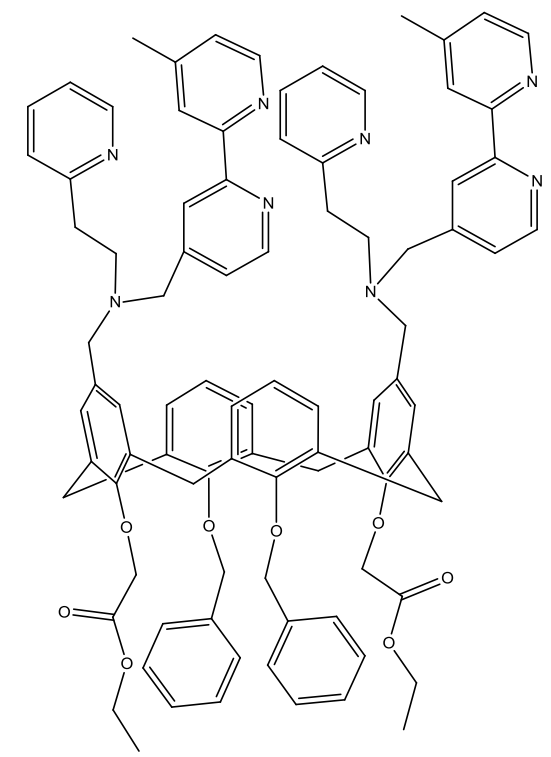

Compound (22) (0.2 g, $0.76 \mathrm{mmol})$, compound (49) $(0.1 \mathrm{~g}, 0.12 \mathrm{mmol})$, and triethylamine $(0.06 \mathrm{~g}, 0.76 \mathrm{mmol})$ were refluxed in $\mathrm{CH}_{3} \mathrm{CN}(50 \mathrm{~mL})$ overnight under nitrogen gas. The solution was allowed to cool to room temperature, the inorganic salts were removed by filtration and the volatiles removed under reduced pressure to give a dark red waxy solid (42).

Yield $=0.11 \mathrm{~g}, 79 \% ; \mathrm{v}_{\max } / \mathrm{cm}^{-1}(\mathrm{KBr}): 1637,1437,1383,1175,1093,697 ; \delta_{\mathrm{H}} / \mathrm{ppm}:$ Conc. of $10^{-3} \mathrm{~mm} ;\left(\mathrm{CDCl}_{3}\right) ; 8.66$ (d, $4 \mathrm{H}$, bipy-NCH,$\left.J=4.3 \mathrm{~Hz}\right), 8.58(\mathrm{~d}, 4 \mathrm{H}, \mathrm{Py}-\underline{\mathrm{H}}$,

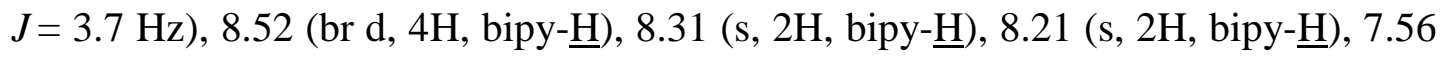
(t, 4H, Py- $\underline{\mathrm{H}}, J=5.6 \mathrm{~Hz}), 7.30$ (d, 4H, Py- $\underline{\mathrm{H}}, J=2.9 \mathrm{~Hz}), 7.26(\mathrm{t}, 4 \mathrm{H}, \mathrm{Py}-\underline{\mathrm{H}}, J=2.6$ $\mathrm{Hz}), 7.07$ (s, 4H, Ar- $\underline{\mathrm{H}}$ ), 6.73 (d, 4H, Ar- $\underline{\mathrm{H}}, J=6.3 \mathrm{~Hz}), 6.51$ (t, $2 \mathrm{H}, \mathrm{Ar}-\underline{\mathrm{H}}, J=6.3$ $\mathrm{Hz}), 5.20$ (s, 4H, $\left.\underline{\mathrm{C}}_{2}-\mathrm{Py}\right), 4.83$ (s, 4H, O-C $\left.\underline{\mathrm{H}}_{2}-\mathrm{CO}\right), 4.79$ (s, 4H, $\left.\underline{\mathrm{C}}_{2}-\mathrm{NR}_{2}\right), 4.15$ (br d, $4 \mathrm{H}, \mathrm{C}_{2}$ ), 3.89 (m, 4H, CO- $\underline{\mathrm{H}}_{2} \mathrm{CH}_{3}, J=2.6 \mathrm{~Hz}$ ), 3.62 (s, 4H, $\underline{\mathrm{C}}_{2}-$ bipy), 3.45 (br d, $4 \mathrm{H}, \mathrm{C}_{2}$ ), 3.10 (t, $\left.4 \mathrm{H}, \mathrm{N}-\mathrm{CH}_{2} \mathrm{C}_{2}, J=3.3 \mathrm{~Hz}\right), 2.99$ (t, $4 \mathrm{H}, \mathrm{N}-\underline{\mathrm{C}}_{2} \mathrm{CH}_{2}, J=4.4$ $\mathrm{Hz}), 2.42$ (s, $6 \mathrm{H}$, bipy- $\left.\underline{\mathrm{C}}_{3}\right), 1.25$ (t, $\left.6 \mathrm{H}, \mathrm{O}-\mathrm{CH}_{2} \mathrm{C}_{3}, J=3.7 \mathrm{~Hz}\right) ; \delta_{\mathrm{C}} / \mathrm{ppm}\left(\mathrm{CDCl}_{3}\right)$; 170.2, 155.8, 155.5, 154.9, 154.4, 153.8, 153.2, 152.1, 148.3, 147.9, 136.9, 136.1, 135.3, 134.6, 133.7, 129.4, 128.4, 128.2, 128.0, 127.7, 127.0, 126.2, 123.9, 123.1, $122.8,122.3,121.9,120.8,74.2,67.6,63.4,59.4,56.5,53.5,36.9,30.0,20.2,13.2$; 
Anal. Calc. for $\mathrm{C}_{90} \mathrm{H}_{88} \mathrm{~N}_{8} \mathrm{O}_{6}$ : C, 76.68; H, 6.29; N, $7.95 \%$, found; C, 76.62; H, 6.25; $\mathrm{N}, 7.91 \%$.

\section{Metal complexes at 1:1 ratio}

All metal ion complexation reactions were performed at room temperature in a solvent mixture of $\mathrm{DCM}-\mathrm{MeOH}$ in a 1:1 ratio. All reactions used $0.07 \mathrm{mmol}$ of (42) and corresponding metal salt. The solution was stirred for $15 \mathrm{~min}$ and then the solvent was slowly evaporated off. A water-DCM wash was performed to remove any uncomplexed metal and the solvent was removed under reduced pressure.

Compound (42i): Zinc acetate; Yield $=0.09 \mathrm{~g}, 79 \% ; \mathrm{v}_{\max } / \mathrm{cm}^{-1}(\mathrm{KBr}): 2923,1756$, 1598, 1455, 1186, 1092, 760; $\delta_{\mathrm{H}} / \mathrm{ppm}$ : Conc. of $10^{-3} \mathrm{~mm} ;\left(\mathrm{CDCl}_{3}\right) ; 8.88(\mathrm{~d}, J=4.9$ $\mathrm{Hz}), 8.73(\mathrm{~d}, J=5.7 \mathrm{~Hz}), 8.67(\mathrm{~d}, J=5.7 \mathrm{~Hz}), 8.28(\mathrm{~d}, J=5.7 \mathrm{~Hz}), 8.24(\mathrm{~s}), 8.20(\mathrm{~s})$, $8.16(\mathrm{~d}, J=5.7 \mathrm{~Hz}), 8.07(\mathrm{~d}, J=9.0 \mathrm{~Hz}), 8.01(\mathrm{~s}), 7.85(\mathrm{t}, J=9.0 \mathrm{~Hz}), 7.70(\mathrm{br} \mathrm{d})$, $7.60(\mathrm{t}, J=5.7 \mathrm{~Hz}), 7.53(\mathrm{~d}, J=9.0 \mathrm{~Hz}), 7.41$ (br t), $7.41($ br t), 7.39 (br d), 7.37 (br d), 7.36 (s), 7.33 (br d), 7.32 (br t), 7.15 (d, $J=7.5 \mathrm{~Hz}$ ), 7.08 (s), 7.04 (d, $J=4.3 \mathrm{~Hz}$ ), $7.02(\mathrm{~d}, J=2.7 \mathrm{~Hz}), 6.88(\mathrm{~d}, J=2.3 \mathrm{~Hz}), 6.85(\mathrm{~d}, J=2.9 \mathrm{~Hz}), 6.82(\mathrm{~d}, J=3.4 \mathrm{~Hz})$, $6.80(\mathrm{~d}, J=1.3 \mathrm{~Hz}), 6.79(\mathrm{~d}, J=3.2 \mathrm{~Hz}), 6.77(\mathrm{~d}, J=2.1 \mathrm{~Hz}), 6.75(\mathrm{~d}, J=2.6 \mathrm{~Hz})$, 6.69 (d, J= $7.7 \mathrm{~Hz}$ ), 6.53 (s), 6.48 (br d), 6.45 (br d), 6.42 (br d), 6.39 (br d), 6.33 (br d), 6.28 (br t), $5.26(\mathrm{~s}), 5.10$ (s), 5.06 (d, $J=8.4 \mathrm{~Hz}), 5.02(\mathrm{~s}), 4.93(\mathrm{~d}, J=3.0 \mathrm{~Hz})$, $4.89(\mathrm{~d}, J=3.8 \mathrm{~Hz}), 4.85(\mathrm{~s}), 4.78(\mathrm{~d}, J=4.6 \mathrm{~Hz}), 4.71(\mathrm{~d}, J=4.6 \mathrm{~Hz}), 4.67(\mathrm{~s}), 4.54$ (br d), 4.49 (s), 4.44 (d, $J=5.9 \mathrm{~Hz}), 4.37$ (d, $J=14.4 \mathrm{~Hz}), 4.32$ (d, $J=3.5 \mathrm{~Hz}), 4.27$ $(\mathrm{d}, J=7.0 \mathrm{~Hz}), 4.22(\mathrm{t}, J=3.1 \mathrm{~Hz}), 4.17(\mathrm{~d}, J=2.4 \mathrm{~Hz}), 4.09(\mathrm{~d}, J=6.3 \mathrm{~Hz}), 4.05(\mathrm{~s})$, 3.99 (s), 3.89 (br d), 3.84 (br t), 3.76 (br t), 3.70 (s), 3.64 (s), 3.59 (d, J=6.4 Hz), $3.57(\mathrm{~d}, J=9.0 \mathrm{~Hz}), 3.50$ (d, $J=5.8 \mathrm{~Hz}), 3.46$ (s), 3.11 (br t), 3.04 (br t), 2.57 (s), 2.55 (s), 2.51 (s); Anal. Calc. for $\mathrm{C}_{94} \mathrm{H}_{94} \mathrm{~N}_{8} \mathrm{O}_{12} \mathrm{Zn}: \mathrm{C}, 70.86$; H, 5.95; N, $7.03 \%$, found; C, 70.64; H, 5.61; N, $6.67 \%$.

Compound (42ii): Zinc chloride; Yield $=0.08 \mathrm{~g}, 77 \%$; $\mathrm{v}_{\max } / \mathrm{cm}^{-1}(\mathrm{KBr}): 2923$, $1731,1614,1562,1455,1090,1092,760 ; \delta_{\mathrm{H}} / \mathrm{ppm}$ : Conc. of $10^{-3} \mathrm{~mm}$; ( $d_{6}$-DMSO); 8.60 (br d), 8.46 (br d), 7.68 (br t), 7.51 (br d), 7.33 (br t), 7.21 (s), 7.04 (br d), 6.72 $(\mathrm{d}, J=7.9 \mathrm{~Hz}), 6.58(\mathrm{~d}, J=8.9 \mathrm{~Hz}), 6.39(\mathrm{br} \mathrm{t}), 6.23(\mathrm{t}, J=6.9 \mathrm{~Hz}), 5.01(\mathrm{~s}), 4.88(\mathrm{~s})$, $4.71(\mathrm{~d}, J=9.6 \mathrm{~Hz}), 4.64(\mathrm{~d}, J=7.7 \mathrm{~Hz}), 4.51(\mathrm{~d}, J=11.6 \mathrm{~Hz}), 4.27(\mathrm{~s}), 4.04(\mathrm{~d}, J=$ 
$7.2 \mathrm{~Hz}), 3.57$ (d, $J=4.9 \mathrm{~Hz}), 3.50$ (d, $J=8.1 \mathrm{~Hz}), 3.06$ (br t), 2.89 (br t), 2.35 (br d), 3.28 (s); Anal. Calc. for $\mathrm{C}_{90} \mathrm{H}_{88} \mathrm{Cl}_{2} \mathrm{~N}_{8} \mathrm{O}_{8} \mathrm{Zn}$ : C, 69.92; H, 5.74; N, $7.25 \%$, found; $\mathrm{C}$, 69.83; H, 5.67; N, $7.03 \%$.

Compound (42iii): Zinc perchlorate; Yield $=0.10 \mathrm{~g}, 84 \% ; \mathrm{v}_{\max } / \mathrm{cm}^{-1}(\mathrm{KBr}): 2923$, 1730, 1614, 1561, 1456, 1190, 1091 (perchlorate anion peak), 761; $\delta_{\mathrm{H}} / \mathrm{ppm}$ : Conc. of $10^{-3} \mathrm{~mm}$; ( $d_{6}$-DMSO); 8.73 (br d), 8.65 (br d), 8.54 (br d), $8.42(\mathrm{~s}), 8.13$ (s), 8.08 (d, $J=7.8 \mathrm{~Hz}$ ), $7.98(\mathrm{~s}), 7.91(\mathrm{t}, J=8.7 \mathrm{~Hz}), 7.83$ (br t), $7.76(\mathrm{~s}), 7.70(\mathrm{~d}, J=6.9 \mathrm{~Hz})$, 7.62 (br d), 7.41 (br d), 7.39 (br d), 7.34 (d, $J=2.0 \mathrm{~Hz}$ ), 7.32 (s), 7.30 (d, $J=4.3 \mathrm{~Hz}$ ), 7.22 (br t), 7.14 (d, $J=7.2 \mathrm{~Hz}), 7.08$ (d, $J=7.2 \mathrm{~Hz}), 7.01$ (d, $J=7.2 \mathrm{~Hz}), 6.96$ (d, $J=$ $7.2 \mathrm{~Hz}), 6.83(\mathrm{~d}, J=6.7 \mathrm{~Hz}), 6.74(\mathrm{~d}, J=7.5 \mathrm{~Hz}), 6.70(\mathrm{~d}, J=1.6 \mathrm{~Hz}), 6.67$ (d, $J=$ $2.5 \mathrm{~Hz}), 6.65$ (br d), 6.62 (d, J=7.1 Hz), 6.54 (s), 6.51 (s), 6.44 (s), 6.41 (s), 6.38 (br d), $6.32(\mathrm{~d}, J=9.2 \mathrm{~Hz}), 6.2(\mathrm{t}, J=7.5 \mathrm{~Hz}), 5.05(\mathrm{~s}), 4.94$ (d, $J=4.8 \mathrm{~Hz}), 4.87$ (d, $J=$ $5.2 \mathrm{~Hz}), 4.81$ (s), 4.79 (s), 4.67 (s), 4.51 (d, J=13.5 Hz), 4.35 (s), 4.27 (s), 4.21 (d, $J$ = $7.1 \mathrm{~Hz}), 4.18(\mathrm{~s}), 4.13(\mathrm{~d}, J=6.5 \mathrm{~Hz}), 4.09(\mathrm{~s}), 4.06(\mathrm{~d}, J=7.6 \mathrm{~Hz}), 3.98(\mathrm{~s}), 3.94$ (s), 3.82 (s), 3.78 (s), 3.75 (br d), 3.69 (br d), 3.17 (s), 3.11 (br t), 3.09 (br t), 2.97 (br t), 2.91 (br t), 2.47 (s); Anal. Calc. for $\mathrm{C}_{90} \mathrm{H}_{88} \mathrm{Cl}_{2} \mathrm{~N}_{8} \mathrm{O}_{16} \mathrm{Zn}$ : C, 64.57; H, 5.30; N, $6.69 \%$, found; C, 64.23; H, 5.17; N, $6.37 \%$. 


\subsection{1,23-Di[ $N, N^{\prime}$ '(2'pyridylethyl)(4-methyl-2,2'-bipyridyl)-4'-}

aminomethyl]-25,27-dibenzyl-26,28-diethoxycarbonyl(methyl-2pyridine)calix[4]arene. (43)

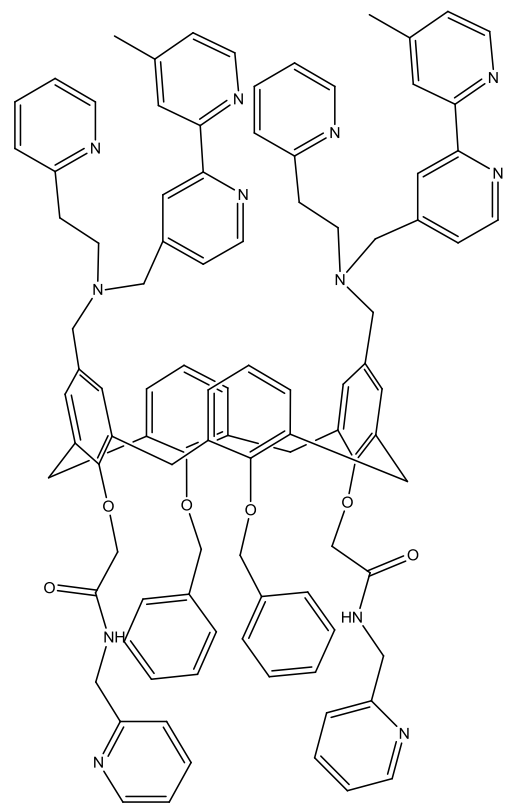

Compound (42) (0.09 g, $0.62 \mathrm{mmol})$ and 2-aminomethylpyridine (0.20 g, 1.24 mmol) were refluxed in $\mathrm{MeOH}$ : toluene (15 mL: $25 \mathrm{~mL}$ ) under nitrogen gas for 6-10 days. The solution was allowed to cool to room temperature and the volatiles removed under reduced pressure to give a dark red waxy solid (43).

Yield $=0.11 \mathrm{~g}, 79 \% ; \mathrm{v}_{\max } / \mathrm{cm}^{-1}(\mathrm{KBr}): 3435,1754,1590,1569,1474,1385,997$, 752; $\delta_{\mathrm{H}} / \mathrm{ppm}$ : Conc. of $10^{-3} \mathrm{~mm} ;\left(\mathrm{CDCl}_{3}\right) ; 8.68(\mathrm{~d}, 2 \mathrm{H}, \mathrm{Py}-\underline{\mathrm{H}}, J=4.8 \mathrm{~Hz}), 8.61$ (br d, 4H, bipy- $\underline{\mathrm{H}}$ ), 8.60 (br d, 2H, Py- $\underline{\mathrm{H}}$ ), 8.58 (br d, 4H, Py- $\underline{\mathrm{H}}$ ), 8.49 (br d, 2H, bipy- $\underline{\mathrm{H}}$ ), 8.42 (br d, 2H, bipy- $\underline{\mathrm{H}}$ ), 8.29 (s, 2H, bipy- $\underline{\mathrm{H}}$ ), 7.68 (t, 4H, Py- $\underline{\mathrm{H}}, J=7.6 \mathrm{~Hz}$ ), 7.60 (t, $4 \mathrm{H}, \mathrm{Bn}-\underline{\mathrm{H}}, J=7.9 \mathrm{~Hz}$ ), 7.54 (br t, 2H, Bn- $\underline{\mathrm{H}}$ ), 7.49 (d, 2H, Py- $\underline{\mathrm{H}}, J=7.4 \mathrm{~Hz}$ ), 7.44 (d, 2H, Py- $\underline{\mathrm{H}}, J=2.6 \mathrm{~Hz}$ ), 7.12 (d, 4H, J= $7.46 \mathrm{~Hz}, \mathrm{Ar}-\underline{\mathrm{H}}$ ), 7.08 (s, 4H, Ar- $\underline{\mathrm{H}}$ ), 7.05 (d, $4 \mathrm{H}, \operatorname{Ar}-\underline{\mathrm{H}}, J=7.4 \mathrm{~Hz}), 6.92(\mathrm{~d}, 4 \mathrm{H}, \mathrm{Ar}-\underline{\mathrm{H}}, J=6.5 \mathrm{~Hz}), 6.84(\mathrm{t}, 4 \mathrm{H}, \operatorname{Ar}-\underline{\mathrm{H}}, J=8.3 \mathrm{~Hz})$, $6.74(\mathrm{t}, 4 \mathrm{H}, \mathrm{Ar}-\underline{\mathrm{H}}, J=7.0 \mathrm{~Hz}), 6.54$ (br t, $2 \mathrm{H}, \mathrm{Ar}-\underline{\mathrm{H}}), 5.11$ (s, 4H, OC$\left.\underline{\mathrm{H}}_{2}-\mathrm{Bn}\right), 4.85$ (br $\left.\mathrm{d}, 4 \mathrm{H}, \underline{\mathrm{C}}_{2} \mathrm{NR}_{2}\right), 4.38\left(\mathrm{~d}, 4 \mathrm{H}, \underline{\mathrm{C}}_{2}, J=12.7 \mathrm{~Hz}\right), 4.29\left(\mathrm{~m}, 4 \mathrm{H}, \mathrm{OC}_{2} \mathrm{CH}_{3}, J=7.0\right.$

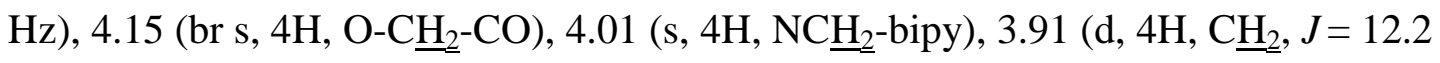

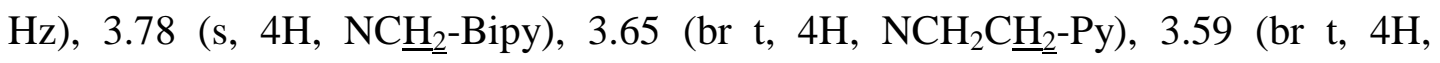
$\left.\mathrm{NC}_{2} \underline{\mathrm{CH}}_{2}-\mathrm{Py}\right), 3.53$ (d, 4H, $\left.\underline{\mathrm{C}}_{2}, J=5.7 \mathrm{~Hz}\right), 3.37$ (d, 4H, $\left.\underline{\mathrm{C}}_{2}, J=7.4 \mathrm{~Hz}\right), 1.32$ (t, $\left.6 \mathrm{H}, \mathrm{OCH}_{2} \underline{\mathrm{C}}_{3}, J=7.9 \mathrm{~Hz}\right) ; \delta_{\mathrm{C}} / \mathrm{ppm}\left(\mathrm{CDCl}_{3}\right) ; 170.0,159.7,157.9,157.8,157.1$, 
$155.4,153.4,149.5,149.6,149.2,148.2,143.4,138.3,136.6,136.5,136.5,133.8$, $129.3,129.3,128.6,128.4,127.6,127.1,125.5,124.8,124.2,123.9,122.8,122.4$, 121.8, 121.5, 120.9, 120.8, 74.4, 63.9, 63.0, 62.5, 61.8, 47.4, 37.1, 31.3, 23.2; Anal. Calc. for $\mathrm{C}_{98} \mathrm{H}_{92} \mathrm{~N}_{12} \mathrm{O}_{6}: \mathrm{C}, 76.74 ; \mathrm{H}, 6.05 ; \mathrm{N}, 10.96 \%$, found; C, 76.52; H, 6.01; N, $10.75 \%$.

\subsection{1,23-Di[N,N'-(2'-pyridylethyl)(4-methyl-2,2-}

bipyridyl)aminomethyl]-25,27-di(methyl-2-pyridine)-26,28diethyoxycarbonylmethoxycalix[4]arene. (44)

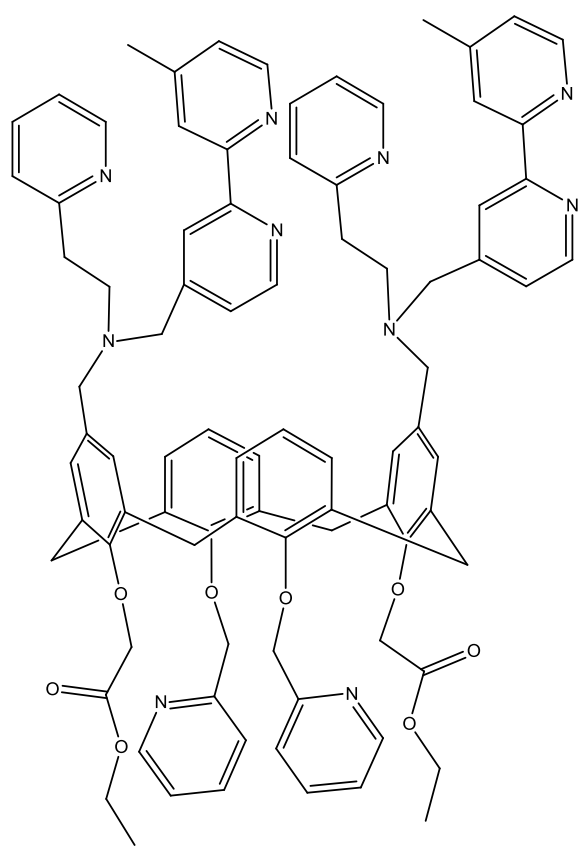

Compound (41) (0.03 g, $0.03 \mathrm{mmol})$, triethylamine $(0.02 \mathrm{~g}, 0.152 \mathrm{mmol})$ and compound (22) (0.04 g, $0.152 \mathrm{mmol})$ were refluxed in chloroform: $\mathrm{CH}_{3} \mathrm{CN}(20 \mathrm{~mL})$ under nitrogen gas for $48 \mathrm{hr}$. The solution was allowed to cool to room temperature, the inorganic salts were removed by filtration and volatiles removed under reduced pressure to give a dark brown-red waxy solid (44).

Yield $=0.02 \mathrm{~g}, 58 \% ; \mathrm{v}_{\max } / \mathrm{cm}^{-1}(\mathrm{KBr}): 1671,1593,1570,1437,1152,1092,998$, 753; $\delta_{\mathrm{H}} / \mathrm{ppm}$ : Conc. of $10^{-3} \mathrm{~mm}$; $\left(\mathrm{CDCl}_{3}\right) ; 8.68(\mathrm{~d}, 2 \mathrm{H}$, bipy- $\underline{\mathrm{H}}, J=4.5 \mathrm{~Hz}), 8.40(\mathrm{~s}$,

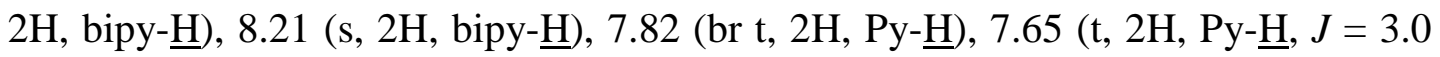


$\mathrm{Hz}), 7.59$ (t, 2H, Py- $\underline{\mathrm{H}}, J=1.7 \mathrm{~Hz}), 7.36$ (d, 2H, Py- $\underline{\mathrm{H}}, J=5.5 \mathrm{~Hz}), 7.33$ (d, 2H, bipy- $\underline{\mathrm{H}}, J=4.8 \mathrm{~Hz}), 7.32$ (t, 2H, Py- $\underline{\mathrm{H}}, J=0.9 \mathrm{~Hz}), 7.31$ (t, 2H, Py- $\underline{\mathrm{H}}, J=0.9 \mathrm{~Hz})$, 7.15 (s, 2H, bipy- $\underline{\mathrm{H}}), 7.08$ (s, 4H, Ar- $\underline{\mathrm{H}}), 6.98$ (d, 4H, Ar- $\underline{\mathrm{H}}, J=6.9 \mathrm{~Hz}), 6.67$ (t, 2H, $\mathrm{Ar}-\underline{\mathrm{H}}, J=7.4 \mathrm{~Hz}), 5.10$ (s, 4H, O-C $\left.\underline{\mathrm{H}}_{2}-\mathrm{Py}\right), 4.79$ (s, 4H, $\left.\underline{\mathrm{C}}_{2} \mathrm{NR}_{2}\right), 4.48$ (s, 4H, $\mathrm{NC}_{2} \underline{2}_{2}$ bipy), 4.30 (q, 4H, O-C $\underline{H}_{2} \mathrm{CH}_{3}, J=12.4 \mathrm{~Hz}$ ), 4.20 (d, 4H, $\underline{\mathrm{C}}_{2}, J=13.1 \mathrm{~Hz}$ ), 3.57 (s, 4H, O-C $\underline{H}_{2}-\mathrm{CO}$ ), 3.24 (d, 4H, $\underline{\mathrm{C}}_{2}, J=13.1 \mathrm{~Hz}$ ), 3.15 (t, 4H, $\mathrm{NCH}_{2} \underline{\mathrm{C}}_{2}-\mathrm{Py}$, $J=3.9 \mathrm{~Hz}), 3.02\left(\mathrm{t}, 4 \mathrm{H}, \mathrm{NC}_{2} \mathrm{CH}_{2}-\mathrm{Py}, J=5.9 \mathrm{~Hz}\right), 2.43\left(\mathrm{~s}, 6 \mathrm{H}, \operatorname{bipy}-\mathrm{CH}_{3}\right), 2.32$ (t, $\left.6 \mathrm{H}, \mathrm{O}-\mathrm{CH}_{2} \mathrm{CH}_{3}, J=8.2 \mathrm{~Hz}\right) ; \delta_{\mathrm{C}} / \mathrm{ppm}\left(\mathrm{CDCl}_{3}\right) ; 169.6,157.9,157.0,156.6,155.9$, 153.2, 150.6, 149.6, 149.1, 148.9, 148.0, 137.8, 136.4, 136.4, 136.0, 134.0, 133.5, $130.7,128.6,128.5,127.8,124.7,124.6,123.5,122.9,122.7,122.5,122.4,119.0$, 77.9, 72.4, 61.4, 60.1, 54.0, 53.8, 37.1, 31.3, 21.4, 14.2; Anal. Calc. for $\mathrm{C}_{88} \mathrm{H}_{86} \mathrm{~N}_{10} \mathrm{O}_{8}:$ C, 74.87; H, 6.14; N, $9.92 \%$, found; C, 74.63; H, 6.20; N, $9.87 \%$.

\section{Metal complexes of (44) at 1:1 ratio}

All metal ion complexation reactions were performed at room temperature in a solvent mixture of DCM-MeOH in a 1:1 ratio. All reactions used $0.07 \mathrm{mmol}$ of (44) and corresponding metal salt. The solution was stirred for $15 \mathrm{~min}$ and then the solvent was slowly evaporated off. A water-DCM wash was performed to remove any uncomplexed metal and the solvent was removed under reduced pressure.

Compound (44i): Zinc acetate; Yield $=0.09 \mathrm{~g}, 79 \% ; \mathrm{v}_{\max } / \mathrm{cm}^{-1}(\mathrm{KBr}): 1737,1614$, 1561, 1413, 1190, 1018, 765; $\delta_{\mathrm{H}} / \mathrm{ppm}$ : Conc. of $10^{-3} \mathrm{~mm} ;\left(\mathrm{CDCl}_{3}\right) ; 8.68$ (br d), 8.56 (br d), 8.45 (br d), 8.33 (br d), 8.24 (br t), 8.20 (br d), 8.13 (br s), 8.04 (br t), 7.72 (br t), $7.60(\mathrm{t}, J=7.1 \mathrm{~Hz}), 7.49$ (br t), 7.35 (br t), 7.14 (d, $J=8.0 \mathrm{~Hz}), 7.08$ (s), 6.63 (br t), 6.46 (br t), 5.22 (s), 4.85 (s), 4.73 (s), 4.39 (d, $J=12.6 \mathrm{~Hz}), 4.21$ (d, $J=3.3 \mathrm{~Hz})$, $4.17(\mathrm{dd}, J=2.3,7.2 \mathrm{~Hz}), 4.14$ (d, $J=2.3 \mathrm{~Hz}$ ), 4.07 (br t), 4.00 (s), 3.89 (br t), 3.79 (d, $J=9.0 \mathrm{~Hz}), 3.72$ (br d), 3.45 (s), 3.24 (d, $J=12.7 \mathrm{~Hz}), 3.14$ (t, $J=6.3 \mathrm{~Hz}), 2.98$ (t, $J=8.1 \mathrm{~Hz}$ ), 2.48 (s), 2.18 (s); Anal. Calc. for $\mathrm{C}_{92} \mathrm{H}_{92} \mathrm{~N}_{10} \mathrm{O}_{12} \mathrm{Zn}$ : C, 68.27; H, 5.81; $\mathrm{N}, 8.78 \%$, found; C, 68.12; H, 5.45; N, $8.64 \%$.

Compound (44ii): Zinc chloride; Yield $=0.08 \mathrm{~g}, 72 \% ; \mathrm{v}_{\max } / \mathrm{cm}^{-1}(\mathrm{KBr}): 1736$, $1613,1561,1437,1189,1017,765 ; \delta_{\mathrm{H}} / \mathrm{ppm}$ : Conc. of $10^{-3} \mathrm{~mm} ;\left(d_{6}\right.$-DMSO); $8.68(\mathrm{~d}$, $J=6.7 \mathrm{~Hz}$ ), 8.60 (d, $J=4.0 \mathrm{~Hz}), 8.53$ (br d), 8.47 (br d), 8.40 (br d), 8.30 (br d), 7.97 $(\mathrm{d}, J=7.4 \mathrm{~Hz}), 7.78(\mathrm{~s}), 7.70(\mathrm{~d}, J=7.4 \mathrm{~Hz}), 7.64(\mathrm{dd}, J=2.0,7.2 \mathrm{~Hz}), 7.52$ (t, $J=$ $3.2 \mathrm{~Hz}), 7.42$ (br t), 7.34 (br t), 7.25 (d, $J=6.6 \mathrm{~Hz}), 7.16$ (d, $J=5.7 \mathrm{~Hz}), 7.15$ (s), 
$6.85(\mathrm{t}, J=6.6 \mathrm{~Hz}), 6.70(\mathrm{t}, J=6.6 \mathrm{~Hz}), 4.90(\mathrm{~s}), 4.64(\mathrm{~s}), 4.39(\mathrm{~s}), 4.15(\mathrm{~d}, J=9.9$ $\mathrm{Hz}$ ), 4.08 (d, $J=7.6 \mathrm{~Hz}$ ), 4.04 (d, $J=3.3 \mathrm{~Hz}$ ), 3.92 (s), 3.81 (br t), 3.53 (s), 2.98 (br t), 3.89 (t, $J=7.6 \mathrm{~Hz}$ ), 2.31 (br t); Anal. Calc. for $\mathrm{C}_{88} \mathrm{H}_{86} \mathrm{Cl}_{2} \mathrm{~N}_{10} \mathrm{O}_{8} \mathrm{Zn}: \mathrm{C}, 68.28 ; \mathrm{H}$, $5.60 ; \mathrm{N}, 9.05 \%$, found; C, 68.10; H, 5.27; N, $8.95 \%$.

Compound (44iii): Zinc perchlorate; Yield $=0.09 \mathrm{~g}, 75 \% ; \mathrm{v}_{\max } / \mathrm{cm}^{-1}(\mathrm{KBr}): 1737$, $1614,1562,1415,1192,1091$ (perchlorate anion peak), 747, 624; $\delta_{\mathrm{H}} / \mathrm{ppm}$ : Conc. of $10^{-3} \mathrm{~mm}$; $\left(d_{6}\right.$-DMSO); 8.52 (br d), 8.43 (br d), 8.34 (br d), 8.29 (s), 7.98 (br t), 7.79 (d, $J=, 9.5 \mathrm{~Hz}$ ), 7.79 (br t), 7.70 (br t), 7.63 (d, $J=7.6 \mathrm{~Hz}$ ), 7.52 (br d), 7.47 (br t), 7.24 (d, $J=8.6 \mathrm{~Hz}), 7.13$ (br d), 6.84 (br t), 4.92 (s), 4.71 (d, $J=4.8 \mathrm{~Hz}), 4.65$ (s), 4.83 (s), 4.22 (d, $J=9.5 \mathrm{~Hz}), 4.09$ (d, $J=4.7 \mathrm{~Hz}), 4.04$ (d, $J=3.0 \mathrm{~Hz}), 4.01$ (s), 3.98 (br s), 3.53 (d, $J=3.4 \mathrm{~Hz}$ ), 2.99 (br t), 2.89 (br t), 2.29 (br t); Anal. Calc. for $\mathrm{C}_{88} \mathrm{H}_{86} \mathrm{Cl}_{2} \mathrm{~N}_{10} \mathrm{O}_{16} \mathrm{Zn}: \mathrm{C}, 63.06 ; \mathrm{H}, 5.17 ; \mathrm{N}, 8.36 \%$, found; $\mathrm{C}, 62.98 ; \mathrm{H}, 4.95 ; \mathrm{N}, 8.09$ $\%$.

\subsection{1,12-Di[ $N, N$ '-(2'pyridylethyl)(4-methyl-2,2'-bipyridyl)-4'-} aminomethyl]-25,27-di(methyl-2-pyrdine)-26,28-di(2'pyridylmethyl)methoxy-calix[4]arene. (45)

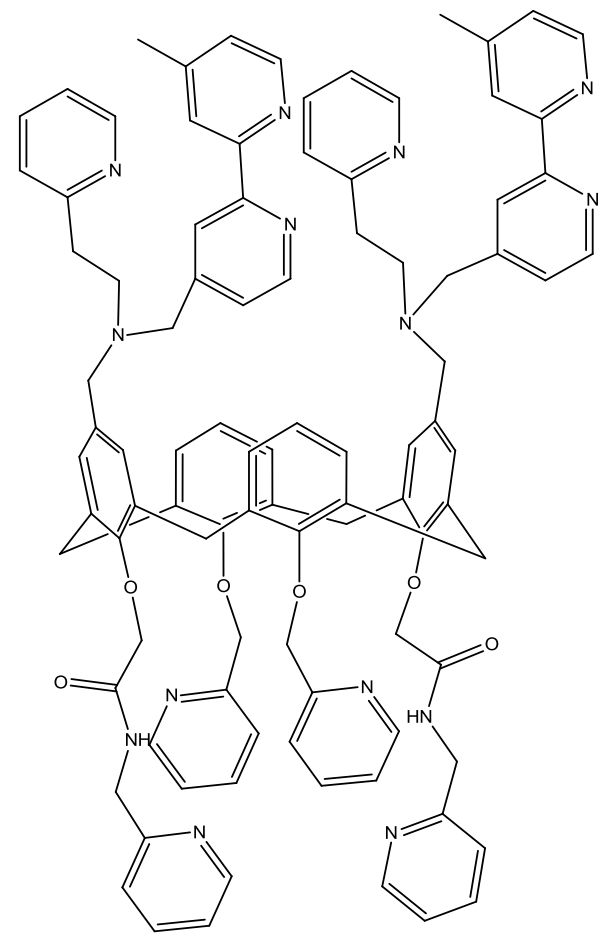


Compound (44) (0.03 g, $0.02 \mathrm{mmol})$ and 2-aminomethylpyridine $(0.01 \mathrm{~mL}, 0.04$ $\mathrm{mmol}$ ), were refluxed in toluene: $\mathrm{MeOH}$ (50: $20 \mathrm{~mL}$ ) under nitrogen gas for 10 days. The solution was allowed to cool to room temperature and the volatiles were removed under reduced pressure to give a dark red waxy solid (45).

Yield $=0.02,49 \% ; \mathrm{v}_{\max } / \mathrm{cm}^{-1}(\mathrm{KBr}): 3446,2364,1754,1677,1590,1569,1460$ 1092, 996, 749; $\delta_{\mathrm{H}} / \mathrm{ppm}$ : Conc. of $10^{-3} \mathrm{~mm} ;\left(\mathrm{CDCl}_{3}\right) ; 8.73$ (d, 2H, Py- $\left.\underline{\mathrm{H}}, J=4.3 \mathrm{~Hz}\right)$, 8.59 (br d, 2H, Py- $\underline{\mathrm{H}}$ ), 8.55 (d, 2H, Py- $\underline{\mathrm{H}} J=5.5 \mathrm{~Hz}$ ), 8.52 (br d, 4H, bipy- $\underline{\mathrm{H}}$ ), 8.33 (d, 4H, bipy- $\underline{\mathrm{H}}, J=8.0 \mathrm{~Hz}$ ), 8.19 (br d, 4H, bipy- $\underline{\mathrm{H}}$ ), 7.93 (t, 2H, Py- $\underline{\mathrm{H}}, J=7.8 \mathrm{~Hz}$ ), 7.83 (t, 2H, Py- $\underline{\mathrm{H}}, J=7.5 \mathrm{~Hz}), 7.65$ (t, 2H, Py- $\underline{\mathrm{H}} J=9.1 \mathrm{~Hz}), 7.58$ (s, 4H, bipy- $\underline{\mathrm{H}}$ ), 7.55 (d, 2H, Py- $\underline{\mathrm{H}}, J=1.5 \mathrm{~Hz}$ ), 7.53 (br d, 2H, Py- $\underline{\mathrm{H}}$ ), 7.51 (d, 2H, Py- $\underline{\mathrm{H}}, J=1.5$ Hz), 7.44 (br t, 2H, Py- $\underline{\mathrm{H}}$ ), 7.42 (t, 2H, Py- $\underline{\mathrm{H}}, J=1.5 \mathrm{~Hz}$ ), 7.40 (br t, 2H, Py- $\underline{\mathrm{H}}$ ), 7.07 (s, 4H, Ar- $\underline{\mathrm{H}}$ ), 6.87 (d, 4H, Ar- $\underline{\mathrm{H}}, J=5.9 \mathrm{~Hz}), 6.75$ (d, 4H, Ar- $\underline{\mathrm{H}}, J=7.5 \mathrm{~Hz}), 6.56$

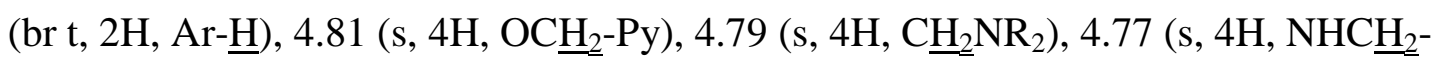
Py), 4.18 (d, 4H, $\underline{\mathrm{H}}_{2}, J=7.2 \mathrm{~Hz}$ ), 3.94 (s, 4H, OC$\underline{\mathrm{H}}_{2} \mathrm{CO}$ ), 3.89 (br m, 4H, $\mathrm{OC}_{2} \underline{C H}_{3}$ ), 3.82 (s, 4H, $\underline{\mathrm{H}}_{2}$-bipy), 3.44 (d, 4H, $\left.\underline{\mathrm{C}}_{2}, J=4.7 \mathrm{~Hz}\right), 3.36$ (d, 4H, $\underline{\mathrm{C}}_{2}$, $J=5.6 \mathrm{~Hz}$ ), 3.21 (d, 4H, $\underline{\mathrm{H}}_{2}, J=13.4 \mathrm{~Hz}$ ), 3.12 (br t, $4 \mathrm{H}, \mathrm{NCH}_{2} \underline{\mathrm{C}}_{2}-\mathrm{Py}$ ), 3.00 (br t, $\left.4 \mathrm{H}, \mathrm{NC}_{2} \mathrm{CH}_{2}-\mathrm{Py}\right), 1.10$ (t, $\left.6 \mathrm{H}, \mathrm{OCH}_{2} \underline{\mathrm{C}}_{3}, J=7.8 \mathrm{~Hz}\right) ; \delta_{\mathrm{C}} / \mathrm{ppm}\left(\mathrm{CDCl}_{3}\right) ; 169.8$, $158.8,158.6,156.3,156.1,155.7,154.5,153.8,149.8,149.7,148.6,148.4,148.2$, $148.1,147.6,139.5,136.4,136.2$, 134.1, 133.7, 128.7, 127.1, 125.4, 125.1, 124.6, 124.1, 124.0, 123.8, 123.5, 123.1, 122.1, 121.2, 120.7, 78.4, 68.5, 61.8, 61.3, 54.7, 48.6, 39.4, 31.1, 21.4; Anal. Calc. for $\mathrm{C}_{96} \mathrm{H}_{90} \mathrm{~N}_{14} \mathrm{O}_{6}$ : C, 75.08; H, 5.91; N, $12.77 \%$, found; C, 74.85; H, 5.77; N, $12.61 \%$. 


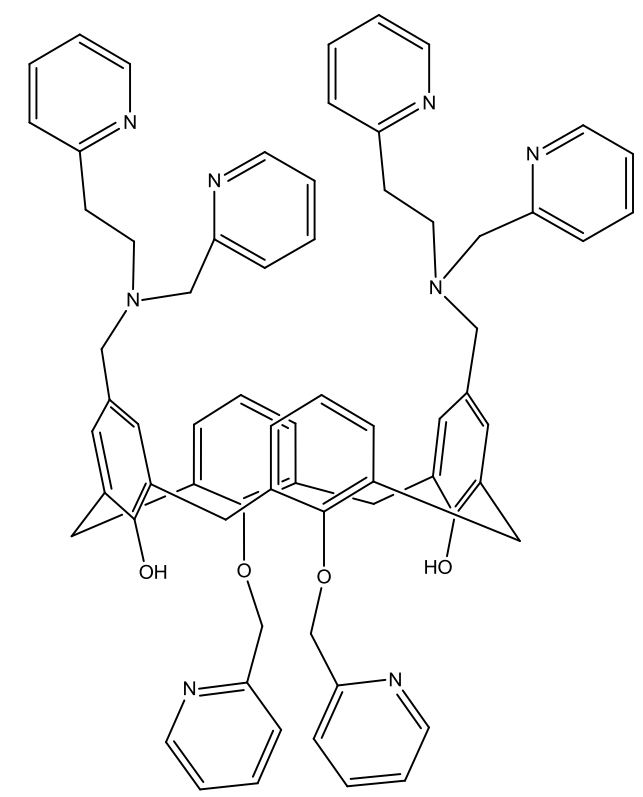

Compound (36) (1.20 g, $1.37 \mathrm{mmol})$, triethylamine $(0.23 \mathrm{~g}, 2.74 \mathrm{mmol})$ and 2aminomethylpyridine $(0.28 \mathrm{~mL}, 2.74 \mathrm{mmol})$, were refluxed in $\mathrm{CHCl}_{3}: \mathrm{CH}_{3} \mathrm{CN}(10$ $\mathrm{mL}$ : $60 \mathrm{~mL}$ ) under argon gas overnight. The solution was allowed to cool to room temperature, the inorganic salts were removed by filtration and the volatiles removed under reduced pressure. The residue was washed with DCM and water to remove any residual triethylamine and the solvents removed under reduced pressure to afford a dark brown, red waxy solid (46).

Yield $=0.95 \mathrm{~g}, 65 \% ; \mathrm{v}_{\max } / \mathrm{cm}^{-1}(\mathrm{KBr}): 3431,2916,1591,1467,1087,995,753$; $\delta_{\mathrm{H}} / \mathrm{ppm}$ : Conc. of $10^{-3} \mathrm{~mm} ;\left(\mathrm{CDCl}_{3}\right) ; 8.70(\mathrm{~d}, 2 \mathrm{H}, \mathrm{Py}-\underline{\mathrm{H}}, J=5.1 \mathrm{~Hz}), 8.63(\mathrm{~d}, 2 \mathrm{H}, \mathrm{Py}-$ $\underline{\mathrm{H}}, J=4.9 \mathrm{~Hz}), 8.52(\mathrm{~d}, 2 \mathrm{H}, \mathrm{Py}-\underline{\mathrm{H}}, J=5.1 \mathrm{~Hz}), 8.22(\mathrm{t}, 2 \mathrm{H}, \mathrm{Py}-\underline{\mathrm{H}}, J=5.8 \mathrm{~Hz}), 8.16$ (t, 2H, Py- $\underline{\mathrm{H}}, J=7.6 \mathrm{~Hz}), 7.86$ (d, 2H, Py- $\underline{\mathrm{H}}, J=1.7 \mathrm{~Hz}), 7.79$ (t, $2 \mathrm{H}, \mathrm{Py}-\underline{\mathrm{H}}, J=6.0$

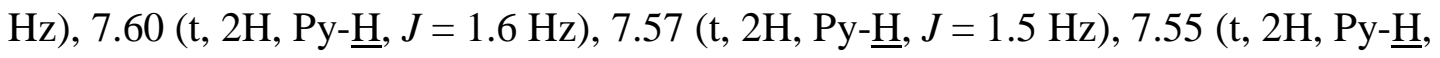

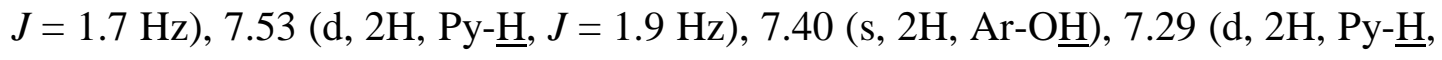
$J=1.9 \mathrm{~Hz}$ ), 7.06 (s, 4H, Ar- $\underline{\mathrm{H}}), 7.04$ (d, 4H, Ar- $\underline{\mathrm{H}}, J=2.8 \mathrm{~Hz}), 6.49$ (t, $2 \mathrm{H}, \mathrm{Ar}-\underline{\mathrm{H}}, J$ $=7.0 \mathrm{~Hz}$ ), 5.18 (s, 4H, $\left.\underline{\mathrm{C}}_{2}-\mathrm{Py}\right), 4.24$ (br d, $\left.4 \mathrm{H}, \underline{\mathrm{C}}_{2}\right), 4.07$ (br s, 4H, $\mathrm{CH}_{2}-\mathrm{NR}_{2}$ ), 3.94 (s, 4H, $\mathrm{R}_{2} \mathrm{NC}_{2} \underline{\mathrm{PY}}$ ), 3.44 (br d, $4 \mathrm{H}, \underline{\mathrm{C}}_{2}$ ), 3.05 (t, $4 \mathrm{H}, \mathrm{CH}_{2} \mathrm{C}_{2}-\mathrm{Py}, J=4.6 \mathrm{~Hz}$ ), 3.00 (t, $\left.4 \mathrm{H}, \mathrm{C}_{2} \mathrm{CH}_{2} \mathrm{Py}, J=3.8 \mathrm{~Hz}\right) ; \delta_{\mathrm{C}} / \mathrm{ppm}\left(\mathrm{CDCl}_{3}\right) ; 156.8,156.6,156.1,154.3$, 149.3, 149.0, 148.7, 137.1, 136.5, 136.3, 133.7, 128.7, 128.5, 128.1, 127.9, 125.3, 
123.8, 123.4, 123.2, 121.8, 121.4, 121.0, 78.6, 63.5, 60.0, 54.3, 38.3, 32.2; Anal. Calc. for $\mathrm{C}_{68} \mathrm{H}_{64} \mathrm{~N}_{8} \mathrm{O}_{4}: \mathrm{C}, 77.25 ; \mathrm{H}, 6.10 ; \mathrm{N}, 10.60 \%$, found; $\mathrm{C}, 76.91 ; \mathrm{H}, 6.05 ; \mathrm{N}$, $10.45 \% ; \lambda_{\max }(\mathrm{DCM} / \mathrm{MeOH}) 260 \mathrm{~nm} @ 0.004 \mathrm{mM}, \varepsilon=969 \mathrm{M}^{-1} \mathrm{~cm}^{-1}$.

\section{Metal complexes of (46) at 1:1 ratio}

All metal ion complexation reactions were performed at room temperature in a solvent mixture of DCM-MeOH in a 1:1 ratio. All reactions used $0.09 \mathrm{mmol}$ of (46) and corresponding metal salt. The solution was stirred for $15 \mathrm{~min}$ and then the solvent was slowly evaporated off. A water-DCM wash was performed to remove any uncomplexed metal and the solvent was removed under reduced pressure.

Compound (46i): Zinc acetate; Yield $=0.09 \mathrm{~g}, 76 \% ; \mathrm{v}_{\max } / \mathrm{cm}^{-1}(\mathrm{KBr}): 3433,2927$, 1593, 1467, 1261, 1088, 756; $\delta_{\mathrm{H}} / \mathrm{ppm}$ : Conc. of $10^{-3} \mathrm{~mm} ;\left(\mathrm{CDCl}_{3}\right) ; 8.83$ (br d), 8.63 (br d), 8.54 (br d), 8.52 (br d), 8.18 (s), 8.05 (t, $J=8.4 \mathrm{~Hz}$ ), 7.97 (t, $J=9.8 \mathrm{~Hz}$ ), 7.92 $(\mathrm{d}, J=9.8 \mathrm{~Hz}), 7.78(\mathrm{t}, J=9.8 \mathrm{~Hz}), 7.72(\mathrm{t}, J=8.4 \mathrm{~Hz}), 7.59(\mathrm{~d}, J=7.0 \mathrm{~Hz}), 7.54$ (t, $J=9.8 \mathrm{~Hz}), 7.48(\mathrm{~d}, J=9.8 \mathrm{~Hz}), 7.45(\mathrm{~d}, J=5.6 \mathrm{~Hz}), 7.34(\mathrm{~d}, J=8.4 \mathrm{~Hz}), 7.30(\mathrm{t}, J$ $=8.4 \mathrm{~Hz}), 7.25(\mathrm{t}, J=8.4 \mathrm{~Hz}), 7.16(\mathrm{~d}, J=8.4 \mathrm{~Hz}), 7.06(\mathrm{~d}, J=8.4 \mathrm{~Hz}), 6.89(\mathrm{~d}, J=$ $5.6 \mathrm{~Hz}), 6.83(\mathrm{t}, J=7.0 \mathrm{~Hz}), 6.61(\mathrm{t}, J=7.0 \mathrm{~Hz}), 5.10(\mathrm{~s}), 4.72(\mathrm{~s}), 4.43(\mathrm{~s}), 4.39(\mathrm{br}$ d), $4.26(\mathrm{~d}, J=13.2 \mathrm{~Hz}), 4.22$ (br s), 4.15 (d, $J=13.2 \mathrm{~Hz}), 3.04$ (t, $J=7.8 \mathrm{~Hz}), 2.88$ (t, $J=7.5 \mathrm{~Hz}$ ); Anal. Calc. for $\mathrm{C}_{72} \mathrm{H}_{70} \mathrm{~N}_{8} \mathrm{O}_{8} \mathrm{Zn}: \mathrm{C}, 69.70 ; \mathrm{H}, 5.69 ; \mathrm{N}, 9.30 \%$, found; $\mathrm{C}, 69.56 ; \mathrm{H}, 5.21 ; \mathrm{N}, 9.11 \% ; \lambda_{\max }(\mathrm{DCM} / \mathrm{MeOH}) 420 \mathrm{~nm} @ 0.4 \mathrm{mM}, \varepsilon=537 \mathrm{M}^{-1} \mathrm{~cm}^{-}$ 1 .

Compound (46ii): Zinc chloride; Yield $=0.08 \mathrm{~g}, 745 \% ; \mathrm{v}_{\max } / \mathrm{cm}^{-1}(\mathrm{KBr}): 3434$, 1606, 1571, 1444, 1088, 1022, 759; $\delta_{\mathrm{H}} / \mathrm{ppm}$ : Conc. of $10^{-3} \mathrm{~mm} ;\left(d_{6}\right.$-DMSO); 8.64 (d, $J=5.6 \mathrm{~Hz}), 8.51(\mathrm{~d}, J=4.3 \mathrm{~Hz}), 8.40(\mathrm{~d}, J=5.6 \mathrm{~Hz}), 8.18(\mathrm{~s}), 8.07(\mathrm{~s}), 8.04(\mathrm{~d}, J=$ $6.0 \mathrm{~Hz}), 7.98(\mathrm{~d}, J=3.4 \mathrm{~Hz}), 7.88(\mathrm{t}, J=6.9 \mathrm{~Hz}), 7.74(\mathrm{~d}, J=6.6 \mathrm{~Hz}), 7.62(\mathrm{~s}), 7.59$ (br t), 7.55 (s), 7.31 (d, 4.67), 6.97 (s), 6.94 (d, J=6.0 Hz), 6.88 (d, J = 6.6 Hz), 6.46 (br t), 6.37 (br t), 504 (s), 4.92 (s), 4.68 (br d), 4.80 (br d), 4.63 (br t), 4.40 (d, J=6.6 $\mathrm{Hz}$ ), 4.36 (d, $J=4.7 \mathrm{~Hz}$ ), 4.33 (s), 4.27 (br t), 4.04 (s), 3.88 (s), 3.83 (br d), 3.78 (br t), $3.74(\mathrm{~s}), 3.69(\mathrm{~d}, J=6.9 \mathrm{~Hz}), 3.52(\mathrm{~s}), 3.04(\mathrm{t}, J=6.6 \mathrm{~Hz}), 2.86(\mathrm{br} \mathrm{t})$; Anal. Calc. for $\mathrm{C}_{68} \mathrm{H}_{64} \mathrm{Cl}_{2} \mathrm{~N}_{8} \mathrm{O}_{4} \mathrm{Zn}$ : C, 68.43; H, 5.40; N, $9.39 \%$, found; C, 68.10; H, 5.29; N, $8.95 \% ; \lambda_{\max }(\mathrm{DCM} / \mathrm{MeOH}) 421 \mathrm{~nm} @ 0.4 \mathrm{mM}, \varepsilon=587 \mathrm{M}^{-1} \mathrm{~cm}^{-1}$. 
Compound (46iii): Zinc perchlorate; Yield $=0.09 \mathrm{~g}, 75 \% ; \mathrm{v}_{\max } / \mathrm{cm}^{-1}(\mathrm{KBr}): 3426$, 2712, 1611, 1446, 1095 (perchlorate anion peak), 762, 624; $\delta_{\mathrm{H}} / \mathrm{ppm}$ : Conc. of $10^{-3}$ mm; $\left(d_{6}\right.$-DMSO); 8.83 (br d), 8.68 (d, $\left.J=4.9 \mathrm{~Hz}\right), 8.52$ (d, $\left.J=4.4 \mathrm{~Hz}\right), 8.41$ (d, $J=$ $4.9 \mathrm{~Hz}$ ), 8.18 (s), 8.13 (br t), 8.09 (br t), 8.07 (s), 8.02 (br d), 7.97 (s), 7.93 (br t), $7.90(\mathrm{~d}, J=2.4 \mathrm{~Hz}), 7.87$ (bt t), 7.79 (d, $J=2.1 \mathrm{~Hz}), 7.87$ (d, $J=1.5 \mathrm{~Hz}), 7.74$ (d, $J=$ $1.3 \mathrm{~Hz}$ ), 7.72 (br t), 7.61 (br d), 7.58 (br t), 7.53 (br s), 7.47 (s), 7.44 (br t), 7.41 (s), 7.39 (s), 7.34 (d, $J=8.8 \mathrm{~Hz}$ ), 7.32 (br t), 7.19 (br t), 7.13 (d, $J=7.3 \mathrm{~Hz}$ ), 7.07 (s), $7.03(\mathrm{~d}, J=6.8 \mathrm{~Hz}), 6.96(\mathrm{br} \mathrm{d}), 6.93(\mathrm{~s}), 6.80(\mathrm{t}, J=7.8 \mathrm{~Hz}), 6.59(\mathrm{t}, J=8.3 \mathrm{~Hz})$, 5.05 (s), 4.87 (s), 4.89 (s), 4.61 (d, $J=7.1 \mathrm{~Hz}), 4.61$ (s), 4.47 (s), 4.45 (d, $J=7.4$ $\mathrm{Hz}$ ), 4.35 (d, J = $4.9 \mathrm{~Hz}), 4.32$ (s), 4.28 (s), 4.04 (s), 3.59 (br d), 3.83 (s), 3.78 (s), 3.74 (s), 3.69 (s), 3.54 (s), 3.24 (br d), 3.04 (t, $J=7.2 \mathrm{~Hz}), 2.95$ (t, $J=6.6 \mathrm{~Hz}), 2.87$ (br t), 2.84 (br t); Anal. Calc. for $\mathrm{C}_{68} \mathrm{H}_{64} \mathrm{Cl}_{2} \mathrm{~N}_{8} \mathrm{O}_{12} \mathrm{Zn}$ : C, 61.80; H, 4.88; N, $4.48 \%$, found; C, 61.60; H, 4.71; N, $4.33 \%$; $\lambda_{\max }(\mathrm{DCM} / \mathrm{MeOH}) 423 \mathrm{~nm} @ 0.4 \mathrm{mM}, \varepsilon=381$ $\mathrm{M}^{-1} \mathrm{~cm}^{-1}$.

\section{Metal complexes of (46) at 1:2 ratio}

Same procedure was used as in the case of the 1:1 with the exception that the ratio of ligand to metal was $0.09 \mathrm{mmol}$ to $0.18 \mathrm{mmol}$.

Compound (46 iv): Zinc acetate; Yield $=0.20 \mathrm{~g}, 78 \% ; \mathrm{v}_{\max } / \mathrm{cm}^{-1}(\mathrm{KBr}): 3437$, $2717,1607,1439,1021,906,757 ; \delta_{\mathrm{H}} / \mathrm{ppm}$ : Conc. of $10^{-3} \mathrm{~mm} ;\left(\mathrm{CDCl}_{3}\right) ; 8.72(\mathrm{~d}, J=$ $4.6 \mathrm{~Hz}), 8.64(\mathrm{~d}, J=5.6 \mathrm{~Hz}), 8.25(\mathrm{~d}, J=7.3 \mathrm{~Hz}), 7.91(\mathrm{t}, J=7.2 \mathrm{~Hz}), 7.77$ (s), 7.70 $(\mathrm{d}, J=6.4 \mathrm{~Hz}), 7.57(\mathrm{~s}), 7.55(\mathrm{~d}, J=1.9 \mathrm{~Hz}), 7.53$ (t, $J=2.8 \mathrm{~Hz}), 7.47$ (s), 7.23 (br d), $7.22(\mathrm{~s}), 7.11(\mathrm{~s}), 7.09(\mathrm{~s}), 7.07(\mathrm{~d}, J=1.4 \mathrm{~Hz}), 7.06(\mathrm{~s}), 7.05(\mathrm{~s}), 7.04(\mathrm{~s}), 7.00$ (t, $J=6.0 \mathrm{~Hz}), 6.92(\mathrm{~d}, J=8.1 \mathrm{~Hz}), 6.79(\mathrm{t}, J=7.4 \mathrm{~Hz}), 6.72(\mathrm{t}, J=8.1 \mathrm{~Hz}), 6.68(\mathrm{~d}, J$ = $7.4 \mathrm{~Hz}), 5.19(\mathrm{~s}), 4.68(\mathrm{~s}), 4.51(\mathrm{~d}, J=14.3 \mathrm{~Hz}), 4.35(\mathrm{~d}, J=13.6 \mathrm{~Hz}), 4.26(\mathrm{~s})$, $4.23(\mathrm{~d}, J=3.9 \mathrm{~Hz}), 4.21(\mathrm{~d}, J=3.1 \mathrm{~Hz}), 4.20$ (s), 4.18 (d, $J=6.2 \mathrm{~Hz}), 3.47$ (s), 3.45 (d, $J=13.3 \mathrm{~Hz}$ ), 3.44 (s), 3.41 (s); Anal. Calc. for $\mathrm{C}_{76} \mathrm{H}_{76} \mathrm{~N}_{8} \mathrm{O}_{12} \mathrm{Zn}_{2}: \mathrm{C}, 64.09 ; \mathrm{H}$, 5.38; N, $7.87 \%$, found; C, 63.96; H, 5.05; N, 7.39\%; $\lambda_{\max }(\mathrm{DCM} / \mathrm{MeOH}) 422 \mathrm{~nm} @$ $0.4 \mathrm{mM}, \varepsilon=660 \mathrm{M}^{-1} \mathrm{~cm}^{-1}$.

Compound (46v): Zinc chloride; Yield $=0.19 \mathrm{~g}, 79 \% ; \mathrm{v}_{\max } / \mathrm{cm}^{-1}(\mathrm{KBr}): 3430$, 2927, 1608, 1571, 1445, 1087, 756; $\delta_{\mathrm{H}} / \mathrm{ppm}$ : Conc. of $10^{-3} \mathrm{~mm} ;\left(d_{6}\right.$-DMSO); 8.70 (d, $J=5.8 \mathrm{~Hz}), 8.52(\mathrm{~d}, J=4.6 \mathrm{~Hz}), 8.41(\mathrm{~d}, J=4.6 \mathrm{~Hz}), 8.21(\mathrm{br} \mathrm{s}), 8.13(\mathrm{~s}), 8.06(\mathrm{~d}, J$ $=5.2 \mathrm{~Hz}), 7.99(\mathrm{t}, J=6.1 \mathrm{~Hz}), 7.94(\mathrm{~d}, J=5.2 \mathrm{~Hz}), 7.94(\mathrm{~s}), 7.88(\mathrm{br} \mathrm{d}), 7.87(\mathrm{br} \mathrm{d})$, 
7.72 (s), 7.69 (d, $J=2.2 \mathrm{~Hz}), 7.67$ (br s), 7.66 (d, $J=2.3 \mathrm{~Hz}$ ), 7.60 (br t), 7.35 (d, $J=$ $1.9 \mathrm{~Hz}), 7.50(\mathrm{~d}, J=1.2 \mathrm{~Hz}), 7.45(\mathrm{br} \mathrm{t}), 7.36(\mathrm{~d}, J=2.8 \mathrm{~Hz}), 7.34(\mathrm{~s}), 7.31(\mathrm{br} \mathrm{t})$, $7.28(\mathrm{~d}, J=1.2 \mathrm{~Hz}), 7.24(\mathrm{br} \mathrm{t}), 7.19(\mathrm{t}, J=3.8 \mathrm{~Hz}), 7.17$ (s), 6.97 (s), 6.88 (d, $J=$ $7.0 \mathrm{~Hz}), 6.80$ (t, $J=7.0 \mathrm{~Hz}), 6.59$ (t, $J=8.3 \mathrm{~Hz}), 6.50$ (br t), 6.38 (br t), 5.04 (s), 4.97 (s), 4.62 (br d), 4.46 (s), 4.40 (d, J=5.8 Hz), 4.35 (d, $J=9.6 \mathrm{~Hz}), 4.05$ (s), 3.87 (s), $3.68(\mathrm{~d}, J=9.6 \mathrm{~Hz}), 3.24(\mathrm{br} \mathrm{d}), 3.04$ (t, $J=7.7 \mathrm{~Hz}), 2.95$ (t, $J=7.7 \mathrm{~Hz}), 2.89$ (br t), 2.84 (br t), 2.73 (t, $J=8.6 \mathrm{~Hz}$ ); Anal. Calc. for $\mathrm{C}_{68} \mathrm{H}_{64} \mathrm{Cl}_{4} \mathrm{~N}_{8} \mathrm{O}_{4} \mathrm{Zn}_{2}: \mathrm{C}, 61.41 ; \mathrm{H}, 4.85$; $\mathrm{N}, 8.43 \%$, found; C, 61.25; H, 4.56; N, $8.39 \%$; $\lambda_{\max }(\mathrm{DCM} / \mathrm{MeOH}) 422 \mathrm{~nm} @ 0.4$ $\mathrm{mM}, \varepsilon=227 \mathrm{M}^{-1} \mathrm{~cm}^{-1}$.

Compound (46vi): Zinc perchlorate; Yield $=0.21 \mathrm{~g}, 73 \% ; \mathrm{v}_{\max } / \mathrm{cm}^{-1}(\mathrm{KBr}): 3428$, 1611, 1571, 1445, 1109 (perchlorate anion peak), 767, 627; $\delta_{\mathrm{H}} / \mathrm{ppm}$ : Conc. of $10^{-3}$ $\mathrm{mm}$; $\left(d_{6}\right.$-DMSO); 8.81 (br d), 8.70 (br d), 8.51 (d, $\left.J=4.5 \mathrm{~Hz}\right), 8.40$ (d, $J=4.5 \mathrm{~Hz}$ ), 8.17 (s), 8.13 (br t), 8.06 (s), 8.03 (br t), 7.95 (d, $J=2.0 \mathrm{~Hz}$ ), 7.92 (d, $J=4.7 \mathrm{~Hz}$ ), $7.90(\mathrm{~d}, J=1.3 \mathrm{~Hz}), 7.85$ (br t), $7.79(\mathrm{~d}, J=2.0 \mathrm{~Hz}), 7.77$ (d, $J=1.8 \mathrm{~Hz}), 7.74$ (d, $J=$ $1.5 \mathrm{~Hz}), 7.71$ (br d), 7.66 (d, $J=6.1 \mathrm{~Hz}), 7.49$ (s), 7.44 (d, $J=7.3 \mathrm{~Hz}), 7.40$ (d, $J=$ $6.8 \mathrm{~Hz}), 7.33(\mathrm{~d}, J=8.2 \mathrm{~Hz}), 7.18(\mathrm{br} \mathrm{d}), 7.13(\mathrm{~d}, J=8.2 \mathrm{~Hz}), 7.03(\mathrm{~d}, J=8.2 \mathrm{~Hz})$, $3.97(\mathrm{~d}, J=8.2 \mathrm{~Hz}), 6.89(\mathrm{~d}, J=6.8 \mathrm{~Hz}), 6.79(\mathrm{t}, J=6.8 \mathrm{~Hz}), 6.59(\mathrm{t}, J=6.8 \mathrm{~Hz})$, 5.09 (s), 4.68 (br s), 4.60 (br d), 4.65 (s), 4.45 (s), 4.39 (s), 4.35 (d, J = 8.8 Hz), 4.33 (d, $J=6.4 \mathrm{~Hz}$ ), 4.28 (br d), 4.10 (s), 4.04 (s), 3.98 (br d), 3.83 (br d), 3.77 (br d), $3.73(\mathrm{~s}), 3.04(\mathrm{t}, J=7.7 \mathrm{~Hz}), 2.94(\mathrm{t}, J=7.2 \mathrm{~Hz}), 2.77$ (bt t); Anal. Calc. for $\mathrm{C}_{68} \mathrm{H}_{64} \mathrm{Cl}_{4} \mathrm{~N}_{8} \mathrm{O}_{20} \mathrm{Zn}_{2}: \mathrm{C}, 51.50 ; \mathrm{H}, 4.07 ; \mathrm{N}, 7.07 \%$, found; C, 51.42; H, 4.01; N, 6.78 $\% ; \lambda_{\max }(\mathrm{DCM} / \mathrm{MeOH}) 420 \mathrm{~nm} @ 0.4 \mathrm{mM}, \varepsilon=338 \mathrm{M}^{-1} \mathrm{~cm}^{-1}$. 


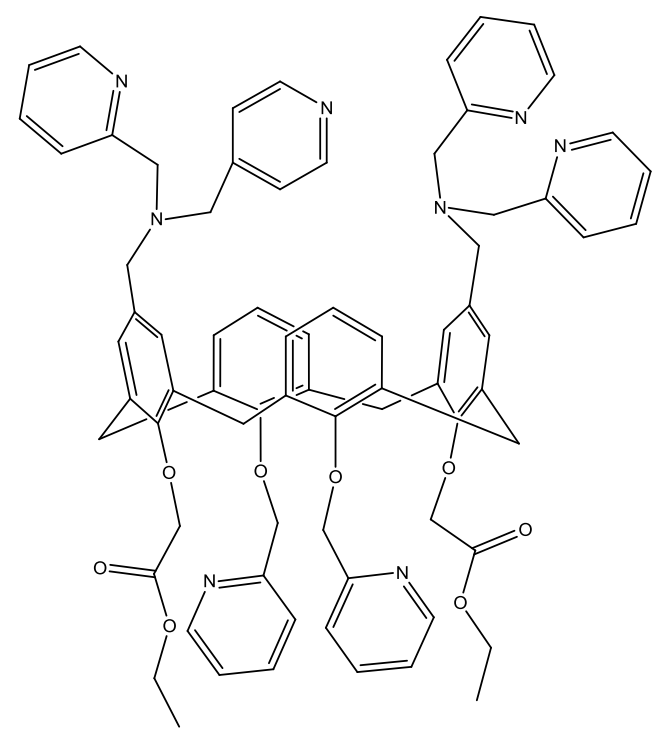

Compound (35) (0.23 g, $0.22 \mathrm{mmol})$, sodium hydride ( $0.02 \mathrm{~g}, 0.45 \mathrm{mmol})$ and ethyl bromoacetate $(0.07 \mathrm{~g}, 0.45 \mathrm{mmol})$ were refluxed under nitrogen gas in DCM: $\mathrm{CH}_{3} \mathrm{CN}$ (20: $60 \mathrm{~mL}$ ) overnight. The solution was allowed to cool to room temperature, the inorganic salts were removed by filtration and the volatiles removed under reduced pressure to afford a dark brown waxy solid (47).

Yield $=0.15 \mathrm{~g}, 56 \% ; \mathrm{v}_{\max } / \mathrm{cm}^{-1}(\mathrm{KBr}): 2924,1748,1627,1591,1463,1190,1092$, 756; $\delta_{\mathrm{H}} / \mathrm{ppm}$ : Conc. of $10^{-3} \mathrm{~mm}$; $\left(\mathrm{CDCl}_{3}\right) ; 8.43$ (d, 6H, Py- $\left.\underline{\mathrm{H}}, J=4.6 \mathrm{~Hz}\right), 7.59(\mathrm{t}$, $2 \mathrm{H}, \mathrm{Py}-\underline{\mathrm{H}}, J=2.2 \mathrm{~Hz}$ ), 7.56 (t, 4H, Py- $\underline{\mathrm{H}}, J=2.6 \mathrm{~Hz}$ ), 7.32 (t, $6 \mathrm{H}, \mathrm{Py}-\underline{\mathrm{H}}, J=9.2 \mathrm{~Hz}$ ), 6.99 (d, 6H, Py- $\underline{\mathrm{H}}, J=6.7 \mathrm{~Hz}), 6.89$ (s, 4H, Ar- $\underline{\mathrm{H}}), 6.86$ (d, 4H, Ar- $\underline{\mathrm{H}}, J=7.5 \mathrm{~Hz}$ ), 6.78 (t, $2 \mathrm{H}, \mathrm{Ar}-\underline{\mathrm{H}}, J=7.1 \mathrm{~Hz}), 5.01$ (s, 4H, OC$\left.\underline{H}_{2} \mathrm{Py}\right), 4.54$ (s, 4H, $\left.\underline{\mathrm{C}}_{2} \mathrm{NR}_{2}\right), 4.34$ (d, $\left.4 \mathrm{H}, \underline{\mathrm{C}}_{2}, J=12.6 \mathrm{~Hz}\right), 4.13\left(\mathrm{~m}, 4 \mathrm{H}, \mathrm{OC}_{2} \mathrm{CH}_{3}, J=4.8 \mathrm{~Hz}\right) 4.10$ (d, 8H, $\underline{\mathrm{C}}_{2}-\mathrm{Py}, J=$ $2.8 \mathrm{~Hz}), 3.94\left(\mathrm{~s}, 4 \mathrm{H}, \mathrm{C}_{2} \mathrm{CO}\right), 3.12\left(\mathrm{~d}, 4 \mathrm{H}, \mathrm{C}_{2}, J=12.2 \mathrm{~Hz}\right), 1.16(\mathrm{t}, 6 \mathrm{H}$, $\left.\mathrm{OCH}_{2} \mathrm{CH}_{3}, J=7.7 \mathrm{~Hz}\right) ; \delta_{\mathrm{c}} / \mathrm{ppm}\left(\mathrm{CDCl}_{3}\right) ; 178.2,163.2,152.5,151.0,149.4,147.2$, 141.0, 137.3, 133.0, 128.7, 128.4, 125.9, 124.6, 122.8, 120.8, 81.1, 78.7, 71.7, 70.6, 58.9, 31.8, 14.1; Anal. Calc. for $\mathrm{C}_{74} \mathrm{H}_{78} \mathrm{~N}_{8} \mathrm{O}_{8}: \mathrm{C}, 73.98 ; \mathrm{H}, 6.04 ; \mathrm{N}, 9.33 \%$, found; $\mathrm{C}$, $73.51 ; \mathrm{H}, 5.88 ; \mathrm{N}, 9.04 \%$. 


\section{Metal complexes of (47) at 1:1 ratio}

All metal ion complexation reactions were performed at room temperature in a solvent mixture of DCM-MeOH in a 1:1 ratio. All reactions used $0.08 \mathrm{mmol}$ of (47) and corresponding metal salt. The solution was stirred for $15 \mathrm{~min}$ and then the solvent was slowly evaporated off. A water-DCM wash was performed to remove any uncomplexed metal and the solvent was removed under reduced pressure.

Compound (47i): Zinc acetate; Yield $=0.09 \mathrm{~g}, 78 \% ; \mathrm{v}_{\max } / \mathrm{cm}^{-1}(\mathrm{KBr}): 2923,1626$, 1438, 1191, 758, 613; $\delta_{\mathrm{H}} / \mathrm{ppm}$ : Conc. of $10^{-3} \mathrm{~mm} ;\left(\mathrm{CDCl}_{3}\right) ; 8.62(\mathrm{~d}, J=5.1 \mathrm{~Hz}), 8.59$ (d, $J=5.1 \mathrm{~Hz}), 8.45(\mathrm{br} \mathrm{d}), 8.32(\mathrm{~s}), 8.27(\mathrm{t}, J=8.8 \mathrm{~Hz}), 8.20(\mathrm{~d}, J=8.1 \mathrm{~Hz}), 8.03$ (br t),7.88 (t, $J=2.9 \mathrm{~Hz}), 7.85$ (t, $J=1.9 \mathrm{~Hz}$ ), 7.83 (br d), 7.79 (br t), 7.75 (br t), 7.74 (br d), 7.69 (br t), 7.65 (br t), 7.62 (br t), 7.54 (br t), 7.84 (br d), 7.64 (d, $J=2.18$ Hz), 7.44 (br d), 7.35 (br d), 7.32 (br d), 7.30 (br t), 7.24 (br d), 7.21 (br d), 7.18 (br t), $7.14($ br t), 7.05 (s), $6.92(\mathrm{~d}, J=2.2 \mathrm{~Hz}), 6.89$ (s), $6.84(\mathrm{br} \mathrm{d}), 6.73$ (t, $J=4.5 \mathrm{~Hz}$ ), 6.63 (br t), 5.07 (s), 4.81 (d, $J=5.7 \mathrm{~Hz}$ ), 4.75 (br d), 4.73 (s), 4.69 (br t), 4.65 (d, $J=$ $4.1 \mathrm{~Hz}), 4.62(\mathrm{~s}), 4.58(\mathrm{~d}, J=5.4 \mathrm{~Hz}), 4.52$ (d, $J=5.4 \mathrm{~Hz}), 4.45(\mathrm{~d}, J=4.8 \mathrm{~Hz}), 4.41$ (s), 4.38 (s), 4.37 (d, $J=4.3 \mathrm{~Hz}), 4.33$ (s), 4.30 (s), 4.17 (s), 4.15 (d, $J=2.3 \mathrm{~Hz}$ ), $4.11(\mathrm{~d}, J=7.8 \mathrm{~Hz}), 4.07$ (s), 3.92 (t, $J=5.1 \mathrm{~Hz}), 3.79$ (br t), 3.47 (d, $J=8.5 \mathrm{~Hz})$, 3.41 (s), 3.29 (br t), 3.22 (br t), 3.16 (br t), 3.05 (t, $J=3.7 \mathrm{~Hz}$ ); Anal. Calc. for $\mathrm{C}_{78} \mathrm{H}_{78} \mathrm{~N}_{8} \mathrm{O}_{12} \mathrm{Zn}: \mathrm{C}, 67.65 ; \mathrm{H}, 5.68 ; \mathrm{N}, 8.09 \%$, found; $\mathrm{C}, 67.44 ; \mathrm{H}, 5.49 ; \mathrm{N}, 8.02 \%$.

Compound (47ii): Zinc chloride; Yield $=0.07 \mathrm{~g}, 63 \% ; \mathrm{v}_{\max } / \mathrm{cm}^{-1}(\mathrm{KBr}): 2924$, 1447, 1626, 1464, 1190, 1092, 759; $\delta_{\mathrm{H}} / \mathrm{ppm}$ : Conc. of $10^{-3} \mathrm{~mm} ;\left(d_{6}\right.$-DMSO); $9.04(\mathrm{~d}$, $J=5.0 \mathrm{~Hz}), 9.01$ (d, $J=4.6 \mathrm{~Hz}), 8.91$ (br d), 8.83 (d, $J=3.9 \mathrm{~Hz}), 8.79$ (d, $J=4.6$ Hz), 8.69 (br t), 8.65 (s), 8.62 (d, $J=4.3 \mathrm{~Hz}$ ), 8.59 (s), 8.58 (br d), 8.17 (br t), 8.06 (br t), 8.02 (s), 7.97 (t, $J=2.6 \mathrm{~Hz}), 7.96$ (br t), 7.90 (d, $J=1.6 \mathrm{~Hz}), 7.07(\mathrm{~d}, J=2.0$ $\mathrm{Hz}), 7.83$ (d, $J=1.6 \mathrm{~Hz}), 7.50$ (d, $J=8.0 \mathrm{~Hz}$ ), 7.47 (s), 7.44 (s), 7.41 (br d), 7.39 (s), $7.36(\mathrm{~d}, J=4.98), 7.16(\mathrm{~d}, J=7.4 \mathrm{~Hz}), 7.10(\mathrm{~s}), 7.04(\mathrm{~d}, J=7.4 \mathrm{~Hz}), 6.82(\mathrm{t}, J=7.4$ $\mathrm{Hz}), 6.61$ (d, $J=8.0 \mathrm{~Hz}$ ), 4.69 (s), 4.66 (br d), 4.62 (d, $J=7.6 \mathrm{~Hz}), 4.49$ (br d), 4.45 $(\mathrm{d}, J=6.4 \mathrm{~Hz}), 4.41(\mathrm{~s}), 4.26(\mathrm{~d}, J=5.6 \mathrm{~Hz}), 4.22(\mathrm{~s}), 4.18(\mathrm{~s}), 4.08$ (t, $J=3.2 \mathrm{~Hz})$, $4.06(\mathrm{~s}), 3.42$ (s), 3.38 (d, $J=4.5 \mathrm{~Hz}), 3.27(\mathrm{~d}, J=7.6 \mathrm{~Hz}), 3.22(\mathrm{t}, J=6.4 \mathrm{~Hz}), 3.14$ (br t); Anal. Calc. for $\mathrm{C}_{74} \mathrm{H}_{72} \mathrm{Cl}_{2} \mathrm{~N}_{8} \mathrm{O}_{8} \mathrm{Zn}$ : C, 66.44; H, 5.43; N, $8.38 \%$, found; $\mathrm{C}$, $66.31 ; \mathrm{H}, 5.35 ; \mathrm{N}, 8.01 \%$. 
Compound (47iii): Zinc perchlorate; Yield $=0.09 \mathrm{~g}, 74 \% ; \mathrm{v}_{\max } / \mathrm{cm}^{-1}(\mathrm{KBr}): 1750$, $1615,1465,1110$ (perchlorate anion peak), 940, 758, 626; $\delta_{\mathrm{H}} / \mathrm{ppm}$ : Conc. of $10^{-3}$ mm; ( $d_{6}$-DMSO); 8.67 (d, $\left.J=4.2 \mathrm{~Hz}\right), 8.64$ (d, $\left.J=4.7 \mathrm{~Hz}\right), 8.65$ (br t), 8.32 (s), 8.17 (s), 8.14 (br t), 7.95 (br t), 7.91 (d, $J=1.9 \mathrm{~Hz}), 7.87$ (d, $J=1.9 \mathrm{~Hz}), 7.85$ (d, $J=1.9$ Hz), 7.70 (br t), 7.95 (d, $J=7.3 \mathrm{~Hz}), 7.52$ (br t), 7.47 (d, $J=8.0 \mathrm{~Hz}), 7.44$ (s), 7.41 (br d), 7.37 (d, $J=6.0 \mathrm{~Hz}), 7.15(\mathrm{~d}, J=8.0 \mathrm{~Hz}), 7.09$ (s), $7.04(\mathrm{~d}, J=6.6 \mathrm{~Hz}), 6.91$ (s), $6.61(\mathrm{br} \mathrm{t}), 5.11(\mathrm{~s}), 4.57(\mathrm{~d}, J=6.6 \mathrm{~Hz}), 4.45$ (d, $J=6.6 \mathrm{~Hz}), 4.43(\mathrm{~s}), 4.24(\mathrm{~s})$, $4.19(\mathrm{~d}, J=5.9 \mathrm{~Hz}), 4.12(\mathrm{~d}, J=9.2 \mathrm{~Hz}), 4.09(\mathrm{~s}), 4.00(\mathrm{~d}, J=7.3 \mathrm{~Hz}), 3.88(\mathrm{~d}, J=$ $7.9 \mathrm{~Hz}), 3.81$ (br t), 3.20 (br t), $3.15(\mathrm{~d}, J=7.9 \mathrm{~Hz}), 3.05$ (t, $J=7.1 \mathrm{~Hz})$; Anal. Calc. for $\mathrm{C}_{74} \mathrm{H}_{72} \mathrm{Cl}_{2} \mathrm{~N}_{8} \mathrm{O}_{16} \mathrm{Zn}$ : C, 60.64; H, 4.95; N, $7.65 \%$, found; C, 60.54; H, 4.89; N, $7.56 \%$.

\section{Metal complexes of (47) at 1:2 ratio}

Same procedure was used as in the case of the 1:1 with the exception that the ratio of ligand to metal was $0.08 \mathrm{mmol}$ to $0.16 \mathrm{mmol}$.

Compound (47iv): Zinc acetate; Analysis of ${ }^{1} \mathrm{H}$ NMR spectrum showed that no reaction had occurred.

Compound (47vi): Zinc chloride; Yield $=0.20 \mathrm{~g}, 72 \% ; \mathrm{v}_{\max } / \mathrm{cm}^{-1}(\mathrm{KBr}): 2924$, 1747, 1626, 1464, 1190, 1093, 760; $\delta_{\mathrm{H}} / \mathrm{ppm}$ : Conc. of $10^{-3} \mathrm{~mm} ;\left(d_{6}\right.$-DMSO); 9.35 (d, $J=8.3 \mathrm{~Hz}$ ), 9.22 (d, $J=6.3 \mathrm{~Hz}), 9.08$ (br d), 9.04 (br d), 8.91 (d, $J=5.3 \mathrm{~Hz}), 8.81$ (d, $J=6.8 \mathrm{~Hz}), 8.76$ (d, $J=7.8 \mathrm{~Hz}), 8.70$ (br t), 8.43 (br d), 8.39 (br t), 8.34 (br t), 8.31 (br d), 8.28 (br d), 8.24 (br t), 8.21 (d, $J=1.7 \mathrm{~Hz}$ ), 8.17 (br d), 8.08 (br t), 7.97 (s), 7.75 (br t), 7.63 (s), 7.33 (s), 7.30 (s), 7.28 (s), 7.21 (br d), 7.16 (t, J= $4.8 \mathrm{~Hz}$ ), 7.10 (s), 7.08 (br d), 6.99 (s), 6.95 (d, J = $7.8 \mathrm{~Hz}$ ), 6.87 (br t), 5.19 (s), 5.82 (br d), 5.77 (br t), 5.57 (d, $J=8.0 \mathrm{~Hz}), 5.44$ (d, $J=6.3 \mathrm{~Hz}$ ), 4.97 (s), 4.98 (s), 4.83 (s), 4.80 (s), 5.59 (s), 4.50 (d, J = 7.7 Hz), 4.45 (s), 4.42 (br t), 4.37 (br d), 4.36 (s), 4.34 (d, $J$ $=4.75 \mathrm{~Hz}), 4.31$ (br t), 4.29 (s), $4.28(\mathrm{~d}, J=1.4 \mathrm{~Hz}), 4.26(\mathrm{~d}, J=2.2 \mathrm{~Hz}), 4.24(\mathrm{br} \mathrm{t})$, 4.22 (br t), 4.21 (s), 4.19 (br d), 4.16 (s), 4.14 (d, J=3.6 Hz), 4.11 (s), 4.05 (s), 3.97 (s), 3.94 (s); Anal. Calc. for $\mathrm{C}_{74} \mathrm{H}_{72} \mathrm{Cl}_{4} \mathrm{~N}_{8} \mathrm{O}_{8} \mathrm{Zn}_{2}$ : C, 60.30; $\mathrm{H}, 4.92 ; \mathrm{N}, 7.60 \%$, found; C, 60.24; H, 4.88; N, $7.55 \%$.

Compound (47v): Zinc perchlorate; Yield $=0.02 \mathrm{~g} 76 \% ; \mathrm{v}_{\max } / \mathrm{cm}^{-1}(\mathrm{KBr}): 2925$, 1749, 1615, 1465, 1088 (perchlorate anion peak), 940, 759, 626; $\delta_{\mathrm{H}} / \mathrm{ppm}$ : Conc. of 
$10^{-3} \mathrm{~mm}$; $\left(d_{6}\right.$-DMSO); 9.35 (d, $\left.J=7.7 \mathrm{~Hz}\right), 9.02(\mathrm{~d}, J=6.9 \mathrm{~Hz}), 9.08(\mathrm{br} \mathrm{d}), 8.91$ (br d), 8.78 (br t), 8.72 (d, $J=6.9 \mathrm{~Hz}), 8.66$ (br d), 8.40 (d, $J=7.2 \mathrm{~Hz}), 8.30$ (t, $J=7.2$ $\mathrm{Hz}), 8.21(\mathrm{t}, J=7.7 \mathrm{~Hz}), 8.16$ (br d), 8.14 (br d), 8.10 (d, $J=2.5 \mathrm{~Hz}), 8.07$ (br d), 7.98 (s), 7.80 (br t), 7.73 (br t), 7.65 (s), 7.47 (br t), 7.35 (d, J = 7.3 Hz), 7.30 (d, $J=$ $3.7 \mathrm{~Hz}), 7.24$ (s), 7.18 (s), 7.15 (s), 7.12 (s), 7.10 (d, J= 5.6 Hz), 6.98 (s), 6.94 (d, J = $7.5 \mathrm{~Hz}), 6.69$ (t, $J=7 . \mathrm{Hz}$ 3), 5.89 (s), 5.83 (s), 5.78 (s), $5.64(\mathrm{~s}), 5.59$ (br d), 5.44 (br d), 5.18 (s), 4.97 (s), 4.89 (br d), 4.82 (s), 4.50 (d, $J=7.8 \mathrm{~Hz}), 4.41$ (d, $J=7.0$ Hz), 4.37 (br d), 4.33 (s), 4.30 (br t), 4.28 (br d), 4.24 (br t), 4.20 (s), 4.15 (s), 4.14 (d, $J=3.1 \mathrm{~Hz}), 4.11(\mathrm{~s}), 4.05(\mathrm{~s}), 3.96(\mathrm{~d}, J=6.6 \mathrm{~Hz})$; Anal. Calc. for $\mathrm{C}_{74} \mathrm{H}_{72} \mathrm{Cl}_{4} \mathrm{~N}_{8} \mathrm{O}_{24} \mathrm{Zn}_{2}$ : C, 51.38; H, 4.19; N, $6.48 \%$, found; C, 51.07; H, 4.13; N, 6.20 $\%$.

\subsection{1,23-dibromomethyl-25,27-dibenzyl-26,28-} diethoxycarbonylmethoxy-calix[4]arene. (48)

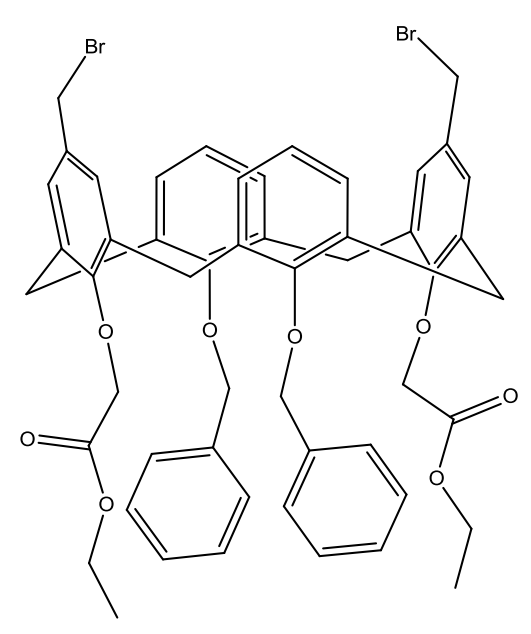

Compound (29) (0.89 g, $1.13 \mathrm{mmol})$, potassium carbonate $(0.31 \mathrm{~g}, 2.26 \mathrm{mmol})$ and ethyl bromoacetate $(0.25 \mathrm{~mL}, 2.26 \mathrm{mmol})$ were refluxed for $48 \mathrm{hr}$. in $\mathrm{CH}_{3} \mathrm{CN}$ under nitrogen gas. The solution was allowed to cool to room temperature, the inorganic salts were removed by filtration and the volatiles removed under reduced pressure to afford a dark brown waxy solid (48). 
Yield $=0.72 \mathrm{~g}, 66 \% ; \mathrm{v}_{\max } / \mathrm{cm}^{-1}(\mathrm{KBr}): 2922,1758,1672,1586,1455,1192,1093$, 761; $\delta_{\mathrm{H}} / \mathrm{ppm}$ : Conc. of $10^{-3} \mathrm{~mm} ;\left(\mathrm{CDCl}_{3}\right) ; 7.41(\mathrm{~d}, 4 \mathrm{H}, \mathrm{Bn}-\underline{\mathrm{H}}, J=5.3 \mathrm{~Hz}), 7.38(\mathrm{t}$, $6 \mathrm{H}, \mathrm{Bn}-\underline{\mathrm{H}}, J=5.0 \mathrm{~Hz}), 7.23$ (s, 4H, Ar- $\underline{\mathrm{H}}), 7.09$ (d, 4H, Ar- $\underline{\mathrm{H}}, J=6.6 \mathrm{~Hz}), 7.16$ (s, 4H, Ar- $\underline{\mathrm{H}}$ ), 7.13 (d, 4H, Ar- $\underline{\mathrm{H}}, J=3.7 \mathrm{~Hz}), 7.09$ (s, 4H, Ar- $\underline{\mathrm{H}}$ ), 7.06 (s, 4H, Ar- $\underline{\mathrm{H}}$ ), 7.03 (s, 4H, Ar- $\underline{\mathrm{H}}), 6.98$ (d, 2H, Ar- $\underline{\mathrm{H}}, J=3.7 \mathrm{~Hz}), 6.97$ (d, 2H, Ar- $\underline{\mathrm{H}}, J=3.1 \mathrm{~Hz}$ ), 6.89 (t, $2 \mathrm{H}, \operatorname{Ar}-\underline{\mathrm{H}}, J=2.7 \mathrm{~Hz}), 6.87$ (br t, $2 \mathrm{H}, \operatorname{Ar}-\underline{\mathrm{H}}), 6.84$ (d, 2H, Ar- $\underline{\mathrm{H}} J=2.7 \mathrm{~Hz}$ ), 6.81 (s, 2H, Ar- $\underline{\mathrm{H}}$ ), 6.80 (br d, 4H, Ar- $\underline{\mathrm{H}}$ ), 6.87 (br d, 4H, Ar- $\underline{\mathrm{H}}$ ), 6.73 (s, 2H, Ar- $\underline{\mathrm{H}}$ ), 6.71 (br d, 4H, Ar- $\underline{\mathrm{H}}$ ), 6.68 (br d, 4H, Ar- $\underline{\mathrm{H}}$ ), 6.53 (t, 2H, Ar- $\underline{\mathrm{H}}, J=3.8 \mathrm{~Hz}$ ), 6.46 (br t, $2 \mathrm{H}, \mathrm{Ar}-\underline{\mathrm{H}}$ ), 6.42 (br t, $2 \mathrm{H}, \mathrm{Ar}-\underline{\mathrm{H}}), 5.06$ (s, 4H, OC$\left.\underline{\mathrm{H}}_{2}-\mathrm{Bn}\right), 4.94$ (s, 4H, $\underline{\mathrm{C}}_{2}-\mathrm{Br}$ ), $4.87(\mathrm{~d}, 4 \mathrm{H}, J=3.2 \mathrm{~Hz}), 4.83$ (s, 4H, OC$\left.\underline{H}_{2} \mathrm{CO}\right), 4.79$ (d, 4H, $\underline{\mathrm{H}}_{2}-\mathrm{Br}, J=3.2 \mathrm{~Hz}$ ), $4.76\left(\mathrm{~d}, 4 \mathrm{H}, \underline{\mathrm{C}}_{2}-\mathrm{Br}, J=3.8 \mathrm{~Hz}\right), 4.65$ (d, 4H, $\left.\underline{\mathrm{C}}_{2}-\mathrm{Br}, J=3.2 \mathrm{~Hz}\right), 4.37$ (d, $4 \mathrm{H}$, $\left.\mathrm{C}_{2}, J=5.1 \mathrm{~Hz}\right), 4.33$ (br s, $\left.4 \mathrm{H}, \mathrm{OC}_{2} \underline{\mathrm{CO}}\right), 4.26$ (d, $\left.4 \mathrm{H}, \underline{\mathrm{C}}_{2}, J=14.7 \mathrm{~Hz}\right), 4.23$ (q, $\left.4 \mathrm{H}, \mathrm{OC}_{2} \mathrm{CH}_{3}, J=2.2 \mathrm{~Hz}\right), 4.05$ (s, $4 \mathrm{H}, \mathrm{OC}_{2} \mathrm{CO}$ ), 3.89 (s, 2H), 3.83 (q, 4H, $J=4.3$ Hz), 3.68 (s, 4H), 3.64 (t, 6H, J = 3.6 Hz), $3.41(\mathrm{~s}, 2 \mathrm{H}), 3.39$ (s, 4H,), 3.33 (d, 4H, $\left.\mathrm{C}_{\underline{H}}, J=10.8 \mathrm{~Hz}\right), 3.10\left(\mathrm{~d}, 4 \mathrm{H}, \underline{\mathrm{CH}}_{2}, J=12.9 \mathrm{~Hz}\right), 2.88\left(\mathrm{~d}, 4 \mathrm{H}, \underline{\mathrm{C}}_{\underline{2}}, J=14.4 \mathrm{~Hz}\right)$, $1.29\left(\mathrm{t}, 6 \mathrm{H}, \mathrm{OCH}_{2} \mathrm{C}_{3}, J=1.9 \mathrm{~Hz}\right) ; \delta_{\mathrm{C}} / \mathrm{ppm}\left(\mathrm{CDCl}_{3}\right) ; 170.4,155.8,154.6,138.3$, $134.3,134.2$, 131.2, 129.5, 128.6, 127.6, 127.1, 126.8, 125.4, 72.2, 71.7, 66.6, 35.9, 31.4, 23.4; Anal. Calc. for $\mathrm{C}_{52} \mathrm{H}_{50} \mathrm{Br}_{2} \mathrm{O}_{4}$ : C, 64.87; H, $5.23 \%$, found; C, 64.51; H, $5.15 \%$. 


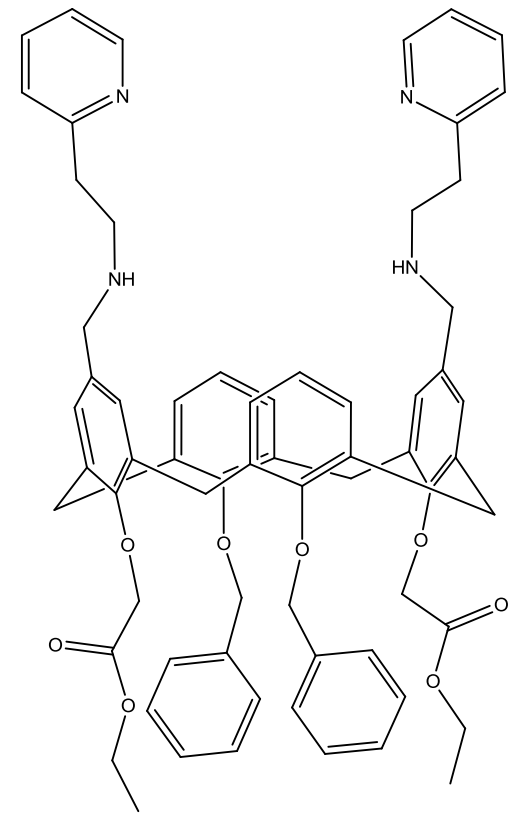

Compound (48) (0.72 g, $0.75 \mathrm{mmol})$, triethylamine $(0.15 \mathrm{~g}, 1.49 \mathrm{mmol})$ and 2aminoethylpyridine $(0.18 \mathrm{~mL}, 1.49 \mathrm{mmol})$ were refluxed overnight under nitrogen gas in $\mathrm{CH}_{3} \mathrm{CN}(80 \mathrm{~mL})$. The solution was allowed to cool to room temperature, the inorganic salts were removed by filtration and the volatiles removed under reduced pressure to give a dark brown waxy solid (49).

Yield $=0.78 \mathrm{~g}, 98 \% ; \mathrm{v}_{\max } / \mathrm{cm}^{-1}(\mathrm{KBr}): 3291,1656,1593,1456,1195,1092,762 ;$ $\delta_{\mathrm{H}} / \mathrm{ppm}$ : Conc. of $10^{-3} \mathrm{~mm} ;\left(\mathrm{CDCl}_{3}\right) ; 8.49(\mathrm{~d}, 2 \mathrm{H}, \mathrm{Py}-\underline{\mathrm{H}}, J=3.2 \mathrm{~Hz}), 7.60$ (t, $2 \mathrm{H}, \mathrm{Py}-$ $\underline{\mathrm{H}}, J=5.5 \mathrm{~Hz}), 7.44(\mathrm{~d}, 4 \mathrm{H}, \mathrm{Bn}-\underline{\mathrm{H}}, J=6.3 \mathrm{~Hz}), 7.35(\mathrm{t}, 6 \mathrm{H}, \mathrm{Bn}-\underline{\mathrm{H}}, J=4.8 \mathrm{~Hz}), 7.31$ (d, 2H, Py- $\underline{\mathrm{H}}, J=3.9 \mathrm{~Hz}), 7.18$ (t, 2H, Py- $\underline{\mathrm{H}}, J=4.8 \mathrm{~Hz}), 7.12(\mathrm{~s}, 2 \mathrm{H}, \mathrm{Ar}-\underline{\mathrm{H}}), 6.91$ (d, $4 \mathrm{H}, \mathrm{Ar}-\underline{\mathrm{H}}, J=6.9 \mathrm{~Hz}), 6.43$ (t, $2 \mathrm{H}, \mathrm{Ar}-\underline{\mathrm{H}}, J=7.3 \mathrm{~Hz}), 5.03$ (s, 4H, OC$\left.\underline{H}_{2}-\mathrm{Bn}\right), 4.55$ (s, $\left.4 \mathrm{H}, \mathrm{OC}_{2}-\mathrm{NHR}\right), 4.29$ (d, $\left.4 \mathrm{H}, \underline{\mathrm{C}}_{2}, J=12.3 \mathrm{~Hz}\right), 4.16\left(\mathrm{q}, 4 \mathrm{H}, \mathrm{OC}_{2} \mathrm{CH}_{3}, J=5.4\right.$ $\mathrm{Hz}), 3.46\left(\mathrm{~s}, 4 \mathrm{H}, \mathrm{OC}_{\underline{2}} \mathrm{CO}\right), 3.35\left(\mathrm{~d}, 4 \mathrm{H}, \mathrm{C}_{2}, J=12.3 \mathrm{~Hz}\right), 3.08(\mathrm{t}, 4 \mathrm{H}$, $\mathrm{NHCH}_{2} \mathrm{CH}_{2}, J=6.4 \mathrm{~Hz}$ ), 2.90 (t, $4 \mathrm{H}, \mathrm{NHCH}_{2} \underline{\mathrm{CH}}_{2}, J=6.4 \mathrm{~Hz}$ ), 1.19 (t, $6 \mathrm{H}$, $\left.\mathrm{OCH}_{2} \mathrm{C}_{3}, J=6.0 \mathrm{~Hz}\right) ; \delta_{\mathrm{C}} / \mathrm{ppm}\left(\mathrm{CDCl}_{3}\right) ; 172.1,159.8,153.4,151.8,149.2,148.6$, 136.5, 136.2, 134.0, 133.5, 129.5, 127.4, 124.2, 123.1, 123.3, 122.5, 121.4, 121.1, 78.4 ,72.8, 60.8, 53.9, 46.7, 38.1, 36.7, 14.2; Anal. Calc. for $\mathrm{C}_{66} \mathrm{H}_{68} \mathrm{~N}_{4} \mathrm{O}_{8}$ : C, 75.84; $\mathrm{H}, 6.56 ; \mathrm{N}, 5.36 \%$, found; C, 75.78; H, 6.44; N, $5.13 \%$. 


\section{Metal complexes of (49) at 1:1 ratio}

All metal ion complexation reactions were performed at room temperature in a solvent mixture of DCM-MeOH in a 1:1 ratio. All reactions used $0.09 \mathrm{mmol}$ of (49) and corresponding metal salt. The solution was stirred for $15 \mathrm{~min}$ and then the solvent was slowly evaporated off. A water-DCM wash was performed to remove any uncomplexed metal and the solvent was removed under reduced pressure.

Compound (49i): Zinc acetate; Yield $=0.08 \mathrm{~g}, 71 \% ; \mathrm{v}_{\max } / \mathrm{cm}^{-1}(\mathrm{KBr}): 3418,2925$, 1756, 1586, 1448, 1188, 1022, 760; $\delta_{\mathrm{H}} / \mathrm{ppm}$ : Conc. of $10^{-3} \mathrm{~mm} ;\left(\mathrm{CDCl}_{3}\right) ; 8.65$ (br t), 8.50 (br t), 7.85 (s), 7.64 (dd, $J=3.4,7.2 \mathrm{~Hz}), 7.38$ (d, $J=1.8 \mathrm{~Hz}), 7.35$ (br d), 7.34 (s), $7.16(\mathrm{~d}, J=7.7 \mathrm{~Hz}), 7.12(\mathrm{~d}, J=5.8 \mathrm{~Hz}), 7.05(\mathrm{~d}, J=7.1 \mathrm{~Hz}), 6.91(\mathrm{~d}, J=7.7$ $\mathrm{Hz}), 6.77$ (t, $J=8.4 \mathrm{~Hz}), 6.65$ (t, $J=7.1 \mathrm{~Hz}), 5.06$ (s), 4.85 (s), 4.78 (d, $J=4.1 \mathrm{~Hz}$ ), 4.70 (br d), 4.54 (s), 4.30 (d, $J=11.6 \mathrm{~Hz}), 4.23$ (d, $J=8.6 \mathrm{~Hz}), 4.19$ (d, $J=10.8 \mathrm{~Hz})$, 4.13 (t, $J=9.1 \mathrm{~Hz}$ ), 3.99 (br t), 3.91 (br t), 3.85 (br d), 3.63 (br d), 3.59 (s), 3.50 (br d), $3.33(\mathrm{~d}, J=12.1 \mathrm{~Hz}), 3.26$ (br t), 3.18 (br t), 3.15 (br t), 3.06 (br d), 3.29 (t, $J=$ $6.6 \mathrm{~Hz}$ ), 2.89 (br t), 1.21 (br t); Anal. Calc. for $\mathrm{C}_{70} \mathrm{H}_{74} \mathrm{~N}_{4} \mathrm{O}_{12} \mathrm{Zn}: \mathrm{C}, 68.42 ; \mathrm{H}, 6.07 ; \mathrm{N}$, $4.56 \%$, found; C, 68.22; H, 5.83; N, $4.63 \%$.

Compound (49ii): Zinc chloride; Yield $=0.09 \mathrm{~g}, 75 \%$; $\mathrm{v}_{\max } / \mathrm{cm}^{-1}(\mathrm{KBr}): 3467$, $2920,1755,1607,1448,1188,1092,760 ; \delta_{\mathrm{H}} / \mathrm{ppm}$ : Conc. of $10^{-3} \mathrm{~mm} ;\left(d_{6}\right.$-DMSO); $8.69(\mathrm{~d}, J=6.6 \mathrm{~Hz}), 8.50(\mathrm{br} \mathrm{d}), 7.88(\mathrm{~s}), 7.83(\mathrm{~d}, J=8.4 \mathrm{~Hz}), 7.63(\mathrm{~d}, J=7.3 \mathrm{~Hz})$, $7.43(\mathrm{t}, J=5.8 \mathrm{~Hz}), 7.38(\mathrm{~d}, J=2.6 \mathrm{~Hz}), 7.35(\mathrm{~d}, J=2.6 \mathrm{~Hz}), 7.34(\mathrm{~s}), 7.31(\mathrm{~d}, J=$ $2.1 \mathrm{~Hz}), 7.16(\mathrm{~d}, J=7.9 \mathrm{~Hz}), 7.12(\mathrm{br} \mathrm{d}), 7.05(\mathrm{~d}, J=7.9 \mathrm{~Hz}), 6.92(\mathrm{~d}, J=7.2 \mathrm{~Hz})$, $6.75(\mathrm{t}, J=7.2 \mathrm{~Hz}), 6.67(\mathrm{t}, J=7.7 \mathrm{~Hz}), 6.50(\mathrm{br} \mathrm{t}), 5.06(\mathrm{~s}), 4.85(\mathrm{~s}), 4.77(\mathrm{~s}), 4.33$ (d, $J=12.4 \mathrm{~Hz}), 4.27(\mathrm{~d}, J=13.8 \mathrm{~Hz}), 4.13(\mathrm{t}, J=6.2 \mathrm{~Hz}), 3.86($ br t ), 3.72 (s), 3.60 (br t), 3.39 (d, $J=13.3 \mathrm{~Hz}), 3.21$ (s), 3.14 (br t), 3.08 (br t), 2.78 (br t), 1.23 (br t); Anal. Calc. for $\mathrm{C}_{66} \mathrm{H}_{68} \mathrm{Cl}_{2} \mathrm{~N}_{4} \mathrm{O}_{8} \mathrm{Zn}$ : C, 67.09; H, 5.80; N, $4.74 \%$, found; C, 66.94; $\mathrm{H}$, $5.73 ; \mathrm{N}, 4.39 \%$.

Compound (49iii): Zinc perchlorate; Yield $=0.09 \mathrm{~g}, 76 \% ; \mathrm{v}_{\max } / \mathrm{cm}^{-1}(\mathrm{KBr}): 3434$, 2922, 1755, 1606, 1448, 1188, 1091 (perchlorate anion peak), 760; $\delta_{\mathrm{H}} / \mathrm{ppm}$ : Conc. of $10^{-3} \mathrm{~mm}$; $\left(d_{6}\right.$-DMSO); $8.50(\mathrm{~d}, J=5.7 \mathrm{~Hz}), 8.30(\mathrm{~s}), 8.14(\mathrm{~s}), 7.81(\mathrm{t}, J=5.7 \mathrm{~Hz})$, $7.65(\mathrm{~d}, J=8.6 \mathrm{~Hz}), 7.43$ (d, $J=4.0 \mathrm{~Hz}), 7.41(\mathrm{~d}, J=2.1 \mathrm{~Hz}), 7.39$ (s), 7.35 (d, $J=$ $4.2 \mathrm{~Hz}$ ), 7.31 (br t), 7.20 (br t), 7.14 (d, $J=8.1 \mathrm{~Hz}), 7.07$ (d, $J=7.6 \mathrm{~Hz}), 7.03$ (d, $J=$ $8.1 \mathrm{~Hz}), 6.80$ (t, $J=7.6 \mathrm{~Hz}), 6.61(\mathrm{t}, J=7.6 \mathrm{~Hz}), 5.05$ (s), 4.79 (s), 4.69 (br t), 4.17 
(d, $J=11.4 \mathrm{~Hz}), 4.13(\mathrm{~d}, J=5.7 \mathrm{~Hz}), 4.10$ (d, $J=5.2 \mathrm{~Hz}), 4.07$ (br t), 3.77 (s), 3.59 (s), 3.51 (s), 3.43 (d, $J=5.8 \mathrm{~Hz}), 3.39$ (s), 3.14 (t, $J=7.0 \mathrm{~Hz}), 3.03$ (t, $J=7.0 \mathrm{~Hz}$ ), 3.29 (s), 2.73 (t, $J=1.1 \mathrm{~Hz}$ ), 1.20 (br t); Anal. Calc. for $\mathrm{C}_{66} \mathrm{H}_{68} \mathrm{Cl}_{2} \mathrm{~N}_{4} \mathrm{O}_{16} \mathrm{Zn}: \mathrm{C}$, $60.53 ; \mathrm{H}, 5.23 ; \mathrm{N}, 4.28 \%$, found; C, 60.41; H, 4.93; N, $4.19 \%$.

\section{Metal complexes of (49) at 1:2 ratio}

Same procedure was used as in the case of the 1:1 with the exception that the ratio of ligand to metal was $0.09 \mathrm{mmol}$ to $0.19 \mathrm{mmol}$.

Compound (49iv): Zinc acetate; Yield $=0.20 \mathrm{~g}, 74 \% ; \mathrm{v}_{\max } / \mathrm{cm}^{-1}(\mathrm{KBr}): 3432$, 2923, 1748, 1604, 1456, 1189, 1093, 760; $\delta_{\mathrm{H}} / \mathrm{ppm}$ : Conc. of $10^{-3} \mathrm{~mm} ;\left(\mathrm{CDCl}_{3}\right) ; 8.68$ (d, $J=4.9 \mathrm{~Hz}), 8.52$ (br d), 7.87 (s), 7.65 (d, $J=8.1 \mathrm{~Hz}), 7.39$ (s), 7.38 (d, $J=2.3$ $\mathrm{Hz}), 7.36$ (d, $J=3.2 \mathrm{~Hz}), 7.34$ (s), 7.17 (d, $J=7.1 \mathrm{~Hz}), 7.12$ (br t), 7.05 (d, $J=7.4$ $\mathrm{Hz}), 6.91(\mathrm{~d}, J=7.5 \mathrm{~Hz}), 6.75(\mathrm{t}, J=7.5 \mathrm{~Hz}), 6.65(\mathrm{t}, J=7.5 \mathrm{~Hz}), 5.06(\mathrm{~s}), 4.85(\mathrm{~s})$, 4.79 (t, $J=6.3 \mathrm{~Hz}), 4.70$ (br d), 4.54 (s), 4.30 (d, $J=13.0 \mathrm{~Hz}), 4.15(\mathrm{t}, J=6.9 \mathrm{~Hz})$, 3.99 (s), 3.93 (br t), 3.85 (br d), 3.71 (s), 3.63 (s), 3.49 (s), 3.34 (d, J=13.9 Hz), 3.21 (br t), 3.14 (br t), 2.91 (br t), 1.22 (br t); Anal. Calc. for $\mathrm{C}_{74} \mathrm{H}_{80} \mathrm{~N}_{4} \mathrm{O}_{16} \mathrm{Zn}_{2}$ : C, 62.94; $\mathrm{H}, 5.71 ; \mathrm{N}, 3.97 \%$, found; C, 62.61; H, 5.63; N, $3.63 \%$.

Compound (49v): Zinc chloride; Yield $=0.21 \mathrm{~g}, 83 \% ; \mathrm{v}_{\max } / \mathrm{cm}^{-1}(\mathrm{KBr}): 3440$, 3062, 1750 1608, 1465, 1189, 1092, 762; $\delta_{\mathrm{H}} / \mathrm{ppm}$ : Conc. of $10^{-3} \mathrm{~mm}$; ( $d_{6}$-DMSO); 8.48 (br t), 8.16 (s), 7.78 (t, $J=8.9 \mathrm{~Hz}), 7.66$ (d, $J=8.0 \mathrm{~Hz}), 7.45$ (s), 7.43 (d, $J=$ $3.8 \mathrm{~Hz}), 7.42$ (d, $J=2.2 \mathrm{~Hz}), 7.38(\mathrm{~d}, J=2.5 \mathrm{~Hz}), 7.36$ (s), 7.33 (s), 7.32 (s), 7.14 (d, $J=7.7 \mathrm{~Hz}), 7.07(\mathrm{~d}, J=7.7 \mathrm{~Hz}), 7.04(\mathrm{~d}, J=7.1 \mathrm{~Hz}), 6.82(\mathrm{t}, J=7.1 \mathrm{~Hz}), 6.74(\mathrm{br}$ d), $6.61(\mathrm{t}, J=8.3 \mathrm{~Hz}), 6.43(\mathrm{br} \mathrm{t}), 5.05(\mathrm{~s}), 4.79(\mathrm{~s}), 4.16(\mathrm{~d}, J=13.4 \mathrm{~Hz}), 4.09(\mathrm{t}, J$ $=7.0 \mathrm{~Hz}), 4.03(\mathrm{~d}, J=7.0 \mathrm{~Hz}), 3.98(\mathrm{br} \mathrm{d}), 3.77(\mathrm{~d}, J=8.4 \mathrm{~Hz}), 3.60(\mathrm{~s}), 3.53(\mathrm{~s})$, 3.44 (s), 3.40 (d, $J=3.9 \mathrm{~Hz}), 3.16$ (d, $J=5.1 \mathrm{~Hz}$ ), 3.15 (br t), 3.07 (br t), 3.92 (s), 3.72 (br t), 2.92 (br t), 1.18 (br t); Anal. Calc. for $\mathrm{C}_{66} \mathrm{H}_{68} \mathrm{Cl}_{4} \mathrm{~N}_{4} \mathrm{O}_{8} \mathrm{Zn}_{2}: \mathrm{C}, 60.15 ; \mathrm{H}$, $5.20 ; \mathrm{N}, 4.25 \%$, found; C, 59.67; H, 5.13; N, $4.19 \%$.

Compound (49vi): Zinc perchlorate; Yield $=0.24 \mathrm{~g}, 80 \%$; $\mathrm{v}_{\max } / \mathrm{cm}^{-1}(\mathrm{KBr}): 3436$, 3027, 1749, 1603, 1496, 1374, 1248, 1188 (perchlorate anion peak), 1022, 916, 732; $\delta_{\mathrm{H}} / \mathrm{ppm}$ : Conc. of $10^{-3} \mathrm{~mm}$; $\left(d_{6}\right.$-DMSO); $8.52(\mathrm{~d}, J=4.7 \mathrm{~Hz}), 8.17(\mathrm{~s}), 7.77$ (t, $J=7.8$ $\mathrm{Hz}$ ), 7.66 (d, $J=7.5 \mathrm{~Hz}), 7.48$ (s), 7.44 (d, $J=4.2 \mathrm{~Hz}$ ), 7.41 (br d), 7.38 (d, $J=2.3$ $\mathrm{Hz}), 7.36(\mathrm{~d}, J=1.3 \mathrm{~Hz}), 7.35$ (s), 7.32 (d, $J=6.2 \mathrm{~Hz}), 7.22(\mathrm{~s}), 7.14(\mathrm{~d}, J=7.9 \mathrm{~Hz})$, 
$7.09(\mathrm{~d}, J=8.2 \mathrm{~Hz}), 7.04(\mathrm{~d}, J=7.2 \mathrm{~Hz}), 6.80(\mathrm{t}, J=7.6 \mathrm{~Hz}), 6.73(\mathrm{~d}, J=7.6 \mathrm{~Hz})$, $6.62(\mathrm{t}, J=7.9 \mathrm{~Hz}), 5.05(\mathrm{~s}), 4.79(\mathrm{~s}), 4.18(\mathrm{~d}, J=3.8 \mathrm{~Hz}), 4.14(\mathrm{~s}), 4.06(\mathrm{t}, J=7.0$ Hz), 3.95 (br t), 3.84 (s), 3.78 (d, $J=8.1 \mathrm{~Hz}), 3.56$ (d, $J=7.1 \mathrm{~Hz}), 3.44$ (s), 3.18 (br t), 3.09 (br t), 2.99 (s), 1.20 (br t); Anal. Calc. for $\mathrm{C}_{66} \mathrm{H}_{68} \mathrm{Cl}_{4} \mathrm{~N}_{4} \mathrm{O}_{24} \mathrm{Zn}_{2}$ : C, 50.37; $\mathrm{H}$, $4.35 ; \mathrm{N}, 3.56 \%$, found; C, 50.13; H, 4.23; N, 3.37\%.

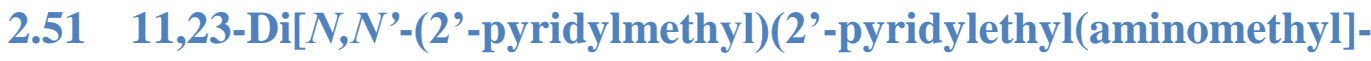
25,27-dibenzyl-26,28-diethoxycarbonylmethoxycalix[4]arene. (50)

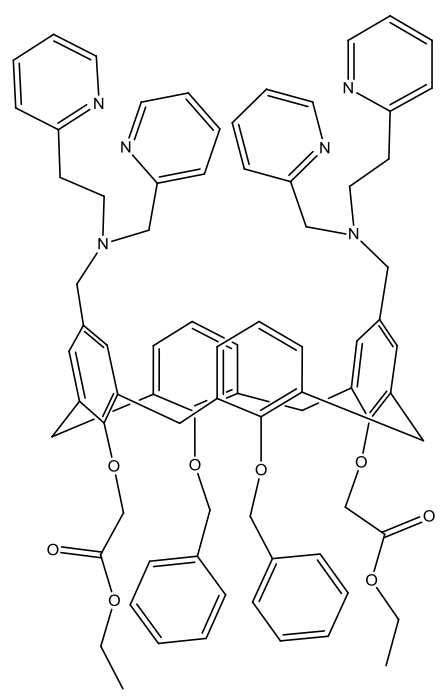

Compound (49) (1.00 g, $0.96 \mathrm{mmol})$, triethylamine $(0.19 \mathrm{~g}, 1.91 \mathrm{mmol})$ were stirred in $\mathrm{CH}_{3} \mathrm{CN}(60 \mathrm{~mL})$ in a $100 \mathrm{~mL}$ round bottom flask for $10 \mathrm{~min}$. 2-Picolylchloride hydrochloride $(0.31 \mathrm{~g}, 1.91 \mathrm{mmol})$ and sodium carbonate $(0.20 \mathrm{~g}, 1.91 \mathrm{mmol})$ were mixed in $\mathrm{CH}_{3} \mathrm{CN}(20 \mathrm{~mL})$, filtered to remove hydrochloric acid salts and the filtrate was added to the stirring solution. The resulting mixture was then refluxed under nitrogen gas overnight. The solution was allowed to cool to room temperature; the inorganic salts were removed by filtration the volatiles removed under reduced pressure to give a brown waxy solid (50).

Yield $=0.98 \mathrm{~g}, 84 \% ; \mathrm{v}_{\max } / \mathrm{cm}^{-1}(\mathrm{KBr}): 2926,1655,1590,1569,1455,1191,1092$, 761; $\delta_{\mathrm{H}} / \mathrm{ppm}$ : Conc. of $10^{-3} \mathrm{~mm} ;\left(\mathrm{CDCl}_{3}\right) ; 8.47$ (d, 2H, Py- $\left.\underline{\mathrm{H}}, J=5.3 \mathrm{~Hz}\right), 8.44$ (br d, 2H, Py- $\underline{\mathrm{H}}$ ), 7.60 (t, 2H, Py- $\underline{\mathrm{H}}, J=2.0 \mathrm{~Hz}$ ), 7.57 (br t, 2H, Py- $\underline{\mathrm{H}}$ ), 7.54 (br d, 4H, Bn- 
H), 7.51 (br t, 6H, Py- $\underline{\mathrm{H}}$ ), 7.37 (br t, 2H, Py- $\underline{\mathrm{H}}$ ), 7.33 (br t, 2H, Py- $\underline{\mathrm{H}}$ ), 7.30 (br d, $2 \mathrm{H}, \mathrm{Py}-\underline{\mathrm{H}}), 7.25$ (d, 2H, Py- $\underline{\mathrm{H}}, J=2.2 \mathrm{~Hz}), 7.10$ (s, 4H, Ar- $\underline{\mathrm{H}}), 6.84$ (d, 4H, Ar- $\underline{\mathrm{H}}, J=$ $7.7 \mathrm{~Hz}$ ), 6.59 (br t, $2 \mathrm{H}, \mathrm{Ar}-\underline{\mathrm{H}}$ ), 4.79 (s, 4H, OC$\underline{\mathrm{H}}_{2} \mathrm{Bn}$ ), 4.54 (s, 4H, $\mathrm{NC}_{2} \mathrm{Py}$ ), 4.44 (s, $4 \mathrm{H}, \underline{\mathrm{C}}_{2} \mathrm{NR}_{2}$ ), 4.24 (d, $4 \mathrm{H}, \underline{\mathrm{CH}}_{2}, J=13.3 \mathrm{~Hz}$ ), 4.13 (br t, $4 \mathrm{H}, \underline{\mathrm{OCH}}_{2} \mathrm{CH}_{3}$ ), 3.66 (s, $4 \mathrm{H}, \mathrm{OC}_{2} \underline{\mathrm{CO}}$ ), 3.29 (d, 4H, $\left.\underline{\mathrm{C}}_{2}, J=13.7 \mathrm{~Hz}\right), 2.93\left(\mathrm{t}, 4 \mathrm{H}, \mathrm{CH}_{2} \underline{\mathrm{C}}_{2} \mathrm{Py}, J=6.2 \mathrm{~Hz}\right.$ ), 2.85 (t, $4 \mathrm{H}, \mathrm{C}_{2} \mathrm{CH}_{2} \mathrm{Py}, J=6.6 \mathrm{~Hz}$ ), 2.03 (br t, $\left.6 \mathrm{H}, \mathrm{OCH}_{2} \underline{\mathrm{H}}_{3}\right) ; \delta_{\mathrm{c}} / \mathrm{ppm}\left(\mathrm{CDCl}_{3}\right)$; $171.4,159.5,155.8,155.4,155.0,149.4,148.8,137.1$, 136.7, 136.5, 133.8, 133.4, $129.1,128.8,128.4,127.7,127.2,125.3,123.5,123.2,122.8,121.7,121.3,72.9$, 71.9, 64.9, 60.5, 60.1, 54.3, 37.3, 31.4, 14.1; Anal. Calc. for $\mathrm{C}_{78} \mathrm{H}_{78} \mathrm{~N}_{6} \mathrm{O}_{8}$ : C, 76.32; H, 6.40; N, $6.85 \%$, found; C, 76.03; H, 6.34; N, $6.65 \%$.

\section{Metal complexes of (50) at 1:1 ratio}

All metal ion complexation reactions were performed at room temperature in a solvent mixture of DCM-MeOH in a 1:1 ratio. All reactions used $0.08 \mathrm{mmol}$ of (50) and corresponding metal salt. The solution was stirred for $15 \mathrm{~min}$ and then the solvent was slowly evaporated off. A water-DCM wash was performed to remove any uncomplexed metal and the solvent was removed under reduced pressure.

Compound (50i): Zinc acetate; Yield $=0.09 \mathrm{~g}, 78 \%$; $\mathrm{v}_{\max } / \mathrm{cm}^{-1}(\mathrm{KBr}): 1608,1447$, 1191, 1022, 761, 698; $\delta_{\mathrm{H}} / \mathrm{ppm}$ : Conc. of $10^{-3} \mathrm{~mm} ;\left(\mathrm{CDCl}_{3}\right) ; 8.93$ (br d), 8.87 (d, $J=$ $6.6 \mathrm{~Hz}$ ), 8.65 (br d), 8.53 (br d), 7.87 (br t), 7.80 (s), 7.72 (br t), 7.63 (d, J=7.6 Hz), 7.39 (s), 7.36 (d, $J=4.7 \mathrm{~Hz}), 7.31$ (s), 7.17 (s), 7.15 (d, J= $7.1 \mathrm{~Hz}), 7.11$ (br d), 7.04 $(\mathrm{d}, J=7.1 \mathrm{~Hz}), 6.87(\mathrm{~d}, J=7.1 \mathrm{~Hz}), 6.71(\mathrm{t}, J=4.7 \mathrm{~Hz}), 6.67(\mathrm{t}, J=7.1 \mathrm{~Hz}), 6.46(\mathrm{br}$ t), $5.06(\mathrm{~s}), 4.93(\mathrm{~d}, J=3.2 \mathrm{~Hz}), 4.86(\mathrm{~d}, J=7.2 \mathrm{~Hz}), 4.78(\mathrm{~d}, J=4.0 \mathrm{~Hz}), 4.72(\mathrm{~d}, J$ $=4.0 \mathrm{~Hz}), 4.66(\mathrm{~d}, J=8.0 \mathrm{~Hz}), 4.31(\mathrm{~d}, J=13.5 \mathrm{~Hz}), 4.16(\mathrm{~d}, J=8.0 \mathrm{~Hz}), 4.05(\mathrm{~s})$, $3.85(\mathrm{~d}, J=10.1 \mathrm{~Hz}), 3.80(\mathrm{~d}, J=4.0 \mathrm{~Hz}), 3.62(\mathrm{~d}, J=3.3 \mathrm{~Hz}), 3.56(\mathrm{~d}, J=8.0 \mathrm{~Hz})$, 3.53 (br t), 3.50 (s), 3.33 (d, $J=12.8 \mathrm{~Hz}$ ), 3.14 (br t), 3.06 (br t), 2.87 (t, $J=7.42$ ), 1.98 (br t); Anal. Calc. for $\mathrm{C}_{82} \mathrm{H}_{84} \mathrm{~N}_{6} \mathrm{O}_{12} \mathrm{Zn}$ : C, 69.80; H, 6.00; N, $5.96 \%$, found; $\mathrm{C}$, $69.54 ; \mathrm{H}, 5.96 ; \mathrm{N}, 5.79 \%$.

Compound (50ii): Zinc chloride; Yield $=0.08 \mathrm{~g}, 76 \% ; \mathrm{v}_{\max } / \mathrm{cm}^{-1}(\mathrm{KBr}): 1607$, 1447, 1186, 1022, 762, 697; $\delta_{\mathrm{H}} / \mathrm{ppm}$ : Conc. of $10^{-3} \mathrm{~mm}$; $\left(d_{6}\right.$-DMSO); 9.28 (d, $J=6.3$ $\mathrm{Hz}), 8.95$ (br d), 8.63 (br d), 8.54 (d, $J=6.8 \mathrm{~Hz}), 8.48$ (d, $J=6.8 \mathrm{~Hz}), 8.24$ (s), 8.14 (s), 8.02 (t, $J=7.9 \mathrm{~Hz}), 7.93(\mathrm{br} \mathrm{t}), 7.83$ (t, $J=6.8 \mathrm{~Hz}), 7.78$ (d, $J=6.8 \mathrm{~Hz}), 7.65$ (d, $J=6.8 \mathrm{~Hz}), 7.56(\mathrm{~d}, J=5.6 \mathrm{~Hz}), 7.50(\mathrm{~d}, J=6.8 \mathrm{~Hz}), 7.50(\mathrm{~d}, J=7.1 \mathrm{~Hz}), 7.24(\mathrm{br}$ 
d), $7.17(\mathrm{~d}, J=7.9 \mathrm{~Hz}), 7.12(\mathrm{~d}, J=7.9 \mathrm{~Hz}), 7.80(\mathrm{~s}), 7.01(\mathrm{~d}, J=7.1 \mathrm{~Hz}), 6.88(\mathrm{br}$ t), $6.80(\mathrm{~d}, J=7.1 \mathrm{~Hz}), 6.60(\mathrm{br} \mathrm{t}), 5.02$ (s), 4.87 (s), 4.79 (s), 4.65 (s), 4.23 (d, $J=$ $13.6 \mathrm{~Hz}), 4.17$ (d, $J=14.6 \mathrm{~Hz}), 4.06$ (d, $J=9.7 \mathrm{~Hz}), 4.01$ (br d), 3.94 (br d), 3.40 (d, $J=12.6 \mathrm{~Hz}$ ), 3.09 (br t), 3.01 (br t), 2.89 (br t), 2.01 (br t); Anal. Calc. for $\mathrm{C}_{78} \mathrm{H}_{78} \mathrm{Cl}_{2} \mathrm{~N}_{6} \mathrm{O}_{8} \mathrm{Zn}: \mathrm{C}, 68.69 ; \mathrm{H}, 5.76 ; \mathrm{N}, 6.16 \%$, found; C, 68.42; H, 5.68; N, 6.09 $\%$.

Compound (50iii): Zinc perchlorate; Yield $=0.01 \mathrm{~g}, 83 \% ; \mathrm{v}_{\max } / \mathrm{cm}^{-1}(\mathrm{KBr}): 1610$, 1449, 1383, 1090 (perchlorate anion peak), 761, 698, 626; $\delta_{\mathrm{H}} / \mathrm{ppm}$ : Conc. of $10^{-3}$ mm; ( $d_{6}$-DMSO); 9.05 (br d), 8.78 (br d), 8.67 (br d), 8.48 (br d), 8.24 (s), 8.13 (s), 8.03 (t, $J=7.5 \mathrm{~Hz}), 7.95$ (br t), 7.86 (t, $J=9.2 \mathrm{~Hz}$ ), 7.74 (br t), 7.63 (d, $J=8.4 \mathrm{~Hz}$ ), 7.56 (t, $J=8.4 \mathrm{~Hz}), 7.49$ (br t), 7.43 (s), 7.30 (s), 7.25 (s), 7.17 (d, $J=6.7 \mathrm{~Hz}), 7.12$ (d, $J=7.6 \mathrm{~Hz}), 7.05$ (s), $7.02(\mathrm{~d}, J=6.7 \mathrm{~Hz}), 6.89$ (br d), $6.81(\mathrm{~d}, J=8.4 \mathrm{~Hz}), 6.62$ (br t), 6.51 (s), 6.23 (t, $J=6.7 \mathrm{~Hz}), 5.02$ (s), 4.87 (br d), 4.75 (br d), 4.69 (br d), 4.63 (d, $J=6.6 \mathrm{~Hz}), 4.32$ (d, $J=12.2 \mathrm{~Hz}), 4.32$ (d, $J=14.2 \mathrm{~Hz}), 4.41$ (s), 4.12 (s), 4.07 $(\mathrm{d}, J=5.5 \mathrm{~Hz}), 4.02(\mathrm{~d}, J=5.5 \mathrm{~Hz}), 3.96(\mathrm{br} \mathrm{d}), 3.76(\mathrm{~s}), 3.50$ (s), 3.44 (d, $J=12.2$ $\mathrm{Hz}$ ), 3.38 (s), 3.09 (br t), 2.85 (br t), 2.04 (br t); Anal. Calc. for $\mathrm{C}_{78} \mathrm{H}_{78} \mathrm{Cl}_{2} \mathrm{~N}_{6} \mathrm{O}_{16} \mathrm{Zn}$ : C, 62.80; H, 5.27; N, $5.63 \%$, found; C, 62.51; H, 5.14; N, $5.52 \%$.

\section{Metal complexes of (50) at 1:2 ratio}

Same procedure was used as in the case of the 1:1 with the exception that the ratio of ligand to metal was $0.08 \mathrm{mmol}$ to $0.16 \mathrm{mmol}$.

Compound (50iv): Zinc acetate; Yield $=0.2 \mathrm{~g}, 78 \%$; $\mathrm{v}_{\max } / \mathrm{cm}^{-1}(\mathrm{KBr}): 1738,1610$, 1372, 1216, 1022, 912, 761; $\delta_{\mathrm{H}} / \mathrm{ppm}$ : Conc. of $10^{-3} \mathrm{~mm}$; $\left(\mathrm{CDCl}_{3}\right) ; 8.93$ (br d), 8.84 (br d), 8.65 (br d), 8.58 (br d), 7.84 (d, J= $7.1 \mathrm{~Hz}$ ), 7.79 (s), 7.73 (br t), 7.64 (br d), 7.58 (br t), 7.39 (s), 7.37 (br d), 7.31 (s), 7.17 (br t), 7.16 (s), 7.04 (d, J=4.7 Hz), $6.87(\mathrm{~d}, J=8.7 \mathrm{~Hz}), 6.81(\mathrm{br} \mathrm{t}), 6.75(\mathrm{t}, J=2.4 \mathrm{~Hz}), 6.70(\mathrm{~d}, J=3.0 \mathrm{~Hz}), 6.67(\mathrm{br} \mathrm{t})$, $5.06(\mathrm{~s}), 4.93(\mathrm{~d}, J=3.8 \mathrm{~Hz}), 4.86(\mathrm{~d}, J=4.4 \mathrm{~Hz}), 4.78(\mathrm{dd}, J=1.6,3.8 \mathrm{~Hz}), 4.72(\mathrm{~d}$, $J=5.1 \mathrm{~Hz}), 4.65$ (d, $J=8.1 \mathrm{~Hz}), 4.30$ (d, $J=12.5 \mathrm{~Hz}), 4.15(\mathrm{br} \mathrm{t}), 4.04$ (s), 3.91 (br s), $3.83(\mathrm{~d}, J=10.2 \mathrm{~Hz}), 3.78(\mathrm{~d}, J=4.7 \mathrm{~Hz}), 3.62(\mathrm{br} \mathrm{t}), 3.57(\mathrm{~d}, J=3.4 \mathrm{~Hz}), 3.52$ (br t), 3.49 (s), 3.33 (d, $J=13.6 \mathrm{~Hz}$ ), 3.09 (br t), 2.87 (br t), 1.97 (br t); Anal. Calc. for $\mathrm{C}_{86} \mathrm{H}_{90} \mathrm{~N}_{6} \mathrm{O}_{16} \mathrm{Zn}_{2}$ : C, 64.78; H, 5.69; N, $5.27 \%$, found; C, 64.63; H, 5.54; N, 5.20 $\%$. 
Compound (50v): Zinc chloride; Yield $=0.21 \mathrm{~g}, 74 \% ; \mathrm{v}_{\max } / \mathrm{cm}^{-1}(\mathrm{KBr}): 1611$, 1448, 1189, 1092, 1022, 934, 762, 699; $\delta_{\mathrm{H}} / \mathrm{ppm}$ : Conc. of $10^{-3} \mathrm{~mm} ;\left(d_{6}\right.$-DMSO); 9.28 (d, $J=5.6 \mathrm{~Hz}), 8.93$ (br d), 8.82 (br d), 8.75 (br d), 8.54 (d, $J=4.5 \mathrm{~Hz}), 8.46$ (br d), $8.23(\mathrm{~s}), 8.13(\mathrm{~s}), 8.01$ (t, $J=7.8 \mathrm{~Hz}), 7.92$ (t, $J=5.6 \mathrm{~Hz}), 7.83(\mathrm{t}, J=7.8 \mathrm{~Hz}), 7.60$ $(\mathrm{d}, J=9.0 \mathrm{~Hz}), 7.53(\mathrm{t}, J=6.7 \mathrm{~Hz}), 7.30($ br t), 7.27 (s), $7.23($ br t), 7.16 (d, $J=7.0$ Hz), $7.11(\mathrm{~d}, J=8.3 \mathrm{~Hz}), 7.06(\mathrm{~d}, J=6.6 \mathrm{~Hz}), 7.00(\mathrm{~d}, J=8.0 \mathrm{~Hz}), 6.88(\mathrm{br} \mathrm{t}), 6.80$ (br d), 6.62 (br t), 5.01 (s), 4.87 (br d), 4.74 (s), 4.64 (br d), 4.33 (d, J=9.9 Hz), 4.26 (br d), $4.14(\mathrm{~d}, J=12.6 \mathrm{~Hz}), 4.04(\mathrm{~d}, J=6.3 \mathrm{~Hz}), 3.97(\mathrm{~s}), 3.46(\mathrm{~d}, J=11.1 \mathrm{~Hz}), 3.40$ (d, $J=11.9 \mathrm{~Hz}), 3.31$ (d, $J=4.4 \mathrm{~Hz}), 3.08$ (br t), 2.94 (br t), 2.85 (br t), 2.05 (br t); Anal. Calc. for $\mathrm{C}_{78} \mathrm{H}_{78} \mathrm{Cl}_{4} \mathrm{~N}_{6} \mathrm{O}_{8} \mathrm{Zn}_{2}: \mathrm{C}, 62.45 ; \mathrm{H}, 5.24 ; \mathrm{N}, 5.60 \%$, found; $\mathrm{C}, 62.38 ; \mathrm{H}$, $5.16 ; \mathrm{N}, 5.42 \%$.

Compound (50vi): Zinc perchlorate; Yield $=0.19 \mathrm{~g}, 79 \% ; \mathrm{v}_{\max } / \mathrm{cm}^{-1}(\mathrm{KBr}): 1611$. 1448, 1375, 1146, 1089 (perchlorate anion peak), 761, 626; $\delta_{\mathrm{H}} / \mathrm{ppm}$ : Conc. of $10^{-3}$ mm; $\left(d_{6}\right.$-DMSO); 9.00 (d, $\left.J=6.0 \mathrm{~Hz}\right), 8.75$ (d, $\left.J=5.2 \mathrm{~Hz}\right), 8.65$ (br d), 8.46 (br d), $8.23(\mathrm{~s}), 8.12$ (s), 8.07 (t, $J=6.9 \mathrm{~Hz}), 7.95$ (t, $J=6.9 \mathrm{~Hz}), 7.87$ (d, $J=3.4 \mathrm{~Hz}), 7.75$ (br t), 7.64 (d, $J=6.8 \mathrm{~Hz}), 7.56$ (d, $J=5.8 \mathrm{~Hz}), 7.49$ (br d), 7.40 (t, $J=3.1 \mathrm{~Hz}), 7.33$ (t, $J=7.3 \mathrm{~Hz}), 7.30(\mathrm{~s}), 7.17(\mathrm{~d}, J=7.6 \mathrm{~Hz}), 7.12(\mathrm{~d}, J=6.6 \mathrm{~Hz}), 7.06(\mathrm{~d}, J=8.5$ Hz), $7.01(\mathrm{~d}, J=8.5 \mathrm{~Hz}), 6.88(\mathrm{br} \mathrm{d}), 6.81$ (d, $J=9.4 \mathrm{~Hz}), 6.78($ br t ), 6.63 (s), 6.61 (br t), 6.30 (br t), 5.03 (s), 4.86 (br d), 4.75 (br d), 4.66 (d, $J=5.3 \mathrm{~Hz}), 4.33$ (d, $J=$ $12.8 \mathrm{~Hz}), 4.24$ (br d), 4.13 (d, J=12.1 Hz), 4.05 (s), 4.02 (d, $J=6.0 \mathrm{~Hz}), 3.97$ (s), $3.44(\mathrm{~d}, J=13.0 \mathrm{~Hz}), 3.37$ (d, $J=13.7 \mathrm{~Hz}), 3.10$ (br t), 2.88 (br t), 2.01 (br t); Anal. Calc. for $\mathrm{C}_{78} \mathrm{H}_{78} \mathrm{Cl}_{4} \mathrm{~N}_{6} \mathrm{O}_{14} \mathrm{Zn}_{2}$ : C, 53.35; H, 4.48; N, $4.79 \%$, found; C, 53.19; H, $4.38 ; \mathrm{N}, 4.64 \%$. 


\subsection{1,23-Di[ $N, N^{\prime}$-(2'-pyridylmethyl)(2'-pyridylethyl)aminomethyl]-}

25,27-di(methyl-2-pyridine)-26,28-

diethoxycarbonylmethoxycalix[4]arene. (51)

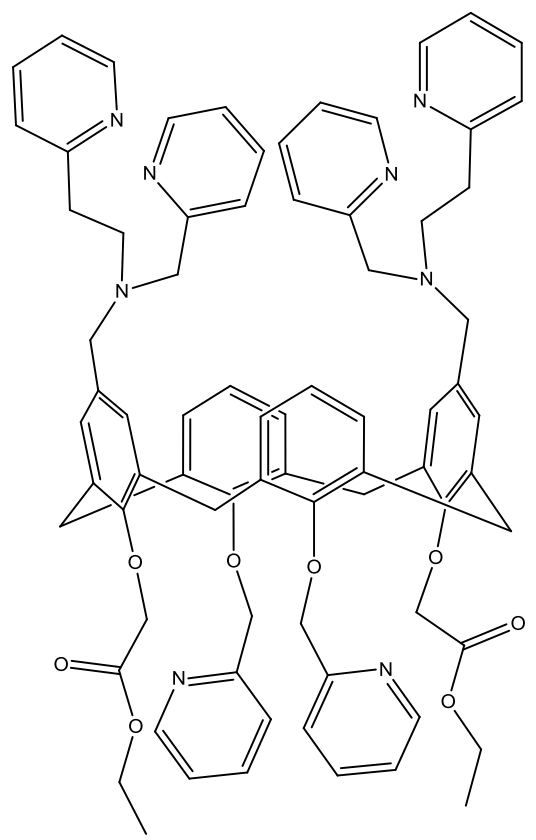

Compound (41) (1.27 g, $1.21 \mathrm{mmol})$, triethylamine $(0.24 \mathrm{~g}, 2.42 \mathrm{mmol})$, and 2aminomethylpyridine $(0.25 \mathrm{~mL}, 2.42 \mathrm{mmol})$ were refluxed under nitrogen gas in $\mathrm{CH}_{3} \mathrm{CN}(80 \mathrm{~mL}$ ) for $48 \mathrm{hr}$. The solution was allowed to cool to room temperature, the inorganic salts were removed by filtration and the volatiles removed under reduced pressure to give a brown waxy solid (51).

Yield $=0.75 \mathrm{~g}, 51 \% ; \mathrm{v}_{\max } / \mathrm{cm}^{-1}(\mathrm{KBr}): 1654,1591,1436,1190,1092,763 ; \delta_{\mathrm{H}} / \mathrm{ppm}:$ Conc. of $10^{-3} \mathrm{~mm} ;\left(\mathrm{CDCl}_{3}\right) ; 8.62(\mathrm{~d}, 4 \mathrm{H}, \mathrm{Py}-\underline{\mathrm{H}}, J=4.0 \mathrm{~Hz}), 8.53$ (br d, 4H, Py- $\left.\underline{\mathrm{H}}\right)$, 8.44 (t, 2H, Py- $\underline{\mathrm{H}}, J=4.9 \mathrm{~Hz}), 8.31$ (d, 2H, Py- $\underline{\mathrm{H}}, J=4.3 \mathrm{~Hz}), 8.23$ (t, 2H, Py- $\underline{\mathrm{H}}, J=$ 7.8 Hz), 8.07 (s, 1H), 7.85 (br t, 2H, Py- $\underline{\mathrm{H}}$ ), 7.73 (br t, 2H, Py- $\underline{\mathrm{H}}$ ), 7.64 (t, 2H, Py- $\underline{\mathrm{H}}$, $J=2.1 \mathrm{~Hz}), 7.61(\mathrm{~d}, 4 \mathrm{H}, \mathrm{Py}-\underline{\mathrm{H}}, J=1.6 \mathrm{~Hz}), 7.58$ (dd, 4H, Py- $\underline{\mathrm{H}}, J=1.7,4.6 \mathrm{~Hz})$, 7.55 (br t, 4H, Py- $\underline{\mathrm{H}}$ ), 7.54 (br t, 2H, Py- $\underline{\mathrm{H}}$ ), 7.40 (d, 2H, Py- $\underline{\mathrm{H}}, J=9.2 \mathrm{~Hz}$ ), 7.25 (s, 2H, Ar-H), 7.14 (br t, 4H, Py- $\underline{\mathrm{H}}$ ), 7.09 (d, 4H, Ar- $\underline{\mathrm{H}}, J=6.2 \mathrm{~Hz}), 6.89$ (t, 4H, Ar- $\underline{\mathrm{H}}, J$ $=6.8 \mathrm{~Hz}), 6.71(\mathrm{t}, 2 \mathrm{H}, \mathrm{Ar}-\underline{\mathrm{H}}, J=6.2 \mathrm{~Hz}), 6.66(\mathrm{~d}, 2 \mathrm{H}, \mathrm{Ar}-\underline{\mathrm{H}}, J=9.1 \mathrm{~Hz}), 5.18(\mathrm{~s}, 2 \mathrm{H}$, $\left.\mathrm{OC}_{2}-\mathrm{Py}\right), 4.73\left(\mathrm{~d}, 2 \mathrm{H}, \underline{\mathrm{C}}_{2} \mathrm{NR}_{2}, J=7.6 \mathrm{~Hz}\right), 4.59\left(\mathrm{~d}, 2 \mathrm{H}, \underline{\mathrm{C}}_{2} \mathrm{NR}_{2}, J=4.2 \mathrm{~Hz}\right)$, 
$4.35\left(\mathrm{~d}, 4 \mathrm{H}, \underline{\mathrm{C}}_{2}, J=12.7 \mathrm{~Hz}\right), 4.20\left(\mathrm{q}, 2 \mathrm{H}, \mathrm{O}-\underline{\mathrm{C}}_{2}{ }_{2} \mathrm{CH}_{3}, J=6.8 \mathrm{~Hz}\right), 4.03(\mathrm{~s}, 4 \mathrm{H}$,

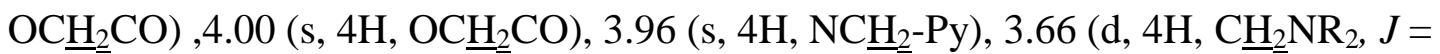
$7.4 \mathrm{~Hz}), 3.41$ (d, 4H, $\left.\underline{\mathrm{H}}_{2}, J=13.3 \mathrm{~Hz}\right), 3.25\left(\mathrm{t}, 4 \mathrm{H}, \mathrm{NCH}_{2} \underline{\mathrm{C}}_{2}-\mathrm{Py}, J=7.4 \mathrm{~Hz}\right), 3.04$ $\left(\mathrm{t}, 4 \mathrm{H}, \mathrm{NCH}_{2} \underline{\mathrm{C}}_{2}-\mathrm{Py}, J=5.9 \mathrm{~Hz}\right), 2.98\left(\mathrm{t}, 4 \mathrm{H}, \mathrm{NC}_{2} \mathrm{CH}_{2}-\mathrm{Py}, J=6.6 \mathrm{~Hz}\right), 1.31$ (br t, $\left.6 \mathrm{H}, \mathrm{OCH}_{2} \underline{\mathrm{C}}_{3}\right) ; \delta_{\mathrm{c}} / \mathrm{ppm}$ : Conc. of $10^{-3} \mathrm{~mm} ;\left(\mathrm{CDCl}_{3}\right) ; 170.2,157.9,156.9,156.4$, $155.8,154.0,148.5,148.2,137.3,136.6,134.5,134.0,128.8,128.5,125.5,123.5$, 123.2, 122.9, 121.1, 119.9, 78.7, 71.3, 61.0, 60.6, 58.6, 57.5, 38.9, 31.6, 14.2; Anal. Calc. for $\mathrm{C}_{76} \mathrm{H}_{76} \mathrm{~N}_{8} \mathrm{O}_{8}: \mathrm{C}, 74.24 ; \mathrm{H}, 6.23 ; \mathrm{N}, 9.11 \%$, found; C, 73.86; H, 5.97; N, $9.01 \%$.

\section{Metal complexes of (51) at 1:1 ratio}

All metal ion complexation reactions were performed at room temperature in a solvent mixture of $\mathrm{DCM}-\mathrm{MeOH}$ in a 1:1 ratio. All reactions used $0.08 \mathrm{mmol}$ of (51) and corresponding metal salt. The solution was stirred for $15 \mathrm{~min}$ and then the solvent was slowly evaporated off. A water-DCM wash was performed to remove any uncomplexed metal and the solvent was removed under reduced pressure.

Compound (51i): Zinc acetate: Yield $=0.09 \mathrm{~g}, 78 \% ; \mathrm{v}_{\max } / \mathrm{cm}^{-1}(\mathrm{KBr}): 1755,1658$, 1591, 1436, 1191, 1094, 761; $\delta_{\mathrm{H}} / \mathrm{ppm}$ : Conc. of $10^{-3} \mathrm{~mm}$; $\left(\mathrm{CDCl}_{3}\right) ; 8.62$ (br d), 8.54 (br d), 8.24 (d, J=8.6 Hz), 7.80 (s), 7.76 (br d), 7.62 (br d), 7.65 (t, $J=7.2 \mathrm{~Hz}$ ), 7.92 (br t), 7.21 (d, $J=8.1 \mathrm{~Hz}$ ), 7.16 (br d), 7.11 (s), 7.08 (br d), 7.03 (br t), 6.97 (s), 6.94 (s), $6.89(\mathrm{~d}, J=6.1 \mathrm{~Hz}), 6.82(\mathrm{~d}, J=10.1 \mathrm{~Hz}), 6.76(\mathrm{~d}, J=7.9 \mathrm{~Hz}), 6.71(\mathrm{~d}, J=7.5$ $\mathrm{Hz}$ ), 6.63 (br d), 6.50 (br t), 6.43 (t, $J=7.0 \mathrm{~Hz}$ ), 6.25 (br t), 5.19 (s), 4.94 (br s), 4.73 (s), 4.63 (d, $J=10.8 \mathrm{~Hz}), 4.54(\mathrm{~d}, J=7.9 \mathrm{~Hz}), 4.51$ (s), 4.40 (s), 4.37 (s), 4.33 (s), $4.28(\mathrm{~d}, J=13.0 \mathrm{~Hz}), 4.20(\mathrm{~d}, J=7.2 \mathrm{~Hz}), 4.08(\mathrm{br} \mathrm{d}), 4.01(\mathrm{~d}, J=10.1 \mathrm{~Hz}), 3.93(\mathrm{~s})$, 371 (br s), 3.42 (d, $J=13.5 \mathrm{~Hz}), 3.31(\mathrm{~d}, J=13.5 \mathrm{~Hz}), 3.23$ (d, $J=13.5 \mathrm{~Hz}), 3.14$ (br d), 3.30 (br t), 2.83 (t, $J=5.73$ ), 1.33 (br t); Anal. Calc. for $\mathrm{C}_{80} \mathrm{H}_{82} \mathrm{~N}_{8} \mathrm{O}_{12} \mathrm{Zn}: \mathrm{C}$, $68.00 ; \mathrm{H}, 5.85 ; \mathrm{N}, 7.93 \%$, found; C, 67.71; H, 5.46; N, $7.65 \%$.

Compound (51ii): Zinc chloride; Yield $=0.07 \mathrm{~g}, 63 \% ; \mathrm{v}_{\max } / \mathrm{cm}^{-1}(\mathrm{KBr}): 1743$, 1616, 1440, 1191, 1094, 1023, 763; $\delta_{\mathrm{H}} / \mathrm{ppm}$ : Conc. of $10^{-3} \mathrm{~mm} ;\left(d_{6}\right.$-DMSO); $8.71(\mathrm{br}$ d), $8.66(\mathrm{~d}, J=7.7 \mathrm{~Hz}), 8.65$ (br d), $8.43(\mathrm{~d}, J=4.7 \mathrm{~Hz}), 8.25$ (s), 7.99 (d, $J=7.1$ $\mathrm{Hz}), 7.76(\mathrm{t}, J=7.7 \mathrm{~Hz}), 7.52(\mathrm{t}, J=6.9 \mathrm{~Hz}), 7.41(\mathrm{~d}, J=8.3 \mathrm{~Hz}), 7.36(\mathrm{~d}, J=4.8$ $\mathrm{Hz}), 7.33$ (s), 7.24 (s), 7.22 (s), 7.20 (d, J = 2.4 Hz), 7.16 (s), 7.12 (d, J = $7.2 \mathrm{~Hz}$ ), $7.06(\mathrm{~d}, J=7.6 \mathrm{~Hz}), 6.99$ (s), $6.91(\mathrm{br} \mathrm{t}), 6.87(\mathrm{~d}, J=8.0 \mathrm{~Hz}), 6.67(\mathrm{t}, J=8.0 \mathrm{~Hz})$, 
6.56 (br t), 5.17 (s), 4.93 (s), 4.75 (s), 4.65 (s), 4.55 (s), 4.46 (br t), 4.33 (d, $J=12.6$ $\mathrm{Hz}), 4.28$ (s), 4.22 (d, $J=8.1 \mathrm{~Hz}), 3.11$ (t, $J=7.3 \mathrm{~Hz}), 2.95$ (t, $J=8.3 \mathrm{~Hz}), 1.30$ (br t); Anal. Calc. for $\mathrm{C}_{76} \mathrm{H}_{76} \mathrm{Cl}_{2} \mathrm{~N}_{8} \mathrm{O}_{8} \mathrm{Zn}$ : C, 66.84; H, 5.61; N, 8.20\%, found; C, 66.54; $\mathrm{H}, 5.37 ; \mathrm{N}, 8.15 \%$.

Compound (51iii): Zinc perchlorate; Yield $=0.07 \mathrm{~g}, 62 \% ; \mathrm{v}_{\max } / \mathrm{cm}^{-1}(\mathrm{KBr}): 1742$, 1618, 1464, 1146, 1088 (perchlorate anion peak), 763, 626; $\delta_{\mathrm{H}} / \mathrm{ppm}$ : Conc. of $10^{-3}$ mm; $\left(d_{6}\right.$-DMSO); 8.65 (d, $\left.J=6.0 \mathrm{~Hz}\right), 8.52$ (br d), 8.37 (br t), 8.18 (d, $J=5.0 \mathrm{~Hz}$ ), $7.91(\mathrm{~d}, J=9.0 \mathrm{~Hz}), 7.77(\mathrm{t}, J=9.0 \mathrm{~Hz}), 6.68(\mathrm{t}, J=9.0 \mathrm{~Hz}), 7.52(\mathrm{br} \mathrm{t}), 7.44(\mathrm{t}, J=$ $5.3 \mathrm{~Hz}), 7.35(\mathrm{~d}, J=6.9 \mathrm{~Hz}), 7.31(\mathrm{~d}, J=8.4 \mathrm{~Hz}), 7.15(\mathrm{~d}, J=8.4 \mathrm{~Hz}), 7.09$ (s), 7.04 (d, $J=8.4 \mathrm{~Hz}), 6.91(\mathrm{~s}), 6.81(\mathrm{br} \mathrm{d}), 6.62(\mathrm{t}, J=6.0 \mathrm{~Hz}), 5.10(\mathrm{~s}), 4.83(\mathrm{~s}), 4.67$ (br s), 4.60 (s), 4.47 (s), 4.39 (br t), 4.25 (d, $J=13.4 \mathrm{~Hz}), 4.17$ (s), 4.14 (d, $J=8.0 \mathrm{~Hz}$ ), $4.01(\mathrm{~d}, J=8.0 \mathrm{~Hz}), 3.95($ br t), $3.23($ br d), $3.05(\mathrm{t}, J=7.9 \mathrm{~Hz}), 2.94(\mathrm{t}, J=8.7 \mathrm{~Hz})$, $2.87\left(\mathrm{t}, J=7.9 \mathrm{~Hz}\right.$ ); 1.29 (br t); Anal. Calc. for $\mathrm{C}_{76} \mathrm{H}_{76} \mathrm{Cl}_{2} \mathrm{~N}_{8} \mathrm{O}_{16} \mathrm{Zn}: \mathrm{C}, 61.11 ; \mathrm{H}$, $5.13 ; \mathrm{N}, 7.50 \%$, found; C, 60.85; H, 5.02; N, 7.44\%.

\section{Metal complexes of (51) at 1:2 ratio}

Same procedure was used as in the case of the 1:1 with the exception that the ratio of ligand to metal was $0.08 \mathrm{mmol}$ to $0.16 \mathrm{mmol}$.

Compound (51iv): Zinc acetate; Yield $=0.20 \mathrm{~g}, 78 \% ; \mathrm{v}_{\max } / \mathrm{cm}^{-1}(\mathrm{KBr}): 1756$, $1729,1591.88,1436,1191,1094,1024,762 ; \delta_{\mathrm{H}} / \mathrm{ppm}$ : Conc. of $10^{-3} \mathrm{~mm} ;\left(\mathrm{CDCl}_{3}\right)$; $8.71(\mathrm{~d}, J=6.3 \mathrm{~Hz}), 8.64(\mathrm{~d}, J=4.6 \mathrm{~Hz}), 8.57$ (br d), 8.27 (d, $J=8.5 \mathrm{~Hz}), 8.19$ (d, $J$ =6. Hz), $7.87(\mathrm{br} \mathrm{t}), 7.77(\mathrm{~d}, J=12.4 \mathrm{~Hz}), 7.67(\mathrm{br} \mathrm{t}), 7.56(\mathrm{t}, J=7.0 \mathrm{~Hz}), 7.31(\mathrm{~s})$, 7.22 (s), 7.16 (s), 7.11 (s), 7.08 (s), 7.07 (d, J = 3.3 Hz), 7.04 (br d), 7.02 (br d), 6.93 (s), 6.89 (d, $J=7.7 \mathrm{~Hz}), 6.86$ (s), 6.76 (s), 6.73 (br t), 6.70 (s), 6.67 (d, J=5.5 Hz), 6.64 (s), 6.50 (br t), 6.43 (br t), 4.90 (br d), 4.84 (br d), 4.73 (s), 4.64 (br d), 4.56 (s), $4.51(\mathrm{~s}), 4.46(\mathrm{~d}, J=12.1 \mathrm{~Hz}), 4.38(\mathrm{~s}), 4.33(\mathrm{~s}), 4.28(\mathrm{~d}, J=12.1 \mathrm{~Hz}), 4.21$ (t, $J=$ $8.8 \mathrm{~Hz}), 4.13$ (s), 4.08 (br t), 3.96 (d, $J=14.3 \mathrm{~Hz}), 3.71$ (br t), 3.42 (d, $J=14.3 \mathrm{~Hz}$ ), $3.32(\mathrm{~d}, J=14.3 \mathrm{~Hz}), 3.27$ (s), 3.20 (br t), 3.07 (br t), 2.85 (t, $J=6.6 \mathrm{~Hz}), 1.32$ (br t); Anal. Calc. for $\mathrm{C}_{84} \mathrm{H}_{88} \mathrm{~N}_{8} \mathrm{O}_{16} \mathrm{Zn}_{2}$ : C, 63.26; H, 5.56; N, $7.02 \%$, found; C, 63.12; H, $5.33 ; \mathrm{N}, 6.97 \%$.

Compound (51v): Zinc chloride; Yield $=0.18 \mathrm{~g}, 74 \% ; \mathrm{v}_{\max } / \mathrm{cm}^{-1}(\mathrm{KBr}): 1743$, $1608,1567,1440,1192,1094,764,644 ; \delta_{\mathrm{H}} / \mathrm{ppm}$ : Conc. of $10^{-3} \mathrm{~mm} ;\left(d_{6}\right.$-DMSO); 
$8.70(\mathrm{~d}, J=4.3 \mathrm{~Hz}), 8.57$ (br d), 8.45 (br d), 8.25 (br t), 7.99 (t, $J=9.3 \mathrm{~Hz}), 7.77$ (br t), 7.49 (br t), 7.32 (br d), 7.22 (br d), 7.16 (s), 7.10 (br d), 7.01 (br d), 6.88 (br t), 6.66 (br t), 6.58 (br t), 5.17 (d, $J=9.0 \mathrm{~Hz}$ ), 4.92 (br d), 4.75 (br d), 4.66 (br t), 4.54 (d, $J=7.5 \mathrm{~Hz}$ ), 4.47 (br d), 4.36 (s), 4.32 (br d), 4.27 (d, $J=9.0 \mathrm{~Hz}$ ), 4.21 (br t), 4.08 (br s), 4.01 (br d), 3.74 (br d), 3.12 (br t); Anal. Calc. for $\mathrm{C}_{76} \mathrm{H}_{76} \mathrm{Cl}_{4} \mathrm{~N}_{8} \mathrm{O}_{8} \mathrm{Zn}_{2}:$ C, 60.77; H, 5.10; N, $7.46 \%$, found; C, 6.49; H, 4.87; N, $7.36 \%$.

Compound (51vi): Zinc perchlorate; Yield $=0.20 \mathrm{~g}, 71 \% ; \mathrm{v}_{\max } / \mathrm{cm}^{-1}(\mathrm{KBr}): 1747$, $1630,1567,1464,1193,1090,763,625 ; \delta_{\mathrm{H}} / \mathrm{ppm}$ : Conc. of $10^{-3} \mathrm{~mm}$; (d $d_{6}$-DMSO); $8.65(\mathrm{~d}, J=5.0 \mathrm{~Hz}), 8.52$ (br d), 8.37 (d, $J=7.0 \mathrm{~Hz}), 8.31$ (s), 8.17 (br d), 7.91 (d, $J$ $=7.6 \mathrm{~Hz}), 7.77(\mathrm{t}, J=7.0 \mathrm{~Hz}), 7.69(\mathrm{t}, J=7.6 \mathrm{~Hz}), 7.52(\mathrm{~d}, J=6.3 \mathrm{~Hz}), 7.45(\mathrm{br} \mathrm{t})$, 7.30 (br d), 7.15 (d, $J=6.3 \mathrm{~Hz}), 7.08$ (s), 7.04 (d, $J=8.2 \mathrm{~Hz}), 6.92$ (s), 6.81 (t, $J=$ $6.9 \mathrm{~Hz}), 6.60(\mathrm{t}, J=6.8 \mathrm{~Hz}), 5.09$ (s), 4.67 (s), 4.47 (s), 4.39 (br t), 4.25 (d, $J=13.6$ $\mathrm{Hz}), 4.17(\mathrm{~s}), 4.13(\mathrm{t}, J=7.2 \mathrm{~Hz}), 3.05(\mathrm{t}, J=6.4 \mathrm{~Hz}), 2.93(\mathrm{t}, J=6.9 \mathrm{~Hz}), 2.87(\mathrm{t}, J$ $=6.4 \mathrm{~Hz}$ ), 2.74 (br t), 1.31 (br t); Anal. Calc. for $\mathrm{C}_{76} \mathrm{H}_{76} \mathrm{Cl}_{4} \mathrm{~N}_{8} \mathrm{O}_{24} \mathrm{Zn}_{2}: \mathrm{C}, 51.92 ; \mathrm{H}$, $4.36 ; \mathrm{N}, 6.37 \%$, found; C, 51.73; H, 4.08; N, $6.21 \%$.

2.53 11,23-Di[N-(2'-pyridylethyl)aminomethyl]-25,27-dibenzyl-26,28di(2'-pyridylmethylmethoxy)calix[4]arene (52)

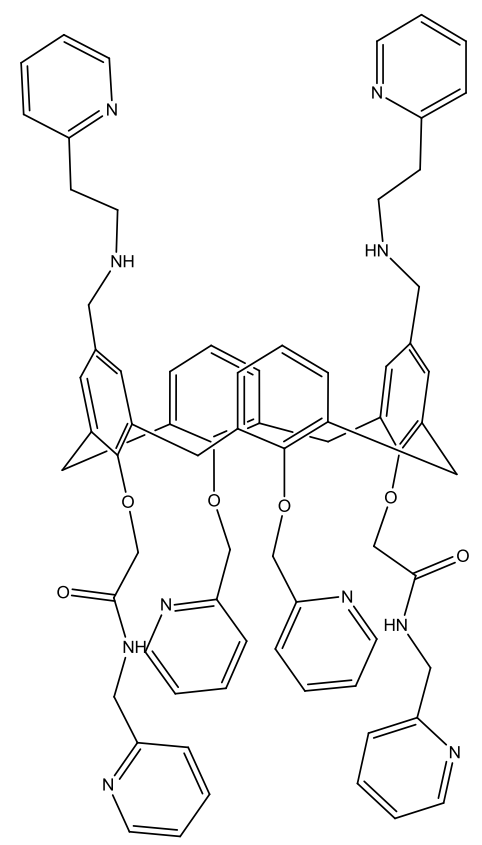


Compound (41) (0.1 g, $0.09 \mathrm{mmol})$, potassium carbonate $(0.03 \mathrm{~g}, 0.19 \mathrm{mmol})$ and 2aminomethylpyridine $(0.02 \mathrm{~mL}, 0.19 \mathrm{mmol})$ were refluxed in $\mathrm{CH}_{3} \mathrm{CN}$ :DCM (40 mL) under nitrogen gas overnight. The solution was allowed to cool to room temperature, the inorganic salts were removed by filtration and the volatiles removed under reduced pressure. The residue was then washed with DCM and water to remove excess reagents and the volatiles were once again removed under reduced pressure to give a brown waxy solid (52).

Yield $=0.82 \mathrm{~g}, 75 \% ; \mathrm{v}_{\max } / \mathrm{cm}^{-1}(\mathrm{KBr}): 2625,1711,1589,1439,1295,1153,1052$, 999, 763; $\delta_{\mathrm{H}}$ /ppm: Conc. of $10^{-3} \mathrm{~mm} ;\left(\mathrm{CDCl}_{3}\right) ; 8.62$ (br d, 2H, Py- $\left.\underline{\mathrm{H}}\right), 8.59$ (br d, 2H, Py- $\underline{\mathrm{H}}$ ), 8.54 (br d, 2H, Py- $\underline{\mathrm{H}}$ ), 7.86 (br t, 2H, Py- $\underline{\mathrm{H}}$ ), 7.79 (t, 2H, Py- $\underline{\mathrm{H}}, J=1.8 \mathrm{~Hz}$ ), 7.74 (t, 2H, Py- $\underline{\mathrm{H}}, J=1.8 \mathrm{~Hz}), 7.61$ (d, 2H, Py- $\underline{\mathrm{H}}, J=1.4 \mathrm{~Hz}), 7.59$ (d, 2H, Py- $\underline{\mathrm{H}}, J$ $=1.4 \mathrm{~Hz}$ ), 7.55 (t, 2H, Py- $\underline{\mathrm{H}}, J=1.92 \mathrm{~Hz}$ ), 7.48 (br t, 2H, Py- $\underline{\mathrm{H}}$ ), 7.34 (br t, 2H, Py-

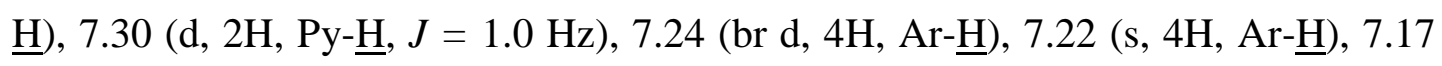
(br d, 4H, Ar- $\underline{\mathrm{H}}$ ), 7.15 (br d, 4H, Ar- $\underline{\mathrm{H}}$ ), 7.12 (s, 4H, Ar- $\underline{\mathrm{H}}$ ), 7.10 (d, 4H, Ar- $\underline{\mathrm{H}}, J=$ $5.6 \mathrm{~Hz}), 7.05$ (d, 4H, Ar- $\underline{\mathrm{H}}, J=7.5 \mathrm{~Hz}), 6.84(\mathrm{~d}, 4 \mathrm{H}, \mathrm{Ar}-\underline{\mathrm{H}}, J=7.5 \mathrm{~Hz}), 6.67$ (t, 2H, $\operatorname{Ar}-\underline{\mathrm{H}}, J=7.5 \mathrm{~Hz}), 6.61$ (t, $2 \mathrm{H}, \mathrm{Ar}-\underline{\mathrm{H}}, J=7.5 \mathrm{~Hz}), 5.05$ (s, 4H, $\left.\underline{\mathrm{H}}_{2} \mathrm{Py}\right), 4.38$ (s, 8H, $\left.\underline{\mathrm{C}}_{2} \mathrm{Py}\right), 4.30\left(\mathrm{~d}, 4 \mathrm{H}, \mathrm{C}_{2}, J=12.6 \mathrm{~Hz}\right), 3.67$ (br s, 4H, $\left.\underline{\mathrm{H}}_{2} \mathrm{CO}\right), 3.42$ (t, 4H, $\mathrm{CH}_{2} \underline{\mathrm{C}}_{2} \mathrm{Py}, J=6.9 \mathrm{~Hz}$ ), 3.34 (d, 4H, $\left.\underline{\mathrm{C}}_{2}, J=13.7 \mathrm{~Hz}\right), 3.25$ (t, $4 \mathrm{H}, \underline{\mathrm{C}}_{2} \mathrm{CH}_{2} \mathrm{Py}, J$ $=6.7 \mathrm{~Hz}) ; \delta_{\mathrm{C}} / \mathrm{ppm}\left(\mathrm{CDCl}_{3}\right) ; 168.4,161.7,156.3,155.2,154.3,154.1,148.9,148.7$, 148.5, 137.6, 137.0, 136.6, 136.5, 133.0, 130.1, 127.6, 125.2, 124.3, 124.0, 123.7, 122.8, 122.1, 121.9, 121.6, 78.4, 70.5, 68.8, 64.6, 51.1, 43.0, 31.3; Anal. Calc. for $\mathrm{C}_{72} \mathrm{H}_{70} \mathrm{~N}_{10} \mathrm{O}_{6}: \mathrm{C}, 73.82 ; \mathrm{H}, 6.02 ; \mathrm{N}, 11.96 \%$, found; C, 73.64; H, 5.81; N, $11.72 \%$.

\section{Metal complexes of (52) at 1:1 ratio}

All metal ion complexation reactions were performed at room temperature in a solvent mixture of DCM-MeOH in a 1:4 ratio. All reactions used $0.08 \mathrm{mmol}$ of (52) and corresponding metal salt. The solution was stirred for $15 \mathrm{~min}$ and then the solvent was slowly evaporated off. A water-DCM wash was performed to remove any uncomplexed metal and the solvent was removed under reduced pressure.

Compound (52i): Zinc acetate; Yield $=0.09 \mathrm{~g}, 82 \% ; \mathrm{v}_{\max } / \mathrm{cm}^{-1}(\mathrm{KBr}): 3028,1607$, 1591, 1444, 1194, 1091, 1019, 761, 699; $\delta_{\mathrm{H}} / \mathrm{ppm}$ : Conc. of $10^{-3} \mathrm{~mm} ;\left(\mathrm{CDCl}_{3}\right) ; 8.64$ (br d), 8.56 (br d), 7.84 (s), 7.73 (br t), 7.65 (s), 7.62 (d, $J=2.9 \mathrm{~Hz}), 7.54$ (d, $J=8.2$ Hz), 7.47 (d, $J=7.7 \mathrm{~Hz}), 7.41$ (s), 7.39 (s), 7.37 (d, J = $1.7 \mathrm{~Hz}), 7.35$ (s), 7.33 (s), 
7.31 (s), 7.30 (br t), 7.23 (br t), 7.22 (s), 7.17 (br d), 7.13 (d, J = 4.0 Hz), 7.10 (br d), 7.08 (s), 7.05 (d, $J=7.1 \mathrm{~Hz}), 7.02$ (s), 6.99 (br d), 6.86 (d, $J=6.9 \mathrm{~Hz}), 6.78$ (br d), $6.74(\mathrm{~s}), 6.72(\mathrm{~s}), 6.65$ (t, J = $7.2 \mathrm{~Hz}), 6.61$ (s), 6.58 (s), 6.48 (s), 6.44 (br t), 6.41 (s), 5.05 (s), 4.94 (d, J = 3.6 Hz), 4.98 (s), 4.85 (br d), 4.80 (br d), 4.79 (s), 4.77 (br d), 4.72 (d, $J=3.9 \mathrm{~Hz}), 4.69$ (s), 4.63 (br s), 4.54 (s), 4.30 (d, $J=12.7 \mathrm{~Hz}), 4.11$ (d, $J=$ $13.5 \mathrm{~Hz}), 4.04$ (d, $J=8.2 \mathrm{~Hz}), 3.98(\mathrm{br} \mathrm{t}), 3.91$ (s), 3.87 (s), 3.81 (d, $J=3.7 \mathrm{~Hz})$, $3.74(\mathrm{~d}, J=12.0 \mathrm{~Hz}), 3.68$ (s), 3.41 (d, $J=7.5 \mathrm{~Hz}), 3.32$ (d, $J=12.0 \mathrm{~Hz}), 3.19$ (d, $J$ $=6.0 \mathrm{~Hz}$ ), $3.13(\mathrm{~d}, J=6.1 \mathrm{~Hz}), 2.95$ (br t); Anal. Calc. for $\mathrm{C}_{76} \mathrm{H}_{76} \mathrm{~N}_{10} \mathrm{O}_{10} \mathrm{Zn}: \mathrm{C}$, 67.37; H, 5.65; N, $10.34 \%$, found; C, 67.04; H, 5.47; N, $10.22 \%$.

Compound (52ii): Zinc chloride; Yield $=0.08 \mathrm{~g}, 72 \% ; \mathrm{v}_{\max } / \mathrm{cm}^{-1}(\mathrm{KBr}): 3030$, $1709,1607,1571,1445,1193,1091,1022,762,699 ; \delta_{\mathrm{H}} / \mathrm{ppm}$ : Conc. of $10^{-3} \mathrm{~mm} ;\left(d_{6^{-}}\right.$ DMSO); 8.63 (br d), 8.53 (br d), 8.24 (br d), 8.14 (s), 7.88 (t, $J=7.5 \mathrm{~Hz}$ ), 7.79 (br d), 7.66 (br d), 7.49 (br d), 7.41 (d, $J=6.8 \mathrm{~Hz}), 7.34$ (br d), 7.14 (d, $J=6.8 \mathrm{~Hz}$ ), 7.09 (s), 7.03 (d, $J=7.3 \mathrm{~Hz}), 6.90$ (s), 6.79 (t, $J=7.3 \mathrm{~Hz}), 6.60$ (br t), 5.15 (br d), 5.04 (s), 4.89 (s), 4.76 (s), 4.68 (br d), 4.41 (d, J=8.3 Hz), 4.19 (br d), 4.13 (s), 4.00 (s), 3.93 (d, $J=11.9 \mathrm{~Hz}), 3.88$ (s), 3.81 (d, $J=11.9 \mathrm{~Hz}), 3.15$ (br t), 3.06 (br t), 2.96 (br t); Anal. Calc. for $\mathrm{C}_{72} \mathrm{H}_{70} \mathrm{Cl}_{2} \mathrm{~N}_{10} \mathrm{O}_{6} \mathrm{Zn}$ : C, 66.13; H, 5.40; N, $10.71 \%$, found; $\mathrm{C}$, 65.89; H, 5.23; N, $10.69 \%$.

Compound (52iii): Zinc perchlorate; Yield $=0.09 \mathrm{~g}, 73 \% ; \mathrm{v}_{\max } / \mathrm{cm}^{-1}(\mathrm{KBr}): 2923$, 1718, 1609, 1571, 1448, 1093 (perchlorate anion peak), 918, 761, 622; $\delta_{\mathrm{H}} / \mathrm{ppm}$ : Conc. of $10^{-3} \mathrm{~mm}$; $\left(d_{6}\right.$-DMSO); 8.69 (br d), 8.61 (d, $\left.J=5.3 \mathrm{~Hz}\right), 8.52$ (br d), 8.24 (br t), $8.17(\mathrm{~s}), 8.14(\mathrm{~s}), 7.88(\mathrm{t}, J=7.7 \mathrm{~Hz}), 7.79(\mathrm{br} \mathrm{t}), 7.66(\mathrm{~d}, J=6.5 \mathrm{~Hz}), 7.47(\mathrm{~d}, J=$ $8.4 \mathrm{~Hz}), 7.39$ (d, $J=7.0 \mathrm{~Hz}), 7.34$ (d, $J=4.3 \mathrm{~Hz}), 7.2(\mathrm{br} \mathrm{d}), 7.17(\mathrm{~d}, J=8.1 \mathrm{~Hz})$, $7.12(\mathrm{~d}, J=7.5 \mathrm{~Hz}), 7.08(\mathrm{~s}), 7.02(\mathrm{~d}, J=7.5 \mathrm{~Hz}), 6.91(\mathrm{~s}), 6.79(\mathrm{t}, J=7.0 \mathrm{~Hz}), 6.60$ (t, $J=8.1 \mathrm{~Hz}$ ), 5.04 (s), 4.97 (s), 4.88 (s), 4.77 (br d), 4.71 (s), 4.67 (br d), 4.43 (d, $J$ $=5.5 \mathrm{~Hz}), 4.21(\mathrm{br} \mathrm{d}), 4.14(\mathrm{br} \mathrm{d}), 4.00(\mathrm{~s}), 3.95(\mathrm{br} \mathrm{t}), 3.88$ (t, $J=5.9 \mathrm{~Hz}), 3.81$ (d, $J$ $=3.6 \mathrm{~Hz}), 3.76(\mathrm{~s}), 3.13(\mathrm{br} \mathrm{t}), 3.05(\mathrm{t}, J=8.8 \mathrm{~Hz})$; Anal. Calc. for $\mathrm{C}_{72} \mathrm{H}_{70} \mathrm{Cl}_{2} \mathrm{~N}_{10} \mathrm{O}_{14} \mathrm{Zn}: \mathrm{C}, 60.23 ; \mathrm{H}, 4.91 ; \mathrm{N}, 9.76 \%$, found; C, 59.84; H, 4.78; N, 9.65 $\%$. 


\section{Metal complexes of (52) at 1:2 ratio}

Same procedure was used as in the case of the 1:1 with the exception that the ratio of ligand to metal was $0.08 \mathrm{mmol}$ to $0.17 \mathrm{mmol}$.

Compound (52iv): Zinc acetate; Yield $=0.02 \mathrm{~g}, 77 \% ; \mathrm{v}_{\max } / \mathrm{cm}^{-1}(\mathrm{KBr}): 3028$, $1713,1605,1589,1445,1194,1091,1021,762,691 ; \delta_{\mathrm{H}} / \mathrm{ppm}$ : Conc. of $10^{-3} \mathrm{~mm}$; $\left(\mathrm{CDCl}_{3}\right) ; 8.69$ (br d), $8.58(\mathrm{~d}, J=5.1 \mathrm{~Hz}), 8.27$ (s), 7.84 (s), $7.77(\mathrm{~d}, J=8.3 \mathrm{~Hz})$, 7.72 (br d), 7.63 (br t), 7.37 (br d), 7.35 (s), 7.33 (s), 7.23 (s), 7.18 (d, $J=7.8 \mathrm{~Hz}$ ), $7.14(\mathrm{~d}, J=3.9 \mathrm{~Hz}), 7.05(\mathrm{~d}, J=7.4 \mathrm{~Hz}), 6.84(\mathrm{~d}, J=7.8 \mathrm{~Hz}), 6.66(\mathrm{t}, J=7.8 \mathrm{~Hz})$, $5.05(\mathrm{~s}), 4.94(\mathrm{~d}, J=3.8 \mathrm{~Hz}), 4.87(\mathrm{~d}, J=8.6 \mathrm{~Hz}), 4.80(\mathrm{br} \mathrm{d}), 4.77$ (s), 4.73 (d, $J=$ $4.5 \mathrm{~Hz}), 4.68$ (d, $J=3.4 \mathrm{~Hz}), 4.62$ (s), 4.31 (d, $J=13.1 \mathrm{~Hz}), 4.07$ (br t), 4.00 (d, $J=$ $13.9 \mathrm{~Hz}), 3.89$ (d, $J=14.6 \mathrm{~Hz}$ ), 3.77 (s), 3.73 (s), 3.68 (br t), 3.60 (br t), 3.40 (d, $J=$ $8.0 \mathrm{~Hz}$ ), 3.33 (d, $J=13.9 \mathrm{~Hz}), 3.17$ (s), 3.12 (br t), 3.06 (br t), 2.95 (br t); Anal. Calc. for $\mathrm{C}_{80} \mathrm{H}_{82} \mathrm{~N}_{10} \mathrm{O}_{14} \mathrm{Zn}_{2}: \mathrm{C}, 62.46 ; \mathrm{H}, 5.37 ; \mathrm{N}, 9.11 \%$, found; $\mathrm{C}, 62.28 ; \mathrm{H}, 5.21 ; \mathrm{N}$, $9.07 \%$.

Compound (52v): Zinc chloride; Yield $=0.01 \mathrm{~g}, 73 \% ; \mathrm{v}_{\max } / \mathrm{cm}^{-1}(\mathrm{KBr}): 3029$, $1707,1607,1571,1446,1193,1091,1022,762,699 ; \delta_{\mathrm{H}} / \mathrm{ppm}\left(d_{6}\right.$-DMSO); $8.63(\mathrm{~d}, J$ $=5.8 \mathrm{~Hz}), 8.52($ br d), $8.24($ br t), $8.17(\mathrm{~s}), 8.13(\mathrm{~s}), 8.02(\mathrm{t}, J=8.1 \mathrm{~Hz}), 7.87(\mathrm{t}, J=$ 7. Hz), 7.77 (br t), 7.65 (d, $J=7.0 \mathrm{~Hz}), 7.53$ (s), 7.47 (d, $J=7.4 \mathrm{~Hz}), 7.40$ (d, $J=$ $7.4 \mathrm{~Hz}), 7.36$ (d, $J=4.3 \mathrm{~Hz}), 7.31$ (br t), 7.19 (d, $J=6.8 \mathrm{~Hz}), 7.13$ (d, $J=7.4 \mathrm{~Hz})$, 7.08 (s), 7.02 (d, $J=7.4 \mathrm{~Hz}), 6.91(\mathrm{~s}), 6.79$ (t, $J=7.4 \mathrm{~Hz}), 6.59(\mathrm{t}, J=6.8 \mathrm{~Hz}), 5.04$ (s), 4.88 (s), 4.76 (d, $J=5.8 \mathrm{~Hz}), 4.70$ (d, $J=4.9 \mathrm{~Hz}), 4.67$ (br d), 4.63 (d, $J=5.8$ Hz), 4.49 (s), $4.41(\mathrm{~d}, J=7.4 \mathrm{~Hz}), 4.38(\mathrm{~d}, J=4.1 \mathrm{~Hz}), 4.32(\mathrm{~d}, J=11.4 \mathrm{~Hz}), 4.21$ (br d), $4.16(\mathrm{~d}, J=12.0 \mathrm{~Hz}), 4.06(\mathrm{~d}, J=5.0 \mathrm{~Hz}), 4.00$ (s), 3.94 (br d), 3.87 (s), 3.81 (t, $J=6.8 \mathrm{~Hz}), 3.76(\mathrm{~s}), 3.72(\mathrm{~d}, J=6.4 \mathrm{~Hz}), 3.66(\mathrm{br} \mathrm{d}), 3.15(\mathrm{br} \mathrm{t}), 3.06$ (t, $J=7.2$ $\mathrm{Hz}), 2.94(\mathrm{t}, J=6.9 \mathrm{~Hz})$; Anal. Calc. for $\mathrm{C}_{72} \mathrm{H}_{70} \mathrm{Cl}_{4} \mathrm{~N}_{10} \mathrm{O}_{6} \mathrm{Zn}_{2}$ : C, 59.89; H, 4.89; N, $9.70 \%$, found; C, 59.62; H, 4.65; N, $9.59 \%$.

Compound (52vi): Zinc perchlorate; Yield $=0.02 \mathrm{~g}, 72 \% ; \mathrm{v}_{\max } / \mathrm{cm}^{-1}(\mathrm{KBr}): 2925$, 1622, 1611, 1571, 1448, 1248, 1091 (perchlorate anion peak), 762, 626; $\delta_{\mathrm{H}} / \mathrm{ppm}$ : Conc. of $10^{-3} \mathrm{~mm}$; $\left(d_{6}\right.$-DMSO); 8.62 (d, $J=6.3 \mathrm{~Hz}$ ), 8.52 (br d), 8.22 (br t), 8.16 (s), 8.13 (s), 7.86 (d, $J=8.9 \mathrm{~Hz}), 7.80$ (br t), 7.65 (d, $J=10.1 \mathrm{~Hz}), 7.46$ (d, $J=8.9 \mathrm{~Hz})$, 7.42 (s), 7.39 (d, $J=7.5 \mathrm{~Hz}), 7.34$ (d, $J=4.2 \mathrm{~Hz}), 7.24$ (s), 7.13 (d, $J=6.1 \mathrm{~Hz})$, 7.08 (s), 7.01 (d, $J=7.5 \mathrm{~Hz}), 6.91(\mathrm{~s}), 6.78(\mathrm{t}, J=6.9 \mathrm{~Hz}), 6.58(\mathrm{t}, J=7.5 \mathrm{~Hz}), 5.12$ 
(d, $J=4.3 \mathrm{~Hz}), 5.04$ (s), 4.87 (s), 4.75 (br d), $4.70(\mathrm{~d}, J=7.1 \mathrm{~Hz}), 4.66$ (d, $J=7.1$ $\mathrm{Hz}), 4.62(\mathrm{~d}, J=7.8 \mathrm{~Hz}), 4.43(\mathrm{~d}, J=6.4 \mathrm{~Hz}), 4.37(\mathrm{br} \mathrm{d}), 4.14(\mathrm{~d}, J=13.6 \mathrm{~Hz})$, 4.01 (s), 3.94 (s), 3.87 (d, $J=10.0 \mathrm{~Hz}), 3.79$ (br d), 3.14 (t, $J=7.8 \mathrm{~Hz}), 3.03$ (t, $J=$ 7.8 Hz); Anal. Calc. for $\mathrm{C}_{72} \mathrm{H}_{70} \mathrm{Cl}_{4} \mathrm{~N}_{10} \mathrm{O}_{22} \mathrm{Zn}_{2}$ : C, 50.87; H, 4.15; N, $8.24 \%$, found; C, $50.81 ; \mathrm{H}, 4.08 ; \mathrm{N}, 8.19 \%$.

\subsection{1,23-Di[ $N, N^{\prime}$-(2'-pyridylmethyl)(2'-pyridylehtyl)aminomethyl]-} 25,27-dibenzyl-26,28-di(2'pyridylmethyl)methoxycalix[4]arene. (53)

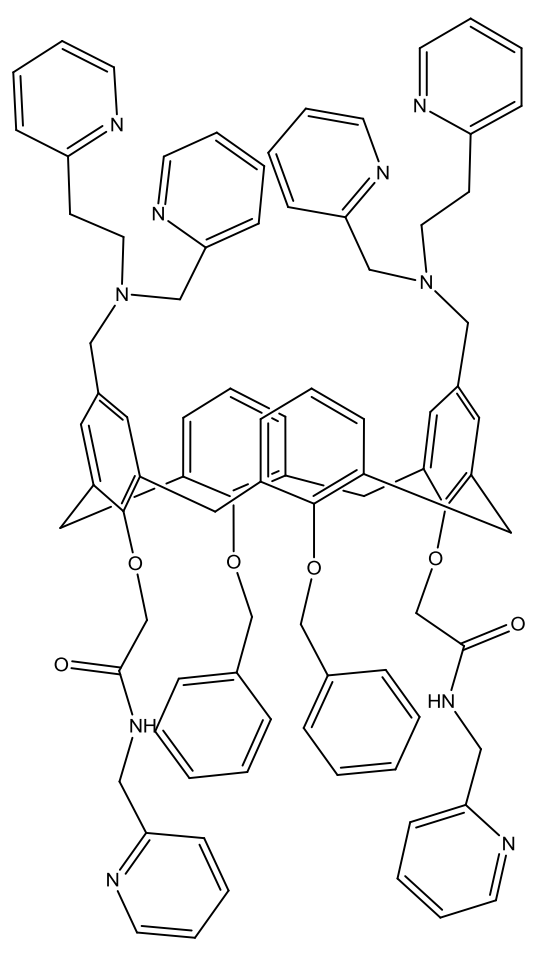

Compound (50) (0.51 g, $0.42 \mathrm{mmol})$, 2-aminomethylpyridine (0.08 mL, $0.83 \mathrm{mmol})$ were refluxed in $\mathrm{MeOH}$ : toluene mixture (10:20 mL) under nitrogen gas for 7 days. The solution was allowed to cool to room temperature and the volatiles were removed under reduced pressure to afford a dark brown-red waxy solid (53).

Yield $=0.26 \mathrm{~g}, 46 \% ; \mathrm{v}_{\max } / \mathrm{cm}^{-1}(\mathrm{KBr}): 3410,1631,1589,1457,1436,1192,1092$, 995, 757; $\delta_{\mathrm{H}} / \mathrm{ppm}$ : Conc. of $10^{-3} \mathrm{~mm} ;\left(\mathrm{CDCl}_{3}\right) ; 8.57$ (br d, 2H, Py- $\underline{\mathrm{H}}$ ), 8.51 (br d, 4H, Py- $\underline{\mathrm{H}}), 8.40$ (t, 2H, Py- $\underline{\mathrm{H}}, J=4.71 \mathrm{~Hz}), 7.83$ (t, 2H, Py- $\underline{\mathrm{H}}, J=8.6 \mathrm{~Hz}$ ), 7.64 (br t, 4H, 
Py- $\underline{\mathrm{H}}$ ), 7.62 (br d, 4H, Py- $\underline{\mathrm{H}}$ ), 7.60 (br d, 4H, Py- $\underline{\mathrm{H}}$ ), 7.50 (br d, 2H, Py- $\underline{\mathrm{H}}$ ), 7.47 (br d, 2H, Py- $\underline{\mathrm{H}}$ ), 7.37 (d, 4H, Bn- $\underline{\mathrm{H}}, J=2.1 \mathrm{~Hz}$ ), 7.35 (br t, 6H, Bn- $\underline{\mathrm{H}}$ ), 7.33 (br d, 4H, Py- $\underline{\mathrm{H}}$ ), 7.30 (br d, 4H, Py- $\underline{\mathrm{H}}$ ), 7.24 (br d, 4H, Py- $\underline{\mathrm{H}}$ ), 7.23 (br t, 4H, Py- $\underline{\mathrm{H}}$ ), 7.05 (d, $4 \mathrm{H}, \operatorname{Ar}-\underline{\mathrm{H}}, J=7.5 \mathrm{~Hz}), 6.85$ (d, 2H, Ar- $\underline{\mathrm{H}}, J=7.0 \mathrm{~Hz}), 6.76(\mathrm{t}, 2 \mathrm{H}, \operatorname{Ar}-\underline{\mathrm{H}}, J=8.0$ $\mathrm{Hz}), 6.63(\mathrm{t}, 2 \mathrm{H}, \mathrm{Ar}-\underline{\mathrm{H}}, J=8.5 \mathrm{~Hz}), 6.46(\mathrm{t}, 2 \mathrm{H}, \operatorname{Ar}-\underline{\mathrm{H}}, J=7.0 \mathrm{~Hz}), 5.05$ (s, 4H, $\left.\mathrm{OC}_{2} \underline{2} \mathrm{Bn}\right), 4.81\left(\mathrm{~s}, 4 \mathrm{H}, \underline{\mathrm{C}}_{2} \mathrm{NR}_{2}\right), 4.73$ (d, 4H, NHC$\left.\underline{H}_{2} \mathrm{Py}, J=7.7 \mathrm{~Hz}\right), 4.31(\mathrm{~d}, 4 \mathrm{H}$,

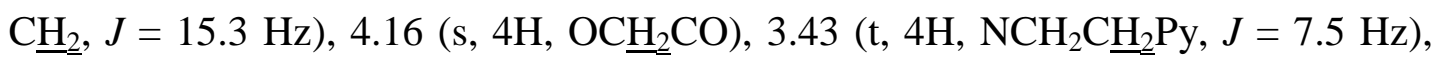

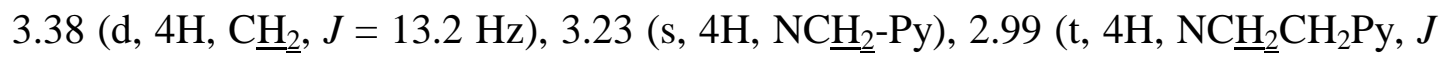
$=5.0 \mathrm{~Hz}) ; \delta_{\mathrm{C}} / \mathrm{ppm}$ : Conc. of $10^{-3} \mathrm{~mm} ;\left(\mathrm{CDCl}_{3}\right) ; 166.9$, 158.4, 156.6, 154.1, 152.9, $152.2,148.9,148.2,140.4,137.1,136.7,133.7,133.0,129.0,128.8,128.3$, 127.7, $127.1,124.4,124.1,123.8,122.9,122.1,119.1,71.8,69.4,65.1,64.5,58.9,49.9$, 39.4, 31.6; Anal. Calc. for $\mathrm{C}_{86} \mathrm{H}_{82} \mathrm{~N}_{10} \mathrm{O}_{6}$ : C, 76.42; H, 6.11; N, $10.36 \%$, found; C, 76.23; H, 6.06; N, $10.27 \%$.

\section{Metal complexes of (53) at 1:1 ratio}

All metal ion complexation reactions were performed at room temperature in a solvent mixture of DCM-MeOH in a 1:1 ratio. All reactions used $0.07 \mathrm{mmol}$ of (53) and corresponding metal salt. The solution was stirred for $15 \mathrm{~min}$ and then the solvent was slowly evaporated off. A water-DCM wash was performed to remove any uncomplexed metal and the solvent was removed under reduced pressure.

Compound (53i): Zinc acetate; Yield $=0.08 \mathrm{~g}, 70 \% ; \mathrm{v}_{\max } / \mathrm{cm}^{-1}(\mathrm{KBr}): 3435,1676$, 1590, 1568, 1443, 1193, 1092, 1020, 762; $\delta_{\mathrm{H}} / \mathrm{ppm}$ : Conc. of $10^{-3} \mathrm{~mm} ;\left(\mathrm{CDCl}_{3}\right) ; 8.69$ (d, $J=4.5 \mathrm{~Hz}), 8.58$ (d, $J=4.5 \mathrm{~Hz}), 8.49$ (br d), 8.35 (br t), 8.31 (s), 8.20 (d, $J=7.7$ $\mathrm{Hz}), 8.15$ (s), 7.89 (d, $J=1.9 \mathrm{~Hz}), 7.86$ (d, $J=1 . \mathrm{Hz}), 7.83$ (d, $J=1.7 \mathrm{~Hz}), 7.71(\mathrm{~d}, J$ $=1.9 \mathrm{~Hz}), 7.69(\mathrm{~d}, J=1.9 \mathrm{~Hz}), 7.65(\mathrm{~d}, J=1.7 \mathrm{~Hz}), 7.62(\mathrm{~d}, J=1.0 \mathrm{~Hz}), 7.56(\mathrm{br} \mathrm{t})$, $7.47(\mathrm{~d}, J=1.4 \mathrm{~Hz}), 7.45(\mathrm{dd}, J=7.2,0.8 \mathrm{~Hz}), 7.43(\mathrm{~d}, J=0.8 \mathrm{~Hz}), 7.38(\mathrm{~d}, J=5.3$ Hz), 7.36 (br t), 7.32 (s), 7.24 (s), 7.20 (d, J=8.0 Hz), 7.16 (s), 7.12 (d, $J=4.9 \mathrm{~Hz}$ ), 7.08 (d, $J=6.7 \mathrm{~Hz}), 7.05$ (br t), 6.97 (d, $7.2 \mathrm{~Hz}), 6.86$ (d, $J=7.8 \mathrm{~Hz}), 6.77$ (d, $J=6.9$ Hz), 6.68 (br t), 6.48 (t, $J=7.5 \mathrm{~Hz}$ ), 6.48 (br t), 5.06 (s), 4.94 (s), 4.87 (d, $J=4.3$ Hz), 4.82 (br d), 4.78 (d, $J=8.7 \mathrm{~Hz}), 4.73$ (d, $J=3.7 \mathrm{~Hz}), 4.64$ (d, $J=5.0 \mathrm{~Hz}), 4.55$ (br t), 4.46 (s), 4.38 (s), $4.31(\mathrm{~d}, J=12.5 \mathrm{~Hz}), 4.05$ (s), 3.72 (t, $J=6.6 \mathrm{~Hz}), 3.66$ (d, $J$ $=6.6 \mathrm{~Hz}), 3.62(\mathrm{br} \mathrm{d}), 3.59(\mathrm{~d}, J=4.5 \mathrm{~Hz}), 3.53(\mathrm{br} \mathrm{t}), 3.39(\mathrm{~s}), 3.33(\mathrm{~d}, J=13.2 \mathrm{~Hz})$, 
3.25 (br t), 3.13 (br t), 3.05 (t, $J=6.0 \mathrm{~Hz}$ ); Anal. Calc. for $\mathrm{C}_{90} \mathrm{H}_{88} \mathrm{~N}_{10} \mathrm{O}_{10} \mathrm{Zn}: \mathrm{C}$, 70.42; H, 5.78; N, 9.12\%, found; C, 70.08; H, 5.58; N, $9.03 \%$.

Compound (53ii): Zinc chloride; Yield $=0.08 \mathrm{~g}, 68 \% ; \mathrm{v}_{\max } / \mathrm{cm}^{-1}(\mathrm{KBr}): 3428$, 1651, 1605, 1571, 1440, 1193, 1092, 1021, 761, 699; $\delta_{\mathrm{H}} / \mathrm{ppm}$ : Conc. of $10^{-3} \mathrm{~mm} ;\left(d_{6^{-}}\right.$ DMSO); 8.69 (d, $J=4.8 \mathrm{~Hz}), 8.62(\mathrm{~d}, J=5.3 \mathrm{~Hz}), 8.54$ (br d), 8.28 (br t), 8.15 (d, $J$ $=10.7 \mathrm{~Hz}), 8.05($ br d), $8.02($ br t), 8.00 (d, $J=1.9 \mathrm{~Hz}), 7.97$ (br t), 7.95 (t, $J=1.9$ $\mathrm{Hz}), 7.92(\mathrm{~d}, J=1.7 \mathrm{~Hz}), 7.90(\mathrm{~d}, J=1.7 \mathrm{~Hz}), 7.87$ (br d), 7.85 (d, $J=1.3 \mathrm{~Hz}), 7.82$ (t, $J=1.9 \mathrm{~Hz}), 7.80(\mathrm{t}, J=2.1 \mathrm{~Hz}), 7.78(\mathrm{~d}, J=1.7 \mathrm{~Hz}), 7.77($ br t), 7.74 (br t), 7.71 (br t), 7.64 (br t), 7.60 (br d), 7.58 (br t), 7.56 (br d), 7.53 (br s), 7.48 (d, J=7.9 Hz), 7.43 (s), 7.41 (d, $J=2.2 \mathrm{~Hz}), 7.39$ (d, $J=3.0 \mathrm{~Hz}), 7.36$ (s), 7.34 (br t), 7.23 (d, $J=$ $5.4 \mathrm{~Hz}), 7.18$ (s), 7.13 (t, $J=3.5 \mathrm{~Hz}), 7.10$ (d, $J=3.5 \mathrm{~Hz}), 7.05$ (s), 7.03 (br d), 7.01 $(\mathrm{s}), 6.97(\mathrm{~d}, J=6.0 \mathrm{~Hz}), 6.16(\mathrm{t}, J=7.1 \mathrm{~Hz}), 6.38(\mathrm{t}, J=7.1 \mathrm{~Hz}), 6.29(\mathrm{t}, J=7.8 \mathrm{~Hz})$, 5.05 (s), 4.89 (s), 4.80 (br d), 4.77 (d, $J=5.6 \mathrm{~Hz}), 4.66$ (s), 4.64 (d, $J=5.6 \mathrm{~Hz}), 4.41$ (d, $J=7.7 \mathrm{~Hz}), 4.36(\mathrm{~s}), 4.31(\mathrm{~s}), 4.20(\mathrm{~s}), 4.01$ (s), 3.80 (s), 3.76 (s), 3.24 (br t), 3.06 (t, $J=7.1 \mathrm{~Hz}$ ), 2.94 (t, $J=7.1 \mathrm{~Hz}$ ), 2.23 (br t); Anal. Calc. for $\mathrm{C}_{86} \mathrm{H}_{82} \mathrm{Cl}_{2} \mathrm{~N}_{10} \mathrm{O}_{6} \mathrm{Zn}: \mathrm{C}$, 69.42; H, 5.55; N, $9.41 \%$, found; C, 69.24; H, 5.34; N, $9.20 \%$.

Compound (53iii): Zinc perchlorate; Yield $=0.08 \mathrm{~g}, 67 \% ; \mathrm{v}_{\max } / \mathrm{cm}^{-1}(\mathrm{KBr}): 3342$, 1636, 1609, 1573, 1445, 1088 (perchlorate anion peak), 762, 622; $\delta_{\mathrm{H}} / \mathrm{ppm}$ : Conc. of $10^{-3} \mathrm{~mm}$; $\left(d_{6}\right.$-DMSO); $8.67(\mathrm{~d}, J=5.3 \mathrm{~Hz}), 8.63(\mathrm{~d}, J=4.1 \mathrm{~Hz}), 8.53(\mathrm{br} \mathrm{d}), 8.16(\mathrm{~s})$, 8.07 (br t), 8.02 (br t), 7.97 (br d), 7.90 (d, $J=2.0 \mathrm{~Hz}$ ), 7.87 (d, $J=2.0 \mathrm{~Hz}), 7.85$ (d, $J=2.0 \mathrm{~Hz}$ ), 7.80 (br t), $7.77(\mathrm{~d}, J=1.7 \mathrm{~Hz}), 7.71($ br t $), 7.64$ (br t), 7.52 (d, $J=7.2$ Hz), 7.48 (s), 7.45 (s), 7.43 (br d), 7.42 (d, $J=2.0 \mathrm{~Hz}$ ), 7.39 (br d), 7.36 (br d), 7.35 (br d), 7.32 (br d), 7.20 (br t), 7.13 (d, $J=7.3 \mathrm{~Hz}$ ), 7.09 (s), 7.00 (d, J= 7.6 Hz), 6.92 (s), 6.60 (br t), 5.05 (s), 4.98 (d, $J=7.0 \mathrm{~Hz}), 4.92$ (d, $J=6.4 \mathrm{~Hz}), 4.89$ (s), 4.77 (d, $J$ $=5.7 \mathrm{~Hz}), 4.72(\mathrm{~d}, J=6.4 \mathrm{~Hz}), 4.67(\mathrm{~d}, J=5.1 \mathrm{~Hz}), 4.42(\mathrm{~d}, J=7.0 \mathrm{~Hz}), 4.38(\mathrm{~d}, J=$ $5.7 \mathrm{~Hz}), 4.00(\mathrm{~s}), 3.80(\mathrm{~s}), 3.05(\mathrm{t}, J=7.0 \mathrm{~Hz}), 2.98(\mathrm{t}, J=7.5 \mathrm{~Hz})$; Anal. Calc. for $\mathrm{C}_{86} \mathrm{H}_{82} \mathrm{Cl}_{2} \mathrm{~N}_{10} \mathrm{O}_{14} \mathrm{Zn}: \mathrm{C}, 63.92 ; \mathrm{H}, 5.11 ; \mathrm{N}, 8.67 \%$, found; C, 63.54; H, 4.97; N, $8.51 \%$.

\section{Metal complexes of (53) at 1:2 ratio}

Same procedure was used as in the case of the 1:1 with the exception that the ratio of ligand to metal was $0.07 \mathrm{mmol}$ to $0.14 \mathrm{mmol}$. 
Compound (53iv): Zinc acetate; Yield $=0.02 \mathrm{~g}, 83 \% ; \mathrm{v}_{\max } / \mathrm{cm}^{-1}(\mathrm{KBr}): 3427$, 1590, 1563, 1438, 1193, 1092, 1018, 758, 700; $\delta_{\mathrm{H}} / \mathrm{ppm}$ : Conc. of $10^{-3} \mathrm{~mm} ;\left(\mathrm{CDCl}_{3}\right)$; 8.86 (br d), 8.68 (d, $J=4.3 \mathrm{~Hz}$ ), 8.62 (br d), 8.49 (br t), 8.26 (br s), 8.11 (s), 8.00 (br t), 7.89 (br t), 7.87 (br d), 7.84 (br d), 7.76 (br d, 7.70 (br t), 7.69 (br d), 7.61 (br t), 7.49 (br t), 7.42 (s), 7.40 (d, J=6.0 Hz), 7.37 (s), 7.35 (s), 7.32 (s), 7.24 (s), 7.20 (br t), $7.11(\mathrm{~s}), 7.09$ (d, $J=3.8 \mathrm{~Hz}), 7.05$ (br t), 6.98 (d, $J=7.1 \mathrm{~Hz}), 6.83(\mathrm{~d}, J=7.6 \mathrm{~Hz})$, 6.77 (br t), 6.69 (d, $J=7.1 \mathrm{~Hz}), 6.62(\mathrm{br} \mathrm{t}), 5.06(\mathrm{~s}), 4.94$ (s), 4.87 (d, $J=4.1 \mathrm{~Hz})$, $4.81(\mathrm{~s}), 4.76$ (d, $J=8.8 \mathrm{~Hz}), 4.73(\mathrm{~d}, J=5.3 \mathrm{~Hz}), 4.64$ (s), 4.34 (d, $J=2.3 \mathrm{~Hz}), 4.29$ (s), 4.25 (s), 4.12 (d, $J=13.6 \mathrm{~Hz}), 4.05$ (s), 3.96 (s), 3.69 (d, $J=6.5 \mathrm{~Hz}), 3.66$ (d, $J=$ $4.0 \mathrm{~Hz}), 3.63(\mathrm{~d}, J=2.1 \mathrm{~Hz}), 3.58(\mathrm{~d}, J=3.4 \mathrm{~Hz}), 3.55(\mathrm{~s}), 3.52(\mathrm{~d}, J=3.4 \mathrm{~Hz}), 3.39$ (s), 335 (s), 3.28 (br d), $3.14(\mathrm{t}, J=5.8 \mathrm{~Hz}), 3.08$ (t, $J=6.1 \mathrm{~Hz})$; Anal. Calc. for $\mathrm{C}_{90} \mathrm{H}_{88} \mathrm{~N}_{10} \mathrm{O}_{10} \mathrm{Zn}_{2}:$ C, 65.69; H, 5.51; N, 8.15\%, found; C, 65.44; H, 5.37; N, $8.03 \%$.

Compound (53v): Zinc chloride; Yield $=0.01 \mathrm{~g}, 72 \% ; \mathrm{v}_{\max } / \mathrm{cm}^{-1}(\mathrm{KBr}): 3429$, $1647,1606,1571,1443,1193,1093,1022,762,700 ; \delta_{\mathrm{H}} / \mathrm{ppm}$ : Conc. of $10^{-3} \mathrm{~mm} ;\left(d_{6^{-}}\right.$ DMSO); 8.69 (d, $J=4.4 \mathrm{~Hz}), 8.62(\mathrm{~d}, J=4.4 \mathrm{~Hz}), 8.57$ (d, $J=2.7 \mathrm{~Hz}), 8.54$ (d, $J=$ $4.4 \mathrm{~Hz}$ ), 8.18 (s), 8.10 (br d), 8.02 (br d), 7.98 (s), 7.94 (d, $J=2.3 \mathrm{~Hz}), 7.92$ (d, $J=$ $2.0 \mathrm{~Hz}), 7.90(\mathrm{~d}, J=1.8 \mathrm{~Hz}), 7.88(\mathrm{~d}, J=1.6 \mathrm{~Hz}), 7.86(\mathrm{~d}, J=1.6 \mathrm{~Hz}), 7.82(\mathrm{t}, J=$ $1.8 \mathrm{~Hz}), 7.80(\mathrm{t}, J=1.6 \mathrm{~Hz}), 7.78(\mathrm{~d}, J=1.6 \mathrm{~Hz}), 7.75(\mathrm{t}, J=2.7 \mathrm{~Hz}), 7.74(\mathrm{br} \mathrm{d})$, 7.71 (br t), 7.67 (br t), 7.60 (br t), 7.57 (br d), 7.53 (s), 7.49 (s), 7.46 (s), 7.44 (s), 7.41 (br d), 7.39 (d, J=3.9 Hz), 7.36 (br d), 7.33 (br d), 7.31 (br t), 7.28 (br d), 7.24 (s), 7.20 (d, $J=7.4 \mathrm{~Hz}), 7.17$ (d, $J=7.4 \mathrm{~Hz}$ ), 7.10 (s), 7.05 (s), 7.03 (br d), 6.99 (d, $J$ = 7.1 Hz), $6.96(\mathrm{~s}), 6.93$ (s), 6.61 (br t), 6.38 (br t), 6.28 (br t), 5.04 (s), 4.88 (s), 4.76 (d, $J=6.2 \mathrm{~Hz}), 6.70$ (br d), 4.66 (br t), 4.63 (s), 4.41 (d, $J=6.0 \mathrm{~Hz}), 4.20$ (s), 4.13 (s), 4.00 (s), 3.87 (s), 3.23 (br d), 3.06 (t, $J=6.0 \mathrm{~Hz}), 2.93$ (t, $J=6.9 \mathrm{~Hz}$ ); Anal. Calc. for $\mathrm{C}_{86} \mathrm{H}_{82} \mathrm{Cl}_{4} \mathrm{~N}_{10} \mathrm{O}_{6} \mathrm{Zn}_{2}: \mathrm{C}, 63.60 ; \mathrm{H}, 5.09 ; \mathrm{N}, 8.62 \%$, found; C, 63.22; $\mathrm{H}$, $4.95 ; \mathrm{N}, 8.54 \%$.

Compound (53vi): Zinc perchlorate; Yield $=0.01 \mathrm{~g}, 72 \% ; \mathrm{v}_{\max } / \mathrm{cm}^{-1}(\mathrm{KBr}): 3450$, 1611, 1627, 1444, 1088 (perchlorate anion peak), 762, 626; $\delta_{\mathrm{H}} / \mathrm{ppm}$ : Conc. of $10^{-3}$ $\mathrm{mm}$; $\left(d_{6}\right.$-DMSO); 8.70 (d, $\left.J=4.2 \mathrm{~Hz}\right), 8.60$ (d, $\left.J=5.1 \mathrm{~Hz}\right), 8.53$ (br d), 8.15 (s), 7.96 (s), 7.90 (d, $J=1.3 \mathrm{~Hz}), 7.87$ (d, $J=1.9 \mathrm{~Hz}), 7.84$ (d, $J=1.6 \mathrm{~Hz}), 7.82$ (br t), 7.80 (d, $J=1.5 \mathrm{~Hz}$ ), 7.73 (br t), 7.66 (br t), 7.47 (d, $J=7.7 \mathrm{~Hz}), 7.43$ (s), 7.41 (br d), 7.39 (s), 7.35 (d, J = $5.9 \mathrm{~Hz}), 7.30$ (s), 7.16 (br d), 7.09 (s), 6.92 (s), 5.04 (s), 4.88 (s), $4.77(\mathrm{~d}, J=5.4 \mathrm{~Hz}), 4.67(\mathrm{~s}), 4.41(\mathrm{~d}, J=6.9 \mathrm{~Hz}), 4.20(\mathrm{~s}), 3.99$ (s), $3.88(\mathrm{~d}, J=3.8$ 
$\mathrm{Hz}$ ), $3.05(\mathrm{t}, J=7.0 \mathrm{~Hz}), 2.97\left(\mathrm{t}, J=6.7 \mathrm{~Hz}\right.$ ); Anal. Calc. for $\mathrm{C}_{86} \mathrm{H}_{82} \mathrm{Cl}_{4} \mathrm{~N}_{10} \mathrm{O}_{22} \mathrm{Zn}_{2}: \mathrm{C}$, 54.94; H, 4.40; N, $7.45 \%$, found; C, 49.85; H, 4.29; N, $7.36 \%$. 


\section{Discussion}




\subsection{Introduction}

Functionalisation of calix[4]arenes at either the upper or lower rims has been widely reported $^{11,69-74}$. Functionalisation of both rims is more difficult and as a result there are less publications in this area ${ }^{130-136}$. The majority of the compounds presented here have both rims functionalized with potential metal binding sites. It was envisioned that one of the binding sites could be able to capture and store solar energy between the calixarene unit and the two metal centres.

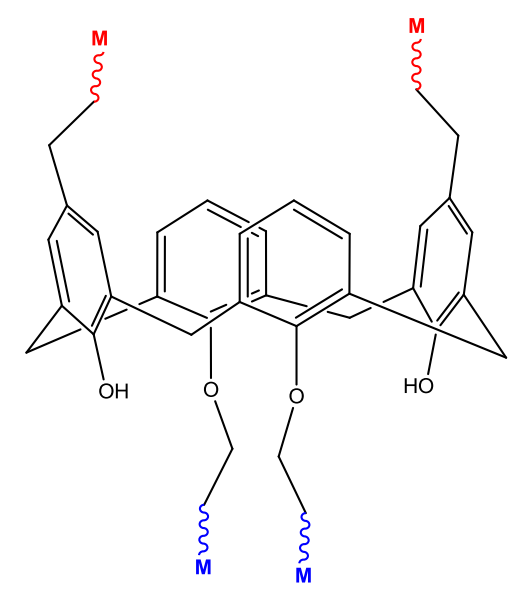

Figure 68: Dual-Metal binding calix[4]arene.

The calix[4]arene template used here contains three main regions, Figure 68. The central unit consisting of four phenol rings joined by methylene carbons provides the main scaffold on which to build the compound. The upper region (in red) is the first metal ion binding site and the lower rim region (in blue) will house the second metal ion binding site. Both of these regions were subject to similar conditions and the results of which are discussed below.

\subsection{Synthesis of calixarenes as potential central scaffolds}

Synthesis of the parent calix[8]arene, compound (1) used herein was prepared using a well-known method developed by Gutsche et al. ${ }^{11,137}$. The resulting calix[8]arene appears as a white precipitate which is then used to form the calix[4]arene, compound (2). The yields for these two steps are generally low, yielding around 50 
$\%$ at best, which is a little less than the published yields of $69 \%$ and $62 \%$ respectively ${ }^{10}$. The ${ }^{1} \mathrm{H}$ NMR spectra of compound (1) and (2) are very straight forward and correspond well to literature values ${ }^{138}$. The spectra are almost identical with the exception that in the calix[8]arene the signal for the hydroxyl proton is below $10 \mathrm{ppm}$. While in the calix[4]arene, due to an increase in the strength of the intramolecular hydrogen bonding observed between the hydroxyl groups on the lower rim, the signal for the hydroxyl proton occurs above $10 \mathrm{ppm}^{11}$.

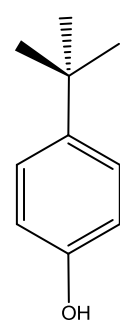

Paraformaldehyde Potassium Hydroxide Xylene

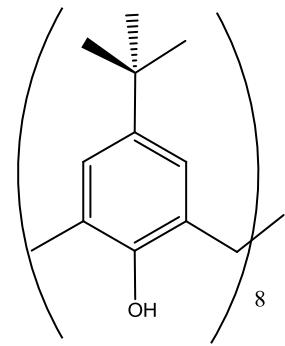

(1)

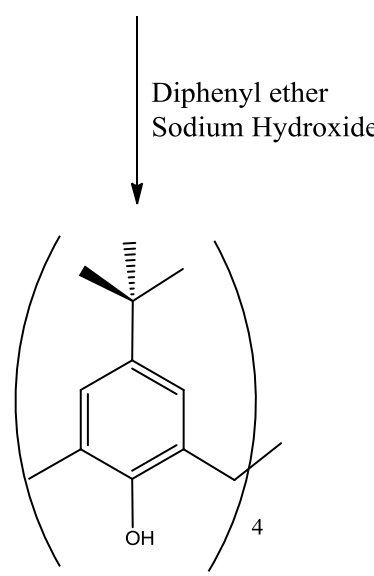

(2)

Figure 69: Synthesis of calix[4]arene

The melting points of both the calix[8]arene (1) and the calix[4]arene (2) were found to be in excess of $300{ }^{\circ} \mathrm{C}$ which corresponds to the literature values ${ }^{137}$. The IR of the two compounds were also found to be quite similar and corresponded to the literature values ${ }^{139}$. The calix[8] arene showing stretching regions for $v_{\mathrm{OH}} 3190 \mathrm{~cm}^{-1}$ and $v_{\mathrm{CH}}$ at $2955 \mathrm{~cm}^{-1}$ while the calix[4] arene showed stretches at $v_{\mathrm{OH}}$ at $3119 \mathrm{~cm}^{-1}$ and $v_{\mathrm{CH}}$ at $2950 \mathrm{~cm}^{-1}$. The ${ }^{1} \mathrm{H}$ NMR spectra of compound (1) and (2) are very straight forward and correspond well to literature values ${ }^{138}$. The spectra are almost identical 
with the exception that in the calix[8]arene the signal for the hydroxyl proton is below $10 \mathrm{ppm}$. While in the calix[4]arene, due to an increase in the strength of the intramolecular hydrogen bonding observed between the hydroxy groups on the lower rim, the signal for the hydroxyl proton occurs above $10 \mathrm{ppm}^{11}$. The ${ }^{1} \mathrm{H}$ NMR spectrum of the calix[8]arene (1), showed a singlet at $9.62 \mathrm{ppm}$ for the phenolic protons, and a set of doublets at 4.36 and $3.48 \mathrm{ppm}$ for the methylene bridging doublets. The doublet at $4.36 \mathrm{ppm}$ corresponds to the axial protons and the doublet at $3.48 \mathrm{ppm}$ corresponds to the protons in the equatorial positions. The axial protons are shifted downfield due to the influence of the hydrogen bonding between the hydroxyl groups on the lower rim. The ${ }^{1} \mathrm{H}$ NMR spectrum of the calix[4]arene (2) also shows a hydroxyl peak but it is shifted downfield to $10.78 \mathrm{ppm}$. This downfield shift is caused by a conformational change in the molecule on going from the calix[8]arene to yield the calix[4]arene. When the construct consists of eight phenolic units as in the calix[8]arene it has a greater degree of flexibility and as such can adopt a pleated loop conformation, ${ }^{11}$ whereas in the calix[4]arene the flexibility is decreased due to halving the number of phenolic units and the molecule adopts a cone conformation. This smaller cone conformation increases intramolecular hydrogen bonding on the lower rim producing the downfield shift. The calix[4]arene also shows the characteristic set of doublets at 4.38 and $3.52 \mathrm{ppm}$ for the axial and equatorial protons respectively. Because of the established difference in the ${ }^{1} \mathrm{H}$ NMR spectrum signal for the hydroxyl group it allows quick determination between the calix[8]arene and the calix[4]arene. The ${ }^{13} \mathrm{C}$ NMR spectra for both compounds were similar with each showing the expected seven different signals for the two compounds.

The plan was now to prepare a series of compounds in which both the upper and lower rims of the calix[4]arene, (2) would be functionalized to create potential metal binding sites. The lower rim was to be functionalised with esters, butoxys, and benzyls to provide conformational stability to the lower rim and to help prevent conformer conversion, along with creating a potential metal ion binding site. The upper rim would be then functionalised with the dipyridyl amine (DPA) moiety or other binding sites containing nitrogen donor atoms, to create the second metal ion binding site. 


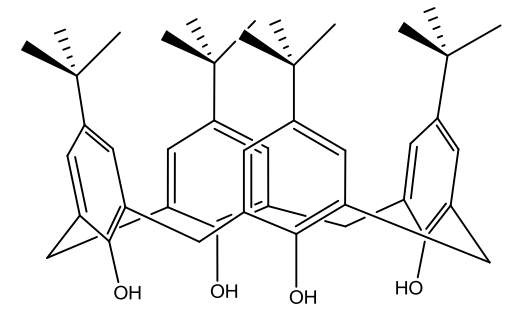

(2)

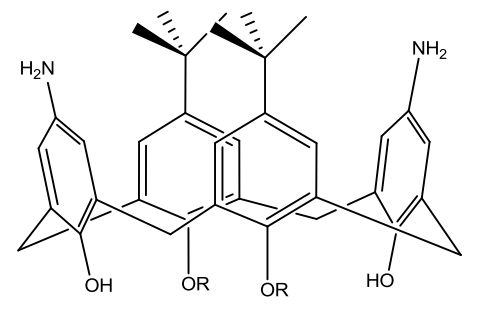

(5) $\mathrm{R}=$ ester
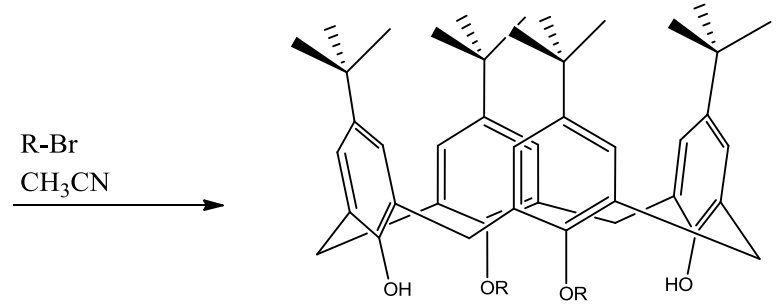

(3) $\mathrm{R}=$ ester

(6) $\mathrm{R}=$ bromoethyl

(8) $\mathrm{R}=$ benzyl

(9) $\mathrm{R}=$ butyl

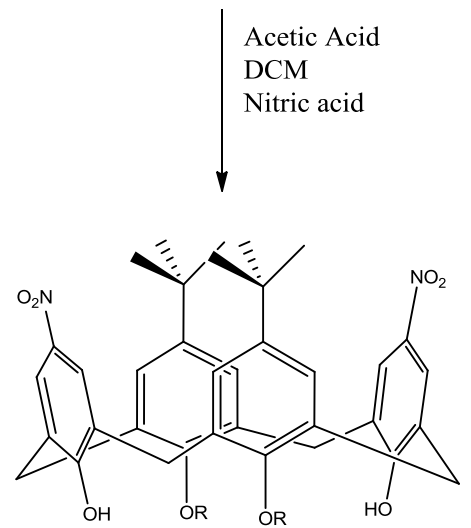

(4) $\mathrm{R}=$ ester

(7) $\mathrm{R}=$ bromoethyl

Figure 70: Synthesis of (3)-(9)

Compound (3) was obtained as a white solid in high yields (90\%). The IR data showed $v_{\mathrm{OH}}$ at $3434 \mathrm{~cm}^{-1}, v_{\mathrm{CH}}$ at $2962 \mathrm{~cm}^{-1}$ and the $v_{\mathrm{CO}}$ at $1745 \mathrm{~cm}^{-1}$. The melting point range of this compound was found to be $178-181{ }^{\circ} \mathrm{C}$ which corresponds with the literature values $\left(182-184{ }^{\circ} \mathrm{C}\right){ }^{140}$. The ${ }^{1} \mathrm{H}$ NMR spectrum of $(\mathbf{3})$ was relatively clean and showed the expected peaks. The hydroxyl peak was found at $7.19 \mathrm{ppm}$ as a singlet, the two aromatic singlets of the calix[4]arene were found at $7.01 \mathrm{ppm}$ and $6.81 \mathrm{ppm}$ respectively. The lower rim $\mathrm{OCH}_{2}$ group of the bridging ester arm was found at $4.71 \mathrm{ppm}$ as a singlet and the two methylene bridging doublets were seen at $4.45 \mathrm{ppm}$ and $3.33 \mathrm{ppm}$ respectively, representing the cone conformation. The final peaks to be observed were the quartet and triplet of the ester ethyl moiety at 4.30 ppm and $1.33 \mathrm{ppm}$ and the two singlets representing the $t$-butyl groups appeared at $1.25 \mathrm{ppm}$ and $1.00 \mathrm{ppm}$. Two singlets are observed for the $t$-butyl groups due to the different groups functionalised at the lower rim. As the ester is a more strongly electron withdrawing group, this causes a greater deshielding effect than the free 
hydroxyl resulting in the signal for the $t$-butyl group attached to the same phenol ring as the ester moiety to occur further downfield. The ${ }^{13} \mathrm{C}$ NMR spectrum showed thirteen signals for the non-equivalent carbons.

Compound (4) was a yellow-orange solid obtained in low yields (18\%). The low yield can be attributed to a large amount of starting materials (2) and (3) being recovered. The melting point range of this solid was found to be between 180-183 ${ }^{\circ} \mathrm{C}$, which is a bit less than the literature values $\left(198{ }^{\circ} \mathrm{C}-200{ }^{\circ} \mathrm{C}\right)^{124}$ most likely due to the excess starting materials. The IR data showed the $v_{\mathrm{OH}}$ at $3356 \mathrm{~cm}^{-1}$, the $v_{\mathrm{CH}}$ at $2966 \mathrm{~cm}^{-1}$, the $v_{\mathrm{CO}}$ at $1769 \mathrm{~cm}^{-1}$ and the new $v_{\mathrm{NO}}$ at $1516 \mathrm{~cm}^{-1}$. The ${ }^{1} \mathrm{H}$ NMR spectrum of (4) showed the lower rim hydroxyl at $8.78 \mathrm{ppm}$ as a singlet, and the two calix[4]arene aromatic singlets at $8.12 \mathrm{ppm}$ and $7.03 \mathrm{ppm}$ respectively. The downfield shift to $8.12 \mathrm{ppm}$ can be attributed to the deshielding effect of the nitro group in the para position on the upper rim. The methylene bridging protons appeared as two doublets at 4.51 and $3.47 \mathrm{ppm}$, indicating the cone conformer. The $\mathrm{OCH}_{2}$ ester protons appeared at $4.81 \mathrm{ppm}$ as a singlet for the bridging arm, $4.33 \mathrm{ppm}$ for the quartet and $1.36 \mathrm{ppm}$ for the triplet. The remaining $t$-butyls were seen at 1.10 ppm. As only one $t$-butyl signal was seen which integrated to eighteen hydrogens, this was evidence along with the IR data that the $\mathrm{NO}_{2}$ groups had attached on the upper rim. The ${ }^{13} \mathrm{C}$ NMR spectrum showed fifteen different signals for the nonequivalent carbon atoms. The final step in these synthesis was to convert the nitro groups on the upper rim of the calix[4]arene to amine groups (5) using tin(II) chloride in ethanol (Figure 70). Unfortunately this step was not successful and complete decomposition of the calix[4]arene was observed

Compound (6) was obtained as a white, sticky solid in good yields (80 \%). The IR data showed the $v_{\mathrm{OH}}$ at $3450 \mathrm{~cm}^{-1}$ and the $v_{\mathrm{CH}}$ at $2963 \mathrm{~cm}^{-1}$. The ${ }^{1} \mathrm{H}$ NMR spectrum showed the lower rim hydroxyl as a singlet at $7.19 \mathrm{ppm}$ and the two calix[4]arene singlets at 7.10 and $6.81 \mathrm{ppm}$. The methylene bridging doublets appeared as two doublets at 4.45 and $3.33 \mathrm{ppm}$ showing that the cone form was present. The protons of the lower rim bromoethyl arms were seen as two triplets at $3.85 \mathrm{ppm}$ and 1.87 ppm. The two $t$-butyl groups appeared at 1.25 and $1.00 \mathrm{ppm}$ respectively 
corresponding to eighteen protons each. The ${ }^{13} \mathrm{C}$ NMR spectrum showed ten different signals for the non-equivalent carbons.

Compound (7) was a light brass coloured solid in low yields (16\%). The IR data showed the $v_{\mathrm{OH}}$ at $3449 \mathrm{~cm}^{-1}$ and the $v_{\mathrm{CH}}$ at $2964 \mathrm{~cm}^{-1}$ and the new $v_{\mathrm{NO}}$ at $1559 \mathrm{~cm}^{-1}$, indicating that functionalisation had occurred at the upper rim. The ${ }^{1} \mathrm{H}$ NMR spectrum showed the hydroxyl group at $8.77 \mathrm{ppm}$ as a singlet and the two calix[4]arene singlets appeared at 8.13 and $6.81 \mathrm{ppm}$. The methylene bridging doublets occurred at 4.45 and $3.33 \mathrm{ppm}$ indicating cone conformation. The ethoxy protons on the lower rim were seen at 3.85 and $1.87 \mathrm{ppm}$ as two triplets. Only one signal for the $t$-butyls was seen at $1.00 \mathrm{ppm}$ indicating that substitution at the upper rim had occurred. The ${ }^{13} \mathrm{C}$ NMR spectrum showed thirteen non-equivalent carbon atoms. An attempt was made to convert the nitro to the amine but as was seen with the previous pathways the reaction did not proceed and the calix[4]arene derivative underwent complete decomposition.

Two other lower rim functionalised calix[4]arene derivatives, compound (8) and compound (9), were also prepared. However due to the harsh conditions and the low yields of the nitro reaction, it was decided to investigate different routes to the required compounds. Compound (8) was obtained as a white solid in quantitative yields $(100 \%)$. The IR data showed the $v_{\mathrm{OH}}$ at $3343 \mathrm{~cm}^{-1}$ and the $v_{\mathrm{CH}}$ at $2959 \mathrm{~cm}^{-1}$. The ${ }^{1} \mathrm{H}$ NMR spectrum showed the lower rim hydroxyl at $7.84 \mathrm{ppm}$ and the singlet for the aromatic protons of the calix[4]arene at $7.03 \mathrm{ppm}$. The lower rim benzyl arm appeared as a singlet at $5.04 \mathrm{ppm}$ and the benzyl aromatic protons were seen as a doublet and triplet at 7.64 and $7.36 \mathrm{ppm}$ respectively. The methylene bridging doublets were observed as a pair of doublets at 4.28 and 3.24 ppm indicating the calix[4]arene is present in the cone conformation. The final two peaks observed represented the $t$-butyl groups and appeared as two singlets at 1.25 and $1.00 \mathrm{ppm}$ respectively. The ${ }^{13} \mathrm{C}$ NMR spectrum showed sixteen individual signals for the nonequivalent carbon atoms. 
Compound (9) was a white precipitate in high yields (86\%). The melting point of this compound was found between $218-22{ }^{\circ} \mathrm{C}$ which corresponds well with literature values $\left(219-221^{\circ} \mathrm{C}\right)^{124}$. The IR analysis showed the $v_{\mathrm{OH}} 3332 \mathrm{~cm}^{-1}$ band and the $v_{\mathrm{CH}}$ band at $2965 \mathrm{~cm}^{-1}$. The ${ }^{1} \mathrm{H}$ NMR spectrum showed all the expected peaks. The hydroxyl groups occurred at $7.95 \mathrm{ppm}$ as a singlet and the aromatics of the calix[4]arene occurred at $7.05 \mathrm{ppm}$ and $6.86 \mathrm{ppm}$ as a singlet and doublet. The bridging methylene protons occurred at $4.31 \mathrm{ppm}$ and $3.28 \mathrm{ppm}$ representing the cone conformation. The new butoxy peaks occurred at 4.00, 2.04, 1.75 and $1.08 \mathrm{ppm}$ as a triplet, two multiplets and a final triplet respectively. The last two peaks to be observed were the $t$-butyl singlets at $1.25 \mathrm{ppm}$ and $1.00 \mathrm{ppm}$. The ${ }^{13} \mathrm{C} \mathrm{NMR}$ spectrum showed sixteen different peaks for the non-equivalent carbons with the butoxy peaks occurring at $69.1,31.38,18.54$ and 13.25 ppm respectively.

Due to the complete decomposition of the calix[4]arene derivatives when the conversion to the amine was attempted, and as the formation of the dinitrocalix[4]arenes, $(4 \& 7)$, proceeded with a low yields, it was decided to investigate other routes for the attachment of the dpa moiety to the upper rim. No further work was carried out using this pathway.

A similar synthesis to compound (3) was employed to attach methoxy groups on the lower rim, to give compound (10), and once the lower rim had been successfully functionalised, the attempted removal of two of the upper rim $t$-butyl groups, (11) was then carried out. 


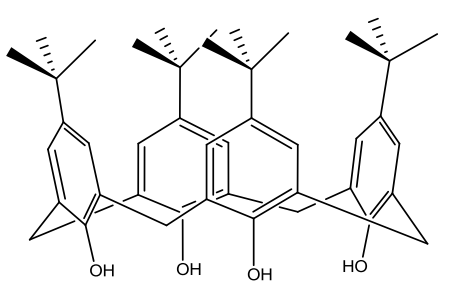

(2)

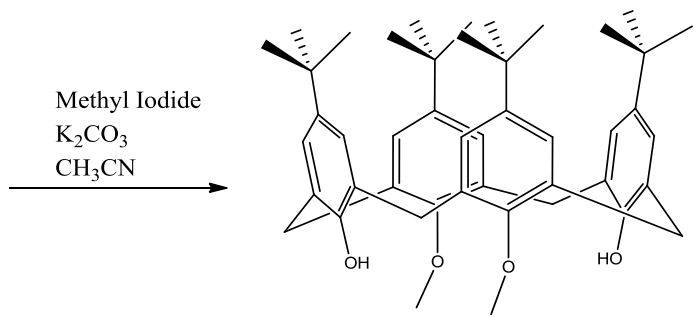

(10)

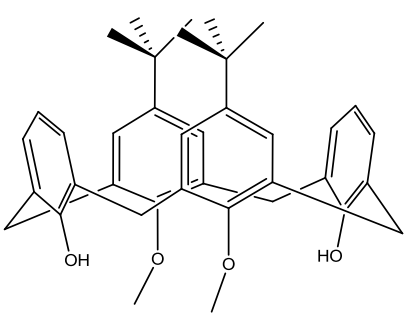

(11)

Figure 71: Synthesis of compound (10) \& (11)

Compound (10) was obtained as a white solid in high yields (95\%). The melting point was found between $215-218{ }^{\circ} \mathrm{C}$ corresponding well with literature values (217$\left.219{ }^{\circ} \mathrm{C}\right)^{127}$. The IR data showed the $v_{\mathrm{OH}}$ band at $3329 \mathrm{~cm}^{-1}$ and the $v_{\mathrm{CH}}$ at $2958 \mathrm{~cm}^{-1}$. The ${ }^{1} \mathrm{H}$ NMR data showed the hydroxyl peak at $7.28 \mathrm{ppm}$ and the aromatic singlets at 7.16 and $6.78 \mathrm{ppm}$. The bridging methylene protons occurred at 4.30 and 3.30 ppm indicating the cone conformation. The lower rim methoxy peak occurred at 3.95 ppm and the two singlets for the $t$-butyl groups on the upper rim appeared at 1.29 and 0.94 ppm respectively. The ${ }^{13} \mathrm{C}$ NMR spectrum showed twelve non-equivalent carbons with the methoxy carbon occurring at $63.51 \mathrm{ppm}$. Compound (11) was produced as a yellow solid in low yields (45\%). The IR analysis showed the $v_{\mathrm{OH}}$ band at $3345 \mathrm{~cm}^{-1}$ and the $v_{\mathrm{CH}}$ band at $2962 \mathrm{~cm}^{-1}$. The ${ }^{1} \mathrm{H}$ NMR spectrum showed that hydroxyl peak had shifted to $10.27 \mathrm{ppm}$ and the aromatic singlet, doublet and triplet were found at 7.15, 7.12 and $6.97 \mathrm{ppm}$ respectively. The bridging methylene doublets were found at 4.31 and $3.75 \mathrm{ppm}$ indicating a distorted cone due to the downfield shift of the equatorial protons. The methoxy protons were found at 4.01 ppm as a singlet and the remaining $t$-butyl groups appeared at $1.27 \mathrm{ppm}$ as a singlet. The ${ }^{13} \mathrm{C}$ NMR spectrum showed twelve non-equivalent carbon atoms. 
Due to the low yields produced by this step and the fact that the methoxy groups are very difficult to remove, a new approach was employed to synthesis the desired tetrahydroxycalix[4]arene (12). It was envisioned that this new method would remove all of the $t$-butyl groups from the upper rim and leave the hydroxy groups free to interact at later stages.

\subsection{Synthesis of core scaffold (18)}

Work began initially with the removal of the $t$-butyl groups from the upper rim of the calix[4]arene using a procedure by Arduini et al. ${ }^{141}$ (Figure 72). Yields for this reaction were generally between $30-50 \%$, with the product appearing as a cream coloured solid.

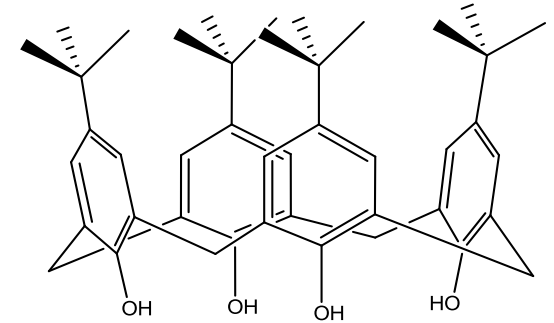

(2)

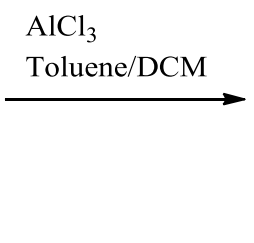

Figure 72: Synthesis of 25,26,28,28-tetrahydroxycalix[4]arene (12)

Upon analysis the compound was found to contain similar peaks to the parent compound. The most obvious difference was the loss of the $t$-butyl peak at $1.25 \mathrm{ppm}$ and the appearance of a triplet at $6.69 \mathrm{ppm}$ corresponding to the para proton on the upper rim of the calix[4]arene (Figure 73). The set of doublets was as expected at 4.21 and 3.24 ppm respectively, indicating that the cone conformation was retained. The IR spectrum showed the $v_{\mathrm{OH}}$ stretching frequency at $3416 \mathrm{~cm}^{-1}$ and the ${ }^{13} \mathrm{C}$ spectra only displays five signals, further indicating that the $t$-butyl groups had been removed. 


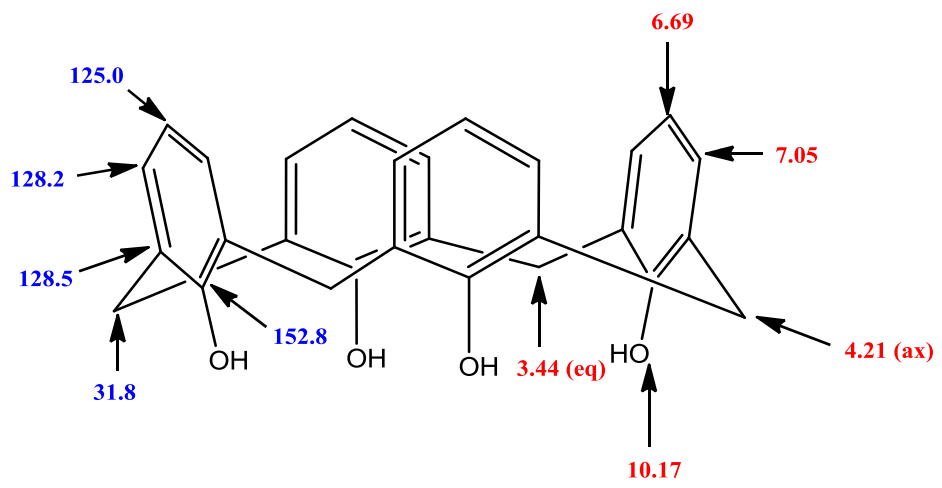

Figure 73: ${ }^{1} \mathrm{H} \&{ }^{13} \mathrm{C}$ NMR data of (12)

Subsequently, the upper rim was functionalised with a bromomethyl substituent, (18), to create a site of attachment for future groups. This procedure was carried out with the help of Dr. Charlotte Williams and her Ph.D. student Emma Bulloughs, at the Chemistry Department, University of Leeds. Compound (18) was obtained as a brown solid in high yields (99\%). The IR analysis showed the $v_{\mathrm{OH}}$ band at $3416 \mathrm{~cm}^{-}$ ${ }^{1}$. In the ${ }^{1} \mathrm{H}$ NMR spectrum, there were three signals present for the aromatic rings, namely a singlet at $7.24 \mathrm{ppm}$, a doublet at $7.12 \mathrm{ppm}$ and a triplet at $6.72 \mathrm{ppm}$. The new singlet at $7.24 \mathrm{ppm}$ indicates a different type of aryl unit is now present and corresponds with what would be expected in the case of substitution para to the phenol group. Another new peak at $4.65 \mathrm{ppm}$ indicated the presence of the new bromomethyl group. It is worth mentioning that this peak was not always visible in the ${ }^{1} \mathrm{H}$ NMR spectra, but was present in all subsequent reactions so it may be possible that some form of ionic interaction is occurring with only a partial bond being formed. Unfortunately as a crystal structure was never obtained this is still open to debate. 


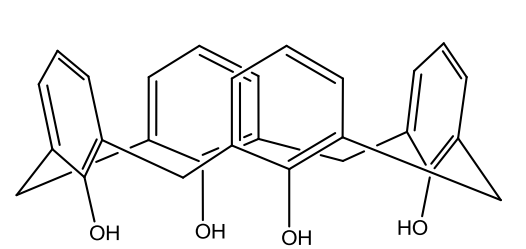

(12)

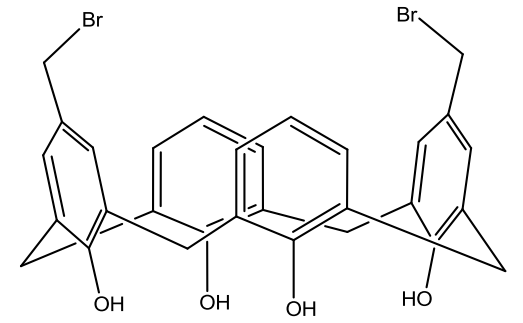

(18)

Figure 74: Synthesis of 11,23-dibromomethyl-25,26,27,28-tetrahydroxycalix[4]arene (18)

Another method to functionalise the lower rim and remove the $t$-butyl groups on the upper rim was attempted following a procedure by Cooper et al. ${ }^{128}$. This involved reacting compound (12), with benzyl and ester groups on the lower rim, to give initial conformational stability to the calixarene. This would then be followed by removal of the benzyl group to selectively functionalise the upper rim with a nitro group (Figure 75). However, removal of the benzyl group was never achieved so attachment with the nitro group was not attempted. 


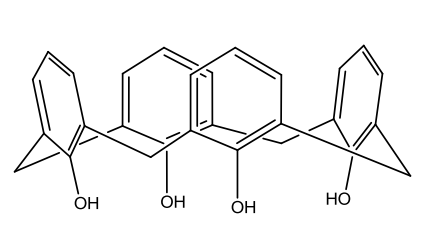

(12)

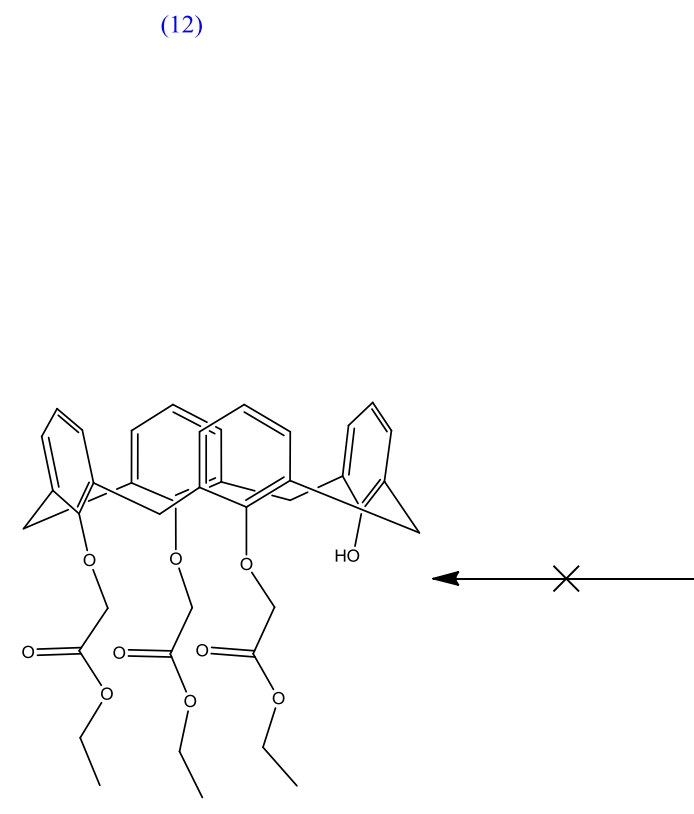

(16)

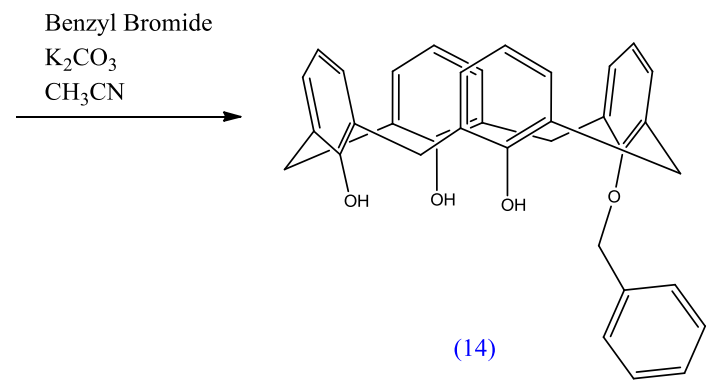

Ethyl Bromoacetate

$\mathrm{K}_{2} \mathrm{CO}_{3}$

$\mathrm{CH}_{3} \mathrm{CN}$

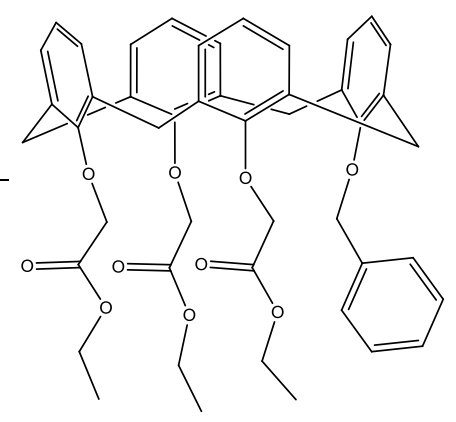

(15)

Figure 75: Synthesis of (14)-(15) and attempted synthesis of (16)

\subsection{Functionalisation of the lower rim}

With the potential binding unit now established on the upper rim, work could continue on the lower rim. Functionalizing the lower rim adds conformational stability to the compounds in solution and provides some rigidity to the unit which helps to retain the cone conformation. Without the addition of bulky groups to add steric hindrance, the calix[4]arene unit would be free to rotate between any of the

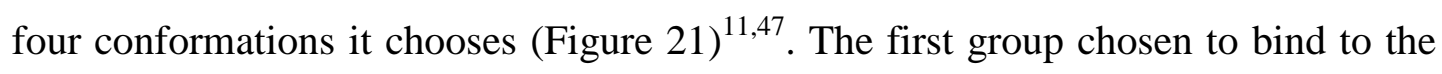
lower rim was the benzyl group as it was bulky enough to aid conformational stability in solution. The synthesis was relatively straight-forward following a procedure similar to Ocak et al. ${ }^{142}$ (Figure 76). This afforded compound (29) as a red-wine waxy solid. The IR of this compound showed a $v_{\mathrm{OH}}$ at $3361 \mathrm{~cm}^{-1}$ as expected. The ${ }^{1} \mathrm{H}$ NMR spectrum showed the new methylbenzyl peak on the lower rim at $5.12 \mathrm{ppm}$ and the upper rim bromomethyl group protons were observed at $4.54 \mathrm{ppm}$. Two clear doublets at 4.28 and $3.42 \mathrm{ppm}$ were observed for the bridging 
methylene protons as well as the singlet at $7.94 \mathrm{ppm}$ for the hydroxyl group. The aromatics appeared as a doublet and triplet at 6.96 and 6.77 ppm respectively along with a singlet at $7.12 \mathrm{ppm}$. The ${ }^{13} \mathrm{C}$ spectrum shows fourteen different signals for the non-equivalent carbons and the peak for the lower rim methyl benzyl linker was observed at $78 \mathrm{ppm}$ and the upper rim bromomethyl carbon at $76 \mathrm{ppm}$.

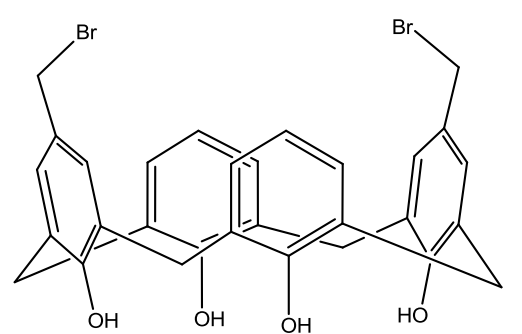

(18)
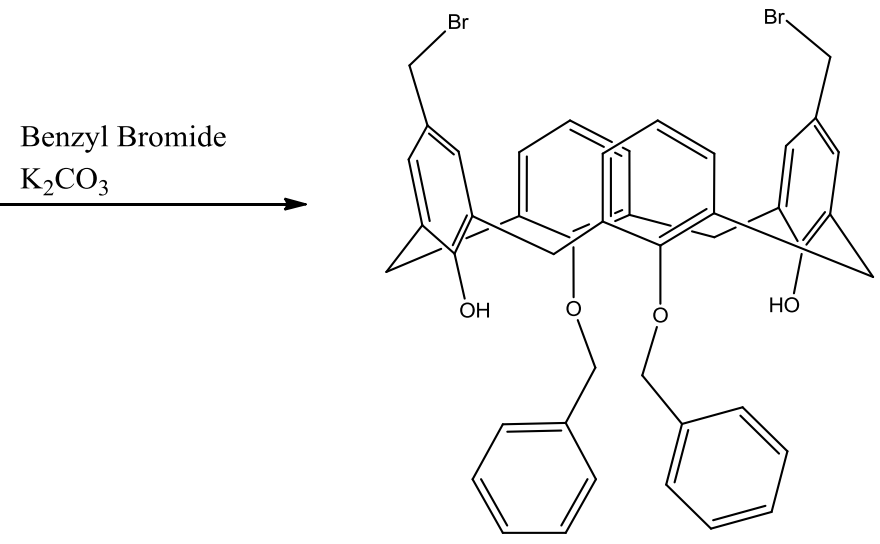

(29)

Figure 76: Synthesis of 11,23-dibromomethyl-25,27-dibenzyl-26,28-dihydroxycalix[4]arene

To determine whether the lower rim benzyl group was attaching to the same phenol ring as the upper rim bromomethyl group Heteronuclear Multiple Bond Correlation (HMBC) NMR spectroscopy was performed. The resulting spectrum that was obtained is shown in (Figure 77). From the spectrum it can be seen that the lower rim hydroxyl group (7.65 ppm) interacts with the upper aromatic para carbon (128.1 ppm) which has been functionalised with the bromomethyl group. Whereas the $\mathrm{CH}_{2}$ group form the benzyl moiety $(5.11 \mathrm{ppm})$, is seen to interact with the free para carbon $(124.1 \mathrm{ppm})$. From this it is clear that the functionalisation of the upper and lower rims occurs on two different phenolic units. 


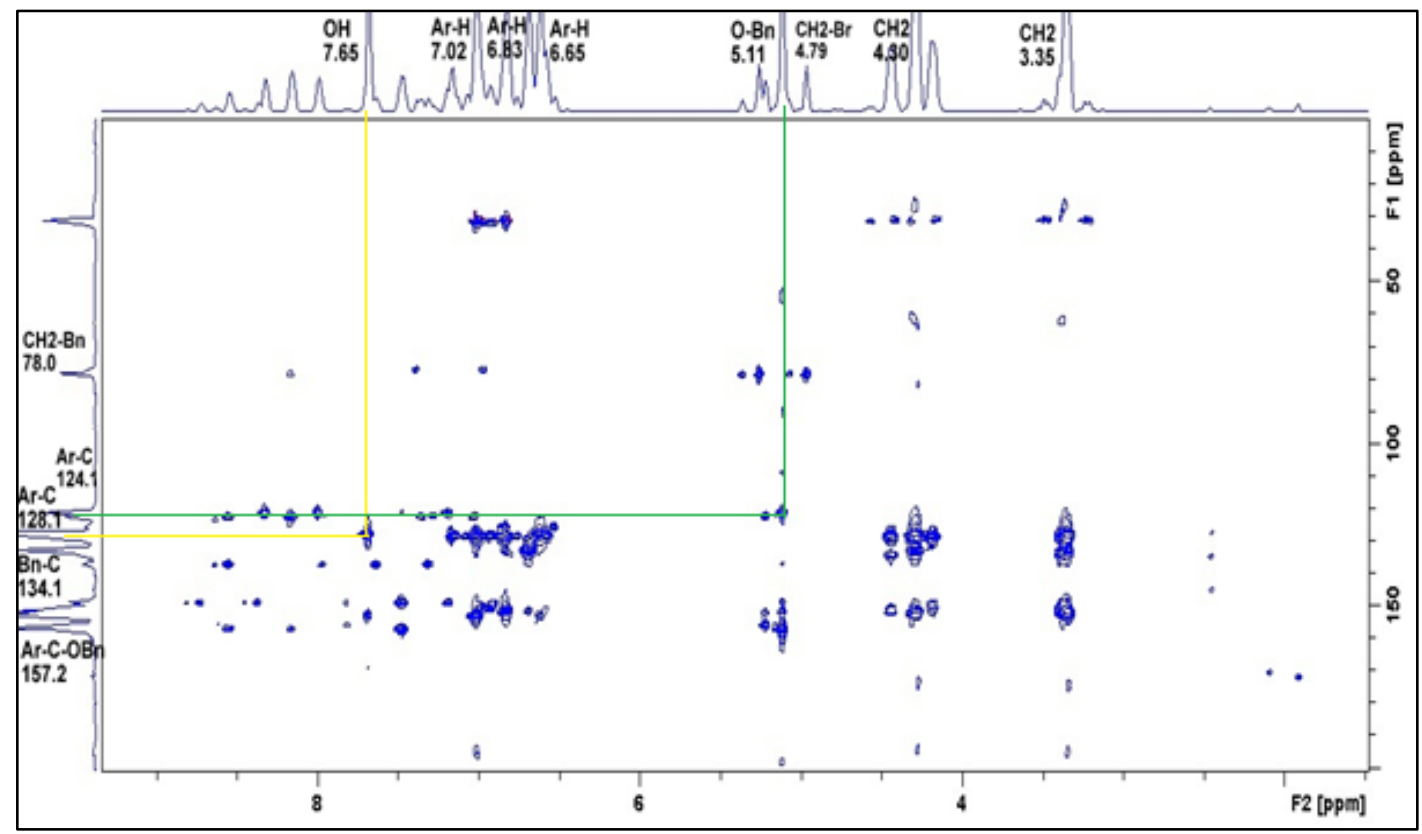

Figure 77: HMBC spectrum of compound (29)

\subsubsection{DPA Synthesis and upper rim Functionalisation}

It was always the plan to attach $\mathrm{N}$-donor ligands to the upper rim of the calix[4]arene unit such as bipy or DPA ligands as potential metal ion binding sites. The synthesis of DPA, (19), began by following a reported method by Incarvito et al. ${ }^{116}$. This synthetic route initially gives an imine (Figure 78) which is then converted in situ to an amine which is obtained as a red waxy solid. 


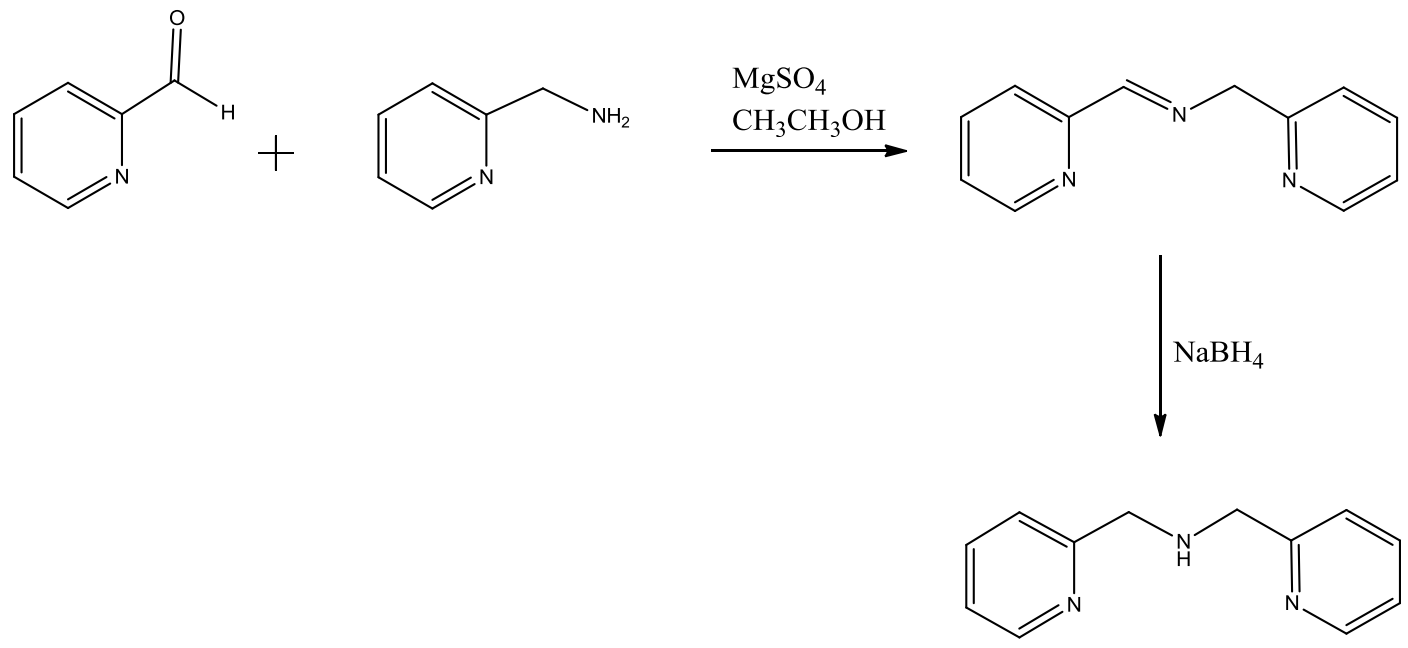

(19)

Figure 78: Synthesis of DPA (19)

While the synthesis of the DPA, Figure 78, afforded decent yields, the complexation of the pure oil to the upper rim of the calix[4]arene gave varying results. This ligand was to be attached to the upper rim of the calix[4]arene in place of the bromo group on (29). Unfortunately this synthetic route lead to varying results involving monoand di-substituted calix[4]arene derivatives as well as low yields, most commonly due to the DPA breaking down in solution. To this end a new approach was devised involving a stepwise synthesis for the addition of the DPA unit to the upper rim. The procedure is a two-step process which involves attaching one arm of the DPA group first, (30), and then attaching the second arm of the DPA moiety (31), to create a cage-like metal binding site (Figure 79). Compound (30) was a brown waxy solid which was always obtained in high yields (>95\%). The IR analysis of the compound showed the $v_{\mathrm{OH}}$ stretching region at $3413 \mathrm{~cm}^{-1}$, the $v_{\mathrm{NH}}$ stretching region at $3388 \mathrm{~cm}^{-1}$ and the pyridyl $\mathrm{NCH}$ at $1590 \mathrm{~cm}^{-1}$. The ${ }^{1} \mathrm{H} \mathrm{NMR}$ analysis of the compound showed the characteristic calix[4] arene cone conformation set of doublets at 4.41 and $3.32 \mathrm{ppm}$ respectively as well as the hydroxyl signal at $7.39 \mathrm{ppm}$. The pyridyl peaks were clearly visible, with a doublet at $8.59 \mathrm{ppm}$ for the ortho protons beside the nitrogen atom, two triplets at 7.73 and $7.42 \mathrm{ppm}$ for the para and meta protons to the nitrogen atom respectively, and a doublet at $7.37 \mathrm{ppm}$ for the remaining pyridyl protons. For the benzyl group the signals were at $7.79 \mathrm{ppm}$ and $7.34 \mathrm{ppm}$ for the doublet and triplet respectively. All of the signals for the linking 
methylene protons were also clearly seen. A singlet for the benzyl methylene protons was observed on the lower rim at $5.13 \mathrm{ppm}$, while the upper rim methylene protons appeared as a singlet at $4.79 \mathrm{ppm}$. There was also a very broad peak for the $\mathrm{NH}$ at $2.43 \mathrm{ppm}$. The ${ }^{13} \mathrm{C}$ NMR spectrum of the compound showed twenty different signals indicating the twenty-one non-equivalent carbons now present.

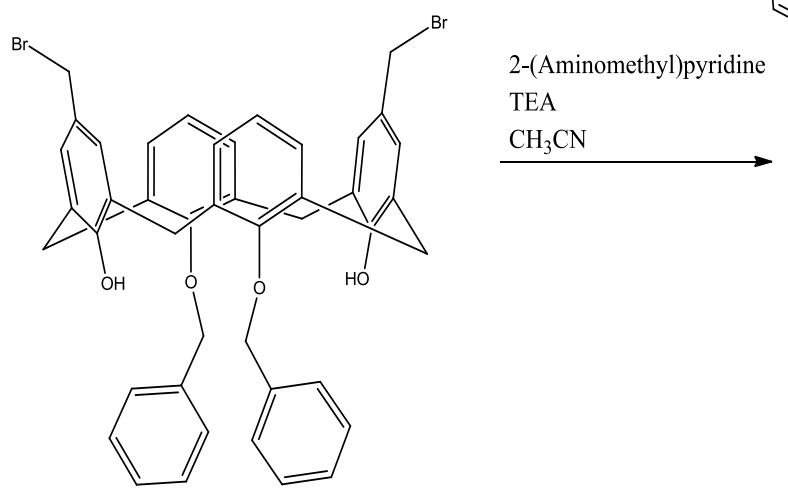

(29)

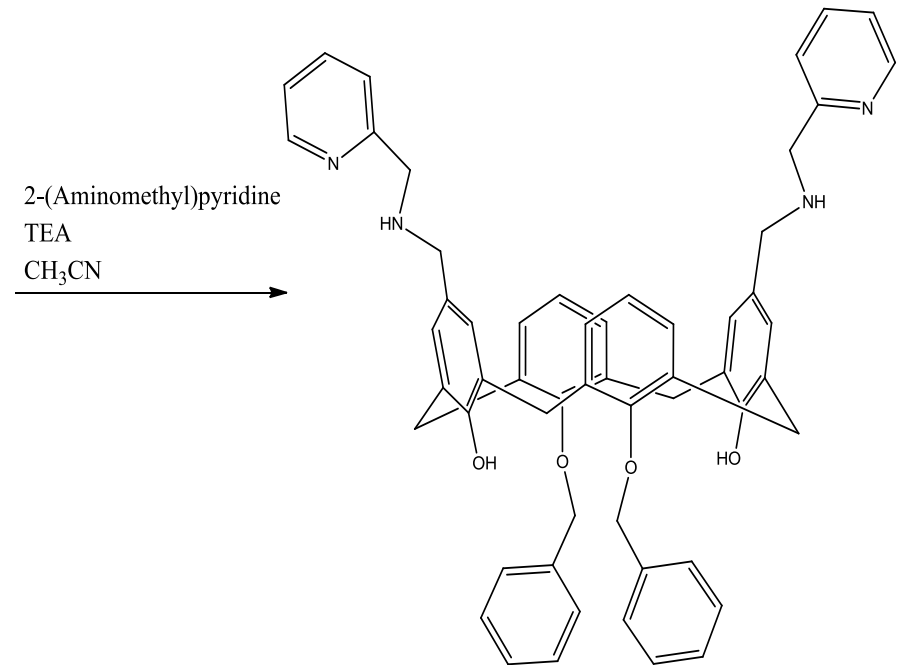

(30)

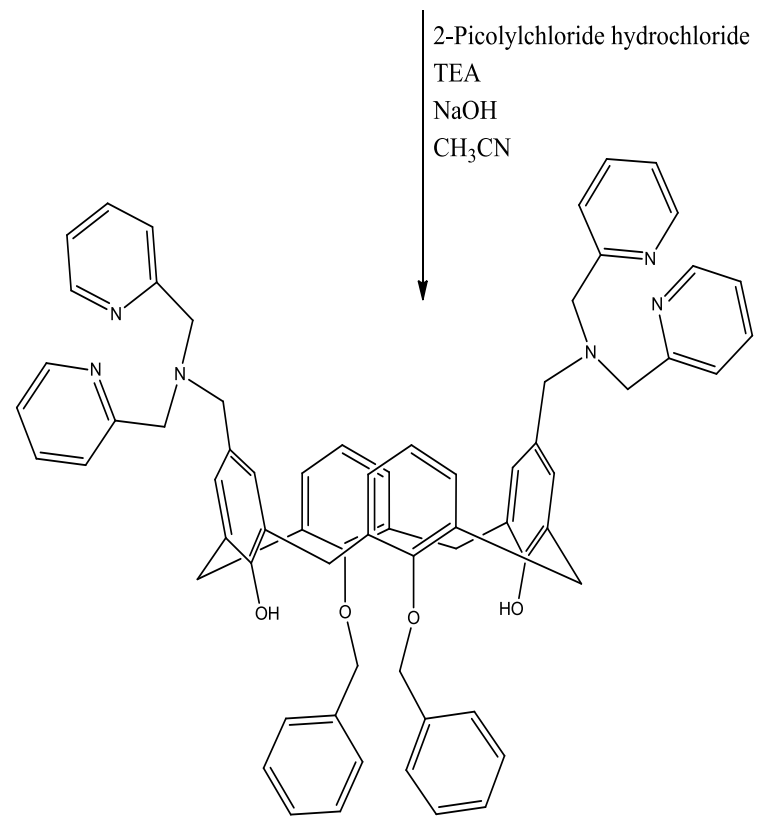

(31)

Figure 79: Synthesis of (30) and (31) 
Compound (31), (Figure 79), was a deep red coloured waxy solid also obtained in high yields (95\%). The IR analysis showed $v_{\mathrm{OH}}$ at $3388 \mathrm{~cm}^{-1}$ and the pyridyl $\mathrm{NCH}$ at $1590 \mathrm{~cm}^{-1}$. As the hydroxy band was very broad it is quite possible that the NH band was there but could not be discerned. The ${ }^{1} \mathrm{H}$ NMR data was quite surprising in that there were many more peaks than were originally expected (Figure 80). The pyridyl protons occurred at $8.56,7.65,7.63$ and $7.58 \mathrm{ppm}$ for the ortho, para, ortho and meta protons respectively, in relation to the nitrogen atom. The benzyl protons were found at $7.43,7.37$ and $7.19 \mathrm{ppm}$ respectively. For the calix[4]arene unit itself several new peaks were seen. While the $\mathrm{OH}$ signal appeared around $7.85 \mathrm{ppm}$, corresponding to the two hydroxyl protons, several singlets, doublets and triplets were also observed for the aromatic signals. Singlets integrating for four hydrogens appeared at 7.24 and $7.10 \mathrm{ppm}$, doublets corresponding to four hydrogens were seen at $6.86,6.80$ and at $6.71 \mathrm{ppm}$ and triplets for two hydrogen's appeared at 6.41 (broad), 6.36 and $6.27 \mathrm{ppm}$ respectively. The bridging methylene region also showed a large number of extra peaks such as doublets at 4.30, 4.24, 3.99, 3.81, 3.60, 3.53, $3.34,3.16$ and $3.06 \mathrm{ppm}$ all corresponding to four hydrogens, and several singlets at 4.39, 3.77 and $3.65 \mathrm{ppm}$. All of the linking arms were accounted for at 5.06, 4.74, $3.85 \mathrm{ppm}$ for the benzyl, methyl amine and the aminomethyl pyridyl groups. Clearly the proton NMR spectrum was showing that the desired compound had been made, but even if some residual starting materials remained it still couldn't account for the huge increase in peaks. It began to look like something else was occurring in solution. Referring once again to works done by Gutsche et al. ${ }^{138}$ it was suggested that perhaps that at an earlier stage, the free phenol rings were rotating into different conformation states, and then the upper rim of these phenol rings were being functionalised. Different conformers could account for these numerous, albeit lesser intense peaks, but again without a crystal structure this is still open to debate. 


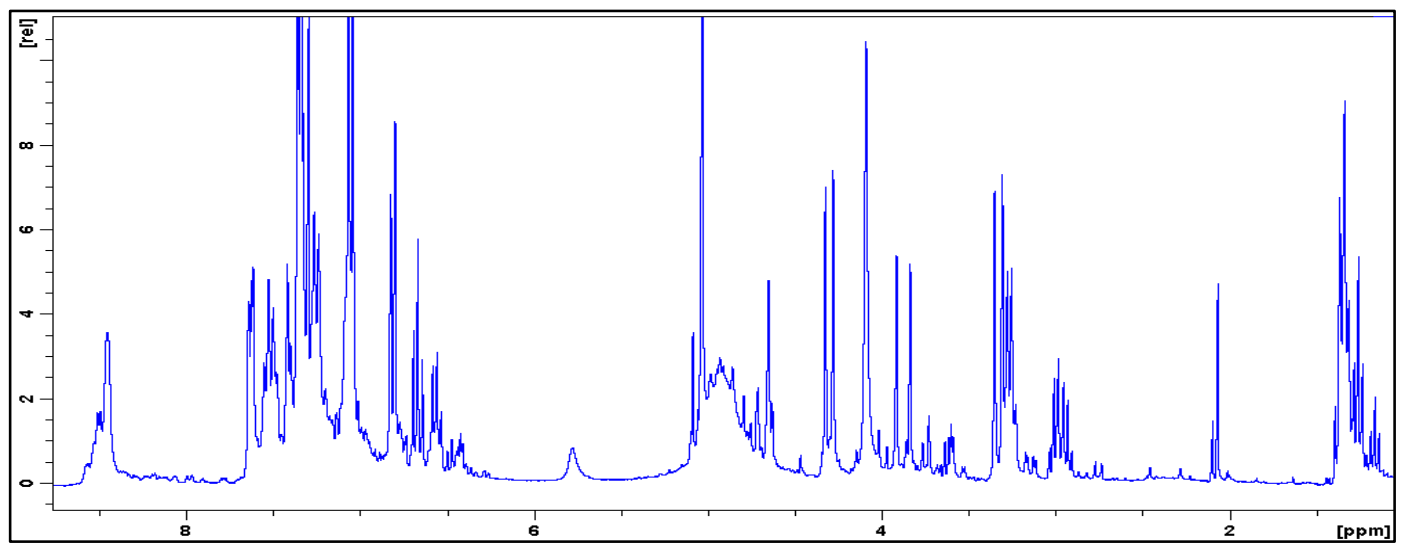

Figure 80: ${ }^{1} \mathrm{H}$ NMR spectrum of (31)

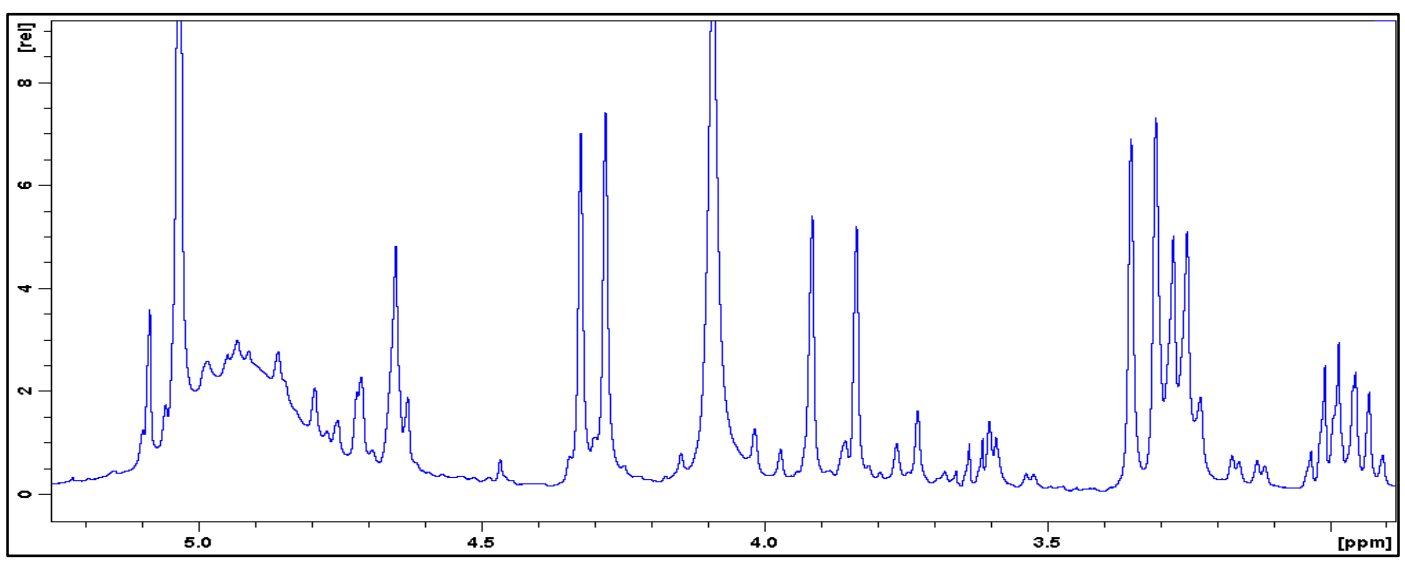

Figure 81: ${ }^{1} \mathrm{H}$ NMR spectrum showing the bridging region of (31)

As previously mentioned there are four possible conformers that the calix[4]arene can adopt the cone, partial cone, 1,2-alternate and the 1,3-alternate (Figure 21). Gutsche et al. ${ }^{143}$ have provided a general table (Table 1) which assists in assigning NMR spectral peaks to the different conformers and using this, and the colourcoding therein as a guide, it can be said that in the bridging region (Figure 81) the peaks around 4.30 and $3.34 \mathrm{ppm}$ represent the cone conformation, the peaks at 4.39, 3.81, 3.16 and 3.06 ppm could represent the partial cone form and the 3.77 and 3.53 ppm (first set of broad doublets), 2.94 and $2.85 \mathrm{ppm}$ (second set of doublets) could represent the 1,3-alternate form. This corresponds well with what is observed in the aromatic region (Figure 82) were the cone singlet, doublets and triplets appear at 7.10, 6.86 and $6.41 \mathrm{ppm}$, the partial cone at 7.02, 6.80, 6.71, 6.41 and $6.27 \mathrm{ppm}$ or 
were the 1,3-alternate appears at 7.32, 6.83, and $6.27 \mathrm{ppm}$ for its singlet, doublet and triplet respectively.

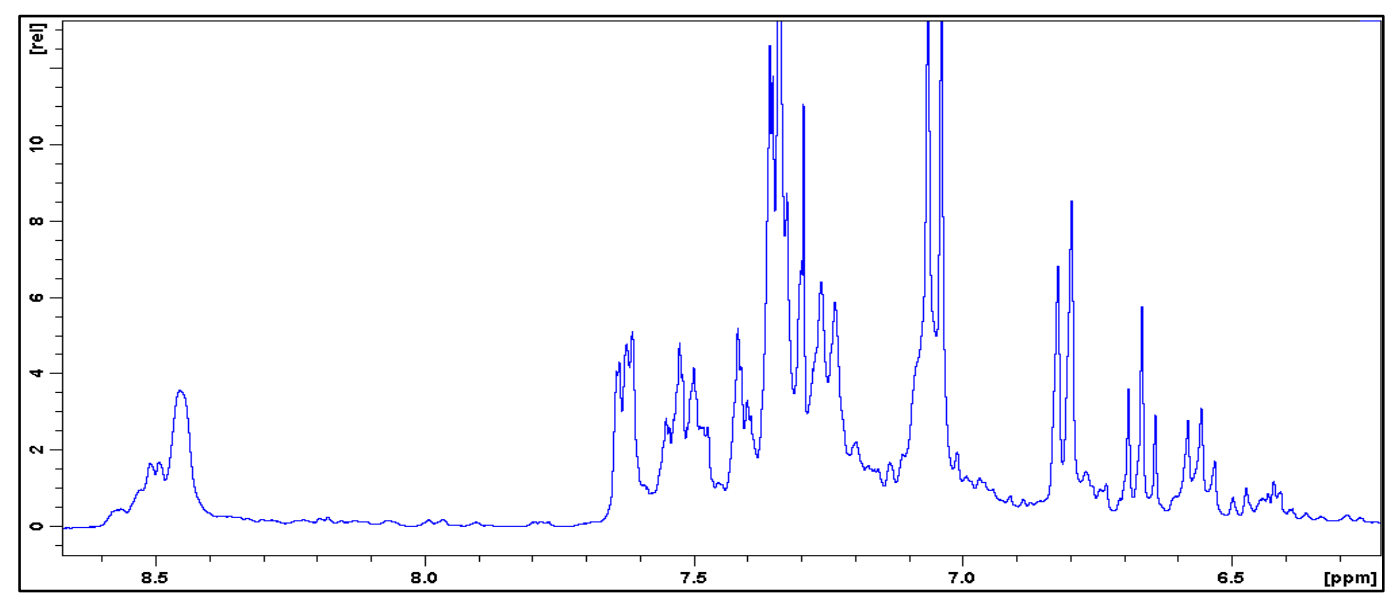

Figure 82: ${ }^{1} \mathrm{H}$ NMR spectrum of the aromatic region of (31)

The idea that the conformers are occurring seems plausible. However, there are still several peaks unaccounted for and these will be discussed later. It would be quite possible for the hydroxyl groups to pass through the endo pathway of conformational change, as the hydroxyl group is small. By passing through the center of the ring it allows the rest of the phenol ring to pass by on the outside of the calix[4]arene unit, thereby avoiding steric hindrance (Figure 83). 

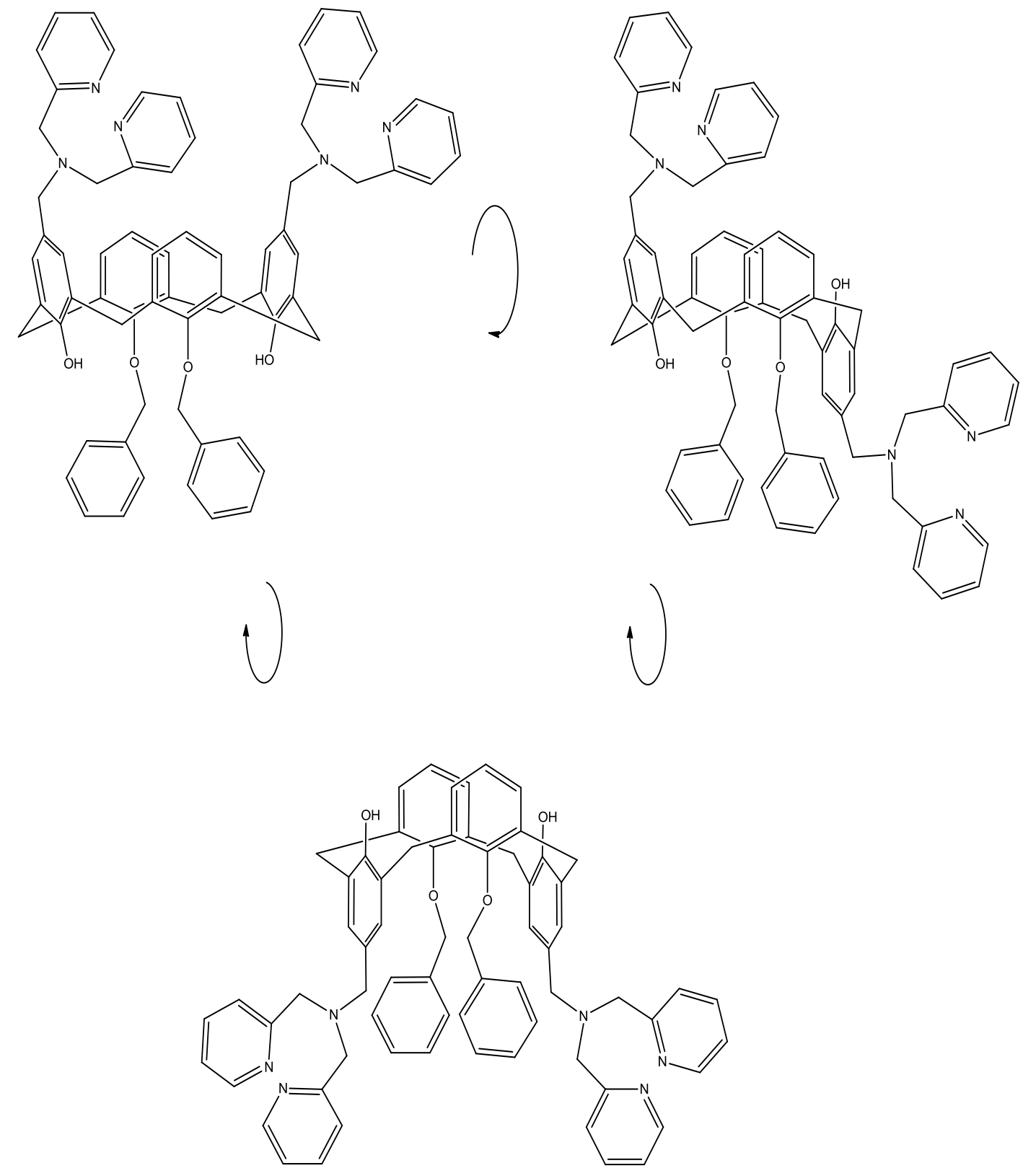

Figure 83: Three possible conformations, cone (top left), partial cone (top right) and 1,3-Alt (bottom)

\subsubsection{Lower rim functionalised pyridyl complexes}

Given the success of attaching the pyridyl group to the upper rim of the calix[4]arene, the pyridyl group was then selected to see if it would bind to the lower rim equally as well, to give compound (33). Before this reaction could take place the 2-picolylchloride hydrochloride was treated with sodium carbonate in acetonitrile to remove the hydrochloride salts. This was done as the untreated acid salts can prevent attachment to the calix[4]arene unit. The acid salts precipitated out of solution as 
pink salts and were removed by filtration. Compound (33) was afforded as a red waxy solid in high yields (95\%) (Figure 84). The IR data showed a new peak at $1591 \mathrm{~cm}^{-1}$ indicating the appearance of the pyridyl group. The ${ }^{1} \mathrm{H}$ NMR spectrum showed eleven peaks, with the pyridyl peaks occurring at 8.51, 7.59, 7.39 and 7.15 ppm, the hydroxyl signal at $7.51 \mathrm{ppm}$ along with a singlet, doublet and triplet at $7.09,6.89$ and $6.17 \mathrm{ppm}$ representing the calix[4]arene unit aromatic protons. The new $\mathrm{OCH}_{2}$ signal of the lower rim pyridyl arm was observed at $5.16 \mathrm{ppm}$. This is slightly higher than the benzyl arm, probably due to the presence of two electronegative oxygen atoms deshielding the peak slightly further downfield. The bromomethyl peak appeared at $4.64 \mathrm{ppm}$ and only one set of doublets was seen for the methylene bridging carbons at 4.37 and $3.42 \mathrm{ppm}$. The ${ }^{13} \mathrm{C}$ NMR spectrum showed sixteen different peaks for the non-equivalent carbons.
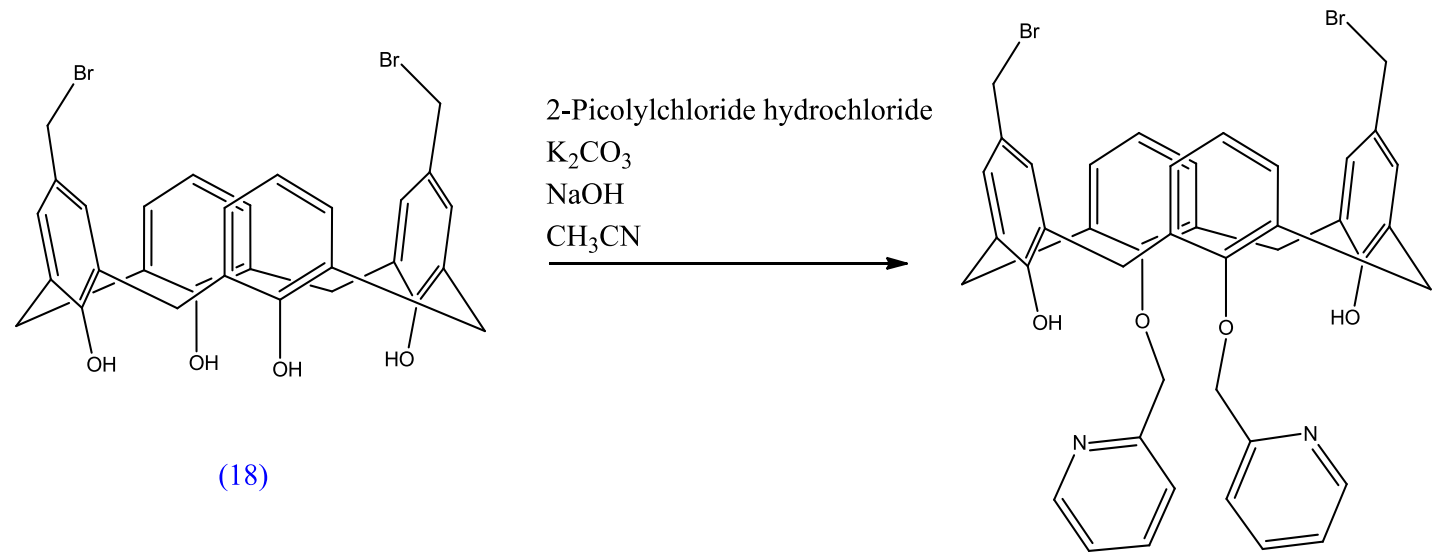

(33)

Figure 84: Synthesis of (33)

Growing crystals of (33) was attempted from various solvents. However, the crystals obtained were always found to be unsuitable for X-ray analysis. Instead scanning electron micrograph (SEM) was performed on the obtained crystals. The crystals were obtained from a 1:4 ratio of dichloromethane: methanol. Two very interesting pictures were obtained in that their overall images represented a horse's head (Figure 85) and what looks like two meteors crashing through space (Figure 86). The first image shows a very smooth texture and when examining an alternate site of the 
crystal, clear rows of material appears to have formed throughout. The second images also show the smooth rippled effect, except for the two 'meteorites' which appear to have a rougher texture.

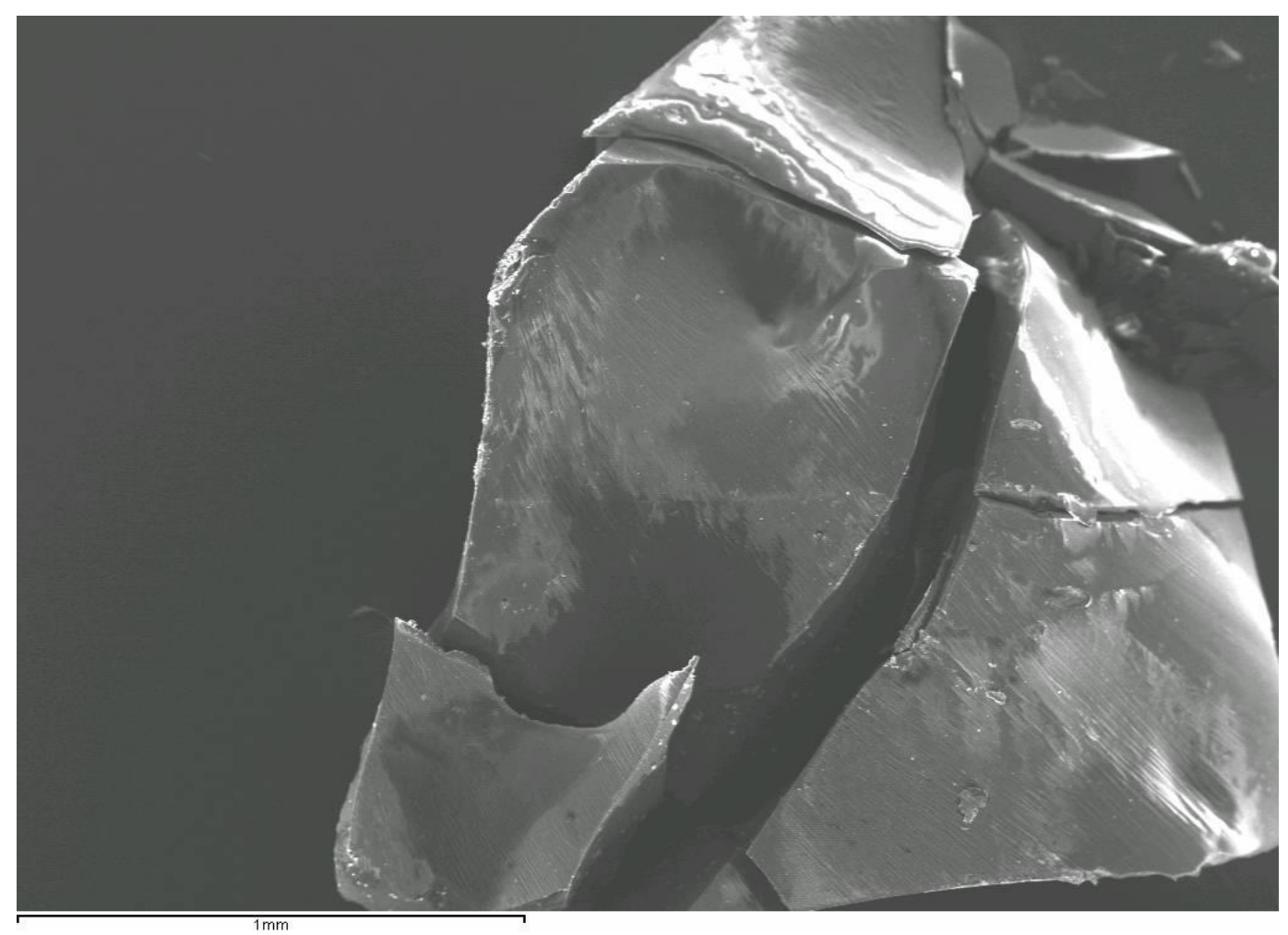

Figure 85: SEM of (33) X 400 


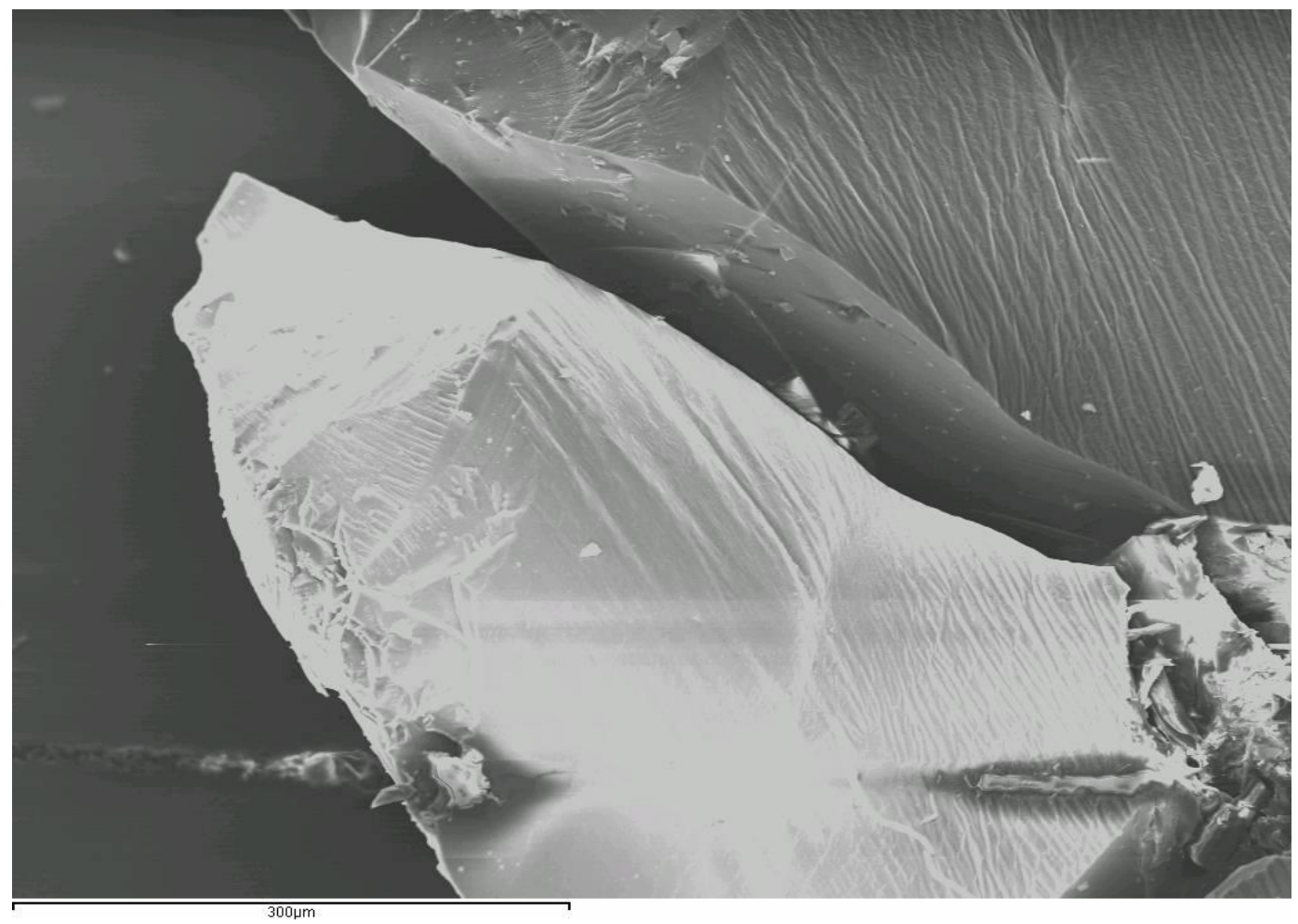

Figure 86: SEM of (33) X 450

The next step was to functionalise the upper rim, with 2-(aminomethyl)-pyridine, (34). Compound (34) was produced as a brown-red waxy solid in high yields (99\%) (Figure 88). The IR analysis showed the pyridyl band at $1591 \mathrm{~cm}^{-1}$ and the new NH band at $2923 \mathrm{~cm}^{-1}$. The ${ }^{1} \mathrm{H}$ NMR spectrum showed the expected peaks as well as several extra peaks (Figure 87). The lower rim pyridyl peaks occurred at 8.59, 7.63, 7.58 and $7.56 \mathrm{ppm}$ as a doublet, triplet, doublet and triplet respectively. The upper rim pyridyl was present at $8.49,7.52,7.47$ and $7.41 \mathrm{ppm}$ following the same pattern as the lower rim in terms of splitting signals. Several additional peaks were viewed for the phenol units of the calix[4]arene aromatic unit, doublets appeared at 7.09, 6.98, 6.94 and $6.81 \mathrm{ppm}$, two singlets at $7.05 \mathrm{ppm}$ and $7.03 \mathrm{ppm}$, and two clear triplets at 6.71 and $6.54 \mathrm{ppm}$ with the possibility of two more around $6.64 \mathrm{ppm}$ but due to signal overlap this could not be clearly distinguished. In the bridging region the upper and lower rim linker arms appeared as two singlets quite close together, with the lower rim slightly higher at $5.24 \mathrm{ppm}$ and the upper at $5.16 \mathrm{ppm}$. The new aminomethyl pyridyl arm on the upper rim was located as a singlet at $3.95 \mathrm{ppm}$. The methylene bridging protons exhibited several doublets. The two methylene protons 
appeared at $4.35 \mathrm{ppm}$ as a doublet and at $3.38 \mathrm{ppm}$ appearing as doublets within doublets. Two additional doublets at 4.50 and $4.25 \mathrm{ppm}$, as well as a singlet at 3.86 ppm were also observed which could indicate either the partial cone or the 1,3alternate form. The ${ }^{13} \mathrm{C}$ NMR spectrum showed the twenty-two desired peaks along with multiple peaks that even conformers cannot account fully for.

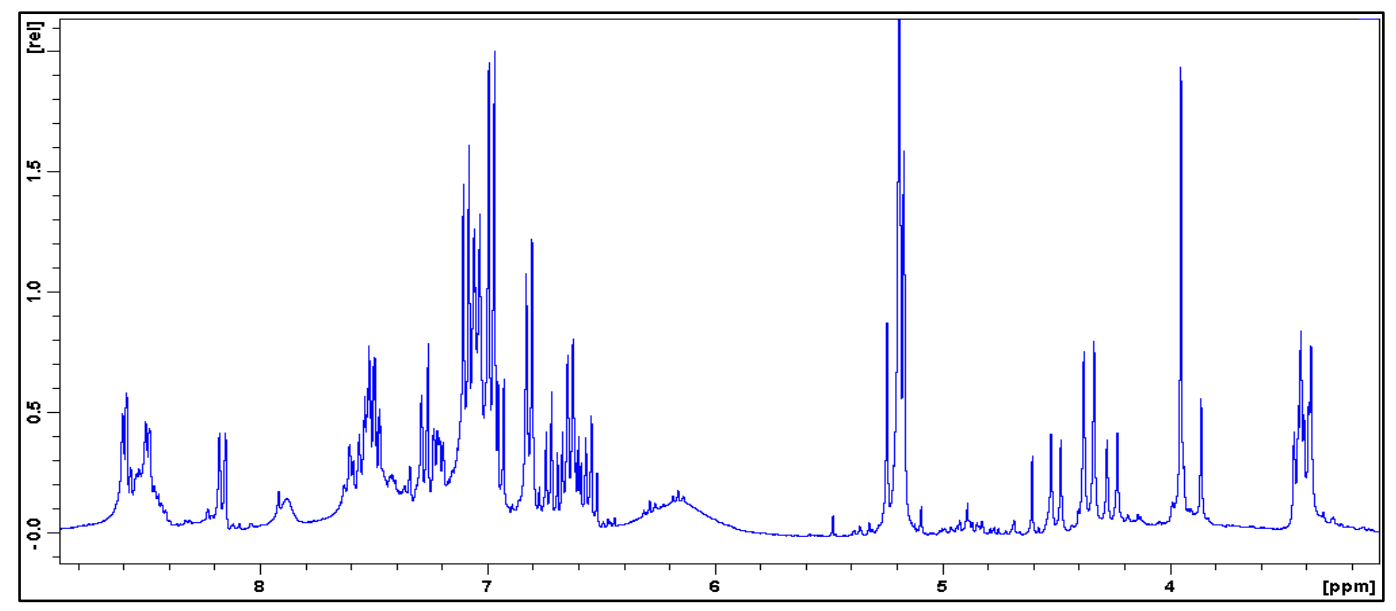

Figure 87: ${ }^{1} \mathrm{H}$ NMR spectrum of (34) 


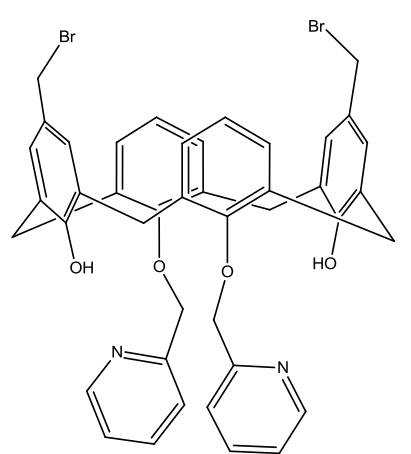

(33)

2-(Aminomethyl)pyridine

TEA

$\mathrm{CH}_{3} \mathrm{CN}$

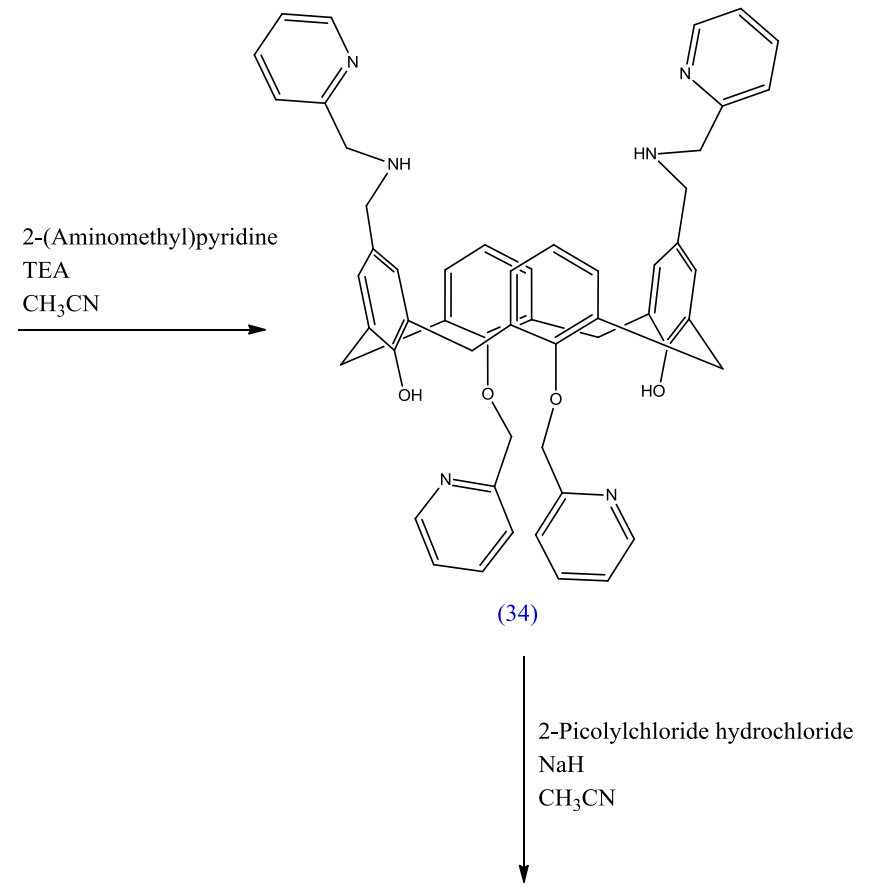

(34)

2-Picolylchloride hydrochloride $\mathrm{NaH}$ $\mathrm{CH}_{3} \mathrm{CN}$

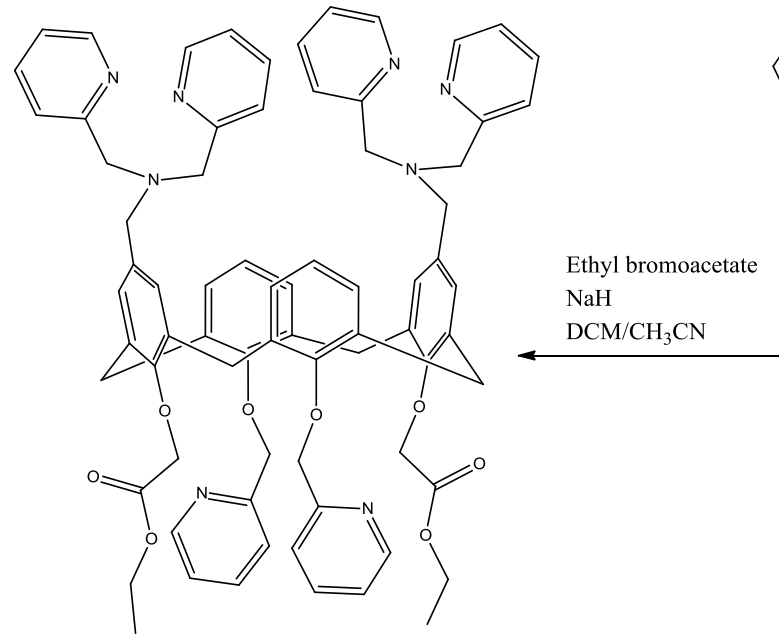

(47)

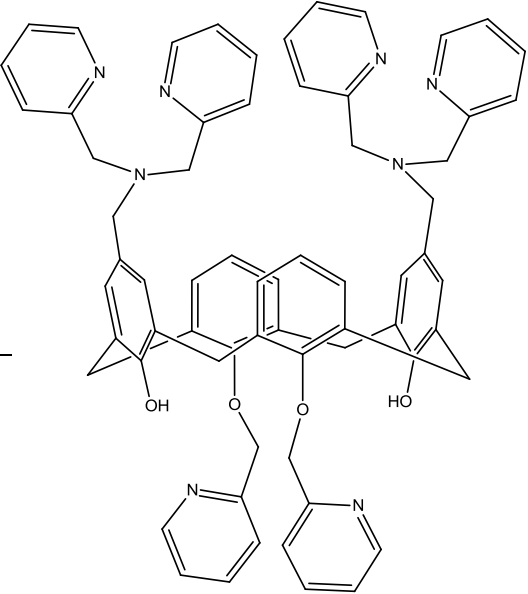

(35)

Figure 88: Synthesis of (34), (35) \& (47)

Continuing on in this synthesis was to attach another pyridyl arm to the upper rim to complete the metal binding site, to give compound (35). Compound (35) was obtained as a red waxy solid in moderate yields (76\%) (Figure 88). The ${ }^{1} \mathrm{H}$ NMR spectrum (Figure 89) showed a huge number of peaks. As was noted previously a large number of doublets and triplets were seen for the aromatic region of the calixarene itself suggesting that conformers may again be present. The lower rim pyridyls occurred at $8.53,7.72,7.61$ and $7.48 \mathrm{ppm}$ as a doublet, triplet, doublet and 
triplet respectively. The upper rim pyridyl peaks arose at 8.44, 7.56, 7.33 and 7.14 ppm and followed the same splitting pattern as the lower rim pyridyl. The lower rim pyridyl signals are shifted further downfield than the upper rim pyridyl signals, due to the presence of the electronegative deshielding oxygen atom on the lower rim. On looking at the bridging region the lower rim arm was seen at $5.12 \mathrm{ppm}$ as a singlet, the upper rim arms at $4.59 \mathrm{ppm}$ for the methyl amine and at $4.38 \mathrm{ppm}$ for the pyridyl arm. Two clear doublets could be distinguished, indicating the cone conformation, as well as three other doublets which may indicate alternate conformations. One signal in particular, at $3.36 \mathrm{ppm}$, appears as if a doublet is forming inside a doublet. This is slightly unusual even for conformer behaviour and suggests that something else, other than conformational change, may be occurring as well. The ${ }^{13} \mathrm{C}$ NMR spectrum showed that there were twenty-two clearly visible peaks for the non-equivalent carbon atoms (Figure 90).

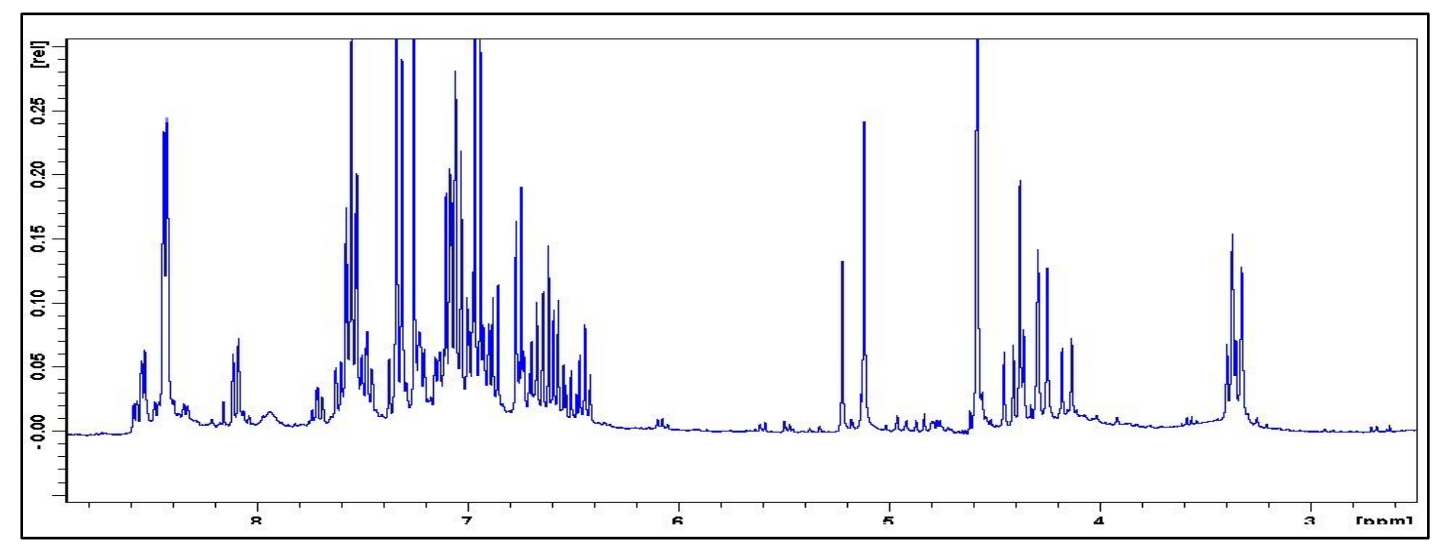

Figure 89 : ${ }^{1} \mathrm{H}$ NMR spectrum of (35) 


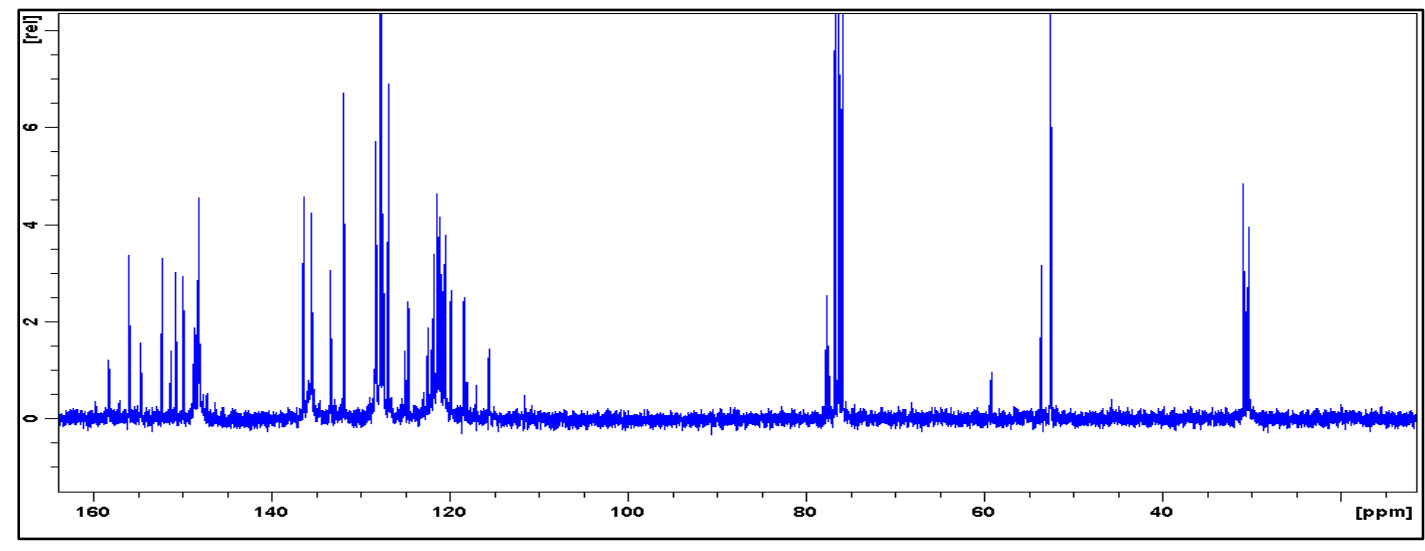

Figure 90: ${ }^{13} \mathrm{C}$ NMR spectrum of (35)

The final step in this synthesis was to attach an ester functionality onto the two remaining free hydroxyl groups on the lower rim to create another metal ion binding site on the lower rim, resulting in compound (47), (Figure 88). Compound (47) was obtained as a brown waxy solid in moderate yields (56\%). The IR data showed the $v_{\mathrm{CO}}$ at $1591 \mathrm{~cm}^{-1}$ and the $v_{\mathrm{NCH}}$ at $1591 \mathrm{~cm}^{-1}$. The ${ }^{1} \mathrm{H}$ NMR spectrum showed an overlap of the pyridyl peaks at 8.43, 7.59, 7.56 and $7.32 \mathrm{ppm}$ in the doublet, triplet, doublet, triplet, fashion respectively (Figure 91). The calix[4]arene aromatics occurred at 6.99, 6.89, 6.86, and $6.78 \mathrm{ppm}$ as a doublet, singlet, doublet, triplet respectively. The lower rim bridging arm was observed as a singlet at $5.01 \mathrm{ppm}$, and the upper rim pendant arms occurred at $4.54 \mathrm{ppm}$ and $4.10 \mathrm{ppm}$. The two methylene bridging doublets were found at 4.34 and $3.12 \mathrm{ppm}$. The new ester linkages were observed at 4.13, 3.94 and $1.16 \mathrm{ppm}$ as a multiplet, singlet and triplet respectively. The ${ }^{13} \mathrm{C}$ NMR spectrum showed twenty-two non-equivalent carbons with the new ester carbonyl carbon appearing at $178 \mathrm{ppm}$. 


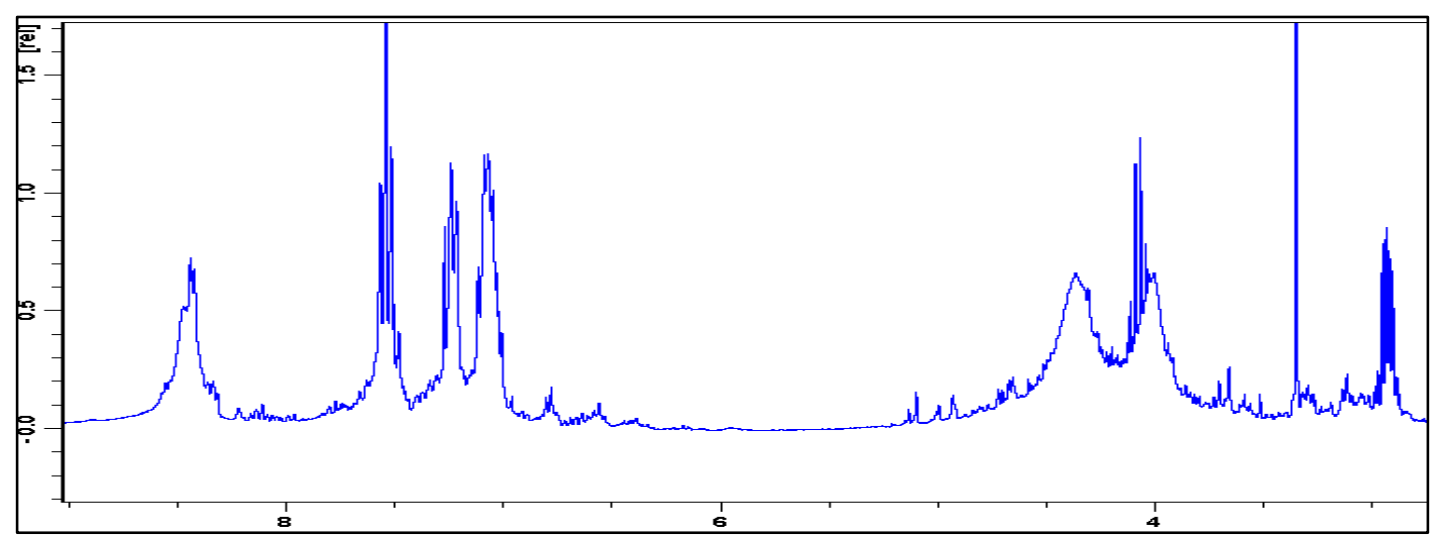

Figure 91: ${ }^{1} \mathrm{H}$ NMR spectrum of (47)

\subsection{Reactions of the bipyridyl unit}

Intrigued by what was occurring, work continued with an attempt to attach a bipy unit to the upper rim of some of the compounds. This was to investigate the effect this would have in terms of a potential metal binding site, as well as its effect on conformational stability. The synthesis of the desired bipy unit required three steps. Firstly, it is necessary to form 4-methyl-4-carbaldehyde-2,2-bipyridine (20), and then to reduce it to 4-methyl-4-hydroxymethyl-2,2-bipyridine (21) and finally to halogenate (21) to 4-methyl-4-bromomethyl-2,2-bipyridine (22) (Figure 92). A method similar to Dorta et al. was employed ${ }^{144}$. Compound (20) was obtained from the reaction of 4,4-dimethylpyridine with selenium dioxide, as a cream-white solid in high yields (96\%). The ${ }^{1} \mathrm{H}$ NMR spectrum for this compound was very straightforward, with the aldehyde proton occurring as a singlet at $10.19 \mathrm{ppm}$. The para protons to the nitrogen atom appeared as two distinct doublets at 8.90 and $8.84 \mathrm{ppm}$. The meta protons to the nitrogen atom also appeared as two doublets at 8.58 and $7.73 \mathrm{ppm}$, while the final ortho protons to the nitrogen atom occurred as two singlets at 8.82 and $8.28 \mathrm{ppm}$ respectively. The methyl group was located at $2.47 \mathrm{ppm}$ as a singlet. The ${ }^{13} \mathrm{C}$ NMR spectrum showed thirteen distinct peaks for the nonequivalent carbons with the aldehyde peak dominating at 191.7 ppm. Compound (20) was then reduced using sodium borohydride to produce compound (21) as a yellow solid in high yields. The ${ }^{1} \mathrm{H}$ NMR spectrum for this compound showed a total of nine peaks, six corresponding to the aromatic rings, a new singlet at $4.83 \mathrm{ppm}$ for the new $\mathrm{CH}_{2}$ protons and the methyl protons appeared as a singlet at $2.44 \mathrm{ppm}$. 
Unfortunately the hydroxyl proton was masked by the chloroform solvent peak. The ${ }^{13} \mathrm{C}$ NMR spectrum showed a total of thirteen peaks with the loss of the carbonyl peak at $191 \mathrm{ppm}$. Compound (22) was afforded from the reaction of (21) with hydrobromic acid to form the halogenated species, which was obtained as a rich red oil in moderate to high yields. The ${ }^{1} \mathrm{H}$ NMR spectrum showed eight resonance signals, six for the aromatics, one singlet at $4.49 \mathrm{ppm}$ for the bromomethyl group and one singlet at $2.43 \mathrm{ppm}$ for the methyl protons. The ${ }^{13} \mathrm{C}$ NMR data produced thirteen peaks with the new bromomethyl peak occurring at $30 \mathrm{ppm}$. It should be noted that the bipy formed often contained the acid salt, which was not always visible in the ${ }^{1} \mathrm{H}$ NMR spectrum, and as a result 1:1 reactions were rarely performed with the bipy group. It was found that in order to successfully attach (22) to the upper rim of the calix[4]arene derivative 6 equivalents of $(\mathbf{2 2})$ was required.<smiles>Cc1ccnc(-c2cc(C)ccn2)c1</smiles><smiles>Cc1ccnc(-c2cc(C=O)ccn2)c1</smiles><smiles>Cc1ccnc(-c2cc(CBr)ccn2)c1</smiles>

(22)

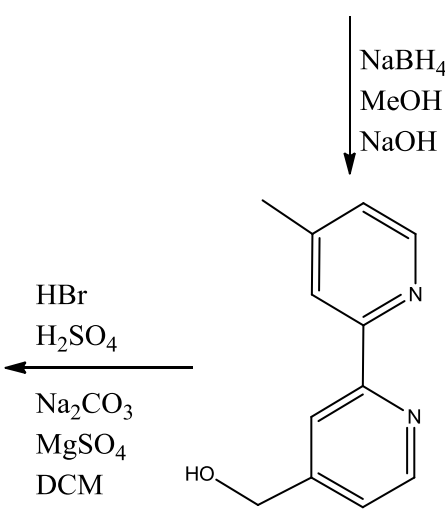

(21)

Figure 92: Synthesis of (20) - (22)

With (22) synthesised, the next step was to attach it to the upper rim of the (30) to produce a new metal binding site in compound (32). Compound (32) was produced 
as a brown waxy solid in moderate yields (66\%), (Figure 93). The first time this reaction was attempted it was carried out at a 2:1 bipy to calix[4]arene ratio but it was noted that the reaction did not proceed as expected. From the ${ }^{1} \mathrm{H}$ NMR spectrum it appeared that only one of the upper rim arms had been functionalised with the bipy unit. This was seen by the integration of the bipy linking arm which only integrated to two hydrogens, had the bipy unit attached on both upper rim sites the integration would have indicated four hydrogens. When this was increased to a 6:1 ratio, of bipy to calix[4]arene derivative, functionalisation at both arms was observed as indicated by the integrations in the ${ }^{1} \mathrm{H}$ NMR spectrum. It is suggested that this is due to the formation of acid salts around the bipy unit during its formation which did not appear in the ${ }^{1} \mathrm{H}$ NMR spectrum. Due to the excessive number of peaks present in the following compounds, a short-hand notation of $\mathrm{s}, \mathrm{d}$ and $\mathrm{t}$ will be used to designate singlet, doublet and triplet respectively.

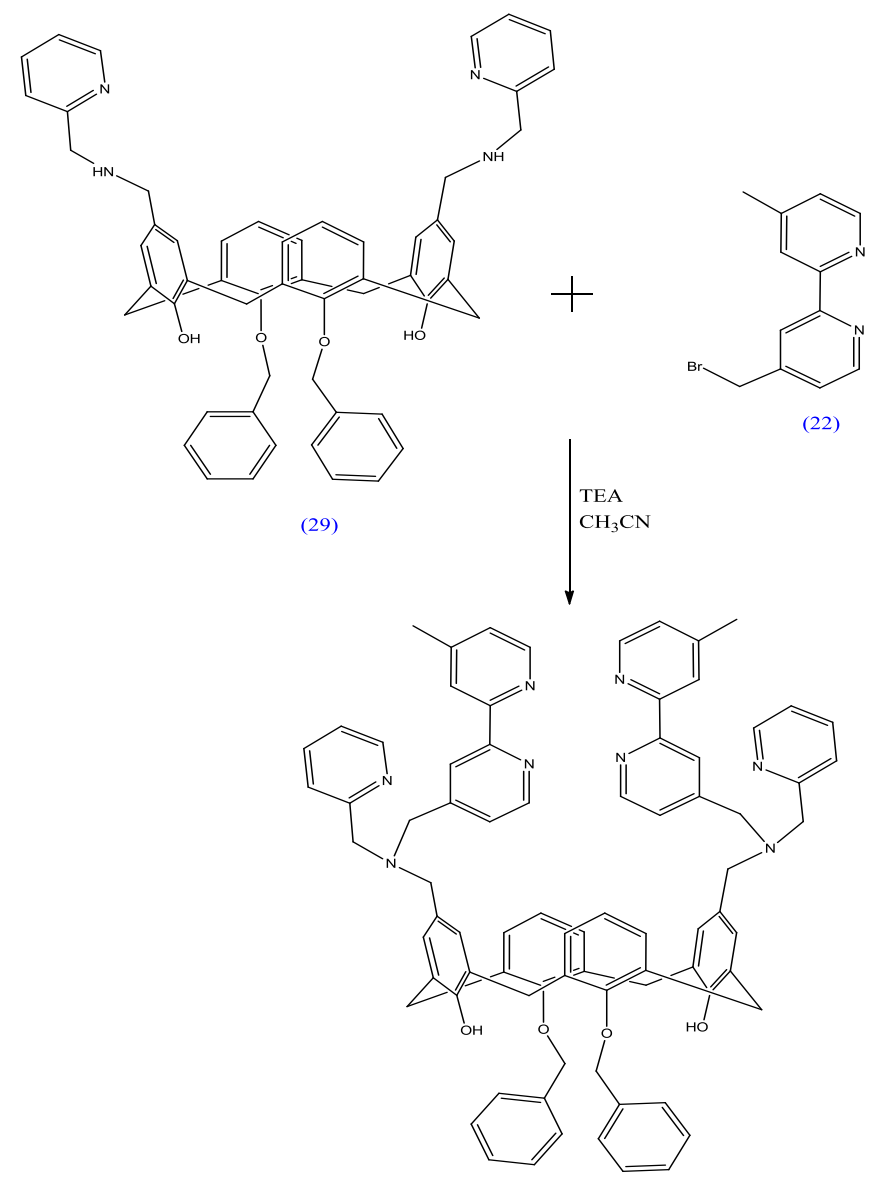

(32) 
The ${ }^{1}$ H NMR spectrum of (32), (Figure 94), showed the bipy peaks at 8.63 (d), 8.54 (d), 8.41 (s), 8.22 (d), 7.38 (d), 7.14 (s) ppm, the pyridyl at 8.63 (d), $7.65(\mathrm{t}), 7.63(\mathrm{t})$, 7.36 (d) ppm. The lower rim benzyl peaks were observed at 7.48 (d), 7.31 (t) and $7.14(\mathrm{t}) \mathrm{ppm}$ respectively. The hydroxyl singlet was seen at $7.82 \mathrm{ppm}$ the appearance of two doublets and two triplets occurred again for the aromatic calix[4]arene protons suggesting conformers may be present. The lower rim benzyl arm appeared as a singlet at $5.03 \mathrm{ppm}$. The upper rim pyridyl arms occurred as two singlets at 4.83 and $4.63 \mathrm{ppm}$, while the new bipy arm, also a singlet, appeared at $3.95 \mathrm{ppm}$. The methyl group was seen as a singlet at $2.44 \mathrm{ppm}$. Three doublets were clearly distinguished for the methylene protons along with another peak which appears to be a distorted triplet. However this could also be said to be a singlet which is undergoing conversion to a doublet i.e. in the rotation from the partial cone to the 1,3-alternate form. Unfortunately, low temperature NMR studies were not carried out to further examine this due to lack of specialised equipment so this cannot be proved at this time. Considering the peak integrates to eight hydrogens, it is possible that we have captured a 'snapshot' in mid-rotation. The ${ }^{13} \mathrm{C}$ NMR spectrum showed thirty different signals for the non-equivalent carbons.

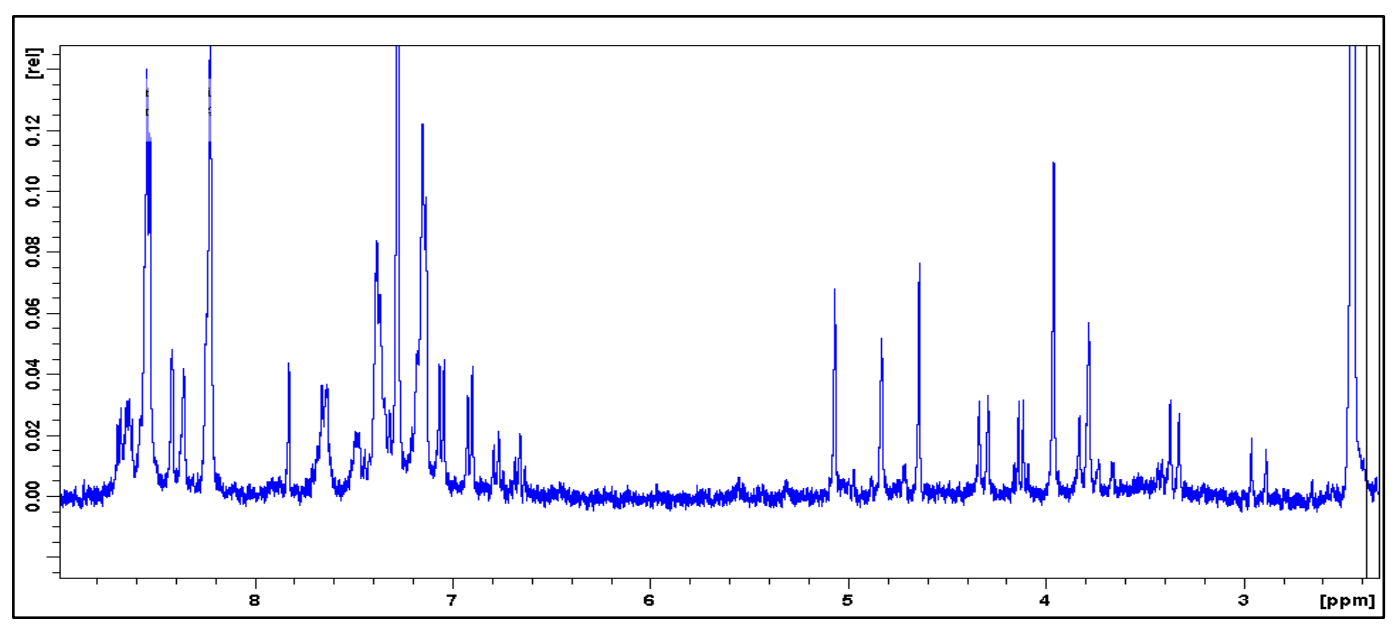

Figure 94: ${ }^{1} \mathrm{H}$ NMR spectrum of (32)

The major disadvantage with this synthesis is that the bipy group was not seen to attach to the calix[4]arene derivative every time. Quite often the reaction would not proceed and if attachment was achieved the compound broke down after a few days. 
This is believed to be due to the steric hindrance between the pyridyl and bipy groups on the upper rim as both are connected to the amine by only one carbon linker and when any metal ion complexation was attempted (will be discussed in more detail at a later stage) the bipy group was found to detach itself from the calix[4]arene unit.

Following the success of this line of reactions it was decided to repeat the basic idea but with longer linking groups on the upper rim. It was hoped that the addition of the extra carbon atom would give more flexibility to the upper rim and allow the bipy unit to attach, and remain attached, with more ease. To this end compounds (36) and (37) were synthesised by reacting the precursor with 2-(aminoethyl)-pyridine overnight under inert conditions (Figure 95).

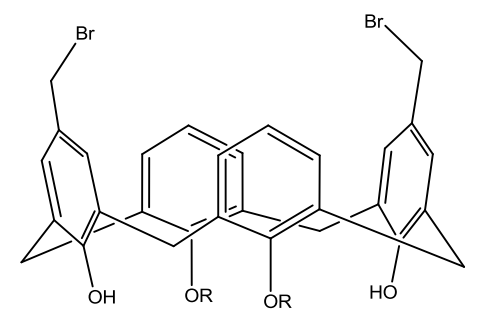

(29) $\mathrm{R}=$ benzyl

(33) $\mathrm{R}=$ pyridyl

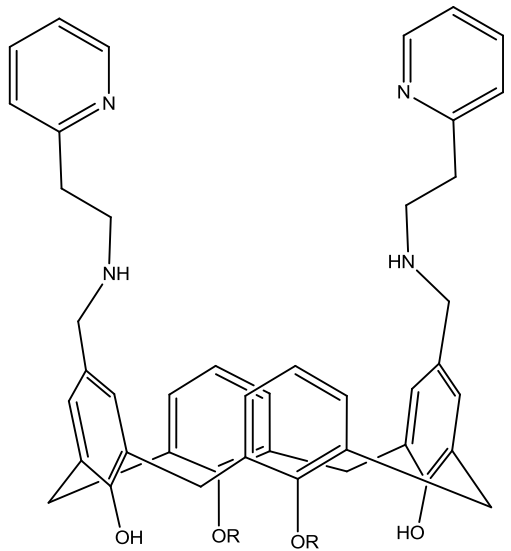

(36) $\mathrm{R}=$ pyridyl (37) $\mathrm{R}=$ benzyl

Figure 95: Synthesis of (36) \& (37)

Compound (36) was obtained as a brown-red waxy solid in high yields (95\%). The IR analysis showed the hydroxy band at $3404 \mathrm{~cm}^{-1}$, the $v_{\mathrm{NH}}$ at $2916 \mathrm{~cm}^{-1}$ and two pyridyl NCH bands at $1591 \mathrm{~cm}^{-1}$ and $1569 \mathrm{~cm}^{-1}$. The ${ }^{1} \mathrm{H}$ NMR spectrum exhibited the eighteen desired peaks as well as several extra peaks indicating that something was occurring in solution. The lower and upper rim pyridyl's appeared at 8.62, 7.75, 7.63, $7.41 \mathrm{ppm}$ and 8.51, 7.59, 7.08 and $7.06 \mathrm{ppm}$ respectively in the doublet, triplet, 
doublet, triplet fashion. The aromatic calix[4]arene signals were observed at 6.99, $6.89,6.62 \mathrm{ppm}$, but several extra signals were also observed. In the bridging region the lower and upper rim linking arms were seen as singlets at 5.14 ppm and 3.94 ppm. The two new triplets for the aminoethyl linkage were seen at 3.04 and 2.94 ppm and the methylene doublets were located at 4.36 and $3.33 \mathrm{ppm}$. Again several more signals due to the bridging methylene proton were also present. The ${ }^{13} \mathrm{C}$ NMR spectrum showed the desired twenty-three non-equivalent carbon signals as well as extra peaks which could be indicating the presence of conformers.

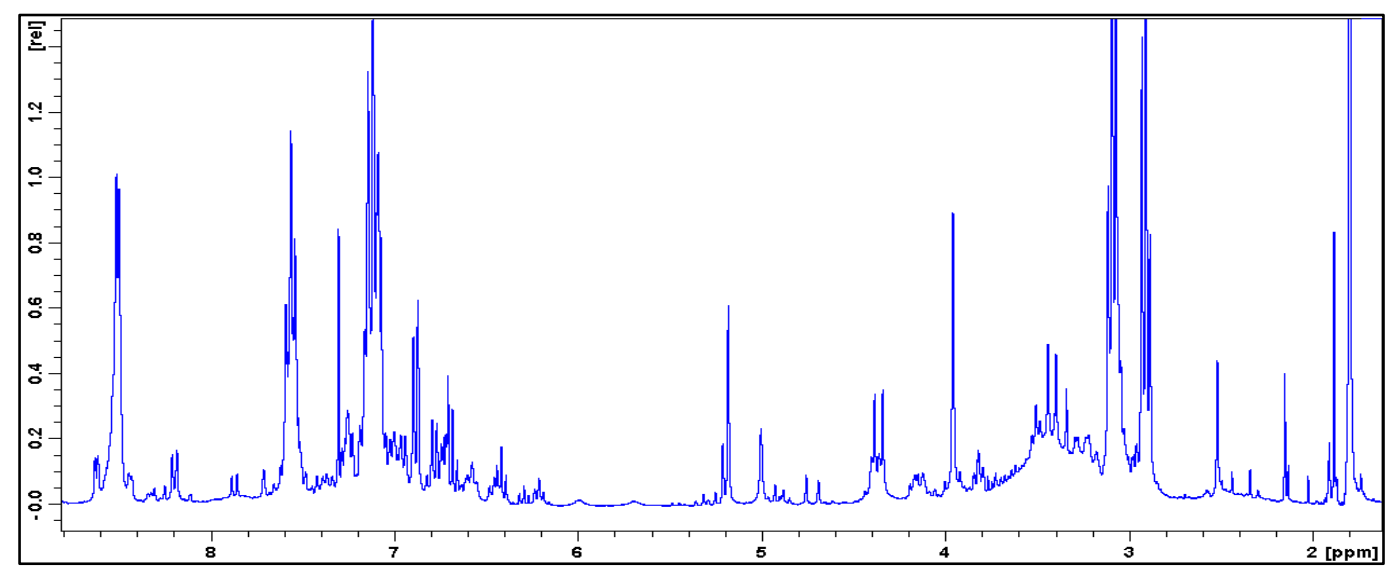

Figure 96: ${ }^{1} \mathrm{H}$ NMR spectrum of (36)

Compound (37) was obtained as a dark yellow waxy solid also in high yields (95\%). The IR analysis of (37) was similar to (36); the hydroxyl band occurred at $3394 \mathrm{~cm}^{-1}$, the $v_{\mathrm{NH}}$ at $3351 \mathrm{~cm}^{-1}$ and the upper rim pyridyl $v_{\mathrm{NCH}}$ at $1591 \mathrm{~cm}^{-1}$. The ${ }^{1} \mathrm{H}$ NMR spectrum (Figure 97) showed the upper rim pyridyl peaks at 8.53, 7.57, 7.49 and $7.46 \mathrm{ppm}$, the benzyl peaks at $7.70,7.43$ and $7.37 \mathrm{ppm}$ respectively. Once more multiple peaks were observed for the calix[4]arene aromatic signals at 7.07, 7.05, $7.00 \mathrm{ppm}$ for the doublets, $7.15 \mathrm{ppm}$ and $7.03 \mathrm{ppm}$ for the singlet's, and 6.95, 6.66 and $6.50 \mathrm{ppm}$ for the triplets. Signal overlap was abundant therefore it's likely that further duplicates may be found, however, only clear signals are listed here. In the bridging region of the spectrum, the lower rim benzyl linking arm was located as a singlet at $5.02 \mathrm{ppm}$ and the upper rim arms, also as singlets at 4.71 and $3.79 \mathrm{ppm}$. 
The two new triplets for the upper rim pyridyl arm were seen at 3.07 and $2.99 \mathrm{ppm}$. A final broad singlet was observed at $1.78 \mathrm{ppm}$ for the $\mathrm{NH}$ of the upper rim arm. The ${ }^{13} \mathrm{C}$ NMR spectrum showed the expected twenty different peaks along with multiple others.

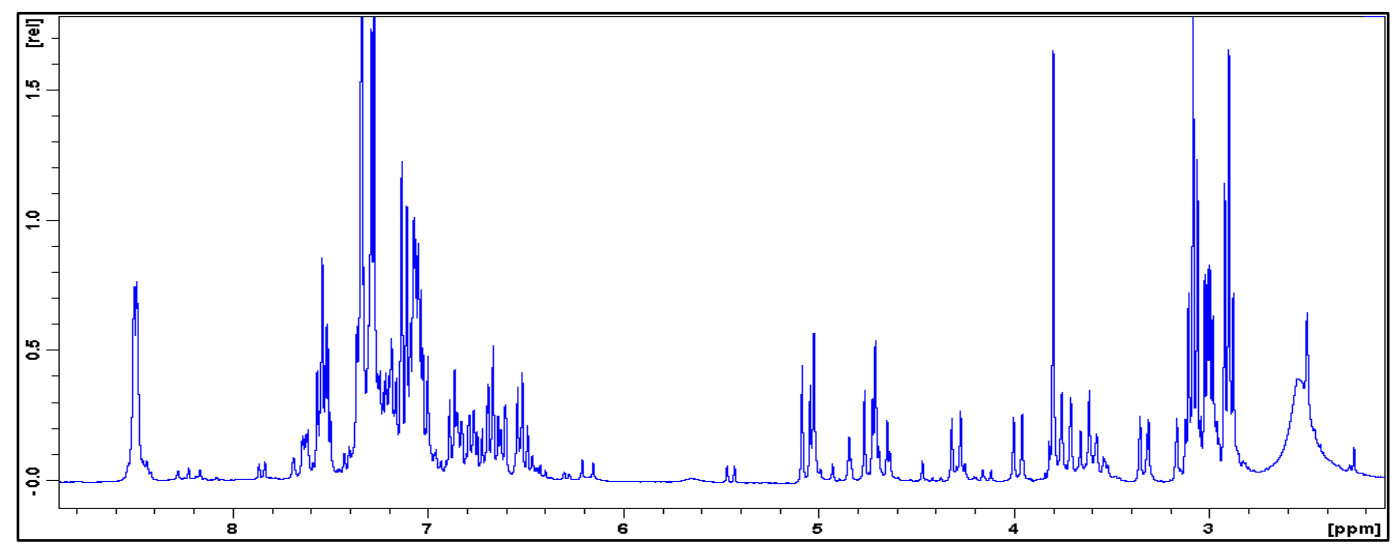

Figure 97: ${ }^{1} \mathrm{H}$ NMR spectrum of (37)

Seeing how the presence of multiple peaks was not a once off occurrence and that more than just conformer peaks were being observed it was decided to conduct further investigations into the compounds.

\subsection{Structural Characterisation of (37)}

Given the excessive number of peaks in the proton NMR spectra it was proposed that some form of self-assembly was occurring in solution. To investigate this further crystal growing was attempted. All obtained crystals were recovered as long branchlike fibres and as such were unsuitable for X-ray studies. Instead, SEM was performed on the crystals to try to clarify the situation. The samples were observed by JSM-5610LV SEM at $20 \mathrm{kV}$. Several of the compounds were examined under SEM and compound (31) was found to give a very detailed image (Figure 98) which clearly shows a unique 'stacking' structure that is occurring throughout the calix[4]arene unit. 


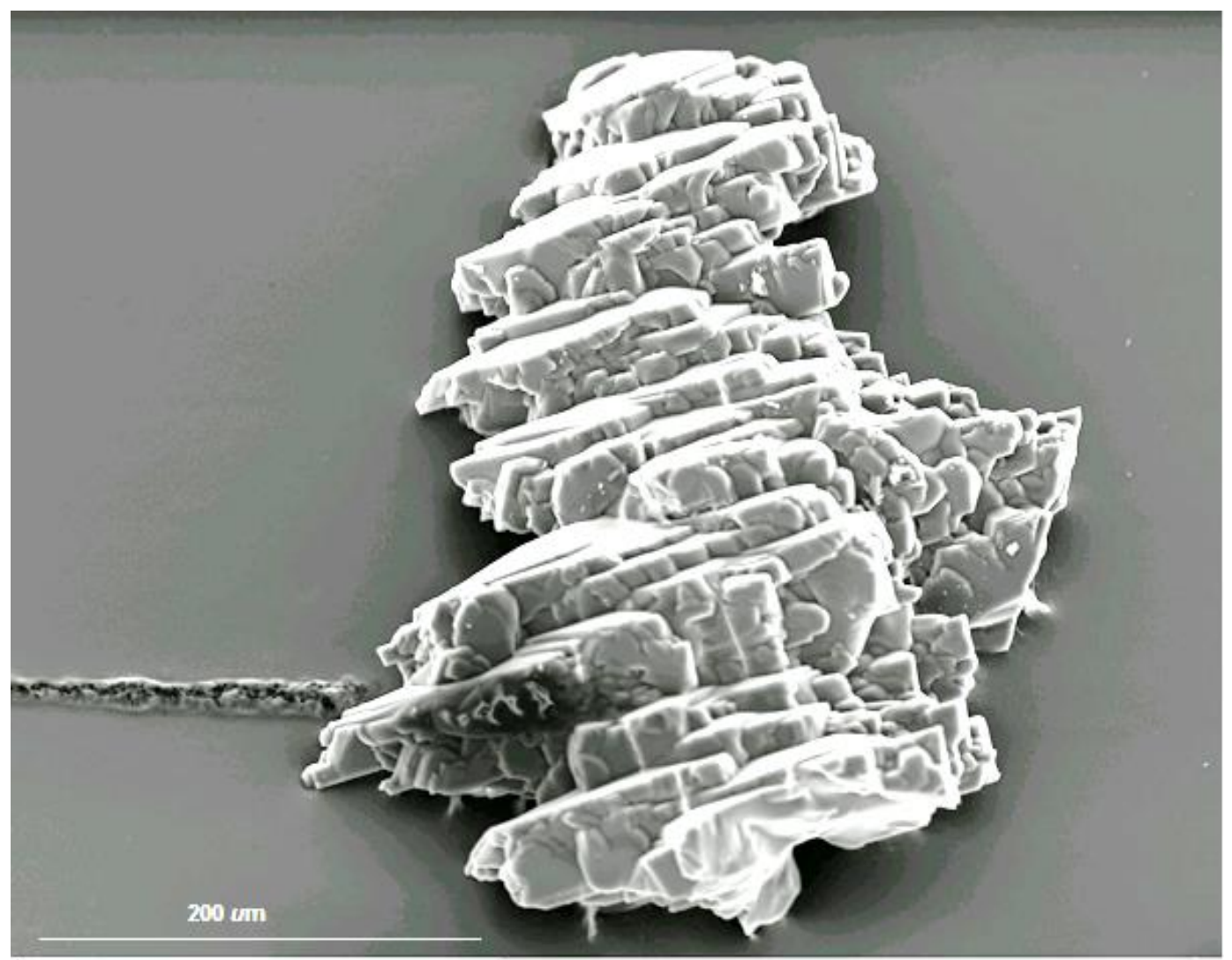

Figure 98: SEM image of (31) @ 1.5Kv, WD 14 mm, X 100

Following on from this further NMR analysis was carried out. The first compound chosen for further analysis was compound (37). nOe NMR, serial dilution and high temperature studies were carried out in IT Tallaght Dublin on the $500 \mathrm{MHz}$ Bruker NMR machine.

\subsection{Self-assembly investigations of (37) and other benzyl compounds}

Analysis of compound (37) began with a series of nOe NMR experiments in which several of the main peaks from the proton NMR (Figure 99) were chosen to be irradiated and their interaction observed. 


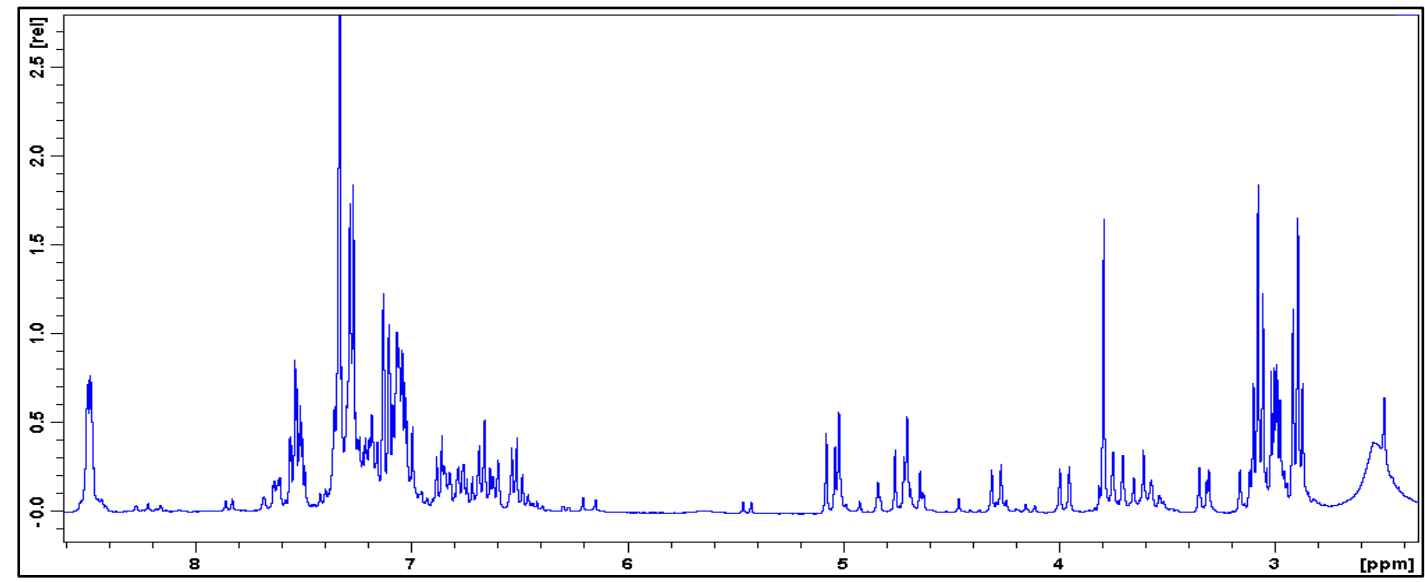

Figure 99: ${ }^{1} \mathrm{H}$ NMR spectrum of (37)

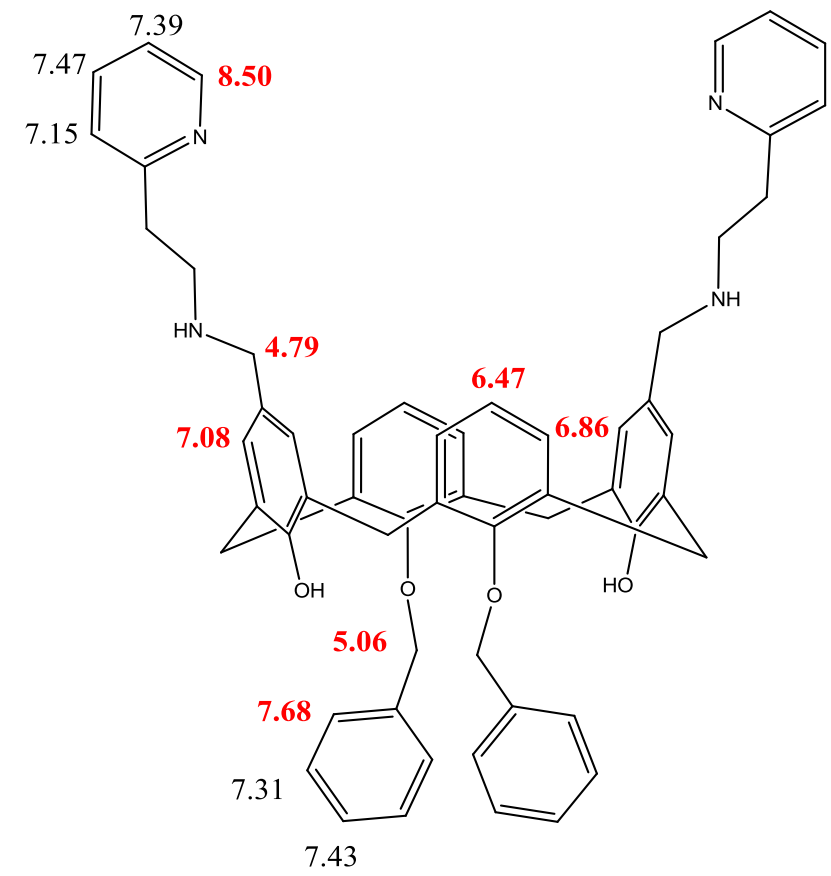

Figure 100: Compound (37) peaks chosen for nOe NMR studies. Numbers in red indicate points chosen for nOe studies.

Several points of interest were examined under nOe NMR using a $500 \mathrm{MHz}$ NMR (Figure 100). These tests were conducted to investigate if the calixarene itself was able to self-assemble, which would explain the presence of 'string-like' crystals. The first point chosen was related to the pyridyl $\mathrm{N}-\mathrm{CH}$ at $8.50 \mathrm{ppm}$. This point was irradiated with 256,000 scans. It was observed (Figure 101) that this peak interacts with both the bridging doublets at 4.35 and $3.39 \mathrm{ppm}$ indicating that the upper rim 
pyridyl group may either be folding back down into the calix[4]arene cavity itself or through binding with another molecule, or it could be interacting with the other molecules methylene bridges. The latter explanation seems more plausible as it is possible that through $\mathrm{H}$-bonding with the pyridyl ring of one molecule and the $\mathrm{OH}$ of another molecule this could bring the two molecules close enough together to exhibit this kind of interaction. It was also seen that this peak interacts with the doublet of the pyridyl at $7.15 \mathrm{ppm}$. Interaction is also seen with the peak at $5.10 \mathrm{ppm}$ which corresponds to the lower rim benzyl arm. This again supports the theory of selfassembly of two molecules via the interaction between the pyridyl and the hydroxyl. It is also possible that some interaction exists between the pyridyl and the bridge of another pyridyl, as there appears to be some interaction with the peak at $3.02 \mathrm{ppm}$ which marks the position of the first $\mathrm{CH}_{2}$ triplet of the $\mathrm{NH}-\mathrm{CH}_{2}-\mathrm{CH}_{2}$-pyridyl arm. This could simply be due to the flexibility of the group itself, or interaction between another molecule or a combination of both. Further peaks on the pyridyl were not investigated due to time restraints, but it is possible that some of the pyridyl peaks may interact with the benzyl ortho to the $\mathrm{OH}$ which would further confirm this hypothesis.

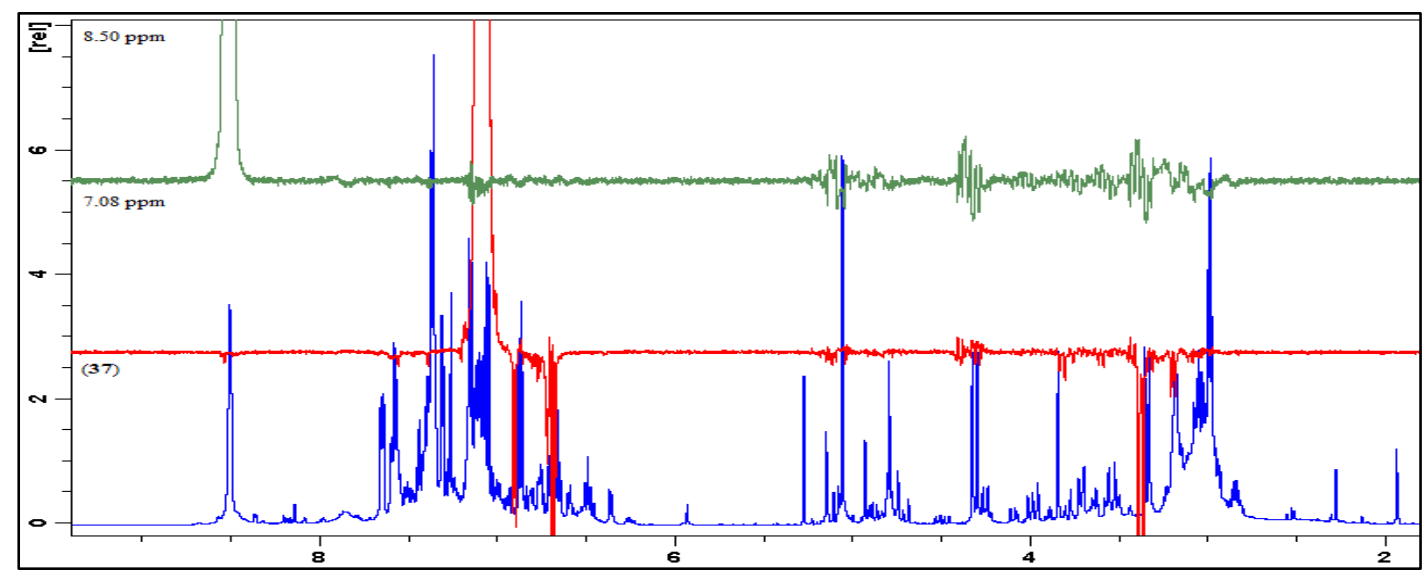

Figure 101: nOe NMR spectra for (37) at 8.50 and $7.08 \mathrm{ppm}$. The green trace represents the interactions at $8.50 \mathrm{ppm}$ and red trace represents the interactions at $7.08 \mathrm{ppm}$.

The second peak examined was at $7.08 \mathrm{ppm}$ which corresponds to the aromatic singlet of the calix[4]arene ring. This showed the expected interactions with the doublet at about $6.9 \mathrm{ppm}$ and the triplet at $6.69 \mathrm{ppm}$ of the neighbouring ring in the 
calix[4]arene complex. Strong interactions with the equatorial bridging $\mathrm{CH}_{2}$ group located at $3.37 \mathrm{ppm}$, and minor interactions with the axial bridging unit were observed (Figure 101).

The third peak was at $6.86 \mathrm{ppm}$ corresponding to the doublet on calixarene (Figure 102). Interactions were seen at $6.73 \mathrm{ppm}$ which could represent another doublet through self-assembly, as well as interaction at $7.11 \mathrm{ppm}$ indicating interaction with the singlet of the calixarene ring. Small interactions at $7.19 \mathrm{ppm}$ and $7.47 \mathrm{ppm}$ were noticed most likely to the doublet and triplet of the pyridyl ring respectively. A strong interaction at $8.39 \mathrm{ppm}$ corresponding to the pyridyl $\mathrm{N}-\mathrm{CH}$ doublet was observed. In the bridging region, a strong interaction was seen at the $3.20 \mathrm{ppm}$, and a small interaction at the $4.20 \mathrm{ppm}$ representing the methylene bridging protons. Small interactions are observable at $5.08 \mathrm{ppm}$ which is the lower rim benzyl and the upper rim pyridyl-methylene protons at $4.77 \mathrm{ppm}\left(\mathrm{C}_{2}-\mathrm{NH}\right)$, indicating that some interaction is occurring between these and the calix[4]arene aromatic doublet.

The fourth peak for this compound that was tested was at $6.48 \mathrm{ppm}$ corresponding to the triplet of the calixarene (Figure 102). A small interaction is observed at $6.63 \mathrm{ppm}$ corresponding to the doublet of the calixarene and another interaction at $6.82 \mathrm{ppm}$ corresponding to the another doublet of a calix[4]arene unit. A small interaction was seen at $5.04 \mathrm{ppm}$ indicating interaction with the lower rim benzyl linking arm. Several interactions were seen in the bridging region, interactions with two sets of doublets at 4.39, 4.29 and 3.42, $3.32 \mathrm{ppm}$ respectively which may indicate conformers as well as self-assembly.

The fifth peak of this compound to be irradiated was at $5.06 \mathrm{ppm}$ corresponding to the $\mathrm{CH}_{2}$ of the benzyl moiety (Figure 102). This showed several interactions in the aromatic region as well as the bridging region. As expected it interacts with the ortho $\mathrm{CH}_{2}$ of the benzyl group itself at $7.68 \mathrm{ppm}$. Smaller interactions at 7.56 and 7.42 ppm were also seen corresponding to the two pyridyl triplets. This may suggest arylaryl interactions between the pyridine ring and the benzyl ring. 


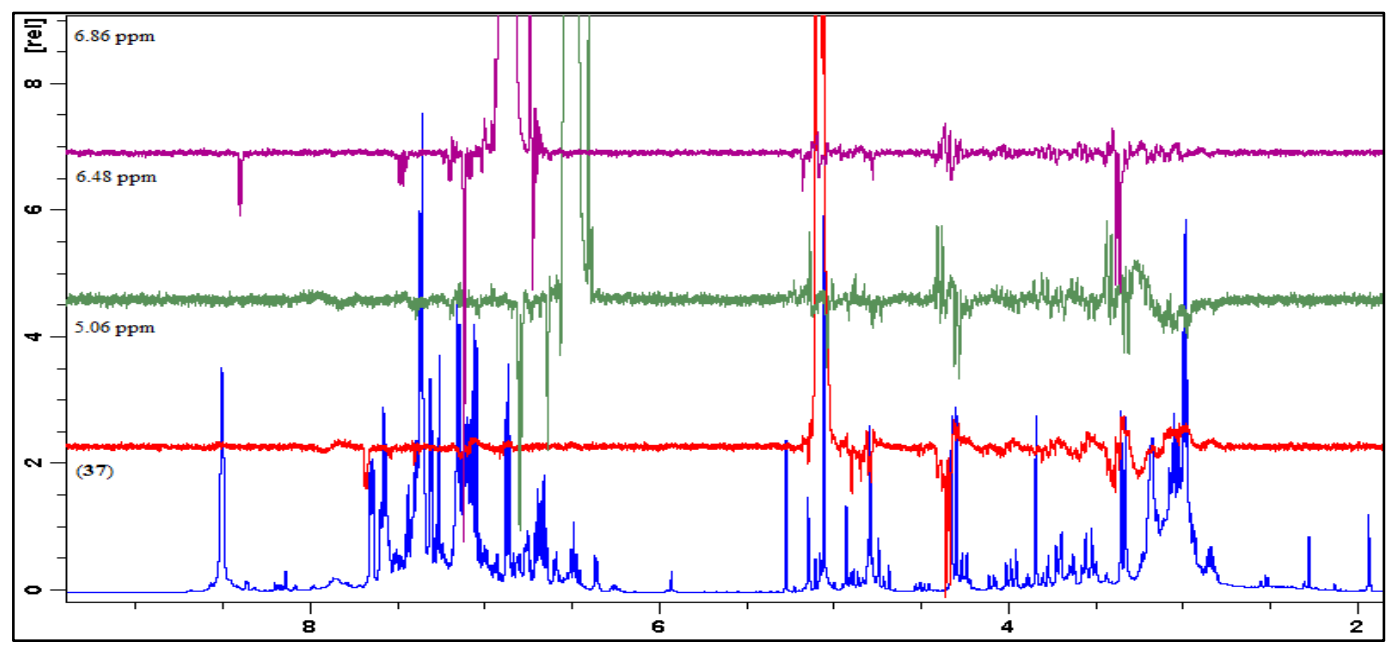

Figure 102: nOe NMR for (37) at 6.86, 6.48 and $5.06 \mathrm{ppm}$. The purple trace represents the interactions observed at $6.86 \mathrm{ppm}$, the green represents interactions at $6.48 \mathrm{ppm}$ and the red interactions at $5.06 \mathrm{ppm}$.

The final peak chosen was 4.79 ppm representing the upper rim $\mathrm{C}_{2}-\mathrm{NHR}$ methylene peak. It showed an interaction with $5.06 \mathrm{ppm}$ indicating the lower rim benzyl group. An interaction was also seen at $6.62 \mathrm{ppm}$ and $6.86 \mathrm{ppm}$ which is the doublet region of the aromatics. Interaction was also observed with the singlet of the ring at $7.08 \mathrm{ppm}$. This is the expected interaction for this peak with the ring aromatics. A small interaction was observed with $7.15 \mathrm{ppm}$, which represents the doublet of the pyridyl as well as the triplets of the pyridyl at $7.47 \mathrm{ppm}$ and at 7.39 ppm. This interaction between the upper rim arm and the upper rim pyridyl group could be caused by either the increased flexibility or by self-assembly with another calix[4]arene unit.

Figure 103 shows a summary of all the nOe NMR spectroscopy interactions seen for compound (37). It is clear from this that some of the interactions cannot be happening within the molecule, e.g. the interaction between the protons giving the doublet of the phenol ring and the lower rim benzyl linking arm due to steric hindrance. If this interaction was to occur within the molecule itself, then it would suggest that the benzyl unit would have to pass up through the annulus of the calix[4]arene ring itself, which would not be favoured ${ }^{11}$. 


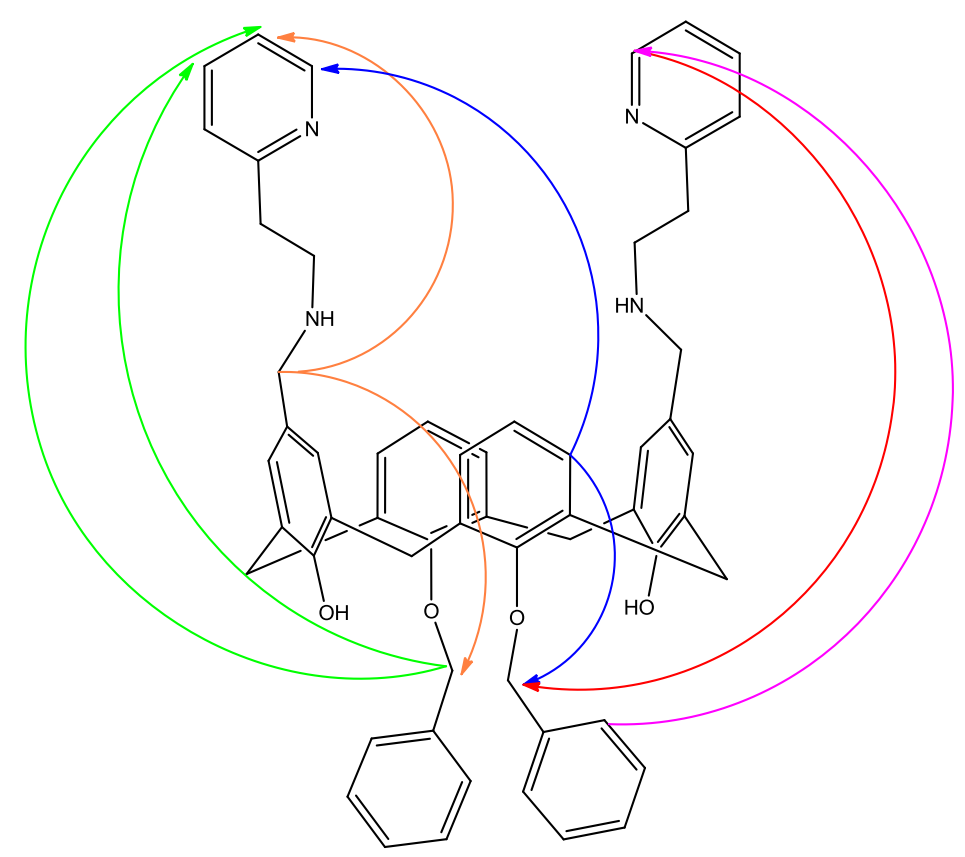

Figure 103: Summary of nOe NMR interactions for (37)

From the above illustration it can be seen that the lower rim benzyl linking arm (green) interacts with the upper rim pyridyl unit, the upper rim aminomethyl arm (orange) interacts with the pyridyl unit and the lower rim benzyl linking arm. The aromatic protons of the phenol ring (blue) interacts with the pyridyl group and the benzyl arm, the pyridyl NCH (red) interacts with the lower rim benzyl linking arm and the benzyl doublet (purple) interacts with the upper rim pyridyl group. Interactions with the bridging methylene protons have been omitted for clarity. It is much more plausible that self-assembly occurs between two molecules and that the self-assembly is occurring in either a heterodimerisation (hetero) or homodimerisation (homo) manner ${ }^{54,145}$ as demonstrated in Figure 104. 

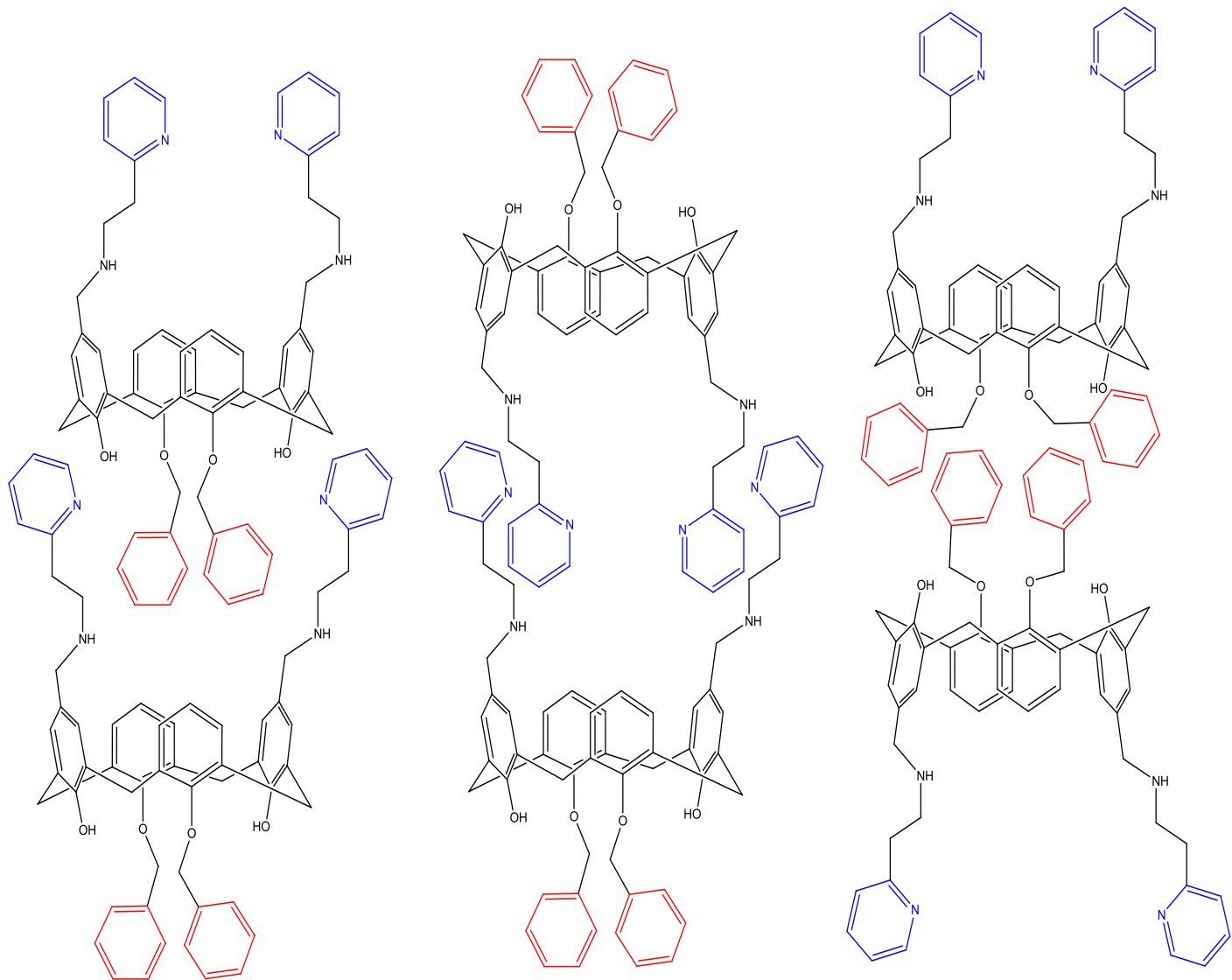

Figure 104: Heterodimerisation form (left) and Homodimerisation form (middle \& right) for compound (37)

Now that the occurrence of self-assembly is a strong possibility for (37), the next step was to see if it could be controlled with temperature. To this end high temperature proton NMR spectroscopy studies were conducted and it was found that even at $140{ }^{\circ} \mathrm{C}$ no change was seen in the spectrum, compared to the one obtained at $20{ }^{\circ} \mathrm{C}$ (Figure 105) which rules out temperature as a major contributor to conformational change. 


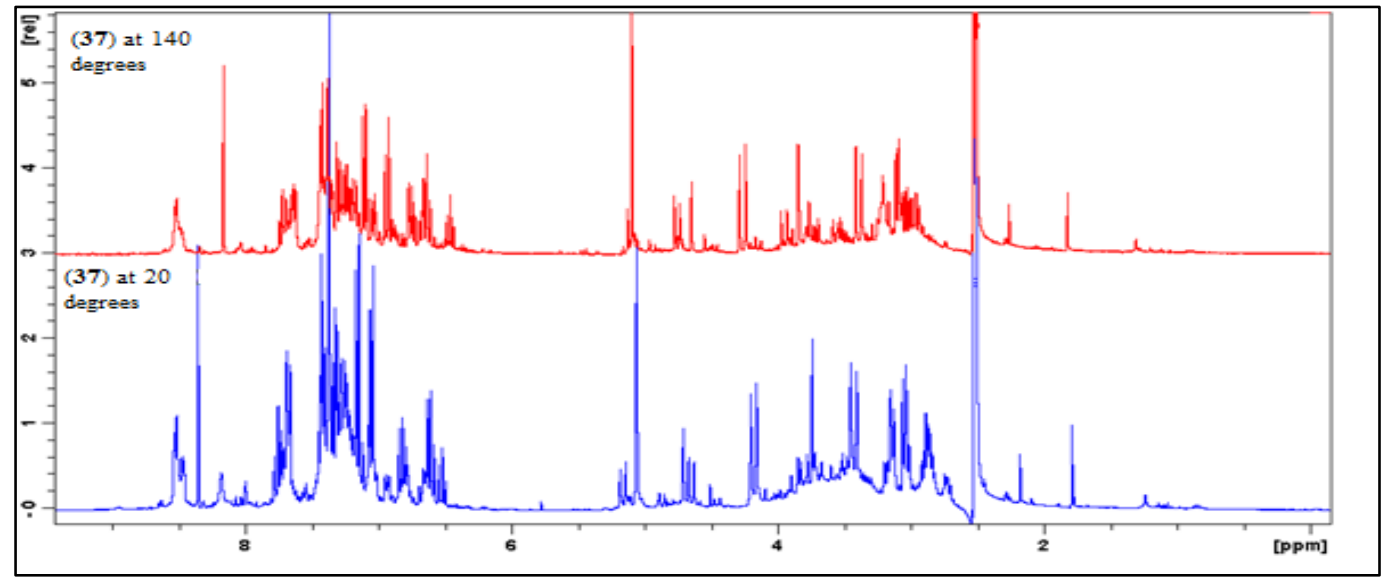

Figure 105: High temperature studies for compound (37)

As previously mentioned the three possible conformers are the cone, 1,3-alternate and the partial cone (Figure 106) and it is possible that any of these three may be undergoing self-assembly in solution. However as the doublet for the cone conformer is the most dominant in the majority of the proton NMR spectra for the compounds, it will be the focus of attention in this discussion.
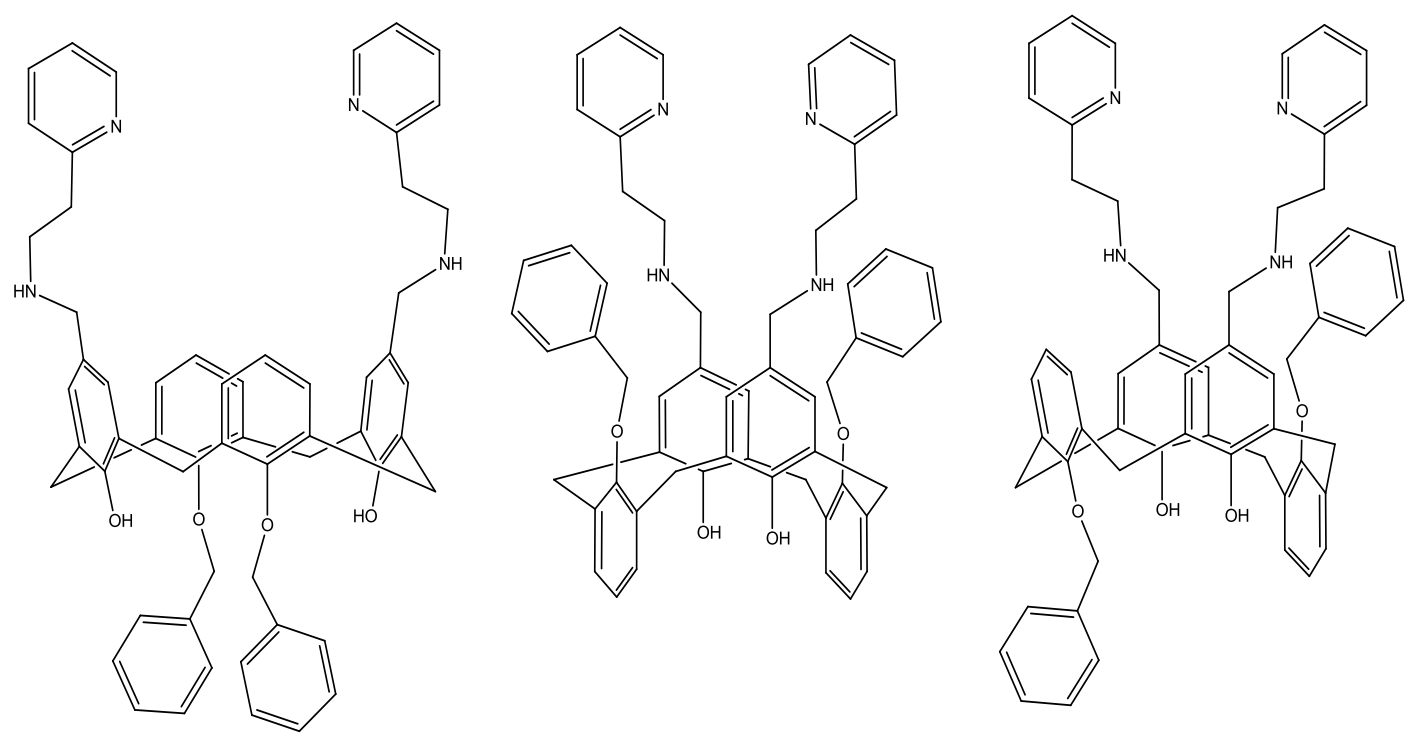

Figure 106: Three possible conformations in solution capable of undergoing self-assembly.

Following on from the high temperature studies a series of serial dilution proton NMR's were performed to see the effects of concentration. Solutions from 
millimolar to micromolar were prepared and examined using ${ }^{1} \mathrm{H}$ NMR spectroscopy (Figure 107).

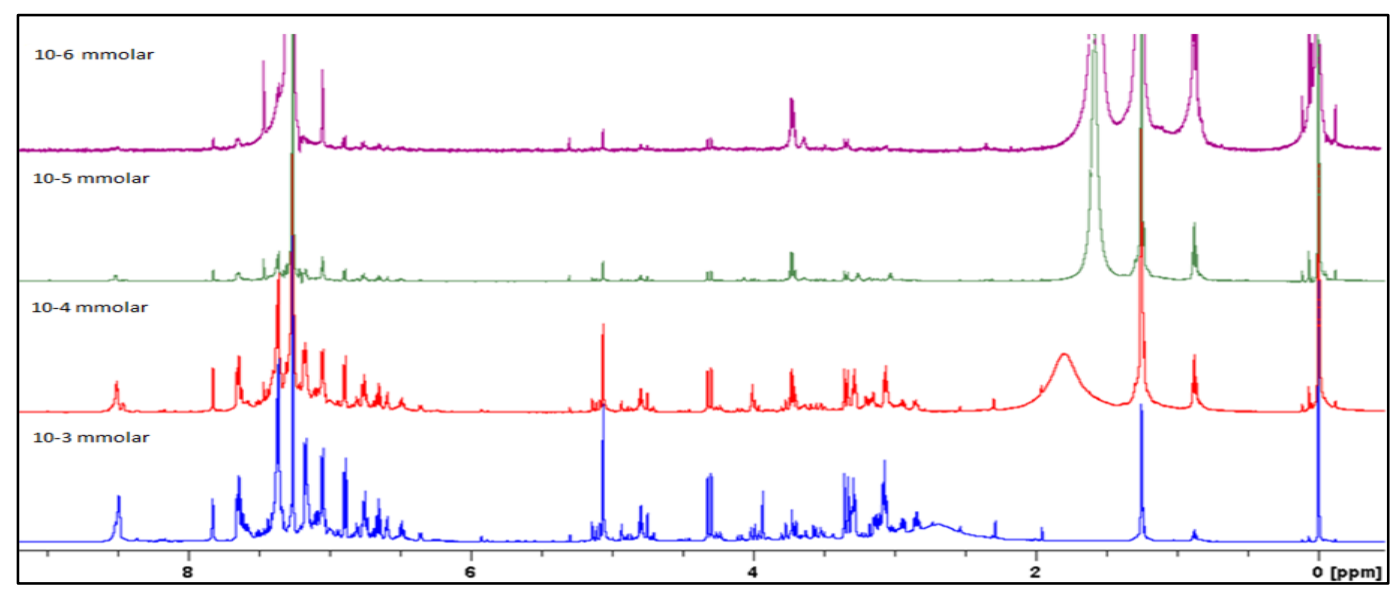

Figure 107: Serial dilution studies for compound (37) examined under ${ }^{1} \mathrm{H}$ NMR spectroscopy.

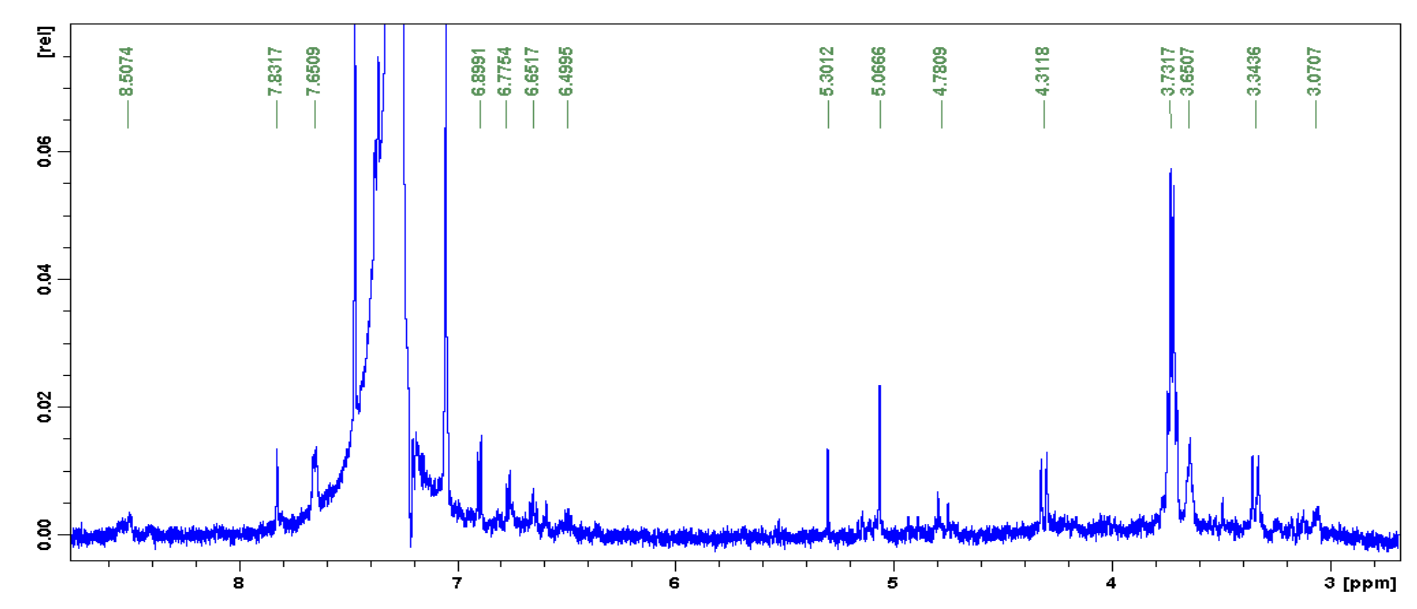

Figure 108: ${ }^{1} \mathrm{H}$ NMR spectrum of micromolar range of (37)

It can be clearly seen from the proton NMR spectra that as the concentration becomes lower the ${ }^{1} \mathrm{H}$ NMR spectra becomes much simpler with the 'disappearance' of several peaks (Figure 108). From this it is proposed that at high concentrations (millimolar) the calix[4]arene are close enough together (abundant in solution) to interact and form self-assembly constructs in the homodimerisation and heterodimerisation manner. As the concentration is lowered, the molecules move 
further apart from one another and as a result self-assembly becomes more difficult and the monomer form of the calix[4]arene unit dominants. From the NMR spectroscopy investigations it can be concluded so far that some form of selfassembly is occurring (homodimerisation, heterodimerisation or both), temperature seems to have no role in the assembly and concentration is the main contributor for self-assembly to occur. These serial dilution studies have also shown that the main conformer in solution at the micromolar level is the cone conformation. We believe that this is the favoured conformer in all these compounds and so we can exclude the possibility of conformer exchange in these compounds. Therefore, the extra signals that are observed in the more concentrated solutions of our compounds are due to self-assembly only.

Now with a fair insight into what was occurring the ${ }^{1} \mathrm{H}$ NMR spectra for the previous compounds were reviewed. Their complex spectra can now be attributed to selfassembly. nOe NMR spectroscopy studies were performed on some of these compounds and will be discussed later, (pg. 224 onwards).

Following on from the synthesis of compounds (36) and (37), work continued to attach another pyridyl arm to the upper rims of both of these compounds to create the two metal ion binding cages, compounds (46) and (39), (Figure 109). 


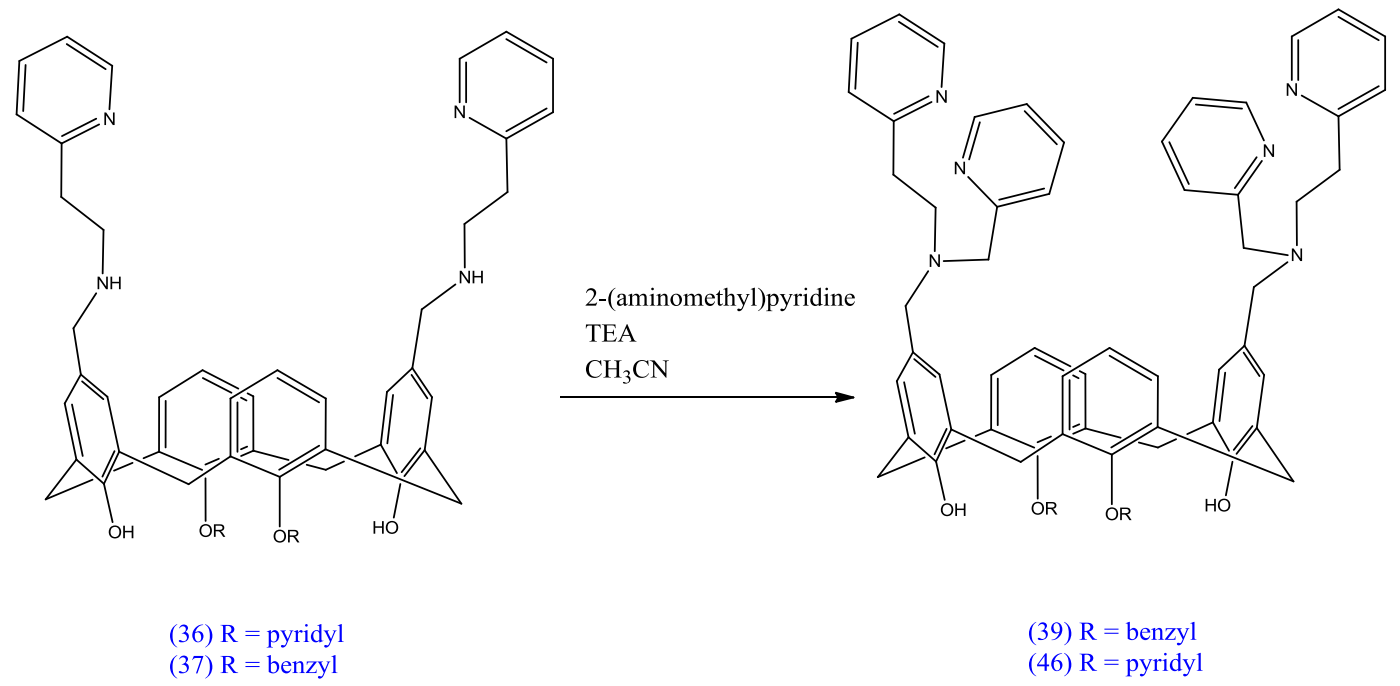

Figure 109: Synthesis of compounds (39) \& (46)

Compound (46) was obtained as a brown waxy solid in moderate yields (65\%). The IR data showed the $v_{\mathrm{OH}}$ band at $3431 \mathrm{~cm}^{-1}$ and the $v_{\mathrm{NCH}}$ band at $1591 \mathrm{~cm}^{-1}$. The ${ }^{1} \mathrm{H}$ NMR spectrum (Figure 110) showed the lower rim pyridyl protons at 8.70, 8.22, 7.86 and $7.60 \mathrm{ppm}$ as a doublet, triplet, doublet, triplet, the upper rim methylpyridyl protons were seen at $8.63,8.16$ and $7.57 \mathrm{ppm}$ as a doublet, triplet, triplet, while the other pyridyl doublet could not be clearly distinguished due to peak overlap. The upper rim pyridyl protons of the ethylpyridyl arm were viewed at 8.52, 7.55 and 7.53 as a doublet, triplet, doublet, again the remaining triplet was not clearly visible due to peak overlap. The calix[4]arene aromatic protons appeared at 7.06, 7.04 and 6.49 as a singlet, doublet, and triplet respectively. The pendant arms occurred at 5.18, 4.07 and $3.94 \mathrm{ppm}$ respectively for the lower rim pyridyl, upper arm linker and the upper rim methylpyridyl arm. The methylene triplets for the ethylpyridyl arm were observed at $3.05 \mathrm{ppm}$ and $3.00 \mathrm{ppm}$ and the methylene bridging protons appeared as two doublets at $4.24 \mathrm{ppm}$ and $3.44 \mathrm{ppm}$. The ${ }^{13} \mathrm{C}$ NMR spectrum showed 28 nonequivalent carbon atoms. 


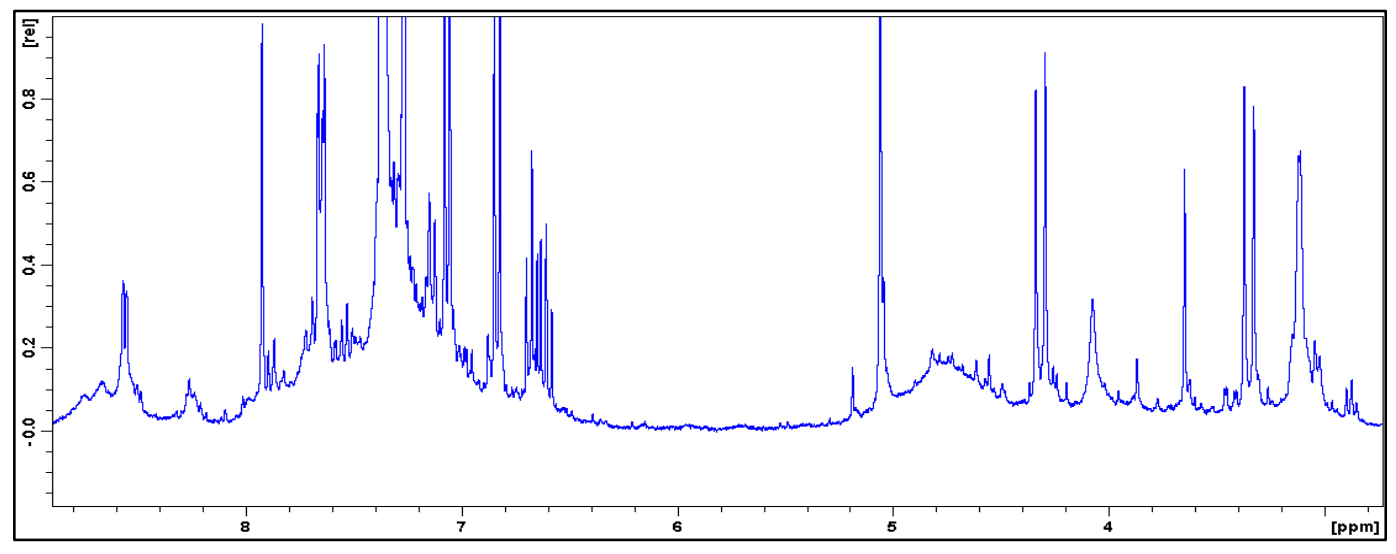

Figure 110: ${ }^{1} \mathrm{H}$ NMR of compound (46)

Compound (39) was afforded as a brown waxy solid in high yields (96\%). The IR data showed the $v_{\mathrm{OH}}$ band at $3429 \mathrm{~cm}^{-1}$ and the $v_{\mathrm{NCH}}$ band at $1591 \mathrm{~cm}^{-1}$. The ${ }^{1} \mathrm{H}$ NMR spectrum (Figure 111) showed the upper rim methylpyridyl protons at 8.50, 7.57, 7.38 and $7.31 \mathrm{ppm}$ as a doublet, triplet, doublet, triplet, respectively. The upper rim pyridine ethylpyridyl protons appeared in the same manner at 8.47, 7.50, 7.43 and $7.25 \mathrm{ppm}$ respectively. The lower rim benzyl protons occurred at $7.65 \mathrm{ppm}$ as a doublet, however, the triplet could not be seen clearly due to peak overlap. The calix[4] arene aromatic protons were viewed at 7.13, 7.08 and $6.76 \mathrm{ppm}$ as a singlet, doublet, and triplet respectively. The lower rim benzyl pendant arm appeared as a singlet at $5.06 \mathrm{ppm}$, and the upper rim pyridyl-methylene arm occurred at $4.75 \mathrm{ppm}$ also as a singlet. The methylpyridyl methylene arm was seen at $3.91 \mathrm{ppm}$ and the ethylpyridyl triplets were seen at 3.15 and $3.02 \mathrm{ppm}$. The bridging methylene protons appeared at $4.31 \mathrm{ppm}$ and $3.34 \mathrm{ppm}$ as two doublets. The ${ }^{13} \mathrm{C}$ NMR spectrum showed twenty-eight non-equivalent carbon atoms. 


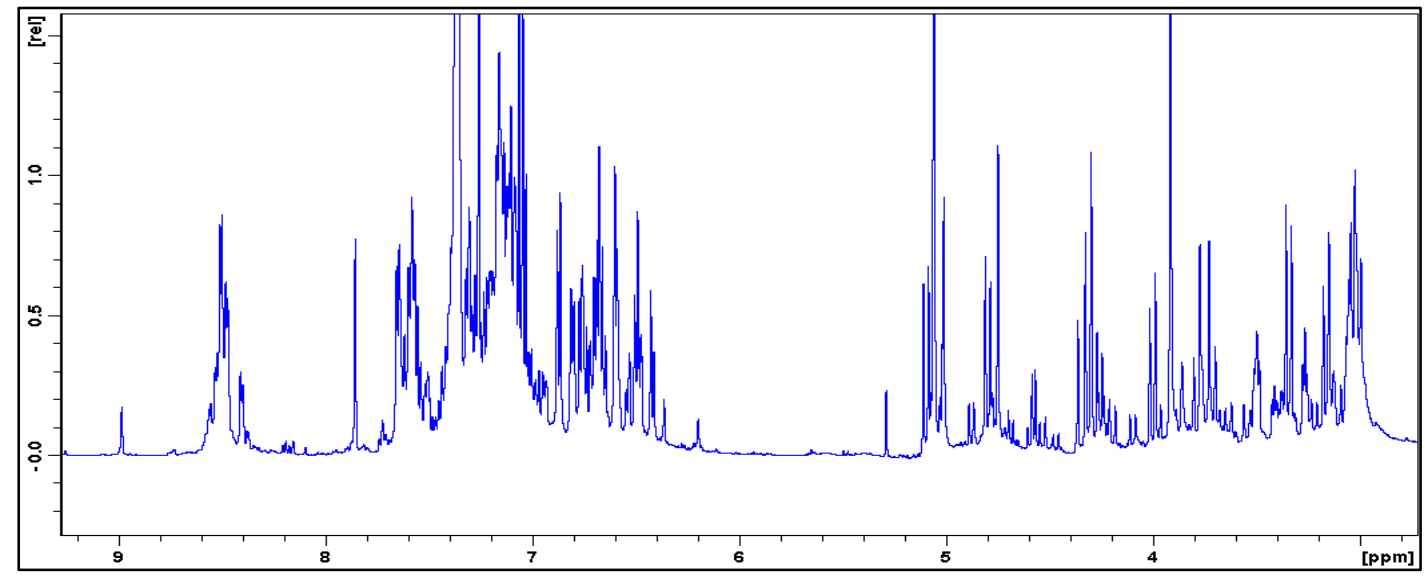

Figure 111: ${ }^{1} \mathrm{H}$ NMR for compound (39)

Serial dilution proton NMR spectroscopy was carried out on compound (39) from millimolar to micromolar and as was seen previously that same trend ensued (Figure 112). At high concentration the dimerisation could be seen quite clearly but as the concentration is lowered the monomer form starts to be the dominant, as the selfassembly process no longer takes place.

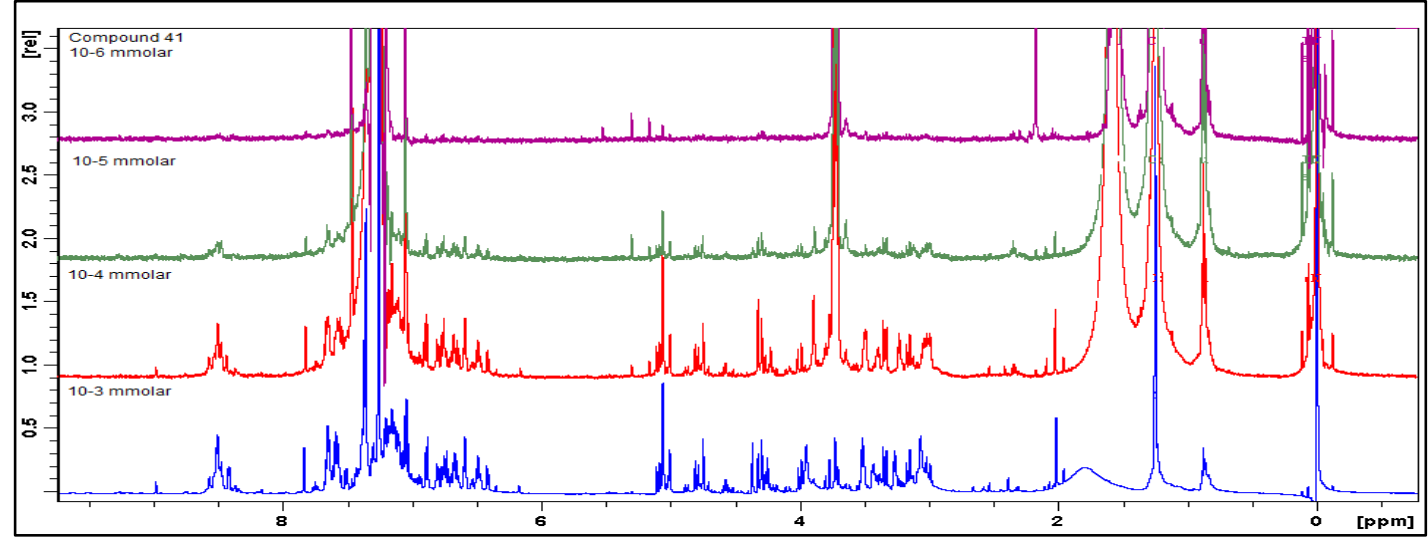

Figure 112: Serial dilution ${ }^{1} \mathrm{H}$ NMR of compound (39)

nOe NMR spectroscopy studies were also carried out on this compound to see if the self-assembly could again be discerned. In this set of nOe NMR studies only certain peaks were chosen such as the linking arms and the aromatic region of the calix[4]arene itself (Figure 113). 


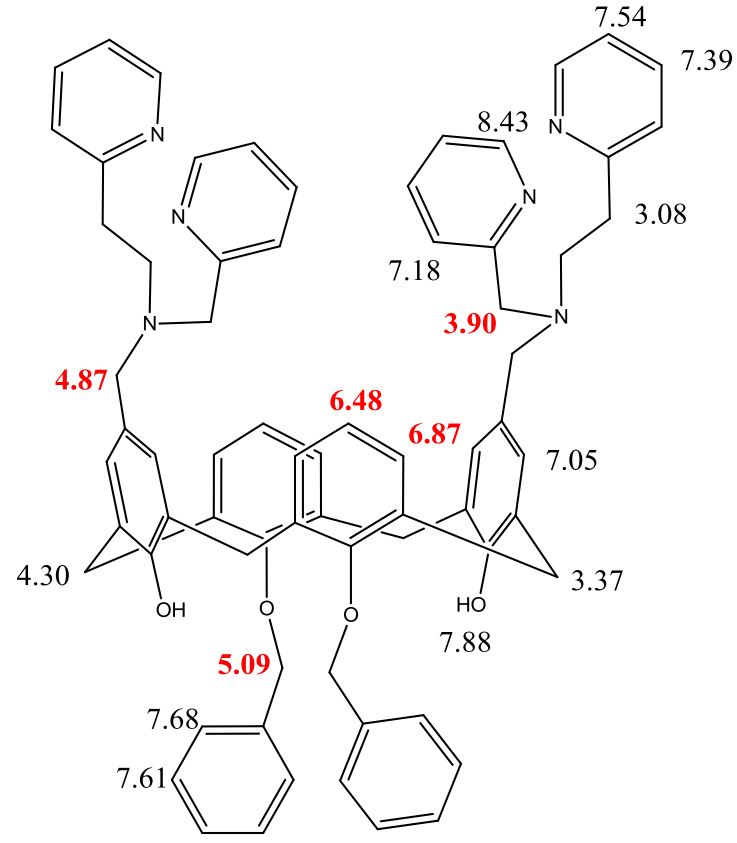

Figure 113: Chosen nOe points of (39)

Starting with the aromatic region, the aromatic proton corresponding to the doublet at $6.87 \mathrm{ppm}$ was initially chosen. Interactions were seen at 6.74 and $6.71 \mathrm{ppm}$ which could be a doublet in a different self-assembled form. There was an interaction with the triplet of the calix[4] arene ring at $6.67 \mathrm{ppm}$ and with the singlet at $7.05 \mathrm{ppm}$ as expected. Interaction seen at $7.18 \mathrm{ppm}$ for the methylpyridyl doublet along with a strong interaction at $3.37 \mathrm{ppm}$ indicating the equatorial bridging doublet. Two final interactions were seen at $4.78 \mathrm{ppm}$ and $5.09 \mathrm{ppm}$ for the upper rim aminomethyl linker and the lower rim benzyl linker. The next peak under investigation was the triplet of the aromatic calix[4]arene ring at $6.48 \mathrm{ppm}$. Interactions occurred at 6.51 , $6.53,6.56,6.63,6.70,6.78,6.79$ and $6.80 \mathrm{ppm}$ all of which would generally represent the aromatic region of the calixarene in various conformations. Interaction with the lower rim benzyl arm was viewed at $5.09 \mathrm{ppm}$ as well as two interactions at 4.82 and $4.79 \mathrm{ppm}$ which could correspond to the upper rim aminomethyl linkers in the self-assembled structure possibly in different conformations. The triplet was seen to interact with both the axial and equatorial bridging protons along with the methylpyridyl linking arm at 3.9 and $3.8 \mathrm{ppm}$ and with the two triplets of the ethylpyridyl group. 
The third peak to be examined was the lower rim benzyl arm at $5.09 \mathrm{ppm}$. It showed strong interactions with the upper rim aminomethyl arm at $4.79 \mathrm{ppm}$ as well as another interaction at $4.89 \mathrm{ppm}$ which could be the pyridyl arm of another calix[4]arene unit. Several interactions were observed in the bridging region at 4.34, 4.29 and $4.26 \mathrm{ppm}$ for the protons of the methylene bridges, indicating that two of these interactions belong to different calixarene molecules. Interaction in the aromatic region is also seen with the doublet of the calix[4]arene at $7.14 \mathrm{ppm}$ and with the pyridyl doublet at $7.34 \mathrm{ppm}$. Three other interactions were noticed at 7.54 , 7.61 and $7.68 \mathrm{ppm}$ representing the triplet of the ethylpyridyl, the benzyl triplet and the benzyl doublet respectively. Two final interactions corresponding to the hydroxy group at $7.88 \mathrm{ppm}$ and the methylpyridyl $\mathrm{NCH}$ at $8.43 \mathrm{ppm}$ were also seen.

The fourth peak to be viewed was the upper rim aminomethyl linker at $4.87 \mathrm{ppm}$. It showed strong interaction with the lower rim benzyl arm at $5.07 \mathrm{ppm}$ as well as the aromatic proton which gave the doublet and triplet of the calix[4]arene unit at 6.83 and $6.63 \mathrm{ppm}$. The final observed in this case was at $3.73 \mathrm{ppm}$ indicating the methylpyridyl linking arm. The fifth and final peak to be looked at was methylpyridyl linker at $3.90 \mathrm{ppm}$ and strong interactions with the triplet of the pyridyl group at $3.08 \mathrm{ppm}$ was seen and expected. Interactions with the bridging doublets are also seen at 3.43 and $4.30 \mathrm{ppm}$, along with another doublet at $4.40 \mathrm{ppm}$ which is possibly the doublet of another calix[4]arene unit. An expected interaction with the aminomethyl arm was observed at $4.82 \mathrm{ppm}$ as well as the lower rim benzyl arm at $5.07 \mathrm{ppm}$. Interaction with a peak at $5.15 \mathrm{ppm}$ leads to the belief that another benzyl unit is interacting nearby. The final peak was located at $7.39 \mathrm{ppm}$ representing the triplet of the ethylpyridyl group. The data from this study can be used to support either the hetero or homo dimerisation form.

Following on from the previous work with the bipy unit whereby steric hindrance seemed to cause the bipy to detach from the upper rim, it was now attempted to attach it to the upper rim of compound (37), to create a cage metal ion binding site on the upper rim, compound (38). It was hoped the extra carbon unit would allow 
greater flexibility and prevent the bipy from detaching (Figure 114). Compound (38) was obtained as a dark yellow waxy solid in low yields (14\%).

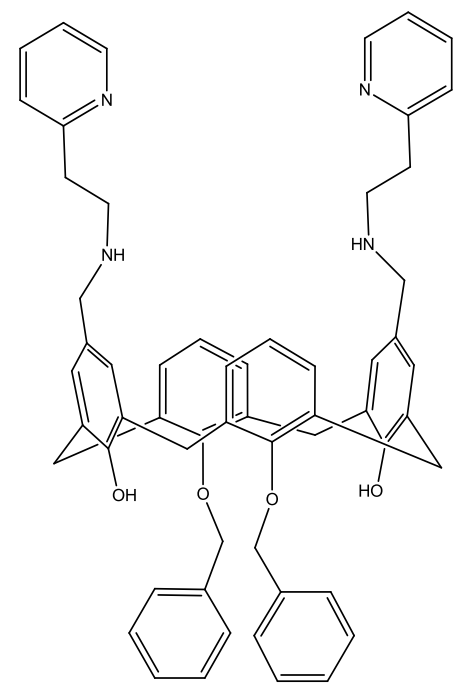

(37)

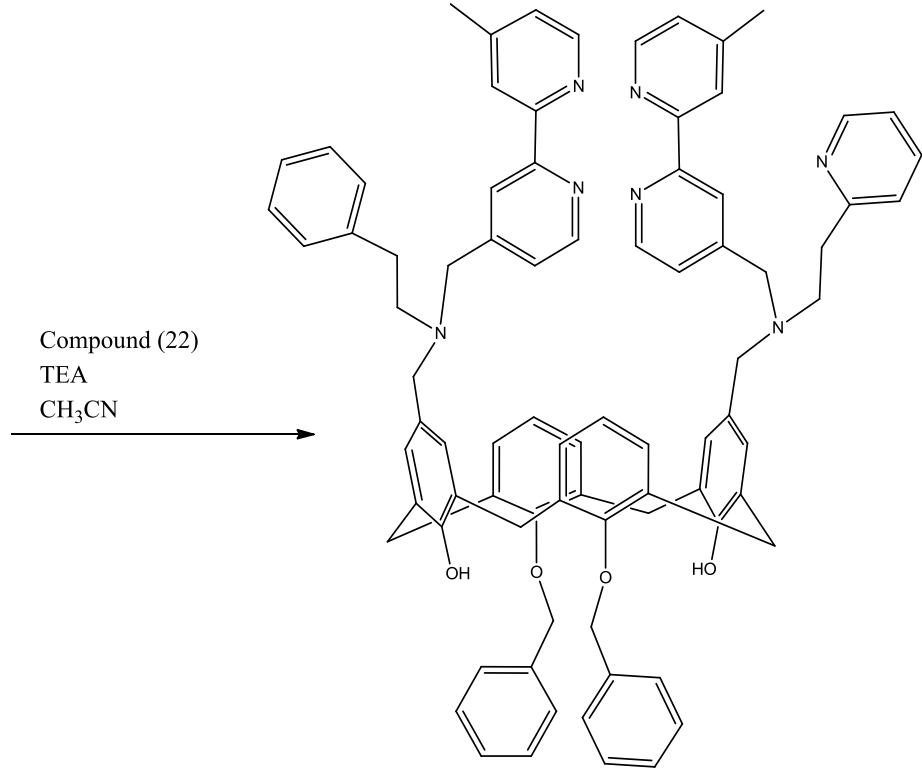

(38)

Figure 114: Synthesis of (38)

The IR analysis showed the position of the $v_{\mathrm{OH}}$ stretching band at $3401 \mathrm{~cm}^{-1}$ and the $v_{\mathrm{NCH}}$ stretching band at $1593 \mathrm{~cm}^{-1}$. The proton NMR spectrum showed a relatively clean spectrum with the bipy complexed to the dominant cone conformer (Figure 115). The upper rim ethylpyridyl protons appeared at 8.64, 7.58, $7.32 \mathrm{ppm}$ respectively as a doublet, triplet, doublet. However the last triplet could not be clearly seen, but due to the large number of peaks it is more than likely being overshadowed by other peaks. The bipyridyl protons were seen at 8.53, 8.34, 8.32, 8.23 and $7.30 \mathrm{ppm}$ as a doublet, singlet, singlet, doublet, doublet respectively. The methyl protons for the bipy group were located at $2.44 \mathrm{ppm}$ as a large singlet. Peaks representing the benzyl group occurred at 7.65, 7.48 and $7.13 \mathrm{ppm}$ as a doublet, triplet, and triplet. The hydroxyl singlet was observed at $7.83 \mathrm{ppm}$, and the aromatic protons appeared at 7.08, 7.05 and $6.65 \mathrm{ppm}$ as a singlet, doublet and triplet. In the bridging region the lower rim benzyl-methylene arm was seen at $5.06 \mathrm{ppm}$, the upper rim pyridyl-methylene arm was observed at $4.81 \mathrm{ppm}$ and the bipyridyl-methylene arm appeared at $3.46 \mathrm{ppm}$. The methylene bridging protons at appeared 4.31 and $3.34 \mathrm{ppm}$ as doublets, and the methylene and ethylene protons of the ethylpyridyl 
arm occurred at 3.06 and $2.94 \mathrm{ppm}$. However it is clear that many more peaks are present in the spectrum than these mentioned. This again is attributed to the presence of self-assembled molecules, but due to the dominant peaks of the bipy in the aromatic region of the spectrum it is not clear whether or not these self-assembled constructs contain the bound bipy unit or if they are simply unreacted starting material forms. The ${ }^{13} \mathrm{C}$ NMR spectrum showed a total of thirty-one non-equivalent carbon peaks.

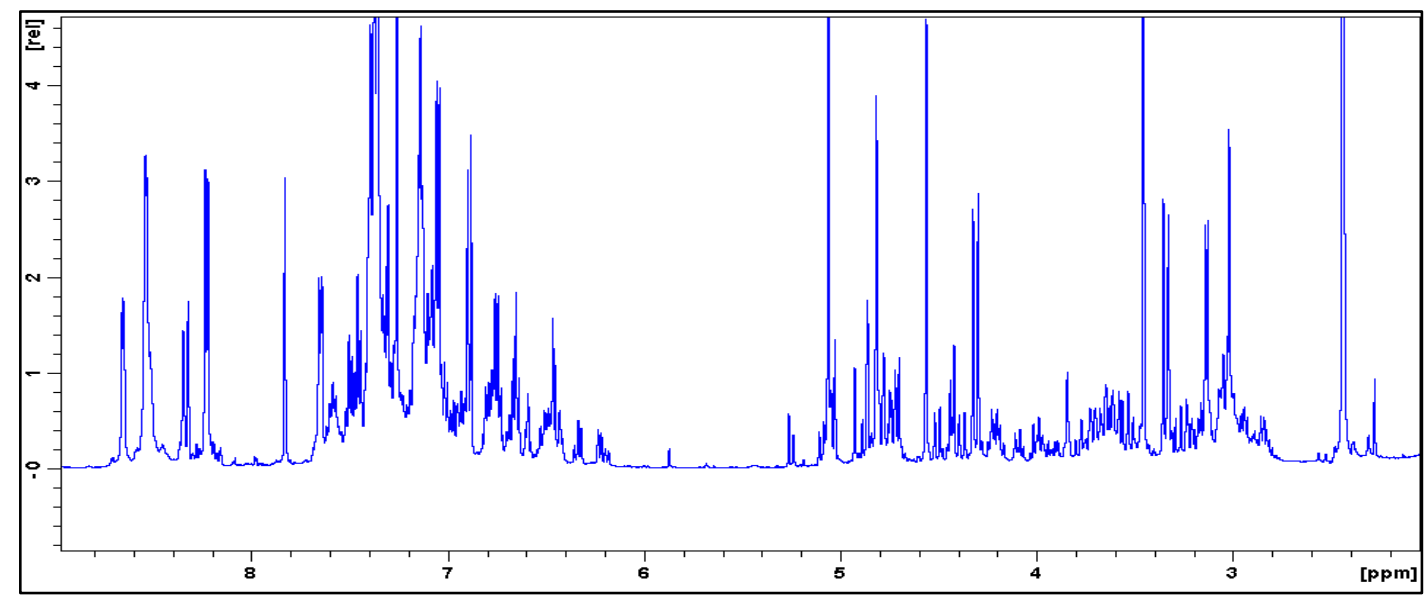

Figure 115: ${ }^{1} \mathrm{H}$ NMR of (38)

nOe NMR spectroscopy was performed on this compound and several of the main peaks were chosen and will be discussed here. 


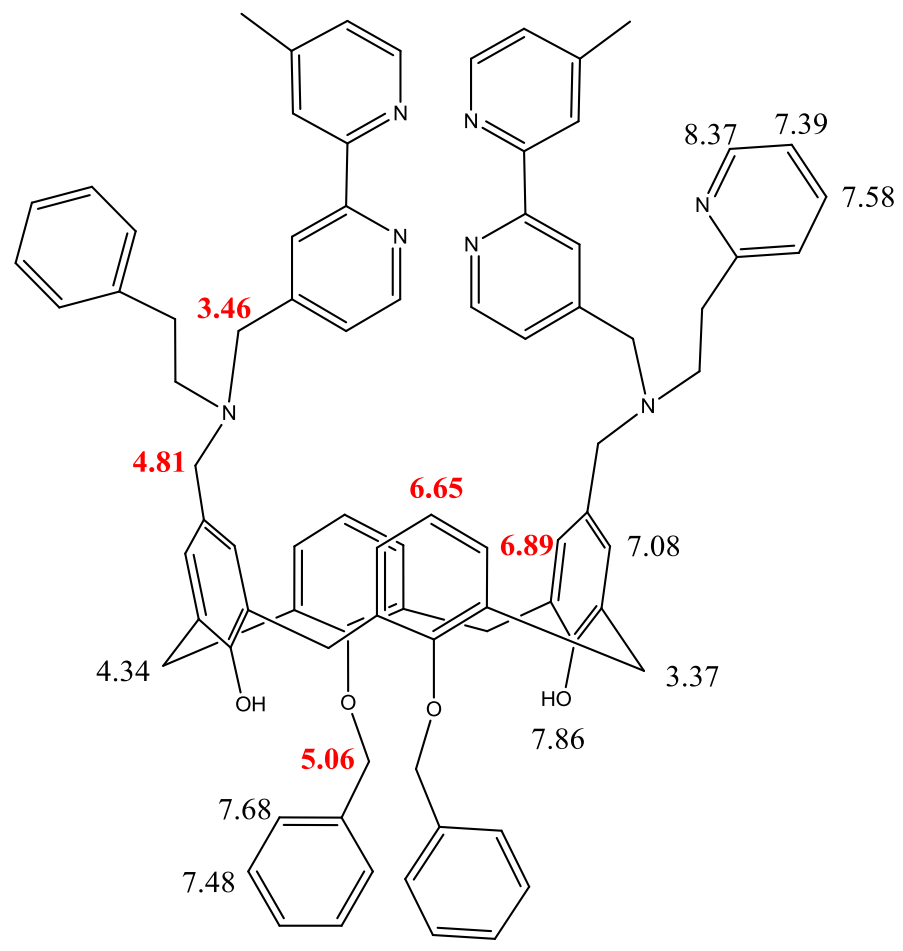

Figure 116: Points chosen for nOe interactions for (38)

The first peak to be irradiated of this compound was the aromatic protons corresponding to the doublet at $6.89 \mathrm{ppm}$. The first interaction to be seen was at 6.78 ppm representing the doublet of another calixarene unit. An interaction was also seen at $6.68 \mathrm{ppm}$ possibly the calix[4]arene triplet along with an interaction at $7.86 \mathrm{ppm}$ indicating interaction with the lower rim hydroxyl group. The final observed peak is at $3.37 \mathrm{ppm}$ representing the equatorial bridging protons.

The second peak to be examined was the aromatic protons corresponding to the triplet of the calix[4]arene at $6.65 \mathrm{ppm}$. Interaction with the aromatic protons was seen at $6.79 \mathrm{ppm}$ and $7.08 \mathrm{ppm}$. Another interaction was seen at $6.97 \mathrm{ppm}$ possibly due to an alternate aromatic doublet. A final interaction for this nOe NMR spectrum was at $7.39 \mathrm{ppm}$ corresponding to the protons of the ethylpyridyl group.

The third peak irradiated was at $5.06 \mathrm{ppm}$ corresponding to the lower rim benzylmethylene arm. Interactions were seen with several peaks at 4.90, 4.84, and 4.80 
ppm which are characteristic of the pyridyl-methylene arm on the upper rim. The interaction at $4.84 \mathrm{ppm}$ can be attributed to the dominant cone conformer selfassembled structure and the other two are more than likely due to the lesser selfassembled structures. With the dominant interaction it is most likely due to selfassembly but with the other peaks it is more difficult to discern whether this interaction is due to conformers or a self-assembled structure or a combination of both. Without a crystal structure, definitive analysis cannot be made at this point. Interactions were also seen with two doublets at 4.50 and $4.34 \mathrm{ppm}$, the latter of which corresponds to the dominant cone conformation, along with interactions at 7.58 and $7.48 \mathrm{ppm}$, corresponding to the ethylpyridyl and benzyl protons accordingly. Another interaction was seen at $7.68 \mathrm{ppm}$ which is most likely due to the benzyl protons and a final interaction at $7.86 \mathrm{ppm}$ representing the hydroxy peak.

The final peaks to be investigated were the upper rim pyridyl-methylene peak at 4.81 ppm, and the bipyridyl-methylene arm at $3.46 \mathrm{ppm}$. The upper rim pyridylmethylene protons were seen to interact with the lower rim benzyl-methylene protons at $5.06 \mathrm{ppm}$ and possibly the equatorial bridging doublet at $3.61 \mathrm{ppm}$. In the aromatic region several peaks are seen; $7.12 \mathrm{ppm}$, corresponding to the aromatic protons, $7.39 \mathrm{ppm}$ possibly representing the benzyl protons and a peak at $7.49 \mathrm{ppm}$ for the ethylpyridyl protons. The final interaction was observed at $8.37 \mathrm{ppm}$ representing the ethylpyridyl protons. For the bipyridyl-methylene arm at $3.46 \mathrm{ppm}$, interactions were seen for the axial bridging doublets at $4.32 \mathrm{ppm}$ along with another interaction at $4.6 \mathrm{ppm}$, which could be an alternate peak for the upper rim pyridylmethylene linking arm. A final interaction was seen with the lower rim benzylmethylene arm at $5.10 \mathrm{ppm}$. To account for these interactions one would adopt a self-assembled structure in which the lower rim benzyl group is in close proximity to the upper rim groups such as that found in the hetero form. 


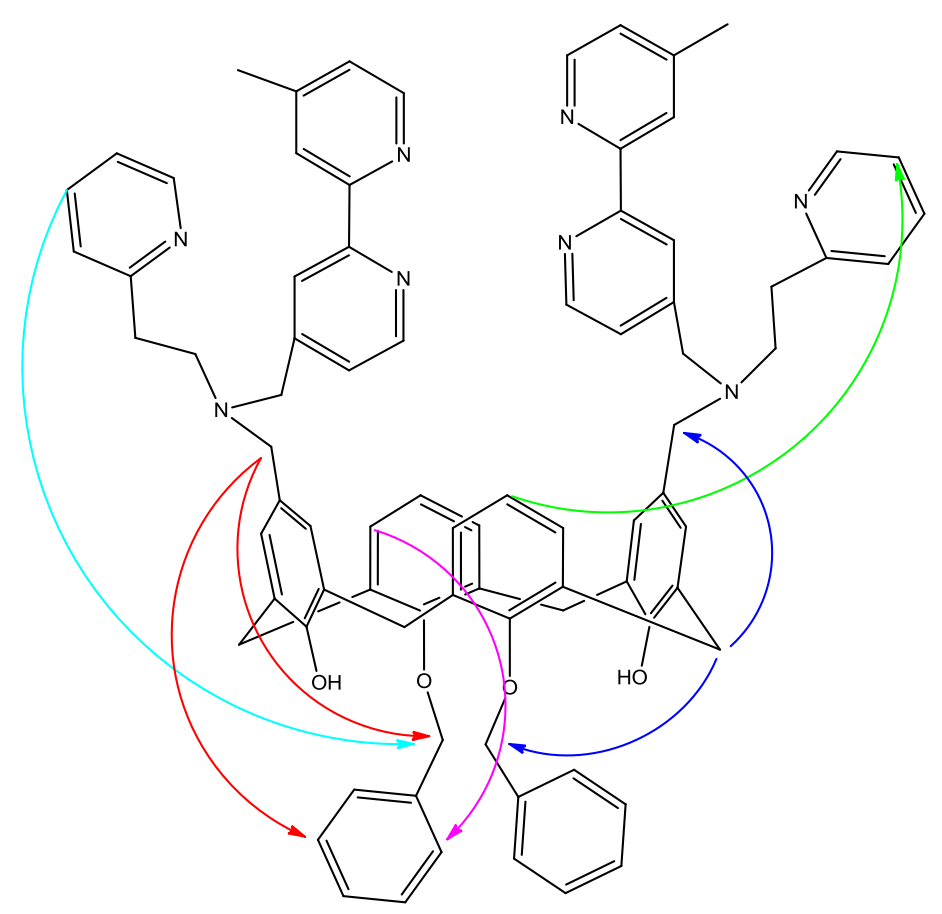

Figure 117: summery of nOe NMR interactions for $(\mathbf{3 8})$

The data obtained from these nOe NMR studies provides strong evidence for the heterodimerisation of the cone conformation (Figure 117). So when compared to the previous attempt to attach the bipy unit to the upper rim, were only the pyridylmethylene was used, it appears that with the pyridyl-ethylene arm this provides additional space and as a result reduces the unfavourable sterics that were previously encountered.

\subsubsection{Self-Assembly of lower rim pyridyl complexes}

With the success in the functionalisation of the lower rim with two groups, the next step was to try and functionalise all four of the lower rim hydroxyls as well as functionalising the upper rim. This was carried out following a method by Creaven et $a l$. , to create compounds (40) and (41), (Figure 118). Compound (40) was obtained as a red waxy solid in moderate yields (74\%) from the reaction of (33) with sodium hydride in a mixture of dichloromethane and acetonitrile (1:2 ratio). The IR data showed the $v_{\mathrm{CO}}$ at $1747 \mathrm{~cm}^{-1}$ and the pyridyl $v_{\mathrm{NCH}}$ at $1590 \mathrm{~cm}^{-1}$. As the ethylbromoacetate starting material produces a $v_{\mathrm{CO}}$ at $1739 \mathrm{~cm}^{-1}$, the shift in the IR provides evidence of attachment. The ${ }^{1} \mathrm{H}$ NMR spectrum showed multiple peaks 
again however not to the same extent, indicating self-assembly. This may be due to the addition of the two extra pendant arms on the lower rim, resulting in a more strict self-assembly alignment. The pyridyl protons were found at 8.66, 7.75, 7.40 and 7.16 ppm as a doublet, triplet, doublet, triplet fashion. The aromatic protons on the calix[4]arene's ring showed the singlet at $7.05 \mathrm{ppm}$, two doublets at 7.00 and 6.86 ppm and two triplets at 6.67 and $6.32 \mathrm{ppm}$. Three clear singlet's were seen in the bridging region of the spectrum, one at $5.12 \mathrm{ppm}$ for the lower rim pyridyl bridging arm, another at $4.68 \mathrm{ppm}$ for the upper rim bromo-methylene arm and a final peak at $4.75 \mathrm{ppm}$ representing the lower rim methyl ester arm. The hydroxyl peak was no longer present indicating that the reaction had occurred on the lower rim. The methylene and ethylene protons of the ester arm were seen at $4.33 \mathrm{ppm}$ and 1.37 ppm as a multiplet and triplet respectively. The bridging methylene protons were observed at $4.22 \mathrm{ppm}$ for the axial protons and $3.22 \mathrm{ppm}$ for the equatorial protons. The ${ }^{13} \mathrm{C}$ NMR spectrum showed twenty individual peaks for the non-equivalent carbons. 


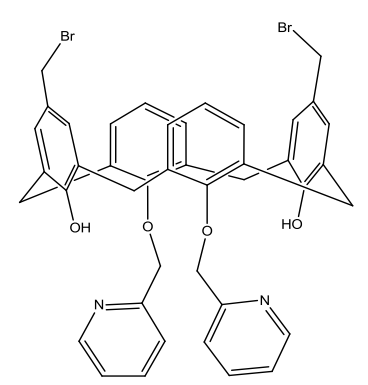

(33)

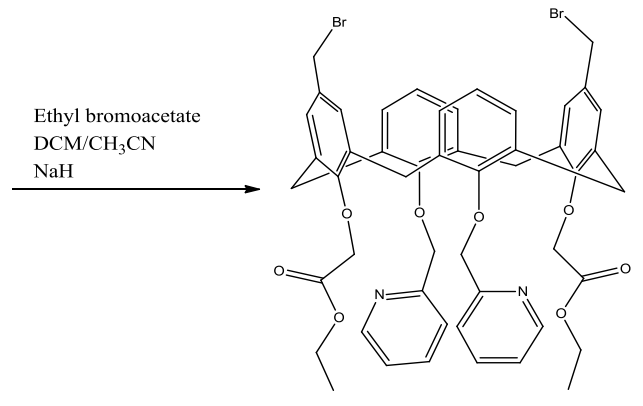

(40)

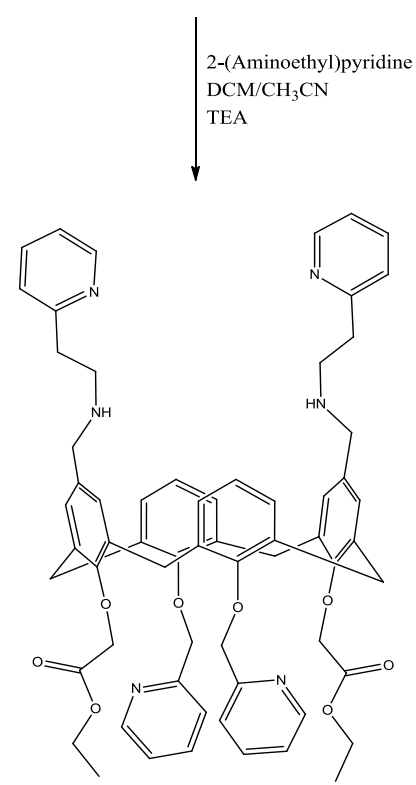

$(41)$

Figure 118 : Synthesis of (40) and (41)

Compound (41) was afforded as a yellow waxy solid in high yields (84\%) from the reaction of (40) with 2-(aminoethyl)pyridine in the presence of triethylamine. As triethylamine is a bulky base it was assumed that the reaction would occur on the upper rim without interfering with the groups already attached in the lower rim. The IR analysis showed that the $v_{\mathrm{CO}}$ stretching band at $1751 \mathrm{~cm}^{-1}$, the pyridyl $v_{\mathrm{NCH}}$ at $1591 \mathrm{~cm}^{-1}$ and the upper rim $v_{\mathrm{NH}}$ at $3419 \mathrm{~cm}^{-1}$. The ${ }^{1} \mathrm{H}$ NMR spectrum showed some overlapping peaks but only the dominant peaks will be discussed here. The pyridyl protons appeared at 8.58, 7.75, 7.56 and $7.20 \mathrm{ppm}$ as a doublet, triplet, doublet, and triplet respectively. The aromatic protons occurred at $6.99,6.89$ and $6.77 \mathrm{ppm}$ as a singlet, doublet and triplet. The lower rim pyridyl-methylene arm was seen as a singlet at $5.19 \mathrm{ppm}$ and the upper rim pyridyl-methylene arm was seen at $4.64 \mathrm{ppm}$ 
also as a singlet. The carbonyl linking arm was observed as a singlet at $3.42 \mathrm{ppm}$ and the protons of the methylene and ethylene of the ester arm were observed at 4.37 ppm and $1.15 \mathrm{ppm}$ as a multiplet and triplet. The bridging methylene doublets were located at $4.32 \mathrm{ppm}$ and $3.37 \mathrm{ppm}$ and the two remaining upper rim pyridyl-ethylene and methylene protons were seen at $3.17 \mathrm{ppm}$ and $3.01 \mathrm{ppm}$ as two triplets. The ${ }^{13} \mathrm{C}$ NMR spectrum showed twenty-seven different peaks as expected for the nonequivalent carbons as well as considerable extras.

Several SEM images were obtained for (41) and on initial viewing (Figure 119) the structure of the SEM contains two different morphologies, one side with a rough 'mountainous' texture and the other a smooth unblemished quality. However on closer analysis (Figure 120) of the smooth side it is revealed that the actual structure appears to be made up of several layers consisting of rough pitted textures. So perhaps the smooth side may be merely due to the flat side of the vial in which the crystal was allowed to grow. What the images do tell us is the morphology of the crystals is not uniform with a rough texture and the second image shows a stacking or layered structure which supports the self-assembly seen in the ${ }^{1} \mathrm{H}$ NMR spectrum. 


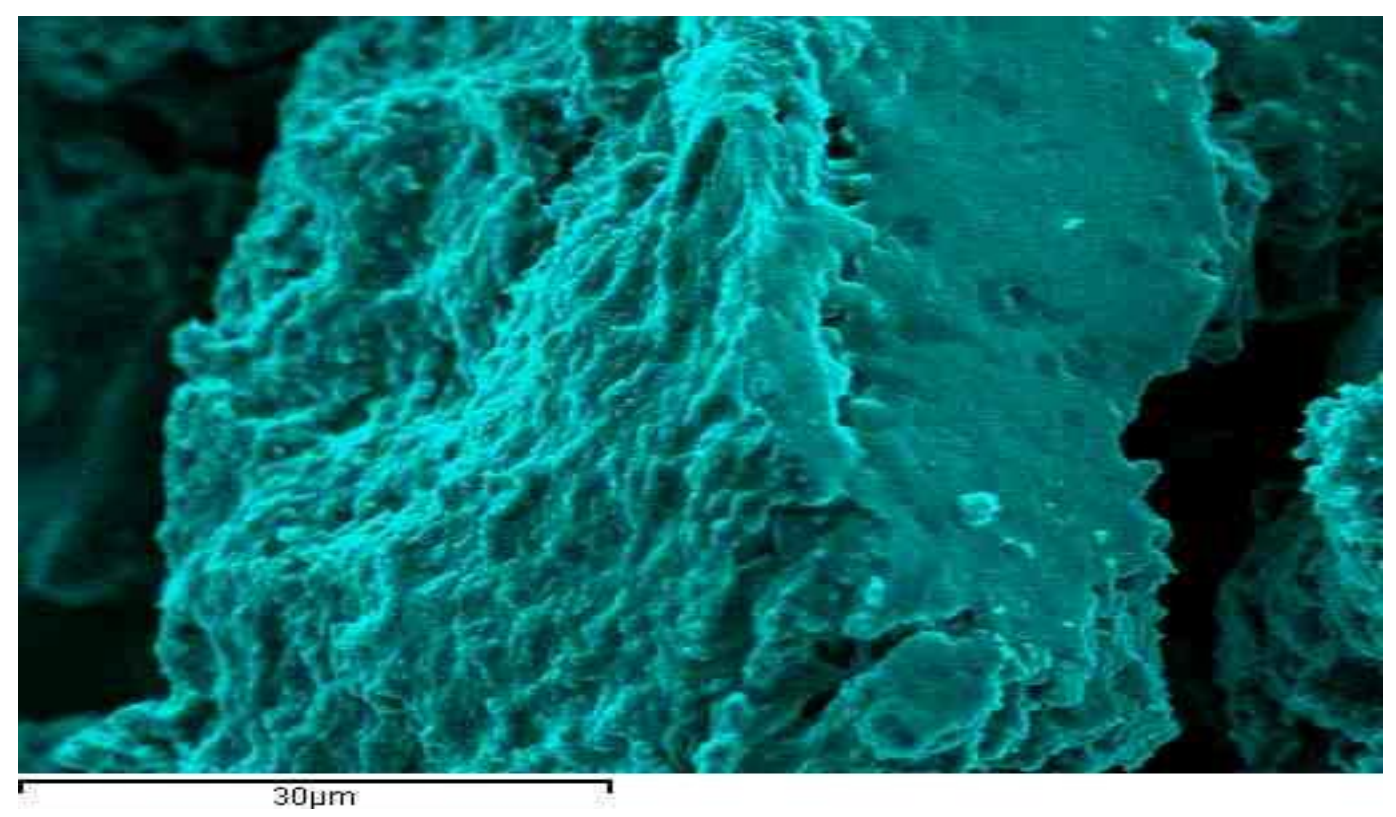

Figure 119: SEM of (41) working distance 14 mm, $16.0 \mathrm{Kv}, \mathrm{X} 350$.

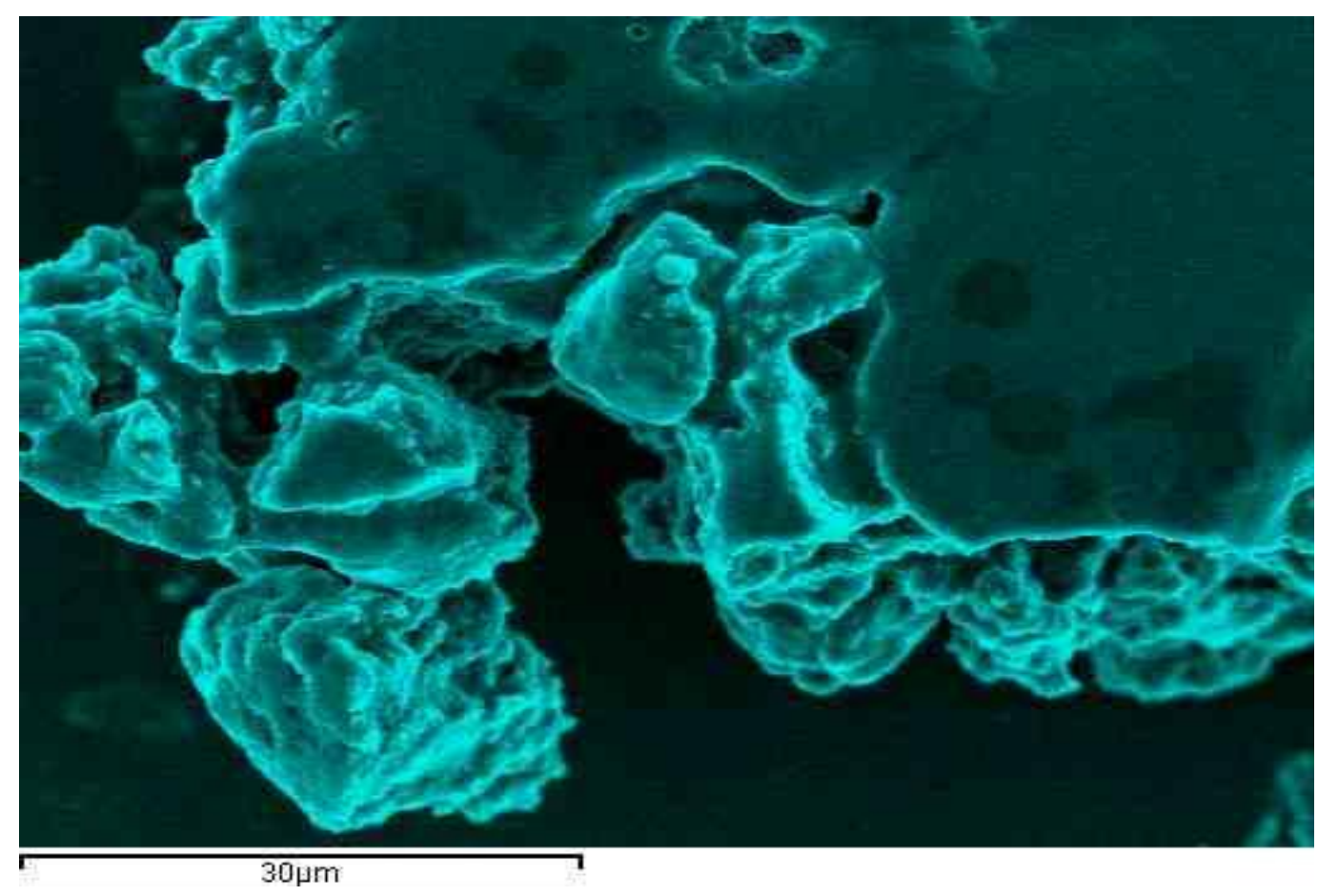

Figure 120: SEM of (41) working distance 14 mm, $16.0 \mathrm{Kv}, \mathrm{X} 100$.

The final step in this synthesis was to attach the second pyridyl arm to the upper rim of the calix[4]arene derivative to form the cage metal ion binding site, compound (51), (Figure 121). Compound (51) was produced as a brown waxy solid in good 
yields $(75 \%)$ by the reaction of (41) with 2-(aminomethyl)-pyridine in the presence of potassium carbonate.

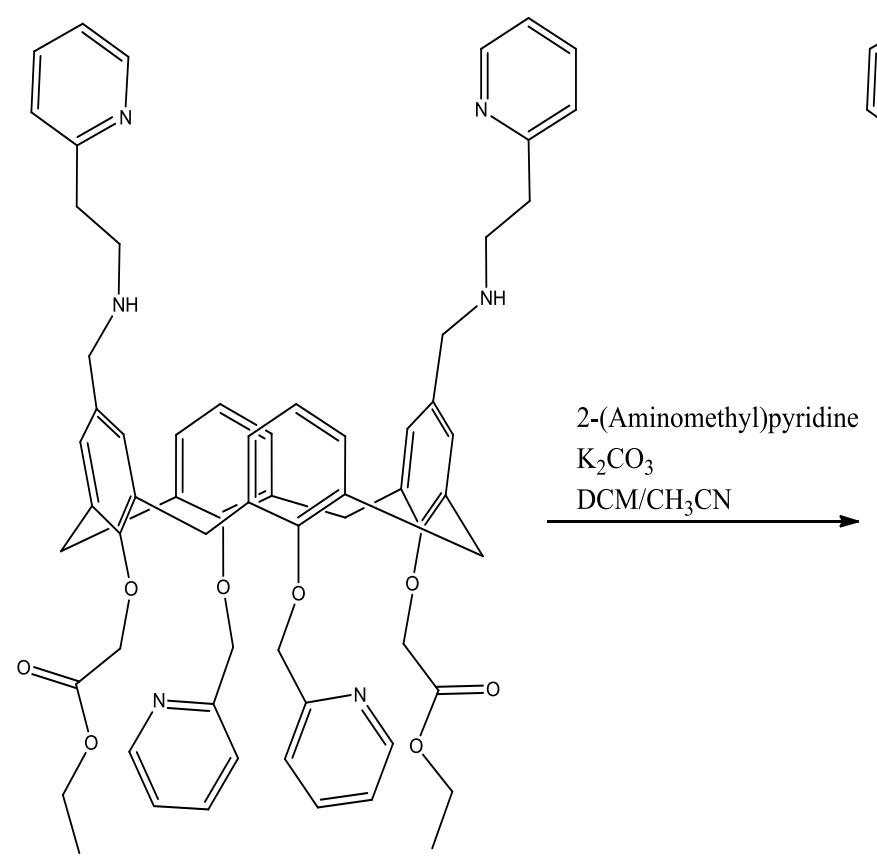

$(41)$

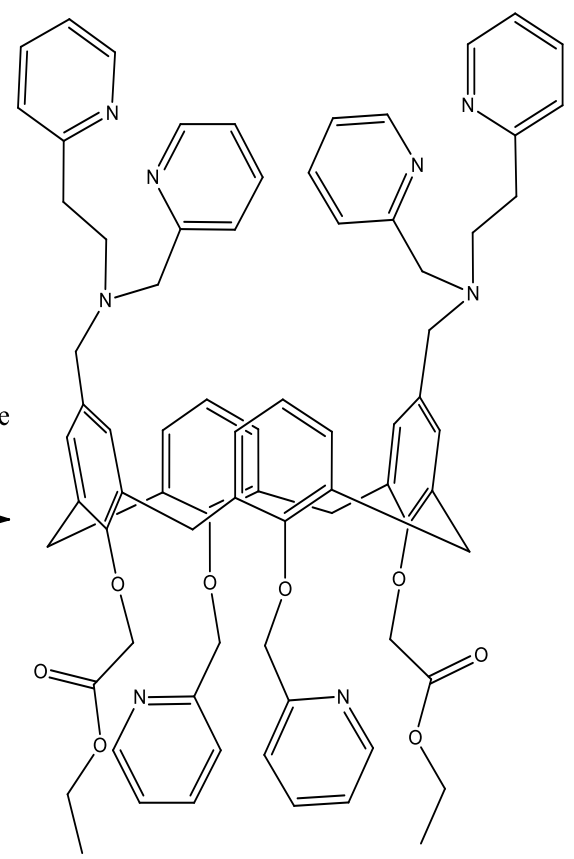

$(51)$

Figure 121: Synthesis of (51)

The IR data showed the $v_{\mathrm{NH}}$ at $2925 \mathrm{~cm}^{-1}$, the $v_{\mathrm{CO}}$ at $1711 \mathrm{~cm}^{-1}$ and the $v_{\mathrm{NCH}}$ at 1589 $\mathrm{cm}^{-1}$. The ${ }^{1} \mathrm{H}$ NMR spectrum showed multiple overlapping peaks confirming the presence of dimers (Figure 122). The lower rim pyridyl protons occurred at 8.62, 7.86, 7.61 and $7.55 \mathrm{ppm}$ as a doublet, triplet, doublet and triplet, while the upper rim ethylene-pyridyl protons occurred at 8.59, 7.79, 7.59, $7.48 \mathrm{ppm}$ respectively also as a doublet, triplet, doublet and triplet. The signals for the upper rim methylene-pyridyl doublet protons occurred slightly further upfield than the other two pyridyl groups, at 8.54, 7.74, 7.34 and $7.30 \mathrm{ppm}$ as a doublet, triplet, triplet, and doublet. A possible explanation for this would be that during the heterodimerisation, were the doublet beside the nitrogen in the upper rim methylene-pyridyl could be facing away from the cavity of the calix[4]arene. In this manner the rest of the protons of the upper rim pyridyl would experience slight shifts due to interactions with the lower rim pyridyl's of another calix[4]arene unit, but as this proton is facing away from the 
interaction its involvement would be minimised, therefore leading to a slightly upfield shift. The aromatic protons were seen at $6.84 \mathrm{ppm}$ as a doublet, at $7.12 \mathrm{ppm}$ as a singlet and a triplet at $6.61 \mathrm{ppm}$. Additional peaks were seen in this region as well as in the bridging region of the spectrum however only the peaks which were clearly defined will be mentioned here. The lower rim pyridyl-methylene arm was seen as a singlet at $5.05 \mathrm{ppm}$, the two upper rim pyridyl-methylene arm also occurred as a singlet at $4.38 \mathrm{ppm}$. The lower rim carbonyl arm was observed at $3.67 \mathrm{ppm}$ as a broad singlet. The two upper rim triplets corresponding to the pyridyl-methylene and pyridyl-ethylene protons were seen at $3.34 \mathrm{ppm}$ and $3.25 \mathrm{ppm}$ which was slightly shifted upfield when compared to the precursor arms of $3.17 \mathrm{ppm}$ and $3.01 \mathrm{ppm}$. This can be accounted for by self-assembly interactions with the lower rim groups. The methylene bridging protons of the calix[4]arene unit which appeared at 4.30 ppm and $3.34 \mathrm{ppm}$ and the final two observed peaks, corresponding to the methylene and ethylene of the ester linkage appeared at $4.20 \mathrm{ppm}$ and 1.31 as a quartet and broad triplet respectively. The ${ }^{13} \mathrm{C}$ NMR spectrum showed thirty-one non-equivalent carbons.

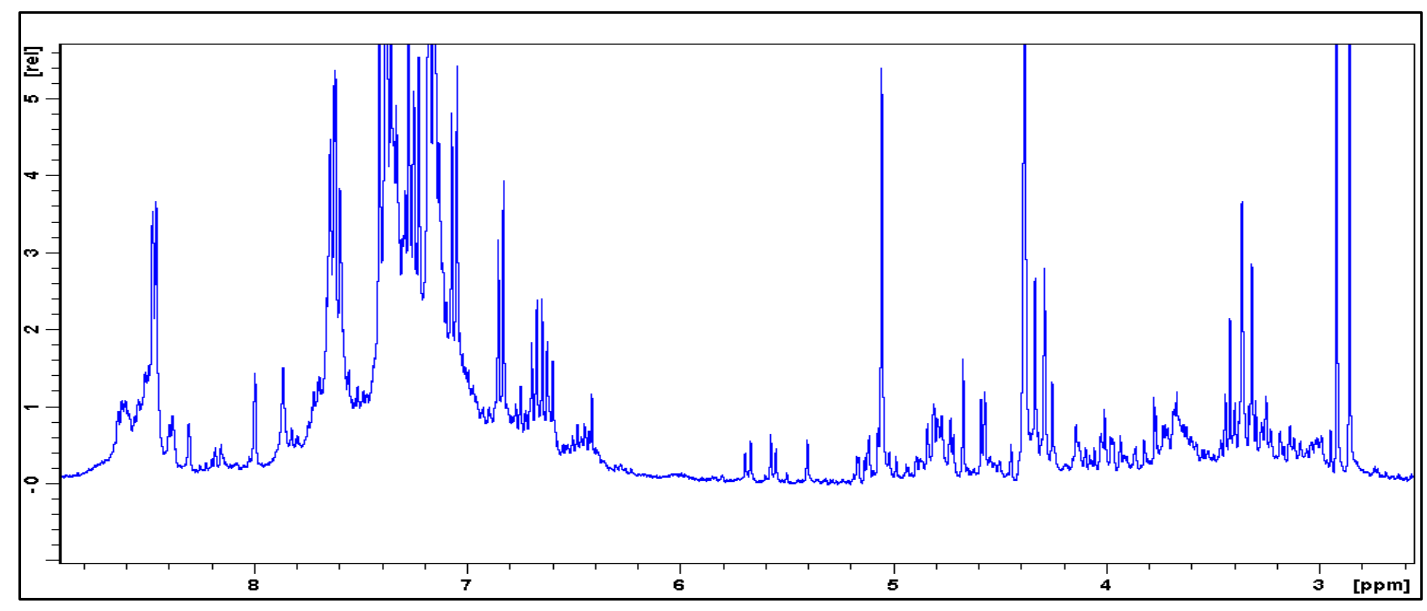

Figure 122: ${ }^{1} \mathrm{H}$ NMR spectrum of (51)

\subsubsection{Bipyridyl complexes}

With compound (41) successfully synthesised, work also began in an attempt to attach the bipyridyl unit to the upper arm to create the potential dual-metal ion binding center, compound (44), (Figure 123). Compound (44) was obtained as a dark red-brown waxy solid in moderate yields $(58 \%)$. IR analysis showed the $v_{\mathrm{CO}}$ 
stretching band at $1754 \mathrm{~cm}^{-1}$ and the pyridyl $v_{\mathrm{NCH}}$ at $1595 \mathrm{~cm}^{-1}$. The ${ }^{1} \mathrm{H}$ NMR spectrum (Figure 124) did show some level of self-assembly however, the peaks indicating this were small and often obscured due to excess solvent and 2(aminoethyl)pyridine most likely trapped within the cavity of the calix[4]arene. The bipyridyl protons occurred at 8.68, 8.53, 8.40, 8.21, 7.33 and $7.15 \mathrm{ppm}$ as a doublet, doublet, singlet, singlet, doublet and singlet respectively. The lower rim pyridyl protons appeared at $8.66,7.82,7.55$ and $7.32 \mathrm{ppm}$ in the expected doublet, triplet, doublet, and triplet fashion. The upper rim pyridyl protons were observed at 8.64, 7.65, 7.36 and $7.31 \mathrm{ppm}$ again in the doublet, triplet, doublet, triplet fashion. The aromatic protons of the calix[4] arene ring were seen at $7.08 \mathrm{ppm}$ for the singlet, 6.98 ppm for the doublet and $6.67 \mathrm{ppm}$ for the triplet. The lower rim pyridyl-methylene arm occurred at $5.10 \mathrm{ppm}$, the upper rim pyridyl-methylene arm was seen at 4.79 ppm, the bipyridyl-methylene arm appeared at $4.48 \mathrm{ppm}$ and the lower rim estermethylene was seen at $3.57 \mathrm{ppm}$. The upper rim methylene and ethylene pyridyl arms appeared as two triplets at 3.15 and $3.02 \mathrm{ppm}$ respectively. The quartet and triplet of the methylene and ethylene ester arms were seen at $4.30 \mathrm{ppm}$ and 2.32 ppm. The bridging methylene protons appeared as two doublets, signifying cone conformation at $4.20 \mathrm{ppm}$ and $3.24 \mathrm{ppm}$. The final peak to be observed was the bipyridyl methyl group which occurred as a singlet at $2.43 \mathrm{ppm}$. The ${ }^{13} \mathrm{C}$ NMR showed thirty-nine non-equivalent carbons with the methylene carbon of the new bipy arm occurring at $61 \mathrm{ppm}$. 


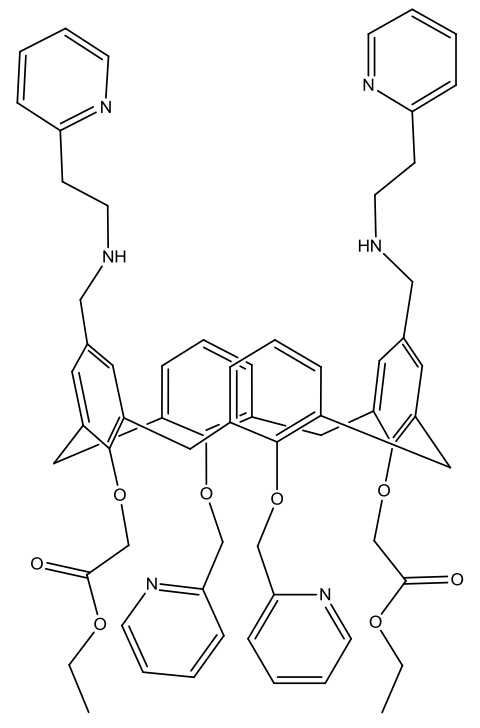

(41)

\section{2-(Aminomethyl)pyridine}

TEA

$\mathrm{CH}_{3} \mathrm{CN}$

$\checkmark$

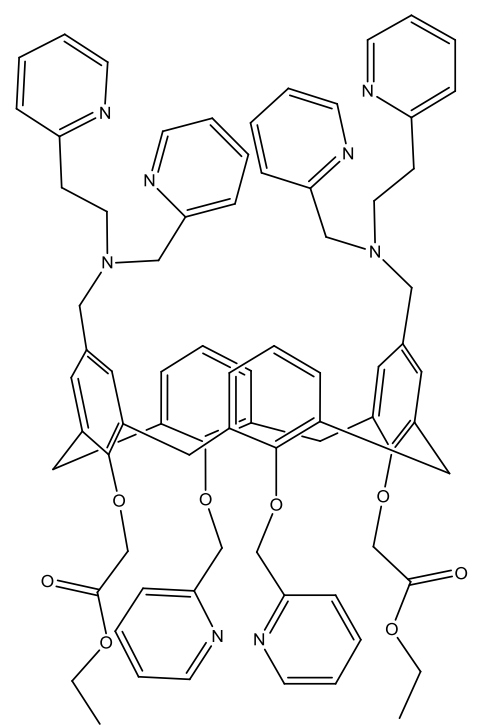

(51)

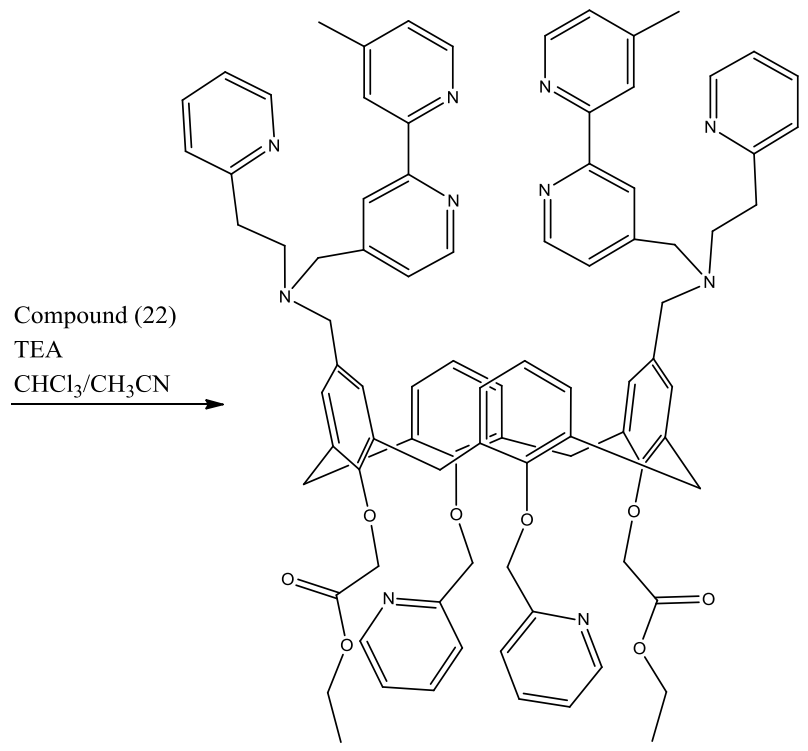

(44)

2-(aminomethyl)pyridine Toluene $/ \mathrm{MeOH}$

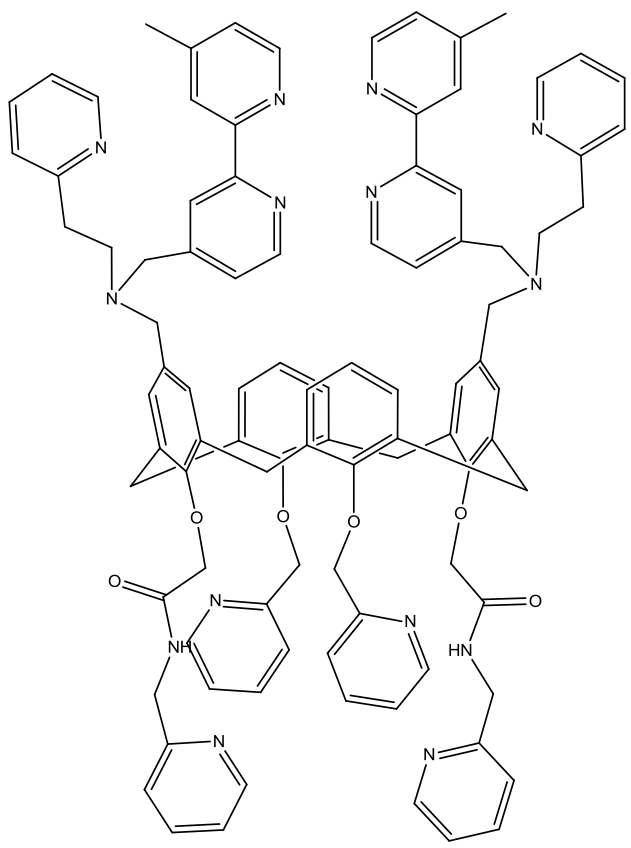

(45)

Figure 123: Synthesis of (44), (45) and (51) 


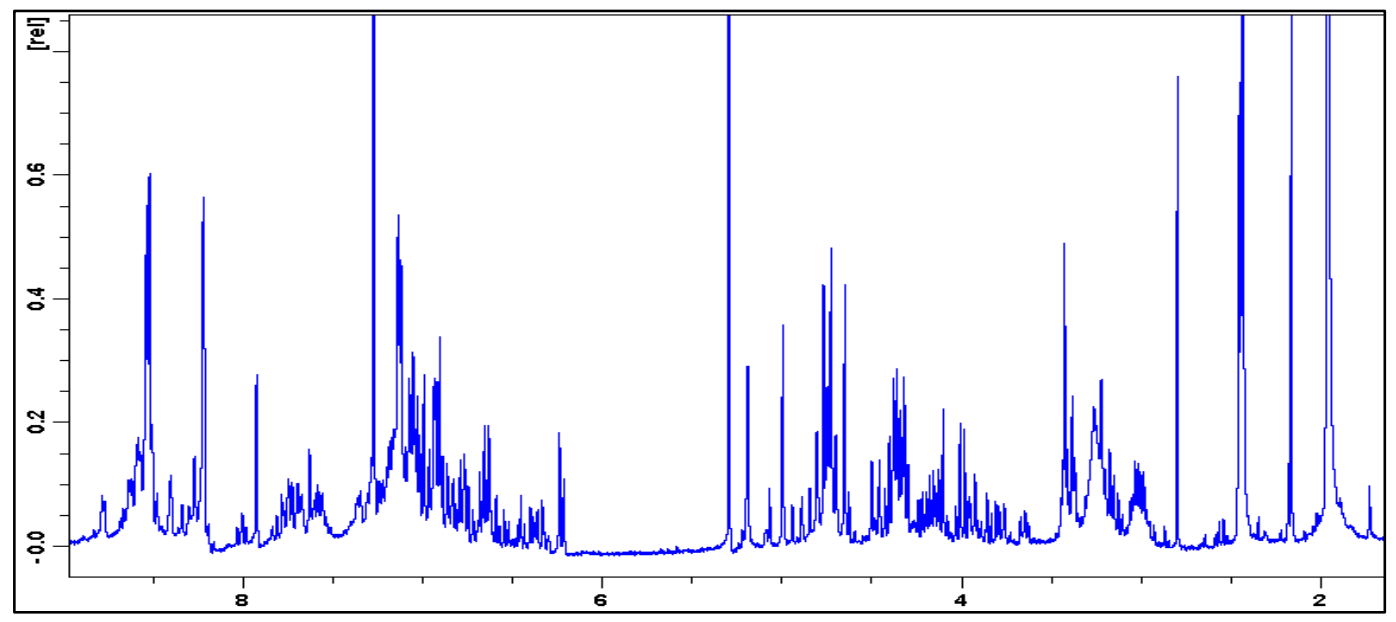

Figure 124: ${ }^{1} \mathrm{H}$ NMR of (44)

A further synthesis of compound (44) was attempted in order to try and further functionalise the lower rim to create a stronger metal binding center, compound (45). Compound (45) was obtained as a dark-red waxy solid in moderate yields (49\%). The IR data showed the $v_{\mathrm{CO}}$ stretching band at $1754 \mathrm{~cm}^{-1}$ and the pyridyl $v_{\mathrm{NH}}$ stretching band at 1677 and $1590 \mathrm{~cm}^{-1}$. The ${ }^{1} \mathrm{H}$ NMR spectrum again showed the self-assembly playing a dominant role, but the majority of the peaks could be clearly seen. The bipyridyl protons occurred as a doublet, doublet, doublet, singlet, at 8.52, $8.33,8.19$ and $7.58 \mathrm{ppm}$ respectively. The lower rim pyridyl protons appeared as a doublet, triplet, doublet, triplet, at 8.73, 7.93, 7.55 and $7.44 \mathrm{ppm}$ and the lower rim amide pyridyl protons occurred also in the doublet, triplet, doublet, triplet, fashion at $8.59,7.83,7.53,7.42 \mathrm{ppm}$. The upper rim pyridyl protons again followed the same format as the lower rim pyridyl protons, appearing at 8.55, 7.65, 7.51 and $7.40 \mathrm{ppm}$. The aromatics protons of the calix[4]arene were seen as a singlet, doublet, and triplet, at 7.07, 6.87, $6.56 \mathrm{ppm}$. Additional peaks were seen in both the aromatic and bridging region but inly the main peaks are mentioned here. The upper rim pyridylmethylene arm was seen at $4.79 \mathrm{ppm}$ as a singlet, the pyridyl-methylene and ethylene protons appeared at 3.12 and 3.00 ppm as two triplets and the bipyridylmethylene arm occurred at $3.82 \mathrm{ppm}$ also as a singlet. The lower rim pyridylmethylene arm appeared at $4.81 \mathrm{ppm}$ as a singlet, and the ester singlet appeared at $3.94 \mathrm{ppm}$. The lower rim amide methylene arm was seen at $4.77 \mathrm{ppm}$ as a singlet. The bridging methylene protons appeared as two broad doublets at 4.18 and 3.21 ppm. The final two observed peaks correspond to the methylene and ethylene 
protons of the ester arm occurring at $4.30 \mathrm{ppm}$ and $2.32 \mathrm{ppm}$ as a quartet and triplet respectively. The ${ }^{13} \mathrm{C}$ NMR spectra showed forty-two different carbon peaks for the non-equivalent carbon atoms.

As well as the bipy unit, another pyridyl arm was also attached to the upper rim as an alternate metal ion binder compound (51) (Figure 123). Compound (51) was produced as a brown waxy solid in moderate yields (51\%) from the reaction of (41) with 2-(aminomethyl)-pyridine in the presence of triethylamine. The IR data showed the $v_{\mathrm{NH}}$ band at $2625 \mathrm{~cm}^{-1}$, the $v_{\mathrm{CO}}$ band at $1711 \mathrm{~cm}^{-1}$ and the $v_{\mathrm{NCH}}$ band at $1589 \mathrm{~cm}^{-1}$. The ${ }^{1} \mathrm{H}$ NMR spectrum showed lower rim pyridyl protons at 8.62, 8.44, 7.64 and $7.40 \mathrm{ppm}$ following the doublet, triplet, doublet, triplet pattern. The protons of the methyl-pyridyl arm occurred at $8.53 \mathrm{ppm}, 7.85,7.61$ and $7.54 \mathrm{ppm}$ as a doublet, triplet, doublet and triplet. It should be noted here that the peak at $8.53 \mathrm{ppm}$ is quite broad and as it integrates for four hydrogens, this peak most likely also represents the corresponding protons of the ethyl-pyridyl group. The protons of the ethylpyridyl group were found at $8.53 \mathrm{ppm}, 7.73,7.40$ and $7.14 \mathrm{ppm}$ also as a doublet, triplet, doublet and triplet. The aromatic protons of the calix[4]arene ring were found at $7.25 \mathrm{ppm}$ for the singlet, $7.09 \mathrm{ppm}$ for the doublet and $6.89 \mathrm{ppm}$ for the triplet. The pendant arms appeared as singlets at $5.18 \mathrm{ppm}$ for the lower rim pyridylmethylene, at $4.73 \mathrm{ppm}$ for the upper rim pyridyl-methylene arm, at $4.03 \mathrm{ppm}$ for ester-methylene arm and $3.96 \mathrm{ppm}$ for final pyridyl-methylene arm. The upper rim pyridyl-ethylene protons were seen at 3.25 and 3.04 ppm as two triplets respectively. The methylene bridging protons were observed at 4.35 and $3.41 \mathrm{ppm}$ along with the quartet for the ester-ethylene protons at $4.20 \mathrm{ppm}$. Unfortunately the final peak for the ester methyl protons wasn't seen due to acetonitrile over-shadowing its region. The ${ }^{13} \mathrm{C}$ NMR spectrum showed twenty-nine non-equivalent carbon peaks with the new methylpyridyl linker carbon appearing at $60 \mathrm{ppm}$.

\subsubsection{Lower rim benzyl complexes containing additional ester groups on the lower rim}

A similar set of reactions to (44-51) were carried out using the lower rim functionalised benzyl compounds, again to expand further the number of potential metal ion binding compounds. The reaction conditions that were used were the same 
to the previous set, except that the starting compound contained the benzyl group on the lower rim instead of the pyridyl to create compounds (48)-(50), (Figure 125). Compound (48) was obtained as a brown waxy solid in moderate yields (66\%) from the reaction of (29) with ethyl bromoacetate in the presence of potassium carbonate. The IR analysis showed the carbonyl stretching band at $1758 \mathrm{~cm}^{-1}$ indicating ester attachment. The ${ }^{1} \mathrm{H}$ NMR spectrum showed multiple peaks again indicating that selfassembly was occurring. The lower rim benzyl protons appeared at $7.41 \mathrm{ppm}$ and $7.38 \mathrm{ppm}$ as a doublet and triplet respectively. The aromatic protons of the calix[4]arene ring were observed at 7.23, 7.09 and $6.89 \mathrm{ppm}$ for the singlet, doublet and triplet accordingly. The lower rim benzyl-methylene protons were seen at 5.06 ppm as a singlet and the upper rim bromo-methylene protons were found at 4.94 ppm also as a singlet. The bridging methylene protons were found at $4.37 \mathrm{ppm}$ for the axial protons and at $3.33 \mathrm{ppm}$ for the equatorial protons. The ester-methylene protons appeared as a singlet at $4.33 \mathrm{ppm}$, and the ester-methylene and ethylene protons appeared at $4.23 \mathrm{ppm}$ and $1.29 \mathrm{ppm}$ as a quartet and triplet. Several other peaks were also observed in the ${ }^{1} \mathrm{H}$ NMR spectrum. In the aromatic region several peaks were seen at $7.16 \mathrm{ppm}(\mathrm{s}), 7.13 \mathrm{ppm}(\mathrm{t}), 7.09 \mathrm{ppm}(\mathrm{s}), 7.06 \mathrm{ppm}(\mathrm{s}), 7.03 \mathrm{ppm}$ (s), 6.97 ppm (d), $6.87 \mathrm{ppm}(\mathrm{t}), 6.84 \mathrm{ppm}(\mathrm{d}), 6.80 \mathrm{ppm}(\mathrm{d}), 6.53 \mathrm{ppm}(\mathrm{t}), 6.46 \mathrm{ppm}$ $(\mathrm{t})$ and $6.42 \mathrm{ppm}(\mathrm{t})$. In the bridging region of the spectrum peaks appeared at 4.87 ppm and $4.83 \mathrm{ppm}$ as broad singlets, $4.79,4.76$ and $4.65 \mathrm{ppm}$ as doublets, a doublet at 4.26 were seen, singlets at $4.05,3.89,3.68,3.42$ and $3.39 \mathrm{ppm}$ were also observed. With this abundance of peaks, several self-assembled structures can easily be supported. The ${ }^{13} \mathrm{C}$ NMR spectrum shows nineteen different carbon signals for the non-equivalent carbon atoms with the new ester-methylene carbon appearing at 71 ppm. 


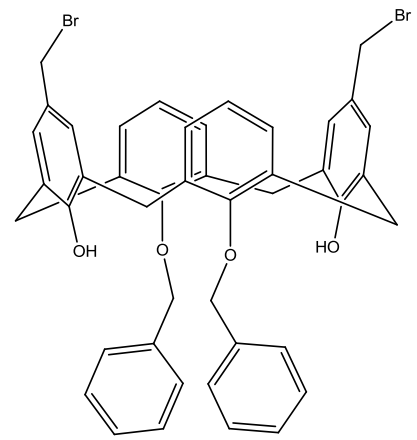

(29)
Ethylbromoacetate

$\mathrm{K}_{2} \mathrm{CO}_{3}$

$\mathrm{CH}_{3} \mathrm{CN}$

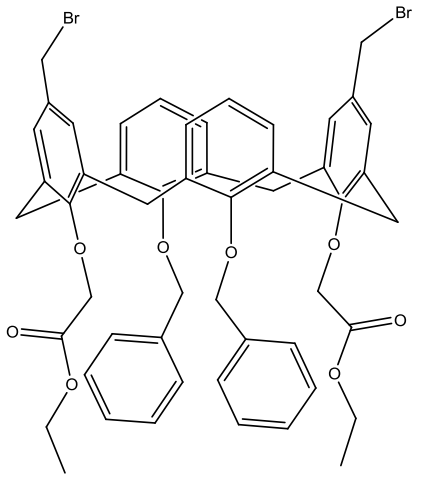

(48)

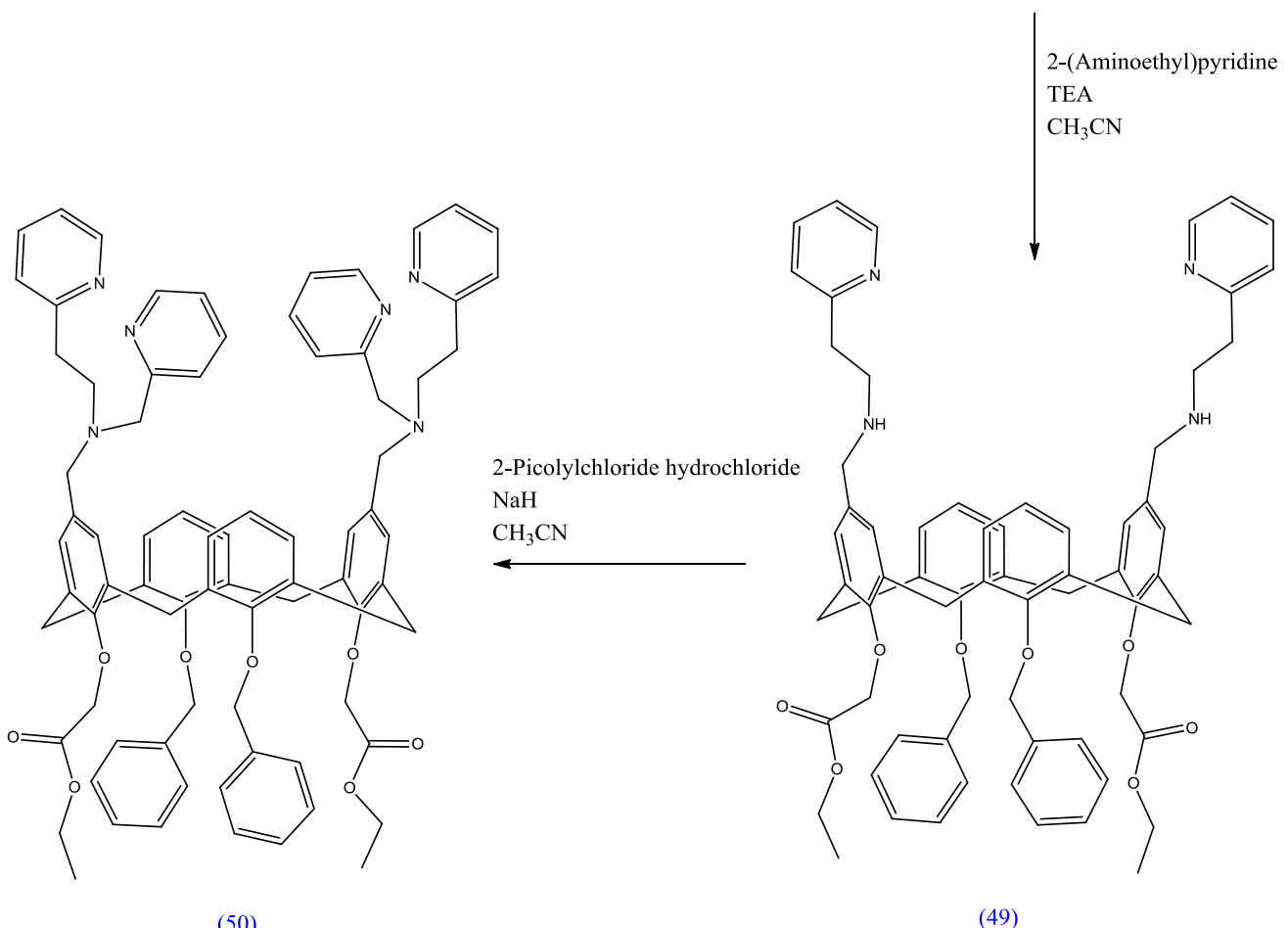

Figure 125: Synthesis of (48)-(50)

Following on from compound (48), attachment of the first upper rim pyridyl arm was carried out to form compound (49), (Figure 125). This was done by reacting (48) with 2-(aminoethyl)-pyridine in the presence of triethylamine. Compound (49) was obtained as a brown waxy solid in high yields $(98 \%)$. The IR data showed the $v_{\mathrm{CO}}$ stretching band at $1656 \mathrm{~cm}^{-1}$, the pyridyl $v_{\mathrm{NCH}}$ stretching band at $1593 \mathrm{~cm}^{-1}$ and the pyridyl $v_{\mathrm{NH}}$ stretching band at $3410 \mathrm{~cm}^{-1}$. The ${ }^{1} \mathrm{H}$ NMR spectrum showed expected peaks as well as self-assembly peaks. The protons of the ethyl-pyridyl occurred at $8.49,7.60,7.31$ and $7.18 \mathrm{ppm}$ in a doublet, triplet, doublet, triplet manner. The benzyl protons occurred at $7.44 \mathrm{ppm}$ and $7.35 \mathrm{ppm}$ as a doublet and triplet 
respectively. The aromatic protons of the calix[4]arene ring appeared at 7.12, 6.91 and $6.43 \mathrm{ppm}$ as a singlet, doublet and triplet respectively. The lower rim benzylmethylene arm occurred at $5.03 \mathrm{ppm}$ as a singlet, and the lower rim ester-methylene arm was seen at $3.46 \mathrm{ppm}$. The upper rim pyridyl-methylene arm was seen at 4.55 ppm. The two methylene bridging doublets were found at $4.29 \mathrm{ppm}$ and $3.35 \mathrm{ppm}$. The remaining ester-methylene and ethylene protons were found at $4.16 \mathrm{ppm}$ and at $1.19 \mathrm{ppm}$ as a quartet and triplet. The final peaks to be observed were the two upper rim pyridyl-methylene and ethylene arms at $3.08 \mathrm{ppm}$ and $2.90 \mathrm{ppm}$ as two triplets. Several additional peaks were seen in the aromatic and in the bridging region of the spectrum supporting the formation self-assembled structures. The ${ }^{13} \mathrm{C} N M R$ spectrum showed twenty-six non-equivalent carbons with the new upper rim pyridylmethylene and pyridyl-ethylene carbons appearing at $46 \mathrm{ppm}$ and $36 \mathrm{ppm}$ respectively.

The final step in this synthesis is to attach the second arm of the cage to the upper rim to form the desired dual-metal binding complex compound (50) (Figure 125). Compound (50) was produced as a brown waxy solid in high yields $(83 \%)$ from the reaction of (49) with 2-picolylchloride hydrochloride in the presence of sodium hydride. The IR data showed the $v_{\mathrm{CO}}$ stretching band at $1655 \mathrm{~cm}^{-1}$ and the pyridyl $v_{\mathrm{NCH}}$ stretching band at $1590 \mathrm{~cm}^{-1}$. The ${ }^{1} \mathrm{H}$ NMR spectrum showed the methylpyridyl protons were seen at 8.47, 7.60, 7.51 and $7.30 \mathrm{ppm}$ and the ethylpyridyl protons were seen at $8.44,7.57,7.33$ and $7.25 \mathrm{ppm}$ both sets of signals appearing in the doublet, triplet, doublet, triplet, manner. The benzyl protons were found at $7.54 \mathrm{ppm}$ and $7.37 \mathrm{ppm}$ as a doublet and triplet respectively. The aromatic protons of the calix[4]arene ring were seen at 7.08, 6.92 and $6.62 \mathrm{ppm}$ as a singlet, doublet and triplet respectively. Again peaks indicating self-assembly were also seen in the aromatic region, other doublets were seen at $6.99 \mathrm{ppm}$ and $6.79 \mathrm{ppm}$ all representing four hydrogens each. Further doublets representing two hydrogens were observed at 6.44, 6.42, 6.40, 6.38 and $6.36 \mathrm{ppm}$. Several singlets were seen at 7.10, 7.06, 7.02 and $6.97 \mathrm{ppm}$ each corresponding to four hydrogens and another singlet at $6.88 \mathrm{ppm}$ was also seen corresponding to two hydrogens. Triplets were seen at 6.72, 6.70, $6.59,6.56,6.47$ and $6.32 \mathrm{ppm}$ all corresponding to two hydrogens each. The lower rim benzyl-methylene arm was seen at $4.87 \mathrm{ppm}$ as a singlet, the upper rim pyridyl- 
methylene protons were seen at $4.54 \mathrm{ppm}$ as a singlet and the final pyridylmethylene protons appeared at $4.44 \mathrm{ppm}$. The upper rim pyridyl-methylene and ethylene protons were found at 2.93 and $2.85 \mathrm{ppm}$ as two triplets respectively. The bridging methylene doublets appeared at 4.24 and 3.29 ppm and the lower rim estermethylene protons were seen at $3.66 \mathrm{ppm}$. The ester-methylene protons were seen at $4.13 \mathrm{ppm}$ as a multiplet and the ester-ethylene was located at $2.03 \mathrm{ppm}$ as a triplet. The ${ }^{13} \mathrm{C}$ NMR spectrum showed thirty-two non-equivalent carbon peaks, with the new pyridyl-methylene carbon appearing at $64 \mathrm{ppm}$.

Further functionalisation of the lower rim was also attempted with compound (50). The same conditions that were used for compound (45) were employed here. This included refluxing (50) with 2-(aminomethyl)-pyridine in a mixture of toluene and methanol (1:2) for 7 days under inert conditions. Compound (53) was obtained as a dark red-brown waxy solid in moderate yields (Figure 126).

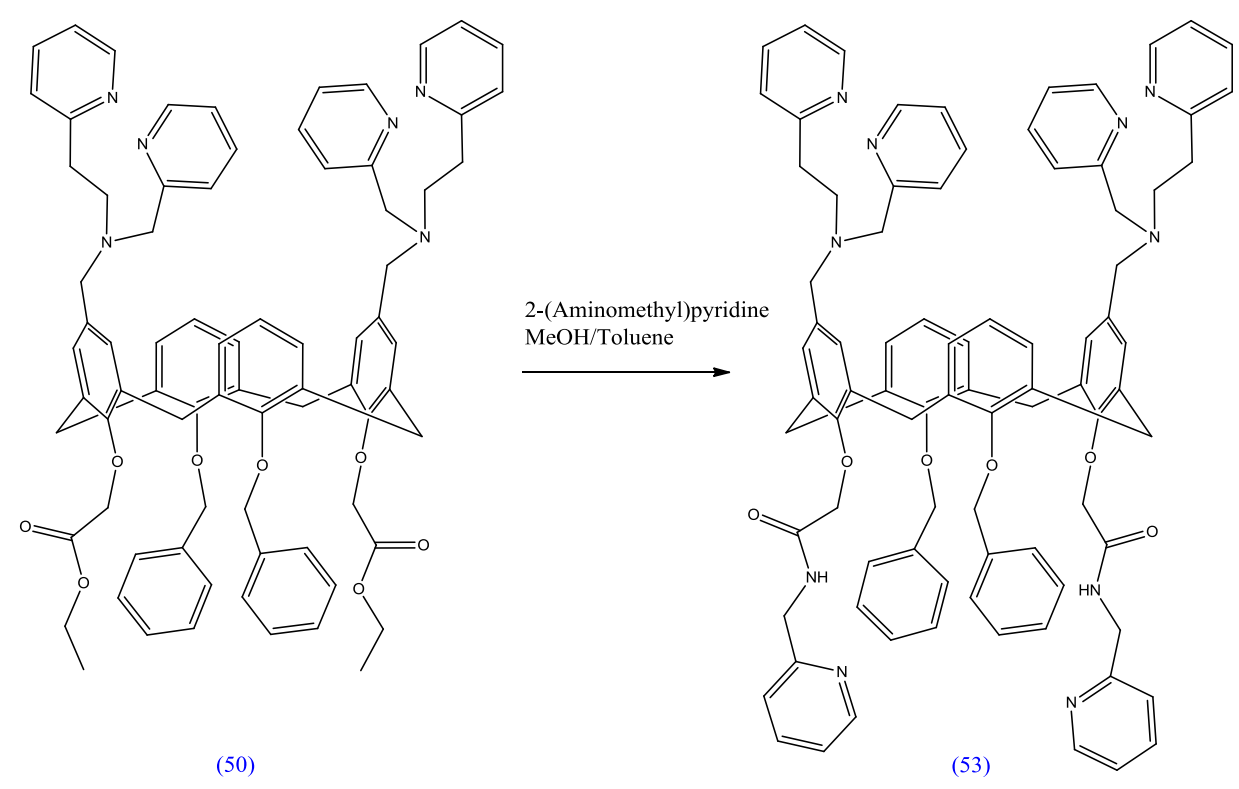

Figure 126: Synthesis of compound (53)

The IR data showed several peaks, including the $v_{\mathrm{NH}}$ band at $3410 \mathrm{~cm}^{-1}$ indicating that the amide group had been attached successfully, the $v_{\mathrm{CO}}$ band at $1631 \mathrm{~cm}^{-1}$ and the pyridyl $v_{\mathrm{NCH}}$ at 1589 and $1570 \mathrm{~cm}^{-1}$. The ${ }^{1} \mathrm{H}$ NMR spectrum showed only a small 
level of self-assembly occurring. It was seen that the lower rim pyridyl protons and upper rim methylpyridyl protons overlapped. They were found to occur in the doublet, triplet, doublet, triplet fashion at 8.51, 7.64, 7.33 and 7.23 ppm respectively. The upper rim ethylpyridyl protons occurred at 8.57, 7.83, $7.50 \mathrm{ppm}$ in the same doublet, triplet, doublet. The last triplet for the pyridyl group could not be clearly distinguished due to peak overlap but is expected at $7.21 \mathrm{ppm}$. The lower rim benzyl protons were found at $7.37 \mathrm{ppm}$ and $7.35 \mathrm{ppm}$ as a doublet and triplet respectively. The aromatic protons for the calix[4] arene ring showed a clear doubling of the peaks suggesting self-assembly, possibly in the hetero form as the upper rim pyridyl occurred further upfield than the lower rim pyridyl. In general the lower rim should be the highest so this suggests that stacking is occurring in a hetero manner where the upper rim ethylpyridyl protons must be able to interact with the lower rim groups of another calix[4]arene molecule causing the downfield shift. The aromatic protons occurred at 6.85 and $6.46 \mathrm{ppm}$ as a doublet and triplet respectively. Other signals occurred at 7.05, 6.76 and $6.63 \mathrm{ppm}$. Unfortunately due to the abundance of peaks in the aromatic region of the spectrum the singlets for the aromatic protons could not be located. The lower rim benzyl-methylene protons occurred at $5.05 \mathrm{ppm}$ as a singlet, the upper rim pyridyl-methylene protons occurred at $4.81 \mathrm{ppm}$ as a singlet, and the final pyridyl-methylene protons appeared at $4.81 \mathrm{ppm}$ also as a singlet. This peak however appeared as a doublet suggesting that the self-assembly is altering the environment the arm is in so that the two hydrogens are no longer equivalent. The lower-rim ester-methylene arm appeared at $4.16 \mathrm{ppm}$ as a singlet and the upper rim ethylpyridyl-methylene and -ethylene protons were found at $3.43 \mathrm{ppm}$ and at 2.99 ppm as two triplets. Both of these triplets had been shifted slightly downfield with respect to the precursor further supporting the heterodimerisation. The new lower rim amide-methylene arm was found at $4.96 \mathrm{ppm}$ as a broad singlet. The methylene bridging doublets were found at $4.31 \mathrm{ppm}$ and $3.38 \mathrm{ppm}$. The ${ }^{13} \mathrm{C}$ NMR spectrum (Figure 127) showed thirty-two non-equivalent carbon signals with the new amidemethylene carbon appearing at $49 \mathrm{ppm}$. 


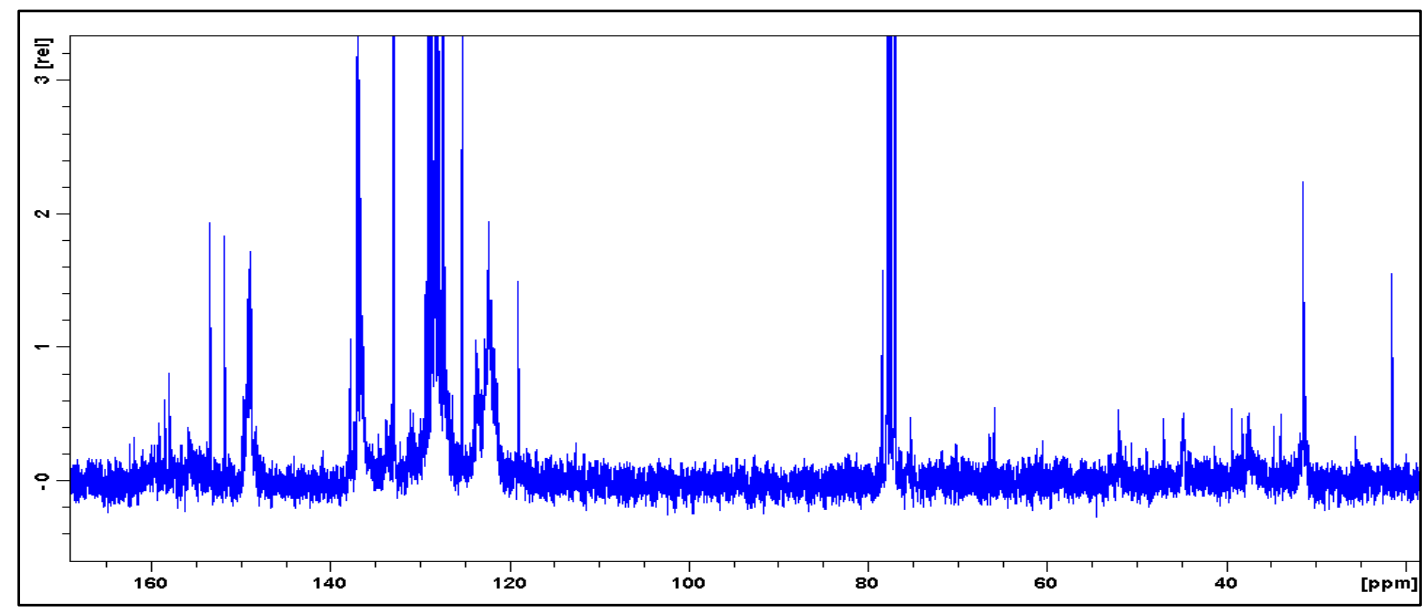

Figure 127: ${ }^{13} \mathrm{C}$ NMR spectrum of $(\mathbf{5 3})$

\subsection{Metal ion complexation reactions}

The metal ion binding ability of the majority of these compounds was examined with ${ }^{1} \mathrm{H}$ NMR and IR spectroscopy. In the calix[4]arene compounds which exhibited selfassembly, the metal ion binding experiments would serve two roles, firstly; would the compound have the ability to bind the metal ion without disrupting the selfassembly process, and secondly, would metal ion complexation break up the selfassembly process to give discrete metallo-calix[4]arene compounds. The metal salts (acetate, chloride and perchlorate) of zinc(II), mercury(II), copper(II), nickel(II) and cobalt(II) were used and the products of these reactions were initially examined by IR spectroscopy. The zinc and the mercury complexes were also examined by ${ }^{1} \mathrm{H}$ NMR spectroscopy as they are diamagnetic compounds. As diamagnetic compounds consist of paired electrons they don't create a local magnetic field as the relative spins of the paired electrons cancel out. Paramagnetic compounds on the other hand have lone electrons which can create their own local magnetic field which opposes the applied field from the NMR machine, resulting in shortening the relaxation time and ultimately leading to line broadening. Unless otherwise stated the metal ion complexation reactions were carried out in a 4:1 methanol to DCM mixture. The short-hand $1: 1$ or $1: 2$ etc. will be used to indicate the number of ligands to metal ions, where the first digit represents the ligand and the second digit the metal ion. 
Initially metal ion complexation reactions began with compound (37), (Figure 128). Good structural information has already been obtained for this compound using nOe NMR spectroscopy (section 3.7) and so it seemed like the logical choice for examination with metal salts.

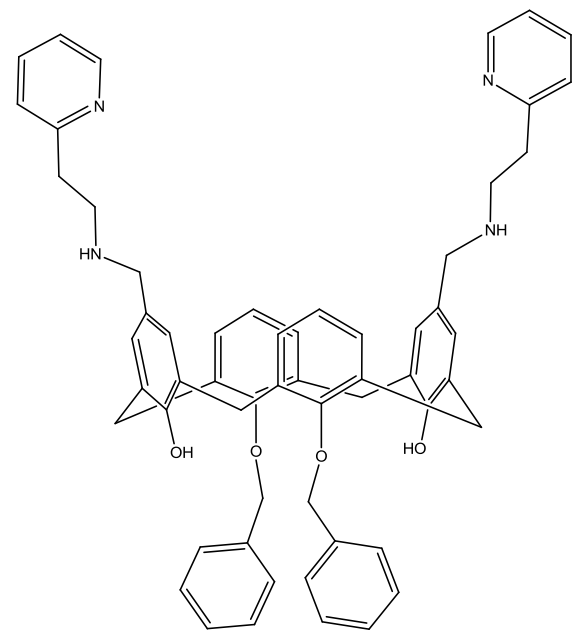

Figure 128: Compound (37)

The NMR spectroscopy examinations were recorded in IT Tallaght on a $500 \mathrm{MHz}$ Bruker Avance III NMR machine. 1:1 Metal ion complexation reactions were done with the acetate, chloride and perchlorate salts of mercury(II) and zinc(II) metals. From the figures below it can be seen that only a small amount of complexation is occurring with the mercury salts (Figure 129). In the aromatic region the pyridyl protons are shifted from $8.49 \mathrm{ppm}$ in the parent compound to $8.53 \mathrm{ppm}$ and 8.61 ppm in the chloride and perchlorate spectra respectively. 


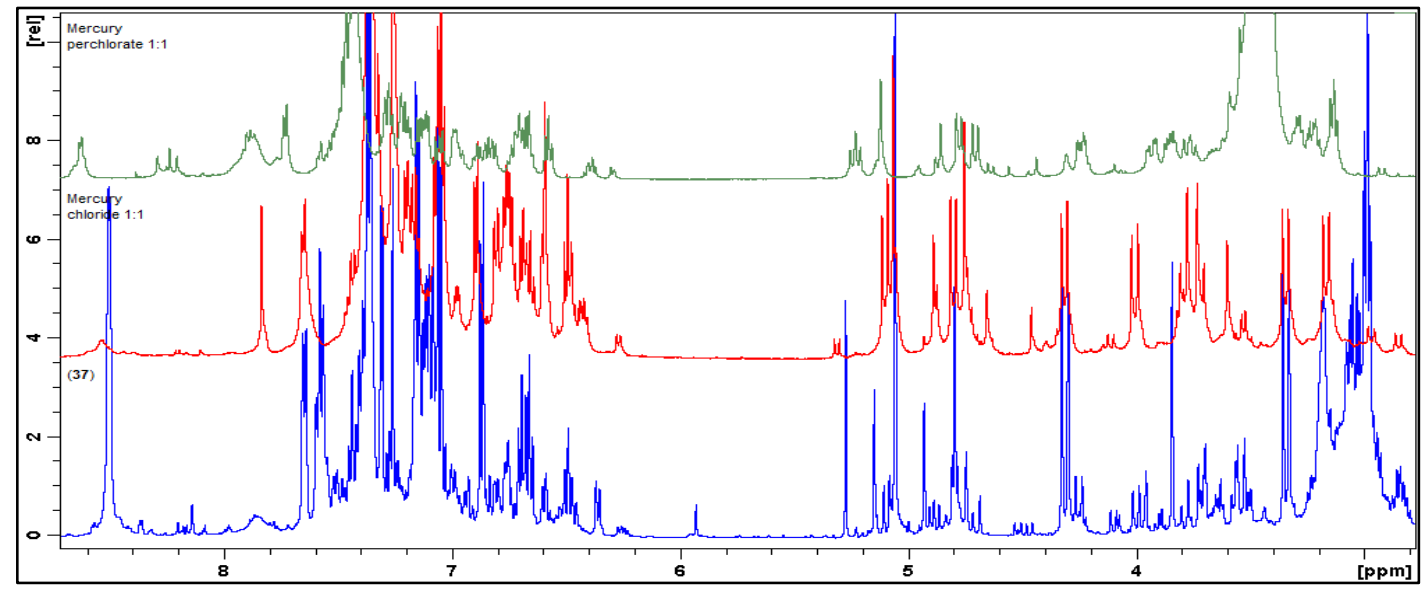

Figure 129: ${ }^{1} \mathrm{H}$ NMR of the mercury metal ion complexation with (37). Red trace $=$ Mercury(II) Chloride, Green trace $=$ Mercury $($ II) perchlorate .

In the bridging region of the spectrum, Figure 130, some minor shifts were seen in the perchlorate spectrum. The axial methylene protons were shifted upfield from $4.30 \mathrm{ppm}$ to $4.24 \mathrm{ppm}$ and the lower rim benzyl-methylene was shifted downfield form $5.05 \mathrm{ppm}$ to $5.11 \mathrm{ppm}$. The upper rim amine methylene arm was also shifted, and now appeared as two doublets at 4.71 and $4.70 \mathrm{ppm}$, indicating that the two protons are no longer in the same environment. This can be accounted for if we consider that one set of ethylpyridyl-methylene protons are in close proximity to the metal ion, causing the environment to change, and the other set of ethylpyridylethylene protons are away from the metal ion, such as in the self-assembled chain. A small shift can also be seen in the upper rim ethylpyridyl-methylene and ethylene protons from $3.04 \mathrm{ppm}$ and $2.98 \mathrm{ppm}$ to $3.20 \mathrm{ppm}$ and $3.13 \mathrm{ppm}$ respectively. The chloride spectrum is quite different in that the upper rim ethylpyridyl methylene and ethylene protons are no longer visible. This suggests that these two groups are no longer there. 


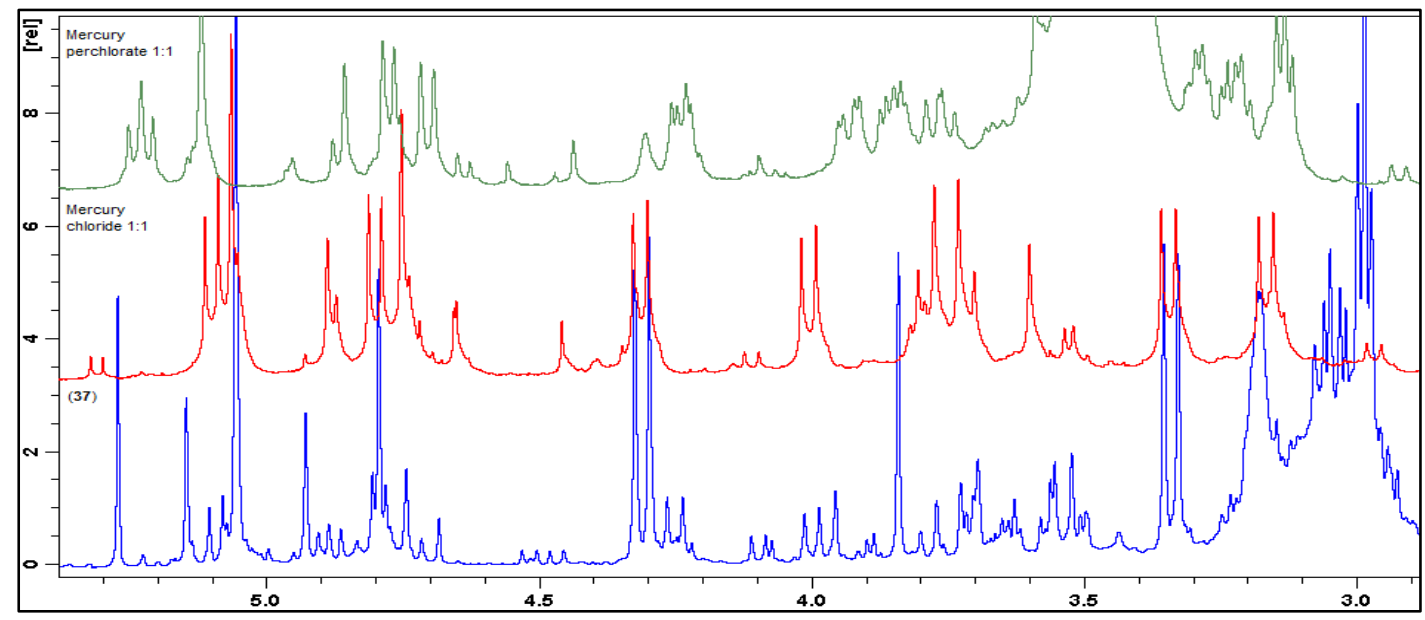

Figure 130: ${ }^{1} \mathrm{H}$ NMR of the bridging region of (37) with 1:1 mercury(II) complexation. Red trace $=$ Mercury $($ II) Chloride, Green trace $=$ Mercury(II) perchlorate .

However with the zinc salts, the chloride salt does not appear to bind as well as the acetate and perchlorate salts, producing only a small degree of chemical shift (0.02 ppm) (Figure 131). All three of the salts appear to bind to the upper rim pyridyl as evident from the downfield shift in the pyridyl proton signals in the ${ }^{1} \mathrm{H}$ NMR spectrum. In the parent compound the pyridyl protons occur at $8.49 \mathrm{ppm}$ and in the zinc acetate spectrum they occur downfield at $8.62 \mathrm{ppm}$, a difference of $0.13 \mathrm{ppm}$. In the zinc perchlorate spectrum a small downfield shift is seen from $8.49 \mathrm{ppm}$ to 8.53 ppm, a difference of $0.04 \mathrm{ppm}$. What can also be seen clearly in the acetate spectrum is another doublet at $8.56 \mathrm{ppm}$. This could be due to an uncomplexed pyridyl in a self-assembled form. It is very clear that the number of peaks in the spectra has not decreased however the spectra have cleaned up. 


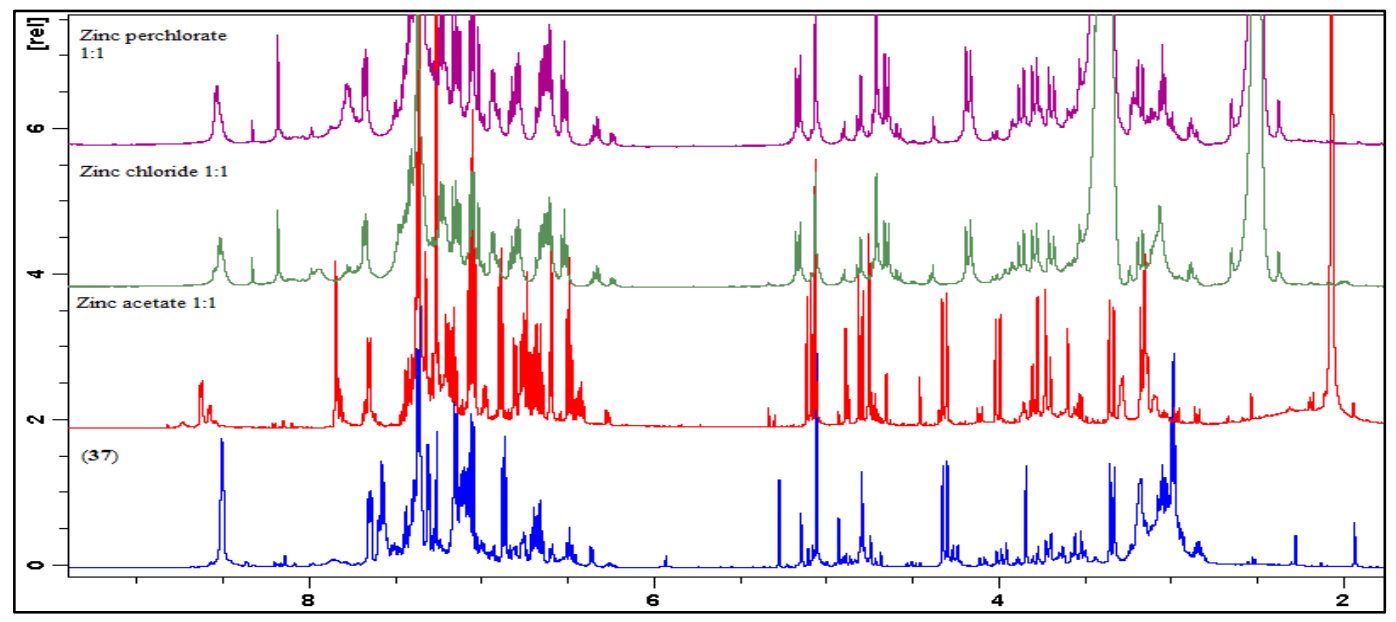

Figure 131: ${ }^{1} \mathrm{H}$ NMR of the 1:1 Zinc metal ion complexation with (37). Red trace = Zinc(II) acetate, Green trace $=$ Zinc(II) chloride, purple trace $=$ Zinc(II) perchlorate .

In the bridging region of the spectrum, (Figure 132) some changes can be seen. In the parent spectrum the methylene bridging protons occur at 4.31 and $3.33 \mathrm{ppm}$ for the axial and equatorial protons. In the acetate spectrum these protons don't experience any chemical shift, but in the chloride and perchlorate spectrum the axial protons shift to $4.17 \mathrm{ppm}$ a difference of 0.14 upfield. In the parent spectrum several peaks can be seen representing the lower rim benzyl-methylene at $5.05 \mathrm{ppm}$ arm as well as the upper rim methylamine methylene arm at $4.79 \mathrm{ppm}$. The peaks at 5.05 and $4.79 \mathrm{ppm}$, which are the most dominant signals don't experience any chemical shift, but the lesser peaks at $5.09 \mathrm{ppm}$, a doublet and a singlet at $4.74 \mathrm{ppm}$ do experience shifts. The peaks at $5.09 \mathrm{ppm}$ are shifted downfield to 5.10, 5.16 and 5.16 ppm in the acetate, chloride and perchlorate spectra respectively, and the singlet at 4.74 is shifted upfield to 4.71 and 4.70 in the chloride and perchlorate spectra. No change was seen in the acetate spectrum. The final observed shifts in this region are seen in the upper rim pyridyl-methylene and ethylene protons, which in the parent compound occur at 3.04 and 2.90 ppm respectively, but upon binding they experience downfield shifts to $3.09,3.17$ and $3.19 \mathrm{ppm}$ and 3.08, 3.05, $3.04 \mathrm{ppm}$ respectively in the acetate, chloride and perchlorate spectra. This suggests that metal ion complexation is having a noticeable effect on this region as well as enhancing some of the less dominant peaks. This could be due to the metal ion deshielding the protons by drawing electron density up through the calix[4]arene system, as well as indicating that metal ion complexation has occurred at the upper rim of (37). 


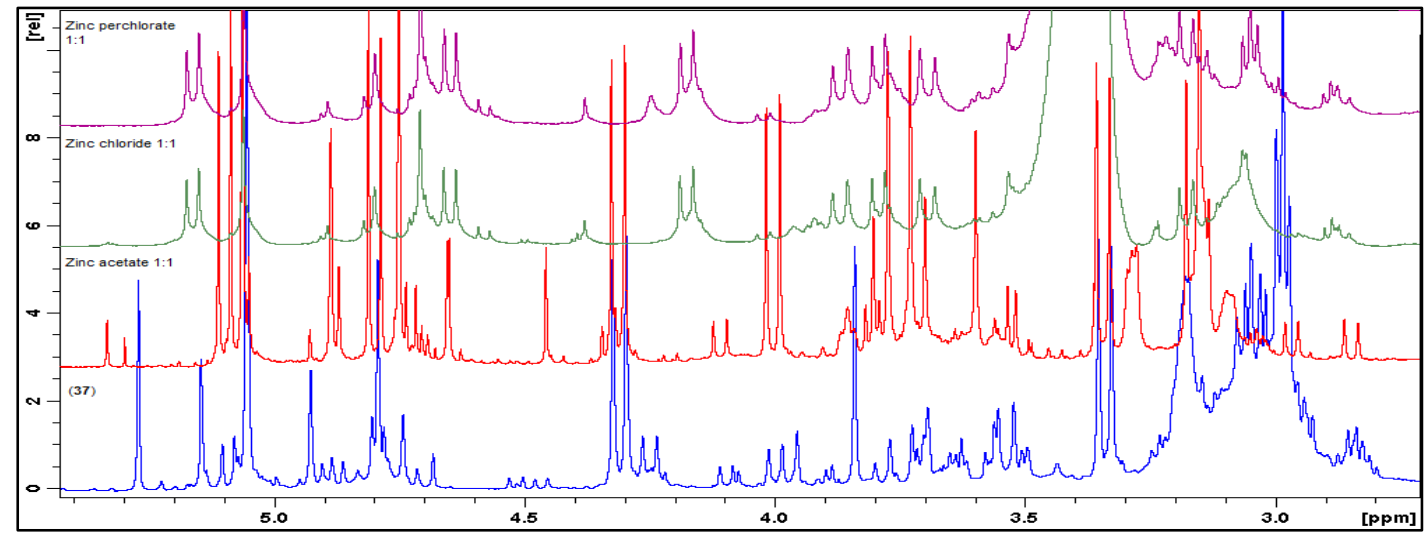

Figure 132: ${ }^{1} \mathrm{H}$ NMR of the bridging region of (37) with 1:1 zinc(II) metal ion complexation. Red trace $=$ Zinc $($ II $)$ acetate, Green trace $=$ Zinc $($ II $)$ chloride, purple trace $=$ Zinc(II) perchlorate.

Overall, these results show that the metal ion binding at a 1:1 concentration is not sufficient to overcome the self-assembly properties of the compound. To see if the self-assembly could be overcome by metal ion complexation, reactions of ligand to metal, from 1:2 up to $1: 4$ concentrations were examined using ${ }^{1} \mathrm{H}$ NMR spectroscopy. In the 1:2 metal ion complexation reactions, the chloride salt didn't appear to bind at all to the parent compound but the acetate and the perchlorate forms were seen to bind (Figure 133). The zinc acetate spectrum saw a downfield shift from $8.49 \mathrm{ppm}$ to $8.63 \mathrm{ppm}$ and the perchlorate shifted further downfield from $8.49 \mathrm{ppm}$ to $8.53 \mathrm{ppm}$ along with a small broad doublet occurring at $8.91 \mathrm{ppm}$. Overall the number of peaks had not decreased indicating that self-assembly was still dominating even though an excess of the metal was present. 


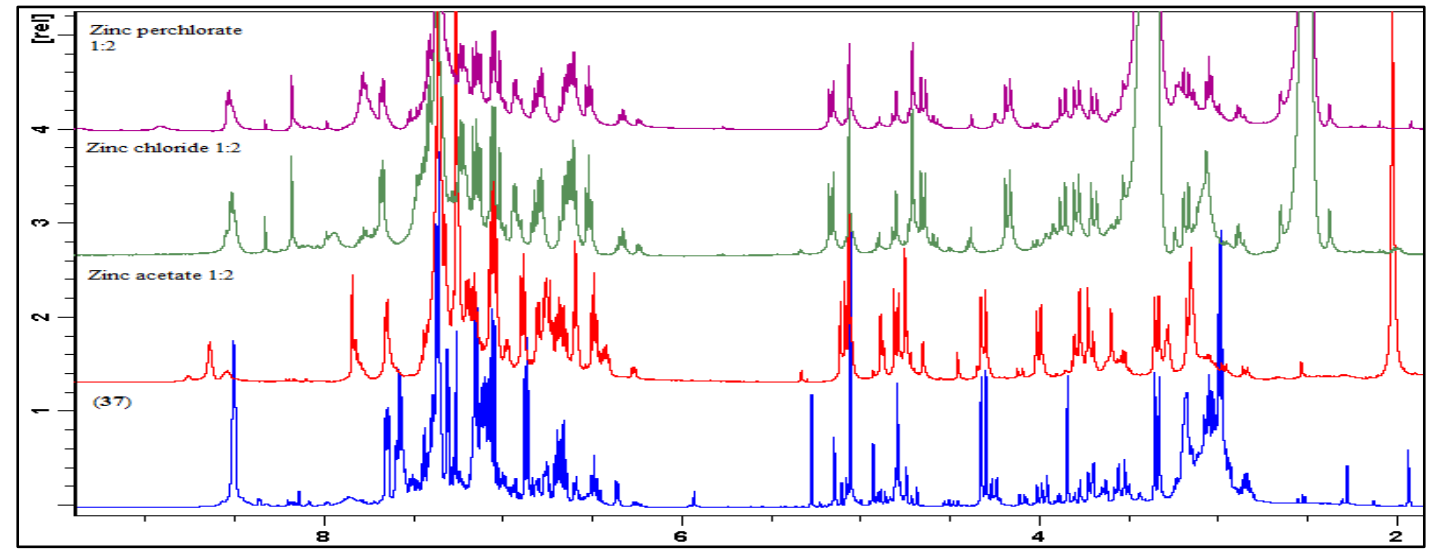

Figure 133: ${ }^{1} \mathrm{H}$ NMR of the 1:2 Zinc metal ion complexation with $(\mathbf{3 7})$. Red trace = Zinc(II) acetate, Green trace $=$ Zinc(II) chloride, purple trace $=$ Zinc(II) perchlorate.

As was seen previously some changes also occurred in the bridging region (Figure 134). Overall the same shifts that were seen for the 1:1 bridging region were seen again for the 1:2. While the number of peaks in the spectrum has decreased selfassembly is still occurring but possibly at a lower rate. A few peaks experienced shifts in the 1:2 complexation reactions, the axial bridging methylene protons experienced a shift to $4.17 \mathrm{ppm}$ in the chloride and perchlorate spectra, the methylamine methylene protons at $4.74 \mathrm{ppm}$ shifted to $4.70 \mathrm{ppm}$ in the chloride and perchlorate spectra and the two triplets corresponding to the ethylpyridyl-methylene and ethylene protons shifted from $3.04 \mathrm{ppm}$ to $3.14,3.17$ and $3.17 \mathrm{ppm}$ and from $2.98 \mathrm{ppm}$ to $3.05,3.05,3.04 \mathrm{ppm}$ respectively in the acetate, chloride and perchlorate spectra. As neither the 1:1 or the 1:2 ratios were able to overcome the self-assembly, $1: 3$ and 1:4 concentration were performed to see if they could overcome the assembly. 


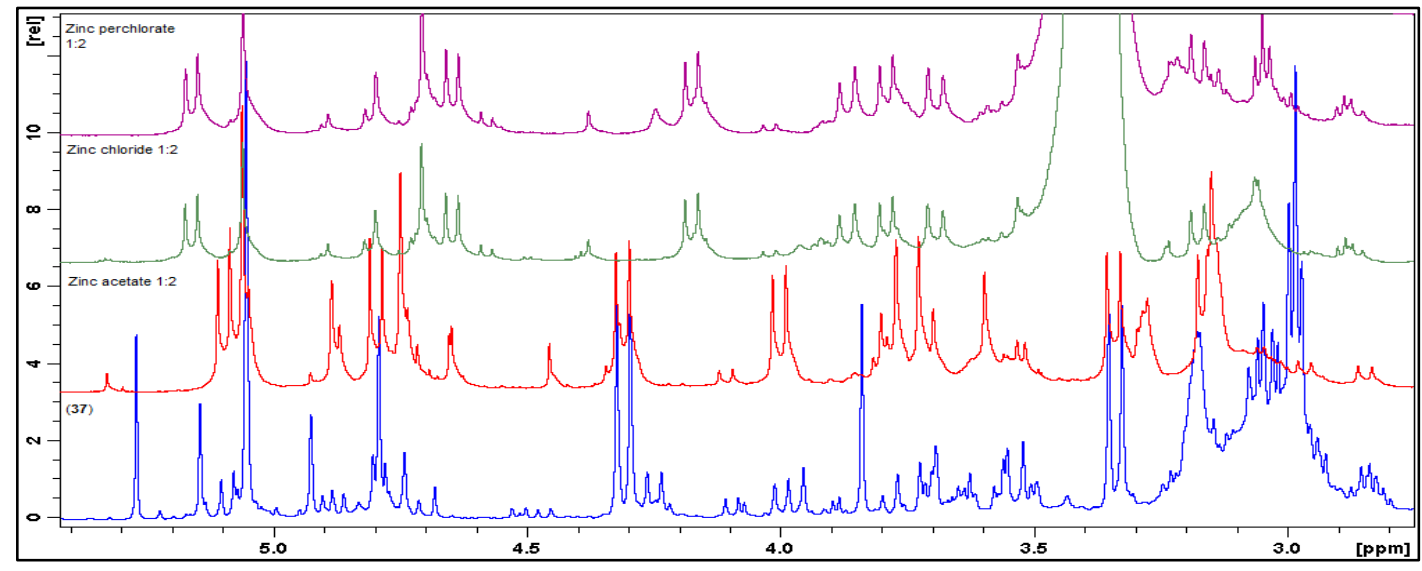

Figure 134: ${ }^{1} \mathrm{H}$ NMR of the bridging region of (37) at 1:2 zinc metal ion complexation. Red trace $=$ Zinc(II) acetate, Green trace $=$ Zinc(II) chloride, purple trace $=$ Zinc(II) perchlorate .

When the 1:3 and 1:4 ${ }^{1} \mathrm{H}$ NMR spectra were examined it was found that the selfassembly still dominated even when excess metal was present (Figure 135). In the 1:3 spectrum binding can be seen at pyridyl proton as well as binding at the other peaks previously seen for this region of the spectrum. In the bridging region, (Figure 136), it is clear that for both the $1: 3$ and 1:4, the spectra are becoming simpler. This might suggest that metal ion complexation is providing some order to the selfassembly but has not overcome it completely.

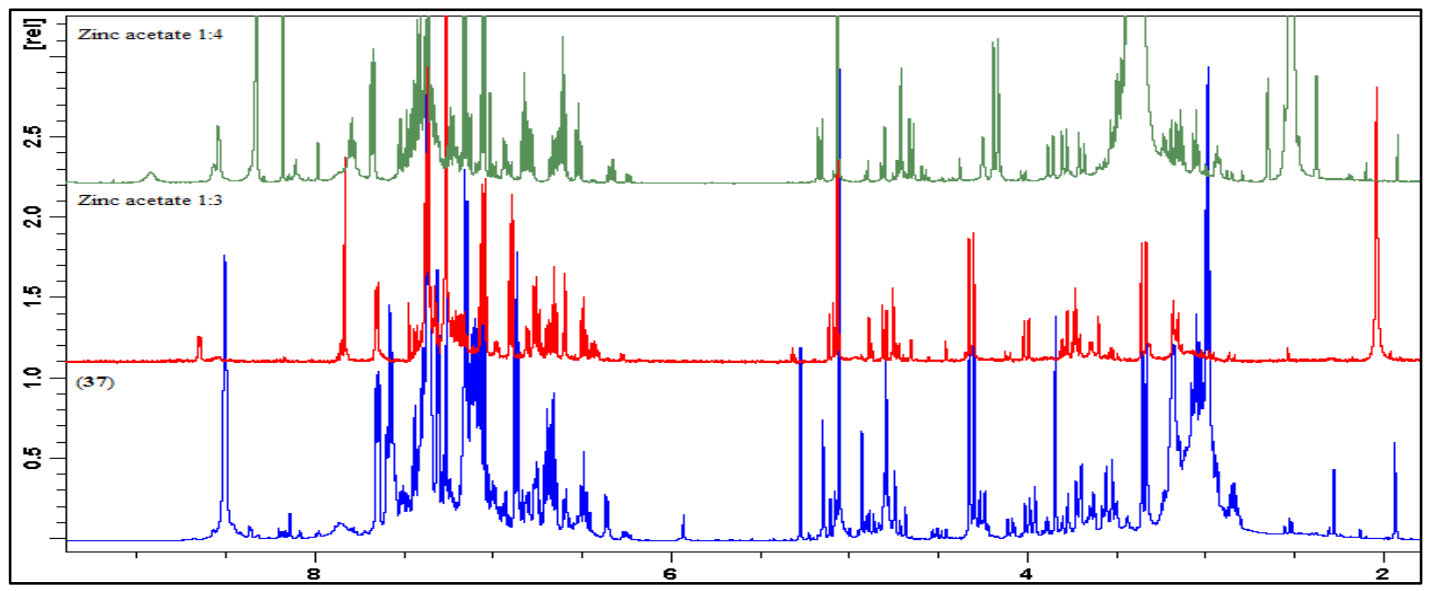

Figure 135: ${ }^{1} \mathrm{H}$ NMR of the 1:3 \& 1:4 Zinc acetate complexation reactions with (37). Red trace $=$ Zinc(II) acetate 1:3, Green trace $=$ Zinc(II) acetate 1:4. 


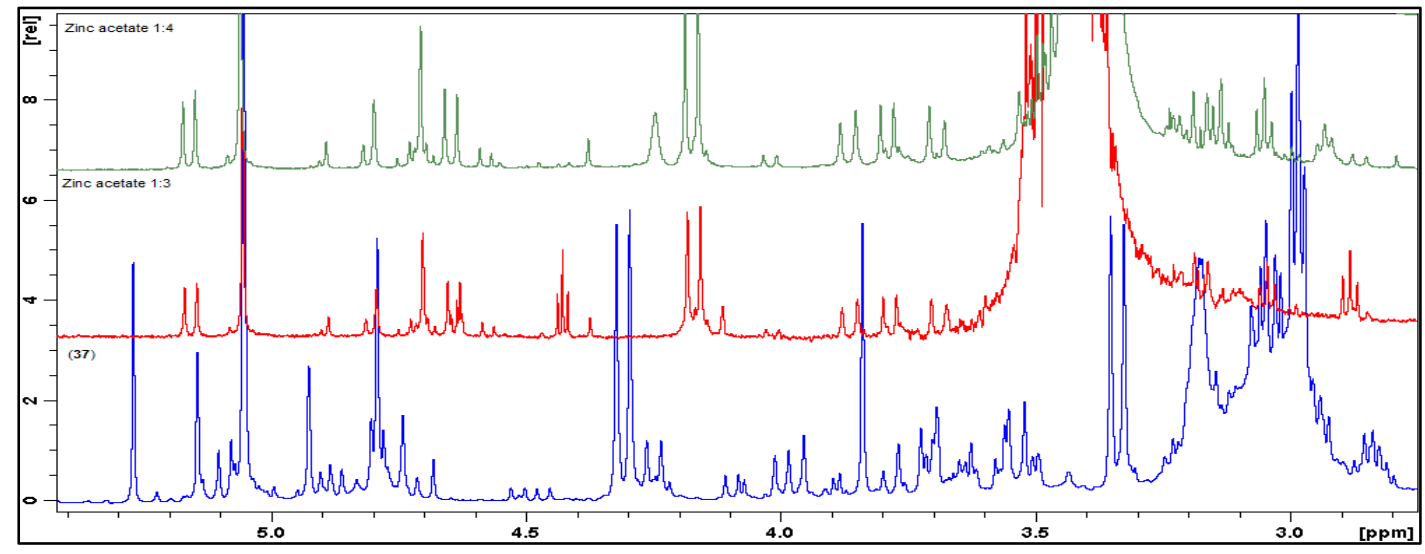

Figure 136: ${ }^{1} \mathrm{H}$ NMR of the bridging region of $1: 3$ and 1:4 Zinc(II) acetate complexation of (37). Red trace $=$ Zinc(II) acetate 1:3, Green trace $=$ Zinc(II) acetate 1:4 .

Serial dilution studies were also performed on the metal complexes and the most noticeable shifts were observed in the aromatic region of the spectrum representing the pyridyl protons as shown in Figure 137. It is clear from the image that most of the pyridyl peaks experience downfield shifts as previously mentioned, except the peak labelled 4, indicating that metal complexation has occurred. The complexation also provides a slight improvement in the spectra in terms of peak clarity, which is seen in peaks labelled 2 and 3, where in the parent spectrum clear assignment is difficult but in the complexed spectrum two triplets can be resolved.

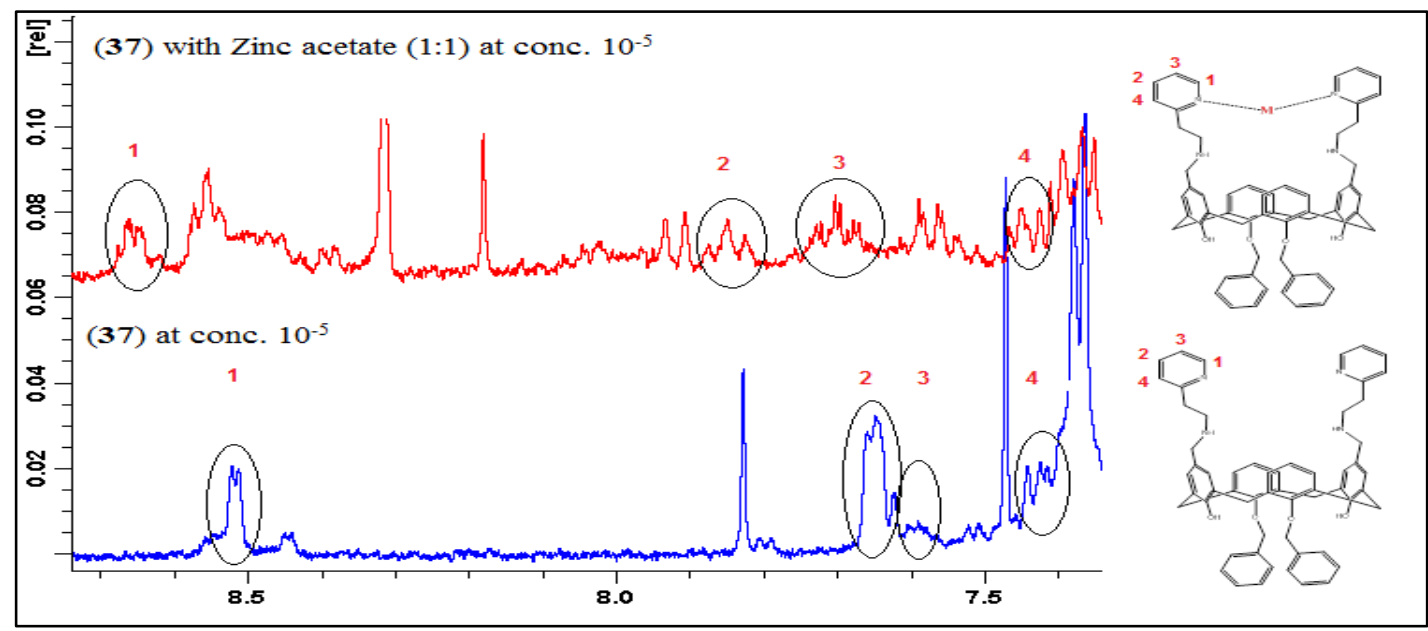

Figure 137: Comparison of pyridyl region of (37) and (37) metal complex at $10^{-5}$ molar solution 
nOe NMR spectroscopy studies were also performed on the metal complexes to see if the self-assembly was following the same trend as the parent compound. Essentially the same peaks that were chosen for the parent compound were chosen again for the metal complex (Figure 138). The nOe NMR spectra for the zinc acetate complex are shown in the figures below.

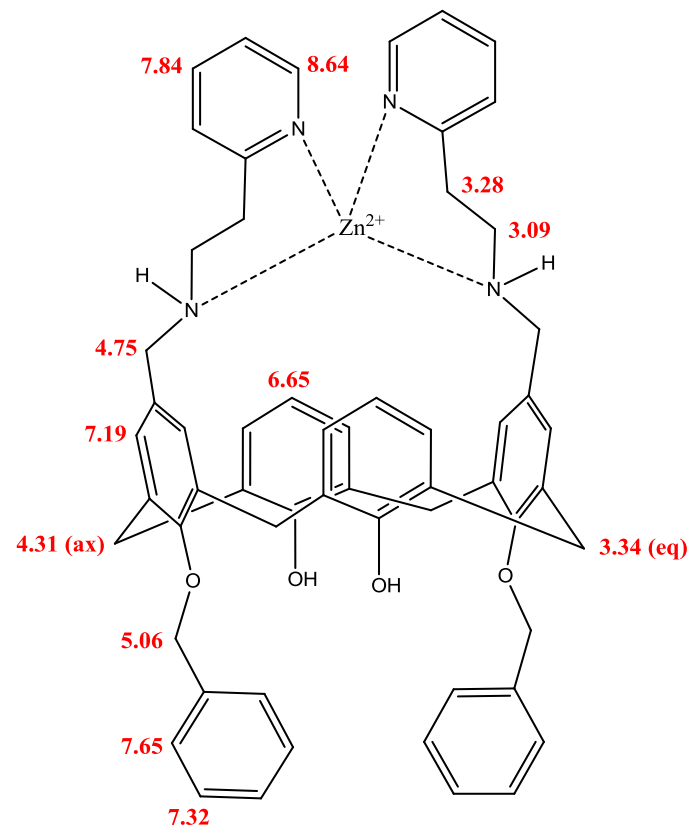

Figure 138: nOe NMR peaks chosen for metal complexed (37)

The first peak chosen was the upper rim pyridyl NCH at $8.64 \mathrm{ppm}$ (Figure 139). It was seen to interact with the pyridyl doublets at $8.65,8.64$ and $8.59 \mathrm{ppm}$, the pyridyl triplet at $6.69 \mathrm{ppm}$, the calix[4]arene triplet at $6.56 \mathrm{ppm}$, the lower rim arm at 5.09 ppm, both bridging doublets at 4.32 and $3.35 \mathrm{ppm}$, along with both of the triplets corresponding to the upper rim pyridyl linking arm. Two other broad doublets were seen at 4.01 and $3.38 \mathrm{ppm}$ as well as a singlet at $3.80 \mathrm{ppm}$. These latter three peaks may be representing another calixarene conformer. The second peak chosen was the upper rim pyridyl triplet at $7.84 \mathrm{ppm}$ (Figure 139), para to the nitrogen atom. It was seen to interact with the pyridyl doublet at $8.66 \mathrm{ppm}$, the lower rim hydroxyl singlet at $7.92 \mathrm{ppm}$, the lower rim benzyl doublet at $7.68 \mathrm{ppm}$ and the triplet at $7.31 \mathrm{ppm}$. Interactions were also seen with the upper rim pyridyl doublet at $7.28 \mathrm{ppm}$. The last 
two observed interactions corresponded to the upper rim aminomethyl bridging arm at $4.87 \mathrm{ppm}$ and the axial bridging doublets at $4.34 \mathrm{ppm}$.

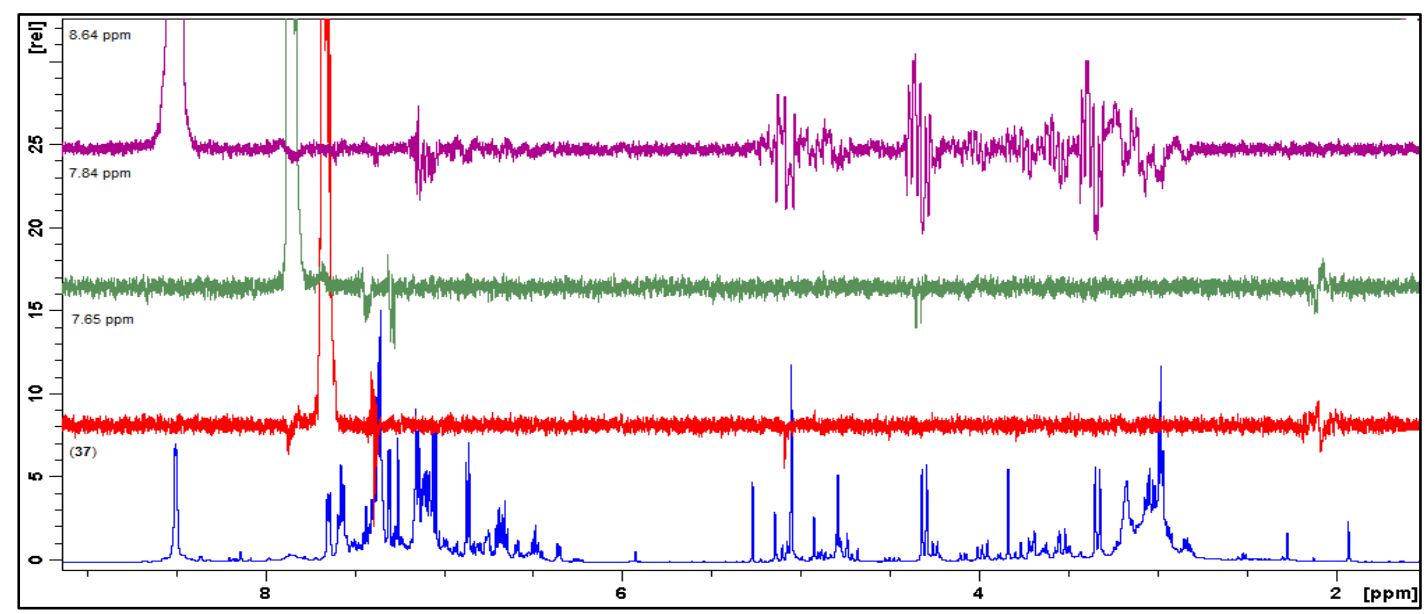

Figure 139: nOe NMR for aromatic protons of $(37)$. Red trace $=7.65 \mathrm{ppm}$, Green trace $=$ 7.84 ppm, Purple trace 8.64 ppm.

The next peak to be examined was the benzyl doublet at $7.65 \mathrm{ppm}$ (Figure 139). It was seen to interact with the upper rim pyridyl triplet at $7.87 \mathrm{ppm}$, the triplet of the benzyl at $7.38 \mathrm{ppm}$, the lower rim pendant arm at $5.09 \mathrm{ppm}$, the upper rim pendant arm at $4.92 \mathrm{ppm}$ and the axial bridging doublet at $4.34 \mathrm{ppm}$. The final observed peak was at $3.81 \mathrm{ppm}$ possibly corresponding to a different conformation. The benzyl triplet at $7.32 \mathrm{ppm}$ (Figure 140) was also examined and it showed to interact with the upper rim pyridyl doublet at $8.65 \mathrm{ppm}$, the benzyl doublet at $7.87 \mathrm{ppm}$, the upper rim pyridyl triplet and doublet at $7.39 \mathrm{ppm}$ and $7.22 \mathrm{ppm}$ respectively. It also interacted with the aromatic singlet, doublet and triplet of the calix[4]arene at 7.01, 6.81 and $6.74 \mathrm{ppm}$ respectively. Interaction with the upper rim linking arm at $4.95 \mathrm{ppm}$ and the upper arm triplet at $3.14 \mathrm{ppm}$ were also seen. Two other interactions were also seen at 3.75 and 3.63 ppm again most likely due to an alternate conformer.

The aromatics of the calix[4]arene were next to be examined, the singlet at $7.19 \mathrm{ppm}$ and the triplet at $6.65 \mathrm{ppm}$ (Figure 140). The singlet interacted with doublets at 7.14 and $7.11 \mathrm{ppm}$ and a singlet at $7.10 \mathrm{ppm}$, possible alternate forms, as well as a triplet at $6.83 \mathrm{ppm}$ for the calix[4] arene triplet. It was also seen to interact with two singlets at 4.91 and $4.78 \mathrm{ppm}$ representing the upper rim aminomethyl pendant arm, possibly 
from itself as well as another conformer. The triplet of the calix[4]arene interacted with two doublets at 6.70 and 6.88 along with the upper rim pendant arm at 4.77 ppm. It also interacted with the axial doublets at $4.28 \mathrm{ppm}$. Other interactions were seen at 4.03, 3.89, 3.80 and 3.73 ppm indicating other possible conformers.

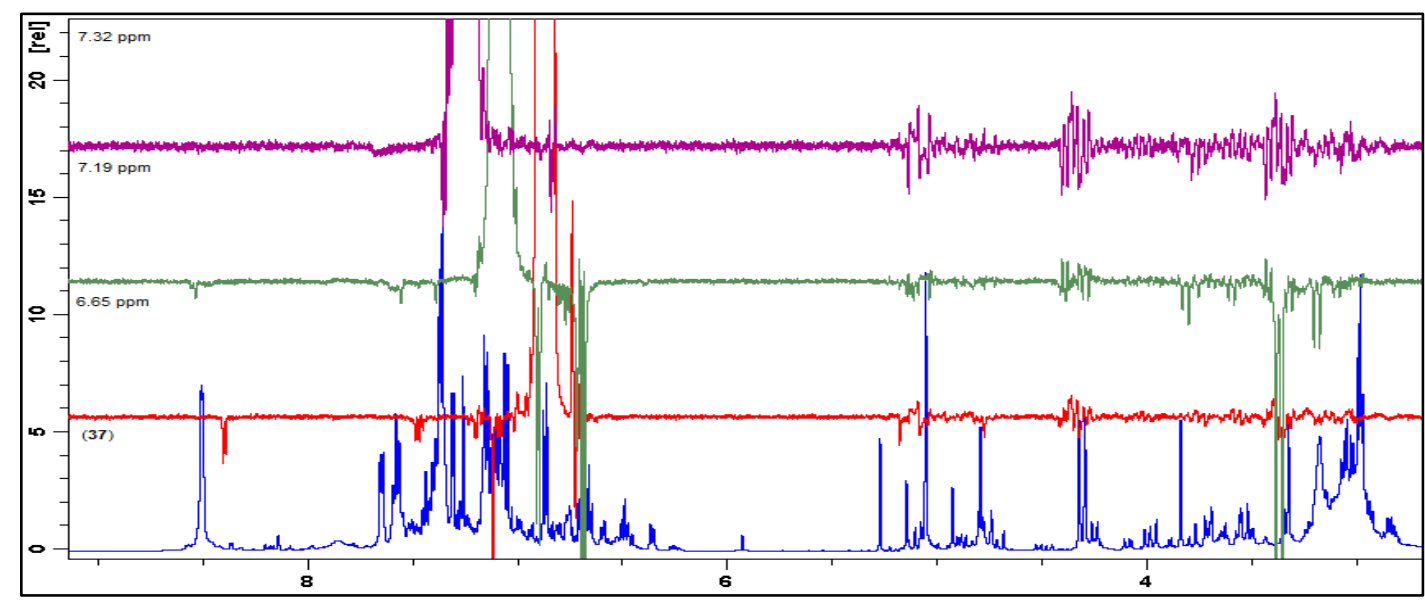

Figure 140: nOe NMR for aromatic protons of $(37)$. Red trace $=6.65 \mathrm{ppm}$, Green trace $=$ $7.19 \mathrm{ppm}$, Purple trace $=7.32 \mathrm{ppm}$.

The two main linking lower and upper rim arms at $5.06 \mathrm{ppm}$ and $4.75 \mathrm{ppm}$ were also investigated (Figure 141). The lower rim arm was found to interact with the lower rim benzyl doublet at $7.68 \mathrm{ppm}$ and the upper rim pyridyl triplet at $7.29 \mathrm{ppm}$ as well as the axial bridging doublets. Other interactions were also observed at 5.15, 5.11 and $5.09 \mathrm{ppm}$ which could indicate other conformations present. The upper rim methylamine arm interacted with the unbound pyridyl doublets at $8.49 \mathrm{ppm}$, the lower rim benzyl doublet at $7.60 \mathrm{ppm}$, and the calix[4]arene doublet and triplet at 6.83 and $6.63 \mathrm{ppm}$ respectively. Interactions with the lower rim pendant arm at 5.04 ppm were seen as well as several other interactions at 4.73, 3.81 and 3.74, again representing other conformers. The bridging doublets were next to be examined under nOe NMR spectroscopy. The axial doublets at $4.31 \mathrm{ppm}$ (Figure 141) were seen to interact with the benzyl doublet and the calix[4]arene singlet, the lower rim pendant arm and the equatorial bridging doublets. The equatorial bridging doublets at $3.34 \mathrm{ppm}$ (Figure 142) interacted with the calix[4]arene singlet at $7.07 \mathrm{ppm}$ as well as a doublet at $6.92 \mathrm{ppm}$. It was also seen to interact with the axial bridging doublet at $4.36 \mathrm{ppm}$. 


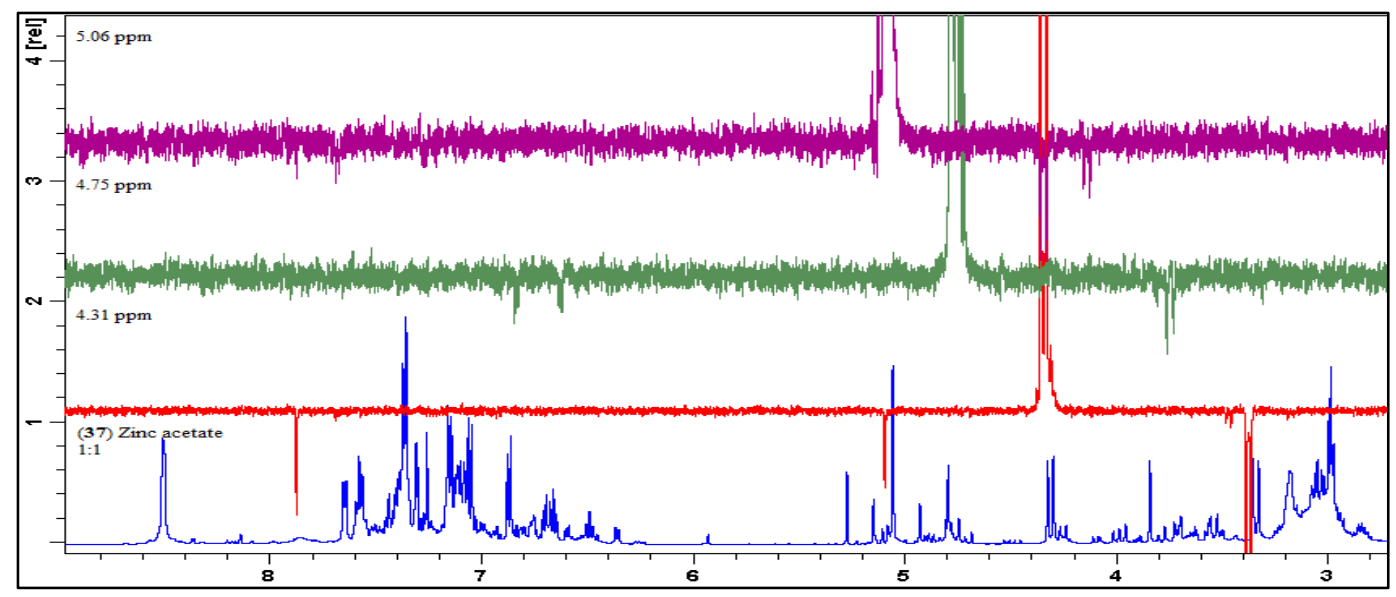

Figure 141: nOe NMR for bridging protons of $(37)$. Red trace $=4.31 \mathrm{ppm}$, Green trace $=$ $4.75 \mathrm{ppm}$, Purple trace $=5.06 \mathrm{ppm}$.

The final two peaks to be examined were the upper rim triplets of the pyridyl linking arm at 3.28 and $3.09 \mathrm{ppm}$ respectively (Figure 142). Both of these seemed only to interact with each other and virtually no other interaction was seen.

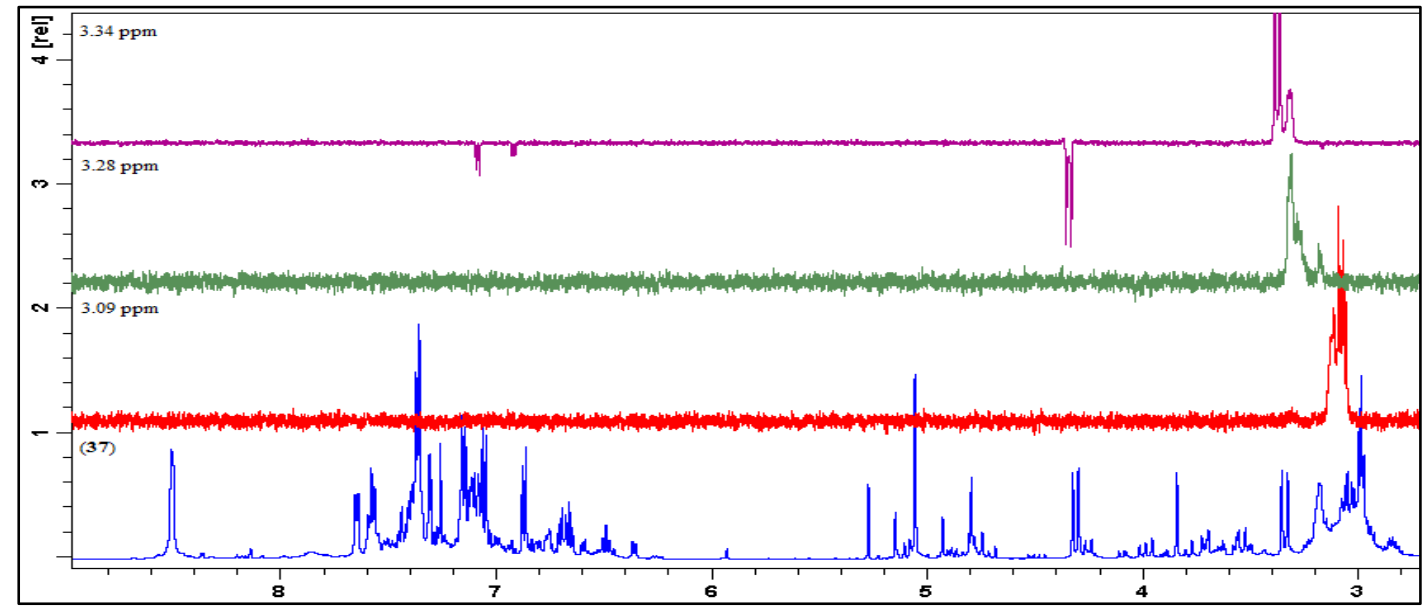

Figure 142: nOe NMR for bridging protons of (37) 


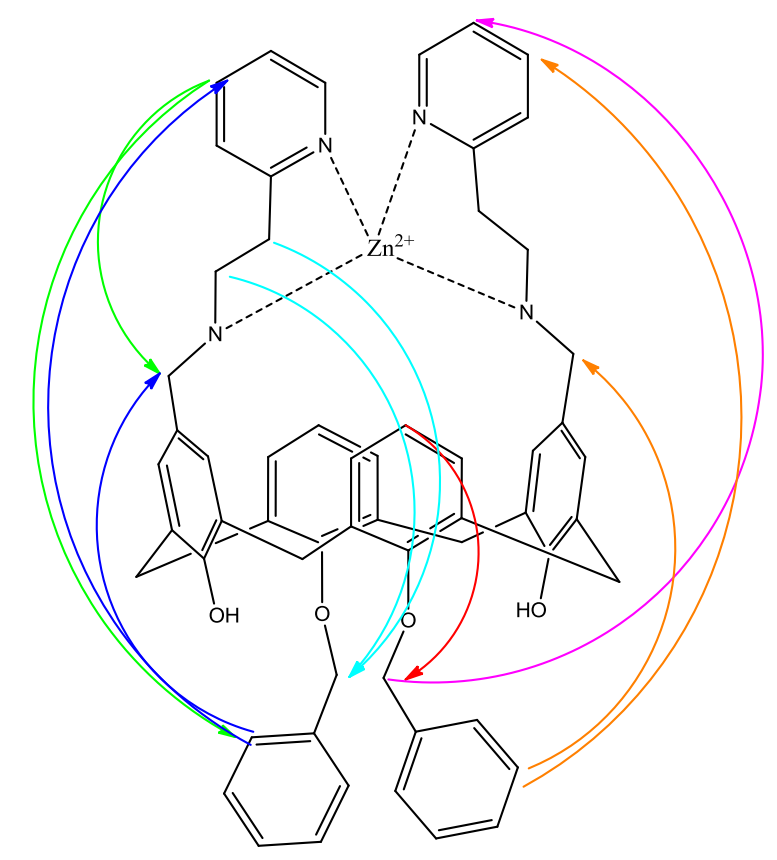

Figure 143: Summery of nOe NMR for metal complexed (37)

Figure 143 shows a summary of some of the interactions seen by the zinc(II) acetate complex of (37). Starting from left to right, the red arrows indicate the upper rim pyridyl NCH interactions, the green arrows the pyridyl para triplet interactions, the purple the lower rim pendant arm, the light blue arrows the upper rim pendant arm, the orange the benzyl triplet and the dark blue representing benzyl doublet interactions. It is clear from this image that both the hetero- and homodimerisation forms are possible. The hetero form can clearly be seen by the fact the lower rim benzyl group is able to interact with the upper rim pyridyl group as well as aromatic protons of the calix[4]arene ring. The homo form may be the less dominant form as the majority of the interactions seem to support the hetero. However, due to the interactions between the upper rim pyridyl-methylene protons and those of the pyridyl triplet the homo form may be present in a lesser amount. Without a crystal structure, however, this is open to debate.

When the 1:2 ligand to metal, metal ion complexation reactions were examined under nOe NMR spectroscopy similar results were obtained to those described above. From this what we propose is that metal ion complexation is occurring with one of the calix[4]arene molecules at the top and that self-assembly is continuing on 
further down the line, in both the hetero- and homodimerisation fashion (Figure 144).

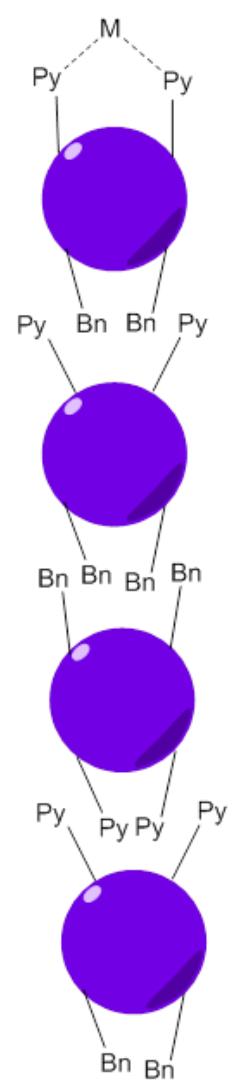

Figure 144: Proposed hetero- and homodimerisation chain for (37)

The IR data for compound (37) corresponds well with the ${ }^{1} \mathrm{H}$ NMR spectra (Table 4). Large downfield shifts were seen for the NCH bands, along with residual bands present for the unbound pyridyl's in the self-assembled chain. Some shifts were seen in the hydroxyl groups which is most likely caused by the self-assembly interactions.

\begin{tabular}{|l|c|c|c|c|}
\hline & $\mathrm{O}-\mathrm{H} \mathrm{cm}^{-1}$ & $\begin{array}{r}\mathrm{NC}-\mathrm{H} \text { (bound) } \\
\mathrm{cm}^{-1}\end{array}$ & $\begin{array}{r}\mathrm{NC}-\mathrm{H} \text { (unbound) } \\
\mathrm{cm}^{-1}\end{array}$ & $\mathrm{~N}-\mathrm{H}$ \\
\hline Compound (37) & 3394 & 1591 & 1587 & 3351 \\
\hline 1:1 reaction & & & & $\mathrm{N} / \mathrm{O}$ \\
\hline Nickel perchlorate & 3419 & 1609 & 1570 & $\mathrm{~N} / \mathrm{O}$ \\
\hline Nickel acetate & 3420 & 1591 & $\mathrm{~N} / \mathrm{O}$ & $\mathrm{N} / \mathrm{O}$ \\
\hline Nickel chloride & 3407 & 1591 & \multicolumn{4}{|l}{} \\
\hline
\end{tabular}




\begin{tabular}{|l|l|l|l|l|}
\hline Copper acetate & 3405 & 1590 & $\mathrm{~N} / \mathrm{O}$ & $\mathrm{N} / \mathrm{O}$ \\
\hline Copper chloride & 3524 & 1607 & $\mathrm{~N} / \mathrm{O}$ & $\mathrm{N} / \mathrm{O}$ \\
\hline Copper perchlorate & 3398 & 1614 & $\mathrm{~N} / \mathrm{O}$ & 3314 \\
\hline Cobalt Chloride & 3415 & 1627 & $1592 / 1569$ & $\mathrm{~N} / \mathrm{O}$ \\
\hline Zinc chloride & 3429 & 1651 & $1592 / 1570$ & 3254 \\
\hline Zinc acetate & 3419 & 1591 & 1570 & $\mathrm{~N} / \mathrm{O}$ \\
\hline Zinc perchlorate & 3431 & 1591 & $\mathrm{~N} / \mathrm{O}$ & $\mathrm{N} / \mathrm{O}$ \\
\hline
\end{tabular}

Table 4: Selected IR data for metal complexes of (37). N/O = not observed, those in green experience shifts compared with parent spectrum in red.

The next self-assembled complexes to be tested for metal ion binding ability were compounds (30) and (31), (Figure 145). For compound (30) the metal ion complexation reactions proceeded with the addition of the metal salt at a 1:1 ratio, as there was only one potential metal binding site located on the upper rim. The solution was allowed to stir at room temperature for two hours and then the solvent was removed under reduced pressure. All of the metal ion complexes were washed with water and dichloromethane to remove any excess metal salt. As mercury salts seemed to display poor binding potential with these compounds, ${ }^{1} \mathrm{H}$ NMR spectroscopy from this point on will focus on the binding of zinc salts.

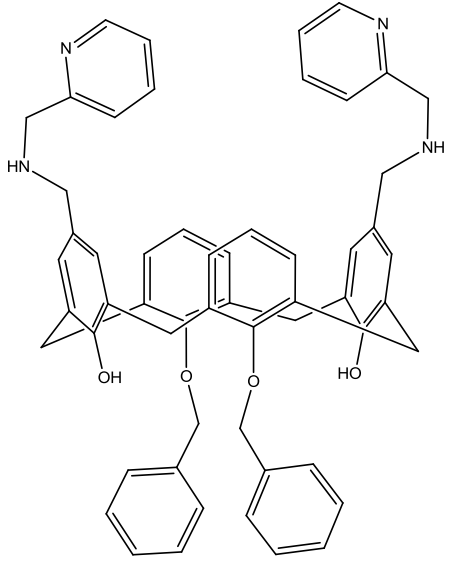

(30)

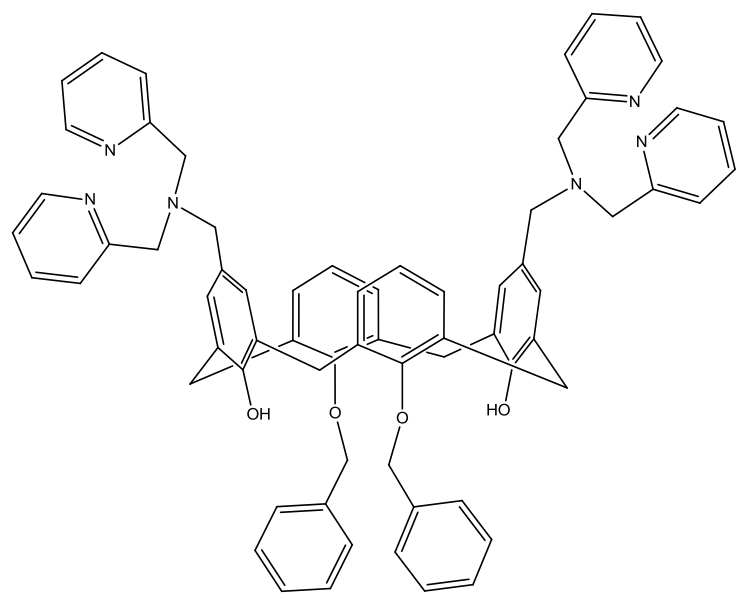

(31)

Figure 145: Compounds (30) \& (31)

For compound (30) the 1:1 ${ }^{1} \mathrm{H}$ NMR spectra for the reactions with the three zinc salts are shown below (Figure 146). In the 1:1 ratio reactions it can be clearly seen that a 
real shift in the peaks can be observed in the aromatic region from approx. $8.60 \mathrm{ppm}$. This is most likely due to the upper rim pyridyl interactions with the zinc(II) metal ion. In the acetate spectrum a shift from $8.49 \mathrm{ppm}$ to $8.57 \mathrm{ppm}$ can be seen for the upper rim pyridyl doublet protons, a difference of $0.08 \mathrm{ppm}$. The chloride spectrum shows a shift of $0.14 \mathrm{ppm}$ on going from 8.49 to $8.63 \mathrm{ppm}$, and the perchlorate spectrum shows a shift to $8.57 \mathrm{ppm}$, a difference of $0.08 \mathrm{ppm}$. From this it can be concluded that the chloride spectrum gave the largest shift and all three displayed a doublet at $8.49 \mathrm{ppm}$ to indicate the unbound pyridyl doublets in the self-assembled chain.

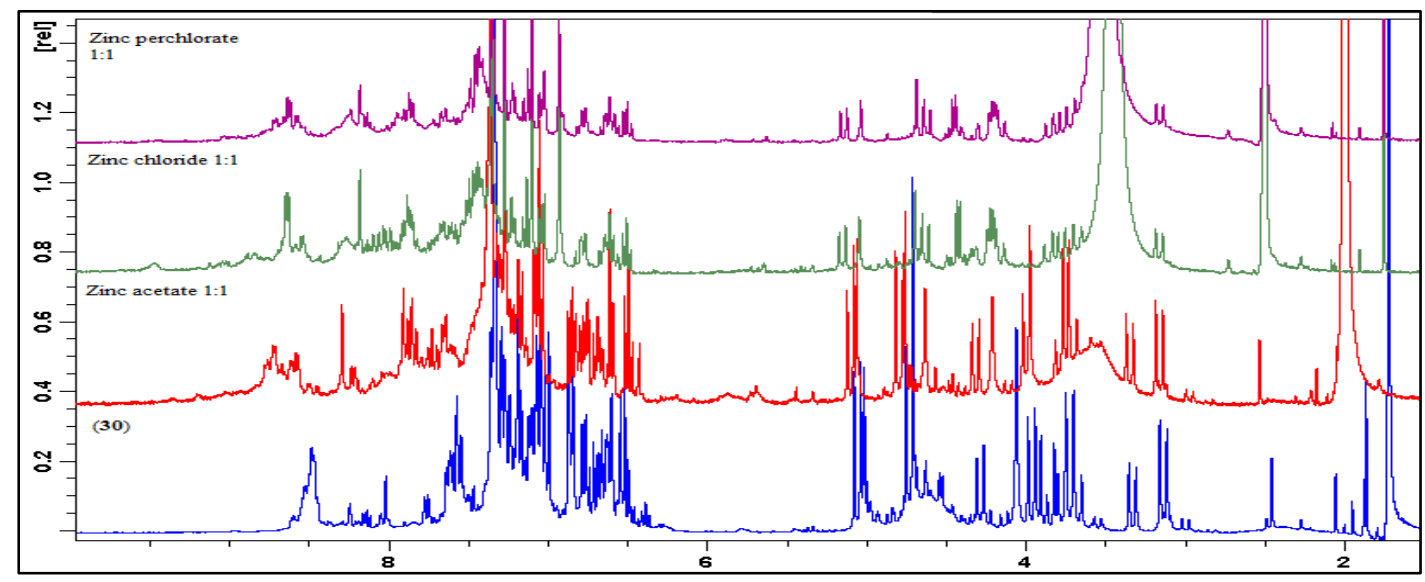

Figure 146: 1:1 ${ }^{1} \mathrm{H}$ NMR spectra for zinc(II) metal salts binding with (30)

Unfortunately during the metal ion complexation reactions methanol was used as the solvent and as a result some methanol has remained trapped within the cavity of the calix[4]arene ring, obscuring the bridging methylene protons. Some clear changes can be seen in the bridging region, (Figure 147), despite this. The most noticeable is that in the parent compound the bridging methylene protons appeared as a doublet within a doublet but upon metal ion complexation they are resolved to a clear single doublet. In the parent spectrum the axial bridging methylene protons appear at 4.49 ppm and are shifted to $4.31,4.41$ and $4.46 \mathrm{ppm}$ in the acetate, chloride and perchlorate spectra respectively. No change is seen in the lower rim benzyl methylene protons which occur at $5.05 \mathrm{ppm}$ in all the spectra. The upper rim methylamine methylene protons occur at $4.54 \mathrm{ppm}$ in the parent spectrum and are shifted to $4.63,4.69$ and $4.69 \mathrm{ppm}$ in the acetate, chloride and perchlorate spectra respectively. This is further support for metal ion complexation at the upper rim, due 
to the deshielding property of the metal. Several other doublets can clearly be seen in the spectrum indicating that self-assembly has not been overcome, but that metal ion complexation has occurred on the upper rim.

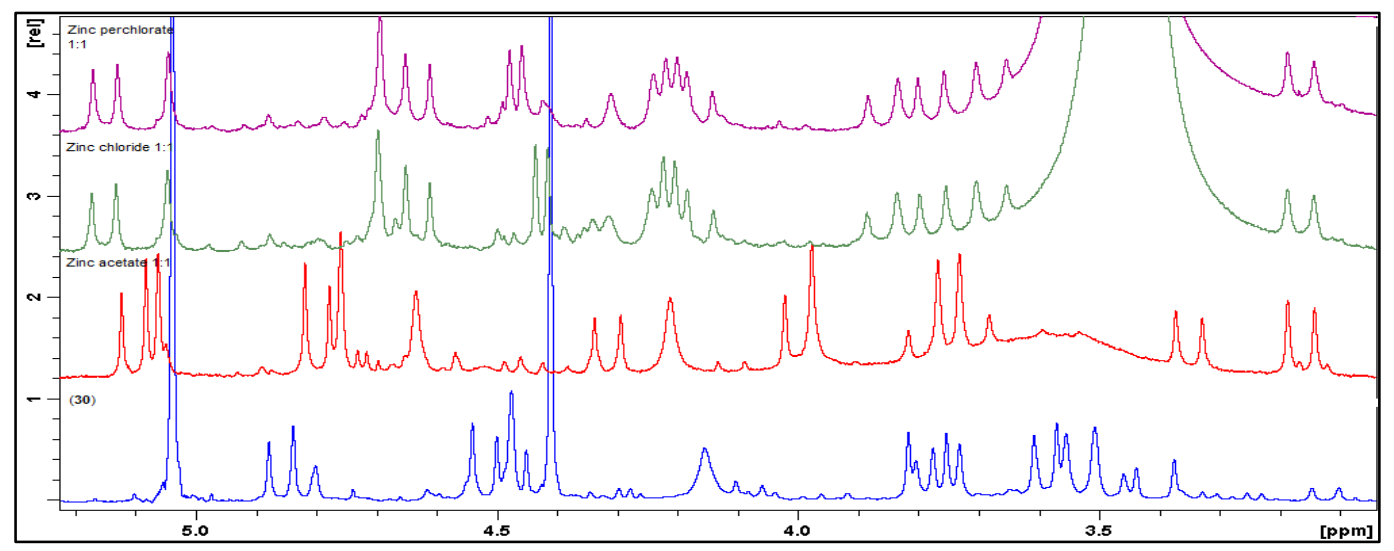

Figure 147: ${ }^{1} \mathrm{H}$ NMR of the bridging region of (30) with 1:1 zinc(II) metal ion complexation. Red trace $=$ Zinc(II) acetate, Green trace $=$ Zinc(II) chloride, purple trace $=$ Zinc(II) perchlorate.

The IR data (Table 5) for compound (30) shows that the $\mathrm{NCH}$ bands experience shifts due to binding the metal ion, and with the hydroxyl group experiencing a small shift in a limited number of the complexed compounds. The $\mathrm{NH}$ band undergoes downfield shifts in the majority of the complexed compounds. This data suggests that binding is occurring on the upper rim of the calix[4] arene and that shifts in the hydroxyl groups are most likely caused by the effects of self-assembly. This corresponds well with the proton NMR spectra which all show shifts in the upper rim pyridyl doublet.

\begin{tabular}{|l|c|c|c|}
\hline & $\mathrm{O}-\mathrm{H} \mathrm{cm}^{-1}$ & $\mathrm{NC}-\mathrm{H} \mathrm{cm}^{-1}$ & $\mathrm{~N}-\mathrm{H} \mathrm{cm}^{-1}$ \\
\hline Compound (30) & 3414 & 1591 & 3314 \\
\hline 1:1 reaction & & & 3257 \\
\hline Zinc acetate & 3426 & 1607 & 3386 \\
\hline Zinc chloride & 3447 & 1607 & 3391 \\
\hline Zinc perchlorate & 3444 & 1607 & 3333 \\
\hline Nickel chloride & 3413 & 1605 & 3355 \\
\hline Cobalt chloride & 3411 & 1591 & 3327 \\
\hline Nickel acetate & 3391 & 1610 & \\
\hline
\end{tabular}




\begin{tabular}{|l|l|l|l|}
\hline Copper acetate & 3424 & 1591 & 3364 \\
\hline Copper perchlorate & 3386 & 1609 & 3328 \\
\hline Nickel perchlorate & 3422 & 1606 & 3349 \\
\hline Copper chloride & 3419 & 1591 & 3346 \\
\hline
\end{tabular}

Table 5: Summery of IR results from metal ion complexation reactions with (30). N/O = not observed, those in green experience shifts compared with parent spectrum in red.

The metal ion complexation reactions of (31) were also examined by ${ }^{1} \mathrm{H}$ NMR spectroscopy. Due to the self-assembly, several doublets representing the upper rim pyridyl doublets can be observed in the aromatic region of the spectrum. Compound (31) showed the upper rim pyridyl doublets at $8.50 \mathrm{ppm}$. This signal at $8.48 \mathrm{ppm}$ could be due to another self-assembled calix[4]arene. This residual starting material has more than likely bound itself inside the cavity as after excessive washings with methanol, dichloromethane and water it still remained bound in the cavity. The 1:1 metal ion complexation reactions were examined first (Figure 148). The zinc acetate spectrum showed a shift for the upper rim pyridyl protons from $8.50 \mathrm{ppm}$ to 8.58 $\mathrm{ppm}$, a difference of $0.08 \mathrm{ppm}$. The spectrum of the zinc chloride complex showed a shift from $8.50 \mathrm{ppm}$ to $8.66 \mathrm{ppm}$, a difference of $0.16 \mathrm{ppm}$. The spectrum of the zinc perchlorate complex showed a shift from $8.50 \mathrm{ppm}$ to $8.66 \mathrm{ppm}$ with the same difference as was seen for the chloride.

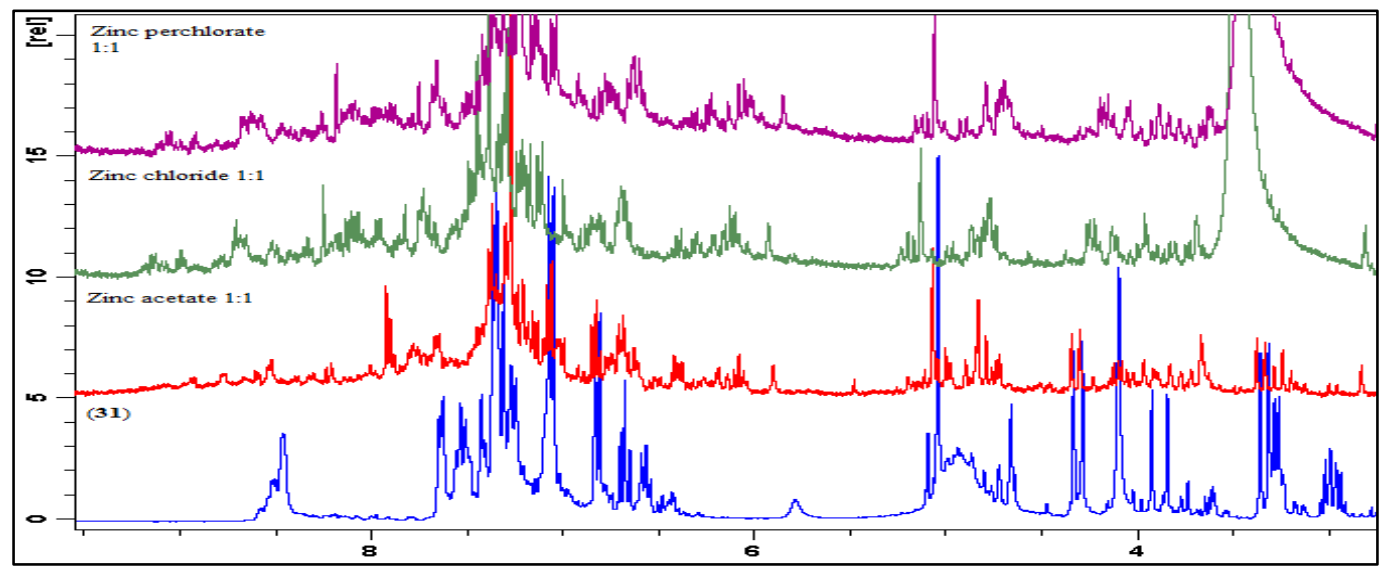

Figure 148: ${ }^{1} \mathrm{H}$ NMR for the 1:1 metal ion complexation reactions for $(\mathbf{3 1})$ 
In the bridging region of the spectrum, (Figure 149), some shifts can be seen, the axial bridging methylene protons shift from $4.30 \mathrm{ppm}$ in the parent compound to $4.23 \mathrm{ppm}$ and $4.16 \mathrm{ppm}$ in the chloride and perchlorate spectra. No change is seen in the acetate spectrum. The lower rim benzyl methylene protons are shifted downfield from $5.03 \mathrm{ppm}$ to $5.06,5.12$ and $5.06 \mathrm{ppm}$ in the acetate, chloride and perchlorate spectra respectively. The upper rim methylamine-methylene protons are shifted from $4.65 \mathrm{ppm}$ to $4.78,4.76$ and $4.78 \mathrm{ppm}$ in the acetate, chloride and perchlorate spectra respectively. The final observed shift is in the methylpyridyl methylene protons which shift from $4.08 \mathrm{ppm}$ in the parent compound to 4.12 and $4.04 \mathrm{ppm}$ in the chloride and perchlorate spectra. This peak is also seen as a broad singlet in the parent compound but appears as two doublets in the metal ion complexed spectra indicating that the protons are no longer in the same environment.

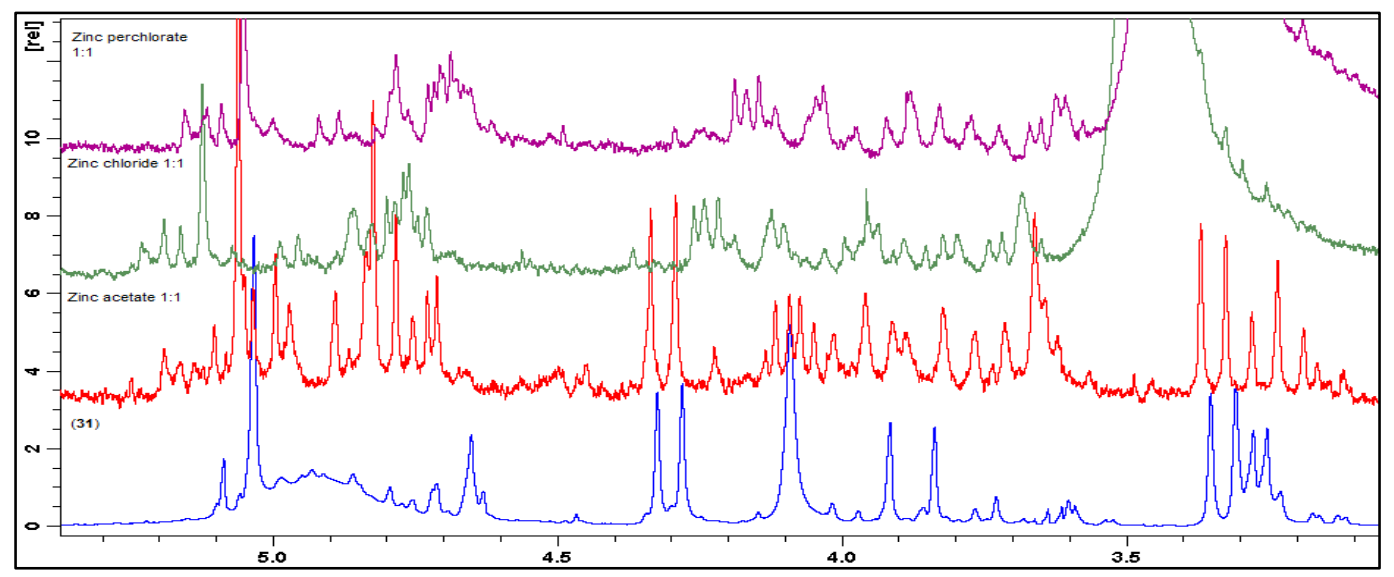

Figure 149: ${ }^{1} \mathrm{H}$ NMR of the bridging region of (31) with 1:1 Zinc(II) metal ion complexation. Red trace $=$ Zinc(II) acetate, Green trace $=$ Zinc(II) chloride, purple trace $=$ Zinc(II) perchlorate.

The 1:2 metal ion complexation reactions were then carried out and examined by ${ }^{1} \mathrm{H}$ NMR spectroscopy (Figure 150). Again the self-assembly peaks dominated most of the spectrum but shifts in the upper rim pyridyl's could be clearly seen. The acetate spectrum showed shifts from $8.50 \mathrm{ppm}$ to $8.69 \mathrm{ppm}$. The chloride showed shifts from $8.50 \mathrm{ppm}$ to $8.61 \mathrm{ppm}$ and the perchlorate showed shifts from $8.50 \mathrm{ppm}$ to 8.69 $\mathrm{ppm}$. In this case the acetate and perchlorate spectra produced the largest shifts. The large peaks in the aromatic region at 8.50, 7.42, 7.31, and 7.21 ppm are most likely due to another self-assembled calix[4]arene. 


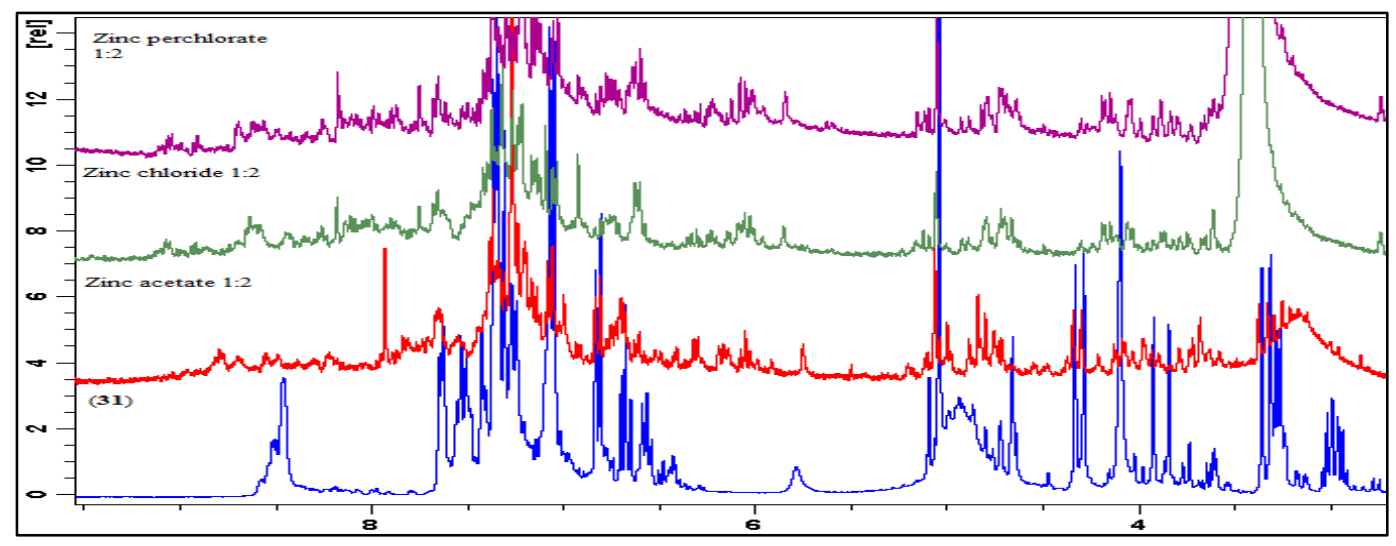

Figure 150: ${ }^{1} \mathrm{H}$ NMR for the 1:2 metal ion complexation reactions for $(\mathbf{3 1})$. Red trace $=$ Zinc(II) acetate, Green trace $=$ Zinc(II) chloride, purple trace $=$ Zinc(II) perchlorate.

Similar shifts in the bridging region, (Figure 151), of the 1:2 metal complexes of (31) as were seen for the 1:1 complexes. The axial bridging protons shifted from $4.30 \mathrm{ppm}$ in the parent spectrum to $4.16 \mathrm{ppm}$ in both the chloride and perchlorate spectra. No change in the acetate spectrum. The lower rim benzyl methylene protons shifted from $5.03 \mathrm{ppm}$ in the parent spectrum to $5.05 \mathrm{ppm}$ in the acetate and chloride spectra, and to $5.04 \mathrm{ppm}$ in the perchlorate spectrum. The upper rim methylpyridyl methylene protons occurred at $4.09 \mathrm{ppm}$ as a broad singlet in the parent spectrum but appeared as doublets or broad doublet in the metal complexes spectra indicating that that the protons are once again in different environments to each other. They also experience small shifts from $4.09 \mathrm{ppm}$ to 4.11 and $4.07 \mathrm{ppm}$ in the acetate spectrum, to 4.17 and $4.03 \mathrm{ppm}$ in the chloride spectrum and to 4.17 and $4.04 \mathrm{ppm}$ in the perchlorate spectrum. Methanol was seen in the cavity of the calix[4]arene ring most likely due to the complexation reaction. 


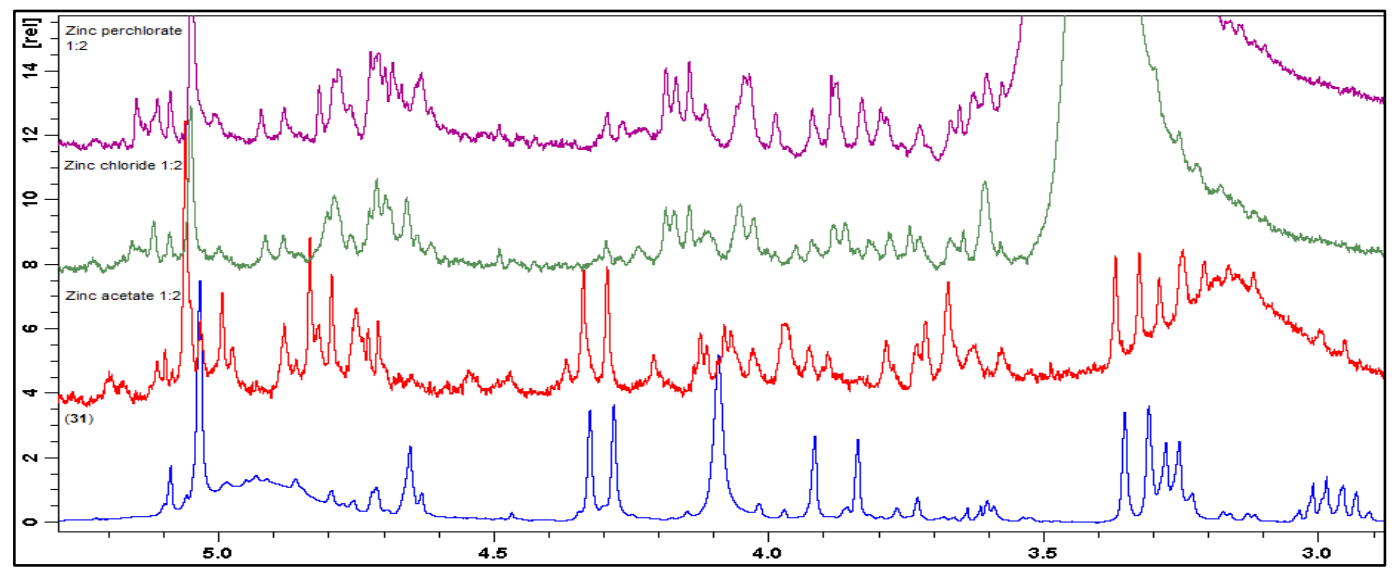

Figure 151: ${ }^{1} \mathrm{H}$ NMR of the bridging region of (31) with 1:2 Zinc(II) metal ion complexation. Red trace $=$ Zinc(II) acetate, Green trace $=$ Zinc(II) chloride, purple trace $=$ Zinc(II) perchlorate.

The IR data was seen to correspond well with the ${ }^{1} \mathrm{H}$ NMR spectra (Table 6). The IR showed that the upper rim pyridyl doublets experience downfield shifts due to the metal ion complexation, which corresponds well with the data from the ${ }^{1} \mathrm{H}$ NMR spectrum. The shifts in the hydroxyl groups can probably be accounted for by the self-assembly process, and not as an indication that metal ion binding is occurring on the lower rim. The hydroxyl shifts are most likely due to the homodimerisation form where the hydroxyl groups on one molecule are close to the hydroxyl group of another calix[4]arene unit.

\begin{tabular}{|l|c|c|}
\hline & $\mathrm{O}-\mathrm{H} \mathrm{cm}^{-1}$ & $\mathrm{NC}-\mathrm{H} \mathrm{cm}^{-1}$ \\
\hline Compound (31) & 3388 & 1590 \\
\hline 1:1 reaction & & \\
\hline Mercury chloride & 3424 & 1597 \\
\hline Mercury perchlorate & 3429 & 1597 \\
\hline Zinc acetate & 3448 & 1606 \\
\hline Zinc chloride & 3426 & 1606 \\
\hline Zinc perchlorate & 3455 & 1608 \\
\hline Copper perchlorate & 3409 & 1610 \\
\hline Copper chloride & 3421 & 1608 \\
\hline Nickel acetate & 3402 & 1607 \\
\hline Nickel chloride & 3378 & 1618 \\
\hline Nickel perchlorate & 3399 & 1604 \\
\hline Cobalt chloride & 3410 & 1605 \\
\hline
\end{tabular}

Table 6: IR data for 1:1 metal ion complexation reactions with (31). N/O = not observed, those in green experience shifts compared with parent spectrum in red. 
The next compound to be examined was (34), (Figure 152), which contained the pyridyl group on the lower rim, whereas (30) \& (31) contained the benzyl group on their lower rims.

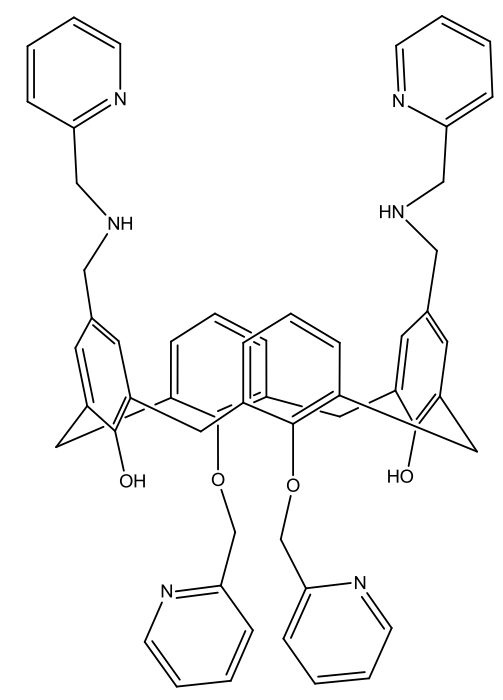

Figure 152: Compound (34)

With compound (34), a small shift in the spectra was observed upon 1:1 metal ion complexation (Figure 153). In the parent form the pyridyl protons (NC-H) occur around $8.49 \mathrm{ppm}$, for the upper pyridyl group and at $8.61 \mathrm{ppm}$ for the lower pyridyl group. In the complexed form, a shift to $8.77 \mathrm{ppm}$ for the lower pyridyl protons and $8.66 \mathrm{ppm}$ for the upper pyridyl protons are seen. It can be seen clearly from the ${ }^{1} \mathrm{H}$ NMR spectra that both the upper and lower rim pyridyl's are being bound, with the lower rim being shifted further downfield from $8.61 \mathrm{ppm}$ to $8.77 \mathrm{ppm}$ and the upper rim pyridyl's shifted from $8.49 \mathrm{ppm}$ to $8.66 \mathrm{ppm}$. However it can also be seen in the three complexed spectra that a broad doublet, still remains at around $8.53 \mathrm{ppm}$. This is most likely the unbound pyridyls involved in self-assembly further down in the construct (Figure 144). Whether these unbound pyridyls are on the upper or lower rim is difficult to say conclusively, however as the peak is slightly closer to the parent upper rim pyridyl this unbound broad doublet may be representing these pyridyl protons. 


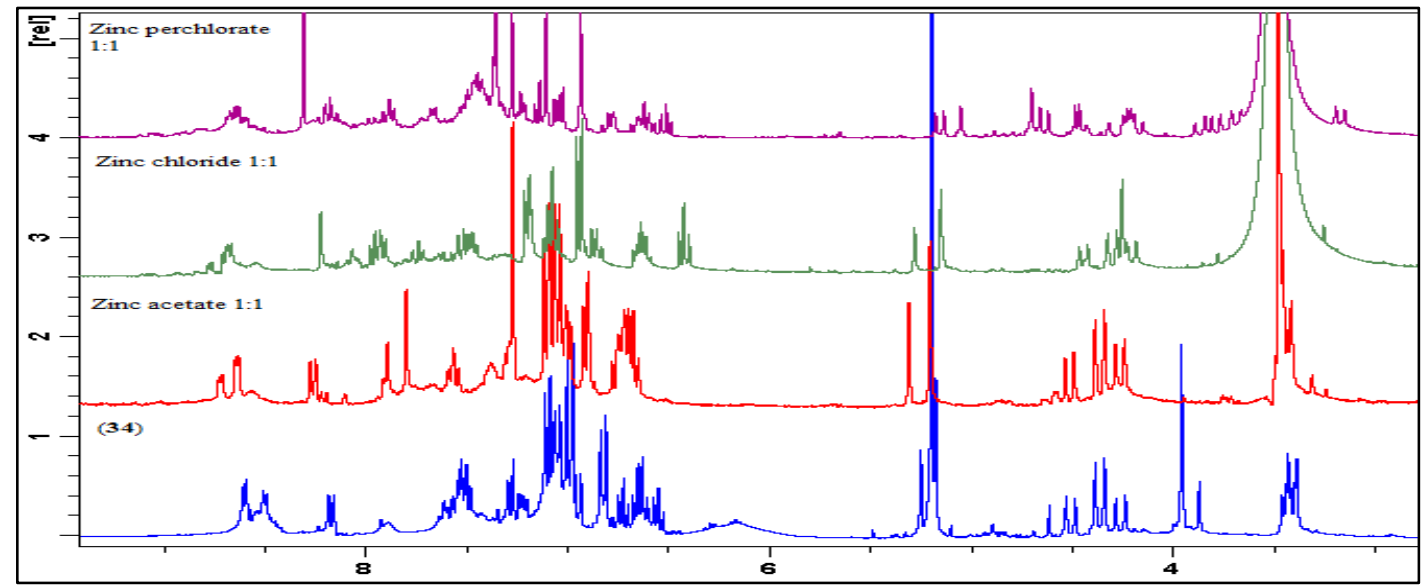

Figure 153: ${ }^{1} \mathrm{H}$ NMR for the 1:1 zinc(II) metal ion complexation reactions for (34). Red trace $=$ zinc $($ II $)$ acetate, Green trace $=$ zinc(II) chloride, Purple trace $=$ zinc(II) perchlorate .

In the bridging region it was found that the upper rim methylpyridyl methylene protons and the lower rim methylpyridyl methylene protons occurred very close together in the parent compound but upon complexation with the metal ion a separation occurred. The lower rim methylpyridyl methylene protons occurred at $5.19 \mathrm{ppm}$ in the parent spectrum but these were shifted to 5.29, 5.26 and $5.26 \mathrm{ppm}$ in the acetate, chloride and perchlorate spectra respectively. The upper rim methylpyridyl protons appeared at $5.17 \mathrm{ppm}$ in the parent spectrum and these were shifted to $5.19,5.14,5.11 \mathrm{ppm}$ in the acetate, chloride and perchlorate spectra respectively. This shift in the upper rim signals would imply that the metal ion is favouring metal ion complexation at the lower rim causing the downfield shifts in the lower rim methylpyridyl methylene protons. Due to the presence of methanol in the cavity the equatorial bridging methylene protons are obscured, however, small shifts can be seen in the axial methylene protons. In the parent compound the methylene protons occur at $4.35 \mathrm{ppm}$ but are shifted to 4.29 and $4.27 \mathrm{ppm}$ in the chloride and perchlorate spectra. No change is seen in the acetate spectrum. Other doublets are also present in the bridging region indicating that self-assembly is still occurring. 


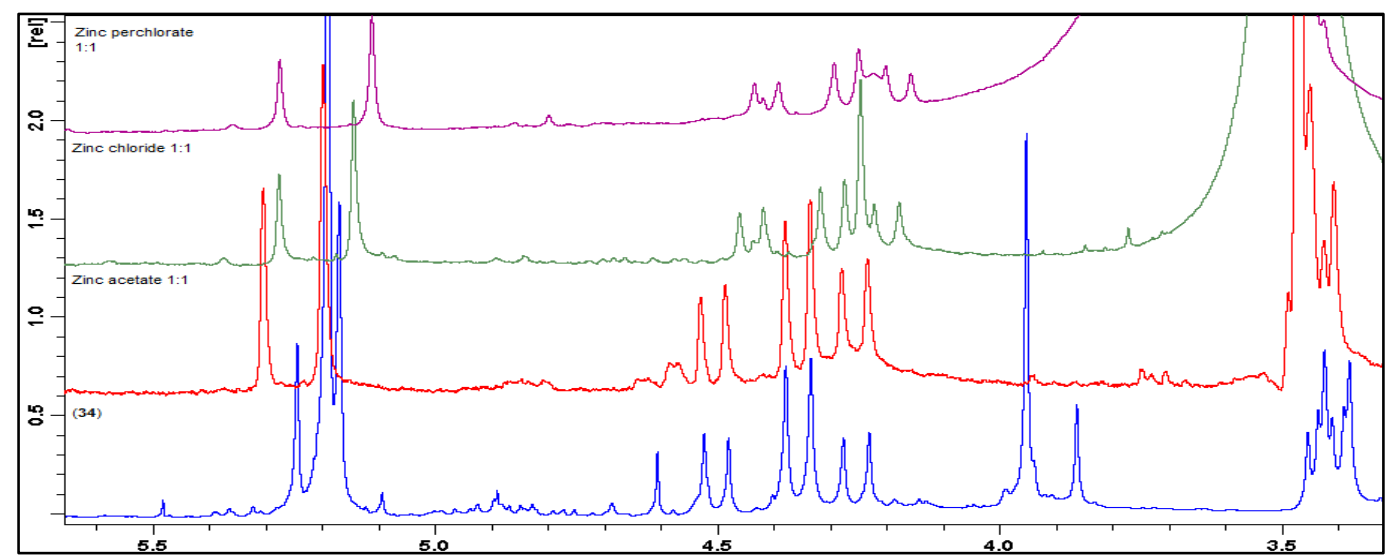

Figure 154: ${ }^{1} \mathrm{H}$ NMR of the bridging region of (34) with 1:2 Zinc(II) metal ion complexation. Red trace $=$ Zinc(II) acetate, Green trace $=$ Zinc(II) chloride, purple trace $=$ Zinc(II) perchlorate.

The 1:2 metal ion complexation reactions also produced a noticeable shift in the spectra (Figure 155). In the zinc acetate spectrum, a shift was seen for the upper rim pyridyl protons from $8.51 \mathrm{ppm}$ to $8.63 \mathrm{ppm}$ and the lower rim pyridyl protons shift from $8.58 \mathrm{ppm}$ to $8.70 \mathrm{ppm}$. This can be clearly seen in the next figure (Figure 156). In zinc chloride a shift from $8.51 \mathrm{ppm}$ to $8.63 \mathrm{ppm}$ for the upper rim pyridyl protons and from $8.58 \mathrm{ppm}$ to $8.71 \mathrm{ppm}$ for the lower rim pyridyl protons is observed. The zinc perchlorate also shows shifts from $8.51 \mathrm{ppm}$ to $8.66 \mathrm{ppm}$ for the upper rim pyridyl protons and a shift from $8.58 \mathrm{ppm}$ to $8.70 \mathrm{ppm}$ for the lower rim pyridyl protons. A broad peak is also seen between 8.53 and $8.55 \mathrm{ppm}$ in the three complexed spectra, most likely representing the unbound pyridyl protons in the selfassembled chain.

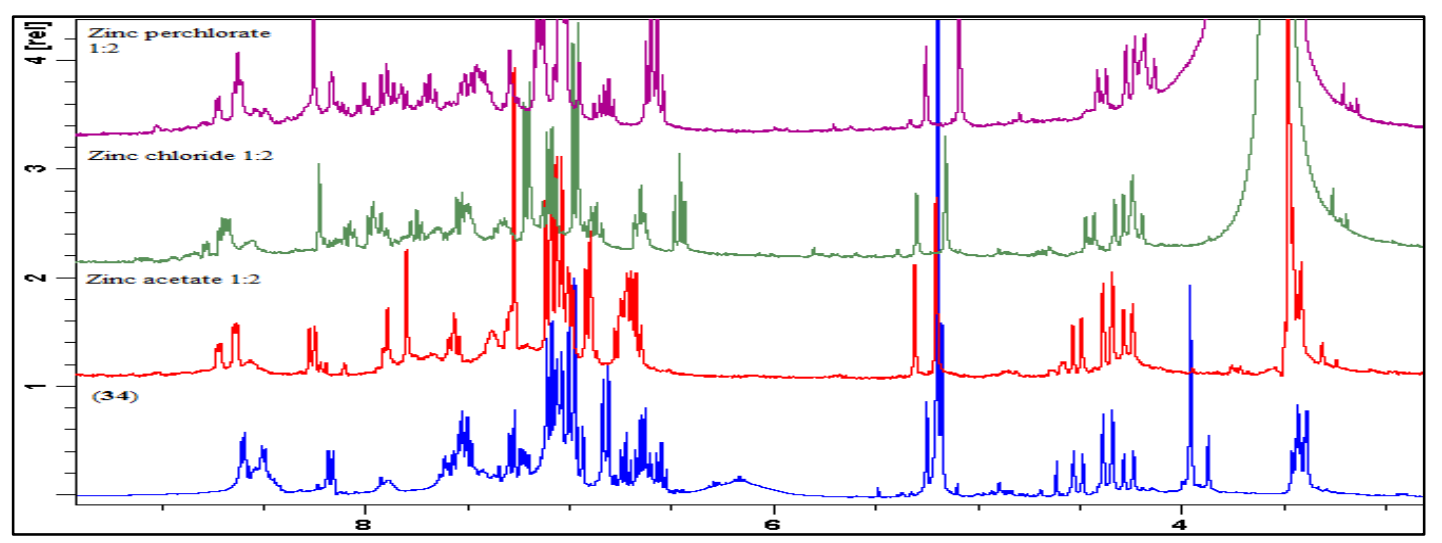

Figure 155: ${ }^{1} \mathrm{H}$ NMR for the 1:2 metal ion complexation reactions for (34) 


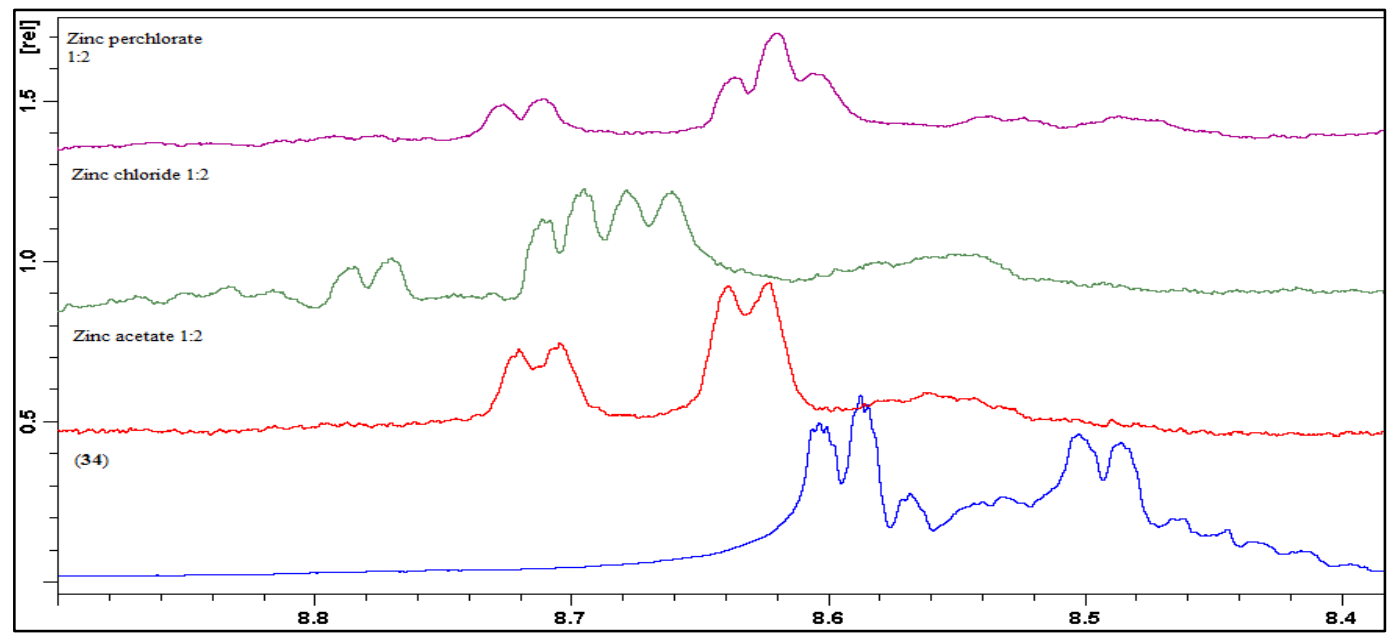

Figure 156: ${ }^{1} \mathrm{H}$ NMR of the aromatic region showing proton shift and residual dimerisation unbound pyridyl's in compound (34).

In the bridging region, (Figure 157), very similar shifts to that of the 1:1 metal complexed spectra were seen and as such will only be briefly discussed here. Shifts in the lower rim methylpyridyl methylene protons occurred from $5.19 \mathrm{ppm}$ in the parent compound to 5.29, 5.28 and $5.24 \mathrm{ppm}$ in the acetate, chloride and perchlorate spectra respectively. Shifts for the upper rim methylpyridyl methylene protons were seen from $5.16 \mathrm{ppm}$ in the parent compound to 5.19, 5.15 and $5.08 \mathrm{ppm}$ for the acetate, chloride and perchlorate spectra respectively. The equatorial bridging methylene protons were obscured by remaining methanol but the axial bridging methylene protons shifted from $4.35 \mathrm{ppm}$ in the parent compound to 4.29 and 4.24 ppm in the chloride and perchlorate spectra. No change was seen in the acetate spectrum. Again several other signals were seen to indicate self-assembly. 


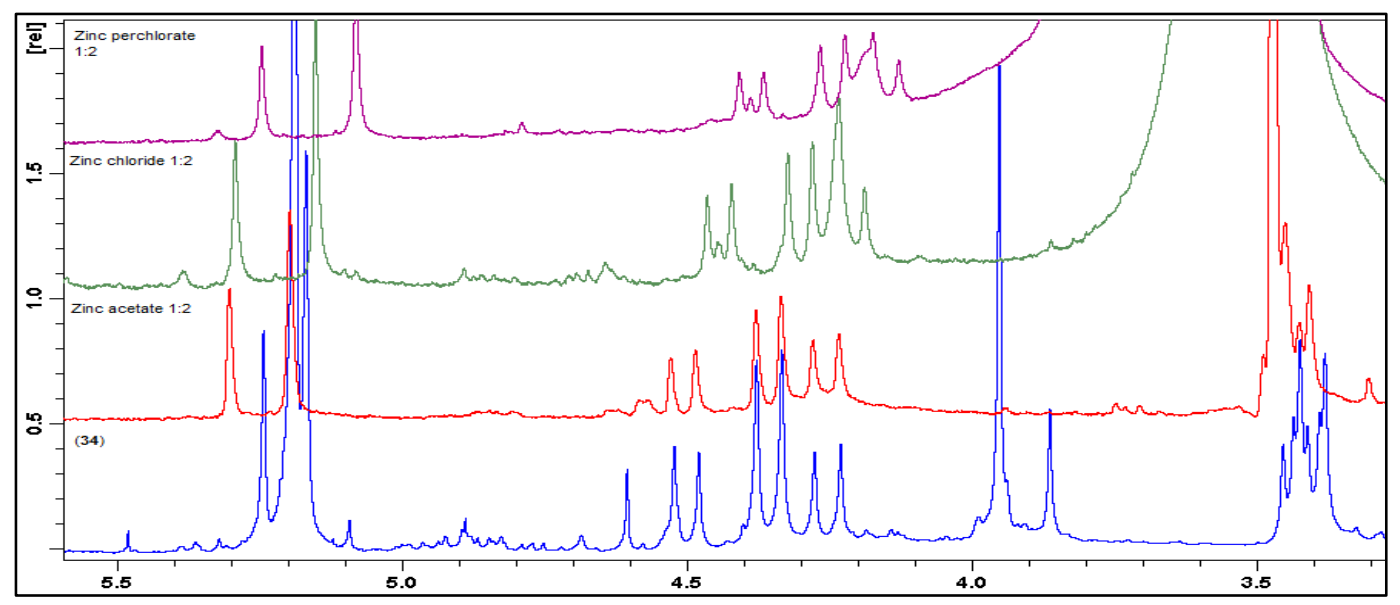

Figure 157: ${ }^{1} \mathrm{H}$ NMR of the bridging region of (34) with 1:2 Zinc(II) metal ion complexation. Red trace $=$ Zinc(II) acetate, Green trace $=$ Zinc(II) chloride, purple trace $=$ Zinc(II) perchlorate.

The IR data of the 1:1 metal ion complexation reactions corresponded well with the ${ }^{1} \mathrm{H}$ NMR spectra (Table 7). The 1:1 metal ion complexation reactions the $\mathrm{NCH}$ of the pyridyl groups experienced a large downfield shift as a result of metal ion complexation. The hydroxyl groups were also shifted downfield most likely to the combined effects of metal ion complexation and self-assembly. The 1:2 metal ion complexation reactions exhibited a similar trend with the pyridyl $\mathrm{NCH}$ band experiencing a large shift due to metal ion complexation.

\begin{tabular}{|l|c|c|c|c|}
\hline & $\mathrm{O}-\mathrm{H} \mathrm{cm}^{-1}$ & $\mathrm{NC}-\mathrm{H} \mathrm{cm}^{-1}$ & $\mathrm{O}-\mathrm{H} \mathrm{cm}^{-1}$ & $\mathrm{NC}-\mathrm{H} \mathrm{cm}^{-1}$ \\
\hline Compound (34) & 3418 & 1591 & & \\
\hline $1: 1$ Reaction & & & $1: 2$ reaction & \\
\hline Zinc acetate & 3420 & 1591 & 3442 & 1608 \\
\hline Zinc chloride & 3436 & 1629 & 3429 & 1607 \\
\hline Zinc perchlorate & 3437 & 1626 & 3513 & 1612 \\
\hline Mercury perchlorate & 3436 & 1599 & 3434 & 1627 \\
\hline Cobalt chloride & 3424 & 1605 & 3422 & 1601 \\
\hline Nickel acetate & 3430 & 1592 & 3417 & 1606 \\
\hline Nickel perchlorate & 3418 & 1627 & 3419 & 1625 \\
\hline Nickel chloride & 3435 & 1592 & 3266 & 1560 \\
\hline Copper acetate & 3431 & 1610 & 3432 & 1610 \\
\hline Copper perchlorate & 3442 & 1626 & 3431 & 1608 \\
\hline Copper chloride & 3414 & 1609 & 3437 & 1628 \\
\hline
\end{tabular}

Table 7: IR data for 1:1 and 1:2 metal ion complexation reactions with (34). N/O = not observed, those in green experience shifts compared with parent spectrum in red. 
A similar trend was seen with compound (35), Figure 158. Unfortunately due to the appearance methanol in the bridging region of the spectrum, especially around the bridging doublets, clear characterization of the compound is difficult.

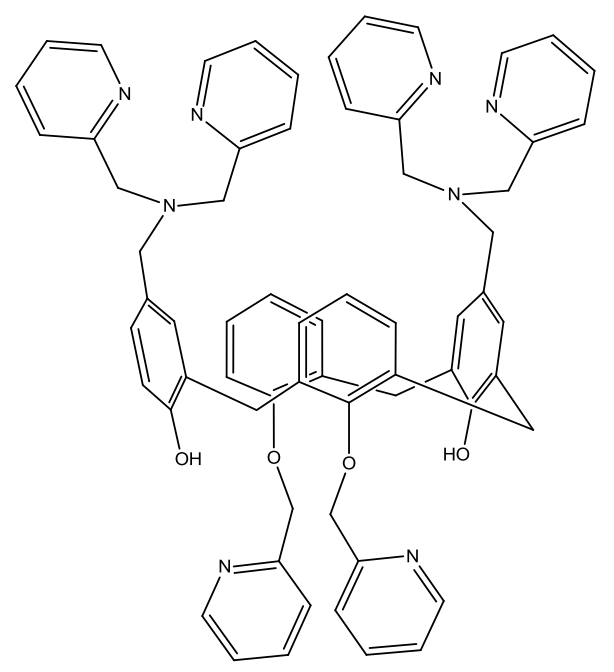

Figure 158: Compound (35)

Metal ion complexation can be seen in the aromatic region with chemical shifts of the pyridyl protons shown below (Figure 159). In the parent compound the lower rim pyridyl peak occurs at $8.53 \mathrm{ppm}$ and the upper rim pyridyl peak at $8.44 \mathrm{ppm}$. In zinc acetate at 1:1 ratio, shifts are seen from $8.53 \mathrm{ppm}$ to $8.69 \mathrm{ppm}$ for the lower rim pyridyl and from $8.44 \mathrm{ppm}$ to $8.58 \mathrm{ppm}$ for the upper rim pyridyl. On closer analysis, a small broad shoulder peak can be observed coming off the peak at $8.58 \mathrm{ppm}$ in the metal complexed spectrum, which may indicate unbound pyridyl protons in the selfassembled chain. A similar occurrence was seen with the zinc chloride and zinc perchlorate ${ }^{1} \mathrm{H}$ NMR spectra. Shifts were observed from $8.53 \mathrm{ppm}$ to $8.97 \mathrm{ppm}$ for the lower rim pyridyl and from $8.44 \mathrm{ppm}$ to $8.72 \mathrm{ppm}$ for the upper rim pyridyl in the chloride. In the perchlorate spectrum a shift from $8.53 \mathrm{ppm}$ to $8.96 \mathrm{ppm}$ was observed for the lower rim pyridyl and from $8.44 \mathrm{ppm}$ to $8.71 \mathrm{ppm}$ for the upper pyridyl. In all of the spectra, due to the abundance of peaks, it is evident that selfassembly is still a dominant factor. 


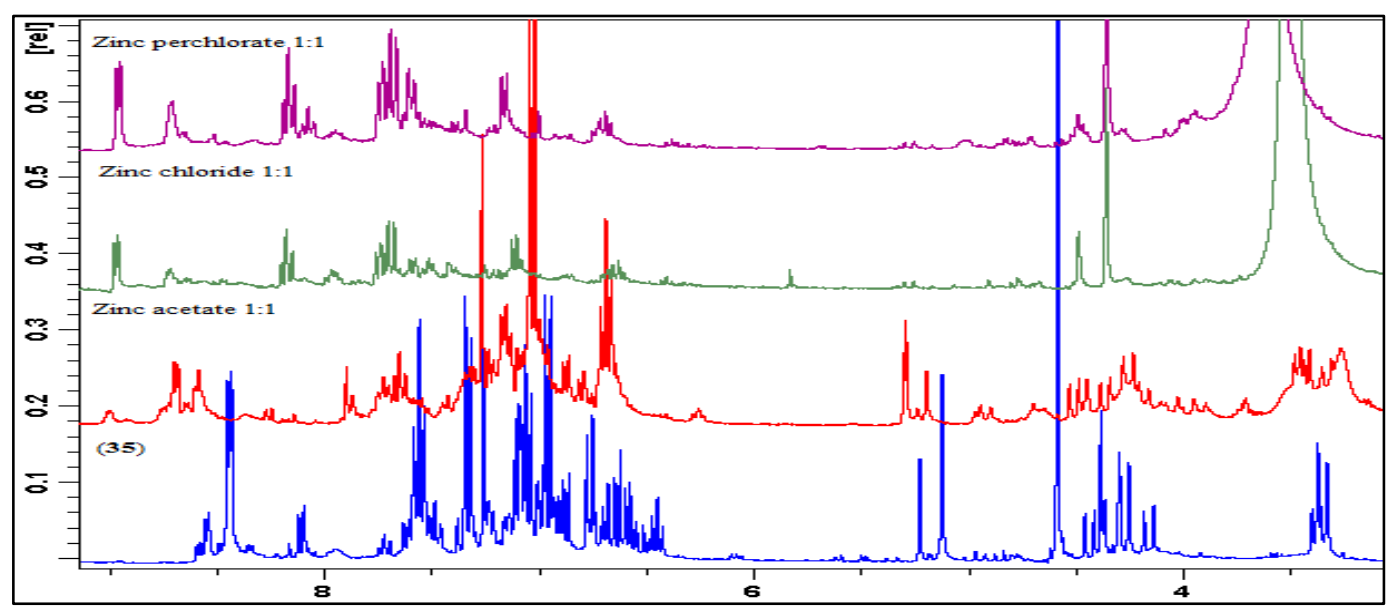

Figure 159: ${ }^{1} \mathrm{H}$ NMR for the 1:1 metal ion complexation reactions for (35)

In the bridging region, (Figure 160), some changes can be seen. Unfortunately the methanol solvent is obscuring the equatorial bridging methylene protons. The axial protons are shifted from $4.27 \mathrm{ppm}$ in the parent spectrum to 4.26, 4.24 and $4.24 \mathrm{ppm}$ in the acetate, chloride and perchlorate spectra respectively. The lower rim methylpyridyl methylene protons shifted from $5.22 \mathrm{ppm}$ in the parent compound to $5.29 \mathrm{ppm}$ in all three of the metal ion complexed spectra. The upper rim methylpyridyl methylene protons shifted from $5.12 \mathrm{ppm}$ in the parent compound to $5.19,5.25$ and $5.25 \mathrm{ppm}$ in the acetate, chloride and perchlorate spectra respectively. Several other peaks were also present indicating that self-assembly was still occurring in solution. 


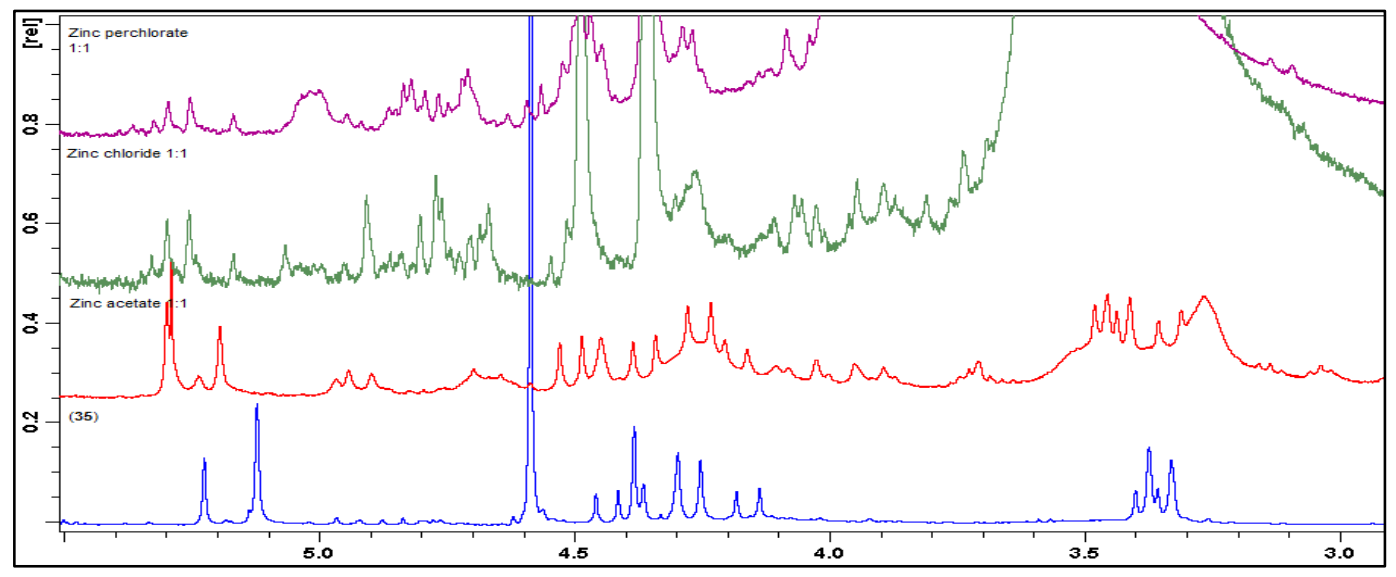

Figure 160: ${ }^{1} \mathrm{H}$ NMR of the bridging region of (35) with 1:1 Zinc(II) metal ion complexation. Red trace $=$ Zinc $($ II) acetate, Green trace $=$ Zinc(II) chloride, purple trace $=$ Zinc(II) perchlorate.

With the 1:2 metal ion complexation reactions, similar results to the $1: 1$ metal ion complexation spectra were obtained (Figure 161). The parent compound maintained the same two peaks at 8.54 and $8.44 \mathrm{ppm}$ for the lower and upper rim respectively. The zinc acetate spectra showed shifts from $8.54 \mathrm{ppm}$ to $8.69 \mathrm{ppm}$ and from 8.44 ppm to $8.58 \mathrm{ppm}$ for the lower and upper rims respectively. The zinc chloride showed shifts from $8.54 \mathrm{ppm}$ to $8.97 \mathrm{ppm}$ for the lower rim pyridyl and from 8.44 ppm to $8.84 \mathrm{ppm}$ for the upper rim pyridyl. Finally the zinc perchlorate also showed shifts from $8.54 \mathrm{ppm}$ to $8.96 \mathrm{ppm}$ and from $8.44 \mathrm{ppm}$ to $8.68 \mathrm{ppm}$ for the lower and upper rim pyridyl's respectively. As was seen with the 1:1 complexation reactions the peaks indicating self-assembly was still occurring.

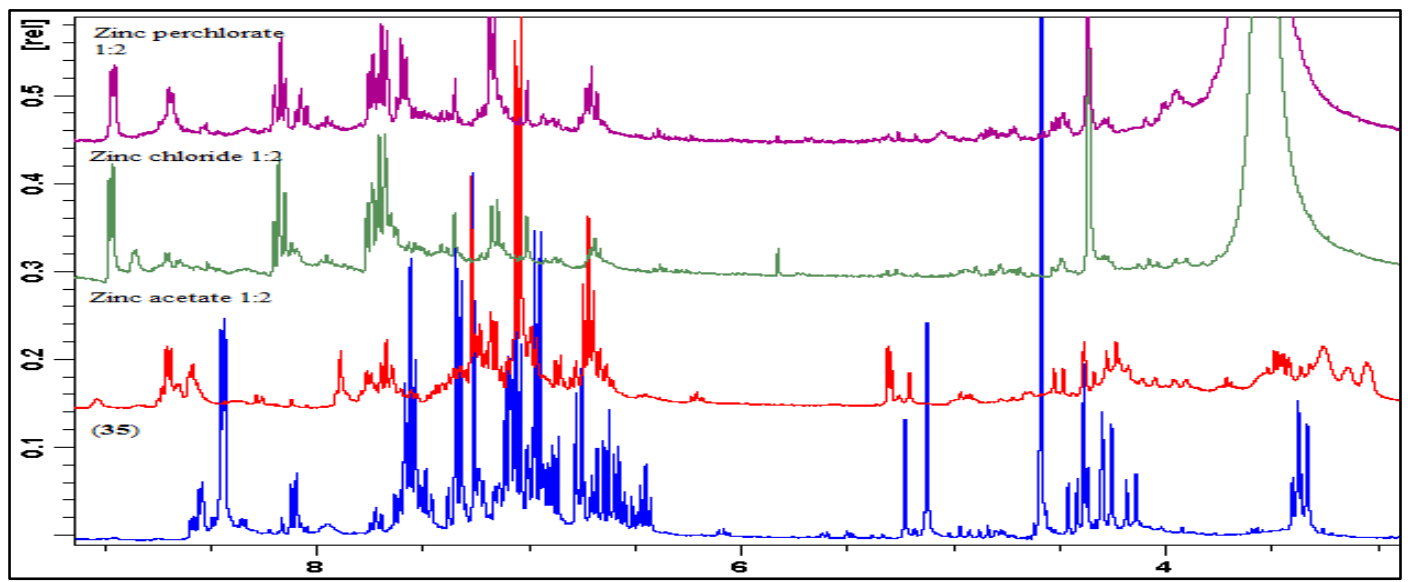

Figure 161: ${ }^{1} \mathrm{H}$ NMR for the 1:2 metal ion complexation reactions for (35) 
In the bridging region, (Figure 162), of the 1:2 metal ion complexed spectra very similar results were obtained when compared to the 1:1 metal ion complexation reactions and so it will only be briefly discussed here. Methanol was once again obscuring the equatorial bridging methylene protons. Only a small shift was seen in the axial bridging protons from $4.27 \mathrm{ppm}$ in the parent compound to 4.25, 4.26 and $4.26 \mathrm{ppm}$ in the acetate, chloride and perchlorate spectra respectively. The lower rim methylpyridyl methylene protons shifted from $5.23 \mathrm{ppm}$ in the parent compound to $5.29 \mathrm{ppm}$ in the three complexed spectra and the upper rim methylpyridyl protons shifted from $5.12 \mathrm{ppm}$ to $5.20,5.26$ and $5.26 \mathrm{ppm}$ in the acetate, chloride and perchlorate spectra respectively.

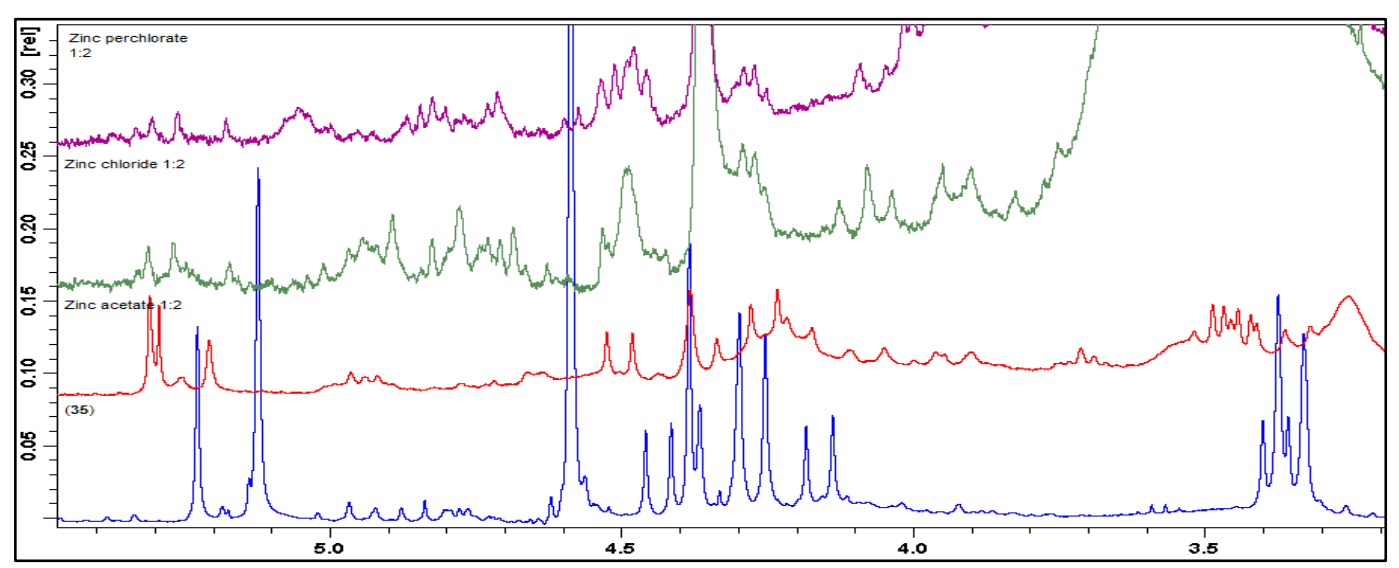

Figure 162: ${ }^{1} \mathrm{H}$ NMR of the bridging region of (35) with 1:2 Zinc(II) metal ion complexation. Red trace $=$ Zinc $($ II) acetate, Green trace $=$ Zinc $($ II $)$ chloride, purple trace $=$ Zinc(II) perchlorate.

The IR data for the $1: 1$ and 1:2 metal ion complexation reactions with (35) are shown below (Table 8). It can be seen from the results that the pyridyl NCH only experiences a small downfield shift as does the hydroxyl in both the 1:1 and 1:2 complexation reactions. This data supports the ${ }^{1} \mathrm{H}$ NMR spectra well in that only a small shift is seen on addition of the metal ion and that self-assembly is still a very dominant effect in this compound. 


\begin{tabular}{|l|c|c|c|c|}
\hline & $\mathrm{O}-\mathrm{H} \mathrm{cm}^{-1}$ & $\mathrm{NC}-\mathrm{H} \mathrm{cm}^{-1}$ & $\mathrm{O}-\mathrm{H} \mathrm{cm}^{-1}$ & $\mathrm{NC}-\mathrm{H} \mathrm{cm}^{-1}$ \\
\hline Compound (35) & 3432 & 1630 & & \\
\hline 1:1 reaction & & & $1: 2$ reaction & \\
\hline Cobalt perchlorate & 3430 & 1627 & 3417 & 1626 \\
\hline Cobalt chloride & 3421 & 1600 & 3428 & 1600 \\
\hline Mercury chloride & 3432 & 1635 & 3422 & 1627 \\
\hline Mercury perchlorate & 3436 & 1624 & 3434 & 1624 \\
\hline Zinc acetate & 3429 & 1621 & 3432 & 1607 \\
\hline Zinc perchlorate & 3443 & 1624 & 3504 & 1619 \\
\hline Zinc chloride & 3434 & 1619 & 3437 & 1609 \\
\hline Nickel acetate & 3421 & 1629 & 3429 & 1591 \\
\hline Nickel chloride & 3429 & 1630 & 3420 & 1630 \\
\hline Nickel perchlorate & 3430 & 1631 & 3421 & 1630 \\
\hline Copper acetate & 3421 & 1591 & 3433 & 1591 \\
\hline Copper perchlorate & 3434 & 1619 & 3441 & 1622 \\
\hline Copper chloride & 3436 & 1627 & 3436 & 1628 \\
\hline
\end{tabular}

Table 8: IR data for 1:1 and 1:2 metal ion complexation reactions with compound (35). N/O

$=$ not observed, those in green experience shifts compared with parent spectrum in red.

Next to be examined by ${ }^{1} \mathrm{H}$ NMR for metal ion binding ability was compound (36), (Figure 163). Methanol was found in the ${ }^{1} \mathrm{H}$ NMR spectra of the complexation reactions, most likely sitting in the cavity.

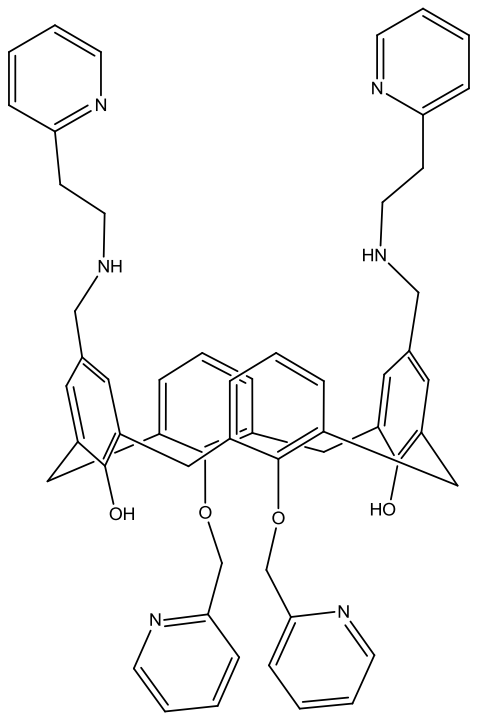

Figure 163: Compound (36) 
The parent proton spectrum showed two peaks for the lower and upper rim pyridyl protons at $8.59 \mathrm{ppm}$ and $8.49 \mathrm{ppm}$ respectively (Figure 164). On looking at the zinc acetate spectrum it appears that complexation of the metal ion has occurred, but on looking at the bridging region the two triplets indicating the upper rim ethylpyridylmethylene and ethylene protons are no longer visible in the spectrum, indicating that the upper arm is no longer attached. The observed shifts in the aromatic may then indicate that metal ion complexation has occurred on the lower rim. The zinc chloride saw shifts from $8.59 \mathrm{ppm}$ to $8.95 \mathrm{ppm}$ for the lower rim methylpyridyl protons and from $8.49 \mathrm{ppm}$ to $8.78 \mathrm{ppm}$ for the upper rim ethylpyridyl protons. The zinc chloride also produced a number of other doublets in this region. This suggests that perhaps two or more separate chains have bound to the metal with each chain in a different orientation. Finally zinc perchlorate exhibited shifts from $8.59 \mathrm{ppm}$ to $8.94 \mathrm{ppm}$ for the lower rim methylpyridyl protons and from $8.49 \mathrm{ppm}$ to $8.79 \mathrm{ppm}$ for the upper rim ethylpyridyl protons. Other doublets were observed in this region, and along with the extra peaks in the bridging region it could be indicative of several different self-assembled chains.

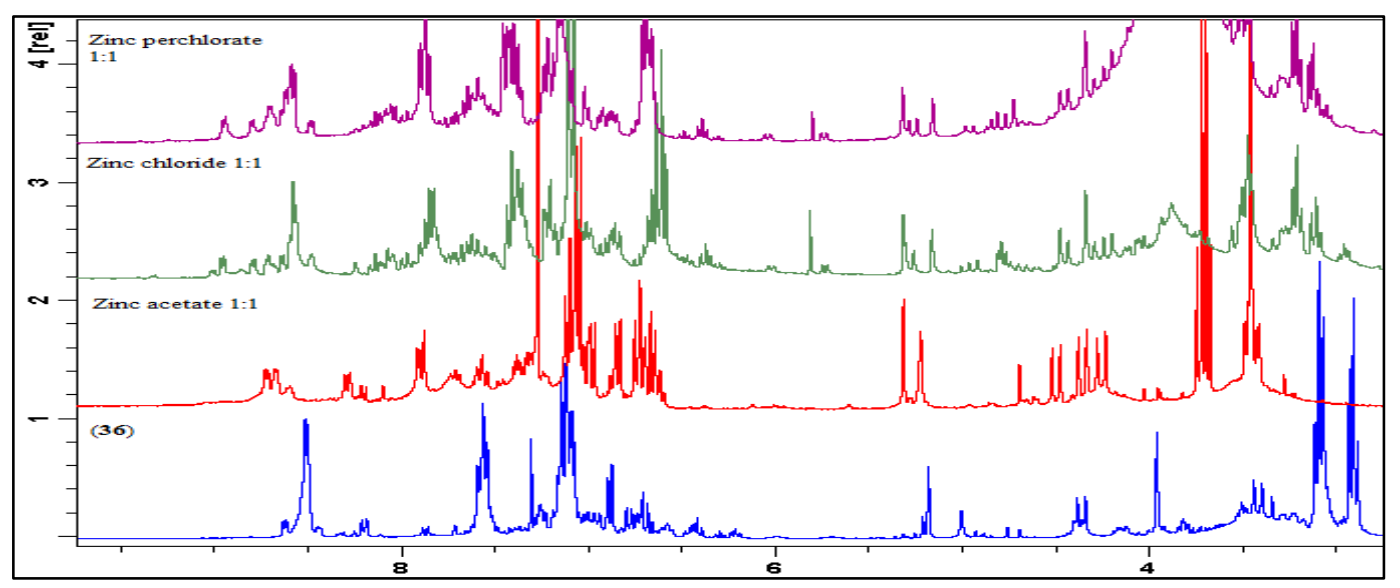

Figure 164: ${ }^{1} \mathrm{H}$ NMR for the 1:1 metal ion complexation reactions for $(\mathbf{3 6})$

In the bridging region, (Figure 165), some changes are also observed. The equatorial bridging methylene protons are shifted from $3.41 \mathrm{ppm}$ in the parent spectrum to 3.46 ppm in the chloride and perchlorate spectra, but they appear as broad peaks due to the presence of the methanol solvent. The axial bridging methylene protons are shifted from $4.36 \mathrm{ppm}$ in the parent spectrum to $4.46 \mathrm{ppm}$ in the chloride and 
perchlorate spectra respectively. The lower rim methylpyridyl methylene protons are shifted from $5.18 \mathrm{ppm}$ to $5.13 \mathrm{ppm}$ in the all three of the spectra. The upper rim methylamine methylene protons ate shifted from $4.99 \mathrm{ppm}$ in the parent spectrum to $5.16 \mathrm{ppm}$ in the chloride and perchlorate spectra respectively. The upper rim ethylpyridyl methylene and ethylene protons are shifted from $3.07 \mathrm{ppm}$ and 2.90 ppm in the parent spectrum to $3.20 \mathrm{ppm}$ and $3.11 \mathrm{ppm}$ in the chloride and perchlorate spectra respectively. In the acetate spectrum these two peaks are no longer visible suggesting that the upper rim ethylpyridyl arm has been lost during metal ion complexation.

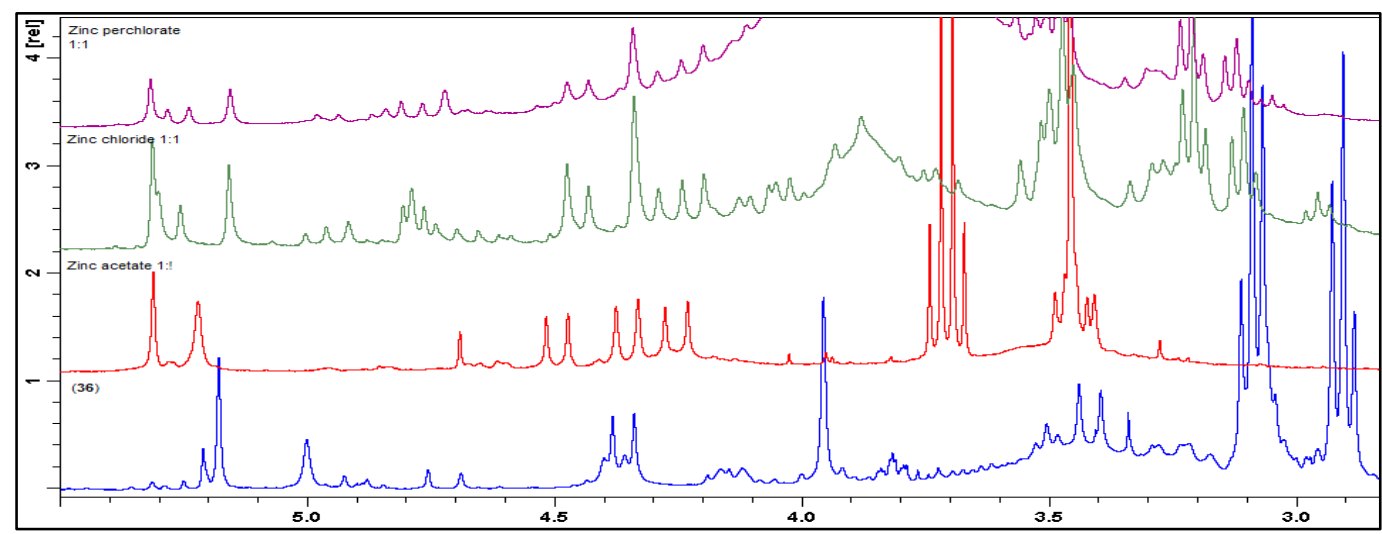

Figure 165: ${ }^{1} \mathrm{H}$ NMR of the bridging region of (35) with 1:1 Zinc(II) metal ion complexation. Red trace $=$ Zinc(II) acetate, Green trace $=$ Zinc(II) chloride, purple trace $=$ Zinc(II) perchlorate.

With the 1:2 metal ion complexation reactions similar shifts were seen (Figure 166). The parent compound pyridyl protons were seen at $8.61 \mathrm{ppm}$ and $8.51 \mathrm{ppm}$ for the lower rim methylpyridyl and upper rim ethylpyridyl protons respectively. Zinc acetate produced a shift to 8.71 and $8.66 \mathrm{ppm}$ in the lower and upper rim pyridyl's respectively, as well as leaving a broad doublet at $8.57 \mathrm{ppm}$ to represent the unbound pyridyl's. Zinc chloride produced shifts to 8.95 and $8.78 \mathrm{ppm}$ for the two pyridyl doublets along with a number of other doublets. Again this suggests that perhaps two or more separate chains have bound to the metal, all in different orientations, and the bridging region supports this theory with the abundance of peaks. The zinc perchlorate spectrum shows shift to $8.94 \mathrm{ppm}$ for the lower rim and $8.79 \mathrm{ppm}$ for the upper rim. Again other doublets were seen in the region, not as many as the chloride 
spectra, which could indicate different self-assembled chains in solution. Unfortunately from the solvent in the bridging region, support for this is difficult but from the few visible peaks the theory seems plausible.

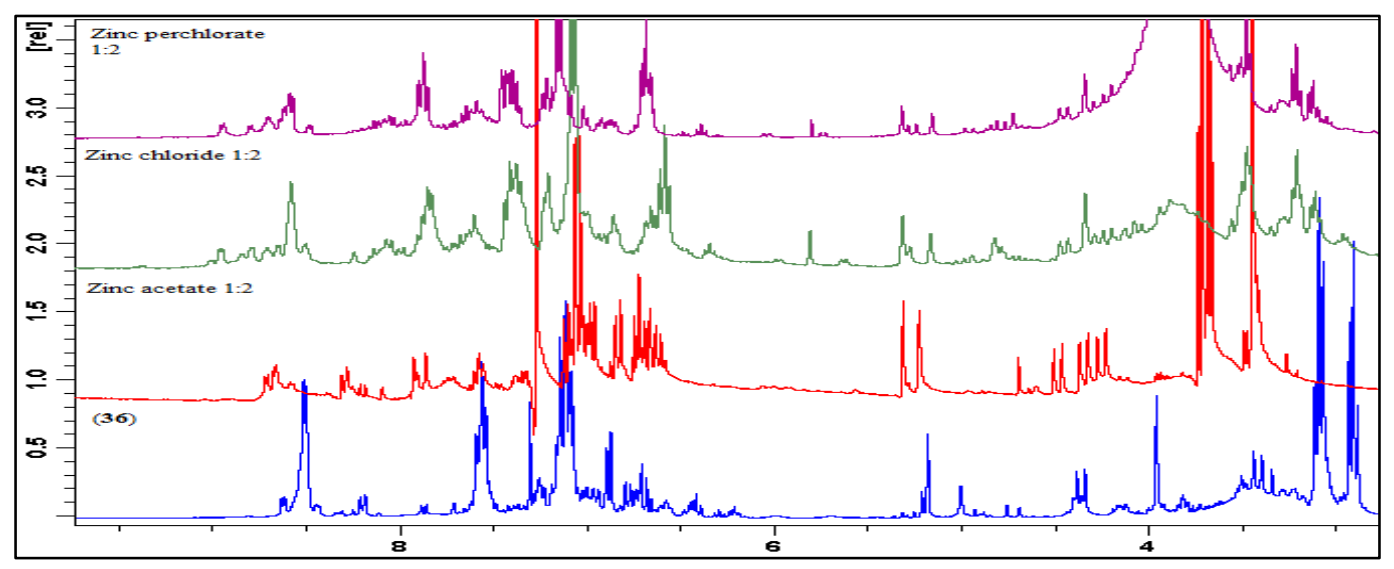

Figure 166: ${ }^{1} \mathrm{H}$ NMR for the 1:2 metal ion complexation reactions for (36)

The bridging region of (36), (Figure 167) of the 1:2 metal ion complexation reactions was very similar to that of the 1:1 metal ion complexation reactions. Again methanol obscured the bridging protons in the chloride and perchlorate spectra. In the acetate spectrum a small shift was seen from $4.36 \mathrm{ppm}$ in the parent compound to $4.34 \mathrm{ppm}$ in the acetate spectrum. Again the acetate spectrum seems to have lost its upper arm by the loss of the ethylpyridyl methylene and ethylene triplet peaks. Small shifts were seen in the lower rim methylpyridyl methylene protons from $5.18 \mathrm{ppm}$ to 5.31 ppm in all three of the spectra. The upper rim methylamine methylene protons also shifted slightly from $5.02 \mathrm{ppm}$ to $5.22,5.15$ and $5.15 \mathrm{ppm}$ in the acetate, chloride and perchlorate spectra respectively. With the continued appearance of the additional peaks it is clear that self-assembly is still occurring. 


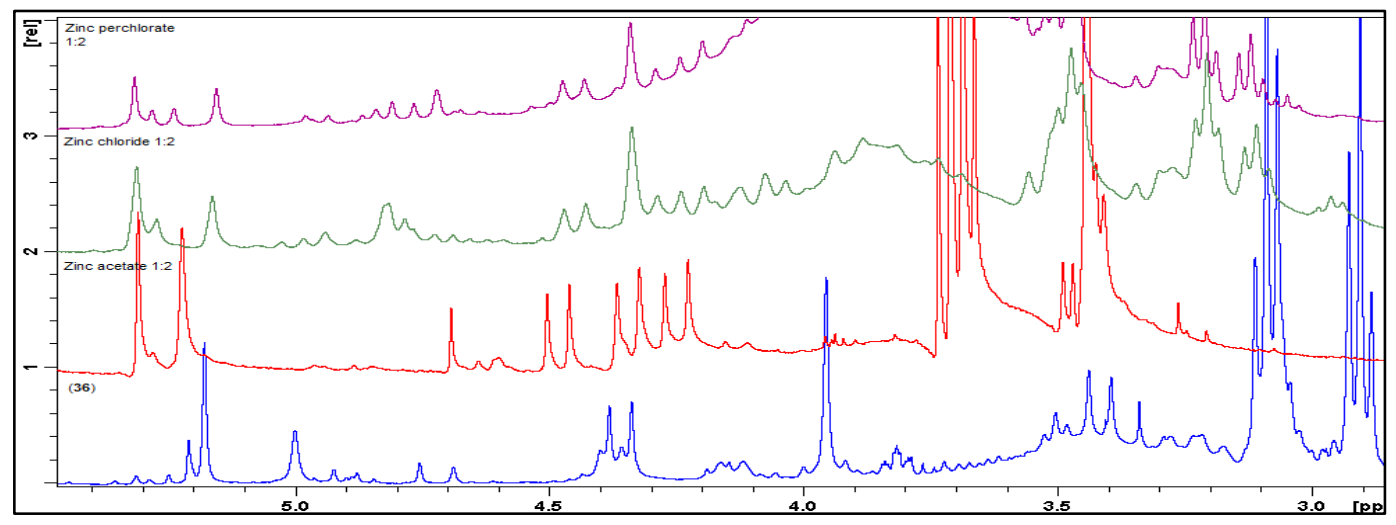

Figure 167: ${ }^{1} \mathrm{H}$ NMR of the bridging region of (36) with 1:2 Zinc(II) metal ion complexation. Red trace $=$ Zinc(II) acetate, Green trace $=$ Zinc(II) chloride, purple trace $=$ Zinc(II) perchlorate.

From the 1:1 metal ion complexation IR data (Table 9) it can be seen that in the majority of cases the $\mathrm{NCH}$ bands experience little to no downfield shifts in the presence of the metal ion. However the free $\mathrm{NH}$ groups on the upper rim, experiences downfield shifts along with the $\mathrm{OH}$ groups on the lower rim. The IR data for the zinc complexed reactions corresponded well with the ${ }^{1} \mathrm{H}$ NMR spectra. The IR data shows that the zinc chloride and zinc perchlorate metal ions induce a larger shift than the acetate ion, which mimics the ${ }^{1} \mathrm{H}$ NMR spectra and residual bands for unbound $\mathrm{NCH}$ groups are present in all three, as was seen in the ${ }^{1} \mathrm{H}$ NMR spectra.

\begin{tabular}{|l|c|c|c|c|}
\hline & $\mathrm{O}-\mathrm{H} \mathrm{cm}^{-1}$ & $\mathrm{NC}-\mathrm{H} \mathrm{cm}^{-1}$ & $\mathrm{NC}-\mathrm{H} \mathrm{cm}^{-1}$ & $\mathrm{~N}-\mathrm{H} \mathrm{cm}^{-1}$ \\
\hline Compound (36) & 3404 & 1591 & 1569 & 2916 \\
\hline $1: 1$ reaction & & & & 2922 \\
\hline Cobalt chloride & 3421 & 1543 & $\mathrm{~N} / \mathrm{O}$ & 2929 \\
\hline Nickel perchlorate & 3405 & 1593 & 1571 & 2920 \\
\hline Nickel acetate & 3414 & 1591 & $\mathrm{~N} / \mathrm{O}$ & 2923 \\
\hline Nickel chloride & 3414 & 1592 & 1570 & 2917 \\
\hline Copper acetate & 3429 & 1591 & $\mathrm{~N} / \mathrm{O}$ & 2923 \\
\hline Copper chloride & 3411 & 1591 & 1569 & 2930 \\
\hline $\begin{array}{l}\text { Copper } \\
\text { perchlorate }\end{array}$ & 3430 & $1609 / 1593$ & 157 & 2924 \\
\hline Mercury chloride & 3399 & $1630 / 1592$ & 1572 & 2924 \\
\hline $\begin{array}{l}\text { Mercury } \\
\text { perchlorate }\end{array}$ & 3410 & 1594 & 1571 & $\mathrm{~N} / \mathrm{O}$ \\
\hline Zinc chloride & 3430 & 1607 & 1570 & 2922 \\
\hline Zinc acetate & 3429 & 1591 & $\mathrm{~N} / \mathrm{O}$ & 2928 \\
\hline Zinc perchlorate & 3429 & $1647 / 1610$ & 1571 & 212 \\
\hline
\end{tabular}

Table 9: 1:1 Metal ion complexation reaction with (36). IR data for 1:1 and 1:2 metal ion complexation reactions with compound (36). $\mathrm{N} / \mathrm{O}=$ not observed, those in green experience shifts compared with parent spectrum in red. 
The 1:2 metal ion complexation IR data (Table 10) was very similar to the metal ion complexation 1:1 data, in that shifts were seen more in the $\mathrm{NH}$ bands than the $\mathrm{NCH}$ ones. The zinc perchlorate was shown to give the largest shift in the $\mathrm{NCH}$ region which corresponded well with the ${ }^{1} \mathrm{H}$ NMR data. Unfortunately due to the presence of broad bands the peak to indicate the unbound pyridyl $\mathrm{NCH}$ groups could not be clearly seen in the in the acetate and perchlorate spectra, but as they are present in the chloride IR spectrum and the ${ }^{1} \mathrm{H}$ NMR spectra they are believed to be there.

\begin{tabular}{|l|c|c|c|c|}
\hline $1: 2$ reaction & $\mathrm{O}-\mathrm{H} \mathrm{cm}^{-1}$ & $\mathrm{NC}-\mathrm{H} \mathrm{cm}^{-1}$ & $\mathrm{NC}-\mathrm{H} \mathrm{cm}^{-1}$ & $\mathrm{~N}-\mathrm{H} \mathrm{cm}^{-1}$ \\
\hline Cobalt chloride & 3431 & 1592 & 1570 & 2926 \\
\hline Nickel perchlorate & 3437 & 1631 & $\mathrm{~N} / \mathrm{O}$ & $\mathrm{N} / \mathrm{O}$ \\
\hline Nickel chloride & 3399 & 1606 & 1571 & $\mathrm{~N} / \mathrm{O}$ \\
\hline Nickel acetate & 3429 & 1592 & $\mathrm{~N} / \mathrm{O}$ & $\mathrm{N} / \mathrm{O}$ \\
\hline Mercury perchlorate & 3435 & 1610 & 1572 & 2930 \\
\hline Mercury chloride & 3401 & 1594 & 1570 & 3059 \\
\hline Zinc acetate & 3426 & 1592 & $\mathrm{~N} / \mathrm{O}$ & 2926 \\
\hline Zinc chloride & 3427 & 1592 & 1571 & 2922 \\
\hline Zinc perchlorate & 3428 & 1610 & $\mathrm{~N} / \mathrm{O}$ & 2928 \\
\hline Copper chloride & 3423 & 1607 & 1570 & 2927 \\
\hline Copper perchlorate & 3435 & 1630 & $\mathrm{~N} / \mathrm{O}$ & $\mathrm{N} / \mathrm{O}$ \\
\hline Copper acetate & 3428 & 1624 & $\mathrm{~N} / \mathrm{O}$ & 2926 \\
\hline
\end{tabular}

Table 10: IR data for 1:2 metal ion complexation reactions with (36). IR data for 1:1 and 1:2 metal ion complexation reactions with compound (36). N/O = not observed, those in green experience shifts compared with parent spectrum in red.

Compound (40) was next to be examined using ${ }^{1} \mathrm{H}$ NMR spectroscopy (Figure 168). All the 1:1 metal ion complexation reactions proceeded well with the self-assembly intact. 


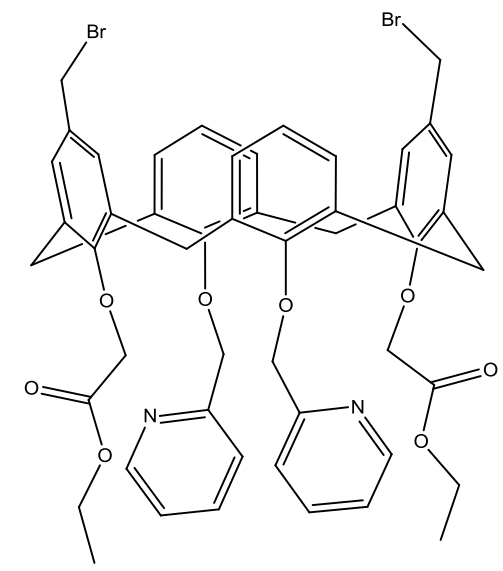

Figure 168: Compound (40)

Due to solubility issues of the zinc acetate complexes in both $\mathrm{CDCl}_{3}$ and $d_{6}$-DMSO, a high quality spectrum was difficult to obtain, but it looks as if the pyridyl group on the lower rim has been lost. The parent compound showed the lower rim pyridyl $\mathrm{NCH}$ protons as a broad doublet at $8.61 \mathrm{ppm}$ (Figure 169). Zinc chloride showed shifts to $9.12 \mathrm{ppm}$ for the lower rim pyridyl protons from $8.61 \mathrm{ppm}$ in the parent spectrum. Zinc perchlorate also showed shifts from $8.61 \mathrm{ppm}$ to $9.08 \mathrm{ppm}$ for the lower rim pyridyl protons. Both spectra exhibited broad doublets between 8.55-8.57 ppm which could be indicating the unbound pyridyl doublets in the self-assembled chain.

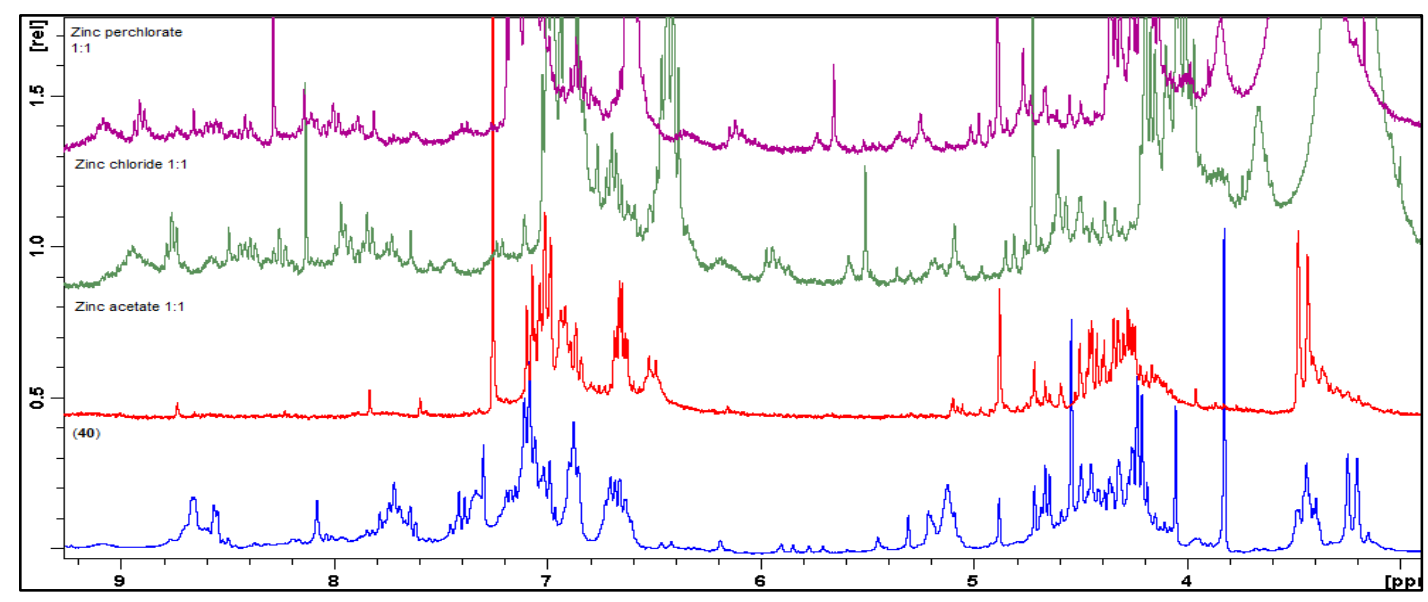

Figure 169: ${ }^{1} \mathrm{H}$ NMR for the 1:1 metal ion complexation reactions for $(\mathbf{4 0})$. 
The bridging region of the spectrum, (Figure 170), showed a few shifts, but again the bridging methylene protons were overshadowed by methanol. Several doublets were seen in the region of the axial bridging methylene protons indicating either selfassembly or different conformers. This could easily be supported with the presence of a large singlet at $3.82 \mathrm{ppm}$ which could be indicating the 1,3-alternate form. This peak is broadened and shifted to 3.76 and $3.84 \mathrm{ppm}$ in the chloride and perchlorate spectra respectively. The loss of this signal in the acetate could be used as evidence that only the cone conformation is present in the self-assembled form. The upper rim methylbromo-methylene protons are seen at $4.88 \mathrm{ppm}$ in the parent spectrum and are shifted to $4.72 \mathrm{ppm}$ and $4.93 \mathrm{ppm}$ in the chloride and perchlorate spectra. The abundance of peaks clearly indicated that self-assembly is occurring.

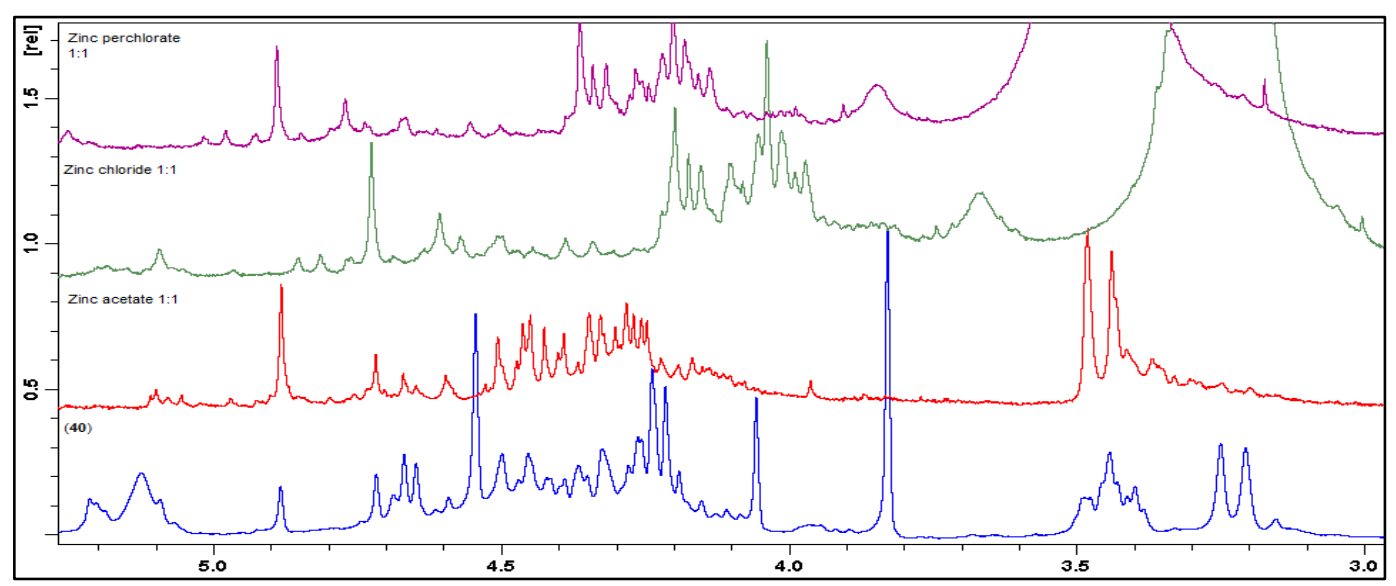

Figure 170: ${ }^{1} \mathrm{H}$ NMR of the bridging region of (40) with 1:1 Zinc(II) metal ion complexation. Red trace $=$ Zinc(II) acetate, Green trace $=$ Zinc(II) chloride, purple trace $=$ Zinc(II) perchlorate.

The IR data for the 1:1 metal ion complexation reactions showed that both the carbonyl and the pyridyl NCH groups experienced a shift due to the metal ion, indicating that both groups are involved in its complexation. However as the shift was more predominately seen in the pyridyl bands it is suggested that this is the preferred location for the metal ion to bind and that perhaps the carbonyl only plays a minor role in metal ion complexation reactions. 


\begin{tabular}{|l|c|c|}
\hline & $\mathrm{C}=\mathrm{O} \mathrm{cm}$ & $\mathrm{NC}-\mathrm{H} \mathrm{cm}^{-1}$ \\
\hline Compound (40) & 1747 & 1590 \\
\hline 1:1 reaction & & \\
\hline Zinc perchlorate & 1747 & 1628 \\
\hline Zinc chloride & 1746 & 1628 \\
\hline Zinc acetate & 1750 & 1629 \\
\hline
\end{tabular}

Table 11: IR data for 1:1 \& 1:2 metal ion complexation reactions with (40). N/O = not observed, those in green experience shifts compared with parent spectrum in red.

Continuing on with the metal ion complexation reactions compound (41) was next to be examined under ${ }^{1}$ H NMR spectroscopy, (Figure 171).

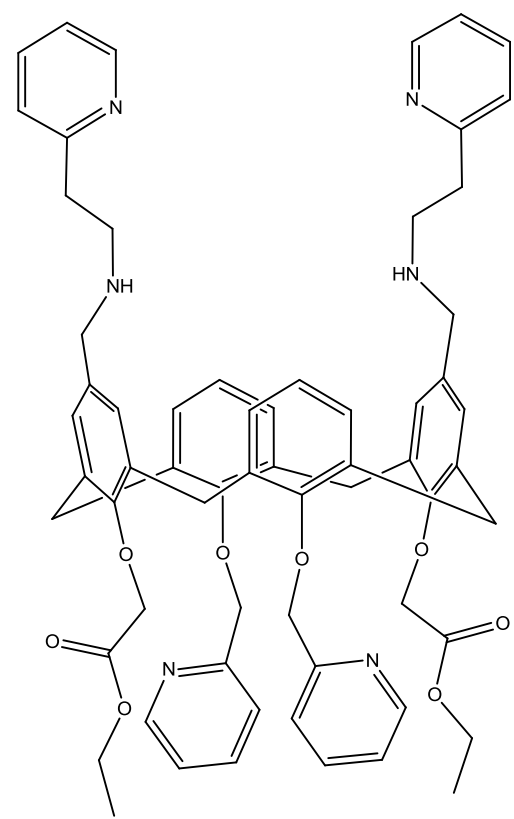

Figure 171: Compound (41)

The parent compound methylpyridyl protons occurred at $8.50 \mathrm{ppm}$ and at $8.40 \mathrm{ppm}$ for the upper rim ethylpyridyl protons (Figure 172). Unfortunately during the 1:1 metal ion complexation reaction, zinc acetate did not show any binding ability at this time. The zinc chloride spectrum showed binding, and shifts in the pyridyl protons were observed. The lower rim methylpyridyl protons shifted from $8.50 \mathrm{ppm}$ to 8.63 ppm and the upper rim ethylpyridyl protons shifted from $8.40 \mathrm{ppm}$ to $8.58 \mathrm{ppm}$. A small broad shoulder peak was observed indicating the unbound pyridyl protons. The 
perchlorate spectrum showed shifts from $8.50 \mathrm{ppm}$ to $8.60 \mathrm{ppm}$ for the lower rim methylpyridyl protons and from $8.40 \mathrm{ppm}$ to $8.56 \mathrm{ppm}$ for the upper rim ethylpyridyl protons. The self-assembly peaks were still dominant throughout these ${ }^{1} \mathrm{H}$ NMR experiments.

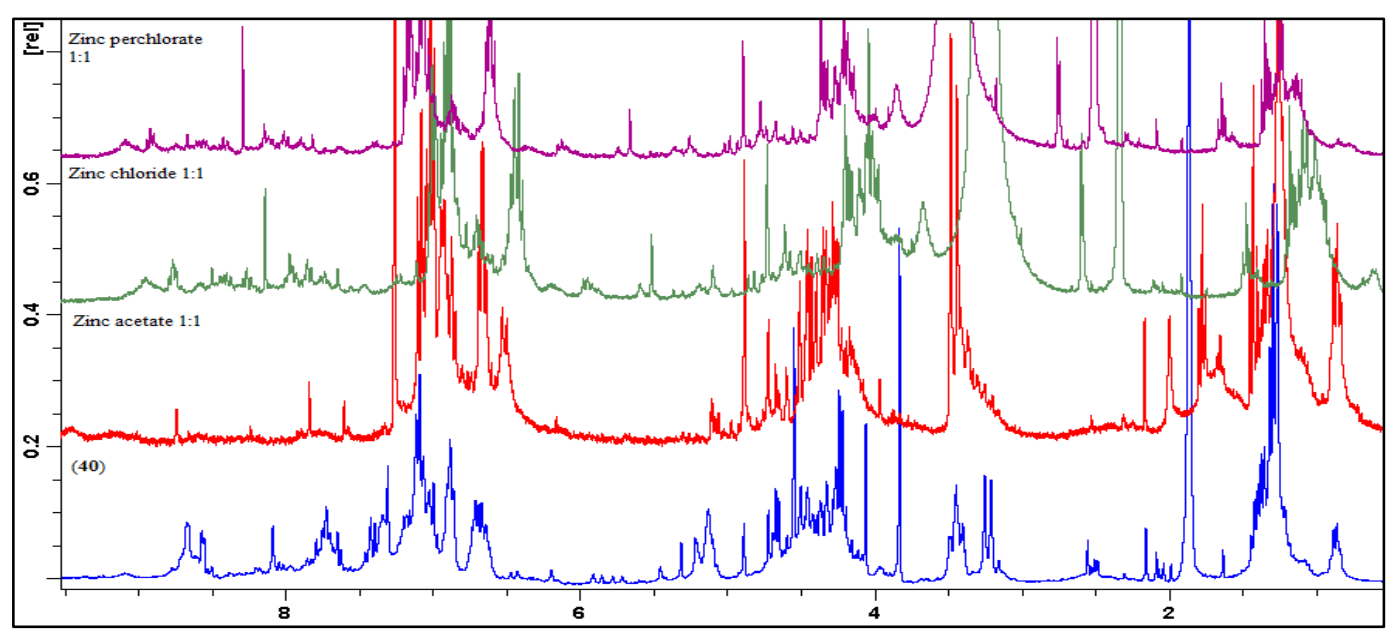

Figure 172: ${ }^{1} \mathrm{H}$ NMR for the 1:1 metal ion complexation reactions for (41)

The bridging region of the spectrum showed some chemical shifts, Figure 173. Again the equatorial bridging methylene protons were obscured by methanol in the chloride and perchlorate spectra, virtually no shift was seen in the acetate spectrum. The lower rim methylpyridyl-methylene protons were seen at $5.18 \mathrm{ppm}$ which shifted to $5.21,5.12$ and $5.12 \mathrm{ppm}$ in the acetate, chloride and perchlorate spectra respectively. The upper rim methylamine methylene protons were seen at $4.64 \mathrm{ppm}$ which shifted to $4.69 \mathrm{ppm}$ in the chloride and perchlorate spectra, no change was seen in the acetate spectrum. Due to the large overlap in the axial bridging region it is difficult to see if any shifts have occurred. 


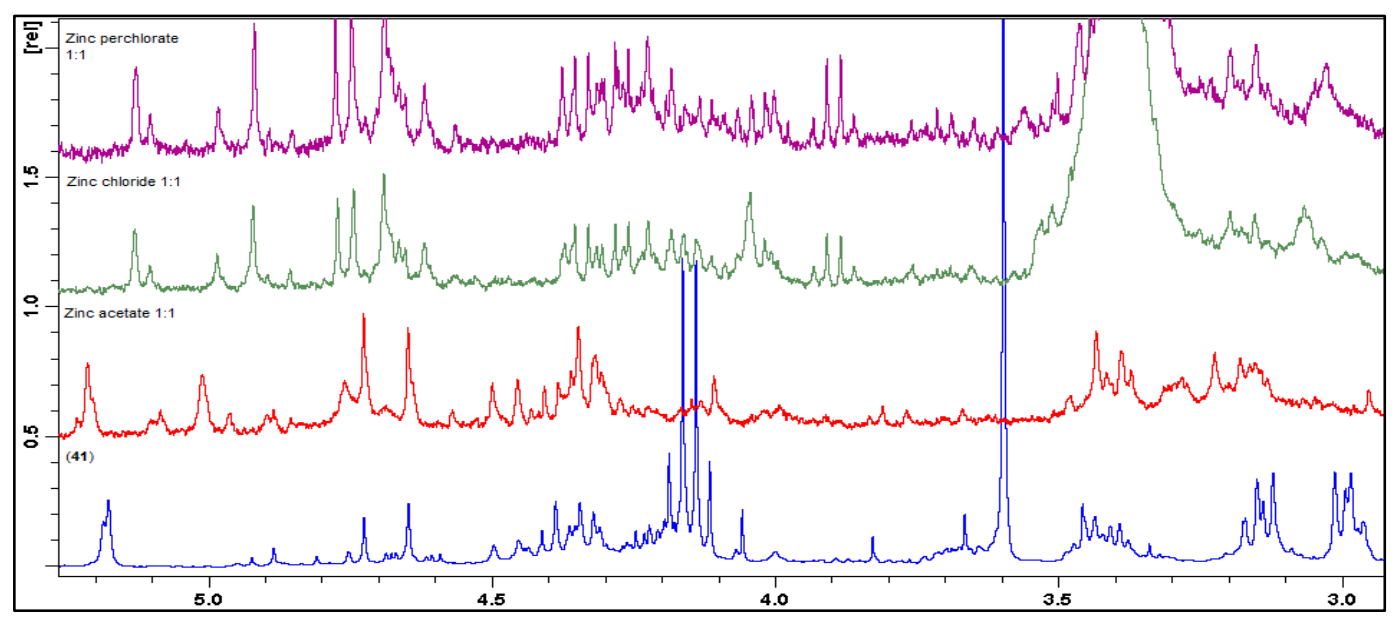

Figure 173: ${ }^{1} \mathrm{H}$ NMR of the bridging region of (41) with 1:1 Zinc(II) metal ion complexation. Red trace $=$ Zinc(II) acetate, Green trace $=$ Zinc(II) chloride, purple trace $=$ Zinc(II) perchlorate.

With the 1:2 metal ion complexation reactions, all of the zinc ions were bound efficiently, with the self-assembly peaks dominating (Figure 174). The parent compound pyridyl doublets were the same as before and the zinc acetate exhibited a shift from $8.50 \mathrm{ppm}$ to $8.63 \mathrm{ppm}$ for the lower rim and from $8.40 \mathrm{ppm}$ to $8.59 \mathrm{ppm}$ for the upper rim pyridyl. The chloride spectrum had shifts from $8.50 \mathrm{ppm}$ to 8.61 ppm for the lower rim pyridyl and from $8.40 \mathrm{ppm}$ to $8.56 \mathrm{ppm}$ for the upper rim pyridyl. The perchlorate also had shifts from $8.50 \mathrm{ppm}$ to $8.60 \mathrm{ppm}$ for the lower rim and from $8.40 \mathrm{ppm}$ to $8.56 \mathrm{ppm}$ for the upper rim pyridyl's. All three were seen to have broad shoulder peak's, representing the unbound pyridyl protons. 


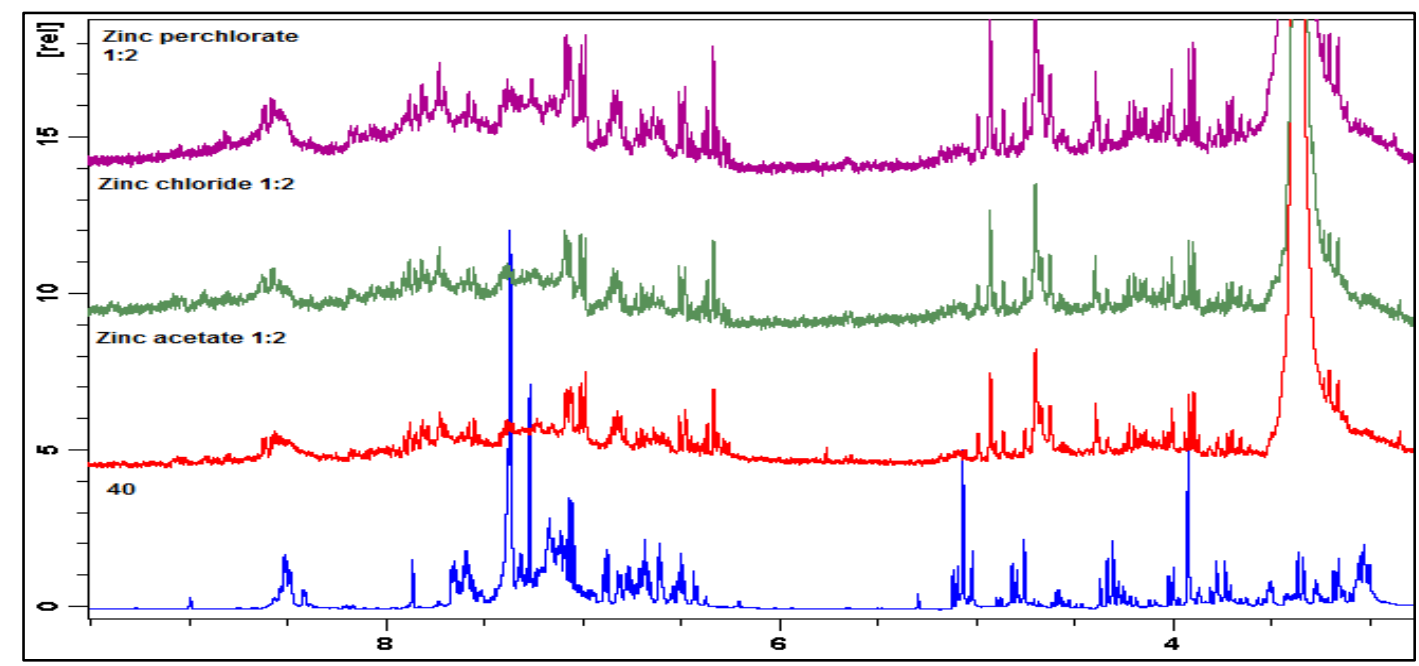

Figure 174: ${ }^{1} \mathrm{H}$ NMR for the 1:2 metal ion complexation reactions for (41)

Some shifts were also seen in the bridging region of the spectrum, (Figure 175). The lower rim pyridyl methylene protons were shifted from $5.18 \mathrm{ppm}$ in the parent spectrum to 5.21, 5.12 and $5.12 \mathrm{ppm}$ in the acetate, chloride and perchlorate spectra respectively. The upper rim methylamine methylene protons shifted from $4.65 \mathrm{ppm}$ in the parent to $4.69 \mathrm{ppm}$ in the chloride and perchlorate spectra with no change was seen in the acetate spectrum. The axial bridging methylene protons shifted from 4.33 ppm to $4.35 \mathrm{ppm}$ in the perchlorate spectrum. No change in the acetate or chloride spectra. The ester methylene protons were not observed in the acetate spectrum, possibly indicating the loss of the ester group, and in the chloride and perchlorate spectra the protons were obscured by the residual methanol solvent in the spectrum. The equatorial methylene protons were shifted to $3.28,3.17$ and $3.17 \mathrm{ppm}$ in the acetate, chloride and perchlorate spectra respectively. The last two peaks to experience shifts in this region were the upper rim ethylpyridyl methylene and ethylene protons. The ethylpyridyl methylene protons occurred at $3.14 \mathrm{ppm}$ in the parent spectrum and shifted to $3.28,3.17$ and $3.17 \mathrm{ppm}$ in the acetate, chloride and perchlorate spectra respectively. The ethylpyridyl ethylene protons appeared at 2.99 ppm in the parent spectrum and shifted to $3.15,3.06$ and $3.03 \mathrm{ppm}$ in the acetate, chloride and perchlorate spectra respectively. These interactions support upper rim metal ion complexation and, along with the abundance of peaks, also indicate that self-assembly is occurring. 


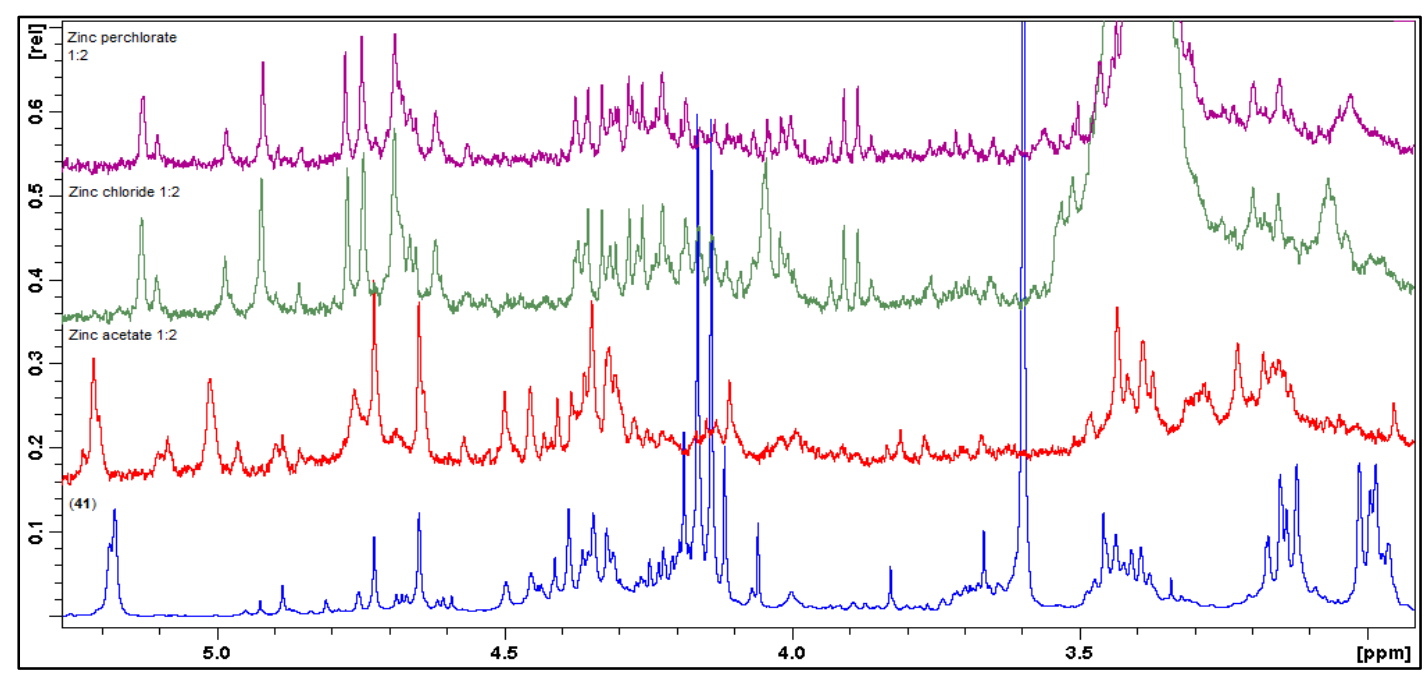

Figure 175: ${ }^{1} \mathrm{H}$ NMR of the bridging region of (41) with 1:2 Zinc(II) metal ion complexation. Red trace $=$ Zinc(II) acetate, Green trace $=$ Zinc(II) chloride, purple trace $=$ Zinc(II) perchlorate.

The IR data (Table 12) for the 1:1 and 1:2 metal ion complexation reactions with compound (41) corresponded well with the ${ }^{1} \mathrm{H}$ NMR data. The zinc complexes showed large downfield shifts in the NCH bands as well as shifts in the hydroxyl groups. As the shifts in the hydroxyl groups are quite large it is believed that metal ion complexation as well as the self-assembly effects are responsible for these downfield shifts.

\begin{tabular}{|l|c|c|c|c|}
\hline & $\begin{array}{c}\mathrm{C}=\mathrm{O} \\
\mathrm{cm}^{-1}\end{array}$ & $\begin{array}{c}\mathrm{NCH} \text { (Bound) } \\
\mathrm{cm}^{-1}\end{array}$ & $\begin{array}{c}\mathrm{NCH} \text { (Unbound) } \\
\mathrm{cm}^{-1}\end{array}$ & $\mathrm{OH} \mathrm{cm}^{-1}$ \\
\hline Compound (41) & 1751.67 & 1591.31 & & 3390.31 \\
\hline 1:1 Reaction & & & & \\
\hline Zinc chloride & 1743.38 & 1608.06 & $\mathrm{~N} / \mathrm{O}$ & 3407.68 \\
\hline Zinc perchlorate & 1747.34 & 1611.14 & $\mathrm{~N} / \mathrm{O}$ & 3413.92 \\
\hline Zinc acetate & 1755.07 & 1591.57 & $\mathrm{~N} / \mathrm{O}$ & 3422.04 \\
\hline Mercury chloride & 1736.25 & 1591.52 & $\mathrm{~N} / \mathrm{O}$ & 3414.37 \\
\hline $\begin{array}{l}\text { Mercury } \\
\text { perchlorate }\end{array}$ & 1746.88 & 1629.64 & 1592.41 & 3420.12 \\
\hline Nickel chloride & 1754.34 & 1591.44 & $\mathrm{~N} / \mathrm{O}$ & 3408.16 \\
\hline Nickel acetate & 1754.75 & 1590.77 & $\mathrm{~N} / \mathrm{O}$ & 3415.15 \\
\hline Nickel perchlorate & 1750.78 & 1674.67 & 1608.62 & 3399.28 \\
\hline Copper perchlorate & 1744.31 & 1610.57 & $\mathrm{~N} / \mathrm{O}$ & 3419.62 \\
\hline Copper acetate & 1754.99 & 1631.86 & 1591.21 & 3413.04 \\
\hline Copper chloride & 1744.52 & 1631.86 & 1591.25 & 3427.93 \\
\hline Cobalt chloride & 1751.8 & $\mathrm{~N} / \mathrm{O}$ & $\mathrm{N} / \mathrm{O}$ & 3424.25 \\
\hline
\end{tabular}




\begin{tabular}{|l|c|c|c|c|}
\hline & & & & \\
\hline $1: 2$ reaction & & & & \\
\hline Zinc chloride & 1747.39 & 1595.02 & $\mathrm{~N} / \mathrm{O}$ & 3429.37 \\
\hline Zinc perchlorate & 1744.28 & 1610.8 & $\mathrm{~N} / \mathrm{O}$ & 3423.05 \\
\hline Zinc acetate & 1751.61 & 1610.15 & $\mathrm{~N} / \mathrm{O}$ & 3429.25 \\
\hline Mercury chloride & 1740.32 & 1627.42 & 1593.6 & 3425.4 \\
\hline $\begin{array}{l}\text { Mercury } \\
\text { perchlorate }\end{array}$ & 1744.08 & 1606.58 & $\mathrm{~N} / \mathrm{O}$ & 3397.62 \\
\hline Copper chloride & 1746.65 & 1606.78 & $\mathrm{~N} / \mathrm{O}$ & 3414.33 \\
\hline Copper acetate & 1749.4 & 1591.54 & $\mathrm{~N} / \mathrm{O}$ & 3433 \\
\hline Copper perchlorate & 1742.74 & 1610.26 & $\mathrm{~N} / \mathrm{O}$ & 3435.16 \\
\hline Cobalt chloride & 1747.69 & 1606.82 & $\mathrm{~N} / \mathrm{O}$ & 3425.59 \\
\hline Nickel chloride & 1753.72 & 1631.86 & 1592.41 & 3413.49 \\
\hline Nickel perchlorate & 1746.5 & 1607.86 & $\mathrm{~N} / \mathrm{O}$ & 3432.56 \\
\hline
\end{tabular}

Table 12: IR data for 1:1 \& 1:2 metal ion complexation reactions with compound (41). N/O

$=$ not observed, those in green experience shifts compared with parent spectrum in red.

SEM images of the compound (41) along with some of the metal complexed forms were obtained and the images are quite interesting. The parent compound (Figure 176) consisted of what appeared to be a circular, rough inter texture encased in a smooth outer exterior.

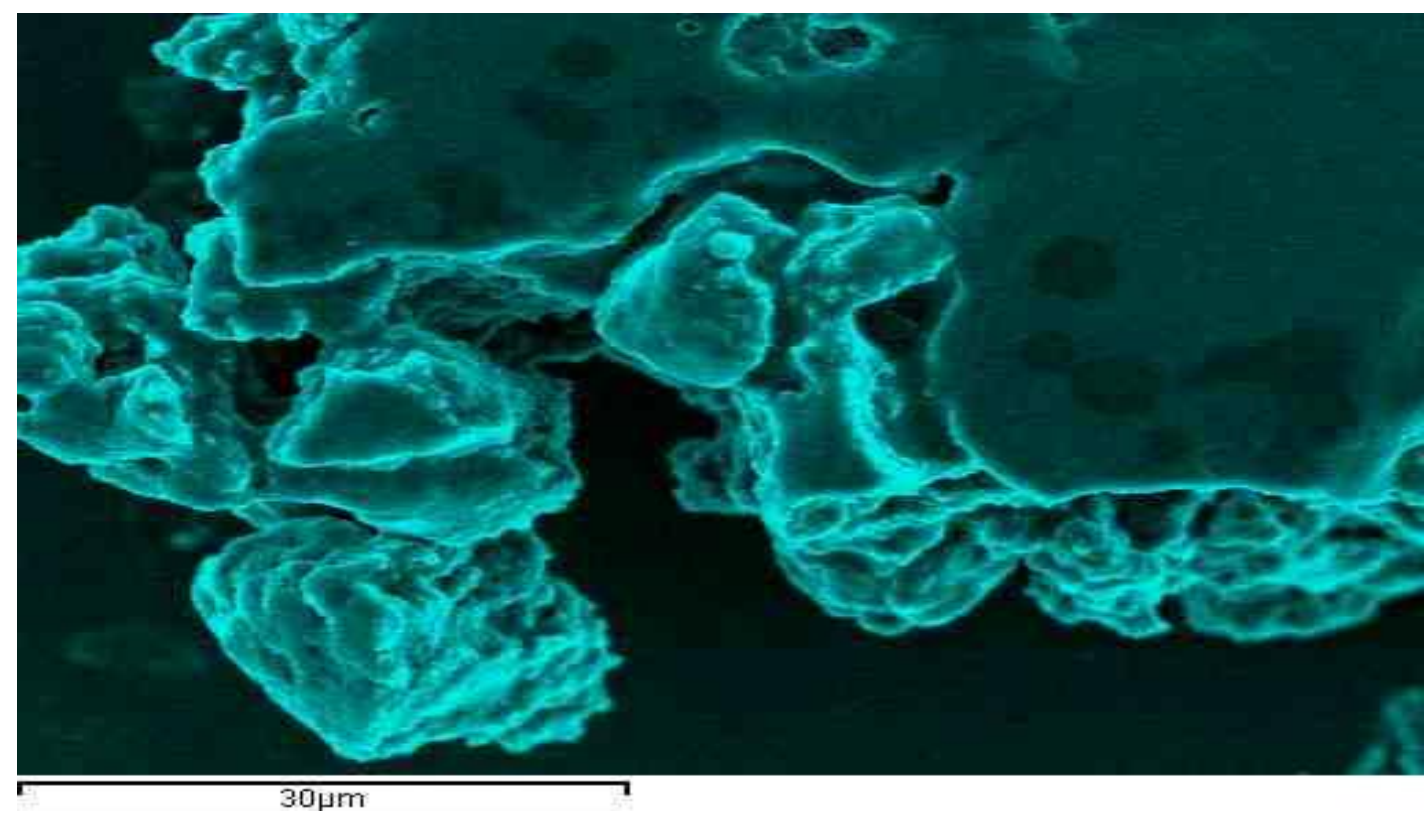

Figure 176: SEM of compound (41), WD 14 mm, $16.0 \mathrm{Kv}$. X 100. 
However when metal ion complexation occurred, a drastic change in structure was seen. What was once a bubble-like effect changed to a spiky, almost ivy like effect (Figure 177).

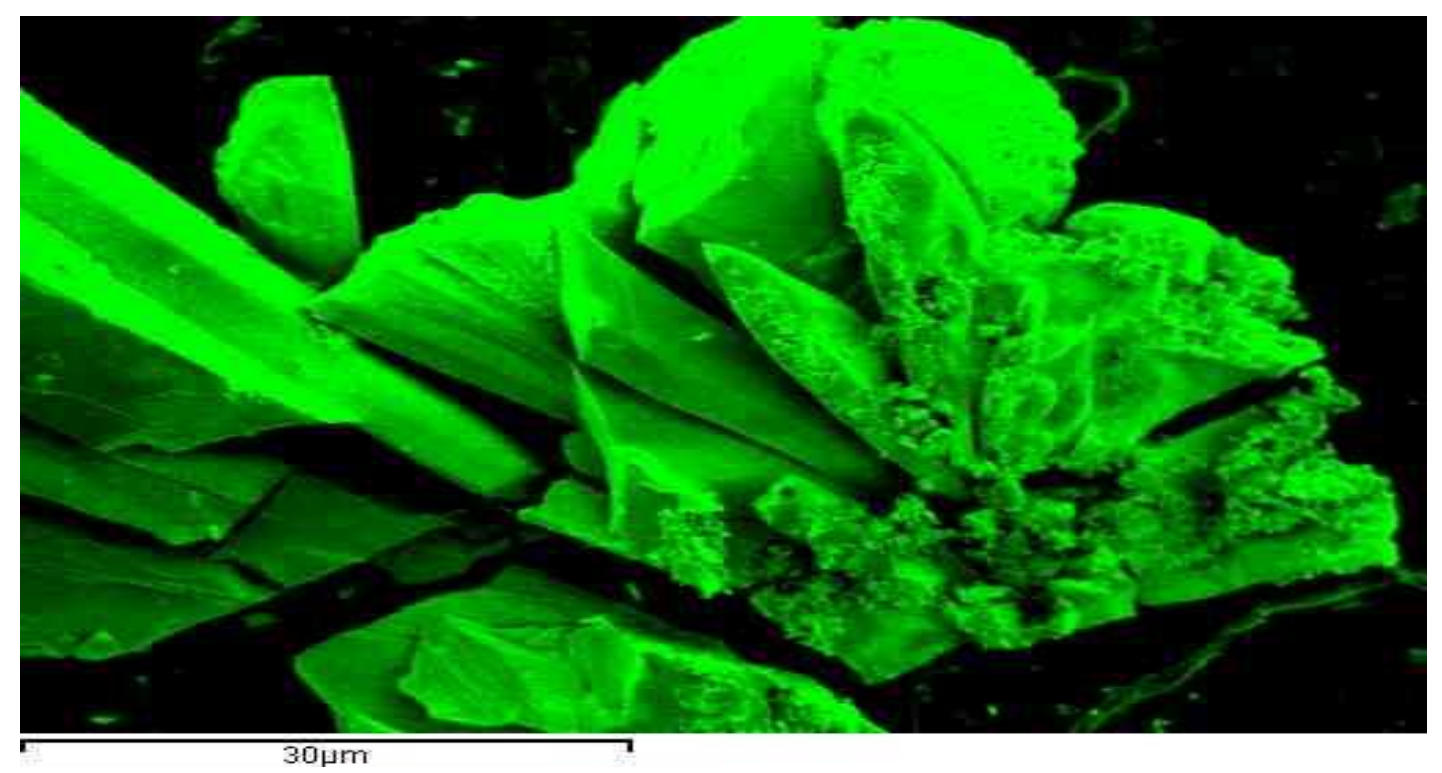

Figure 177: SEM of zinc(II) perchlorate complexed (41). WD 14 mm, 16.0 Kv. X 400.

Upon closer viewing (Figure 178), the edges of the crystals almost look 'plant-like'. It also appears to have retained some characteristics of the parent, in that the inter texture is a lot rougher than the exterior. 


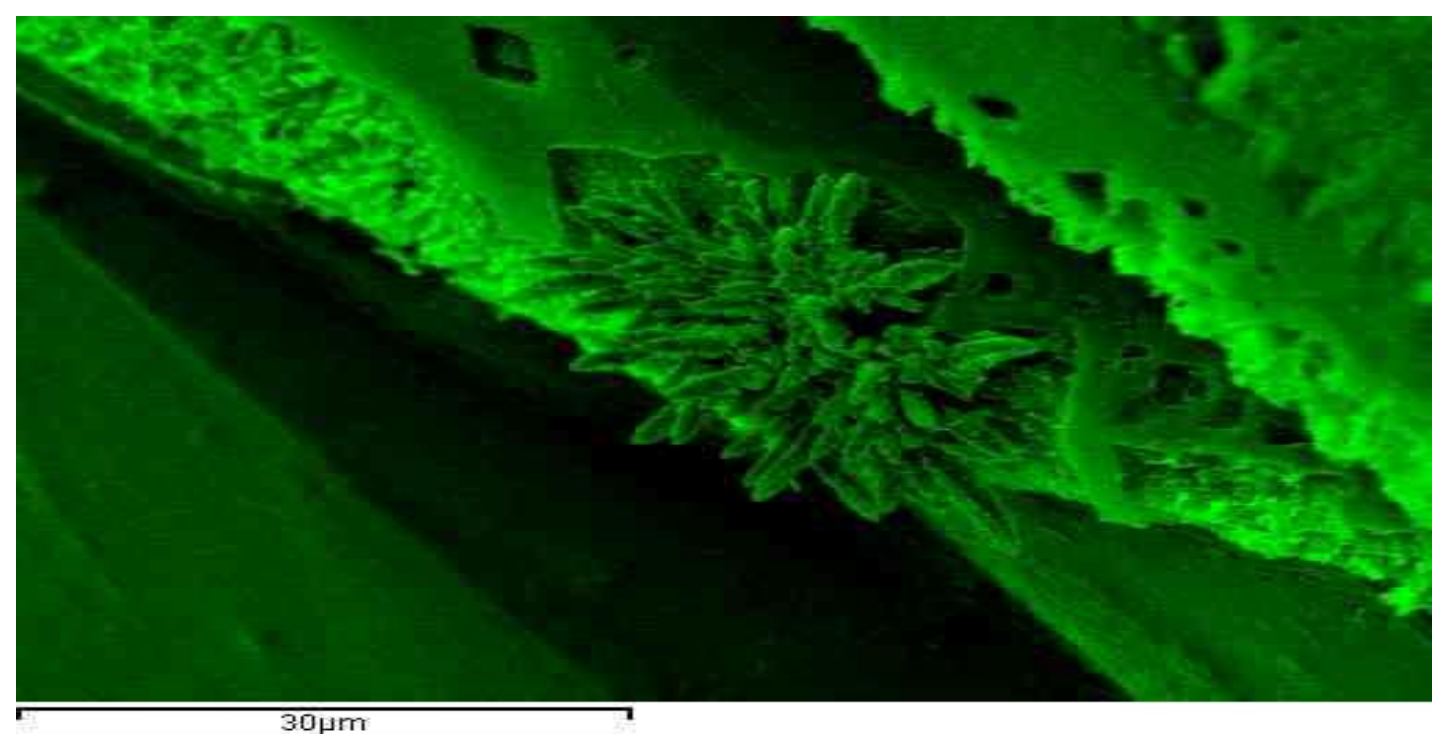

Figure 178: SEM of zinc(II) perchlorate metal complexed (41). WD 14 mm, $16.0 \mathrm{kv}, \mathrm{X}$ 350.

Metal ion complexation reactions were continued with compound (42), (Figure 179). Unfortunately the initial spectrum of the parent compound was quite close-packed, and accurate analysis proved quite difficult. However upon metal ion complexation the spectrum cleaned up dramatically and appeared to show a decrease in the selfassembly. This suggests that while metal ion complexation didn't overcome the selfassembly property of the compound, it did help to establish some order in the selfassembly. Quite possibly it began to 'cut -up' the chain from one long continuous fragment into several shorter metal complexed ones. Still, without a crystal structure this theory cannot be proven. Thankfully the pyridyl and bipy metal binding protons could be determined without difficulty. 


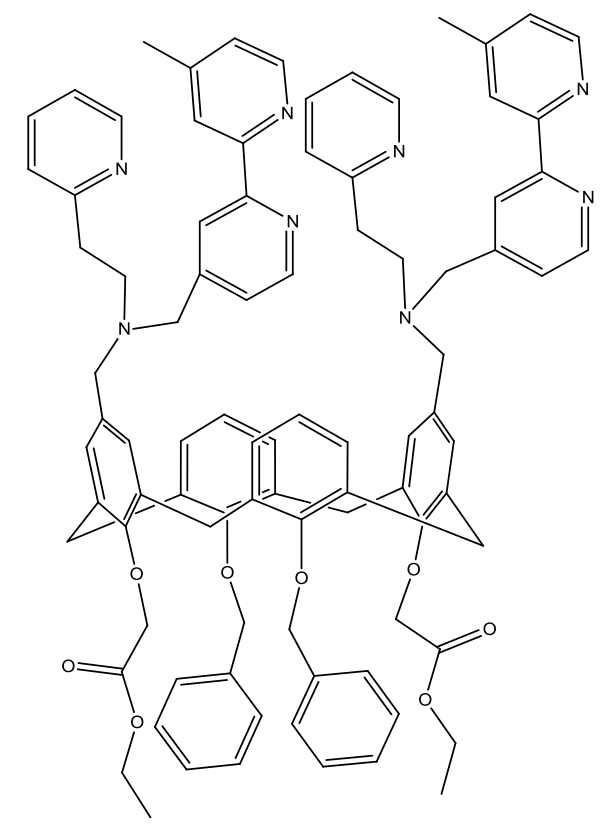

Figure 179: Compound (42)

In the parent compound (42) the pyridyl protons occurred at $8.60 \mathrm{ppm}$ and the bipy protons occurred at $8.53 \mathrm{ppm}$ and $8.50 \mathrm{ppm}$ (Figure 180). Both of these groups occur on the upper rim of the compound. In the 1:1 metal ion complexation reactions zinc acetate and perchlorate ions seemed to bind well. Zinc chloride produced a broadening of the peaks which suggests that the metal may be bound but as no shift is seen it cannot be conclusively said that the metal ion has bound. In the acetate spectrum a shift from $8.60 \mathrm{ppm}$ to $8.88 \mathrm{ppm}$ occurs for the pyridyl. Shifts are also seen from $8.53 \mathrm{ppm}$ to $8.73 \mathrm{ppm}$ and from $8.50 \mathrm{ppm}$ to $8.67 \mathrm{ppm}$ for the bipy protons. These shifts are quite large and are a good indication that the metal has bound successfully. 


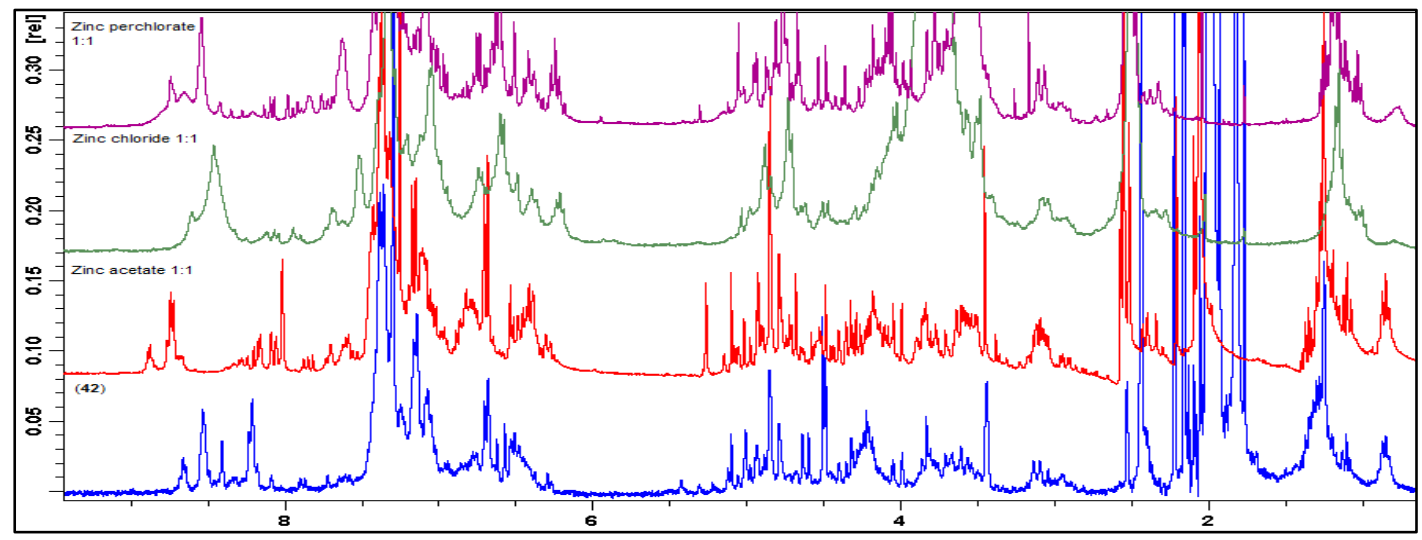

Figure 180: ${ }^{1} \mathrm{H}$ NMR of 1:1 Metal ion complexation reactions for (42). Red trace = Zinc(II) acetate, Green trace $=$ Zinc(II) chloride, purple trace $=$ Zinc(II) perchlorate .

The bridging region, (Figure 181) was abundant with peaks to the point where overlapping of peaks was unavoidable. As a result clear distinction and possible shifts of peaks is difficult to view. What is evident is that self-assembly is still occurring, and that the chloride spectrum has broadened significantly due to metal ion complexation.

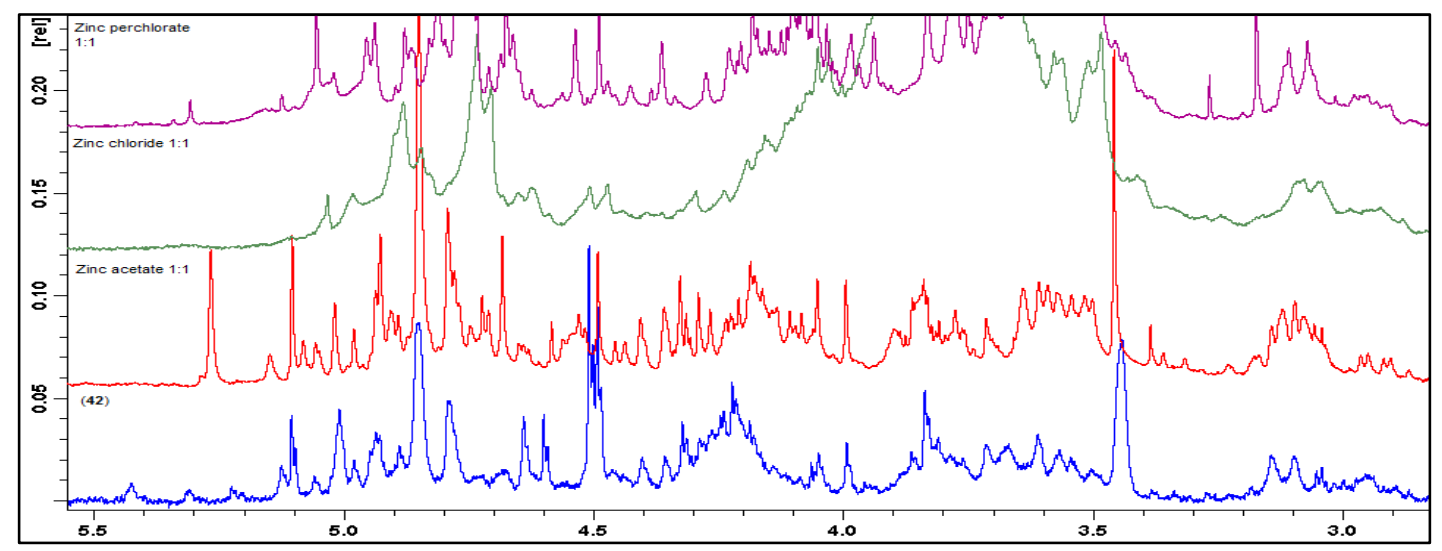

Figure 181: ${ }^{1} \mathrm{H}$ NMR of the bridging region of (42) with 1:1 Zinc(II) metal ion complexation. Red trace $=$ Zinc(II) acetate, Green trace $=$ Zinc $($ II) chloride, purple trace $=$ Zinc(II) perchlorate.

The IR data for compound (42) is shown below (Table 13). It can be seen that the carbonyl groups experience a shift in wavenumber when metal ion complexation occurs, however this shift is not clearly seen in the ${ }^{1} \mathrm{H}$ NMR spectrum. This may be due to a residual effect of the metal causing deshielding through the molecule or 
perhaps, in the parent compound form, it existed purely as the hetero, but on metal ion complexation rearrangement occurred to the homo form. In this form the two ester linkages would be able to interact with each other and cause this deshielding. The NCH band was quite broad and may be encompassing the three NCH's (one from the pyridyl and two from the bipy unit), but it is clear that shifts have occurred and they correspond well with the ${ }^{1} \mathrm{H}$ NMR data.

\begin{tabular}{|l|c|c|c|}
\hline & $\begin{array}{c}\mathrm{C}=\mathrm{O} \\
\mathrm{cm}^{-1}\end{array}$ & $\begin{array}{c}\mathrm{NC}-\mathrm{H} \text { (Bound) } \\
\mathrm{cm}^{-1}\end{array}$ & $\begin{array}{c}\mathrm{NC}-\mathrm{H} \text { (Unbound) } \\
\mathrm{cm}^{-1}\end{array}$ \\
\hline Compound (42) & 1637 & 1562 & 1560 \\
\hline 1:1 reaction & & & $\mathrm{N} / \mathrm{O}$ \\
\hline Zinc acetate & 1756 & 1598 & 1562 \\
\hline Zinc chloride & 1731 & 1614 & 1561 \\
\hline Zinc perchlorate & 1730 & 1614 & \\
\hline
\end{tabular}

Table 13: IR data for 1:1 metal ion complexation reactions with (42). N/O = not observed, those in green experience shifts compared with parent spectrum in red.

Compound (44), (Figure 182), was also examined by ${ }^{1} \mathrm{H}$ NMR spectroscopy to test its binding ability. The spectra for these studies were quite congested, but binding could still be observed.

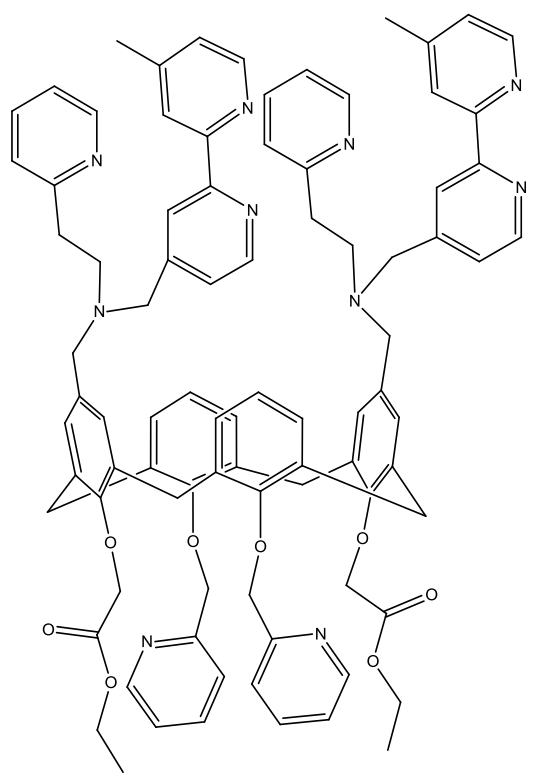

Figure 182: Compound (44) 
The 1:1 metal ion complexation reactions were examined first (Figure 183). The parent compound (44) exhibited a doublet for the bipy $\mathrm{NCH}$ at $8.52 \mathrm{ppm}$. This peak was very broad and it is believed that the upper rim ethylpyridyl protons are hidden under this. The lower rim methylpyridyl protons occurred at $8.64 \mathrm{ppm}$ in the parent compound. In the acetate spectrum a shift from $8.52 \mathrm{ppm}$ to $8.57 \mathrm{ppm}$ was seen for the upper rim ethylpyridyl protons, and a shift from $8.64 \mathrm{ppm}$ to $8.68 \mathrm{ppm}$ was seen for the lower rim methylpyridyl protons. As these shifts are almost the same $(0.05$ and $0.04 \mathrm{ppm}$ respectively) it is likely that both are involved in the complexation of the metal. This could happen in two ways; first there could be two separate selfassembled chains (previously mentioned) or that the metal is in the middle of a chain with one half binding to it by the lower rim and the other half binding by its upper rim. Both seem plausible but without X-ray analysis it cannot be stated conclusively. The chloride spectrum shows a shift in the upper rim ethylpyridyl protons from 8.52 ppm to $8.63 \mathrm{ppm}$ and a small shift in the lower rim methylpyridyl protons from 8.64 ppm to $8.68 \mathrm{ppm}$. This suggests that the majority of the binding is occurring on the upper rim. The perchlorate spectrum shows shifts for the upper rim ethylpyridyl protons from 8.52 to $8.62 \mathrm{ppm}$ and for the lower rim methylpyridyl protons from $8.64 \mathrm{ppm}$ to $8.67 \mathrm{ppm}$. This again suggests that the binding is favouring the upper rim over the lower rim, which isn't too surprising given the extra number of donating atoms on the upper rim. All three exhibited peaks to indicate the unbound selfassembled NCH doublets.

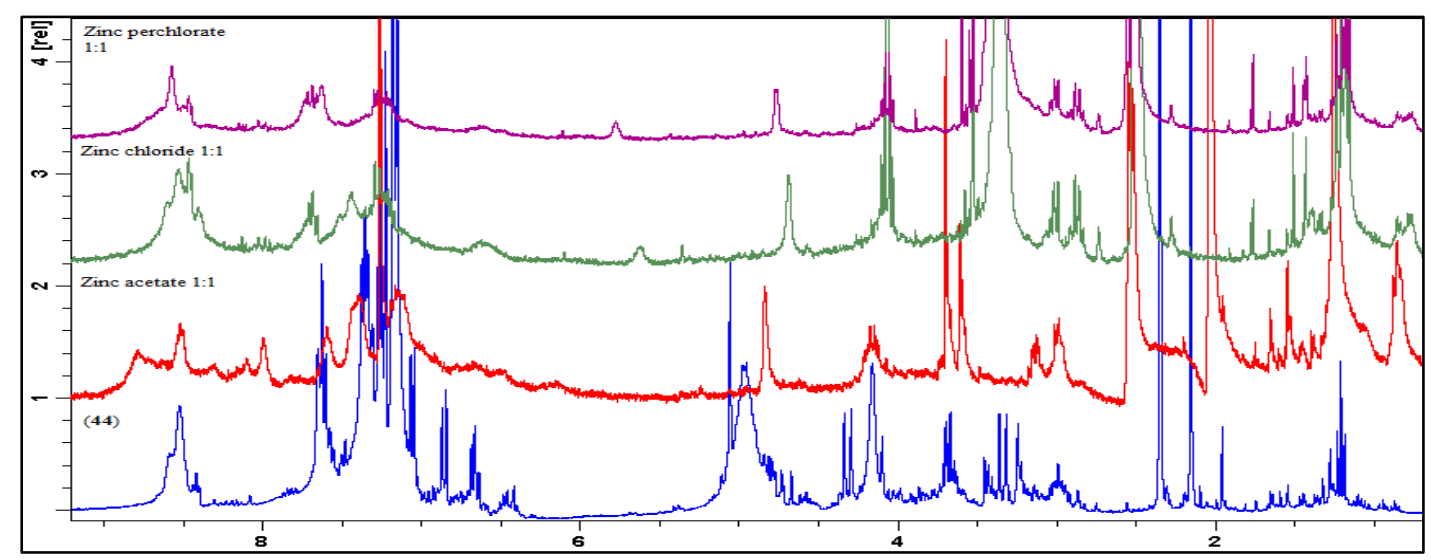

Figure 183: 1:1 Metal ion complexation ${ }^{1} \mathrm{H}$ NMR for (44). Red trace $=$ Zinc(II) acetate, Green trace $=$ Zinc(II) chloride, purple trace $=$ Zinc(II) perchlorate . 
The bridging region was again seen to be quite broad and resembles starting material. This may indicate that the complex has broken down upon metal ion complexation or that due to the broadening the desired peaks are no longer visible.

The 1:2 metal ion complexation reactions were also carried out and investigated under ${ }^{1} \mathrm{H}$ NMR spectroscopy (Figure 184). The parent compound showed a doublet for the upper rim bipy $\mathrm{NCH}$ at $8.53 \mathrm{ppm}$, again the upper rim ethylpyridyl protons are hidden under the broad bipy peak, and a doublet for the lower rim methylpyridyl $\mathrm{NCH}$ at $8.64 \mathrm{ppm}$. The acetate spectrum showed a shift for the upper rim ethylpyridyl protons from $8.53 \mathrm{ppm}$ to $8.69 \mathrm{ppm}$ and the lower rim methylpyridyl protons shifted from $8.64 \mathrm{ppm}$ to $8.69 \mathrm{ppm}$. The chloride spectrum had shifts for the upper rim ethylpyridyl protons from $8.53 \mathrm{ppm}$ to $8.63 \mathrm{ppm}$ and the methylpyridyl protons shifted to $8.68 \mathrm{ppm}$. The perchlorate also showed a similar trend with a shift for the upper rim ethylpyridyl protons from $8.53 \mathrm{ppm}$ to $8.62 \mathrm{ppm}$ and for the lower rim methylpyridyl protons from $8.64 \mathrm{ppm}$ to $8.70 \mathrm{ppm}$. Once again these three spectra support binding at the upper rim over the lower rim. All three exhibited peaks to indicate the unbound self-assembled NCH doublets.

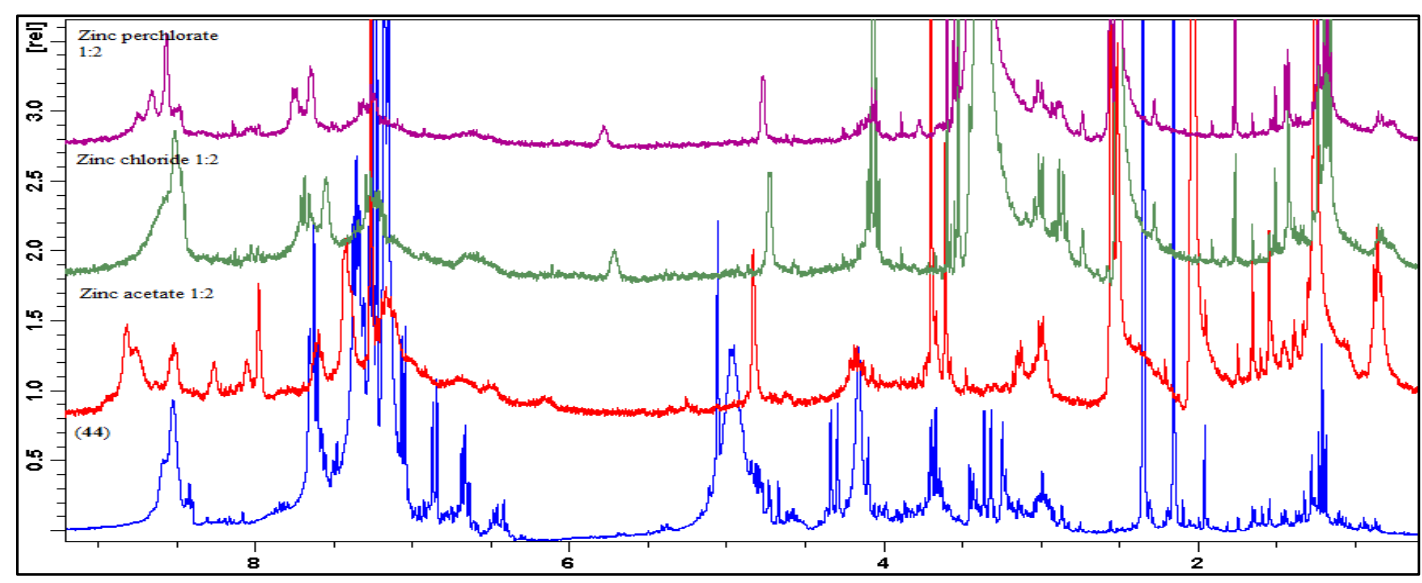

Figure 184: ${ }^{1} \mathrm{H}$ NMR for 1:2 Metal ion complexation reactions of (44). Red trace = Zinc(II) acetate, Green trace $=$ Zinc(II) chloride, purple trace $=$ Zinc(II) perchlorate . 
Again the bridging region was quite broad as previously seen with the 1:1 metal complexation reaction and due to the presence of solvent accurate shifts cannot be determined.

The IR data for the 1:1 metal ion complexation reactions of (44) is shown below (Table 14). Shifts can be seen in the carbonyl bands but this could just be due to the self-assembly. The NCH band was quite broad in both the parent and metal complexed forms, so likely encompassing both the available $\mathrm{NCH}$, but shifts can be seen due to complexation of the metal ion.

\begin{tabular}{|l|c|c|c|}
\hline & $\mathrm{C}=\mathrm{O} \mathrm{\textrm {cm } ^ { - 1 }}$ & $\mathrm{NC}-\mathrm{H}$ (Bound) $\mathrm{cm}^{-1}$ & $\mathrm{NC}-\mathrm{H}$ (Unbound) $\mathrm{cm}^{-1}$ \\
\hline Compound (44) & 1671 & 1593 & 1570 \\
\hline $1: 1$ reaction & & & 1560 \\
\hline Zinc acetate & 1737 & 1614 & 1562 \\
\hline Zinc chloride & 1737 & 1614 & 1561 \\
\hline Zinc perchlorate & 1736 & 1613 & \\
\hline
\end{tabular}

Table 14: IR data for 1:1 metal ion complexation reaction of (44). N/O = not observed, those in green experience shifts compared with parent spectrum in red.

Following on from compound (44), compound (46), (Figure 185), was the next to be examined under ${ }^{1} \mathrm{H}$ NMR and IR spectroscopy.

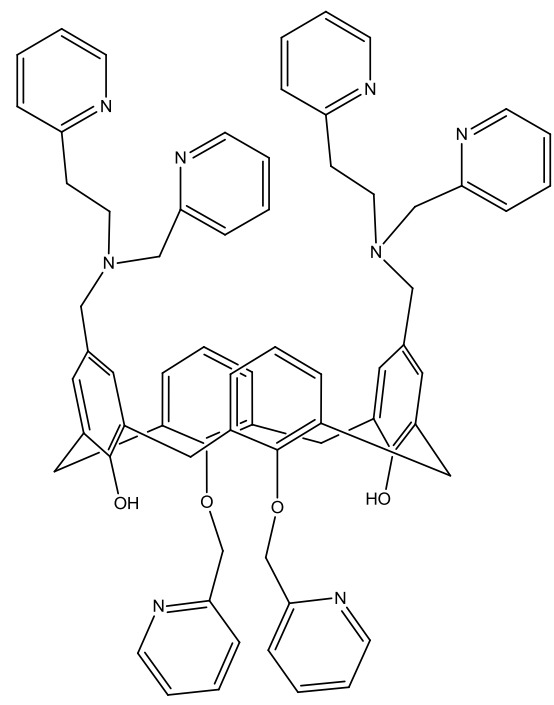

Figure 185: Compound (46) 
Due to the self-assembly and the excess 2-(aminoethyl)-pyridine in the cavity the ${ }^{1} \mathrm{H}$ NMR spectra was complicated (Figure 186). Even with this abundance of peaks, metal ion complexation can still be seen. The parent compound contains two pyridyl arms on the upper rim, the ethylpyridyl and the methylpyridyl, and has a methylpyridyl group on the lower rim. The lower rim methylpyridyl methylene protons were found as a doublet at $8.40 \mathrm{ppm}$ and the two upper rim pyridyl protons over-shadowed each other to create one large doublet at $8.28 \mathrm{ppm}$. The acetate spectrum showed a shift for the lower rim methylpyridyl protons from $8.40 \mathrm{ppm}$ to a very broad doublet at $8.67 \mathrm{ppm}$. The upper rim doublet representing the two upper rim pyridyl groups was shifted to $8.56 \mathrm{ppm}$ upon metal ion binding. The chloride spectrum showed a shift for the lower rim methylpyridyl protons from $8.40 \mathrm{ppm}$ to $8.63 \mathrm{ppm}$ and the upper rim pyridyl protons were shifted to $8.51 \mathrm{ppm}$. The perchlorate spectrum saw a shift for the lower rim methylpyridyl protons from 8.40 ppm to $8.68 \mathrm{ppm}$ and the upper rim to $8.52 \mathrm{ppm}$. All three showed doublets to indicate the unbound pyridyl's in the self-assembled chain. The ${ }^{1} \mathrm{H}$ NMR data suggests that metal ion complexation is preferred on the lower rim and not on the upper rim. This may be due to the increased flexibility on the upper rim, whereby the long arm moves into a position that is unfavourable to metal ion complexation allowing only a small amount of metal ion complexation to occur at the upper rim.

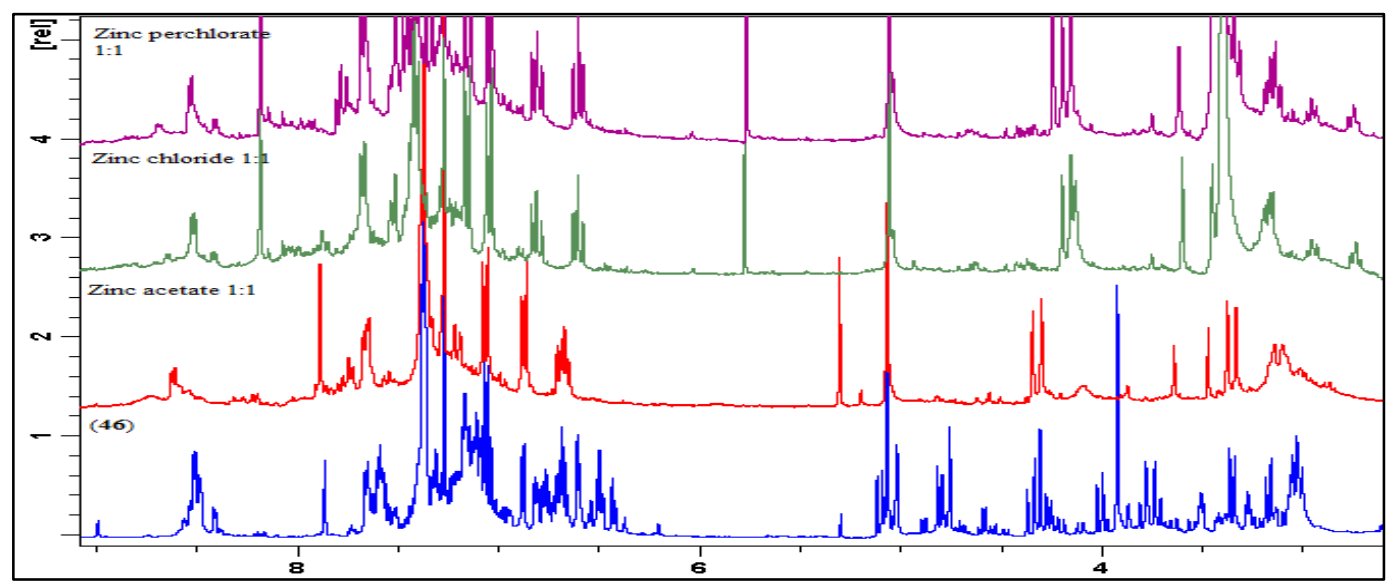

Figure 186: ${ }^{1} \mathrm{H}$ NMR for 1:1 Metal ion complexation reactions of (46). Red trace $=$ Zinc(II) acetate, Green trace $=$ Zinc(II) chloride, purple trace $=$ Zinc(II) perchlorate . 
In the bridging region of the spectra, (Figure 187), it appears that the level of selfassembly has diminished with metal ion complexation. In the acetate spectrum it appears as if the complex has broken up entirely to return starting materials. So shifts can be seen in the chloride and perchlorate spectra. The lower rim methylpyridyl methylene protons occurred at $4.94 \mathrm{ppm}$ in the parent spectrum and were shifted to $5.04 \mathrm{ppm}$ in the chloride and perchlorate spectra. The upper rim methylamine methylene protons occurred at $4.69 \mathrm{ppm}$ in the parent compound which shifted to 4.63 and $4.67 \mathrm{ppm}$ in the chloride and perchlorate spectra respectively. The methylpyridyl methylene protons shifted from $3.69 \mathrm{ppm}$ in the parent spectrum to 3.59 and $3.64 \mathrm{ppm}$ in the chloride and perchlorate spectra. The last two observed shifts in this region belonged to the ethylpyridyl methylene and ethylene protons. The ethylpyridyl methylene protons shifted from $2.82 \mathrm{ppm}$ in the parent spectrum to $2.95 \mathrm{ppm}$ in both the chloride and perchlorate spectra and the ethylene protons shifted from $2.66 \mathrm{ppm}$ to 2.72 and $2.76 \mathrm{ppm}$ in the chloride and perchlorate spectra.

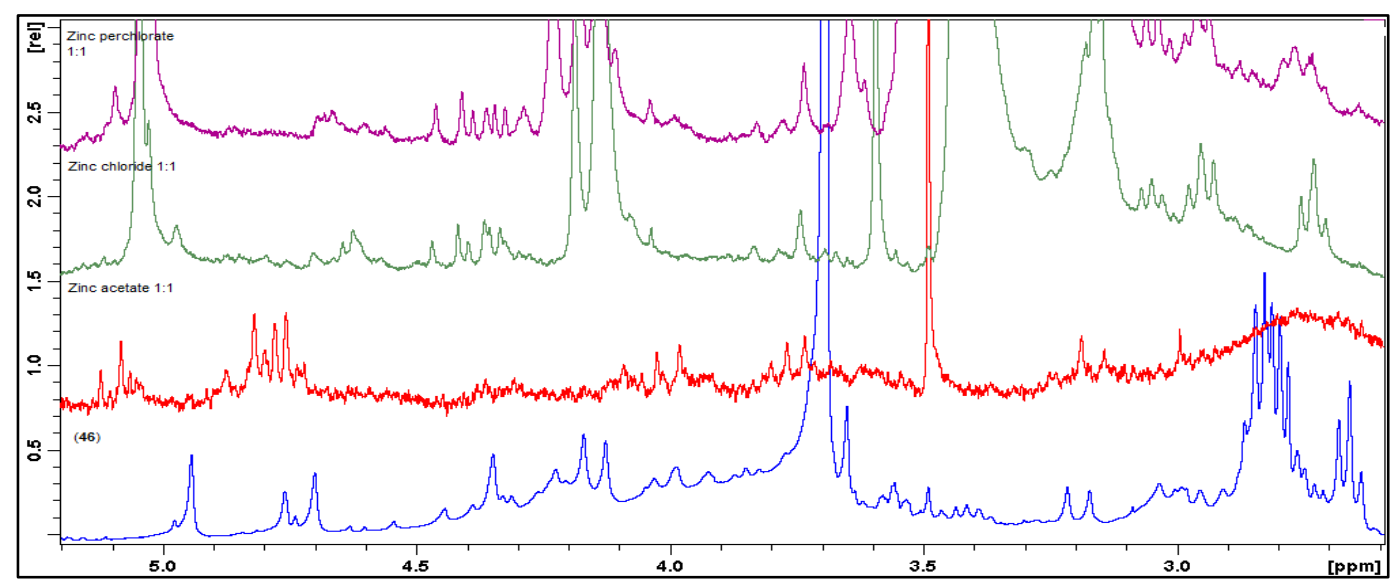

Figure 187: ${ }^{1} \mathrm{H}$ NMR of the bridging region of (46) with 1:1 Zinc(II) metal ion complexation. Red trace $=$ Zinc(II) acetate, Green trace $=$ Zinc(II) chloride, purple trace $=$ Zinc(II) perchlorate.

The 1:2 metal ion complexation reactions were also examined (Figure 188). The acetate spectrum showed shifts for the lower rim methylpyridyl protons from 8.40 ppm to $8.67 \mathrm{ppm}$ were it appeared as a broad doublet. The upper rim methylpyridyl and ethylpyridyl protons were shifted to $8.56 \mathrm{ppm}$, were it continued to represent both of the upper rim pyridyl group protons. The chloride spectrum showed a shift for the lower rim methylpyridyl protons from $8.40 \mathrm{ppm}$ to $8.64 \mathrm{ppm}$ and the upper 
rim pyridyl protons shifted to $8.51 \mathrm{ppm}$. The perchlorate spectrum showed a shift for the lower rim methylpyridyl protons from $8.40 \mathrm{ppm}$ to $8.70 \mathrm{ppm}$ and the upper rim pyridyl protons shifted to $8.51 \mathrm{ppm}$. All three displayed peaks to indicate the unbound pyridyl's in the self-assembled structure. This data suggests that the lower rim is still favoured even when excess metal is available. However as large shifts were seen (from $8.28 \mathrm{ppm}$ up to $8.56 \mathrm{ppm}$ ) this indicated that some metal ion complexation does occur at the upper rim. From the data of the 1:1 and 1:2 ${ }^{1} \mathrm{H}$ NMR spectra it is likely that there are two chains present, one with the upper rim binding the metal ion and the other with the lower rim binding the metal ion.

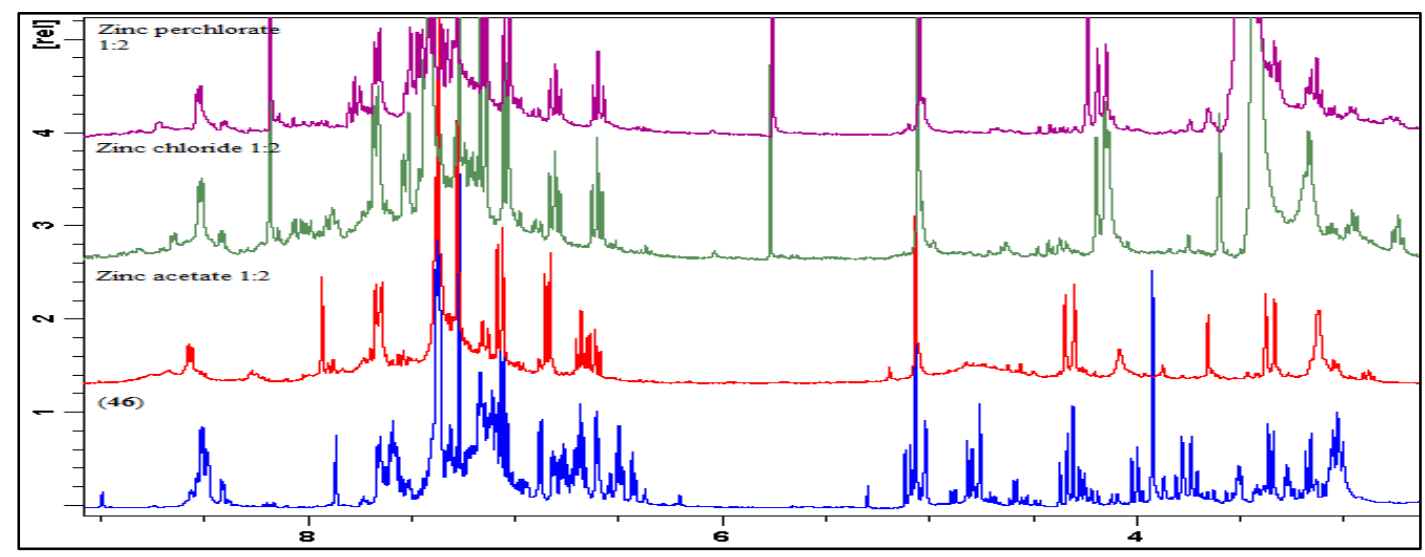

Figure 188: ${ }^{1} \mathrm{H}$ NMR for 1:2 Metal ion complexation reactions of (46). Red trace $=$ Zinc(II) acetate, Green trace $=$ Zinc(II) chloride, purple trace $=$ Zinc(II) perchlorate .

The bridging region of the spectrum, (Figure 189), continued to show additional albeit lesser intense peaks indicating that self-assembly was diminishing. Some shifts were also seen. The lower rim methylpyridyl methylene protons were shifted from $4.94 \mathrm{ppm}$ in the parent spectrum to $5.05 \mathrm{ppm}$ in all three of the metal ion complexed spectra. The axial bridging methylene protons shifted from $4.14 \mathrm{ppm}$ in the parent spectrum to $4.31,4.13$ and $4.13 \mathrm{ppm}$ in the acetate, chloride and perchlorate spectra respectively. The upper rim methylpyridyl methylene protons shifted from $3.67 \mathrm{ppm}$ to $3.63,3.59$ and $3.65 \mathrm{ppm}$ in the acetate, chloride and perchlorate spectra respectively. The upper rim ethylpyridyl methylene protons shifted from $2.82 \mathrm{ppm}$ to 2.98, 2.94 and $2.94 \mathrm{ppm}$ and the ethylpyridyl ethylene protons shifted to 2.86, 2.72 and $2.76 \mathrm{ppm}$ in the acetate, chloride and perchlorate spectra respectively. 


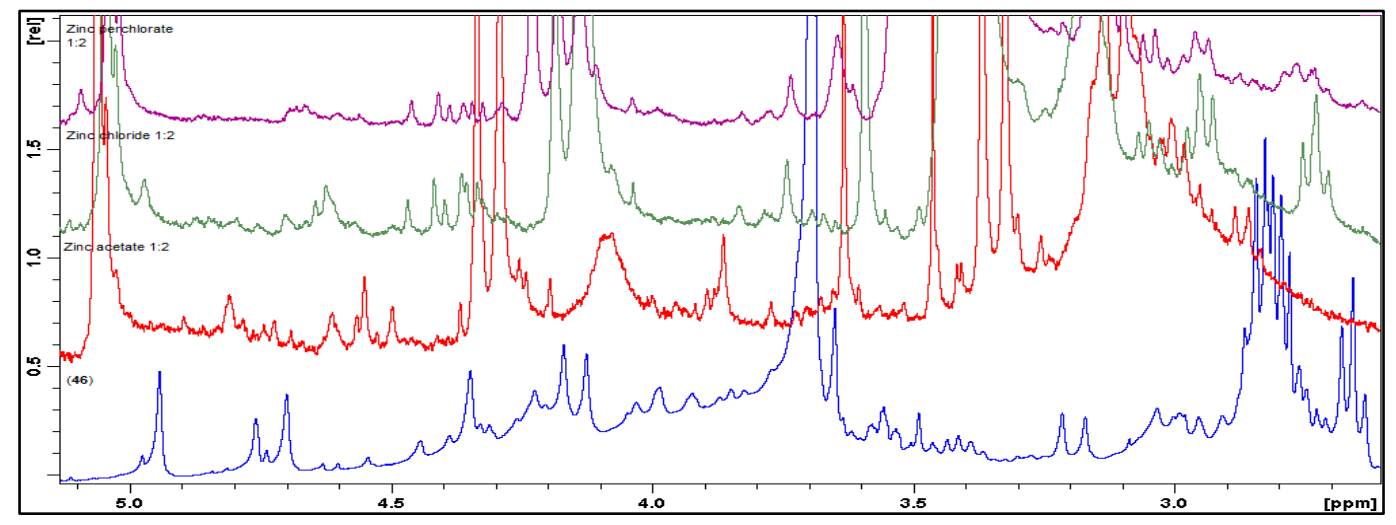

Figure 189: ${ }^{1} \mathrm{H}$ NMR of the bridging region of (46) with 1:2 Zinc(II) metal ion complexation. Red trace $=$ Zinc(II) acetate, Green trace $=$ Zinc(II) chloride, purple trace $=$ Zinc(II) perchlorate.

The IR data for the 1:1 metal ion complexation reactions corresponded well with the ${ }^{1}$ H NMR spectra (Table 15). The lower rim hydroxyl groups only experience small shifts indicating that they were not involved in the complexation of the metal ion. The small shifts can probably be attributed to the self-assembled structure. The pyridyl $\mathrm{NCH}$ bands experience a downfield shift as a result of metal ion complexation, as well as another band present for unbound pyridyl groups.

\begin{tabular}{|l|c|c|c|}
\hline & $\mathrm{O}-\mathrm{H} \mathrm{cm}^{-1}$ & $\mathrm{NC}-\mathrm{H}$ (Bound) $\mathrm{cm}^{-1}$ & $\mathrm{NC}-\mathrm{H}$ (Unbound) $\mathrm{cm}^{-1}$ \\
\hline Compound (46) & 3431 & 1591 & 1569 \\
\hline 1:1 reaction & & & 1571 \\
\hline Mercury perchlorate & 3443 & 1595 & 1571 \\
\hline Mercury chloride & 3426 & 1599 & $\mathrm{~N} / \mathrm{O}$ \\
\hline Zinc acetate & 3433 & 1593 & $\mathrm{~N} / \mathrm{O}$ \\
\hline Zinc perchlorate & 3432 & 1601 & $\mathrm{~N} / \mathrm{O}$ \\
\hline Zinc chloride & 3434 & 1606 & $\mathrm{~N} / \mathrm{O}$ \\
\hline Nickel acetate & 3421 & 1591 & 1571 \\
\hline Nickel chloride & 3421 & 1607 & $\mathrm{~N} / \mathrm{O}$ \\
\hline Nickel perchlorate & 3429 & 1608 & $\mathrm{~N} / \mathrm{O}$ \\
\hline Copper chloride & 3430 & 1609 & $\mathrm{~N} / \mathrm{O}$ \\
\hline Copper perchlorate & 3449 & 1607 & $\mathrm{~N} / \mathrm{O}$ \\
\hline Copper acetate & 3431 & 1592 & $\mathrm{O}$ \\
\hline
\end{tabular}

Table 15: IR data for 1:1 metal ion complexation reactions with (46). N/O = not observed, those in green experience shifts compared with parent spectrum in red.

The 1:2 metal ion complexation reactions were also investigated with IR spectroscopy (Table 16). That data correlated well with the 1:2 ${ }^{1} \mathrm{H}$ NMR data as well 
as the previous IR data. Small shifts were seen in the hydroxyls due to self-assembly. Large downfield shifts were seen in the pyridyl $\mathrm{NCH}$ bands indicating that metal ion complexation had occurred between the pyridyl groups.

\begin{tabular}{|l|c|c|c|}
\hline 1:2 reaction & $\mathrm{O}-\mathrm{H} \mathrm{cm}^{-1}$ & $\mathrm{NC}-\mathrm{H}$ (Bound) $\mathrm{cm}^{-1}$ & $\mathrm{NC}-\mathrm{H}$ (Unbound) $\mathrm{cm}^{-1}$ \\
\hline Compound (46) & 3431 & 1591 & 1569 \\
\hline Mercury perchlorate & 3449 & 1611 & $\mathrm{~N} / \mathrm{O}$ \\
\hline Mercury chloride & 3435 & 1598 & 1570 \\
\hline Copper chloride & 3436 & 1608 & 1569 \\
\hline Copper acetate & 3431 & 1609 & $\mathrm{~N} / \mathrm{O}$ \\
\hline Copper perchlorate & 3444 & 1609 & $\mathrm{~N} / \mathrm{O}$ \\
\hline Zinc acetate & 3437 & 1607 & 1571 \\
\hline Zinc perchlorate & 3428 & 1611 & 1571 \\
\hline Zinc chloride & 3430 & 1608 & $\mathrm{~N} / \mathrm{O}$ \\
\hline Nickel acetate & 3426 & 1570 & $\mathrm{~N} / \mathrm{O}$ \\
\hline Nickel chloride & 3424 & 1630 & 1572 \\
\hline Nickel perchlorate & 3430 & 1609 & 1569 \\
\hline Cobalt chloride & 3412 & 1606 & $\mathrm{~N} / \mathrm{O}$ \\
\hline
\end{tabular}

Table 16: IR data for 1:2 metal ion complexation reactions with (46). N/O = not observed, those in green experience shifts compared with parent spectrum in red.

Compound (47) was next to be examined, Figure 190. The parent compound consisted of the DPA unit on the upper rim and pyridyl and diester groups on the lower rim. 


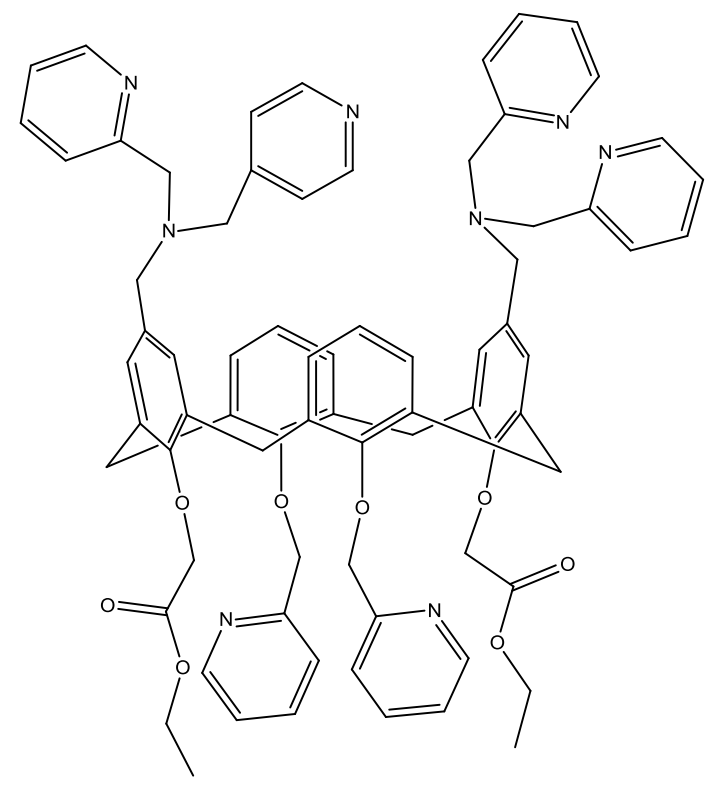

Figure 190: Compound (47)

The upper rim methylpyridyl protons were found at $8.42 \mathrm{ppm}$ and the lower rim methylpyridyl protons were found as a broad doublet at $8.47 \mathrm{ppm}$ (Figure 191). The acetate spectrum showed a shift from $8.42 \mathrm{ppm}$ to $8.58 \mathrm{ppm}$ (difference of $0.16 \mathrm{ppm}$ ) for the upper rim methylpyridyl protons, and a shift from 8.47 to $8.62 \mathrm{ppm}$ (difference of $0.15 \mathrm{ppm}$ ) for the lower rim methylpyridyl protons. The chloride spectrum showed a shift for the lower rim methylpyridyl protons from $8.47 \mathrm{ppm}$ to $8.62 \mathrm{ppm}$ (difference of $0.15 \mathrm{ppm}$ ) and a shift for the upper rim methylpyridyl protons from 8.42 to $8.58 \mathrm{ppm}$ (difference of $0.16 \mathrm{ppm}$ ). The perchlorate spectrum showed a shift for the lower rim methylpyridyl protons from $8.47 \mathrm{ppm}$ to $8.63 \mathrm{ppm}$ (difference of $0.16 \mathrm{ppm}$ ) and a shift for the upper rim methylpyridyl protons from $8.42 \mathrm{ppm}$ to $8.56 \mathrm{ppm}$ (difference of $0.14 \mathrm{ppm}$ ). As these shifts are almost identical in each case, it can be said that both the upper and lower rim groups are equally involved in the binding of the metal ion in either of the two methods mentioned previously. 


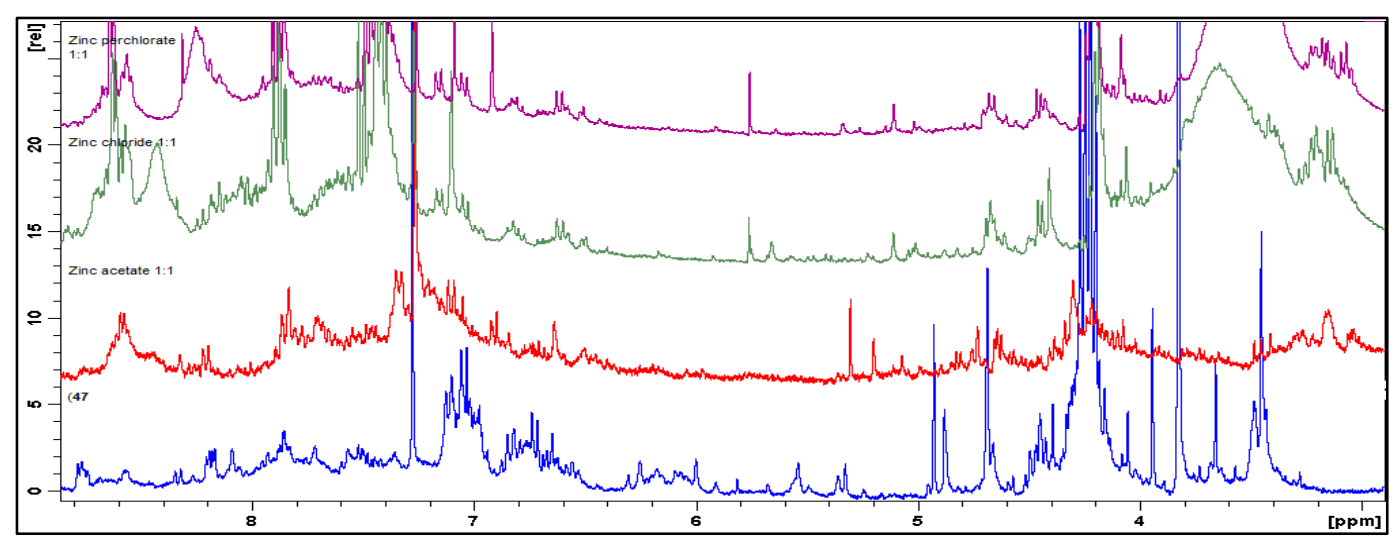

Figure 191: 1:1 metal ion complexation ${ }^{1} \mathrm{H}$ NMR reactions for (47).

The bridging region, (Figure 192) also showed a few shifts in some of the peaks. Unfortunately the bridging methylene protons were obscured under the solvent peak. The lower rim methylpyridyl protons were shifted from $4.93 \mathrm{ppm}$ to 5.07, 5.02, and $5.02 \mathrm{ppm}$ in the acetate, chloride and perchlorate spectra respectively. The upper rim methylamine methylene protons were shifted from $4.69 \mathrm{ppm}$ to $4.73,4.69$ and 4.68 ppm in the acetate, chloride and perchlorate spectra respectively. The final observed shifts were seen in the upper rim methylpyridyl methylene protons from $3.94 \mathrm{ppm}$ to 4.07, 4.06 and $4.08 \mathrm{ppm}$ in the acetate, chloride and perchlorate spectra. While the abundance of peaks has decreased they have not been eliminated altogether indicating that self-assembly is still occurring.

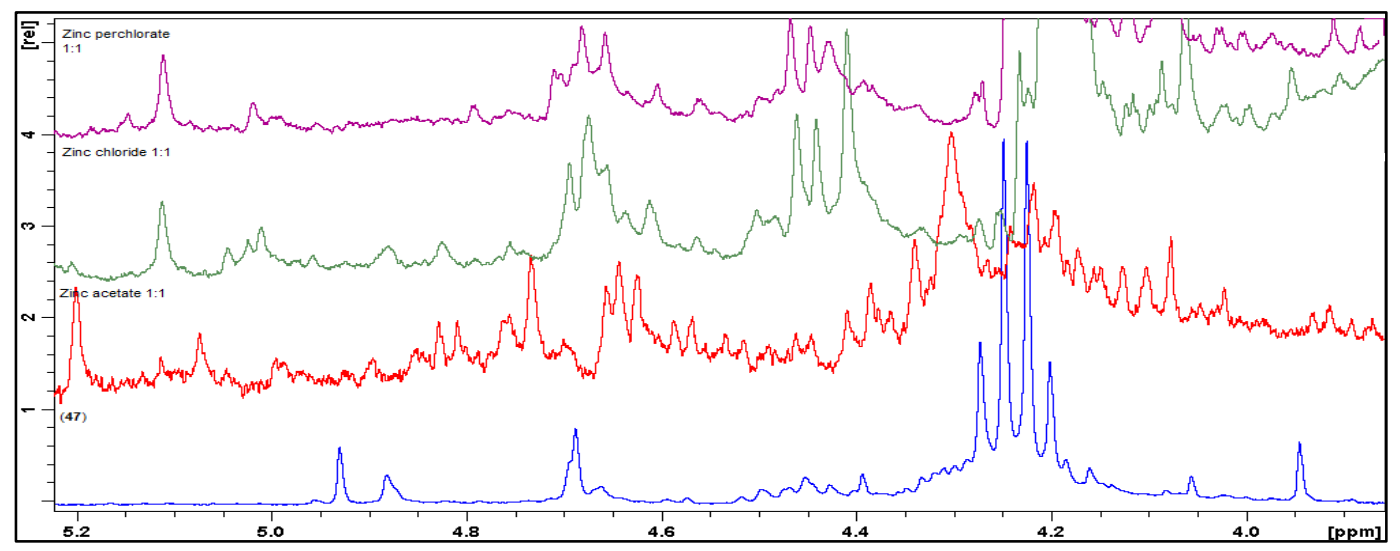

Figure 192: ${ }^{1} \mathrm{H}$ NMR of the bridging region of (47) with 1:1 Zinc(II) metal ion complexation. Red trace $=$ Zinc(II) acetate, Green trace $=$ Zinc(II) chloride, purple trace $=$ Zinc(II) perchlorate. 
The 1:2 metal ion complexation reactions for compound (47) were also investigated with ${ }^{1}$ H NMR spectroscopy (Figure 193). The self-assembly peaks are still clearly dominant in the spectrum. The parent compound showed a doublet for the upper rim methylpyridyl protons at $8.42 \mathrm{ppm}$ and a broad doublet for the lower rim methylpyridyl protons at $8.48 \mathrm{ppm}$. The acetate spectrum showed a shift for the lower rim methylpyridyl protons from $8.48 \mathrm{ppm}$ to $8.62 \mathrm{ppm}$ and a shift from 8.42 ppm to $8.58 \mathrm{ppm}$ for the upper rim methylpyridyl protons. The chloride spectrum shows a shift for the lower rim methylpyridyl protons from $8.48 \mathrm{ppm}$ to $8.76 \mathrm{ppm}$ and a shift from $8.42 \mathrm{ppm}$ to $8.69 \mathrm{ppm}$ for the upper rim methylpyridyl protons, indicating that both rims are involved in metal ion complexation. The perchlorate spectrum showed shifts from $8.48 \mathrm{ppm}$ to $8.79 \mathrm{ppm}$ for the lower rim methylpyridyl protons and from $8.42 \mathrm{ppm}$ to $8.73 \mathrm{ppm}$ for the upper rim methylpyridyl protons. This data indicated that both rims are involved in metal ion complexation. The most likely scenario is that are two different chains, one where the upper rim is binding the metal ion, and the other has the lower rim binding the metal ion, both with selfassembly occurring further down the chain. Other doublet peaks were also seen higher up than those mentioned and these possibly indicate metal ion complexation with different conformers.

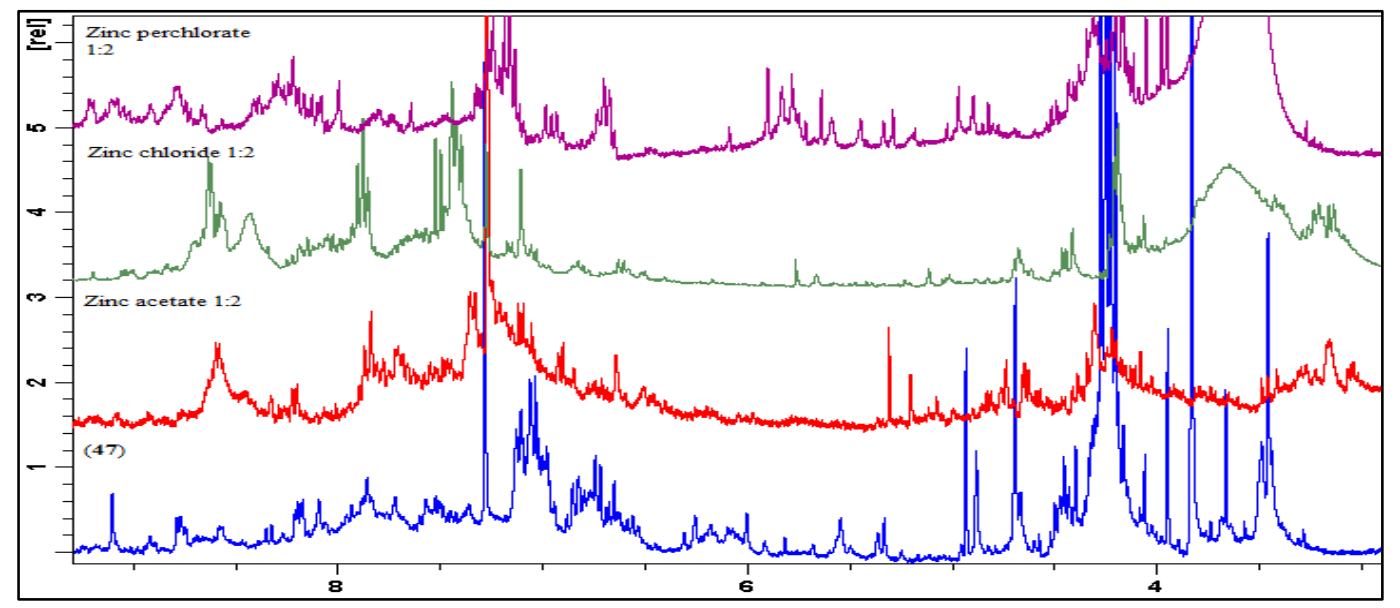

Figure 193: 1:2 metal ion complexation ${ }^{1} \mathrm{H}$ NMR reactions for $(47)$. Red trace $=$ Zinc(II) acetate, Green trace $=$ Zinc(II) chloride, purple trace $=$ Zinc(II) perchlorate .

The bridging region of the 1:2 metal ion complexed ${ }^{1} \mathrm{H}$ NMR spectrum, (Figure 194), was very similar to the 1:1 metal ion complexed spectrum. The equatorial 
bridging methylene protons were once again hidden beneath the solvent peak. The lower rim methylpyridyl methylene protons shifted from $4.93 \mathrm{ppm}$ to $4.96 \mathrm{ppm}$ in the chloride and perchlorate spectra, the signal was not visible in the acetate spectrum indicating that the pyridyl group may have been lost. This could indicate that what was initially thought to be lower rim complexation in this spectrum was actually a separation of the upper rim pyridyl protons into two distinct signals. The upper rim methylamine methylene protons shifted from $4.69 \mathrm{ppm}$ to $4.73,4.80$ and $4.81 \mathrm{ppm}$ in the acetate, chloride and perchlorate spectra respectively. The upper rim methylpyridyl methylene protons shifted from $3.94 \mathrm{ppm}$ to $4.07,4.05$ and $4.05 \mathrm{ppm}$ in all three of the spectra. Again a decrease in the number of peaks is seen indicating that metal complexation is providing some order to the observed self-assembly.

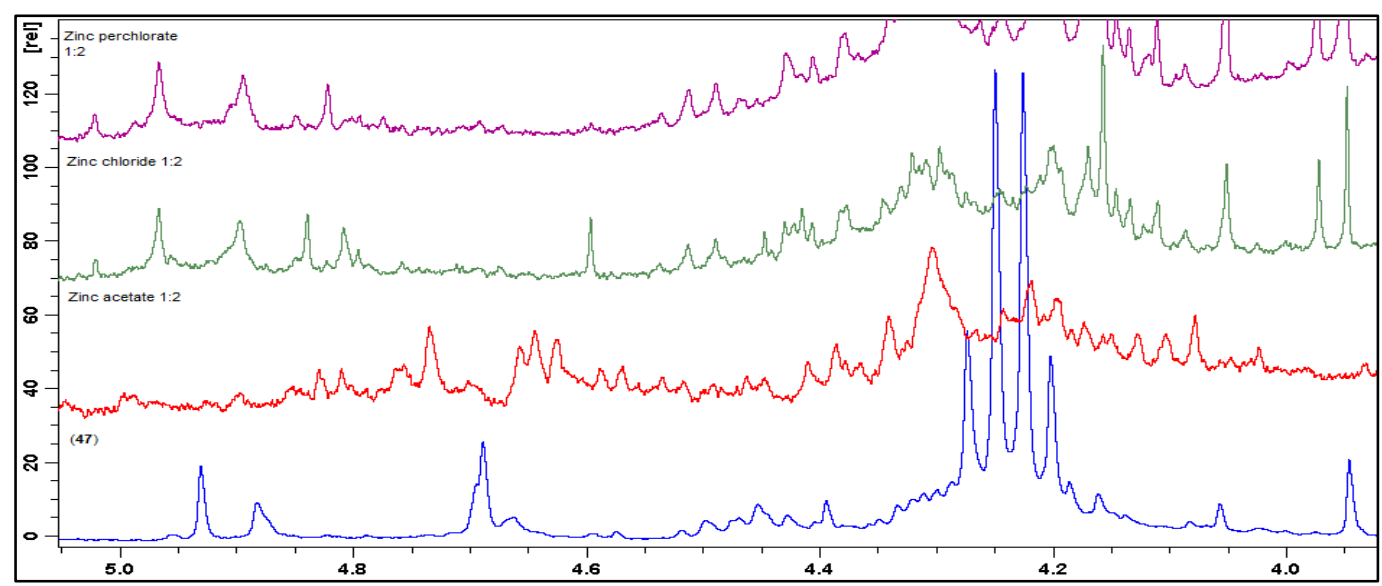

Figure 194: ${ }^{1} \mathrm{H}$ NMR of the bridging region of (47) with 1:2 Zinc(II) metal ion complexation. Red trace $=$ Zinc(II) acetate, Green trace $=$ Zinc(II) chloride, purple trace $=$ Zinc(II) perchlorate

The IR data was consistent with the ${ }^{1} \mathrm{H}$ NMR spectra (Table 17). In the 1:1 metal ion complexation reactions only small shifts were seen in the lower rim carbonyl groups which could be accounted for by the self-assembly. The NC-H bands were seen to experience downfield shifts due to the metal ion complexation. 


\begin{tabular}{|l|c|c|}
\hline & $\mathrm{C}=\mathrm{O} \mathrm{cm}^{-1}$ & $\mathrm{NC}-\mathrm{H} \mathrm{cm}^{-1}$ \\
\hline Compound (47) & 1748 & 1591 \\
\hline 1:1 Reaction & & \\
\hline Zinc acetate & 1749 & 1626 \\
\hline Zinc perchlorate & 1750 & 1615 \\
\hline Zinc chloride & 1747 & 1626 \\
\hline Cobalt chloride & 1747 & 1600 \\
\hline Copper acetate & 1750 & 1602 \\
\hline Copper perchlorate & 1746 & 1628 \\
\hline Copper chloride & 1746 & 1627 \\
\hline Mercury perchlorate & 1742 & 1626 \\
\hline Mercury chloride & 1743 & 1627 \\
\hline
\end{tabular}

Table 17: IR data for 1:1 metal ion complexation reactions with (47). N/O = not observed, those in green experience shifts compared with parent spectrum in red.

The 1:2 metal ion complexation reactions were also investigated under IR spectroscopy (Table 18). The data obtained corresponds well with the ${ }^{1} \mathrm{H}$ NMR spectra as well as what was previously observed for the 1:1 metal ion complexation IR data. Small shifts were seen in the carbonyl bands due to self-assembly and larger shifts were seen in the pyridyl NCH bands due to complexation of the metal ion.

\begin{tabular}{|l|c|c|}
\hline 1:2 reaction & $\mathrm{C}=\mathrm{O} \mathrm{cm}^{-1}$ & $\mathrm{NC}-\mathrm{H} \mathrm{cm}^{-1}$ \\
\hline Zinc acetate & 1752 & 1592 \\
\hline Zinc perchlorate & 1749 & 1615 \\
\hline Zinc chloride & 1747 & 1626 \\
\hline Cobalt chloride & $\mathrm{N} / \mathrm{O}$ & 1601 \\
\hline Copper acetate & 1751 & 1618 \\
\hline Copper perchlorate & 1746 & 1628 \\
\hline Copper chloride & 1747 & 1626 \\
\hline Mercury perchlorate & $\mathrm{N} / \mathrm{O}$ & 1618 \\
\hline Mercury chloride & 1744 & 1614 \\
\hline
\end{tabular}

Table 18: IR data for 1:2 metal ion complexation reactions with (47). N/O = not observed, those in green experience shifts compared with parent spectrum in red.

Compound (49) was also examined by ${ }^{1} \mathrm{H}$ NMR and IR spectroscopy to examine its binding ability, (Figure 195). Compound (49) contained a pyridyl group on the upper rim, and had the benzyl and ester groups on the lower rim. 


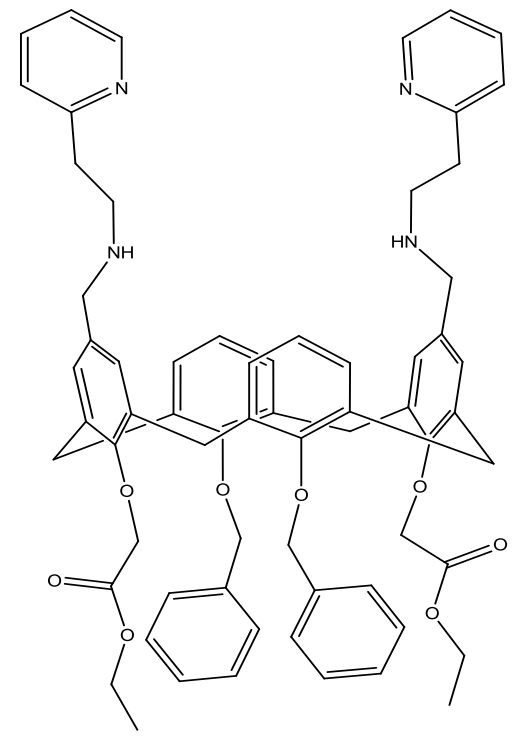

Figure 195: Compound (49)

The upper rim ethylpyridyl protons appeared as a single broad doublet at $8.47 \mathrm{ppm}$ (Figure 196). The 1:1 metal ion complexation reactions were examined first. The acetate spectrum showed that a second broad doublet is beginning to emerge, possibly indicating a different environment. The acetate spectrum shows the two pyridyl doublet at $8.59 \mathrm{ppm}$ and at $8.54 \mathrm{ppm}$. The doublet at $8.59 \mathrm{ppm}$ most likely represents the metal complexed pyridyl. The doublet at $8.54 \mathrm{ppm}$ may indicate a new self-assembled structure, were the parent may have been in the homo form and now with the compound is in the hetero form. The chloride spectrum shows a broad peak between $8.53-8.58 \mathrm{ppm}$. The perchlorate spectrum also shows two doublets at 8.58 ppm and at $8.52 \mathrm{ppm}$, following the same trend as the acetate spectrum. All three metal ion complexation spectra showed a doublet representing the unbound pyridyl protons, around $8.46 \mathrm{ppm}$. 


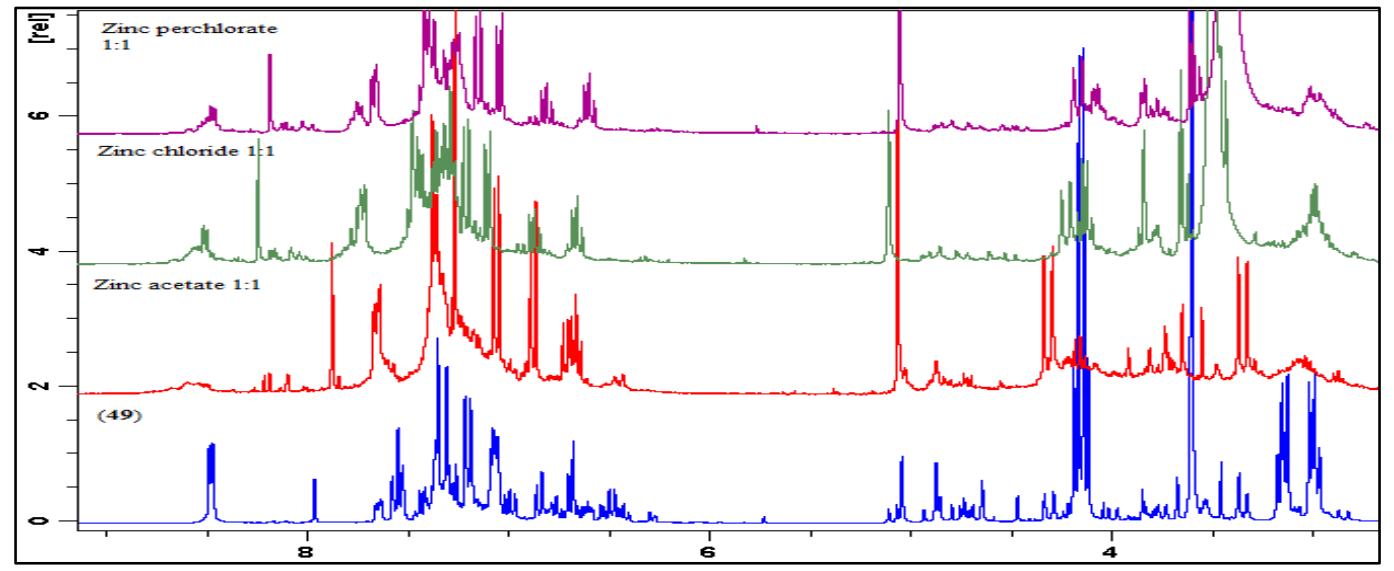

Figure 196: ${ }^{1} \mathrm{H}$ NMR spectra for $1: 1$ metal ion complexation reactions with (49). Red trace $=$ Zinc(II) acetate, Green trace $=$ Zinc(II) chloride, purple trace $=$ Zinc(II) perchlorate

Shifts were also seen in the bridging region of the spectra, (Figure 197). The lower rim methylbenzyl methylene protons shifted from 5.04 ppm to 5.06, 5.10 and 5.06 ppm in the acetate chloride and perchlorate spectra respectively. The axial bridging protons shifted from $4.30 \mathrm{ppm}$ in the parent spectrum to 4.21 and $4.15 \mathrm{ppm}$ in the chloride and perchlorate spectra respectively. No change was seen in the acetate spectrum. Shifts are also seen in the lower rim methylester methylene protons. In the parent compound they appeared at $3.60 \mathrm{ppm}$ and it shifted to $3.73-3.83 \mathrm{ppm}$ upon metal ion complexation. This indicates that the ester groups on the lower are also involved in metal ion complexation. It is possible that the metal is sitting between two calix[4]arene molecules were it is being bound on one side by the upper rim pyridyl's and being bound on the other by the lower rim ester groups. 


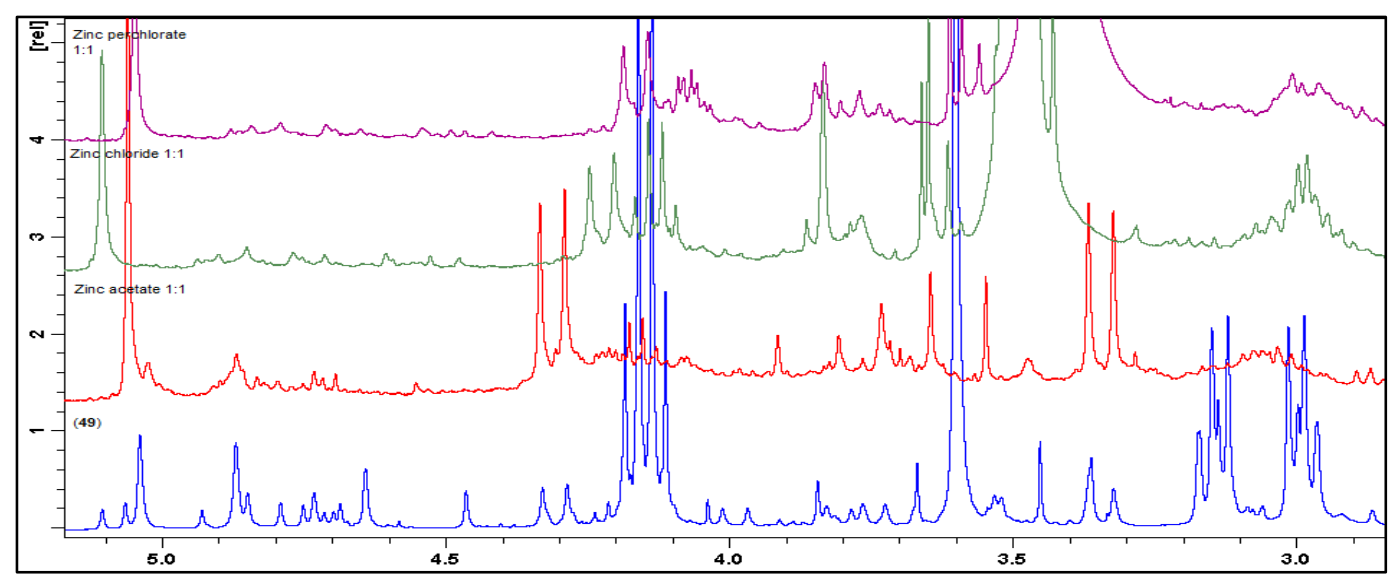

Figure 197: ${ }^{1} \mathrm{H}$ NMR of the bridging region of (49) with $1: 1 \mathrm{Zinc}(\mathrm{II})$ metal ion complexation. Red trace $=$ Zinc $($ II) acetate, Green trace $=$ Zinc(II) chloride, purple trace $=$ Zinc(II) perchlorate.

The IR data for the 1:1 metal ion complexation reactions was seen to correspond well with the ${ }^{1} \mathrm{H}$ NMR data (Table 19). Large downfield shifts are seen in the carbonyl bands indicating that the carbonyls are involved in the metal ion complexation. Downfield shifts were also seen for the upper rim pyridyls indicating their involvement in metal ion complexation.

\begin{tabular}{|l|c|c|}
\hline & $\mathrm{C}=\mathrm{O} \mathrm{cm}^{-1}$ & $\mathrm{NC}-\mathrm{H}$ (Bound) $\mathrm{cm}^{-1}$ \\
\hline Compound (49) & 1656 & 1593 \\
\hline $1: 1$ reaction & & \\
\hline Zinc perchlorate & 1755 & 1606 \\
\hline Zinc chloride & 1755 & 1607 \\
\hline Zinc acetate & 1756 & 1586 \\
\hline
\end{tabular}

Table 19: IR data for 1:1 metal ion complexation reactions with (49). N/O = not observed, those in green experience shifts compared with parent spectrum in red.

Compound (50), (Figure 198), was next to be investigated with ${ }^{1} \mathrm{H}$ NMR and IR spectroscopy. 


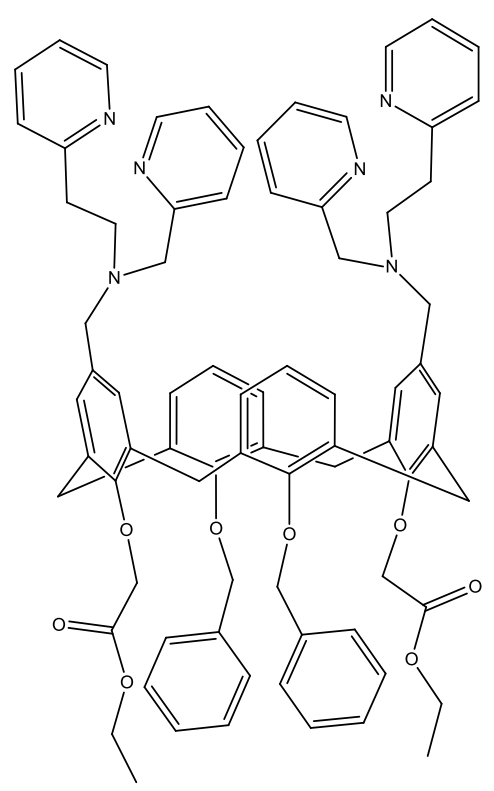

Figure 198: Compound (50)

The upper rim methylpyridyl protons were found at $8.57 \mathrm{ppm}$ and the upper rim ethylpyridyl protons were found at $8.48 \mathrm{ppm}$ (Figure 199). The acetate spectrum showed a shift for the methylpyridyl protons from $8.57 \mathrm{ppm}$ to $8.86 \mathrm{ppm}$ and for the ethylpyridyl protons from $8.48 \mathrm{ppm}$ to $8.54 \mathrm{ppm}$. The chloride spectrum showed a shift from $8.57 \mathrm{ppm}$ to $8.94 \mathrm{ppm}$ for the methylpyridyl protons and from $8.48 \mathrm{ppm}$ to $8.62 \mathrm{ppm}$ for the ethylpyridyl protons. The perchlorate showed shifts from $8.57 \mathrm{ppm}$ to $9.05 \mathrm{ppm}$ for the methylpyridyl protons and from $8.48 \mathrm{ppm}$ to $8.78 \mathrm{ppm}$ for the ethylpyridyl protons. Out of these three the perchlorate produced the largest shift. This may be due to the strength of the perchlorate counter-anion as mentioned previously by McKee et al. ${ }^{84}$ 


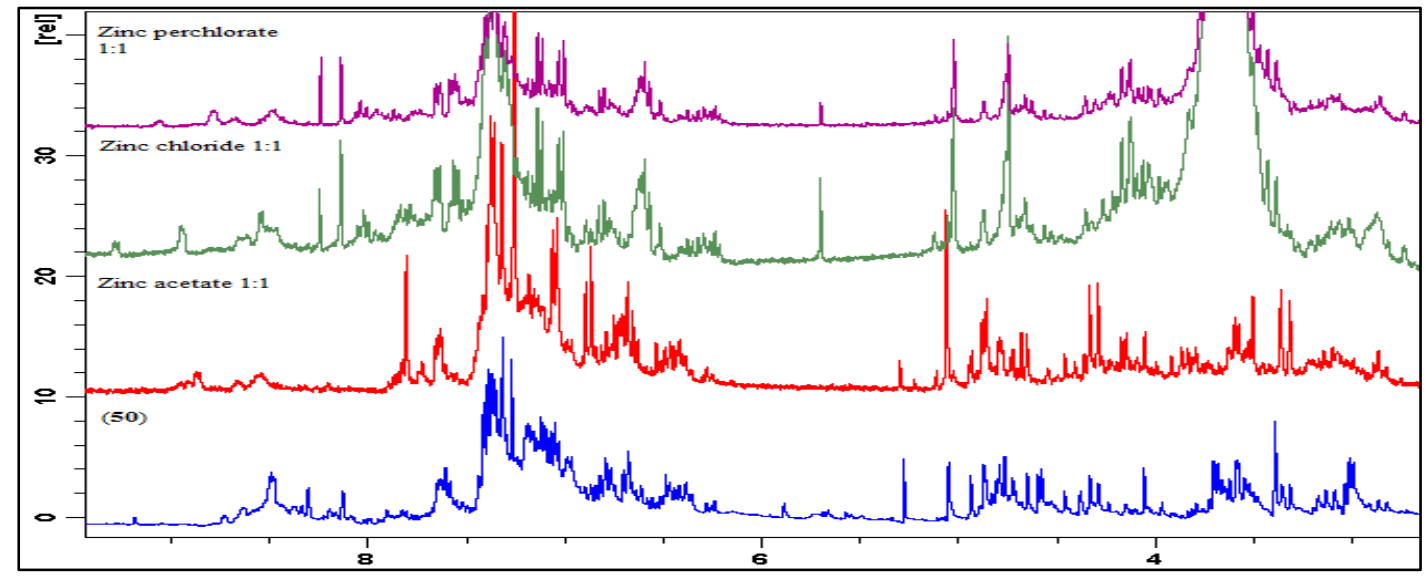

Figure 199: ${ }^{1} \mathrm{H}$ NMR of 1:1 metal ion complexation reactions of $(\mathbf{5 0})$. Red trace $=$ Zinc(II) acetate, Green trace $=$ Zinc(II) chloride, purple trace $=$ Zinc(II) perchlorate .

The bridging region of the spectrum also showed some chemical shifts (Figure 200). The lower rim methylbenzyl methylene protons shifted from $5.03 \mathrm{ppm}$ in the parent spectrum to 5.06, 5.02 and $5.02 \mathrm{ppm}$ in the acetate, chloride and perchlorate spectra respectively. The axial bridging methylene protons occurred at $4.31 \mathrm{ppm}$ in the parent spectrum and shifted to $4.15 \mathrm{ppm}$ in the chloride and perchlorate spectra. No change was seen in the acetate spectrum. The final shifts were seen with the equatorial bridging methylene protons which shifted from $3.33 \mathrm{ppm}$ to $3.40 \mathrm{ppm}$ in the chloride and perchlorate spectra. Again no change was seen in the acetate spectrum. Additional peaks were also seen indicating that self-assembly was still occurring.

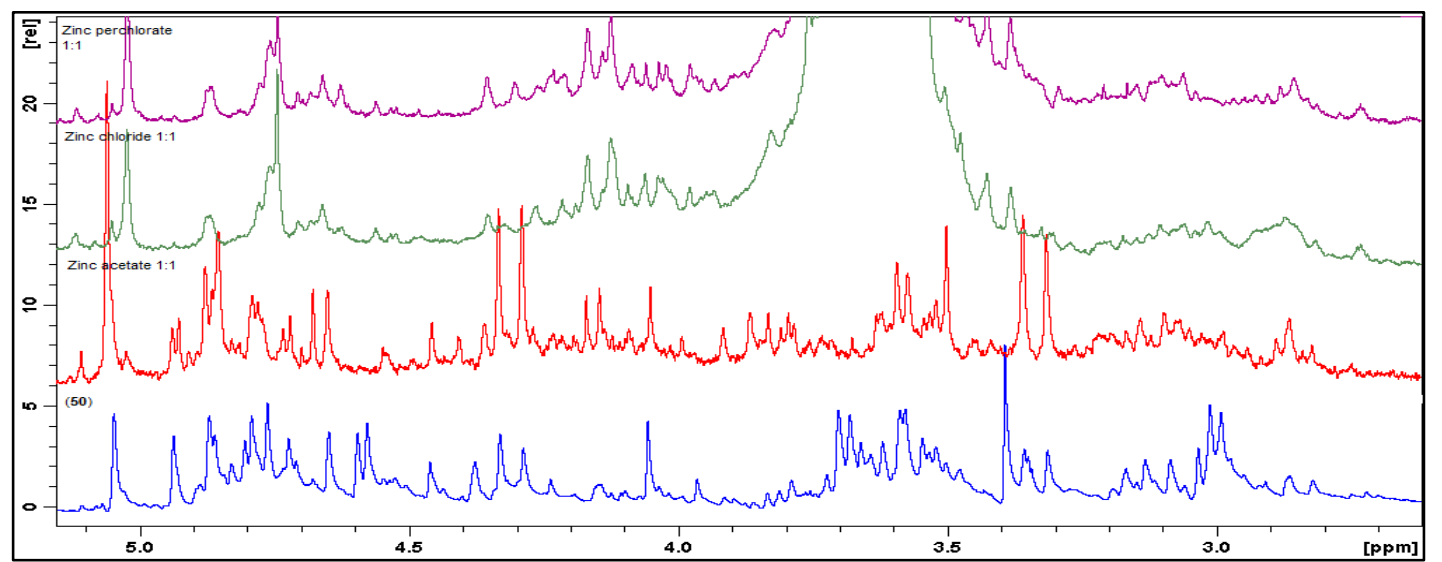

Figure 200: ${ }^{1} \mathrm{H}$ NMR of the bridging region of (50) with 1:1 Zinc(II) metal ion complexation. Red trace $=$ Zinc(II) acetate, Green trace $=$ Zinc(II) chloride, purple trace $=$ Zinc(II) perchlorate. 
The 1:2 metal ion complexation reactions were also investigated. The parent compound peaks were seen at $8.57 \mathrm{ppm}$ for the short arm and $8.48 \mathrm{ppm}$ for the long arm (Figure 201). The acetate spectrum exhibited shifts from $8.57 \mathrm{ppm}$ to $8.83 \mathrm{ppm}$ for the short arm and from $8.48 \mathrm{ppm}$ to $8.65 \mathrm{ppm}$ for the long arm. This latter peak became very broad. The chloride spectrum showed shifts from $8.57 \mathrm{ppm}$ to $9.23 \mathrm{ppm}$ for the short arm and from $8.48 \mathrm{ppm}$ to $8.93 \mathrm{ppm}$ for the long arm. The perchlorate showed shifts from $8.57 \mathrm{ppm}$ to $8.99 \mathrm{ppm}$ for the short arm and from $8.48 \mathrm{ppm}$ to $8.75 \mathrm{ppm}$ for the long arm doublet. In this case the chloride gave the largest shift. All had broad doublet peaks to indicate the unbound pyridyl $\mathrm{NCH}$ doublets in the selfassembled form.

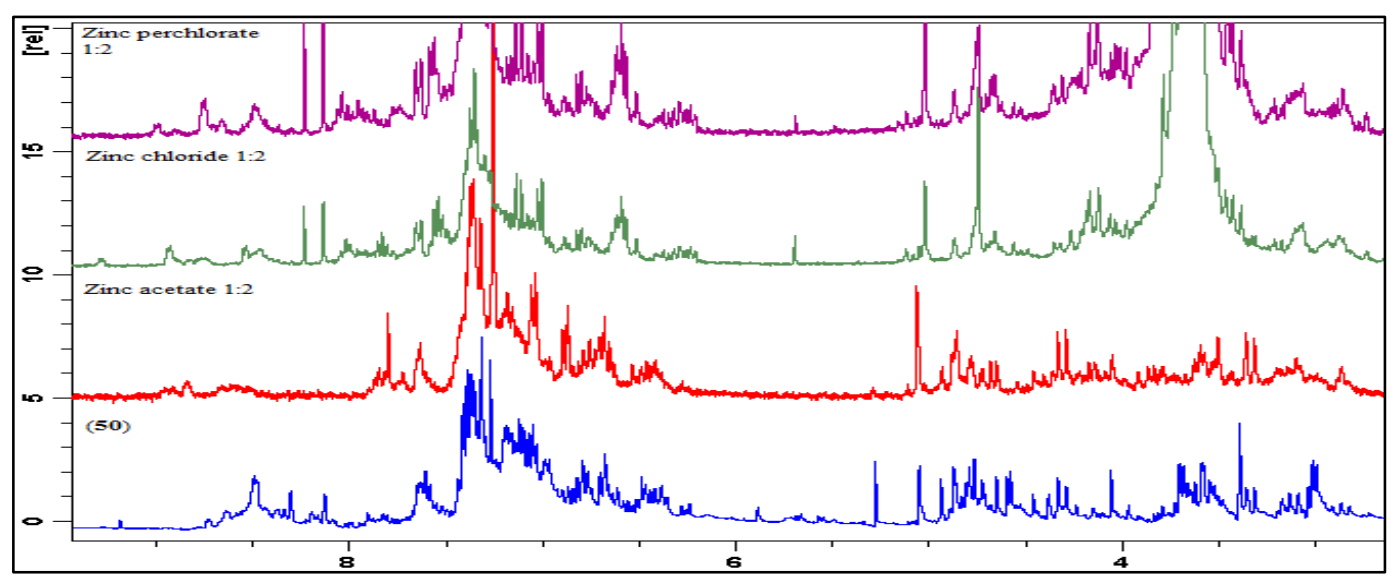

Figure 201: ${ }^{1} \mathrm{H}$ NMR of 1:2 metal ion complexation reactions of (50). Red trace $=$ Zinc(II) acetate, Green trace $=$ Zinc(II) chloride, purple trace $=$ Zinc(II) perchlorate .

The bridging region of the 1:2 metal ion complexation ${ }^{1} \mathrm{H}$ NMR spectra, (Figure 202), were very similar to the $1: 1$ metal ion complexation ${ }^{1} \mathrm{H}$ NMR spectra. Only a few shifts could be clearly discerned. The lower rim methylbenzyl methylene protons shifted from 5.04 ppm to 5.06, 5.02 and $5.02 \mathrm{ppm}$ in the acetate, chloride and perchlorate spectra respectively. The axial bridging methylene protons also shifted from $4.31 \mathrm{ppm}$ to 4.14 in the chloride and perchlorate spectra. No change was seen in the acetate spectrum. Unfortunately the bridging methylene protons were obscured by solvent peaks but some shift may have occurred. Once again the selfassembly has decreased but can still be seen. 


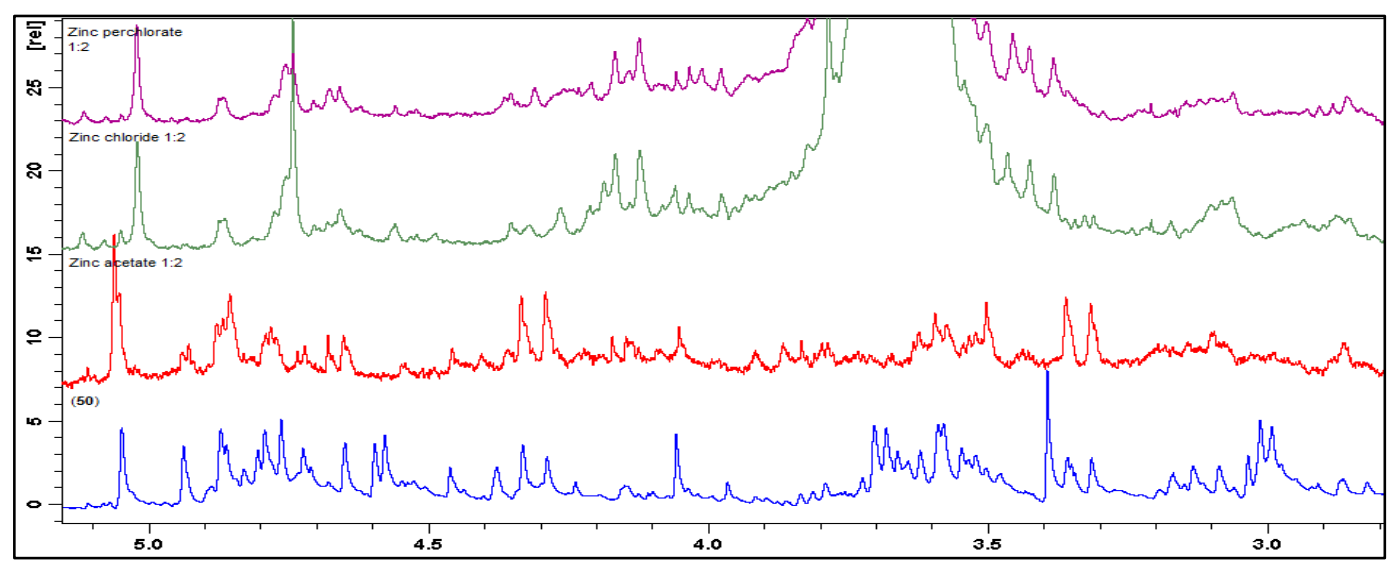

Figure 202: ${ }^{1} \mathrm{H}$ NMR of the bridging region of (50) with 1:2 Zinc(II) metal ion complexation. Red trace $=$ Zinc(II) acetate, Green trace $=$ Zinc(II) chloride, purple trace $=$ Zinc(II) perchlorate.

The IR data showed good shifts in all of the 1:1 metal ion complexation reactions with the NCH band being quite broad to encompass both the upper and lower rim pyridyls (Table 20). The 1:2 metal ion complexation IR data also showed good binding in all most cases (Table 21). Here the counter-anion seemed to overlay the majority of the spectrum leaving detailed analysis of the IR spectrum impossible. Shifts were also seen in the carbonyl groups of both the 1:1 and 1:2 metal ion complexation reactions. These are most likely due to an indirect effect of the metal ion complexation or due to a change in the self-assembled structure as previously mentioned.

\begin{tabular}{|l|c|c|}
\hline & $\mathrm{C}=\mathrm{O} \mathrm{cm}^{-1}$ & $\mathrm{NC}-\mathrm{H} \mathrm{cm}^{-1}$ \\
\hline \multicolumn{1}{|c|}{ Compound (50) } & 1655 & 1590 \\
\hline 1:1 reaction & & \\
\hline Zinc chloride & 1730 & 1607 \\
\hline Zinc acetate & 1725 & 1608 \\
\hline Zinc perchlorate & 1727 & 1610 \\
\hline Cobalt chloride & 1729 & 1607 \\
\hline Copper chloride & 1723 & 1608 \\
\hline Copper acetate & 1727 & 1630 \\
\hline Copper perchlorate & 1726 & 1627 \\
\hline Mercury perchlorate & 1722 & 1634 \\
\hline Mercury chloride & 1729 & 1624 \\
\hline Nickel chloride & 1725 & 1631 \\
\hline Nickel perchlorate & 1721 & 1635 \\
\hline
\end{tabular}


Table 20: IR data for 1:1 metal ion complexation with (50). N/O = not observed, those in green experience shifts compared with parent spectrum in red.

\begin{tabular}{|l|c|c|}
\hline & $\mathrm{C}=\mathrm{O} \mathrm{cm}^{-1}$ & $\mathrm{NC}-\mathrm{H} \mathrm{cm}^{-1}$ \\
\hline Compound (50) & 1655 & 1590 \\
\hline 1:2 reaction & & \\
\hline Zinc chloride & 1732 & 1607 \\
\hline Zinc acetate & 1738 & 1610 \\
\hline Zinc perchlorate & 1728 & 1611 \\
\hline Cobalt chloride & 1724 & 1626 \\
\hline Copper chloride & 1729 & 1626 \\
\hline Copper acetate & 1730 & 1634 \\
\hline Copper perchlorate & 1728 & 1611 \\
\hline Mercury perchlorate & 1728 & 1607 \\
\hline Mercury chloride & 1725 & 1630 \\
\hline Nickel chloride & 1726 & 1636 \\
\hline Nickel perchlorate & 1723 & 1636 \\
\hline Nickel acetate & 1728 & 1607 \\
\hline
\end{tabular}

Table 21: IR data for 1:2 metal ion complexation with (50). N/O = not observed, those in green experience shifts compared with parent spectrum in red.

Following on from this compound (51), (Figure 203), was examined. The parent compound consisted of three pyridyl groups, two on the upper rim, a short arm and a long arm, and one on the lower rim. 


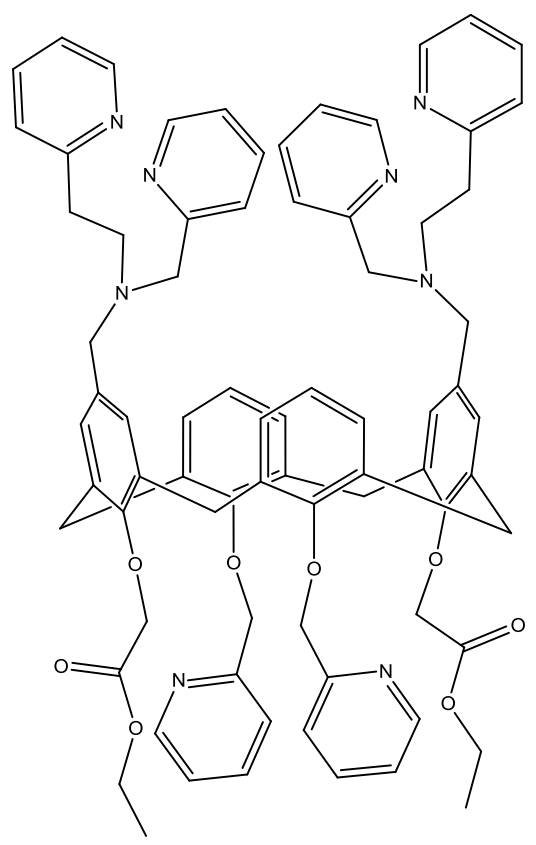

Figure 203: Compound (51)

The lower rim methylpyridyl protons occurred at $8.58 \mathrm{ppm}$, the upper rim methylpyridyl doublet at $8.51 \mathrm{ppm}$ and the ethylpyridyl doublet at $8.48 \mathrm{ppm}$. The 1:1 metal ion complexation reactions were examined first (Figure 204). As these three were almost on top of each other they appeared as broad doublets. The acetate ${ }^{1} \mathrm{H}$ NMR spectrum showed a shift or the lower rim methylpyridyl protons from 8.58 ppm to $8.63 \mathrm{ppm}$, the upper rim methylpyridyl and ethylpyridyl protons were found to be so broad and so close together that they could not be distinguished from each other. An overall broad peak was seen at $8.55 \mathrm{ppm}$ which would account for a shift in both the pyridyl arms. A similar trend was seen in the chloride and perchlorate spectra, where a clear shift was observed for the lower rim but the upper rim pyridyl's have 'merged' once again to produce a broad doublet. In the chloride spectrum the lower rim methylpyridyl protons shifted to $8.71 \mathrm{ppm}$ and the 'merged' broad doublet appears at $8.57 \mathrm{ppm}$. The perchlorate spectrum sees a shift for the lower rim methylpyridyl protons from $8.58 \mathrm{ppm}$ to $8.65 \mathrm{ppm}$ and the 'merged' pyridyl doublet occurs at $8.52 \mathrm{ppm}$. This suggests that the metal is bound between the lower rim pyridyl's as the shift seen is the strongest, and perhaps the small upper rim shift is due to the homo dimerisation form of the self-assembled structure, where the four pyridyl's are able to interact with one another to create this peak 'merging'. 


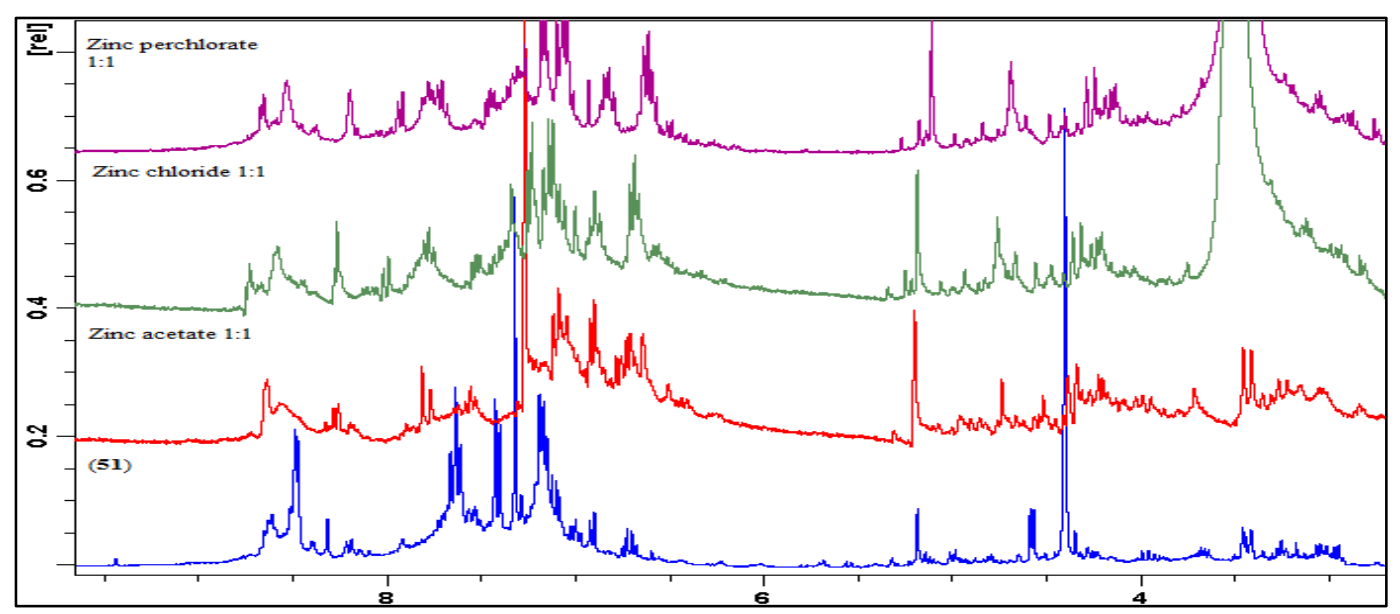

Figure 204: ${ }^{1} \mathrm{H}$ NMR of 1:1 metal ion complexation reactions of $(\mathbf{5 1})$. Red trace $=$ Zinc(II) acetate, Green trace $=$ Zinc(II) chloride, purple trace $=$ Zinc(II) perchlorate .

The bridging region, (Figure 205), also showed some differences from the parent spectrum. A broadening of the peaks was seen in the chloride and perchlorate spectra. The only clear shifts are also seen in these two spectra with the axial bridging methylene protons which have shifted from $4.30 \mathrm{ppm}$ in the parent spectrum to $4.16 \mathrm{ppm}$ in the chloride and perchlorate spectra. What can be clearly seen is that self-assembly has still a dominant role in solution.

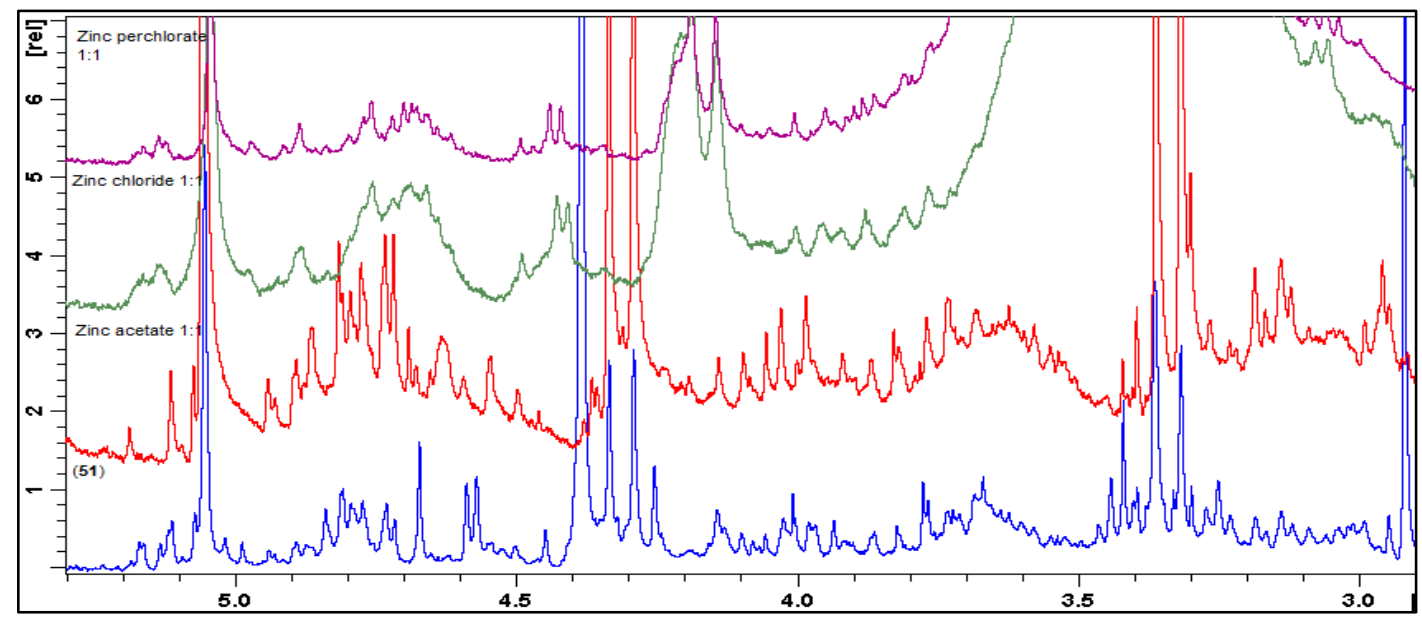

Figure 205: ${ }^{1} \mathrm{H}$ NMR of the bridging region of (51) with 1:1 Zinc(II) metal ion complexation. Red trace $=$ Zinc(II) acetate, Green trace $=$ Zinc(II) chloride, purple trace $=$ Zinc(II) perchlorate. 
The 1:2 metal ion complexation reactions of compound (51) were also investigated (Figure 206). The parent peaks were found at 8.58, 8.51 and $8.48 \mathrm{ppm}$ for the lower rim methylpyridyl protons, the upper rim methylpyridyl protons and the upper rim ethylpyridyl protons respectively. Again this merging of the upper rim pyridyl protons was observed. The acetate spectrum showed a shift for the lower rim methylpyridyl protons from $8.58 \mathrm{ppm}$ to $8.64 \mathrm{ppm}$ and the 'merged' broad doublet for the upper rim pyridyl protons occurred at $8.57 \mathrm{ppm}$. The chloride spectrum showed the lower rim methylpyridyl protons at $8.71 \mathrm{ppm}$ and the 'merged' doublet for the upper rim appeared at $8.58 \mathrm{ppm}$. The perchlorate spectrum showed the lower rim methylpyridyl protons at $8.65 \mathrm{ppm}$ and the 'merged' doublet at $8.51 \mathrm{ppm}$. For all three it seems that the metal ion is favouring the lower rim of the calix[4]arene derivative, which is surprising due to the extra donor nitrogen atoms on the upper rim. This may suggest that self-assembly is so strong, that when the construct is forming the upper rim pyridyl's interact with each other in the homo fashion and even in the presence of the metal ion they do not disengage from each other. Perhaps in the hetero form of this compound, as there is only two pyridyl's on the lower rim, it is not held as strongly as the homo and so in the presence of a metal this form can break to allow binding on the lower rim of (51).

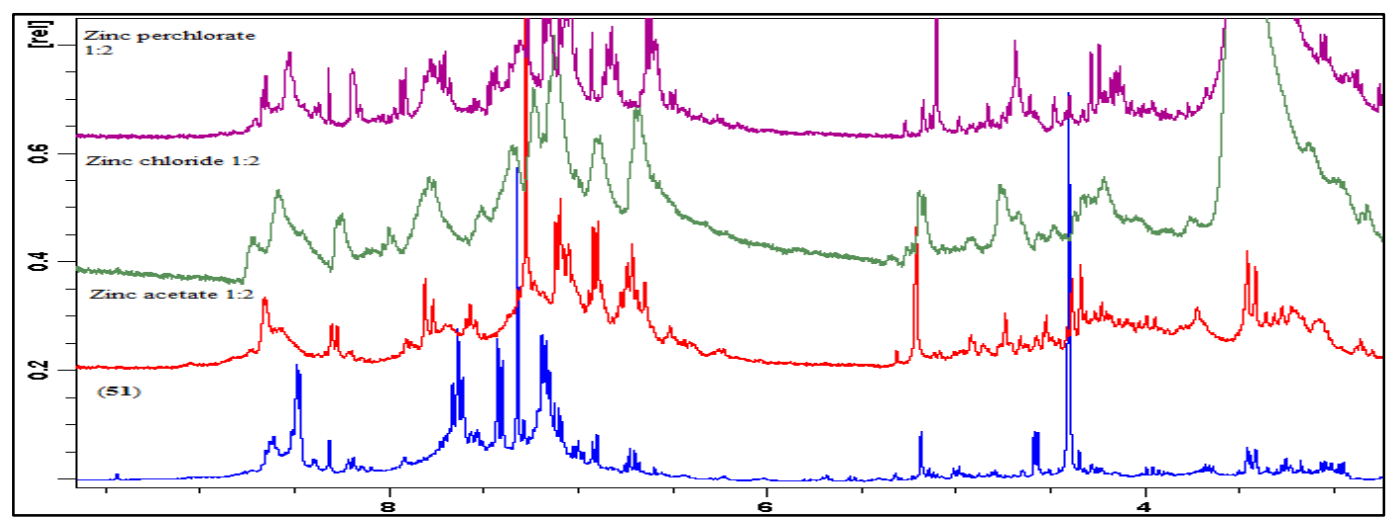

Figure 206: ${ }^{1} \mathrm{H}$ NMR of 1:2 metal ion complexation reactions of (51). Red trace = Zinc(II) acetate, Green trace $=$ Zinc(II) chloride, purple trace $=$ Zinc(II) perchlorate .

The bridging region, (Figure 207) of the 1:2 metal ion complexed spectra was very similar to the 1:1 metal ion complexed spectra in that a broadening of the chloride and perchlorate spectra along with self-assembly could clearly been seen. The axial 
bridging methylene protons were found to give cleaner resolution in the acetate and perchlorate spectra when compared to that of the parent spectrum. A clear shift that was observed belonged to the lower rim methylpyridyl methylene protons which shifted from $5.19 \mathrm{ppm}$ in the parent spectrum to 5.21, 5.18 and $5.09 \mathrm{ppm}$ in the acetate, chloride and perchlorate spectra respectively.

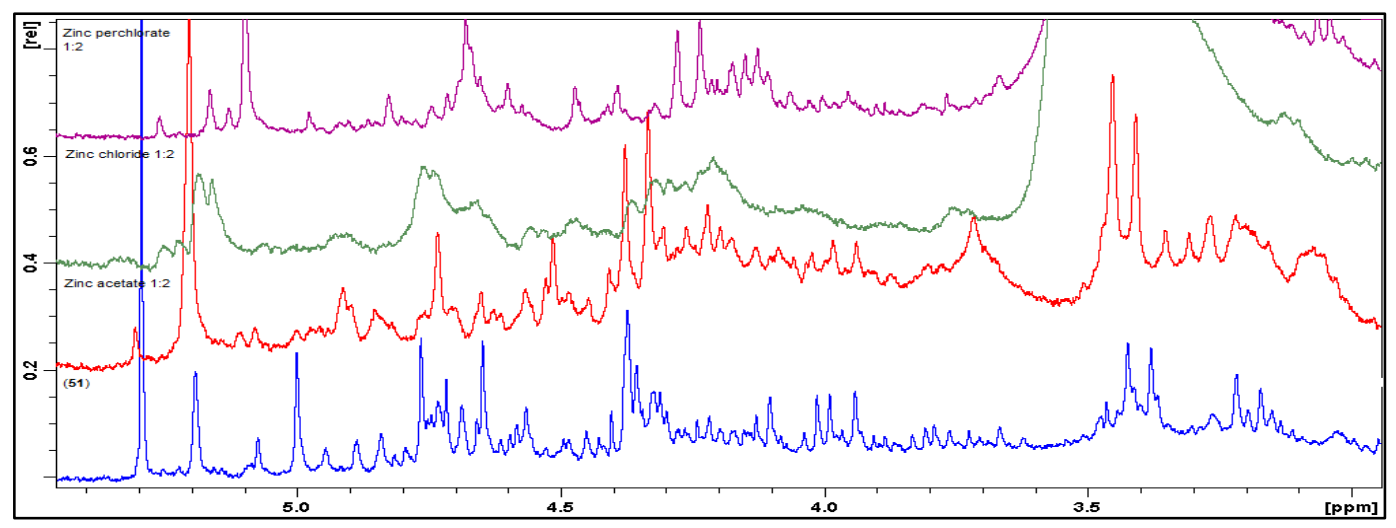

Figure 207: ${ }^{1} \mathrm{H}$ NMR of the bridging region of (51) with 1:2 Zinc(II) metal ion complexation. Red trace $=$ Zinc(II) acetate, Green trace $=$ Zinc(II) chloride, purple trace $=$ Zinc(II) perchlorate.

The IR data for the 1:1 metal ion complexation reactions with compound (51) shows a fair shift in both the carbonyl and pyridyl NCH bands (Table 22). This supports the ${ }^{1} \mathrm{H}$ NMR data well were the metal seems to favour binding on the lower rim of the calix[4]arene derivative.

\begin{tabular}{|l|c|c|c|}
\hline & $\mathrm{C}=\mathrm{O} \mathrm{cm}$ & $\mathrm{NC}-\mathrm{H}$ (Bound) $\mathrm{cm}^{-1}$ & $\mathrm{NC}-\mathrm{H}$ (Unbound) $\mathrm{cm}^{-1}$ \\
\hline Compound (51) & 1654 & 1591 & 1570 \\
\hline 1:1 reaction & & & 1592 \\
\hline Cobalt chloride & 1755 & 1630 & 1591 \\
\hline Mercury perchlorate & 1745 & 1635 & 1569 \\
\hline Mercury chloride & 1742 & 1614 & 1571 \\
\hline Nickel perchlorate & 1747 & 1630 & 1571 \\
\hline Nickel acetate & 1755 & 1591 & 1571 \\
\hline Nickel chloride & 1755 & 1630 & 1540 \\
\hline Copper perchlorate & 1743 & 1618 & 1569 \\
\hline Copper chloride & 1739 & 1608 & $\mathrm{~N} / \mathrm{O}$ \\
\hline Copper acetate & 1756 & 1623 & \\
\hline
\end{tabular}




\begin{tabular}{|l|l|l|l|}
\hline Zinc perchlorate & 1742 & 1618 & N/O \\
\hline Zinc chloride & 1743 & 1616 & N/O \\
\hline Zinc acetate & 1755 & 1658 & 1591 \\
\hline
\end{tabular}

Table 22: IR data for 1:1 metal ion complexation reactions with (51). Those in green experience shifts compared with parent spectrum in red.

The same trend was seen for the IR data of the 1:2 metal ion complexation reactions with compound (51). Both the carbonyl and pyridyl bands experienced large shifts corresponding to the ${ }^{1} \mathrm{H}$ NMR data for the $1: 2$ reactions (Table 23 ).

\begin{tabular}{|l|c|c|c|}
\hline & $\mathrm{C}=\mathrm{O} \mathrm{cm}$ & $\mathrm{NC}-\mathrm{H}$ (Bound) $\mathrm{cm}^{-1}$ & $\mathrm{NC}-\mathrm{H}$ (Unbound) $\mathrm{cm}^{-1}$ \\
\hline Compound (51) & 1654 & 1591 & 1570 \\
\hline 1:2 reaction & & & \\
\hline Cobalt chloride & 1753 & 1627 & 1570 \\
\hline Mercury perchlorate & $\mathrm{N} / \mathrm{O}$ & 1618 & 1571 \\
\hline Mercury chloride & 1746 & 1631 & 1599 \\
\hline Nickel perchlorate & 1744 & 1630 & $\mathrm{~N} / \mathrm{O}$ \\
\hline Nickel chloride & 1754 & 1630 & 1571 \\
\hline Nickel acetate & 1755 & 1591 & 1570 \\
\hline Copper perchlorate & 1742 & 1615 & 1567 \\
\hline Copper chloride & 1735 & 1608 & 1569 \\
\hline Copper acetate & 1756 & 1621 & $\mathrm{~N} / \mathrm{O}$ \\
\hline Zinc perchlorate & 1747 & 1630 & 1567 \\
\hline Zinc chloride & 1743 & 1608 & 1571 \\
\hline Zinc acetate & 1756 & 1729 & 1591 \\
\hline
\end{tabular}

Table 23: IR data for 1:2 metal ion complexation reactions with (51). Those in green experience shifts compared with parent spectrum in red.

Compound (52) was next to be investigated by ${ }^{1} \mathrm{H}$ NMR and IR spectroscopy, (Figure 208). The parent compound consisted of the ethylpyridyl on the upper rim and on the lower rim was the normal lower rim pyridyl along with the amide pyridyl. 


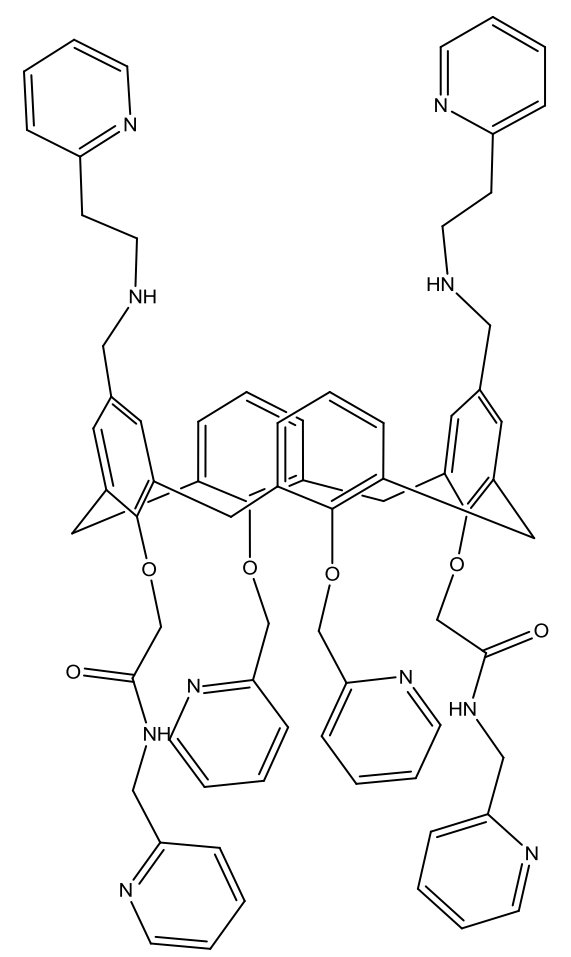

Figure 208: Compound (52)

The ${ }^{1} \mathrm{H}$ NMR spectrum showed that the doublet peak for the lower rim methylpyridyl protons were found at $8.55 \mathrm{ppm}$, the lower rim amidepyridyl protons were found at $8.50 \mathrm{ppm}$ and the upper rim ethylpyridyl protons were seen at 8.46 ppm (Figure 209). The metal ion complexation spectra saw a 'merging' of the lower rim methylpyridyl and amidepyridyl protons, similar to what has been observed in the previous cases. The acetate spectrum saw the 'merged' lower rim pyridyl protons shift to $8.63 \mathrm{ppm}$ and the upper rim ethylpyridyl protons shifted from $8.46 \mathrm{ppm}$ to $8.56 \mathrm{ppm}$. The chloride spectrum saw the 'merged' peak shift to $8.63 \mathrm{ppm}$ and the upper rim ethylpyridyl protons shift from $8.46 \mathrm{ppm}$ to $8.53 \mathrm{ppm}$. The perchlorate had a similar trend, where the 'merged' peak shifted to $8.62 \mathrm{ppm}$ and the upper rim ethylpyridyl protons shifted from $8.46 \mathrm{ppm}$ to $8.53 \mathrm{ppm}$. This suggests that the metal is favouring binding at the lower rim and that the upper rim may only be binding a small fraction of the metal ion. This is similar to what was seen in (51) were binding occurred on the lower rim leaving the upper rim free to self-assemble with neighbouring molecules. Perhaps the lower rim has been favoured in both cases due to the extra rigidity, in that the upper rim long arm may have enough flexibility to move away from the metal, whereas on the lower rim the functionalised groups are 
physically closer together creating a suitable housing for the metal ion. All three again displayed shoulder peaks to indicate the unbound pyridyl's in the selfassembled chain.

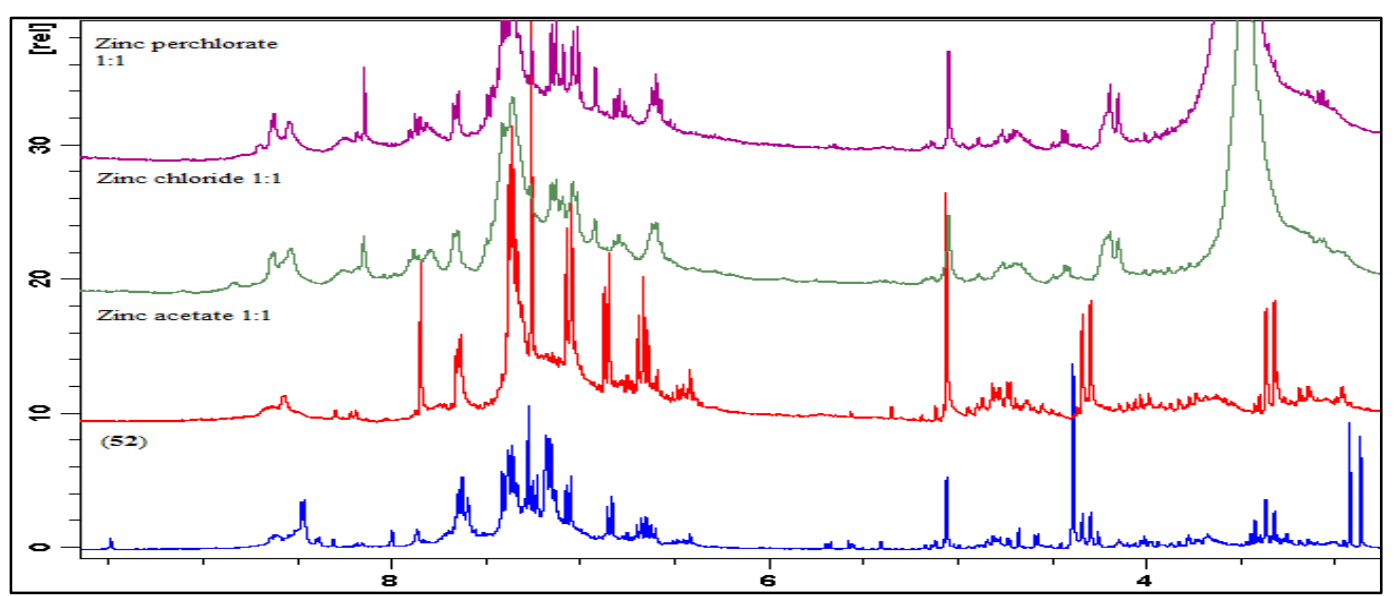

Figure 209: ${ }^{1} \mathrm{H}$ NMR spectra of 1:1 metal ion complexation reactions of (52). Red trace $=$ Zinc(II) acetate, Green trace $=$ Zinc(II) chloride, purple trace $=$ Zinc(II) perchlorate .

The bridging region of the spectrum, (Figure 210), contained an abundance of selfassembled peaks and as a result very few chemical shifts were seen. The only clear shift was in the axial bridging protons, which like (51), experienced shifts in the chlorate and perchlorate spectra from $4.31 \mathrm{ppm}$ in the parent to $4.16 \mathrm{ppm}$ in the two metal complexed spectra. 


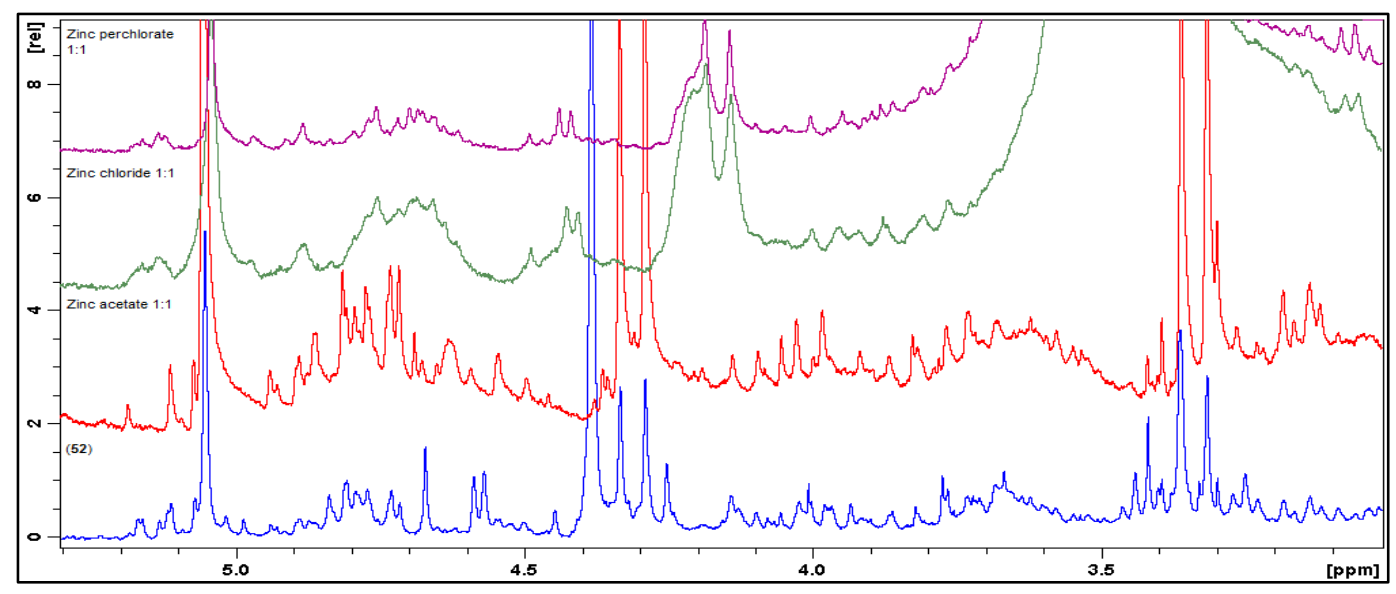

Figure 210: ${ }^{1} \mathrm{H}$ NMR of the bridging region of (52) with 1:1 Zinc(II) metal ion complexation. Red trace $=$ Zinc(II) acetate, Green trace $=$ Zinc(II) chloride, purple trace $=$ Zinc(II) perchlorate.

The 1:2 metal ion complexation reactions were also investigated with ${ }^{1} \mathrm{H}$ NMR spectroscopy (Figure 211). The parent protons occurred at $8.55 \mathrm{ppm}$ for the lower rim methylpyridyl protons, $8.50 \mathrm{ppm}$ for the lower rim amide pyridyl protons and at $8.46 \mathrm{ppm}$ for the upper rim ethylpyridyl protons. The same trend was seen in the 1:2 metal ion complexed spectra as in the 1:1 metal ion complexed spectra, in that the lower rim doublets seem to 'merge' upon metal ion complexation. The acetate spectrum showed a shift to $8.69 \mathrm{ppm}$ for the 'merged' protons and a shift from 8.46 ppm to $8.58 \mathrm{ppm}$ for the upper rim ethylpyridyl protons. The chloride spectrum showed a shift for the 'merged' lower rim protons to $8.63 \mathrm{ppm}$ and a shift from 8.46 ppm to $8.52 \mathrm{ppm}$ for the upper rim ethylpyridyl protons. The perchlorate spectrum showed a shift for the 'merged' protons to $8.62 \mathrm{ppm}$ and a shift from $8.46 \mathrm{ppm}$ to $8.53 \mathrm{ppm}$ for the upper rim ethylpyridyl protons. This data suggests that both the upper and lower rim are involved in the complexation of the metal ion, however not necessarily together. It could be indicating that there are two separate chains in solution were one is binding the metal through the lower rim and the other binding through the upper rim, as previously mentioned. Or the metal could be bound through the two rims, in that the metal is sitting in the middle of a chain and on one side it is bound by the lower rim and on the other it's bound by the upper rim. A crystal structure would be needed to definitively prove these theories. 


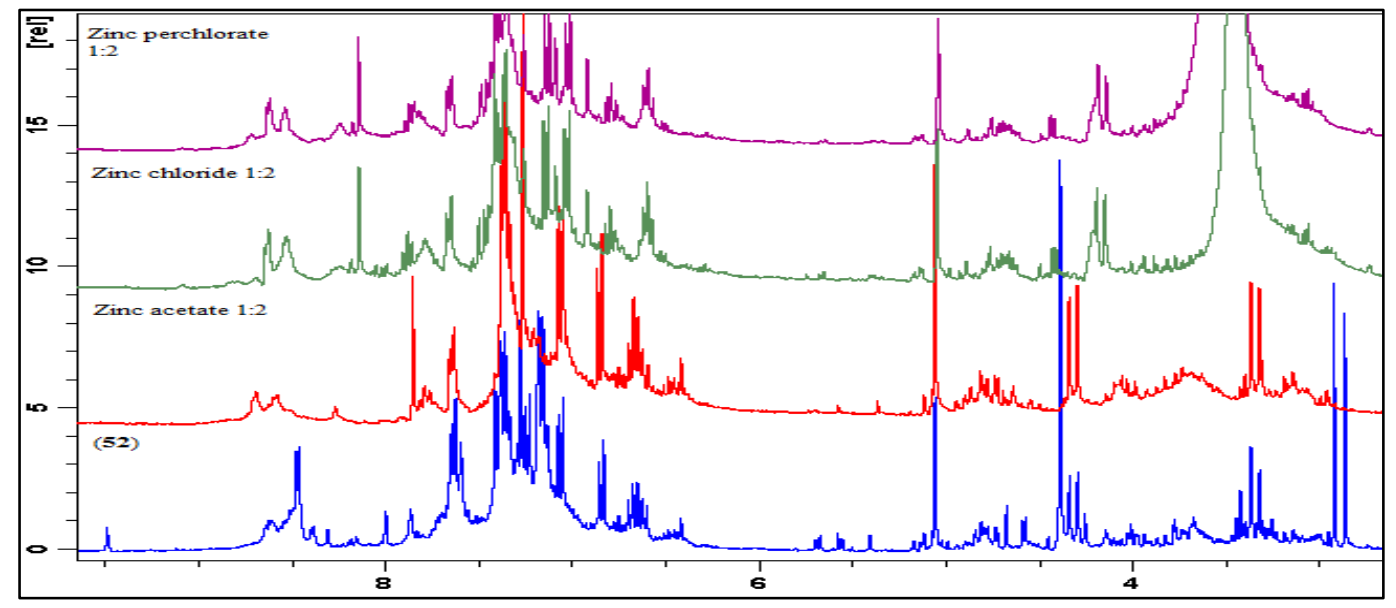

Figure 211: ${ }^{1} \mathrm{H}$ NMR spectra of 1:2 metal ion complexation reactions of $(\mathbf{5 2})$. Red trace $=$ Zinc(II) acetate, Green trace $=$ Zinc(II) chloride, purple trace $=$ Zinc(II) perchlorate .

The bridging region of the 1:2 metal ion complexed spectra, (Figure 212), was very similar to the 1:1 metal ion complexed spectra. Again the same shifts in the axial bridging methylene protons were observed in the chloride and perchlorate spectra and the self-assembly peaks were still quite dominant.

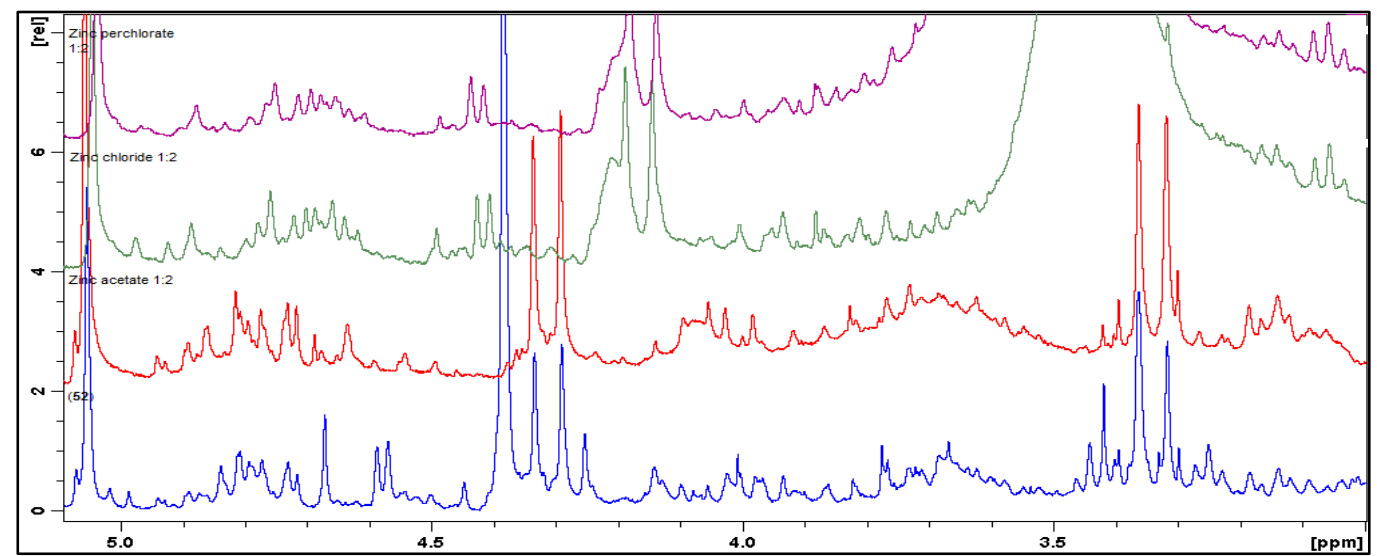

Figure 212: ${ }^{1} \mathrm{H}$ NMR of the bridging region of (52) with 1:2 Zinc(II) metal ion complexation. Red trace $=$ Zinc(II) acetate, Green trace $=$ Zinc(II) chloride, purple trace $=$ Zinc(II) perchlorate.

From the IR data for the 1:1 and 1:2 metal ion complexation reactions (Table $24 \&$ Table 25), it can be seen that the carbonyl group doesn't experience a shift upon metal binding, but the $\mathrm{NH}$ and $\mathrm{NCH}$ bands do experience large shifts. This supports the ${ }^{1} \mathrm{H}$ NMR data for binding at both the upper and lower rims. In binding at the 
lower rim it is likely that the metal is able to bind between the four available nitrogen donor atoms and as such has no need to bind to the carbonyl group. Also the unbound $\mathrm{NCH}$ group is still clearly visible in the IR spectrum indicating that the self-assembly is still occurring. This also corresponds well with the ${ }^{1} \mathrm{H}$ NMR data.

\begin{tabular}{|l|c|c|c|c|}
\hline & $\begin{array}{c}\mathrm{C}=\mathrm{O} \\
\mathrm{cm}^{-1}\end{array}$ & $\mathrm{~N}-\mathrm{H} \mathrm{cm}^{-1}$ & $\begin{array}{c}\mathrm{NC}-\mathrm{H} \text { (Bound) } \\
\mathrm{cm}^{-1}\end{array}$ & $\begin{array}{c}\mathrm{NC}-\mathrm{H} \text { (Unbound) } \\
\mathrm{cm}^{-1}\end{array}$ \\
\hline Compound (52) & 1711 & 2625 & 1589 & 1570 \\
\hline $1: 1$ reaction & & & & 1571 \\
\hline Zinc perchlorate & 1718 & 2923 & 1609 & 1571 \\
\hline Zinc chloride & 1709 & 3030 & 1607 & 1591 \\
\hline Zinc acetate & $\mathrm{N} / \mathrm{O}$ & 3028 & 1607 & \\
\hline
\end{tabular}

Table 24: The IR data for the 1:1 metal ion complexation reactions with (52). Those in green experience shifts compared with parent spectrum in red.

\begin{tabular}{|l|c|c|c|c|}
\hline 1:2 reaction & $\begin{array}{l}\mathrm{C}=\mathrm{O} \\
\mathrm{cm}^{-1}\end{array}$ & $\mathrm{~N}-\mathrm{H} \mathrm{cm}$ & $\begin{array}{c}\mathrm{NC}-\mathrm{H} \text { (Bound) } \\
\mathrm{cm}^{-1}\end{array}$ & $\begin{array}{c}\mathrm{NC}-\mathrm{H} \text { (Unbound) } \\
\mathrm{cm}^{-1}\end{array}$ \\
\hline Zinc perchlorate & 1622 & 2925 & 1611 & 1571 \\
\hline Zinc chloride & 1707 & 3029 & 1607 & 1571 \\
\hline Zinc acetate & 1713 & 3028 & 1605 & 1589 \\
\hline
\end{tabular}

Table 25: The IR data for the 1:1 metal ion complexation reactions with (52). Those in green experience shifts compared with parent spectrum in red.

Compound (53) was the last to be investigated under ${ }^{1} \mathrm{H}$ NMR and IR spectroscopy analysis for binding ability, (Figure 213). The parent compound consisted of two pyridyl's on the upper rim, the short and long arm and the pyridyl amide on the lower rim. 


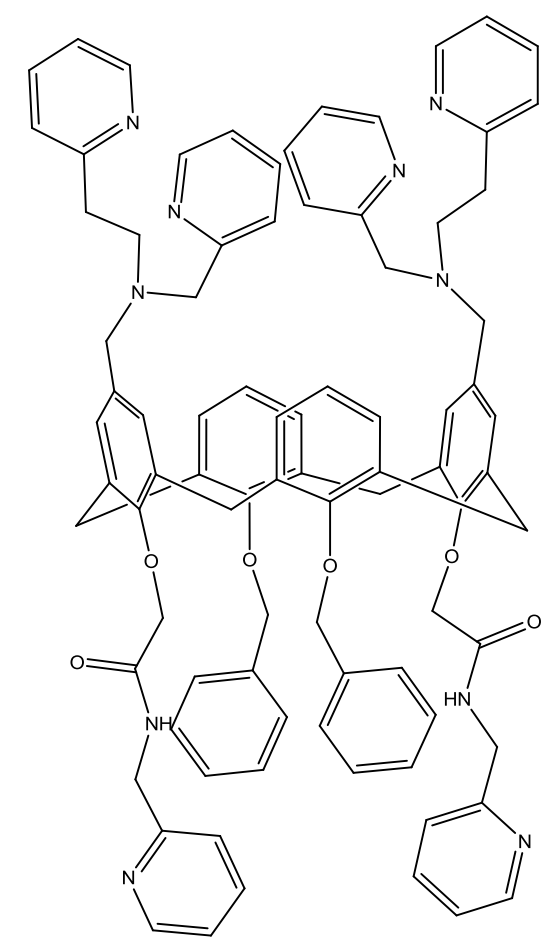

Figure 213: Compound (53)

Again a merging of the upper rim methylpyridyl and ethylpyridyl protons appeared as a broad peak at $8.51 \mathrm{ppm}$ and the lower rim amidepyridyl protons appeared as a broad doublet at $8.57 \mathrm{ppm}$ (Figure 214). The 1:1 metal ion complexation reaction was examined first. When complexed to a metal the upper rim broad doublet was found to split into, what at first looked a like a single doublet, but on closer analysis appeared to be a doublet of doublets each (Figure 215). The metal ion was allowing a distinction, albeit a slight one, between the two upper rim pyridyl doublets. The acetate spectrum showed a shift for the lower rim methylpyridyl protons from 8.57 ppm to $8.68 \mathrm{ppm}$ and the upper rim broad pyridyl signal separated into two doublets of doublets, at $8.59 \mathrm{ppm}$ for the methylpyridyl protons and $8.57 \mathrm{ppm}$ for the ethylpyridyl protons. The chloride spectrum showed a shift from $8.57 \mathrm{ppm}$ to 8.69 ppm for the lower rim methylpyridyl protons and separated the upper rim doublets to $8.63 \mathrm{ppm}$ and $8.62 \mathrm{ppm}$ for the methylpyridyl protons and ethylpyridyl protons respectively. The perchlorate spectrum showed a shift from $8.57 \mathrm{ppm}$ to $8.67 \mathrm{ppm}$ for the lower rim methylpyridyl protons and the upper rim doublet of doublets occurred at $8.63 \mathrm{ppm}$ and $8.62 \mathrm{ppm}$ for the methylpyridyl protons and ethylpyridyl protons. This data suggests that all three of the pyridyl groups are involved in 
binding the metal ion, possibly in two different chains. It also allows a comparison to be made between the two upper rim pyridyl's. This is most likely due to interactions between one of the upper rim pyridyl group and the lower rim methylpyridyl protons.

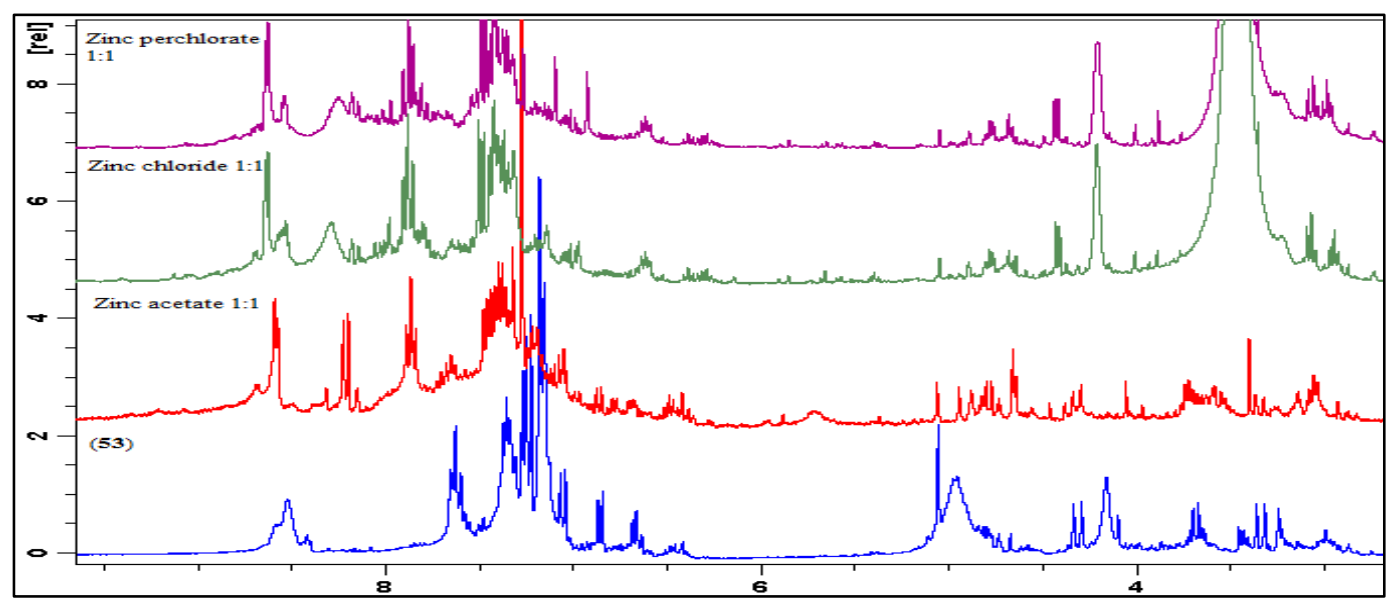

Figure 214: ${ }^{1} \mathrm{H}$ NMR spectra of 1:1 metal ion complexation reactions of $(\mathbf{5 3})$. Red trace $=$ Zinc(II) acetate, Green trace $=$ Zinc(II) chloride, purple trace $=$ Zinc(II) perchlorate

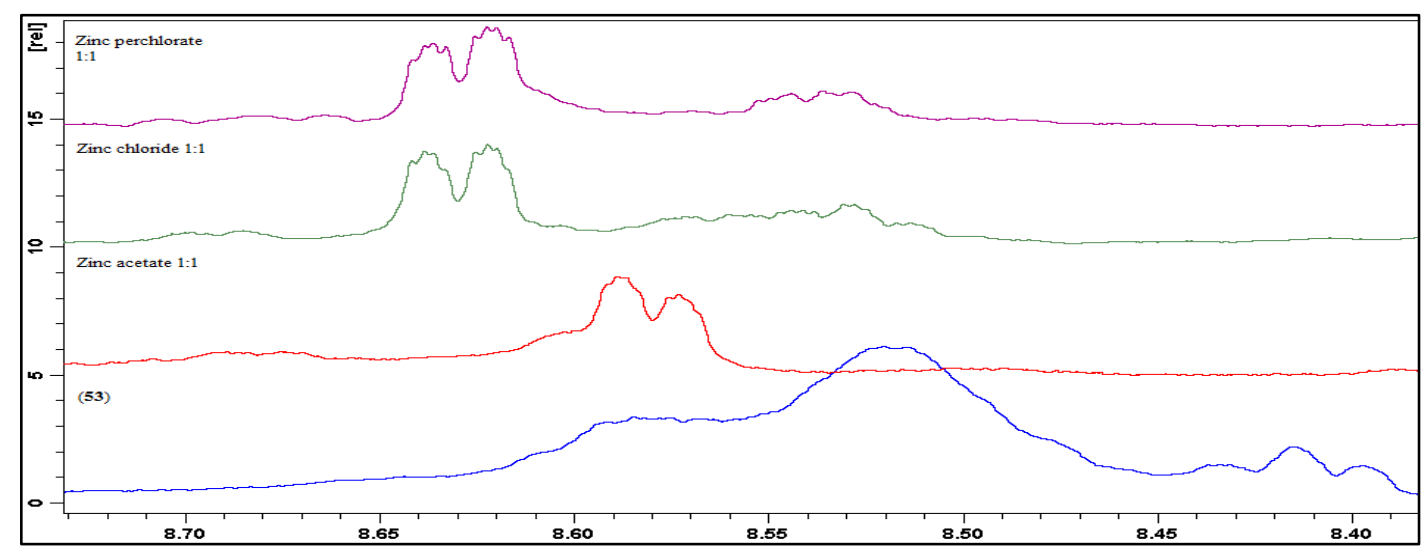

Figure 215: Splitting of upper rim doublet in to doublet of doublets of (53). Red trace = Zinc(II) acetate, Green trace $=$ Zinc(II) chloride, purple trace $=$ Zinc(II) perchlorate.

The bridging region of the spectrum, (Figure 216), shows what looks to be an increase in self-assembly upon metal ion complexation. Very few shifts were seen, one of which was the axial bridging methylene protons which shifted from $4.31 \mathrm{ppm}$ in the parent compound to $4.41 \mathrm{ppm}$ in the chloride and perchlorate spectra respectively. No change was seen in the acetate spectrum. Metal ion complexation 
also allowed a clear distinction between the upper rim ethylpyridyl methylene and ethylene protons which were difficult to distinguish in the parent compound.

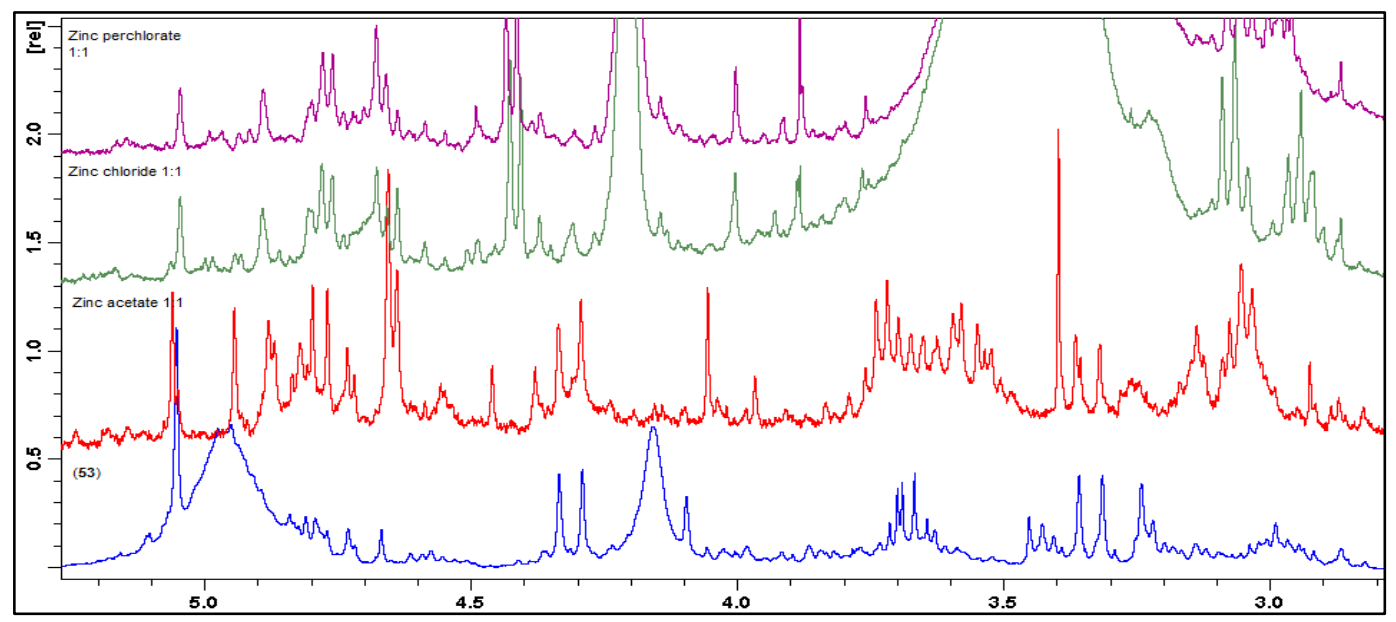

Figure 216: ${ }^{1} \mathrm{H}$ NMR of the bridging region of (53) with 1:1 Zinc(II) metal ion complexation. Red trace $=$ Zinc(II) acetate, Green trace $=$ Zinc(II) chloride, purple trace $=$ Zinc(II) perchlorate.

The 1:2 metal ion complexation reactions were also looked at under ${ }^{1} \mathrm{H}$ NMR spectroscopy (Figure 217). The parent compound had the lower rim amidepyridyl protons at $8.75 \mathrm{ppm}$ and the upper rim pyridyl protons at $8.51 \mathrm{ppm}$, again appearing as a broad doublet encompassing the two upper rim pyridyl groups. The acetate spectrum showed a shift in the lower rim methylpyridyl protons from $8.75 \mathrm{ppm}$ to $8.69 \mathrm{ppm}$ and the upper rim doublets remained on top of each other but shifted to $8.61 \mathrm{ppm}$ and became much broader. The chloride spectrum shifted the lower rim methylpyridyl protons from $8.57 \mathrm{ppm}$ to $8.63 \mathrm{ppm}$ and the broad upper rim doublet seperated to two broad doublets, right beside each other at $8.56 \mathrm{ppm}$ and $8.53 \mathrm{ppm}$. The perchlorate spectrum showed a shift for the lower rim methylpyridyl protons from $8.75 \mathrm{ppm}$ to $8.62 \mathrm{ppm}$, and the two upper rim pyridyl protons remained on top of each other but shifted to $8.52 \mathrm{ppm}$. This suggests that with excess metal present, the lower rim is the favoured site and the upper rim is involved in only a small amount of the binding. This could again be down to how the self-assembled chain forms. If it was to assemble with the upper rim pyridyl groups interacting with one another in a homodimerisation fashion then the $\pi$ - $\pi$ stacking interactions may be more favourable in this scenario than breaking this dimer to allow metal ion complexation. If the chain did stack in the homo form then this would leave the 
lower rim at the very top of the chain, allowing it to bind with the metal thus corresponding with the observed ${ }^{1} \mathrm{H}$ NMR spectra.

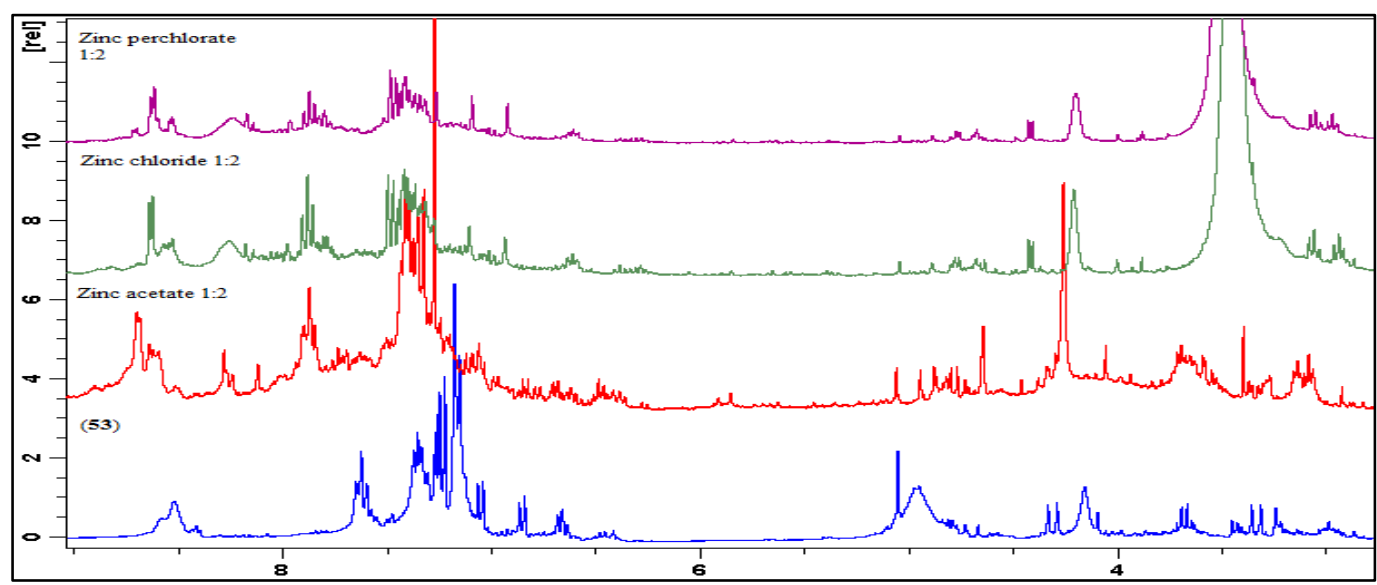

Figure 217: ${ }^{1} \mathrm{H}$ NMR spectra of 1:2 metal ion complexation reactions of $(\mathbf{5 3})$. Red trace $=$ Zinc(II) acetate, Green trace $=$ Zinc(II) chloride, purple trace $=$ Zinc(II) perchlorate .

The bridging region of the 1:2 metal ion complexed ${ }^{1} \mathrm{H}$ NMR spectra, (Figure 218), were very similar to the $1: 1$ metal ion complexed ${ }^{1} \mathrm{H}$ NMR spectra in that selfassembly peaks seemed to increase. Only a few shifts were seen due to peak overlap, with the shift in the axial bridging methylene protons from $4.30 \mathrm{ppm}$ in the parent spectrum to $4.41 \mathrm{ppm}$ in the chloride and perchlorate spectra being the most visible.

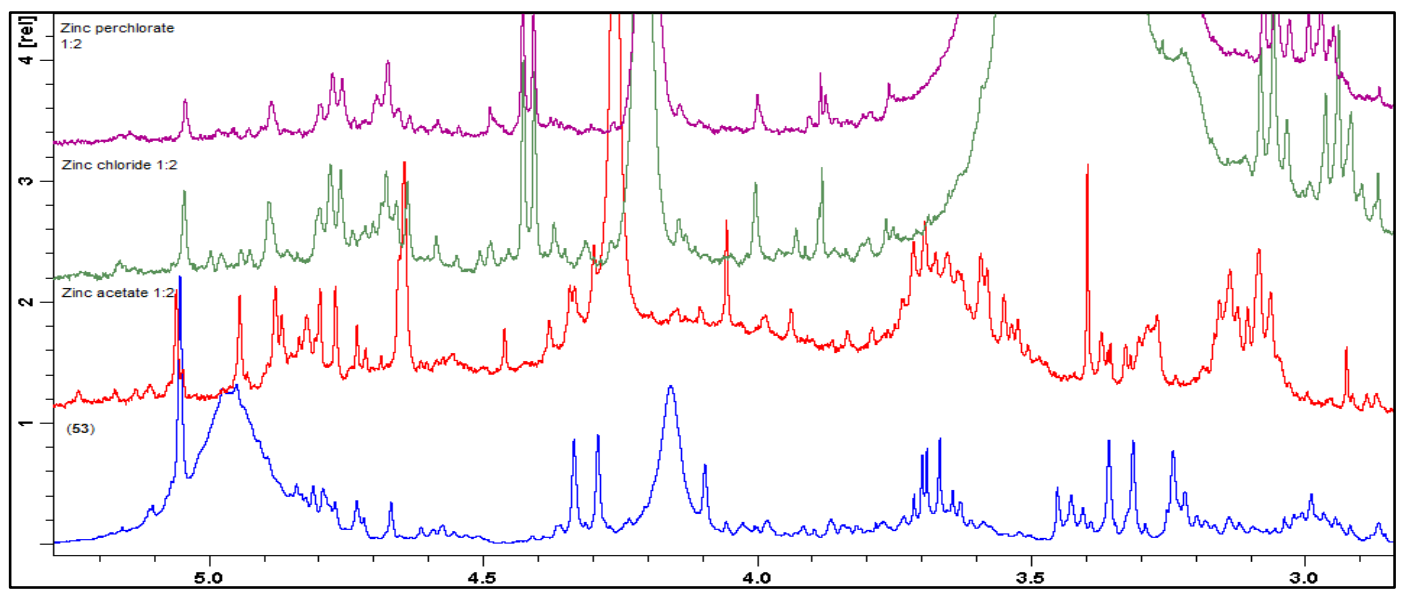

Figure 218: ${ }^{1} \mathrm{H}$ NMR of the bridging region of (53) with 1:2 Zinc(II) metal ion complexation. Red trace $=$ Zinc(II) acetate, Green trace $=$ Zinc(II) chloride, purple trace $=$ Zinc(II) perchlorate. 
The IR data for the 1:1 and 1:2 metal ion complexation reactions are shown below, Table 26. The 1:1 data corresponds well with the ${ }^{1} \mathrm{H}$ NMR spectra, which show a downfield shift in the pyridyl $\mathrm{NCH}$, the lower rim $\mathrm{NH}$ band and the lower rim carbonyl band. This supports the theory that the lower rim is the favoured metal binding site, most likely between the two amide pyridyls. The 1:2 data is a little bit different as only the chloride spectrum seems to show a downfield shift for the carbonyl band. This indicates that there may two different self-assembled chains present were both are capable of metal ion complexation. This corresponds well with the ${ }^{1} \mathrm{H}$ NMR data.

\begin{tabular}{|l|c|c|c|c|}
\hline & $\mathrm{C}=\mathrm{O} \mathrm{cm}^{-1}$ & $\mathrm{~N}-\mathrm{H} \mathrm{cm}^{-1}$ & $\mathrm{NC}-\mathrm{H} \mathrm{cm}^{-1}$ & $\mathrm{NC}-\mathrm{H} \mathrm{cm}^{-1}$ \\
\hline Compound (53) & 1631 & 3410 & 1589 & 1570 \\
\hline 1:1 reaction & & & & \\
\hline Zinc acetate & 1676 & 3435 & 1590 & 1568 \\
\hline Zinc chloride & 1651 & 3428 & 1605 & 1571 \\
\hline Zinc perchlorate & 1636 & 3342 & 1609 & 1573 \\
\hline 1:2 reaction & & & & \\
\hline Zinc acetate & $\mathrm{N} / \mathrm{O}$ & 3427 & 1590 & 1563 \\
\hline Zinc chloride & 1647 & 3429 & 1606 & 1571 \\
\hline Zinc perchlorate & 1611 & 3450 & 1627 & $\mathrm{~N} / \mathrm{O}$ \\
\hline
\end{tabular}

Table 26: IR data for the 1:1 and 1:2 metal ion complexation reactions with (53). Those in green experience shifts compared with parent spectrum in red.

\subsection{Fluorescence studies}

Having investigated the metal ion complexation ability of some of the compounds using ${ }^{1} \mathrm{H}$ NMR and IR spectroscopy, further investigations were conducted using fluorescence spectroscopy to examine metal-ligand interactions. The selection presented in this thesis focus on calix[4]arenes complexed with zinc(II) ion. All spectra were run in a 1:1 mixture of dichloromethane: methanol at $10^{-3}$ millimolar concentration unless otherwise stated. Residual dichloromethane and methanol peaks were seen at 280 and $530 \mathrm{~nm}$ respectively. 
Compound (31), (Figure 219), was the first compound to be examined using fluorescence spectroscopy. The UV absorption spectrum of (31) contained an absorption band at $376 \mathrm{~nm}$.

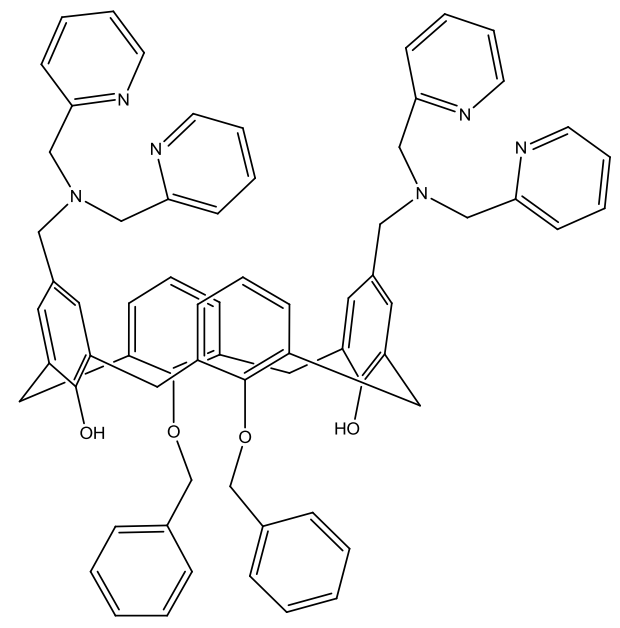

Figure 219: Compound (31)

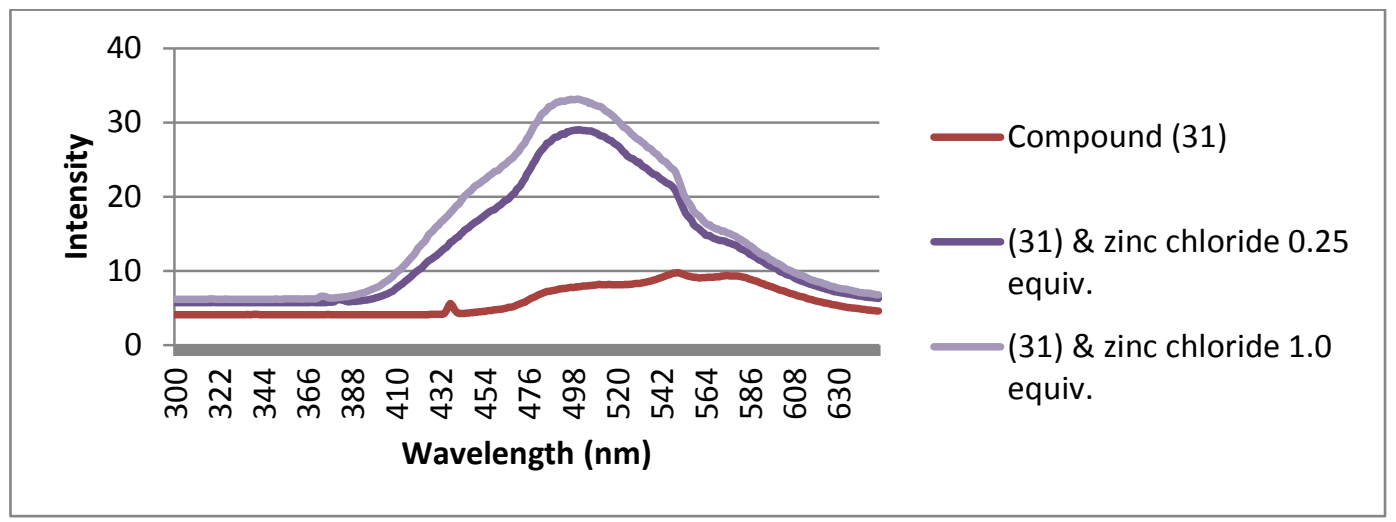

Figure 220: Fluorescecne spectrum of (31) with 0.25 and 1.0 equivalents of zinc chloride.

Excitation of the parent sample (red line) at $376 \mathrm{~nm}$ yielded an emission spectrum with a clear broad band at $543 \mathrm{~nm}$ along with two shoulder peaks at 506 and $572 \mathrm{~nm}$ (Figure 220). Upon addition of the zinc(II) ion an enhancement in fluorescence was seen in the first and second peaks. These peaks were also blue shifted, the first peak at $506 \mathrm{~nm}$ was only slightly blue-shifted but the peak at $543 \mathrm{~nm}$ was shifted much more. The enhancement in fluorescence is believed to be brought about by an 
increase in the interactions in the pendant arms of the calix[4]arene. This interaction is most likely due to interactions with the metal ion which brings the arms closer together, allowing a better orbital overlap resulting in an increase in fluorescence. Only a small difference was seen on going from 0.25 equivalents to 1.0 equivalent which may indicate that only a small amount of the metal ion is required to reorientate the self-assembled chain leading to increases interactions. For the zinc(II) acetate and zinc(II) perchlorate salts enhancement of fluorescence was also seen following the same trend as for the zinc(II) chloride.

The next compound to be investigated was (32), (Figure 221). Zinc(II) acetate produced good quality fluorescence spectra and are shown below in Figure 222. The UV absorption spectrum showed an absorption band at $388 \mathrm{~nm}$.

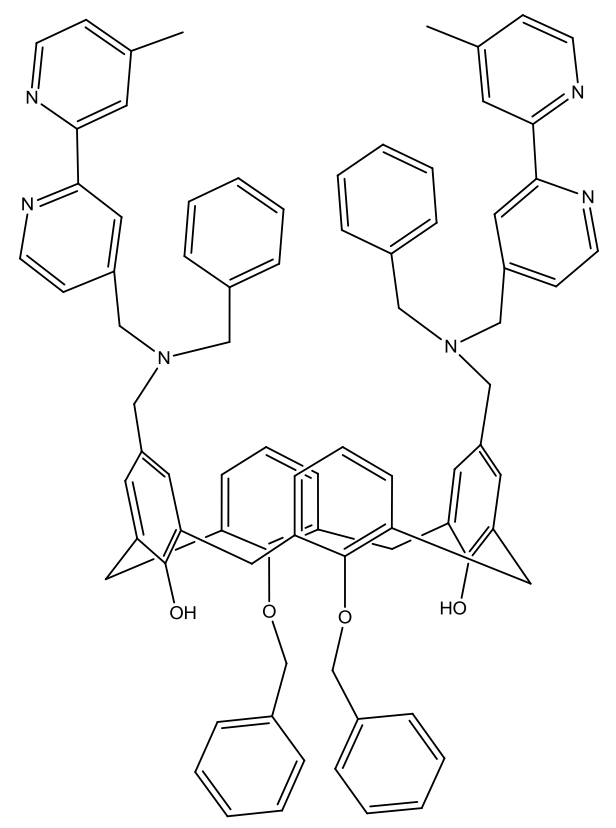

Figure 221: Compound (32)

Excitation of the parent sample at $388 \mathrm{~nm}$ yielded an emission spectrum with broad bands at 504, 540 and $580 \mathrm{~nm}$, (Figure 222). With the addition of zinc(II) acetate the first and second peaks are blue-shifted to 453 and $487 \mathrm{~nm}$ respectively. Overall an increase in the level of fluorescence is seen upon metal ion complexation, whereby 
the metal brings the arms of the calix[4]arene into close proximity with each other to allow good orbital overlap. The same trend is also seen with the metal ion complexation reactions involving two equivalents of the metal ion. Upon metal ion complexation, in this case, an increase in fluorescence is seen and the parent peaks at 504 and $540 \mathrm{~nm}$ are blue-shifted to 452 and $480 \mathrm{~nm}$ respectively. Zinc(II) chloride and zinc(II) perchlorate also produce similar spectra as the acetate spectra upon complexation with the zinc(II) metal ion.

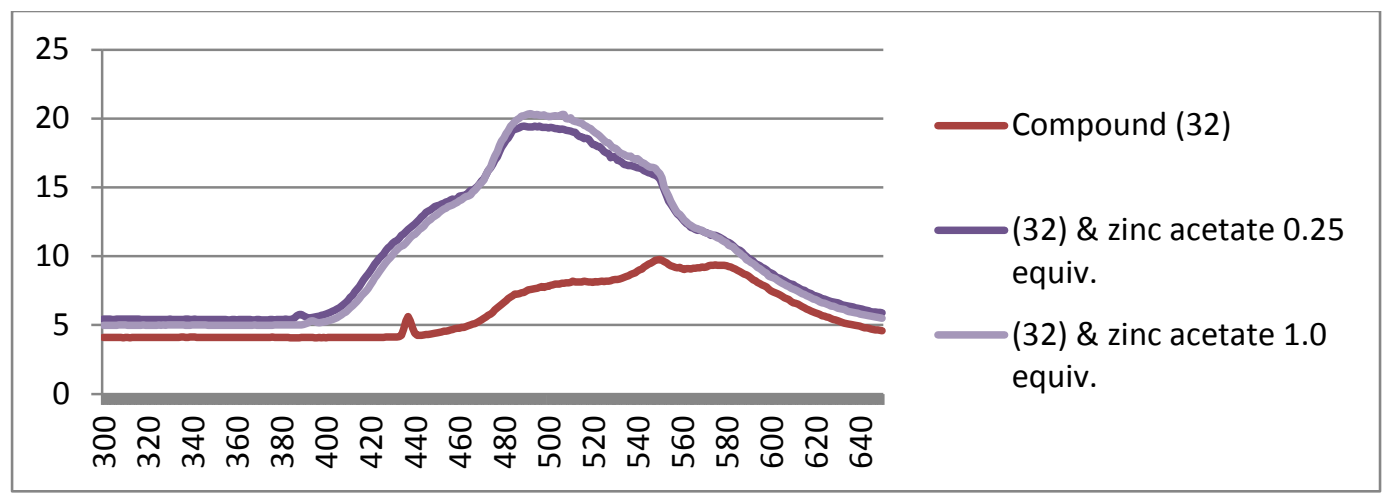

Figure 222: Fluorescecne spectrum of (32) with 0.25 and 1.0 equivalents of zinc(II) acetate.

Serial dilution studies were also performed under fluorescence spectroscopy with (32), Figure 223. The UV absorption spectrum showed an absorption band at $388 \mathrm{~nm}$ for the parent compound. Excitation at $388 \mathrm{~nm}$ produced an emission spectrum with bands at 504, 540 and $580 \mathrm{~nm}$ for the parent compound. When the serial dilution studies were performed with zinc chloride, a clear change in the fluorescence spectra could be observed. In the millimolar spectrum one large intense fluorescence band around $328 \mathrm{~nm}$ is seen, which is blue-shifted with respect to the parent spectrum. But when compared with the micromolar spectrum a decrease in the fluorescence is observed, as well as a separation of the bands at 288, 414 and $426 \mathrm{~nm}$. This indicates that as the concentration is becoming lower the pendant arms are no longer able to interact with each other so a decrease in orbital overlap occurs resulting in decreased fluorescence, even in the presence of a metal ion. This is consistent with what was previously observed in the serial dilution studies of ${ }^{1} \mathrm{H}$ NMR spectroscopy. Serial dilution with zinc acetate and zinc perchlorate also yielded the same results. 


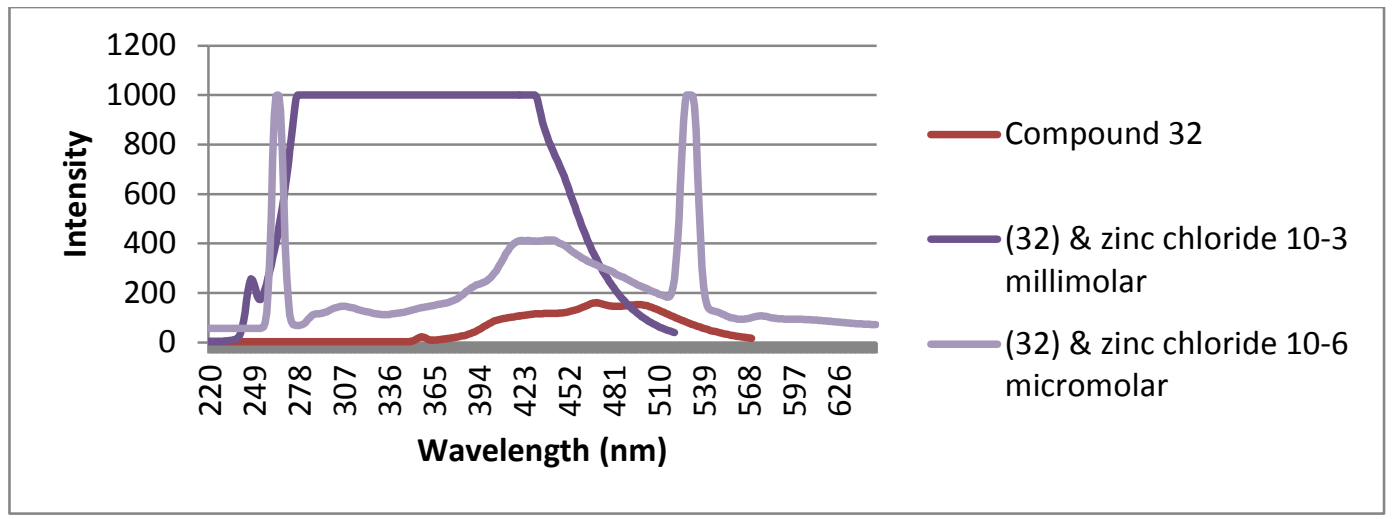

Figure 223: Fluorescecne spectrum of (32), and already complexed forms with zinc chloride at milli- and micro-molar ranges.

Next to be investigated was (35), (Figure 224). The UV absorption spectrum showed an absorption band at $407 \mathrm{~nm}$.

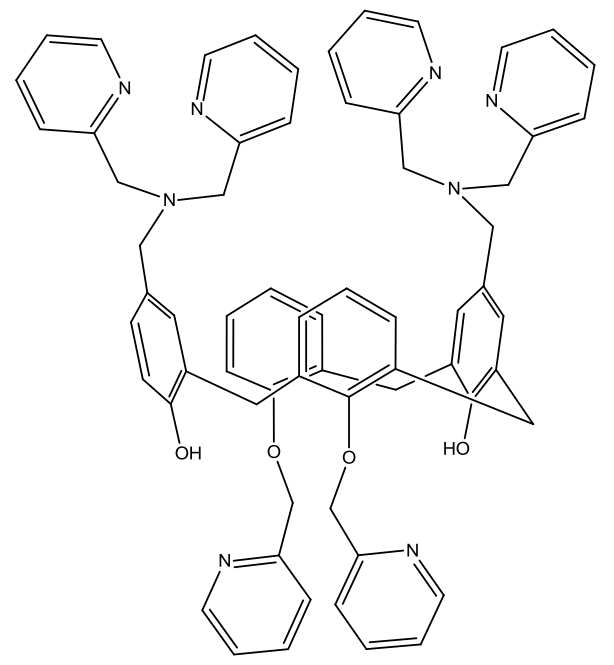

Figure 224: Compound (35)

Excitation at $407 \mathrm{~nm}$ yielded one broad peak at $497 \mathrm{~nm}$ and a shoulder peak at 586 $\mathrm{nm}$ in the parent spectrum, (Figure 225). Upon addition of zinc(II) acetate at 0.25 equivalents virtually no change in fluorescence intensity was seen but the band was blue-shifted to $477 \mathrm{~nm}$. However when 1.0 equivalent was added a small decrease in the fluorescence was seen indicating that the pendant arms were being separated from each other. This band was further red-shifted to $431 \mathrm{~nm}$. 


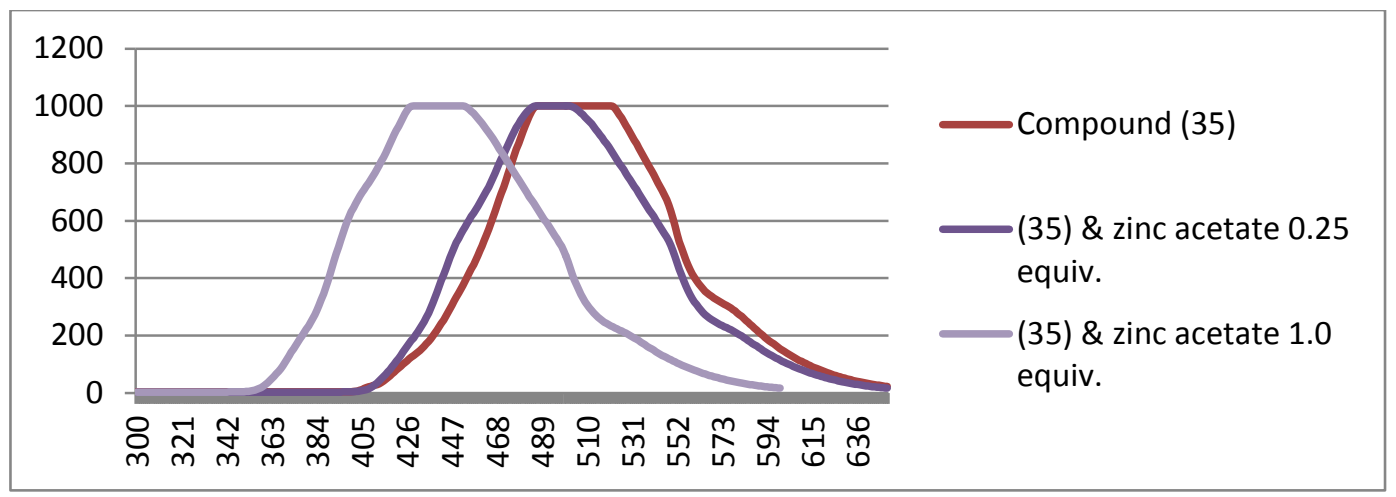

Figure 225: Fluorescecne spectrum of (35) with 0.25 and 1.0 equivalents of zinc acetate.

When excess metal was added the reverse was seen, (Figure 226). When 1.25 equivalents were added the band was blue-shifted to $445 \mathrm{~nm}$ and the fluorescence intensity decreased. But when 2.0 equivalents were added the fluorescence intensity increased but no shift was seen. This indicates that at 1.25 equivalents the pendant arms are moving away from each other, but as more metal is added the arms come together again providing good orbital overlap and increasing fluorescence.

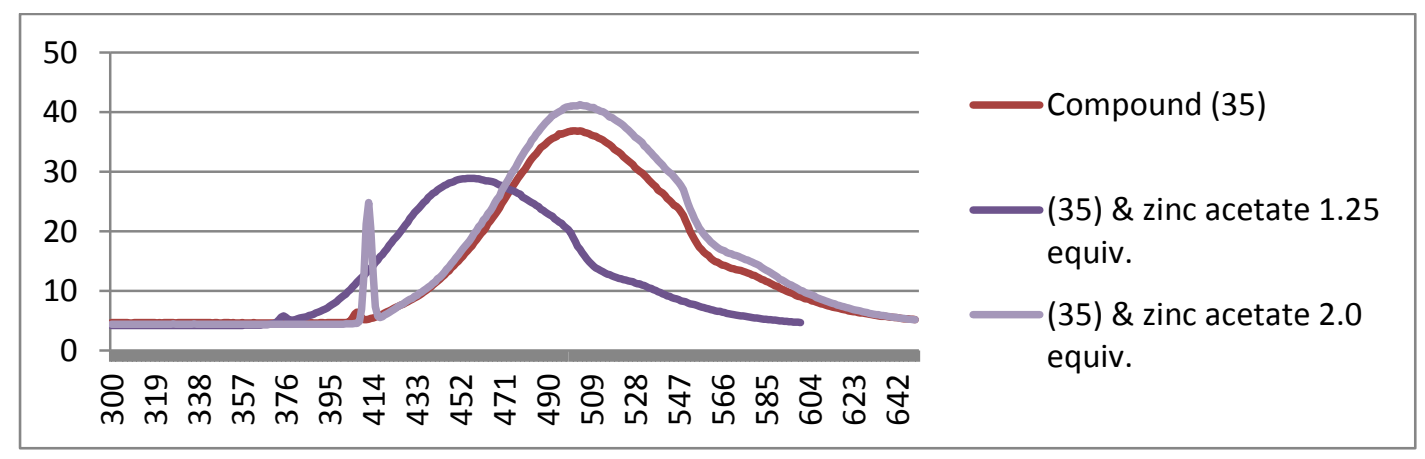

Figure 226: Fluorescecne spectrum of (35) with 1.25 and 2.0 equivalents of zinc chloride.

Serial dilution studies were also done with (35) with the zinc salts, Figure 227. Excitations at $407 \mathrm{~nm}$ on both the milli- and micromolar zinc chloride complexed solutions were performed. The parent compound at each of these concentrations produced a small fluorescence band at $495 \mathrm{~nm}$. The millimolar solution produced one clear band at $380 \mathrm{~nm}$ with two shoulder peaks at 346 and at $467 \mathrm{~nm}$. The band at $467 \mathrm{~nm}$ was the fluorescence maximum. This peak was blue-shifted with respect to the parent compound and was more intense than the parent compound. The 
micromolar spectrum produced a large intense fluorescence band at $426 \mathrm{~nm}$ which was blue-shifted with respect to the parent compound. This data indicates that at lower concentrations the fluorescence is more intense, meaning that the pendant arms are able to interact with one another. As this is opposite to what was seen for (32), where the micromolar spectrum was less intense than the millimolar, it suggests that perhaps the metal is bound more strongly in (35) than in (32).

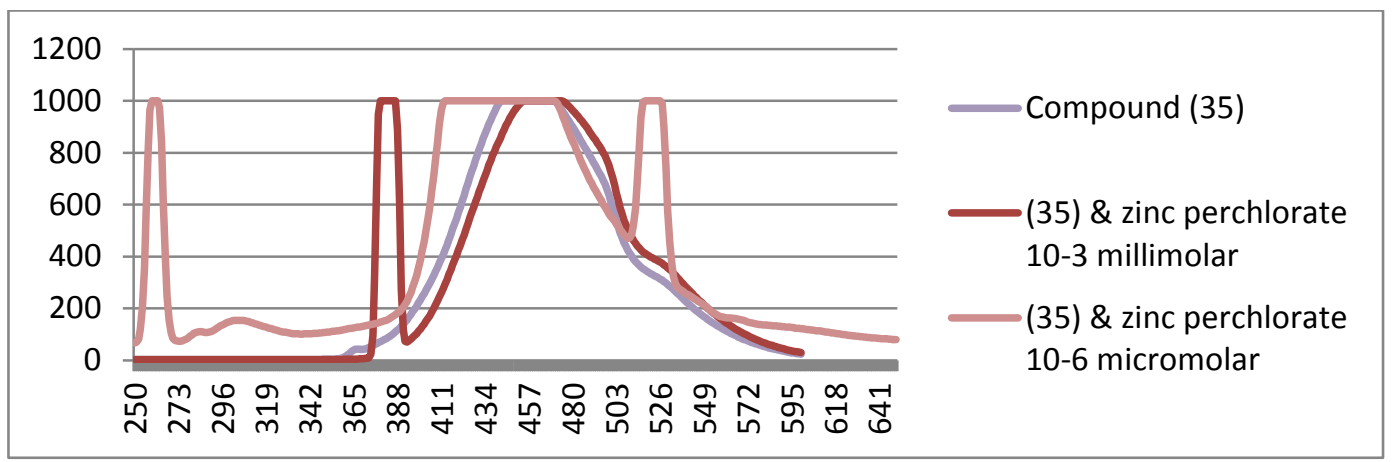

Figure 227: Fluorescecne spectrum of (35), and already complexed forms with zinc perchlorate at milli- and micro-molar ranges.

Following on from (35), (36) was investigated, (Figure 228). The parent compound was viewed under UV absorption spectroscopy and an absorption band at $483 \mathrm{~nm}$ was observed. 


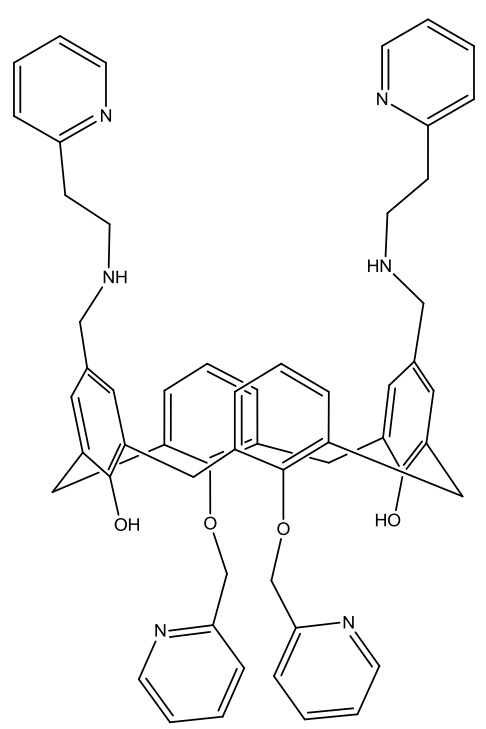

Figure 228: Compound (36)

Excitation at this wavelength produced an emission spectrum for the parent, with a broad band at $575 \mathrm{~nm}$, as well as two shoulder peaks at 541 and $508 \mathrm{~nm}$, (Figure 229). Upon the addition of 0.25 equivalents of zinc(II) acetate a blue shift was seen to wavelengths of lower energy, with a $\lambda_{\max }$ at $480 \mathrm{~nm}$. Three shoulder bands were also seen, one blue-shifted at $455 \mathrm{~nm}$, and two new peaks at 536 and $581 \mathrm{~nm}$ respectively. The intensity levels were the same as the parent compound. When two equivalents were added a small decrease in the intensity of fluorescence was seen indicating that the pendent arms were moving away from each other. The band also seem to be splitting into doublets with two large bands at 444 and $475 \mathrm{~nm}$, and two smaller bands at 538 and $567 \mathrm{~nm}$. The larger two are blue-shifted to lower energy with respect to the parent and 0.5 equivalent spectra, but the smaller bands are redshifted to higher energy in relation to the other two spectra. 


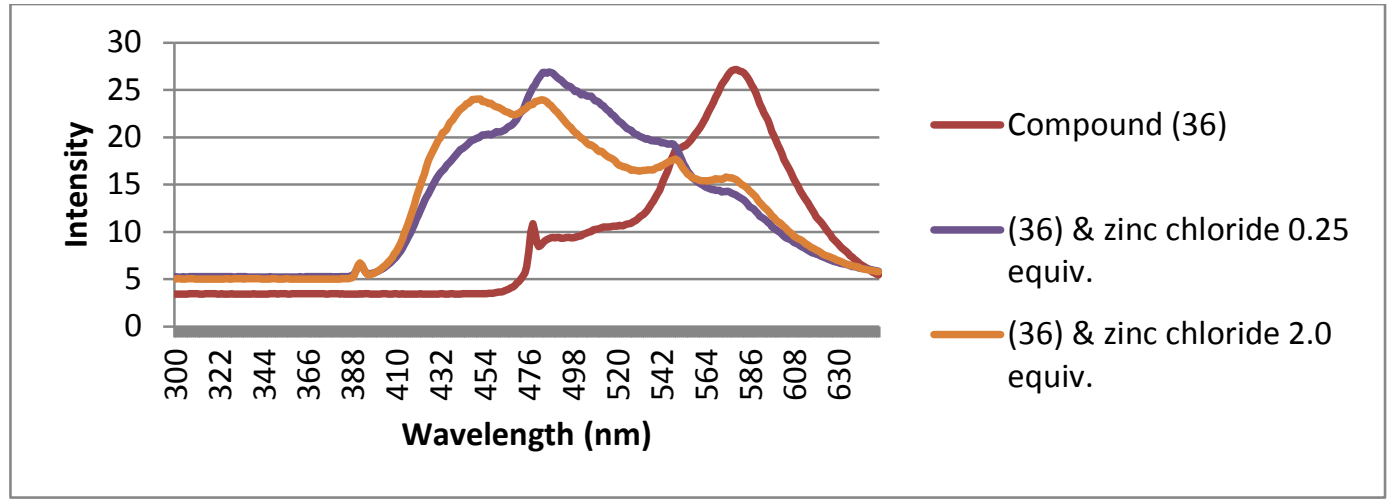

Figure 229: Fluorescence spectrum of (36) with 0.25 and 1.0 equivalents of zinc chloride.

Compound (37) was also examined under fluorescence spectroscopy, (Figure 230). The parent compound was examined under UV absorption spectroscopy and was found to have an absorption band at $420 \mathrm{~nm}$.

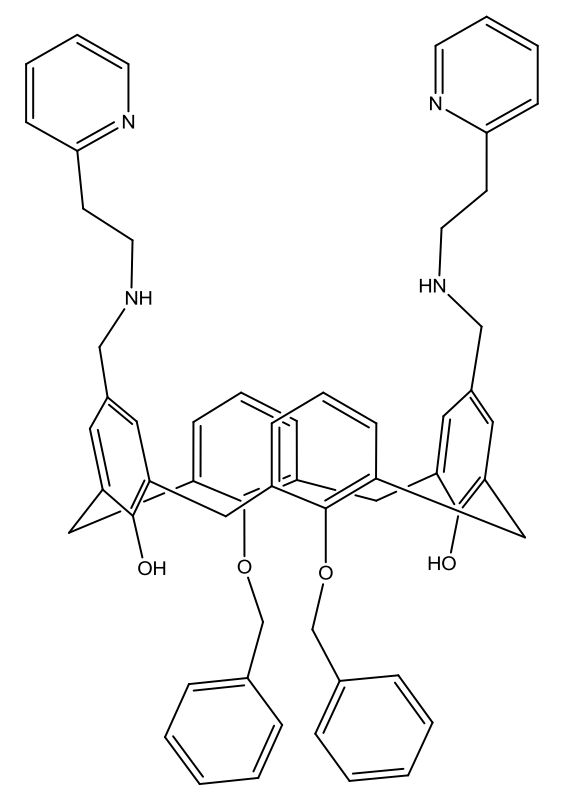

Figure 230: Compound (37)

Excitation at this wavelength resulted in an emission spectrum with a broad band at $491 \mathrm{~nm}$, (Figure 231). When the zinc(II) acetate metal ion was added an increase in the fluorescence was observed which gradually increased as more of the metal was added. The bands were also slightly blue-shifted with respect to the parent compound, going from $419 \mathrm{~nm}$ to $473 \mathrm{~nm}$ and $467 \mathrm{~nm}$ respectively. The zinc(II) chloride and zinc(II) perchlorate exhibited similar spectra. 


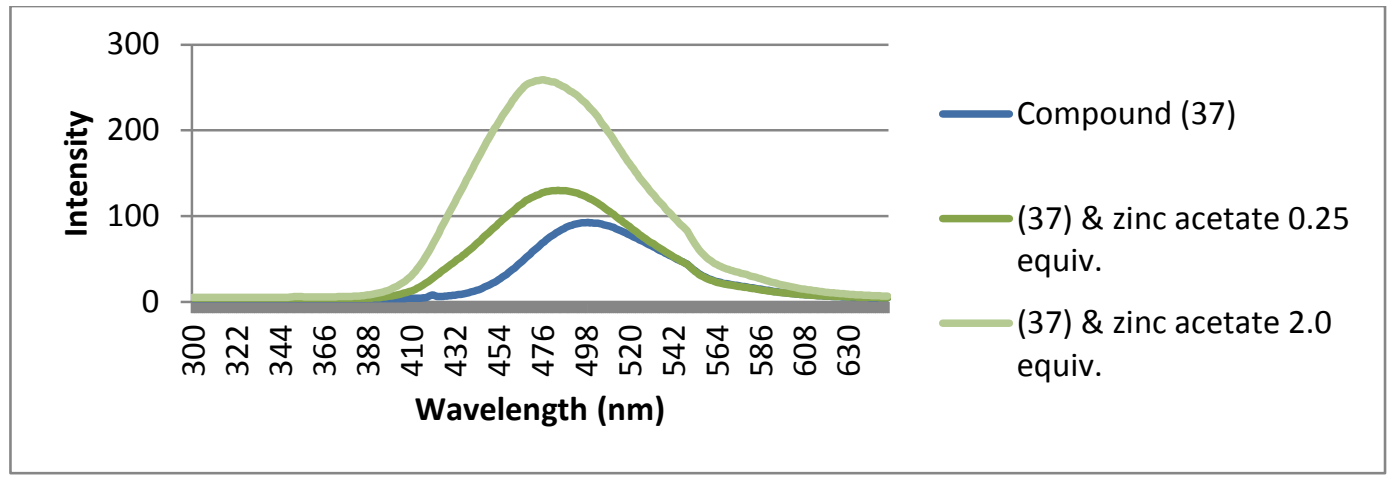

Figure 231: Fluorescecne spectrum of (37) with 0.25 and 1.0 equivalents of zinc chloride.

The remaining compounds (38) (Figure 232), (41) (Figure 235), and (47) (Figure 238), were all examined with fluorescence spectroscopy all contained similar spectra and will only be briefly discussed, (Figure 233, Figure 236 and Figure 239). All resulted in an increase in fluorescence upon metal ion complexation as well as blueshifting of the fluorescence bands. Serial dilution studies were also performed on these compounds (Figure 234, Figure 237 and Figure 240), all of which showed that the micromolar bands were more intense than the millimolar and that all experienced blue-shifts, corresponding with what had previously been seen. The UV absorption spectrum for (38) showed an absorption band at $374 \mathrm{~nm}$.

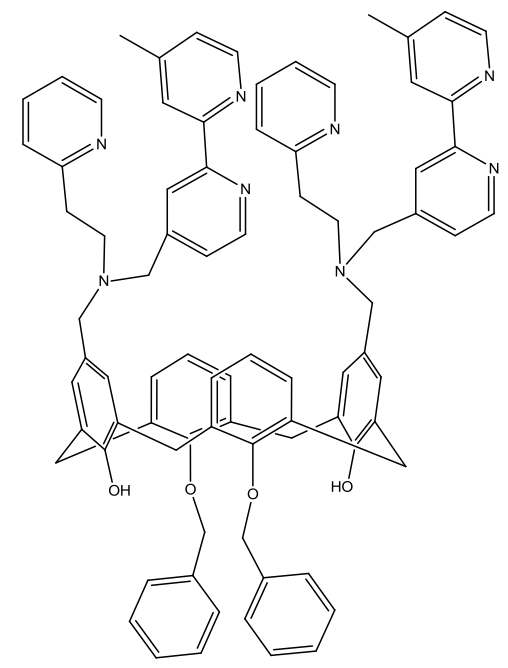

Figure 232: Compound (38) 


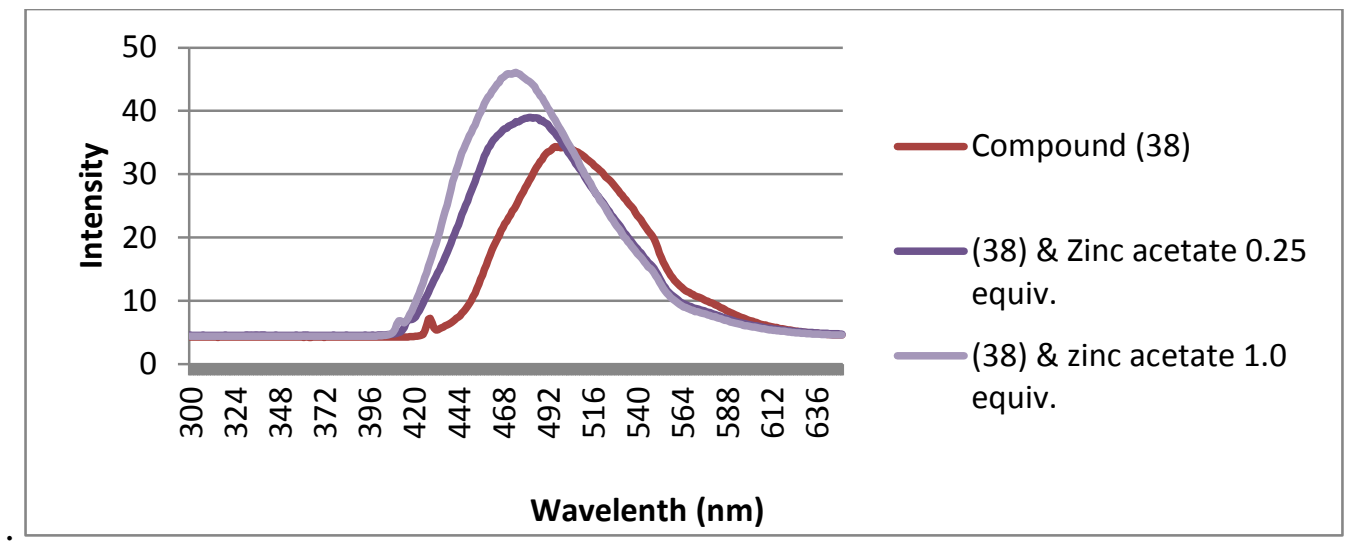

Figure 233: Fluorescecne spectrum of (38) with 0.25 and 1.0 equivalents of zinc chloride.

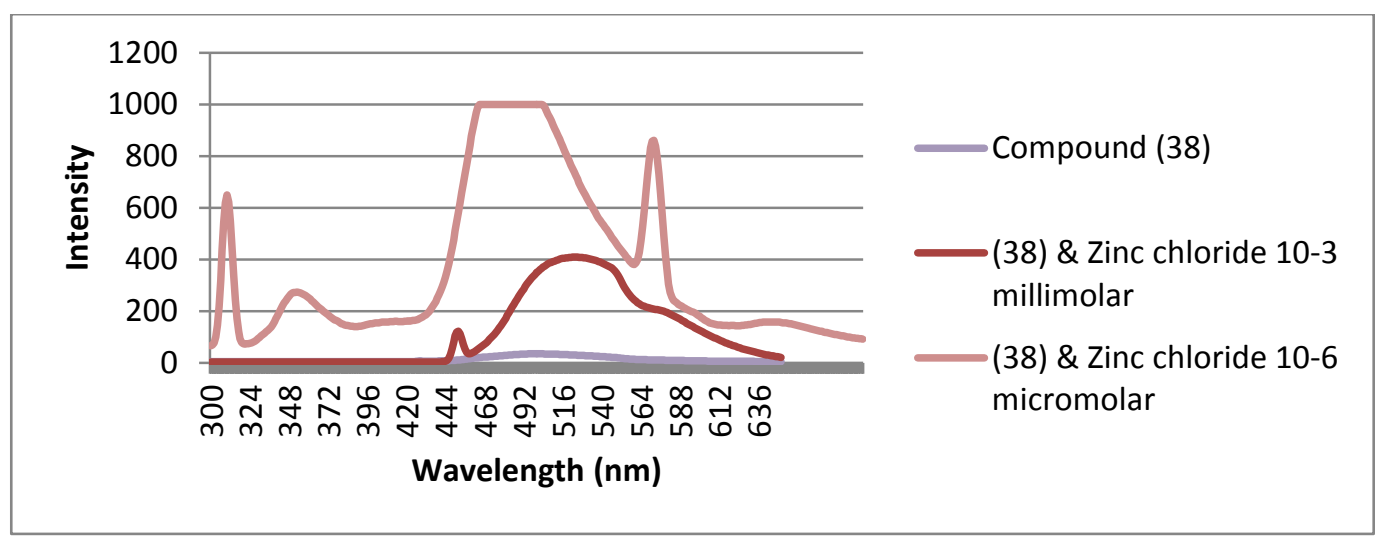

Figure 234: Fluorescecne spectrum of (38), and already complexed forms with zinc perchlorate at milli- and micro-molar ranges.

The UV absorption spectrum for (41) showed an absorption spectrum at $403 \mathrm{~nm}$. 


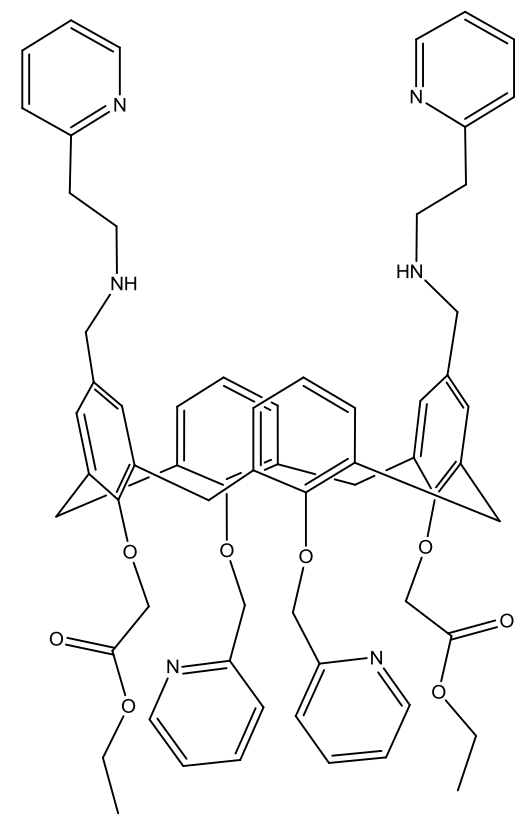

Figure 235: Compound (41)

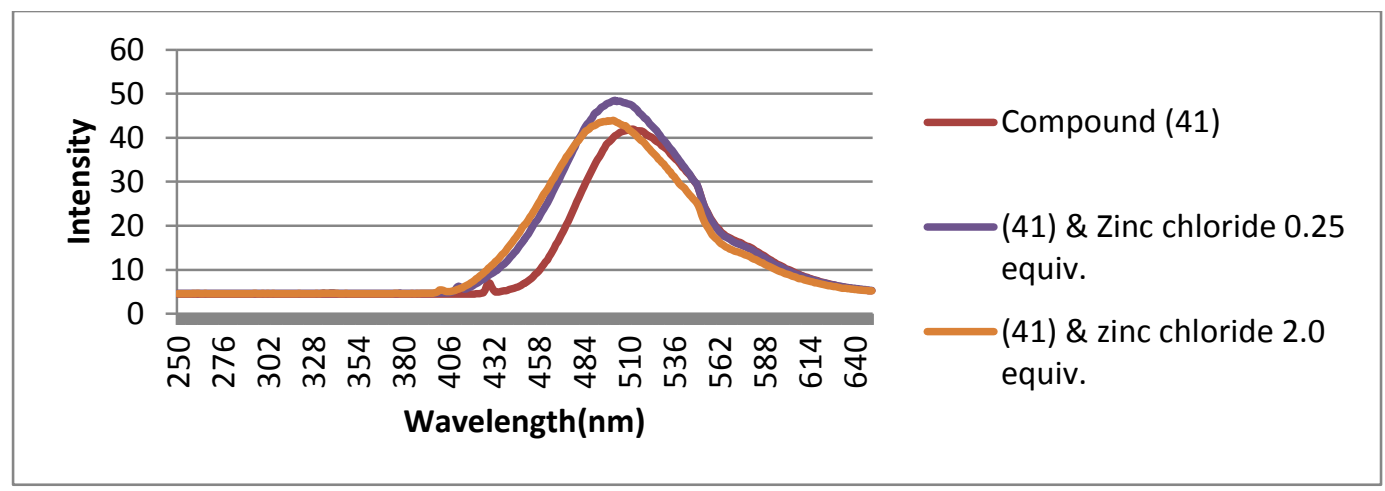

Figure 236: Fluorescecne spectrum of (41) with 0.25 and 1.0 equivalents of zinc chloride.

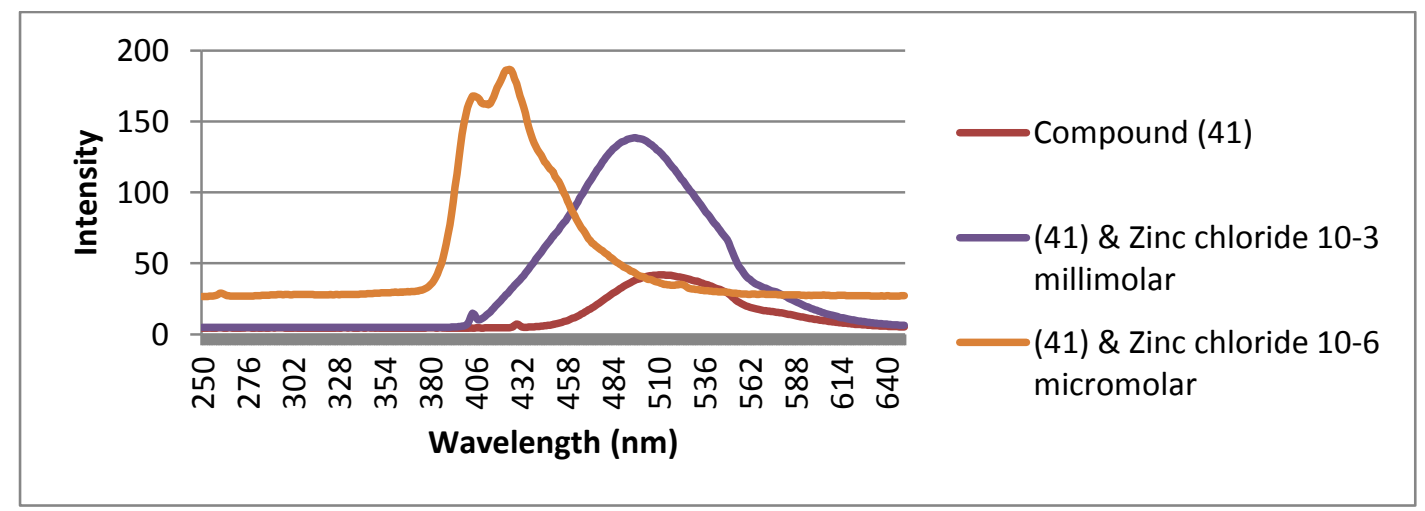

Figure 237: Fluorescecne spectrum of (41), and already complexed forms with zinc perchlorate at milli- and micro-molar ranges. 
The UV absorption spectrum of (47) produced an absorption band at $421 \mathrm{~nm}$.

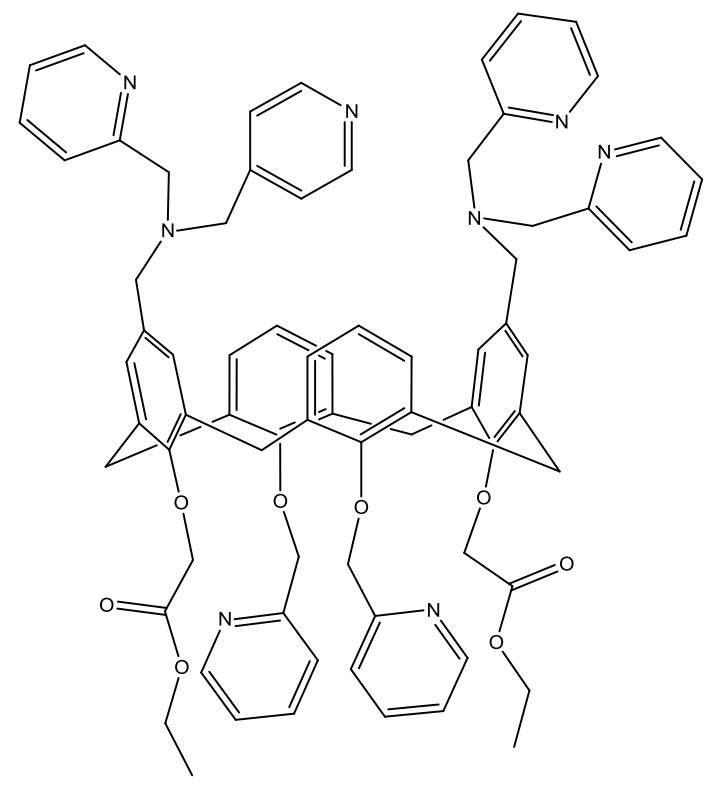

Figure 238: Compound (47)

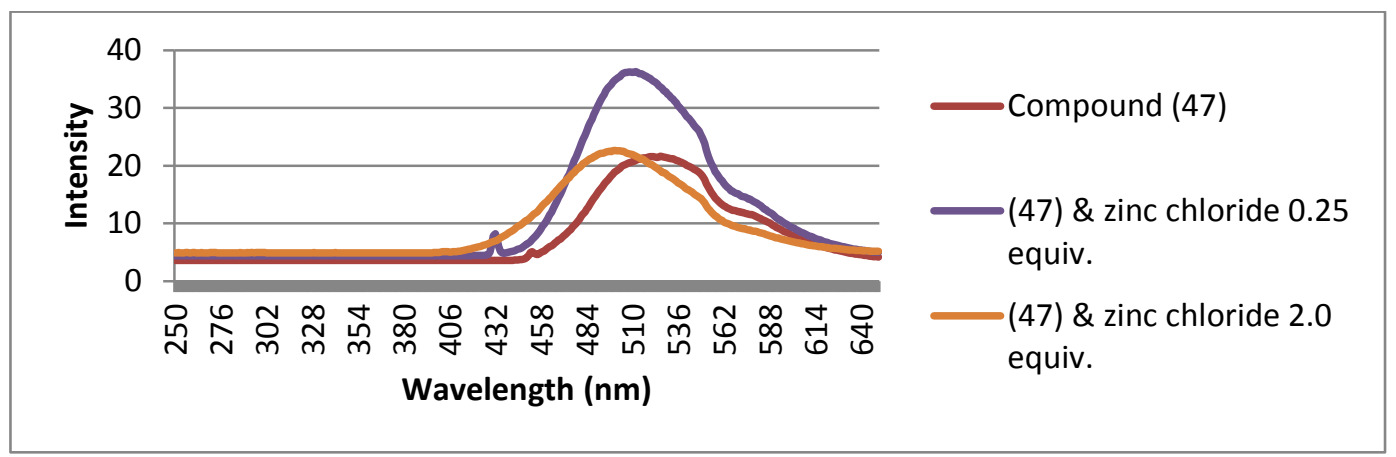

Figure 239: Fluorescecne spectrum of (47) with 0.25 and 1.0 equivalents of zinc chloride. 


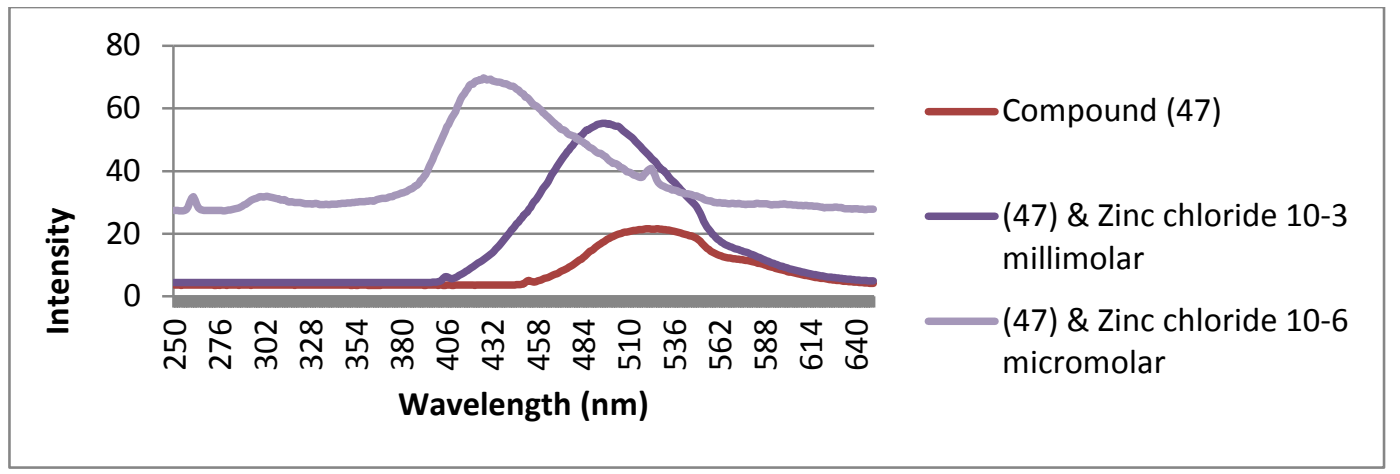

Figure 240: Fluorescecne spectrum of (47), and already complexed forms with zinc perchlorate at milli- and micro-molar ranges.

Upon reaction with the zinc(II) metal a general increase was seen, as well as the spectra being blue-shifted corresponding well to what had been seen for previous samples. This data suggests that as the concentration of the metal increases, pendant arm overlap increases resulting in an increase in fluorescence. 


\section{Conclusions}


The initial aim of this thesis was to develop novel calix[4]arene duel-metal ion binding complexes modelling the calix[4]arene template. This work was carried out stepwise, starting with the development of the parent calix[4]arene. Once the parent had been successfully synthesised work continued to modify both the upper and lower rim of the calix[4]arene to create the two metal ion binding sites in the hopes of establishing a communication between the two by electron transport. These modifications allowed the attachment of amine moieties to the upper rim along with various groups on the lower rim. Where the ester groups were functionalised to the lower rim, it was envisioned to further attach an amide functionality to create the metal ion binding site. In the same way, the upper rim amine allowed the attachment of various nitrogen donor ligands to form different metal ion binding sites.

The previous route to amine formation on the upper rim of the calix[4]arene involved a nitration step, which resulted in very low yields. To this end a novel synthetic route was found for the attachment of the upper rim amine, without the use of the nitration step, resulting in a higher synthetic yield.

This new procedure involved reacting the calix[4]arene with aluminium chloride to remove the upper rim $t$-butyl groups to create the tetrahydroxycalix[4]arene, which was then reacted with hydrobromic acid to attach the bromomethyl arm on the upper rim. Following on from this, attachment of the nitrogen donor ligands to the upper rim could now ensue stepwise. First, either the 2-(aminomethyl)-pyridine or the 2(aminoethyl)-pyridine group was attached and then the second nitrogen donor group was attached to create the upper rim metal ion binding site. The lower rim metal ion binding site was often a combination of two different groups on the lower rim, such as a nitrogen donor ligand (bipy, pyridyl or amide), and non-nitrogen donor ligand such as an ester or amide group.

An unexpected observation was made during the course of this project in that the ${ }^{1} \mathrm{H}$ NMR spectra became much more complex than what had been envisioned. Initially this was believed to be due to alternate conformers of the calix[4]arene molecule, 
owing to the increased flexibility with the loss of the $t$-butyl groups. Compound (37) was the first to undergo detailed investigation with NMR spectroscopy. High temperature NMR spectroscopy was performed but no difference in the ${ }^{1} \mathrm{H}$ NMR spectrum was observed even at $140{ }^{\circ} \mathrm{C}$. Serial dilution NMR spectroscopy studies were carried out and these results provided the first real insight into what was occurring. It was found that as the concentration becomes lower (milli- to micromolar) the spectrum becomes simpler, to what would be initially expected for the compound. From this it was concluded that at high concentrations (millimolar) the calix[4]arene molecules are able to interact with one another to form complex self-assembled structures. But as the solution becomes more dilute, interaction between the molecules becomes increasingly difficult, leading to reduced selfassembly and as a result, a simpler spectrum.

To further investigate this self-assembly nOe NMR spectroscopy studies were conducted. The main linking arms as well as some of the important peaks such as the pyridyl ortho doublet from the nitrogen atom, were chosen to undergo nOe NMR spectroscopy examinations. There are two ways in which the calix[4]arene molecules could be assembling, in a heterodimerisation form where the upper rim groups would interact with the lower rim groups of another molecule, or in the homodimerisation form where groups from the same side rim from two different molecules interact with one another. The results indicated that the self-assembly could be occurring in either the heterodimerisation or the homodimerisation form. It is likely that most of the compounds are in a mixture of the two forms, and it should be noted that the possibility of different conformations of the calix[4]arene may also be involved, in the self-assembled constructs.

Work was also conducted with the bipyridine moiety on the upper rim of the calix[4]arene. Initially the bipy group was to be attached to the upper rim which was already functionalised with the amine (2-aminomethylpyridyl) group, compound (30). However, when this was put into practice the complex was found to be very unstable and often decomposed rapidly to return starting materials. Given the size of both the pyridyl and bipy groups it was suggested that sterics may be the main factor 
causing the break-down of the complex. To this end the upper rim was then functionalised with 2-aminoethyl-pyridine to see if the extra carbon chain would create the space needed to allow a stronger attachment, compound (37). With the addition of this extra chain length the bipy functionalised calix[4]arene was found to be more stable, compound (38). From this it can be said that sterics are a crucial factor in the addition of the bipy unit to the upper rim to maintain a stable construct.

Metal ion complexation reactions were carried out with a range of metal salts, particularly the zinc(II) complexes, and in the majority of cases metal ion complexation was observed. As the majority of the ligands tested for metal ion binding ability maintained the dominant colour of the calix[4]arene derivative, no real colour changes were seen upon complexation, just a lightening or darkening of the solutions. It was hoped that metal ion complexation would help to control the level of self-assembly occurring in solution. 1:1 to 1:4 ligand to metal ratio metal ion complexation reactions were carried out on some of the complexes, but it was found that even with 1:4 ligand to metal ratio, the self-assembly still dominated. But what was clear, is that in the ${ }^{1} \mathrm{H}$ NMR spectroscopy, peaks were still occurring to indicate the unbound calix[4]arene self-assembled structure. This suggests that metal ion complexation is occurring either at the top, and possibly bottom of the chains, and that the self-assembly is occurring between the two. The growing of crystals was attempted in a wide range of solvents and a 1:4 ratio of DCM:MeOH was found to give the best results. Unfortunately, adequate crystals for X-ray analysis were not acquired, but the crystals were viewed under SEM. The crystals themselves were 'tree-like' branches growing up the side of the vial. The images produced from this showed that a range of different surface textures were seen, but all seemed to indicated a stacking structure, supporting the theory of self-assembly.

Fluorescence studies began in the latter part of the project and due to time constraints only a select number of compounds where chosen. The UV-Vis data showed that the organic ligands themselves produced spectra with a $\lambda_{\max }$ around $260 \mathrm{~nm}$. The metal complexes of these produced a much larger $\lambda_{\max }$ above $400 \mathrm{~nm}$. The fluorescence spectra of the parent organic ligands which were less functionalised (compounds $\mathbf{3 1}$ 
\& 32) produced spectra of low intensity $(<10)$ and were found in the red-region of the spectra. But the spectra of the more functionalised complexes (compounds 35 47) produced high intensity spectra $(>30)$ and were also in the red-region of the spectrum. This indicates that the more functionalised the compounds better fluorescence is seen most likely due to greater diversity of overlap in the molecules. In most cases it was found that, upon metal ion complexation, a large increase in intensity was observed as well as the spectra being blue-shifted to lower energy wavelengths. Serial dilution metal ion complexation studies were also performed with fluorescence spectroscopy and the results showed that as the concentration of the metal ion increased the intensity of the fluorescence band increased and was blue shifted. This indicates that the metal ion complexation is having a noticeable effect on the level of fluorescence, possibly by reducing the level of self-assembly and allowing a better overlap of orbitals in the molecule.

The calix[4]arenes discussed in this thesis showed the ability to potentially act as duel metal ion binding agents as well as an unusual ability to self-assemble in solution. Early attempts to directly attach the DPA unit to the upper rim of the calix[4]arene via the nitration route proved to be unsuccessful. This resulted in a new pathway being employed which involved the complete removal of the upper rim $t$ butyl groups followed by the attachment of nitrogen donor groups at the upper rim. The difficulties encountered in Chapter 3 out-line the problems and the unique property of self-assembly associated with the removal of the $t$-butyl groups. It was shown by NMR spectroscopy that temperature had no effect on the level of selfassembly but that the concentration of the solution had a noticeable effect, in that as the solution became more dilute (micromolar) the level of self-assembly decreased. The compounds were also seen to contain fluorescent properties upon metal complexation and this may have use in the detection of toxic metals in various systems. Future work could involve a re-visit to removing only two of the $t$-butyl groups, which would limit the flexibility within the calix[4]arene framework and may establish some control the self-assembly seen here. 
5 Bibliography

350 
(1) A. Baeyer, Ber., 1872, 5, 25.

(2) A. Baeyer, Ber., 1872, 5, 1094.

(3) N. J. L. Megson, Phenolic Resin Chemistry, Butterworths, London, 1958.

(4) L. H. Baekeland, US Patent, 1908, 942, 699.

(5) A. Ziegler and E. Zinke, Ber., 1944, 77, 264.

(6) A. Ziegler and E. Zinke, Ber., 1941, 74, 1729.

(7) B. Hunter and R. Hayes, Chem. Ind.(London, U.K.), 1956, 193.

(8) B. Hunter and R. Hayes, J. Appl. Chem., 1958, 8, 743.

(9) J. W. Cornforth, P. D’Arcy Hart, G.A. Nicholls, R. J. W. Rees and J. A. Stock, Br. J. Pharmacol., 1955, 10, 73.

(10) J. W. Cornforth, E. D. Morgan, K. T. Potts and R. J. W. Rees, Tetrahedron, 1973, 29, 1695.

(11) C.D. Gutsche, Calixarenes: Monographs in Supramolecular Chemistry, The Royal Society of Chemistry, London, 1989.

(12) A. Zinke, G. Zigeuner and K. Hösinger, Monatsh. Chem. 1948, 79, 438.

(13) N. R. I. Megson and R. Oesterr, Chem. Ztg., 1953, 54, 317.

(14) R. Oesterr and A. Zinke, Chem. Ztg., 1954, 55, 156.

(15) C. D. Gutsche, M. Iqbal and D. Stewart, J. Org. Chem., 1986, 51, 742.

(16) M. Iqbal and C. D. Gutsche, Org. Synth., 1990, 68, 4165.

(17) B. Dhawan, S. I. Chen and C. D. Gutsche, Makromol. Chem., 1987, 188, 921.

(18) C. D. Gutsche, B. Dhawan, K. H. No and R. Muthukrishnan, J. Am. Chem. Soc., 1981, 103, 3782.

(19) F. Brittner and K. Ullmann, Ber., 1909, 42, 2523.

(20) A. Zinke, R. Kretz, E. Leggewie and K. Hossinger, Monatsh. Chem., 1952, 83, 1213.

(21) D. J. Cram, S. Karbach, H. E. Kim, C. B. Knobler, E. F. Maverick, J. L. Ericson and R.C.Helgeson, J. Am. Chem. Soc., 1988, 110, 483.

(22) A. Wohl and B. Mylo, Ber., 1912, 45.

(23) K. Hultzsch, Ber., 1942, 75, 106.

(24) H. v. Euler, E. Adler, J. O. Cedwall and O. Törngren, Ark. Kemi. Mineral. Geol., 1941, 15.

(25) P. D. Gardner, H. Sarrafizadeh and R. I. Brandon, J. Am. Chem. Soc., 1959, 81, 5515.

(26) N. Caro, Ber., 1892, 25, 939. 
(27) L. M. Tunstad, J. A. Tucker, E. Dalcanale, J. Weiser, J. A. Bryant, J. C. Sherman, R. C. Helgeson, C. B. Knobler and D. J. Cram, J. Org. Chem., 1989, 54, 1305.

(28) F. Schneider, and H. J. Weinelt, J. Org. Chem., 1991, 56, 5527.

(29) S. R. Izatt, R. T. Hawkins, J. J. Christensen and R. M. Izatt, J. Am. Chem. Soc., 1985, 107, 63.

(30) G. Happel, B. Mathiasch and H. Kammerer, Makromol. Chem., 1975, 176, 3317.

(31) H. Kämmerer, G. Happel and F. Caesar, Makromol. Chem., 1972, 162, 179.

(32) V. Böhmer, P. Chhim and H. Kämmerer, Makromol. Chem., 1979, 180, 2503.

(33) V. Böhmer, L. Merkel and U. Kunz, J. Chem. Soc. Chem. Commun., 1987, 896, 1193. (34) J. deMendoza, P. M. Nieto, P. Prados and C. Sanchez, Tetrahedron, 1990, 46, 671.

(35) H. Tsue, M. Ohmori and K.I. Hirao, J. Org. Chem., 1998, 63, 4866.

(36) H. Tsue, K. Enyo and K.I. Hirao, Org. Lett., 2000, 2, 3071.

(37) D. R. Stewart and C. D. Gutsche, J. Am. Chem. Soc., 1999, 121, 4136.

(38) S. Högberg, S. Abrahamsson and B. Nilsson, Tetrahedron Lett., 1968, 1679.

(39) A. Andreetti, G. D. Ungaro and R. Pochini, J. Chem. Soc., Chem. Commun., 1979, 1005.

(40) G. D. Andreetti, G. Calestani, F. Ugozzoli, A. Arduini, E. Ghidini, A. Pochini and R. J. Ungaro, Incl. Phenom. and Molec. Recognit. Chem., 1987, $5,123$.

(41) C. D. Gutsche, A. E. Gutsche and A. I. Karaulov, J. Incl. Phenom., 1985, 3, 447.

(42) P. D. J. Grootenhuis, P. A. Kollman, I. C. Groenen, D. N. Reinhoudt, G. J. van Hummel, F. Andreetti and G.D. Ugozzoli, J. Am. Chem. Soc., 1990, 112, 4165 .

(43) A. Arduini, A. Pochini, S. Reverberi and R. Ungaro, J. Chem. Soc. Chem. Commun., 1984, 981.

(44) L. J. Bauer and C. D. Gutsche, Tetrahedron Lett., 1981, 22, 4763.

(45) C. Jaime, J. deMendoza, P. Prados, P. M. Nieto and C. Sanchez, J. Org. Chem. 1991, 56, 7269. 
(46) J. O. Magrans, J. deMendoza, M. Pons and P. Prado, J. Org. Chem,. 1997, 62, 4518.

(47) C. D. Gutsche, M. Iqbal, K. C. Nam, K. A. See and I. Alam, Pure Appl. Chem., 1988, 60, 483.

(48) J. M. Harrowfield, M. I. Ogden and A. H. White, Aust. J. Chem., 1991, 44, 1237.

(49) F. Andreetti and G. D. Ugozzoli, J. Incl. Phenom. and Molec. Recognit. Chem., 1992, 13, 337.

(50) V. Bocchi, D. Foina, A. Pochini and R. Ungaro, Tetrahedron, 1982, 38, 373.

(51) I. Flemming, D. Williams, Spectroscopic Methods In Orgainc Chemistry, McGraw-Hill, 2008; Vol. 6th.

(52) N. Singh, M. Kumar and G. Hundal, Tetrahedron, 2004, 60, 5393.

(53) K. Iwamoto, K. Araki and S. Shinkai, J. Org. Chem., 1991, 56, 4955.

(54) Y. Rudzevich, V. Rudzevich and V. Böhmer, Syn. lett, 2009, 1887.

(55) K. Iwamoto, K. Araki and S. Shinkai, Tetrahedron., 1991, 47, 4325.

(56) H. Erdtman, F. Haglid and R. Ryhage, Acta. Chem. Scand., 1964, 18, 1249.

(57) Y. Rudzevich, V. Rudzevich and V. Böhmer, Chem. Eur. J., 2010, 16, 4541.

(58) A.F. Holleman, E. Wiberg, Inorganic Chemistry, Academic Press, 2001.

(59) A. Casnati, G. Cavallo, P. Metrangolo, G. Resnati, F. Ugozzoli and R. Ungaro, Chem. Eur. J., 2009, 15, 7903.

(60) G. M. L. Consoli, G. Granata, R. L. Nigro, G. Malandrino, and C. Geraci, Langmuir, 2008, 24, 6194.

(61) Y. Gauthier and V. A. Langlais, Phys. Rev., 2005, 72, 8.

(62) O. Mogck, E.F. Paulus, V. Bohmer, I.Thondorfc and W. Vogt, Chem. Commun., 1996, 2533.

(63) V. G. Organo, A. V. Leontiev, V. Sgarlata, H. V. R. Dias, and D. M. Rudkevich, Angew. Chem. Int. Ed., 2005, 44, 3043.

(64) J. O. Parker, B. Farrell, K. A. Lahey, and G. N. Moe, Engl. J. Med., 1987, 316, 1635.

(65) G. Cirino, Digestive and Liver Disease, 2003, 35.

(66) J. E Albina and J. S. Reichner, Cancer Metastasis Rev., 1998, 17.

(67) R. Rathore, S. V. Lindeman, K. S. P. Rao, D. Sun, and J. K. Kochi, Angew. Chem. Int. Ed., 2000, 39, 2123. 
(68) B. Botta, I. D’Acquarica, G. Delle Monache, L. Nevola, D. Tullo, F. Ugozzoli and M. J. Pierini, J. Am. Chem. Soc., 2007, 129, 11202.

(69) D. T. Schühle, J.A. Peters and J.Schatz, Coord. Chem. Rev., 2011, 255, 2727.

(70) P. J. Cragg, S. Khayzuran and J. Iqbal, Dalton Trans., 2007, 26, 3951.

(71) S. Khayzuran, J. Iqbal, M. C. Allen, F. Fucassi and P. J. Cragg Chem. Comm., 2007, 3951.

(72) T. Jin, M. Kinjo, Y. Kobayashi, and H. Hirata, Faraday Trans., 1998, 94, 3135 .

(73) N. Maulucci, F. DeRiccardis, C. B. Botta, A. Casapullo, E. Cressina, M. Fregonese, P. Tecilla and I. Izzo, Chem Comm., 2005, 1354.

(74) A. Arduini, A. Credi, G. Faimani, C. Massera, A. Pochini, A. Secchi, M. Semeraro, S. Silvi, and F. Ugozzoli, Chem. Eur. J,. 2008, 14, 98.

(75) M. H. B. Gansey, A. S. DeHaan, E. S. Bos, W. Verboom and D. N. Reinhoudt, Bioconjug. Chem., 1999, 10, 613.

(76) E. M. Georgiev and D. M. Roundhill, Inorg. Chim. Acta., 1997, 258, 93.

(77) L. H. Bryant Jr., A. T. Yordanov, J. J. Linnoila, M. W. Brechbiel, and J. A. Frank, Angew. Chem. Int. Ed., 2000, 39, 1641.

(78) D. Schühle, Calix[4]arenes as Molecular Platforms in Magnetic Resonance Imaging, 2009.

(79) D. W. Sliwa and M. Deska, ARKIVOC, 2008, 87.

(80) F. Chimienti, M. Aouffen, A. Favier, and M. Seve, Curr. Drug Targets 4, $2003,323$.

(81) K. A. McCall, C. Huang and C. A. Fierke, J. Nut., 2000, 130, 1437.

(82) D. K. Perry, M. J. Smyth, H. R. Stennicke, G. S. Salvesen, P. Duriez and G. G.Poirier, J. Biol. Chem,. 1997, 507, 272.

(83) P. O. Brown, K. A. Udachin, G. D. Enrighta and J. A. Ripmeester, Chem. Comm., 2005, 4402.

(84) J. McGinley, V. McKee, J. M. D. Walsh, Inorg. Chim. Acta., 2011, 375, 57.

(85) I. Oueslati, R. Abidi, P. Thuéry, J. Vicens, J. Incl. Phenom. Mol. Recognit. Chem., 2003, 47, 173.

(86) K. Izutsu, Blackwell Scientific Publications (Boston, USA), 1990, Vol. 35.

(87) S. Bhattacharya, New Scientist,; www. newscientist. com: 2003.

(88) C. Exley, Trends in Biochemical Sciences, 2009, 34, 589-593. 
(89) Y. Zhou, J. Chen, Y. Shang, and C. Yong, J. Incl. Phenom. Macrocycl. Chem., 2012, 74, 343.

(90) A. D. Bond, B. S. Creaven, D. F. Donlon, T. L. Gernon, J. McGinley, and H. Toftlund, Eur. J. Inorg. Chem., 2007, 749.

(91) V. Balzani, G. Bergamini, F. Marchioni, P. Ceroni, Coord. Chem. Rev. 2006, 250, 1254.

(92) N. Niklas, F. W. Heinemann, F. Hampel, T. Clark, R. Alsfasser, Inorg. Chem. 2004, 43, 4663.

(93) S. Memon, G. Uysal and M. Yilmaz, Sep. Sci. and Technol., 2000, 35, 1247.

(94) J. Colleran, B. S. Creaven, D. F. Donlon, and J. McGinley, Dalton Trans., 2010, 39, 10928.

(95) R. Joseph, B. Ramanujam, A. Acharya, A. Khutia and C. P. Rao, J. Org. Chem. 2008, 73, 5745.

(96) R. Blaylock, Health and Nutrition Secrets that Can Save Your Life, Health Press NA Inc. Rev Ed. 2006.

(97) A. K. Sharma, A. Rajendra, R. L. Gayathri, Alum. Trans., 1999, 1.

(98) J. P. Celis, D. Drees, M. Z. Huq, P. Q. Wu, and M. De bonte, Surf. Coat. Technol., 1999, 113, 1.

(99) S. S. Tulsi, Trans. Inst. Met. Finish., 1996, 64, 73.

(100) V. Saxena, R. U. Rani and A. K. Sharma, Surf. \& Coat. Technol., 2006, 201, 855 .

(101) J. Sun, D. Meiliu and C. Yan, J. Coord. Chem., 2009, 62, 2337.

(102) A. Chakrabarti, H. M. Chawla, T. Francis, N. Pant and S. Upreti, Tetrahedron, 2006, 62, 1150.

(103) H. Kumagai, M. Hasegawa, S. Miyanari, Y. Sugawa, Y.Sato, T. Hori, S. Ueda, H. Kamiyama, and S. Miyano, Tetrahedron Lett., 1997, 38, 3971.

(104) H. Akdas, L. Bringel, E. Graf, M. Hosseini, J. Pansanel, A. D. Cian, and J. Fischer, Tetrahedron Lett., 1998, 39, 2311.

(105) R. T. F. Jukes, V. Adamo, F. Hartl, P. Belser, and L. DeCola, Coord. Chem. Rev., 2005, 249, 1327.

(106) M. Furue, K. Maruyama, Y. Kanematsu, T. Kushida, M. Kamachi, Coord. Chem. Rev., 1994, 132.

(107) A. D. Bond, B. S. Creaven, D. F. Donlon, T. L. Gernon, J. McGinley, and H. Toftlund Eur. J. Inorg. Chem., 2007, 749. 
(108) B. S. Creaven, D. F. Donlon, J. McGinley, Coord. Chem. Rev., 2009, 253, 893.

(109) F. Cucinotta, F. Carniato, A. Devaux, L. DeCola and L. Marchese, Chem. Eur. J., 2012, 15310.

(110) B. Valeur, Molecular fluorescence: Principles and Applications.,Wiley, 2001.

(111) S. Patra, R. Gunupuru, R. Lo, E. Suresh, and B. Ganguly, Parimal, New J. Chem., 2012, 36, 988.

(112) J. Lee, S. Kim, J. Jung and J. Kim, J. Org. Chem., 2005, 70, 1463.

(113) N. J. vanderVeen, S. Flink, M.A. Deij, R.J.M Egberink, F.C. vanVeggel and D. Reinhoudt, J. Am. Chem. Soc., 2000, 122, 4963.

(114) K. I. Srećko, P. Dubon, T. Weyhermuller, E. Bill and N. Metzler-Nolte, Inorg. Chem., 2005, 44, 5405.

(115) A. Thapper, A. Behrens, J. Fryxelius, M. H. Johansson, F. Prestopino, M. Czaun, D. Rehderb and E. Nordlandera, Dalton Trans., 2005, 3566.

(116) C. Incarvito, M. Lam, B. Rhatigan, A. L. Rheingold, C. Jin Qin, A. Bosnich and B. Gavrilova, J. Chem. Soc., Dalton Trans. 2001, 3478.

(117) D. C. Bebout, A. E. DeLanoy, and D. E. Ehmann, Inorg. Chem., 1997, 37, 2952.

(118) T. Pandiyan, M. Murali, M. Palaniandavar, Transition Metal Chemistry, $1995,20,440$.

(119) H. A. Jenkins, G. P. A. Yap and R. J. Puddephatt, Organometallics, 1997, 16, 1946.

(120) J. Cooper and P. D. Beer, Chem. Commun., 1998, 129, 10.

(121) A. Hazell, J. McGinley and C. J. McKenzie, Acta Cryst., 1997, 53, 723.

(122) C. P Whittle, J. Heterocyclic Chem., 1977, 14, 191.

(123) C. B. Spillane, N. C. Fletcher, S. M. Rountree, H. vandenBerg, S. Chanduloy, J. L. Morgan and F. R.Keene, J. Biol. Inorg. Chem., 2007, 12, 797.

(124) B. S. Creaven, T. L. Gernon, J. McGinley, A. Moore and H. Toftlund, Tetrahedron, 2006, 62, 9066.

(125) C. D. Gutsche, I. Alam, M. Iqbal, T. Mangiafico, K. C. Nam, J. Rogers and K. A. See, J. Incl. Phenom., 1989, 7, 61.

(126) E. M. Collins, M. A. Mckervey and S. J. Harris, J. Am. Chem. Soc., 1989, 2, 372. 
(127) R. K. Castellano, D. M. Rudkevich, and J. Rebek, Jr., J. Am. Chem. Soc., 1996, 118, 10002.

(128) J. B. Cooper, M. Drew and P. D. Beer, J. Chem. Soc., Dalton Trans., 2000, 2721.

(129) D. G. McCafferty, B. M. Bishop, C. G. Wall, S. G. Hughes, S. L. Mecklenberg, T. J. Meyer and B. W. Erickson, Tetrahedron, 1995, 51, 1093.

(130) A. T. Yordanov, N. J. Wolf, E. M. Georgiev, H. Fred Koch, O. M. Falana, D.Roundhill, Comments Inorg. Chem,. 1999, 20, 163.

(131) F. Sansone, M. Dudic, G. Donofrio, C. Rivetti, L. Baldini, A. Casnati, S. Cellai, and R. Ungaro, J. Am.Chem., 2006, 14528.

(132) F. Oueslati, I. Dumazet-Bonnamour and R. Lamartine, New J. Chem., 2003, 27, 644 .

(133) C.D. Gutsche and S. Kanamathareddy, J. Org. Chem. ,1996, 61, 2511.

(134) T. vanderBoom, G. Evmenenko, P. Dutta, and M. R. Wasielewski, Chem. Mater., 2003, 15, 4068.

(135) E. Pinkhassik, I. Stibor, A. Casnati, and R. Ungaro, J. Org. Chem., 1997, 62, 1946.

(136) P. R. A. Webber, M. G. B. Drew, R. Hibbert and P. D. Beer, Dalton Trans., 2004, 1127.

(137) C. D. Gutsche, D. E. Johnston and D. R. Stewart, J. Org. Chem., 1999, 64, 3747.

(138) C. D. Gutsche and L. J. Bauer, Tetrahedron Lett., 1981, 22, 4763.

(139) C. D. Gutsche, Accounts of Chemical Research, 1983, 16, 161.

(140) C. D. Gutsche, I. Alam, M. Iqbal, T. Mangiafico, K. C. Nam, J. Rogers and K. A. See, J. Incl. Phenom., 1989, 7, 61.

(141) A. Arduini, S. Fanni, G. Manfredi, A. Pochini, R. Ungaro, A. R. Sicuri, and F. Ugozzoli, J. Org. Chem. 1995, 60, 1448.

(142) Ü. Ocak, M. Ocak, X. Shen, A. H. Gorman, K. Surowiec and R. A. Bartsch, ARKIVOC, 2010, 8, 81.

(143) C. D. Gutsche, and L. J. Bauer, J. Am.Chem.Soc., 1985, 107, 6052.

(144) R. Dorta, R. Dorta, L. J. W. Shimon, and D. Milstein, Inorg. Chem., 2004, 43, 7180 .

(145) F. Broda, M. O. Vysotsky, V. Böhmer and I. Thondorf, Org. Biomol. Chem., 2006, 4, 2424. 
